Conceptual Models of the Climate 2002 Program of Study Bounds on Turbulent Transport

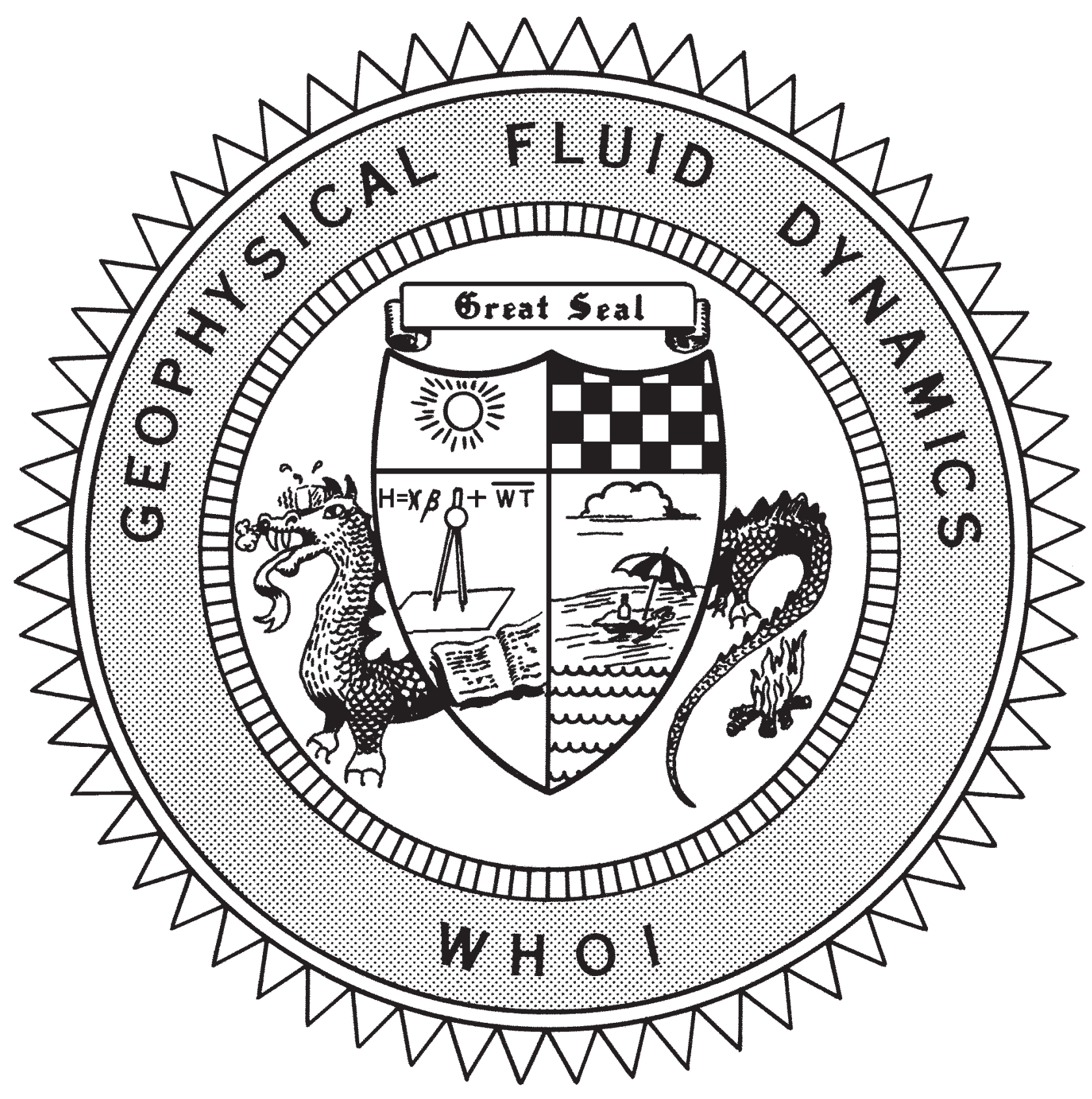

Course Lectures

Fellows Project Reports 


\section{Bounds on Turbulent Transport}

by
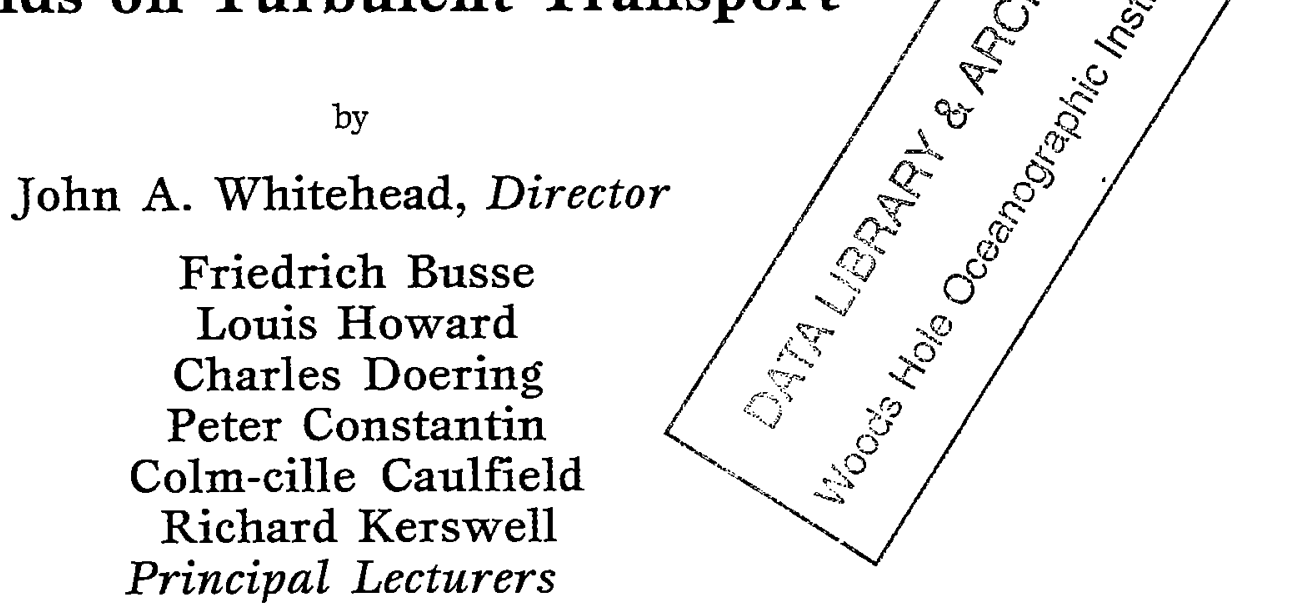

July 2003

\section{Technical Report}

Funding was provided by the Office of Naval Research under contract number N00014-97-1-0934 and the National Science Foundation under contract number OCE 98-10647.

Reproduction in whole or in part is permitted for any purpose of the United States Government. This report should be cited as Woods Hole Oceanog. Inst. Tech. Rept., WHOI-2003-06.

Approved for public release; distribution unlimited.
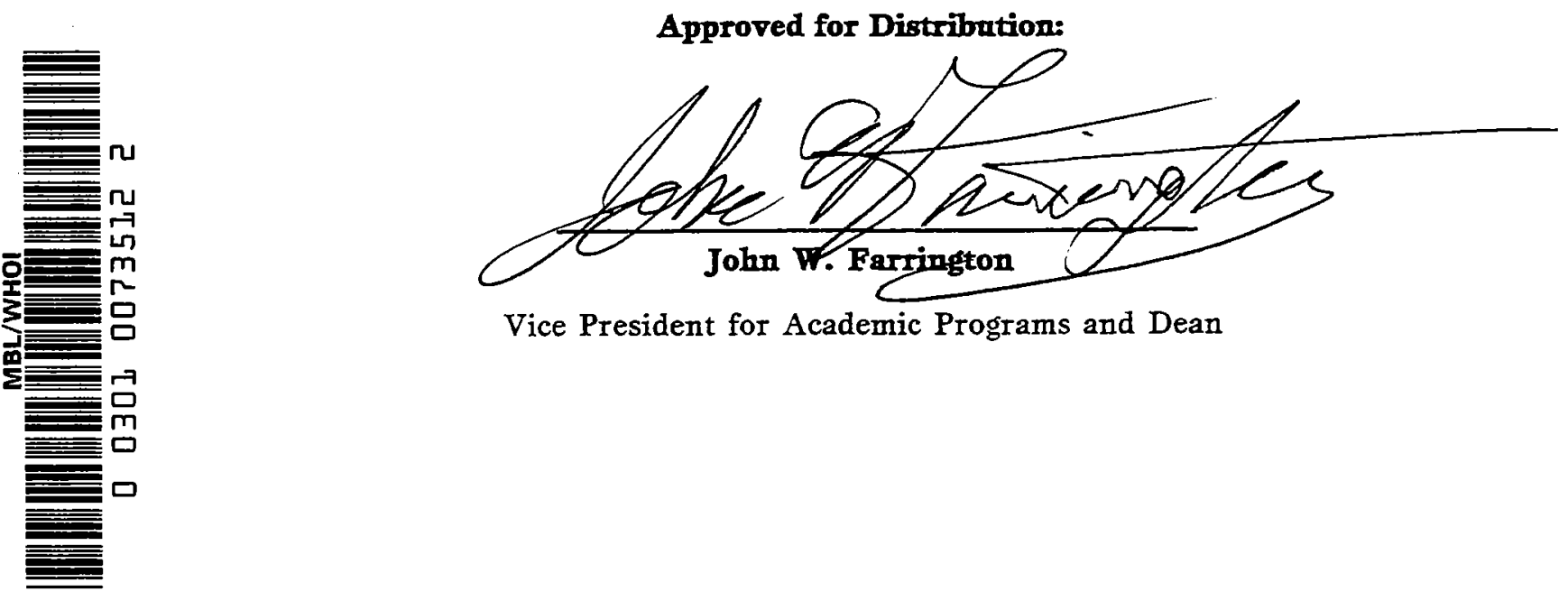

Vice President for Academic Programs and Dean 


\section{PREFACE}

The subject of "Bounds on Turbulent Transport" was introduced in a series of ten lectures. The six lecturers constitute almost all of the contributors to this subject. The subject was introduced and foundations were laid by five lectures by F. H. Busse. In the middle of the first week, L Howard reviewed his historical first approach to this subject and described more recent advances. Additional lectures by $\mathrm{P}$ Constantine, $\mathrm{R}$. Kerswell, C. Caulfield and C. Doering provided modern advances. We trust that the lecture notes will constitute a timely review of this promising subject.

The following weeks had many highlights with approximately 40 additional lectures. The mini symposium on rotating convection in early July included presentations of experimental, ocean, atmospheric, and planetary observations. During the rest of the program, participants and visitors who have studied turbulence, convection, and instability in numerous geophysical situations with application to the ocean, the earth's atmosphere and planetary circulation made numerous contributions.

I want to thank a number of participants. This year's fellows presented ten excellent lectures on their research projects. They clearly enjoyed-and contributed to-a fine and stimulating summer. George Veronis must be thanked for his enthusiastic coaching and to other contributions to this GFD program. Ed. Speigel worked the porch in his usual pedagogic fashion. Eric Chassignet and Glenn Flierl spent many selfless hours with the computers. Jean-Luc Thiffeault, Claudia Pasquero and Jeanne Fleming made important contributions to creating this year's volume. And above all, Janet Fields provided her usual fine hospitality to the visitors and staff, and assisted with many aspects of running a smooth program both during the summer and throughout the year.

Finally, I want to especially thank W.V.R. Malkus, who unselfishly urged boundless bounding efforts throughout the decades. Though seated during his address, he prodded us to raise our sights still higher.

Thank you all,

Jack Whitehead, Director ' 02 


\section{TABLE OF CONTENTS}

PREFACE.

i

CONTENTS

ii

II

PARTICIPANTS

. $\mathrm{v}$

III LECTURE SCHEDULE. vii

IV PRINCIPAL LECTURES - Bounds on Turbulent Transport

Presented by Friedrich Busse, University of Bayreuth, Germany; Louis Howard, Florida State University/Massachusetts Institute of Technology; Charles Doering, University of Michigan; Peter Constantin, University of Chicago; Colm-cille Caulfield, University of California, San Diego; Richard Kerswell, Bristol University, United Kingdom

Lecture One:

Hydrodynamic Stability

Friedrich Busse.

Lecture Two:

Phase Turbulence in Convection

Friedrich Busse.

Lecture Three:

The Sequence-of-Bifurcation Approach for the Transition to Turbulence

Friedrich Busse. .23

Lecture Four:

Simple Approaches to Some Bounding

Louis Howard

Lecture Five:

Upper Bounds for Turbulent Transport

Friedrich Busse.

Lecture Six:

Bounds on Turbulent Transport

Charles Doering.

Lecture Seven:

Multi-Alpha-Solutions

Friedrich Busse. 
Lecture Eight:

Bounds for Rotating Fluid

Peter Constantin.

Lecture Nine:

Bounds on Mixing in Stratified Shear Flows

Colm-cille Caulfield

Lecture Ten:

Unification of Variational Principles for Turbulent Shear Flows:

The Mean-Fluctuation Formulation of Howard-Busse and the

Background Method of Doering-Constantin

Richard Kerswell

FELLOW'S LECTURES

Report One:

Horizontal Convection

Jennifer H. Siggers, University of Cambridge, United Kingdom

Report Two:

Inertial Wave Convection in Rotating Spherical Fluid Shells

Radostin D. Simitev, University of Bayreuth, Germany

Report Three:

Upper Bounds for Convection in an Internally Heated Fluid Layer

$\mathrm{Lu} \mathrm{Lu}$, University of Michigan.

Report Four:

Ball Release Experiment in a Centrifuge

Ulrike Riemenschneider, University of Southampton, United Kingdom

Report Five:

Rearrangement of Annual Rings of High-Vorticity

Huiqun Wang, California Institute of Technology

Report Six:

Bounds on the Dissipation in MHD Problems in Plane Geometry with Magnetic and Velocity Shear

Francois Petrelis, University of Chicago .228 
Report Seven:

Bounds on the Energy Dissipation on the Magnetic and Couette and

Poiseuille (Hartmann) Shear Flow

Alexandros Alexakis, University of Chicago

Report Eight:

On Cyclic and Oscillatory Convection in a Simplified Box Model

with Entrainment

Tomoki Tozuka, University of Tokyo, Japan.....

Report Nine:

Bound on the Heat Transport through a Layer from the Boundary

Layer Theory Perspective: Fixed Heat Flux

Evstati G. Evstatiev, University of Texas, Austin.

Report Ten:

Upper Bounds on the Heat Transport in Infinite Prandtl

Number Convection

Stephen C. Plasting, University of Bristol, United Kingdom. 


\section{GFD FELLOWS, STAFF AND VISITORS}

\section{Fellows}

$\mathrm{Lu} \mathrm{Lu}$

Stephen C. Plasting

Huiqun Wang

Ulrike Riemenschneider

Jennifer H. Siggers

Radostin D. Simitev

Tomoki Tozuka

Alexandros Alexakis

Francois Petrelis

Evstati G. Evstatiev

\section{Staff and Visitors}

James L. Anderson

Helen C. Andersson

Neil J. Balmforth

Gregory Buck

John Bush

Friedrich H. Busse

Colm-cille P. Caulfield

Eric P. Chassignet

Stephen W. Childress

Peter Constantin

Charles Doering

Emmanuel Dormy

Robert Ecke

Glenn Flierl

Pascale Garaud

Chris Hallstrom

Louis N. Howard

Glenn Ierley

Takamitsu Ito

Daniel Joseph

Joseph B. Keller

Richard R. Kerswell

Zhiming Kuang

Norman R. Lebovitz

Sonya Legg

Jennifer A. MacKinnon

Liora Malki-Epshtein

Willem V.R. Malkus

John Marshall

Paul Matthews

Philip J. Morrison

Thomas Mullin
University of Michigan

University of Bristol

California Institute of Technology

University of Southampton

University of Cambridge

University of Bayreuth

University of Tokyo

University of Chicago

Ecole Normale Superieure de Paris

The University of Texas, Austin

Stevens Institute of Technology

Goteborg University

University of California, Santa Cruz

Saint Anselm College

Massachusetts Institute of Technology

University of Bayreuth

University of California, San Diego

University of Miami

New York University

University of Chicago

University of Michigan

Institut de Physique du Globe de Paris

Los Alamos National Lab

Massachusetts Institute of Technology

University of Cambridge

Brown University

Florida State Univ./Massachusetts Institute of Technology

University of California, San Diego

Massachusetts Institute of Technology

University of Minnesota

Stanford University

Bristol University

California Institute of Technology

University of Chicago

WHOI

Scripps

University of Cambridge

Massachusetts Institute of Technology

Massachusetts Institute of Technology

University of Nottingham

University of Texas at Austin

University of Manchester 
Jesse Otero

Francesco Paparella

Thomas Peacock

Michael Proctor

Claes G. Rooth

Wayne H. Schubert

Vitalii Sheremet

Alexander V. Soloviev

Andrew M. Soward

Edward A. Spiegel

Melvin Stern

Jean-Luc Thiffeault

George Veronis

Gosta Walin

Yuan-Nan Young

Jie $\mathrm{Yu}$
Ohio State University

University of Lecce

Massachusetts Institute of Technology

University of Cambridge

University of Miami

Colorado State University

WHOI

NOVA Southeastem University

University of Exeter

Columbia University

Florida State University

Columbia University

Yale University

Goteborg University

Stanford University

Duke University 


\section{GFD 2002 Lecture Schedule}

Week of June 17 - 21, 2002

(All talks held in Walsh cottage unless otherwise noted.)

Monday, June 17

10:00 am

Tuesday, June 18

10:00 am

Wednesday, June 19

10:00 am

2:30 pm

Thursday, June 20

10:00 am

2:00 pm

Friday, June 21

10:00 am

2:00 pm
Fritz Busse, University of Bayreuth, Germany

Introduction to Hydrodynamic Stability Theory

Fritz Busse

Applications of Energy Methods and Linear Theory

Fritz Busse

The Sequence-of-Bifurcations Approach for the Transition to

Turbulence

Louis Howard, Florida State University/M.I.T.

Simple Approaches to Some Bounding Problems

Fritz Busse

Upper Bounds for Turbulent Transports

Charles Doering, University of Michigan

Bounds on Turbulent Transport: Introduction to the

Background Method

Fritz Busse

Multi Alpha Solutions

Peter Constantin, University of Chicago

Bounds for Rotating Fluids

Week of June $24-28,2002$

Monday, June 24

10:00 am

2:00 pm
Colm-cille Caulfied, University of California, San Diego

Bounds on Mixing in Stratified Shear Flows

Richard Kerswell, University of Bristol, United Kingdom

Unification of Variational Approaches 
Tuesday, June 25

10:00 am

Karl Helfrich, Woods Hole Oceanographic Institution

Rotating Hydraulics and Upstream Basin Circulation

3:00 pm

Physical Oceanography Department Seminar

Location - Clark 507

Wednesday, June 26

10:00 am

Vitalii A. Sheremet, Woods Hole Oceanographic Institution Tilted Rotating Convection: Laboratory Experiments on a Centrifuge

2:00 pm

Willem V.R. Malkus, Massachusetts Institute of Technology Observed Turbulence Averages from Upper Bounds Constrained by Nonlinear and Time Dependent Stability Conditions

Thursday, June 27

10:00 am

NO LECTURE

2:00 pm

Charles Doering, University of Michigan

Variational Bounds on Energy Dissipation in

Body-Forced Flows

Friday, June 28

10:00 am

Fritz Busse

Patterns and Phase Turbulence of Centrifugally

Driven Convection

Week of July $1-5,2002$

Monday, July 1

10:00 am

Daniel Joseph, University of Minnesota

Slip Velocity and Lift

Tuesday, July 2

10:00 am

Pascale Garaud, University of Cambridge, United Kingdom Stellar and Solar Rotation

3:00 pm Physical Oceanography Department Seminar

Location - Clark 507

Wednesday, July 3

Thomas Mullin, University of Manchester, United Kingdom Experiments on Gluing Bifurcations 
Thursday, July 4

10:00 am

NO LECTURE - DUE TO INDEPENDENCE DAY HOLIDAY

Friday, July 5

10:00 am

NO LECTURE

2:00 pm Daniel Joseph, University of Minnesota

Viscous Potential Flows and Interfacial Stability Problems

Week of July 8 - 12, 2002 - Mini Symposium - Rotating

Monday, July 8

10:00 am

2:00 pm

Tuesday, July 9

10:00 am

$1: 00$ pm

Wednesday, July 10

10:00 am

$1: 00 \mathrm{pm}$

3:00 pm

Thursday, July 11

10:00 am

2:00 pm
Wayne Schubert, Colorado State University

The Role of Moist Convection in the Hadley Circulation

and in the Development of Hurricanes

Sonya Legg, Woods Hole Oceanographic Institution

Plumes in Rotating Turbulent Convection

Fritz Busse

Convection in the Rotating Cylindrical Annulus

Paul Matthews, University of Nottingham, United Kingdom

Instabilities of Rolls in Rotating Convection

Emmanuel Dormy, Institut de Physique du Globe de Paris, France and Andrew M. Soward, University of Exeter, United Kingdom

The Onset of Thermal Convection in Rotating Spherical Shells

Robert Ecke, Los Alamos National Laboratory

Rotating Convection Experiments: A Tour through Parameter

Space

Alexander Soloviev, NOVA Southeastern University

Sharp Frontal Interfaces in the Near-Surface Layer of the Ocean

Fritz Busse

Convection in Rotating Spherical Shells and the Dynamo Problem

Yuan-Nan Young, Northwestern University

Non-Boussinesq Rotating Convection at Low Prandtl Numbers:

Secondary Instability and Penta-Hepta Defect Chaos 
Friday, July 12

10:00 am

Micharl Proctor, University of Cambridge, United Kingdom

Pulsating Convection Cells at Large Taylor Number in Non-

Boussinesq Convection

2:00 pm

Jean-Luc Thiffeault, Columbia University

The Curvature of Material Lines in Chaotic and Turbulent Flows

Week of July 15-19,2002

Monday, July 15

10:00 am

NO LECTURE

Tuesday, July 16

10:00 am

NO LECTURE

Wednesday, July 17

10:00 am

Francesco Paparella, University of Leece, Italy

Horizontal Convection and the Anti-Turbulence Theorem

Thursday, July 18

10:00 am

Jack Whitehead, Woods Hole Oceanographic Institution

Laboratory Observations of Multiple Equilbrium, Oscillations, and Dynamic Stabilization of an Unstable Point

Friday, July 19

10:00 am

Takamitsu Ito, Massachusetts Institute of Technology

Entropy Production Extremal States for Atmosphere/Climate

System

Week of July 22 - 26, 2002

Monday, July 22

10:00 am

Tuesday, July 23

10:00 am

Stephen Childress, New York University Flapping Flight as a Bifurcation

Melvin Stern, Florida State University

Double Diffusive Intrusions in the Weddell Sea (Antarctica)

Wednesday, July 24

10:00 am

Baylor Fox-Kemper, MIT/WHOI Joint Program

Eddies and Friction: Removal of Vorticity in the Wind-Driven Gyre

Thursday, July 25

10:00 am

Norman Lebovitz, University of Chicago

The Fission Theorey of Binary Star Formation 
Friday, July 26

10:00 am

Chris Hallstrom, Brown University

Bounds for Rotating Infinite Prandtl Number Convection

Week of July 29- August 2, 2002

Monday, July 29

10:00 am

Allen C. Kuo, Massachusetts Institute of Technology

Residual Analysis and Eddy Flux Parameterization in a

Circumpolar Current

Tuesday, July 30

10:00 am

Glenn Ierley, University of California, San Diego

Bound to Improve

Wednesday, July 31

10:00 am

NO LECTURE

Thursday, August 1

10:00 am

Gösta Walin, University of Goteborg

Baroclinic Currents Losing Buoyancy or; What is Going on in

The Northern North Atlantic

Friday, August 2

10:00 am

NO LECTURE

Week of August 5 - 9, 2002

Monday, August 5

Jesse Otero, University of Michigan

TBA

Tuesday, August 6

10:00 am

Nick P. Fofonoff, Woods Hole Oceanographic Institution

Conversion of Gravitational Potential Energy for Mixing

Wednesday, August 7

10:00 am

Joseph B. Keller, Stanford University

Bounds for Slow Flow

2:00 pm

Glenn Flierl, Massachusetts Institute of Technology

Jovian Jets 
Thursday, August 8

10:00 am

2:30 pm

Friday, August 9

10:00 am
Edward A. Spiegel, Columbia University

Derivation of Pattern Equations

Louis Howard, Florida State University/M.I.T.

Video Presentation

Uri Lvov, Rennsalear Polytechnic Institute

Hamiltonian Formalism and the Garrett-Munk Specture of Internal Waves in the Ocean

Week of August 12 - 16, 2002

NO LECTURES

Week of August 19 - 23, 2002 - Fellows' Lectures

Monday, August 19

10:00 am

11:00 am

2:00 pm

Tuesday, August 20

10:00 am

11:00 am

Wednesday, August 21

10:00 am

11:00 am

2:00 pm
Jennifer Siggers, University of Cambridge, U.K.

Bounds for Horizontal Convection

Radostin Simitev, University of Bayreuth, Germany

Inertia Wave Convection in Rotating Spherical Fluid Shells

Lu Lu, University of Michigan

Upper Bounds for Convection in an Internally Heated Fluid Layer

Ulrike Riemenschneider, University of Southampton, U.K.

Ball Release Experiment in a Centrifuge

Huiqun Wang, California Institute of Technology

Rearrangement of Annual Rings of High Vorticity

Francois Petrelis, Ecole Normale Superieure de Paris, France

Bounds in MHD Turbulence I Tearing Models

Alexandros Alexakis, University of Chicagor

Bounds in MHD Turbulence II Magenetic Couette Flow and Hartmann Flow

Tomoki Tozuka, University of Tokyo, Japan

On the Cyclic and Oscillatory Convections in a Simplified Box Model with Entrainment 
Thursday, August 22

10:00 AM

Evstati Evstatiev, The University of Texas, Austin

Boundary Layer Theory for the Fixed Heat Flux Problem

11:00 AM

Stephen Plasting, University of Bristol, U.K.

Infinite Prandtl Number Convection: Bound to Disprovel

Friday, August 23

Fellows' Reports Due 


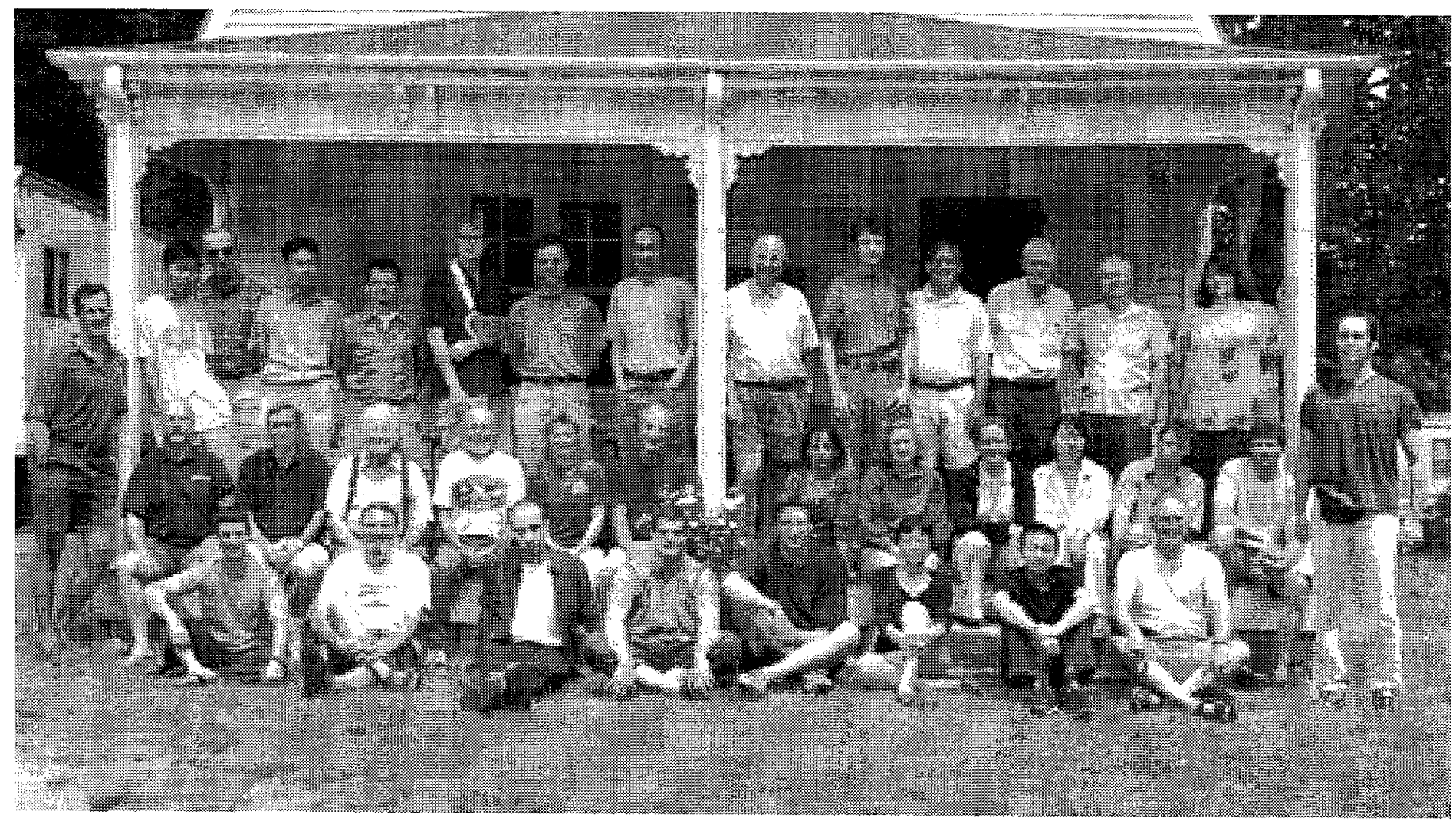

\section{Participants}

Standing (left to right): Richard R. Kerswell, Zhiming Kuang, Edward A. Spiegel, Tomoki Tozuka, JeanLuc Thiffeault, Chris Hallstrom, Colm-cille P. Caulfield, Yuan-Nan Young, Friedrich H. Busse, Vitalii Sheremet, Wayne H. Schubert, Louis N. Howard, Willem V.R. Malkus, Janet Fields, Radostin D. Simitev Seated on porch (left to right): Charles Doering, Peter Constantin, Claes G. Rooth, Joseph B. Keller, Helen C. Andersson, George Veronis, Pascale Garaud, Jennifer H. Siggers, Ulrike Riemenschneider, Huiqun Wang, Neil J. Balmforth, Eric P. Chassignet

Seated on ground (left to right): Evstati G. Evstatiev, Philip J. Morrison, Alexandros Alexakis, Francois Petrelis, Stephen C. Plasting, Jie Yu, Lu Lu, Jack Whitehead

Not Pictured: James L. Anderson, Gregory Buck, John Bush, Stephen W. Childress; Emmanuel Dormy, Robert Ecke, Glenn Flierl, Glenn Ierley, Takamitsu Ito, Daniel Joseph, Norman R. Lebovitz, Sonya Legg, Jennifer A. MacKinnon, John Marshall, Paul Matthews, Thomas Mullin, Jesse Otero, Francesco Paparella, Thomas Peacock, Michael Proctor, Alexander V. Soloviev, Andrew M. Soward, Melvin Stern, Gosta Walin 

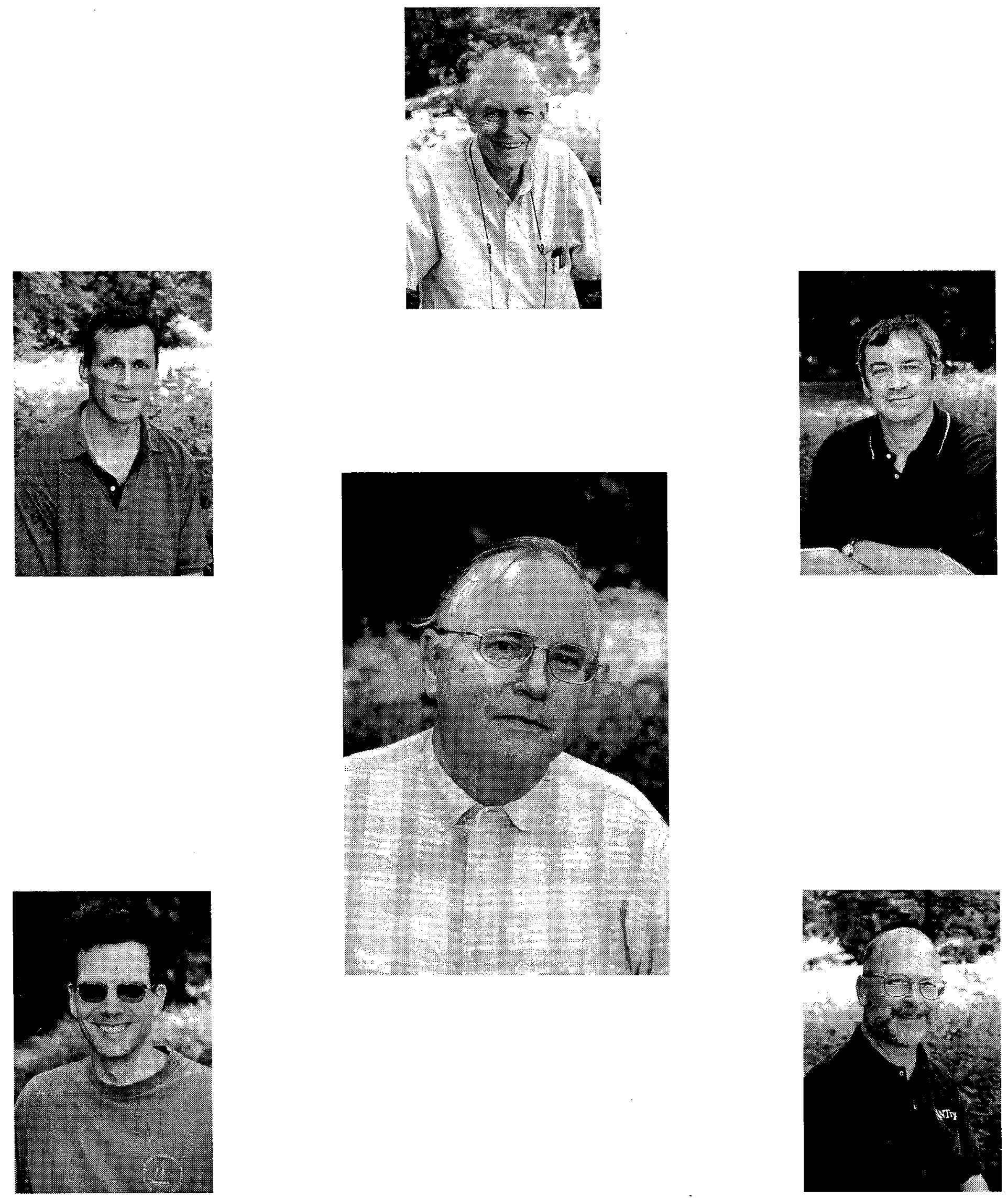

Clockwise: Louis Howard, Peter Constantin, Charles Doering, Colm-cille Caufield, Richard Kerswell Center: Friedrich Busse 


\section{Lecture 1}

\section{Hydrodynamic Stability}

F. H. Busse

Notes by A. Alexakis \& E. Evstatiev

\section{Introduction}

In many cases in nature, like in the Earth's atmosphere, in the interior of stars and planets, one sees the appearance of patterns that seem to be stable and persistent. Those patterns usually correspond to broken symmetries. The purpose of the following lectures is to explain the formation of these patterns and their persistence even under 'strong' turbulent conditions. This first lecture is dealing with onset of turbulence by determining the critical value of the control parameters that the laminar solution becomes unstable.

\section{Linear theory}

\subsection{States of Minimum Energy}

Continuous material systems are subject to conservation laws like mass, energy, momentum and angular momentum. The laws of thermodynamics also tell us that the mechanical energy has a tendency to be converted to thermal energy leading a system to a state of minimum mechanical energy, subject to other conservation laws. Systems that have reached this state are called equilibrium systems. As an example consider a rotational flow in a cylinder. To simplify the problem we consider that the flow has only a radial dependence e.g. $\omega=\omega(r)$. We can then ask the following question: Given an initial condition with angular momentum $\mathcal{A}$, what is the state of minimum mechanical energy our system can have keeping the angular momentum fixed? The kinetic energy $\mathcal{K}$ and the angular momentum can be expressed as functionals of the angular frequency as:

$$
\mathcal{K}[\omega]=\pi \int_{0}^{\tau_{0}}(\omega(r) r)^{2} r d r, \quad \mathcal{A}[\omega]=2 \pi \int_{0}^{r_{0}} \omega(r) r^{2} r d r=\mathcal{A}_{0} \quad \text { (fixed) }
$$

To minimize the energy keeping the angular momentum fixed we have to minimize the functional:

$$
\mathcal{F}[\omega, \lambda]=\mathcal{K}[\omega]+\lambda\left(\mathcal{A}[\omega]-\mathcal{A}_{0}\right)
$$

Where $\lambda$ is a Lagrange multiplier and $\mathcal{A}_{0}$ the angular momentum of the flow. Evaluating the variation of $\mathcal{F}$ with respect to $\omega$ and $\lambda$ we obtain

$$
\delta \mathcal{F}=\delta K+\delta(\lambda \mathcal{A})=\delta\left\{2 \pi \int_{0}^{r_{0}} \frac{1}{2} \omega^{2}(r) r^{3} d r+\lambda\left(2 \pi \int_{0}^{r_{0}} \omega(r) r^{3} d r-\mathcal{A}_{0}\right)\right\}=0
$$




$$
\delta \mathcal{F}=2 \pi\left\{\int_{0}^{r_{0}}\left(\omega(r) r^{3} d r+\lambda r^{3}\right) \delta \omega d r+\delta \lambda\left(2 \pi \int_{0}^{r_{0}} \omega(r) r^{3} d r-\mathcal{A}_{0}\right)\right\}=0
$$

In order for the variation to be equal to zero for every $\delta \omega$ and every $\delta \lambda$ we must have

$$
\omega(r) r^{3}+\lambda r^{3}=0 \quad \text { and } \quad \int_{0}^{r_{0}} \omega(r) r^{3} d r=\mathcal{A}_{0}
$$

which leads to

$$
\omega(r)=-\lambda=\text { constant }=\omega_{0} \quad \text { with } \quad \omega_{0}=\frac{2 \mathcal{A}_{0}}{\pi r_{0}^{4}}
$$

which is a rigid body rotation.

A similar example is if we consider a flow in a cylinder with the velocity being given by $\mathbf{u}=v(r) \mathbf{k}$ where $\mathbf{k}$ is the unit vector parallel to the axis of symmetry. The kinetic energy and the momentum are given by

$$
\mathcal{K}[v]=\pi \int_{0}^{r_{0}} v^{2}(r) r d r, \quad \mathcal{M}[v]=2 \pi \int_{0}^{r_{0}} v(r) r d r=\mathcal{M}_{0} \quad \text { (fixed) }
$$

To minimize the energy, keeping the momentum fixed, we define the functional

$$
\mathcal{F}[\omega]=\mathcal{K}[v]+\lambda\left(\mathcal{M}[v]-\mathcal{M}_{0}\right)
$$

Varying it we get

$$
\delta \mathcal{F}=\delta K+\delta(\lambda \mathcal{M})=\delta\left\{\pi \int_{0}^{r_{0}} v^{2}(r) r d r+\lambda\left(2 \pi \int_{0}^{r_{0}} v(r) r d r-\mathcal{M}_{0}\right)\right\}=0
$$

which leads to

$$
v(r)+\lambda=0 \text { and } 2 \pi \int_{0}^{r_{0}} v(r) r d r=\mathcal{M}_{0}
$$

Which again leads to the motion of a rigid body with velocity given by $\mathbf{u}=\mathcal{M}_{0} / \pi r_{0}^{2} \mathbf{k}$.

The fact that the above states are of minimum energy indicates that they are stable. Any other state with more energy will not be stationery or stable and will decay to the solutions of minimum energy.

As a further example we will examine the flow of a fluid between two infinite parallel plates separated by a distance $d$. A uniform pressure gradient along one of the parallel directions is assumed to keep the flow from being non-zero. The only control parameter of the problem is given by the Reynolds number $R e=U d / \nu$ where $\nu$ is the kinematic viscosity and $U$ is the averaged velocity. The above system has a steady solution given by $\mathbf{u}=\left(\operatorname{Re}\left(1 / 4-z^{2}\right), 0,0\right)$ (Poiseuille flow.). We want to examine for which values of the control parameter $R e$ the Poiseuille flow is stable. It is typical that in stability problems like the one described above four regimes of the flow parameter $R e$ can be distinguished, see Fig. 1. 


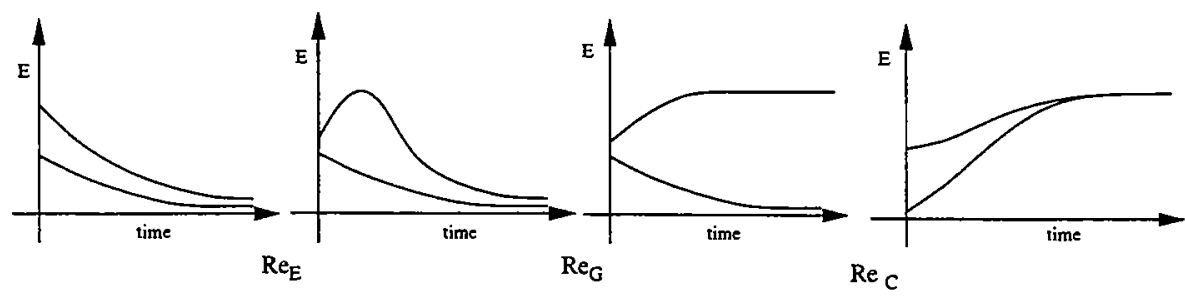

Figure 1: The behavior of perturbations on the laminar solution for different Reynolds numbers.

A) From 0 to $R e_{E}$ all disturbances decay exponentially.

B) From $R e_{E}$ to $R e_{G}$ some infinitesimal or finite disturbances might grow for finite time but all disturbances decay exponentially for $t \rightarrow \infty$

C) From $R e_{G}$ to $R e_{c}$ infinitesimal disturbances decay exponentially but finite disturbances converge to a new solution.

D) From $R e_{c}$ to $\infty$ infinitesimal disturbances grow exponentially.

The following paper is dedicated to estimating the values of $R e_{E}, R e_{c}$.

\subsection{The Energy Method}

We restrict ourselves to an incompressible fluid on a domain $\mathcal{D}$. The equations of motion are given by

$$
\begin{aligned}
\partial_{t} \mathbf{v}+\mathbf{v} \cdot \nabla \mathbf{v} & =-\nabla p+\mathbf{f}+\nu \nabla^{2} \mathbf{v} \\
\nabla \cdot \mathbf{v} & =0
\end{aligned}
$$

with boundary conditions on $\partial \mathcal{D}$

$$
\mathbf{v}=0
$$

or

$$
\mathbf{v} \cdot \mathbf{n}=0 \text { and } \mathbf{n} \times(\nabla \times(\mathbf{n} \times \mathbf{v}))=\mathbf{0}
$$

where $\mathbf{n}$ is the normal unit vector to $\partial \mathcal{D}$.

Denoting the stationary solution of maximum symmetry by $\mathbf{v}_{s}$ and writing the general solution as the stationary solution plus a perturbation $\mathbf{u}$

$$
\mathbf{v}=\mathbf{v}_{s}+\mathbf{u}
$$

The Navier Stokes equation for the velocity $\mathbf{u}$ becomes

$$
\begin{aligned}
\partial_{t} \mathbf{u}+\mathbf{v}_{s} \cdot \nabla \mathbf{u}+\mathbf{u} \cdot \nabla \mathbf{v}_{s} & =-\nabla \pi+\nu \nabla^{2} \mathbf{u} \\
\nabla \cdot \mathbf{u} & =0
\end{aligned}
$$


with the same boundary conditions as (13) or (14). Multiplying the above equation by $\mathbf{u}$ and taking the volume average we obtain

$$
\frac{1}{2} \frac{d}{d t}\langle\mathbf{u} \cdot \mathbf{u}\rangle=-\left\langle|\nabla \mathbf{u}|^{2}\right\rangle-\operatorname{Re}\left\langle\mathbf{u} \cdot(\mathbf{u} \cdot \nabla) \mathbf{v}_{s}\right\rangle
$$

where we have used the boundary conditions to eliminate the surface terms. From the above equation it is obvious that if $\operatorname{Re}\left\langle\mathbf{u}(\mathbf{u} \cdot \nabla) \mathbf{v}_{\mathbf{s}}\right\rangle \geq 0$ then $\frac{1}{2} \frac{d}{d t}\langle\mathbf{u} \cdot \mathbf{u}\rangle \leq 0$ and therefor all perturbations decrease in amplitude with time. On the other hand, if $\operatorname{Re}\left\langle\mathbf{u} \cdot(\mathbf{u} \cdot \nabla) \mathbf{v}_{s}\right\rangle<0$ we can define the functional

$$
\mathcal{R}_{E} \equiv \frac{\left\langle|\nabla \hat{\mathbf{u}}|^{2}\right\rangle-2\langle\pi \nabla \cdot \hat{\mathbf{u}}\rangle}{-\left\langle\hat{\mathbf{u}} \cdot(\hat{\mathbf{u}} \cdot \nabla) \mathbf{v}_{\boldsymbol{s}}\right\rangle}
$$

and look for its minimum.

Let

$$
I_{1}=\left\langle|\nabla \hat{\mathbf{u}}|^{2}\right\rangle, I_{2}=-\left\langle\hat{\mathbf{u}} \cdot(\hat{\mathbf{u}} \cdot \nabla) \mathbf{v}_{s}\right\rangle \text { and } I_{3}=-2\langle\pi \nabla \hat{\mathbf{u}}\rangle
$$

Then

$$
\delta \mathcal{R}_{E}=\frac{\delta I_{1}+\delta I_{3}}{I_{2}}-\frac{\left(I_{1}+I_{3}\right) \delta I_{2}}{I_{2}^{2}}=\frac{\delta I_{1}+\delta I_{3}}{I_{2}}-M \frac{\delta I_{2}}{I_{2}}=0
$$

where $M=\min \left\{\mathcal{R}_{E}(u)\right\}$. Expressing the variations $\delta I_{1}, \delta I_{2}, \delta I_{3}$ as we did in the previous paragraph, we obtain

$$
\frac{1}{2} M\left[\hat{u}_{j} \partial_{j} v_{s i}+\hat{u}_{j} \partial_{i} v_{s j}\right]=-\partial_{i} \pi+\partial_{j} \partial_{j} \hat{u}
$$

and

$$
\partial_{i} \hat{u}_{i}=0
$$

Now, since $M$ is the minimum of the functional $\mathcal{R}_{E}$ we have that for an arbitrary solution of (17) that

$$
M \leq \frac{\left\langle|\nabla \mathbf{u}|^{2}\right\rangle-2\langle\pi \nabla \mathbf{u}\rangle}{-\left\langle\mathbf{u} \cdot(\mathbf{u} \cdot \nabla) \mathbf{v}_{s}\right\rangle}
$$

using the energy equation (18) we have that

$$
\frac{1}{2} \frac{d}{d t}\langle\mathbf{u} \cdot \mathbf{u}\rangle \leq-(M-R e)\left\langle\mathbf{u} \cdot(\mathbf{u} \cdot \nabla) \mathbf{v}_{s}\right\rangle
$$

and since $\left\langle\mathbf{u} \cdot(\mathbf{u} \cdot \nabla) \mathbf{v}_{\mathbf{s}}\right\rangle \leq 0$ we have that the perturbation can grow only if $R e \geq M$. 


\subsection{Linear Stability of Plane Couette Flow}

As a special case illustrating the above general theory we take a flow between two parallel plates moving in opposite directions with relative velocity $\mathbf{U}_{D}$. The distance $d$ between the plates can be used to define Reynolds number $R e=\left|\mathbf{U}_{D}\right| d / \nu$ and the solution can be written in dimensionless form

$$
\mathbf{v}_{s}=-\operatorname{Re} z \mathbf{i}
$$

where we have introduced Cartesian coordinates and the unit vectors in the directions of $(x, y, z)$ are $(\mathbf{i}, \mathbf{j}, \mathbf{k})$, respectively. The velocity $\mathbf{U}_{D}$ is in the direction of $\mathbf{i}$. For the solution of Eqs. (22), (23) we introduce the general representation

$$
\tilde{\mathbf{u}}=\nabla \times(\nabla \varphi \times \mathbf{k})+\nabla \psi \times \mathbf{k}
$$

for a solenoidal vector field $\tilde{\mathbf{u}}$, where $\varphi$ and $\psi$ are some scalar functions. The $z$-components of curl and (curl) $)^{2}$ of Eq. (22) give

$$
\begin{gathered}
\nabla^{4} \Delta_{2} \varphi=\frac{1}{2} M\left(2 \partial_{x} \partial_{z} \Delta_{2} \varphi+\partial_{y} \Delta_{2} \psi\right) . \\
\nabla^{2} \Delta_{2} \psi=\frac{1}{2} M \partial_{y} \Delta_{2} \varphi
\end{gathered}
$$

where $\Delta_{2}=\partial_{x x}^{2}+\partial_{y y}^{2}$. The boundary conditions for this problem are

$$
\varphi=\partial_{z} \varphi=\psi=0 .
$$

If we only consider solutions independent of $x$, the function $\psi$ can be eliminated from Eqs. (28), (29) to give

$$
\left(\nabla^{6}-\frac{1}{4} M_{y}^{2} \partial_{y y}^{2}\right) \Delta_{2} \varphi=0 \quad \text { with } \quad \varphi=\partial_{z} \varphi=\nabla^{4} \varphi=0 \quad \text { at } \quad z= \pm \frac{1}{2} .
$$

Since this eigenvalue problem is similar to the problem of determining the critical Reynolds number in a fluid layer heated from below with rigid boundaries, we can use the latter fact to write

$$
\frac{1}{4} M_{y}^{4}=1708 \quad \text { corresponding to } \varphi=\cos (\alpha y) f(z) \quad \text { with } \quad \alpha_{c}=3.116
$$

where $\alpha_{c}$ is the lowest eigenvalue. It can be prooven that more general solutions $\varphi$ and $\psi$ that depend on $x$ and $y$ do not yield values of $M$ lower than $M_{y}$ [1]. Therefore finally we have $R e_{E}=2 \sqrt{1708} \approx 82.6$ for the plane Couette flow. The values for various non-rotating systems have been determined experimentally and theoretically, and comparison with the linear theory is given ${ }^{1}$ in Table 1.

\footnotetext{
${ }^{1}$ The maximum velocity and the channel width $d$ (radius $d$ in the case of pipe flow) have been used in the definition of Re.
} 


\begin{tabular}{|l|c|c|c|}
\hline & $R e_{E}$ & $R e_{G}$ (from exp.) & $R e_{c}$ \\
\hline Plane Couette Flow & 82.6 & $\approx 1300$ & $\infty$ \\
Poiseuille Flow (Channel Flow) & 99.2 & $\approx 2000$ & 5772 \\
Hagen-Poiseuille Flow (Pipe Flow) & 81.5 & $\approx 2100$ & $\infty$ \\
\hline
\end{tabular}

Table 1: Reynolds Numbers for Shear Flows in Non-Rotating Systems.

\subsection{Linear Stability of Circular Couette Flow}

Consider the flow between coaxial cylinders with radii $r_{1}$ and $r_{2}\left(>r_{1}\right)$ that rotate with angular velocity $\Omega_{1}$ and $\Omega_{2}$, respectively. The basic solution of Eq. (12) for the azimuthal velocity $v_{\varphi}$ is

$$
v_{\varphi}=\frac{r_{2}^{2} \Omega_{2}-r_{1}^{2} \Omega_{1}}{r_{2}^{2}-r_{1}^{2}} r-\frac{r_{1}^{2} r_{2}^{2}\left(\Omega_{2}-\Omega_{1}\right)}{\left(r_{2}^{2}-r_{1}^{2}\right) r}
$$

and is called the circular Couette flow. For simplicity we restrict our analysis to the case $r_{1}-r_{2} \ll r_{1}$ and $0<\Omega_{1}-\Omega_{2} \ll \Omega_{1}$. In this limiting case the solution (33) assumes the form of a plane Couette flow studied in the previous section, with angular velocity $\Omega_{D}=\frac{1}{2}\left(\Omega_{1}+\right.$ $\Omega_{2}$ ). The corresponding coordinate system is oriented so that the $x$-coordinate points in the azimuthal direction, the $y$-coordinate points in the axial direction, and the $z$-coordinate is pointed radially outward. The Reynolds number is defined by $R e=\left(\Omega_{1} r_{1}-\Omega_{2} r_{2}\right) d / \nu$.

Next we study infinitesimal disturbances therefore neglecting the nonlinear term $\tilde{\mathbf{u}} \cdot \nabla \tilde{\mathbf{u}}$ that enters Eq. (17), and add a Coriolis term

$$
\begin{gathered}
\frac{\partial}{\partial t} \tilde{\mathbf{u}}+\mathbf{v}_{s} \cdot \nabla \tilde{\mathbf{u}}+\tilde{\mathbf{u}} \cdot \nabla \mathbf{v}_{s}+2 \Omega \times \tilde{\mathbf{u}}=-\nabla \pi+\nabla^{2} \tilde{\mathbf{u}}, \\
\nabla \cdot \tilde{\mathbf{u}}=0
\end{gathered}
$$

where $\Omega=\Omega_{D} d^{2} / \nu$. Assuming time dependence of the form $\exp (\sigma t)$, boundary conditions $\tilde{\mathbf{u}}=0$ at $z= \pm \frac{1}{2}$ and a representation for $\tilde{\mathbf{u}}$ in the form

$$
\tilde{\mathbf{u}}=\nabla \times(\nabla \times \mathbf{k} \tilde{\varphi})+\nabla \times \mathbf{k} \tilde{\psi}
$$

we obtain the following eigenvalue problem

$$
\begin{gathered}
\nabla^{4} \Delta_{2} \tilde{\varphi}-2 \Omega \cdot \nabla \Delta_{2} \tilde{\psi}=\mathbf{v}_{s} \cdot \nabla \nabla^{2} \Delta_{2} \tilde{\varphi}+\sigma \nabla^{2} \Delta_{2} \tilde{\varphi}-\mathbf{v}_{s}^{\prime \prime} \cdot \nabla \Delta_{2} \tilde{\varphi} \\
\nabla^{2} \Delta_{2} \tilde{\psi}+2 \Omega \cdot \nabla \Delta_{2} \tilde{\varphi}=\mathbf{v}_{s} \cdot \nabla \nabla^{2} \Delta_{2} \tilde{\psi}+\sigma \Delta_{2} \tilde{\psi}+\mathbf{k} \cdot\left(\nabla \Delta_{2} \tilde{\varphi} \times \mathbf{v}_{s}^{\prime}\right) .
\end{gathered}
$$


Again, we are going to focus on disturbances which are $x$-independent and for which the imaginary part of $\sigma$ vanishes. In this case the critical disturbances correspond to $\sigma=0$ and Eqs. (37), (38) reduce to

$$
\begin{gathered}
\nabla^{4} \partial_{y y}^{2} \tilde{\varphi}-2 \Omega \partial_{y} \partial_{y y}^{2} \tilde{\psi}=0, \\
\nabla^{2} \partial_{y y}^{2} \tilde{\psi}-(R e-2 \Omega) \partial_{y} \partial_{y y}^{2} \tilde{\varphi}=0 .
\end{gathered}
$$

In the last formula we have used the expression (26) for $\mathbf{v}_{s}$. Then we observe that the above equations are identical with the ones without $x$-dependence, up to a numerical factor in the second term in Eq. (40). So, we can use the solution (32) to write

$$
R e_{y}=2 \Omega+\frac{1708}{2 \Omega}
$$

A calculation of the minimum of the above expression gives

$$
R e_{c}=2 \sqrt{1708} \text { corresponding to } 2 \Omega=\sqrt{1708} .
$$

It can be shown that the energy stability limit coincides with the result just obtained. Therefore, at this point the stability problem is solved completely because of the relation $R e_{E} \leq R e_{G} \leq R e_{c}$ which in this problem attains strict equalities. We see that for large values of $\Omega$ Eq. (41) that yields

$$
R e<2 \Omega
$$

as a condition for stability.

This also can be shown to follow from the Rayleigh stability criterion, $\frac{d\left(\omega(r) r^{2}\right)^{2}}{d r} \geq$ 0 , which describes the condition for stability of rotating inviscid fluid to axisymmetric disturbances. In our case assumes the form

$$
\left|\Omega_{1} r_{1}^{2}\right| \leq\left|\Omega_{2} r_{2}^{2}\right|
$$

Using the notation in Fig. 2 we can write (44) as

$$
\left(\Omega_{D}+\frac{\Omega_{1}-\Omega_{2}}{2}\right)\left(r_{0}-\frac{d}{2}\right)^{2} \leq\left(\Omega_{D}-\frac{\Omega_{1}-\Omega_{2}}{2}\right)\left(r_{0}+\frac{d}{2}\right)^{2} .
$$

After expanding and regrouping we obtain

$$
\frac{\Omega_{1}-\Omega_{2}}{2}\left(\left(r_{0}-\frac{d}{2}\right)^{2}+\left(r_{0}+\frac{d}{2}\right)^{2}\right) \leq 2 \Omega_{D} r_{0} d
$$




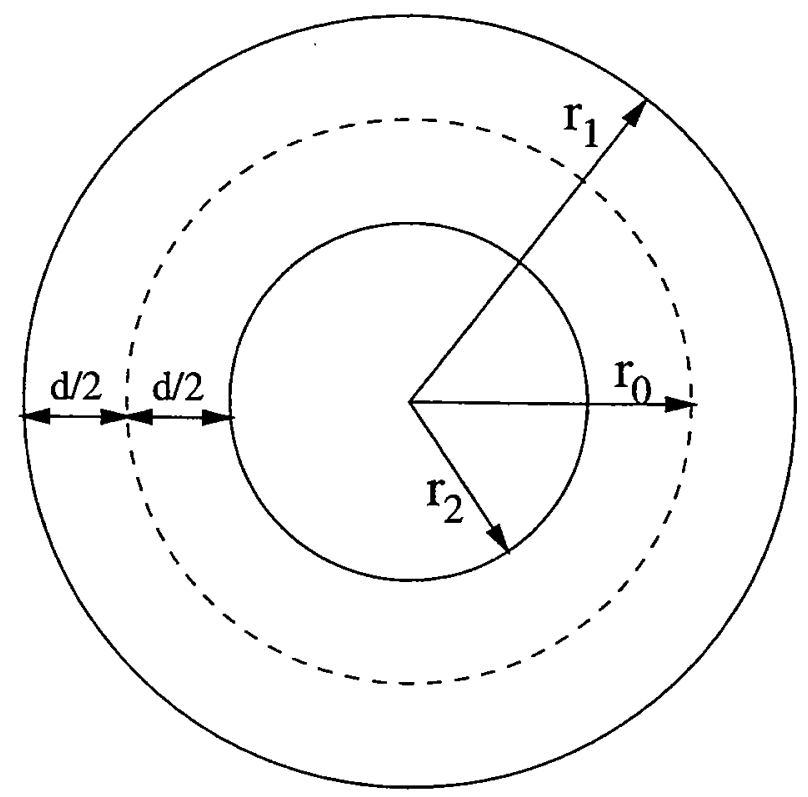

Figure 2: The asymptotic limit for large values of $\Omega$ leads to the inequality $R e<2 \Omega$ as a condition for stability.

Remembering the relation between $\Omega_{D}$ and $\Omega$ we finally obtain

$$
\operatorname{Re} \frac{\nu r_{0}}{d}<2 \Omega \frac{\nu r_{0}}{d}
$$

from which our assertion follows.

\section{References}

[1] Busse, F. H. A property of the energy stability limit for plane parallel shear flow. Arch. Rat. Mech. Anal. 47, pp. 28-35 (1972) 


\title{
Lecture 2
}

\section{Phase Turbulence in Convection}

\author{
F. H. Busse
}

Notes by by L. Lu and F. Pétrélis

\section{Description of the Rayleigh Bénard instability}

\subsection{Mechanism}

When a fluid layer is heated from below, a fluid particle at the bottom of the layer is hotter than the one above her. Consequently it is lighter and has a tendency to go up which is slowed down by the viscous force. This is the mechanism of the Rayleigh Bénard instability which can generate convection movements in a fluid heated from below. By comparing the power of the two forces involved in this mechanism, we can get an idea of a parameter which controls the instability development.

We first have to specify how the density $\rho$ of the fluid depends on its temperature. The simplest hypothesis is to use a first order approximation and to assume a linear dependency that yields

$$
\rho=\rho_{0}\left(1-\gamma\left(T-T_{0}\right)\right)
$$

where $\rho_{0}$ is the density at temperature $T_{0}$ and $\gamma$ is the expansion coefficient

$$
\gamma=\frac{1}{V} \frac{\partial V}{\partial T_{P}}=-\frac{1}{\rho} \frac{\partial \rho}{\partial T_{p}} .
$$

This is part of the Boussinesq approximation, pertinent for almost all common cases of Rayleigh Bénard instability.

We can estimate the buoyancy force $F_{b}$ per unit volume between the bottom of the layer at temperature $T_{2}$ and the top at temperature $T_{1}$ (with $T_{1}<T_{2}$ ). Using the height $d$ between the two surfaces of the layer (see fig 1 ), we get $F_{b}=\rho_{0} \gamma\left(T_{2}-T_{1}\right) g \frac{V d}{\kappa}$. The last coefficient $\frac{V d}{\kappa}$ where $V$ is the velocity of the fluid and $\kappa$ the thermal diffusivity takes into account the effect of the thermal diffusion on the distortion of constant density planes. We compute the power of this buoyancy force per unit volume $L_{p}$ by multiplying it with the velocity and we obtain

$$
L_{p}=c_{1} \rho_{0} \gamma\left(T_{2}-T_{1}\right) g d \frac{V^{2}}{\kappa}
$$

where $c_{1}$ is a numerical coefficient.

The power of the viscous force per unit volume is the product of the force per unit volume $\rho_{0} \nu \nabla^{2} V \simeq \rho_{0} \nu \frac{V}{d^{2}}$ with the velocity

$$
L_{d}=c_{2} \rho_{0} \nu \frac{V}{d^{2}} V
$$




\section{$\mathrm{T}_{1}$}

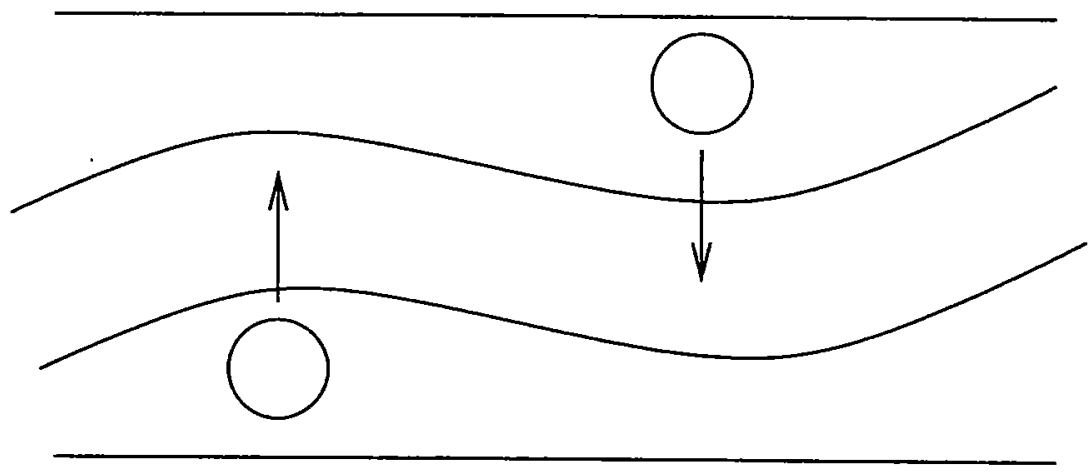

\section{$\mathrm{T}_{2}$}

Figure 1: Sketch of layer heated from below at temperature $T_{2}$ greater than the top surface temperature $T_{1}$.

$c_{2}$ is also a numerical coefficient. When the ratio between these two powers exceeds a critical value convection occurs. We define the Rayleigh number by

$$
R=\frac{L_{p} c_{2}}{L_{d} c_{1}}=\frac{\gamma\left(T_{2}-T_{1}\right) g d^{3}}{\nu \kappa}
$$

and its value at onset is the critical Rayleigh number $R_{c}=\frac{c_{2}}{c_{1}}$.

\subsection{Interesting aspects}

The study of convection phenomena concerns a very wide range of systems. Varying the Prandtl number (ratio between the kinetic viscosity and the thermal diffusivity) and other parameters (related to other effects such as magnetic field or rotation...) lots of situations can arise. Some of them are presented in figure 2.

A particularity of the usual convective instability is that the unstable mode is degenerate at onset. We will show in the following paragraph that the horizontal isotropy of the forcing exists and that the manifold of the unstable modes is characterized by wave vectors of fixed norm but free direction in the $x y$ plane. As we can see in figure 3 , this is really different from an instability generating a unique unstable mode and leads to some new behaviour on which we will now focus on.

\subsection{Onset of Instability}

The full set of equations describing the motion of a fluid is as follows: 


\section{Convection in the presence of (nearly) two-dimensional isotropy under steady external condition}

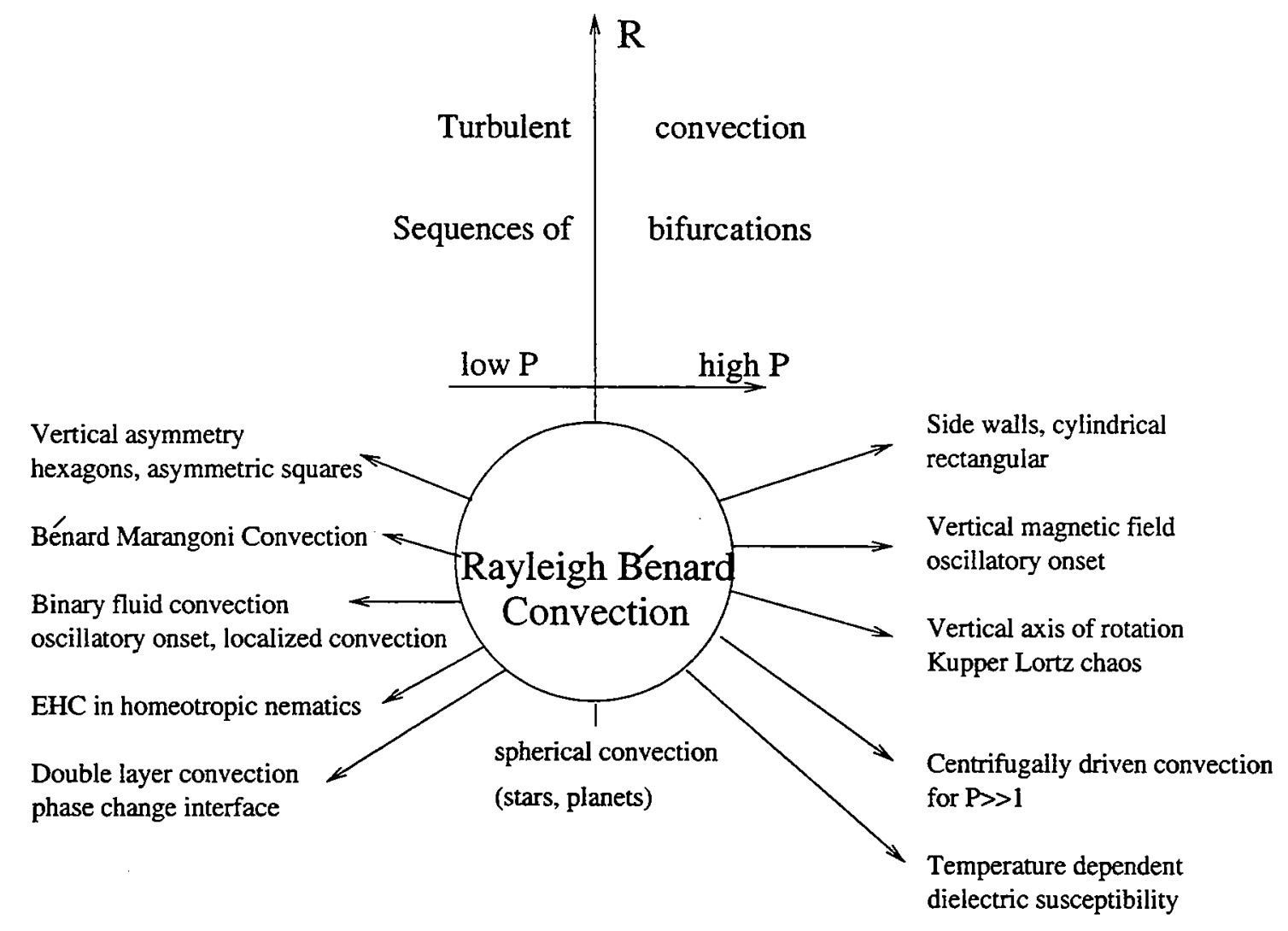

Figure 2: Some effects that occur in Rayleigh-Bénard convection. 

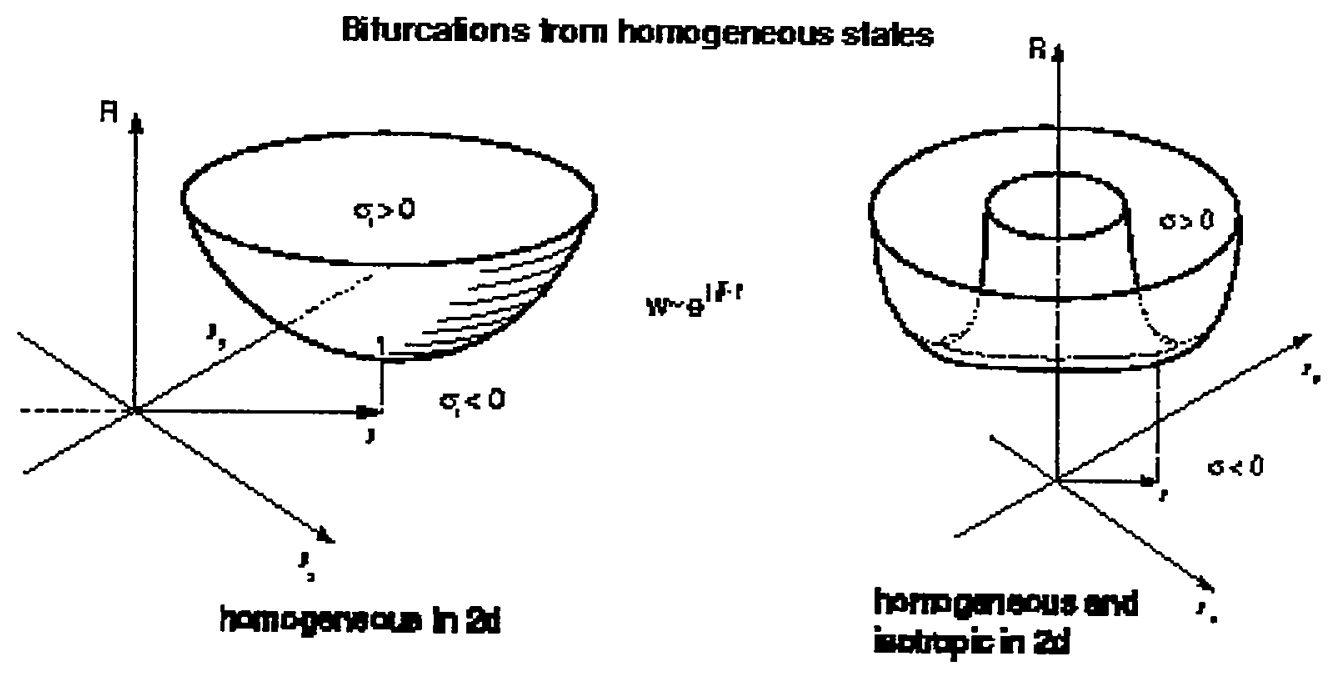

Figure 3: Critical Rayleigh number $R$ at onset of instability for a horizontal wave vector $\left(k_{x}, k_{y}\right)$ in a non-isotropic case and in an isotropic one.

$$
\begin{gathered}
\frac{\partial}{\partial t} \rho+\partial_{j} \rho u_{j}=0 \\
\rho \frac{d}{d t} u_{i}=\rho \frac{\partial}{\partial t} u_{i}+\rho u_{j} \partial_{j} u_{i}=-\partial_{i} p-\rho g k_{i}+\partial_{j}\left[\nu \rho\left(\partial_{i} u_{j}+\partial_{j} u_{i}-\frac{2}{3} \delta_{i j} \partial_{k} u_{k}\right)\right] \\
\rho T \frac{d s}{d t}=\rho c_{p} \frac{d T}{d t}+\frac{T}{\rho}\left(\frac{\partial \rho}{\partial T}\right)_{p} \frac{d p}{d t}=\partial_{j}\left(\lambda \partial_{j} T\right)+\Phi
\end{gathered}
$$

where $\Phi$ is mechanical dissipation and the density $\rho$ has a temperature dependence given by

$$
\rho=\rho_{0}\left(1-\gamma\left(T-T_{0}\right)\right), \quad T_{0}=\frac{T_{1}+T_{2}}{2} .
$$

A static solution exists for equation (6):

$$
\begin{gathered}
\mathbf{u}=0 \\
T_{s}=T_{0}-\frac{T_{2}-T_{1}}{d} z \\
p_{S}=p_{0}-\rho_{0}\left(z+\gamma \frac{T_{2}-T_{1}}{d} \frac{z^{2}}{2}\right) g
\end{gathered}
$$

Using $d$ as the length scale, $\frac{\kappa}{d^{2}}$ as time scale and $T_{2}-T_{1}$ temperature scale, we introduce dimensionless variables

$$
\begin{array}{rlrl}
\left(x^{\prime}, y^{\prime}, z^{\prime}\right) & =\frac{1}{d}(x, y, z), & u_{i}^{\prime}=\frac{d}{\kappa} u_{i} \\
t^{\prime} & =\frac{\kappa}{d^{2}} t, & T^{\prime} & =\frac{T}{T_{2}-T_{1}} .
\end{array}
$$


Assuming the mechanical dissipation $\Phi \approx 0$, and taking into account the temperature dependence of density only in the gravity term (Boussinesq approximation), we obtain from equations (6)

$$
\begin{gathered}
\nabla \cdot \mathbf{u}=0 \\
P^{-1}\left(\frac{\partial}{\partial t}+\mathbf{u} \cdot \nabla\right)=-\nabla \pi+R \Theta \hat{\mathbf{k}}+\nabla^{2} \mathbf{u} \\
(\partial t+\mathbf{u} \cdot \nabla) \Theta=\hat{\mathbf{k}} \cdot \mathbf{u}+\nabla^{2} \Theta
\end{gathered}
$$

where primes have been dropped from the dimensionless variables and $\Theta=T^{\prime}-T_{S}^{\prime}, \pi=$ $p^{\prime}-p_{S}^{\prime}$. Although there are seven dimensional parameters $\left(\rho_{0}, g, \gamma, \Delta T, \nu, \kappa\right)$ that can be measured with four dimensions (namely $m, s, k g, K$ ), the mean density $\rho_{0}$ does not appear in the equations in the Boussinesq approximation so that only two dimensionless parameters are relevant for this problem. A first one is the Rayleigh number $R=\frac{\gamma g\left(T_{2}-T_{1}\right) d^{3}}{\nu \kappa}$. A second dimensionless number $P=\frac{\nu}{\kappa}$ is the Prandtl number. For small amplitude steady convection, we have

$$
\begin{gathered}
\nabla \cdot \mathbf{u}=0 \\
\nabla^{2} \mathbf{u}+R \theta \hat{\mathbf{k}}-\nabla \pi=0 \\
\nabla^{2} \Theta+\mathbf{u} \cdot \hat{\mathbf{k}}=0
\end{gathered}
$$

Operation with $-\hat{\mathbf{k}} \cdot \nabla \times \nabla \times$ on (8b) yields

$$
\nabla^{4} u_{z}+R \Delta_{2} \Theta=0
$$

where

$$
\Delta_{2} f=\left(\frac{\partial^{2}}{\partial x^{2}}+\frac{\partial^{2}}{\partial y^{2}}\right) f
$$

Take $\Delta_{2}$ on equation $8 \mathrm{c}$, and eliminate $\Theta$ from equation 9 , we get a single equation of $u_{z}$ :

$$
\left(\nabla^{6}-R \Delta_{2}\right) u_{z}=0
$$

With the separation ansatz

$$
\Delta_{2} u_{z}=\left(\frac{\partial^{2}}{\partial x^{2}}+\frac{\partial^{2}}{\partial y^{2}}\right) u_{z}=-a^{2} u_{z}
$$

equation (11) becomes

$$
\left(\nabla^{6}+R a^{2}\right) u_{z}=0
$$

For stress-free boundaries, we have

$$
\begin{gathered}
\left.u_{z}\right|_{z= \pm \frac{1}{2}}=0 \\
\left.\partial_{z} u_{x}\right|_{z= \pm \frac{1}{2}}=\left.\partial_{z} u_{y}\right|_{z= \pm \frac{1}{2}}=0 \\
\left.\Theta\right|_{z= \pm \frac{1}{2}}=0
\end{gathered}
$$


Use the continuity equation $\partial_{x} u_{x}+\partial_{y} u_{y}+\partial_{z} u_{z}=0$, we have

$$
\partial_{x} \partial_{z} u_{x}+\partial_{y} \partial_{z} u_{y}+\partial_{z z}^{2} u_{z}=\partial_{z z}^{2} u_{z}=0
$$

at two boundaries. Also, from equation (9)

$$
\left.\left(\nabla^{4} u_{z}+R \Delta_{2} \Theta\right)\right|_{z= \pm \frac{1}{2}}=\partial_{z z z z}^{4} u_{z}=0
$$

In summary, the stress-free boundary conditions are:

$$
\begin{gathered}
u_{z}=0 \\
\partial_{z z}^{2} u_{z}=0, \\
\partial_{z z z z}^{4} u_{z}=0,
\end{gathered}
$$

at $z= \pm \frac{1}{2}$. With these boundary conditions, equation (11) has solutions:

$$
u_{z}=\cos a x \sin n \pi\left(z+\frac{1}{2}\right), \quad n=1,2,3, \ldots
$$

and

$$
R=\frac{\left(n^{2} \pi^{2}+a^{2}\right)^{3}}{a^{2}}, \quad n=1,2,3, \ldots
$$

$a$ is the wavenumber of the unstable mode. The critical Rayleigh number $R_{c}$ is the minimum of $R$, and is given when $n=1$ and $a=\frac{\pi}{\sqrt{2}}$. Hence $R_{c}=\frac{27}{4} \pi^{4}$. Note that $R_{c}$ is independent of the Prandtl number.

A single mode describes a roll of convection as sketched in figure 4 . Because of the degeneracy of the unstable modes, complex behaviour can occur even close to the onset of instability and we will study it through a weakly non-linear analysis.

\section{Weakly Non-linear Analysis}

\subsection{Perturbative Expansion}

To illustrate the ideas of weakly nonlinear analysis, consider the following one dimensional example:

$$
u^{\prime \prime}(z)+R u(z)+u^{\prime}(z) u(z)=0 \quad \text { with } \quad u=0 \text { at } z= \pm \frac{1}{2} .
$$

We make the ansatz

$$
\begin{aligned}
& u=\epsilon u_{1}+\epsilon^{2} u_{2}+\epsilon^{3} u_{3}+, \\
& R=R_{0}+\epsilon R_{1}+\epsilon^{2} R_{2}+\ldots,
\end{aligned}
$$

and the normalization condition

$$
\epsilon \equiv<u_{1}, u>=\epsilon<u_{1}, u_{1}>+\epsilon^{2}<u_{1}, u_{2}>+\ldots,
$$


which is equivalent to

$$
<u_{1}, u_{n}>=\delta_{1 n} \quad n=1,2,3, \ldots
$$

Insert these ansatz into the original equations and collect terms according to the powers of $\epsilon$. To the lowest order $O(\epsilon)$ we have

$$
\begin{gathered}
u_{1}^{\prime \prime}+R_{0} u_{1}=0 \\
\left.u_{1}\right|_{z= \pm \frac{1}{2}}=0
\end{gathered}
$$

The solutions are

$$
u_{1}=\sqrt{2} \sin n \pi\left(z+\frac{1}{2}\right), \quad \text { with } R_{0}=n^{2} \pi^{2}, n=1,2,3, \ldots
$$

To continue the process, and for simplicity, we choose $n=1$ here. Thus

$$
u_{1}=\sqrt{2} \sin \pi\left(z+\frac{1}{2}\right) \text {. }
$$

To the order $O\left(\epsilon^{2}\right)$,

$$
u_{2}^{\prime \prime}+R_{0} u_{2}=-u_{1}^{\prime} u_{1}-R_{1} u_{1}
$$

Multiply both sides with $u_{1}$, integrate over the interval $-\frac{1}{2} \leq z \leq \frac{1}{2}$, we have on the left hand side:

$$
<u_{1}\left(u_{2}^{\prime \prime}+R_{0} u_{2}\right)>=<u_{2}\left(u_{1}^{\prime \prime}+R_{0} u_{1}\right)>=0
$$

where integration by parts has been utilized. This condition yields

$$
<-u_{1}\left(u_{1}^{\prime} u_{1}+R_{1} u_{1}\right)>=0 \Rightarrow R_{1}=-\frac{<\left(u_{1}^{3}\right)^{\prime}>}{3}=0
$$

which is the solvability condition. Now the equation of $u_{2}$ becomes

$$
u_{2}^{\prime \prime}+R_{0} u_{2}=-2 \pi \sin \pi\left(z+\frac{1}{2}\right) \cos \pi\left(z+\frac{1}{2}\right) \quad \text { with }\left.\quad u_{2}\right|_{z= \pm \frac{1}{2}}=0
$$

The solution is

$$
u_{2}=\frac{\pi \sin 2 \pi\left(z+\frac{1}{2}\right)}{4 \pi^{2}-R_{0}}=\frac{\sin 2 \pi\left(z+\frac{1}{2}\right)}{3 \pi}
$$

To the third order of $\epsilon$,

$$
u_{3}^{\prime \prime}+R_{0} u_{3}=-u_{1}^{\prime} u_{2}-u_{2}^{\prime} u_{1}-R_{2} u_{1} \quad \text { with }\left.\quad u_{3}\right|_{z= \pm \frac{1}{2}}=0
$$


Apply the solvability condition, we have

$$
\begin{aligned}
R_{2} & =-<u_{1}\left(u_{1}^{\prime} u_{2}+u_{2}^{\prime} u_{1}\right)> \\
& =-<\frac{1}{2} u_{2}\left(u_{1}^{2}\right)^{\prime}+u_{2}^{\prime}\left(u_{1}^{2}\right)> \\
& =<\frac{1}{2} u_{2}\left(u_{1}^{2}\right)^{\prime}> \\
& =\frac{1}{4}\left\langle\frac{\pi^{2} \sin 2 \pi\left(z+\frac{1}{2}\right) \sin 2 \pi\left(z+\frac{1}{2}\right)}{3 \pi^{2}}\right\rangle \\
& =\frac{1}{24}
\end{aligned}
$$

In this simple example we are able to calculate the amplitude of the mode at saturation as a function of the departure from onset. At order $\epsilon^{2}$ we get $\epsilon^{2} R_{2}=R-R_{c}$, so that

$$
u \approx \epsilon u_{1}=4 \sqrt{3} \sqrt{R-R_{c}} \sin \pi\left(z+\frac{1}{2}\right)
$$

In the case of convection close to onset of instability, the degeneracy of the unstable mode may lead to non trivial behaviour because many modes can interact. We will use a general formalism based on a weakly non-linear analysis similar to the simple one before. We write the Navier-Stokes equations and the temperature equations in the form

$$
(\underline{\mathbf{W}}+R \underline{\mathbf{U}}) \tilde{\mathbf{X}}=\frac{\partial}{\partial t} \underline{\mathbf{V}} \tilde{\mathbf{X}}+\mathbf{Q}(\tilde{\mathbf{X}}, \tilde{\mathbf{X}})
$$

where $\tilde{\mathbf{X}}=\left(\begin{array}{c}u_{z} \\ \theta\end{array}\right)$ is a vector, $\underline{\mathbf{W}}, \underline{\mathbf{U}}$ and $\underline{\mathbf{V}}$ are linear operators, $R$ is the control parameter and $\mathbf{Q}$ the non linear term.

We expand $\tilde{\mathrm{X}}$ and $R$ in the form

$$
\begin{aligned}
& \tilde{\mathbf{X}}=\epsilon \tilde{\mathbf{X}}_{1}+\epsilon^{2} \tilde{\mathbf{X}}_{2}+\ldots, \\
& R=R_{0}+\epsilon R_{1}+\epsilon^{2} R_{2}+\ldots,
\end{aligned}
$$

where $\epsilon$ is a small parameter and the other terms are of order one. We will now describe two aspects of the instability slightly above onset. We first focus on the steady pattern and then we study the dynamic interaction between the different modes that can lead to a kind of turbulence named phase turbulence.

\subsection{Pattern selection}

We write equation (21) at order $\epsilon$ and we recover the linear problem of steady convection. The solution is a linear combination of modes described by equation (17) with wave vectors of norm $\alpha$. Thus, we write

$$
\tilde{\mathbf{X}}_{1}=\tilde{\mathbf{f}}(z) \sum_{n=-N}^{n=N} c_{n} e^{i \vec{k}_{n} \cdot \vec{r}}
$$


where $\vec{k}_{n}$ lies in the $x y$ plane and is of modulus $\alpha$. In order to have real solutions, we impose the two other relations $\vec{k}_{-n}=-\vec{k}_{n}$ and $c_{-n}=c_{n}^{*}$.

At order $\epsilon^{2}$ we get

$$
\left(\underline{\mathbf{W}}+R_{0} \underline{\mathbf{U}}\right) \tilde{\mathbf{X}}_{2}=\mathbf{Q}\left(\tilde{\mathbf{X}}_{1}, \tilde{\mathbf{X}}_{1}\right)-R_{0} \underline{\mathbf{U}} \tilde{\mathbf{X}}_{1}
$$

Then we apply the solvability condition $\left\langle\tilde{\mathbf{X}}_{i}^{*}\right|$ right hand side $\rangle=0$ where $\tilde{\mathbf{X}}_{i}^{*}$ is part of the kernel of the adjoint homogeneous linear operator. If the properties of the layer are symmetric with respect to the $z=0$ plane, we obtain $R_{1}=0$ because $\mathbf{Q}$ is antisymmetric in $z$ while $\tilde{\mathbf{f}}(z)$ and $\tilde{\mathbf{f}}^{*}(z)$ are symmetric. The solution of equation 24 can be written in the form

$$
\tilde{\mathbf{X}}_{2}=\sum_{i, k} \tilde{\mathbf{F}}\left(\vec{k}_{i}+\vec{k}_{k}, z\right) c_{i} c_{k} e^{i\left(\vec{k}_{i}+\vec{k}_{k}\right) \cdot \vec{r}}
$$

We write the equation (21) at order $\epsilon^{3}$ and use the solvability condition. In the most general case, we get an expression of the form

$$
\begin{aligned}
\left(\epsilon R_{1}+\epsilon^{2} R_{2}+\ldots\right) U c_{i}^{*}= & -\beta \epsilon \sum_{n, m} c_{n} c_{m} \delta\left(\vec{k}_{i}+\vec{k}_{n}+\vec{k}_{m}\right) \\
& +\epsilon^{2}\left(\sum_{n=1}^{N} c_{n} c_{n}^{*} A\left(\vec{k}_{i} \cdot \vec{k}_{n}\right)+A_{0}\left|c_{i}\right|^{2}\right) c_{i}^{*}+\ldots \text { for } \mathrm{i}=1,2,3 \ldots
\end{aligned}
$$

Here $\beta$ is an asymmetry coefficient which appears for instance if we consider non Boussinesq effects and write the temperature dependence of the density as $\rho=\rho_{0}\left(1-\alpha\left(T-T_{0}\right)+\beta\left(T-T_{0}\right)^{2}\right)$.

If $\beta=0$, we get $R_{1}=0$ and we recover the symmetric case result.

Looking for regular solutions for which the angle between the $N$ neighbouring q vectors is given by $\pi / N$ and $\left|c_{i}\right|^{2}=1$, we obtain rolls solutions if $N=1$, squares if $N=2$. If $N=3$ many solutions exists depending of the relative phases of the three coefficients $c_{i}$. In figure 4 , these patterns of convection are sketched.

If $N \geq 4$ there are no regular periodic patterns but quasi patterns of higher order can be observed. Depending on the value of $\beta$ and $R$, rolls or hexagons are stable. Without asymmetry or at high Rayleigh number, the rolls are stable whereas hexagons are stable if the asymmetry parameter $\beta$ is high. Note that in some domain of the space parameters, both solution are stable, as can be seen in figure $5[?, ?, ?]$.

\subsection{Phase turbulence}

We will now focus on the case where dynamic effects are present and try to describe how the different modes can interact. Using $C_{i}(t)=\epsilon c_{i}$, we write

$$
\tilde{\mathbf{X}}(x, y, z, t)=\tilde{\mathbf{f}}(z) \sum_{n=-N}^{N} C_{n}(t) e^{i \vec{k}_{n} \cdot \vec{r}}
$$



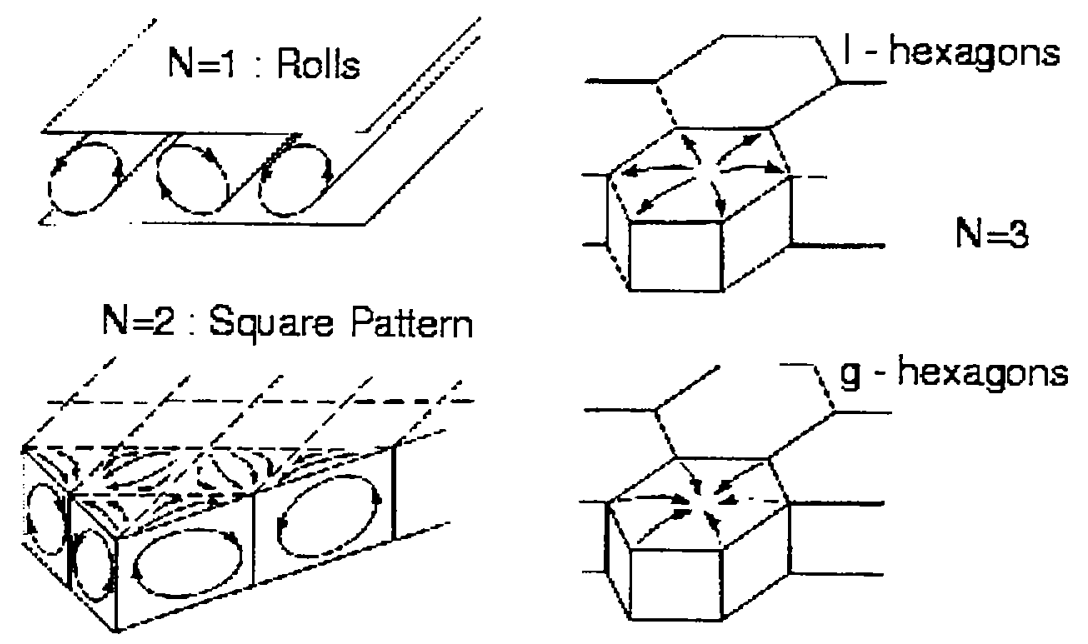

Figure 4: Sketch of patterns of convections: rolls, squares, 1 and g-hexagons.

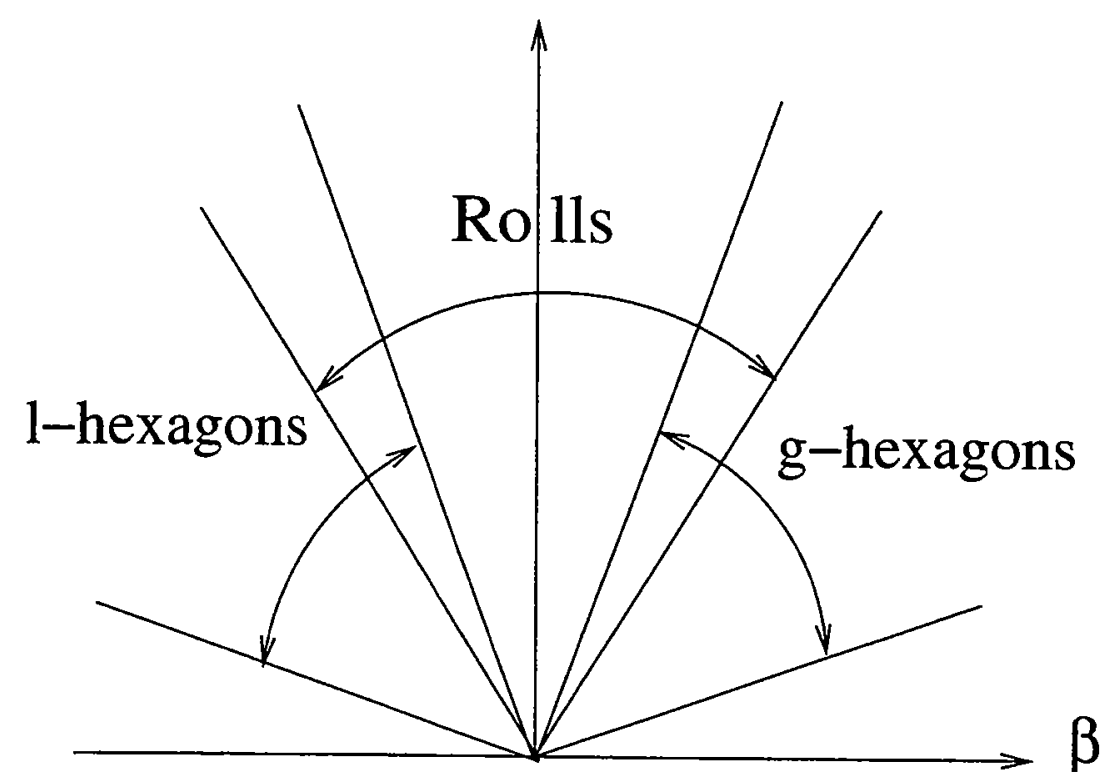

Figure 5: Stability diagram of rolls and hexagons patterns in the $(\beta, R)$ plane. 
Close to onset, one expects that the time dependence of the modes will be slow and of order $\epsilon^{2}$. As before, we write the solvability condition at order three and this yields to dynamical equations for the coefficients

$$
\begin{aligned}
V \frac{d}{d t} C_{i}^{*}= & \left(R-R_{0}\right) U C_{i}^{*}+\beta \sum_{n, m} C_{n} C_{m} \delta\left(\vec{k}_{i}+\vec{k}_{n}+\vec{k}_{m}\right) \\
& +\left(\sum_{n=1}^{N} C_{n} C_{n}^{*} A\left(\vec{k}_{i} \cdot \vec{k}_{n}\right)+E\left(\vec{k}_{i} \cdot \vec{k}_{n}, \vec{\lambda} \cdot \vec{k}_{i} \times \vec{k}_{n}\right)+A_{0}\left|C_{i}\right|^{2}\right) C_{i}^{*}+\ldots \text { with } \mathrm{i}=1,2,3 \ldots
\end{aligned}
$$

If there is no rotation $(E=0)$, and if there is no mean flow, we can write the evolution equations at this order as

$$
V \frac{d}{d t} C_{i}^{*}=-\frac{\partial}{\partial C_{i}} F\left(C_{1}, \ldots, C_{n}\right)
$$

where

$$
\begin{aligned}
F\left(C_{1}, \ldots, C_{n}\right)= & -\frac{1}{2}\left(R-R_{0}\right) U \sum_{i=1}^{N}\left|C_{i}\right|^{2}-\frac{1}{3} \beta \sum_{i, n, m} C_{i} C_{n} C_{m} \delta\left(\vec{k}_{i}+\vec{k}_{n}+\vec{k}_{m}\right) \\
& +\frac{1}{4}\left(\sum_{n=1}^{N}\left|C_{n}\right|^{2} A\left(\vec{k}_{i} . \vec{k}_{n}\right)+A_{0}\left|C_{i}\right|^{2}\right)\left|C_{i}\right|^{2}
\end{aligned}
$$

Thus these are evolution equations of Lyapunov type and the steady stable solutions will correspond to the local minima of $F$. The asymptotic approach is guaranteed and there can not be chaotic behaviour. This is not the case if either $E$ is not equal to zero, or if we consider solutions with non-zero mean flow (stress free boundary) or if we consider terms of higher order. Then, chaotic behaviour can occur.

Indeed, when we consider a horizontal layer heated from below that is rotating about a vertical axis, $E$ is not zero and the evolution equations are not of Lyapunov type. Above a critical value of the rotating parameter, all steady solutions become unstable. The local orientation of the convection rolls changes in time and this phenomenon is called phase turbulence. Experimental evidence have been seen in a rotating convection layer. A typical time evolution of the pattern of convection is shown in figure (6) [?, ?].

Another case in which phase turbulence is present is the convection in the presence of stress-free boundaries. Because no stress is exerted by the boundaries on horizontal motion of the fluid, large scale flow can be generated by a small Reynolds stress. The advection of the pattern by the large scale mean flow must be taken into account in the evolution equations which are no longer of Lyapunov type. Phase turbulence can also occur as presented in figure 7 where the time evolution of the heat flux in convection is calculated numerically for different values of the Rayleigh number [?, ?].

Phase turbulence shares some properties with the asymptotic turbulence of NavierStokes equations in the limit of infinite Reynolds number. A brief characterization of different types of turbulence is given in figure 8 . 

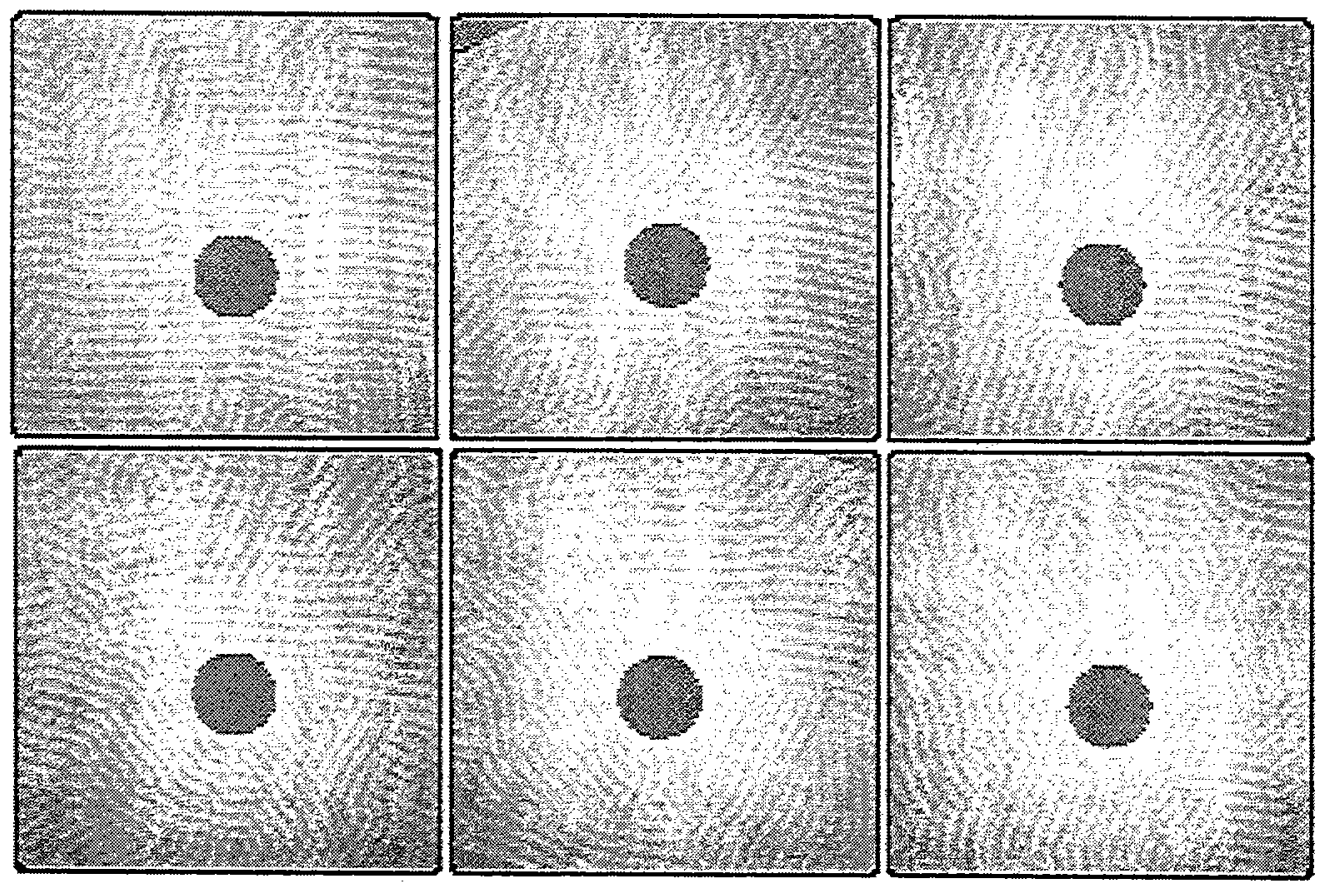

Figure 6: Time evolution of patterns of convection with phase turbulence [?, ?]. 


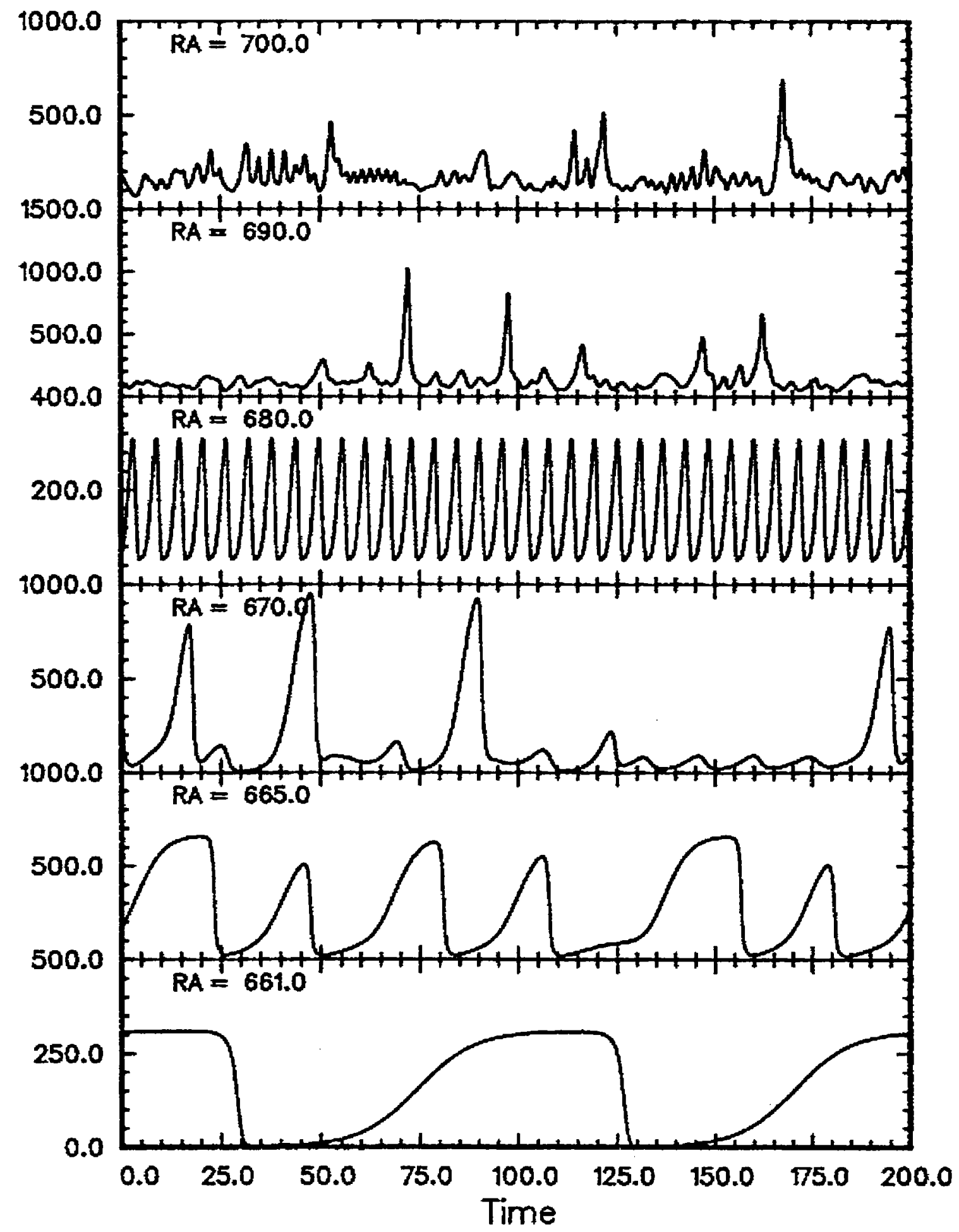

Figure 7: Time evolution of the heat flux for different values of the Rayleigh number and stress-free boundaries. $P_{m}=0.15$. At high Rayleigh numbers the evolution is chaotic. 


\section{Properties of Turbulence}

\begin{tabular}{|c|c|c|c|}
\hline Chaotic time dependence & $\begin{array}{l}\text { Chaotic spatial } \\
\text { dependence }\end{array}$ & $\begin{array}{l}\text { Broad wavenumber } \\
\text { spectrum }\end{array}$ & $\begin{array}{l}\text { Inertial range } \\
\text { Fractal structure }\end{array}$ \\
\hline $\begin{array}{l}\text { Dynamical systems } \\
\text { (few degrees of freedom, } \\
\text { eg convection in a box, } R>R_{4} \text { ) }\end{array}$ & & & \\
\hline \multicolumn{3}{|c|}{$\begin{array}{l}\qquad \text { Phase Turbulence } \\
\text { (many degrees of freedom, isotropy degeneracy; R close to Rc } \\
\text { examples: convection in a large aspect ratio layers, } \\
\text { rotating or non-rotating) }\end{array}$} & \\
\hline \multicolumn{3}{|c|}{$\begin{array}{l}\text { Classical turbulence } \\
\text { (shear-flow turbulence in channels, pipes and boundary layers; high Rayleigh number convection in large } \\
\text { aspect ration layers) }\end{array}$} & \\
\hline
\end{tabular}

Asymptotic Turbulence

(Turbulence in the limit of asymptotically high Reynolds numbers)

Figure 8: Characteristic properties of different kinds of turbulence. 


\title{
Lecture 3
}

\author{
The Sequence-of-Bifurcation Approach \\ for the Transition to Turbulence \\ F. H. Busse \\ Notes by U. Riemenschneider and S. Plasting
}

\section{Introduction}

In this lecture we will discuss fluid systems in which there is a gradual evolution from the basic laminar state toward a turbulent state with increasing Reynolds number. Transition is seen to occur through a sequence of bifurcations. We consider fluid systems with a high degree of symmetries which in the laboratory are observed to undergo transition to complex flow states through supercritical bifurcations that are characterized by the breaking of flow symmetries. We will not consider systems such as pipe flow or plane Couette flow which exhibit strongly subcritical bifurcations from the basic laminar state to a turbulent state. Figure 1 shows some fluid systems with maximum symmetry which undergo gradual transtion from the basic state to ever more complex solutions. For each of these systems the external conditions are homogeneous in two spatial dimensions and in time and since we are dealing with systems far from thermodynamic equilibrium we must have inhomogeneity in the third spatial dimension along which a constant energy flux is applied, thus these systems exhibit maximum symmetry. Although these systems do not represent all important processes in fluid mechanics a large number of system can be idealized or reduced to their physically essential properties such that they conform to this high degree of symmetries.

The sequence-of-bifurcations approach discussed in this lecture has the following advantages

1. In most cases the reduction of inhomogeneity to a single dimension reduces a physical mechanism to its simplest form.

2. The homogeneity in two spatial dimensions and in time provides a maximum of symmetries, the breaking of which identifies the bifurcations in the manifold of solutions for the fluid flow.

3. The relative simplicity of the physical properties is reflected in the simplifications of the numerical analysis. Symmetries can be employed to reduce the numerical effort.

4. Although physically realized systems can only approximate homogeneity in two spatial dimensions, the bifurcations of the ideal system become only slightly imperfect bifurcations in the real system as long as the typical wavelengths introduced by bifurcating solutions are small in comparison to the length scales associated with the deviations from homogerıeity.

5. The spatially and time periodic solutions that are obtained in the sequence-of-bifurcation approach represent only a minute manifold of the realizable solutions of the basic equations. Even if they are stable their basins of attraction decrease with increasing 

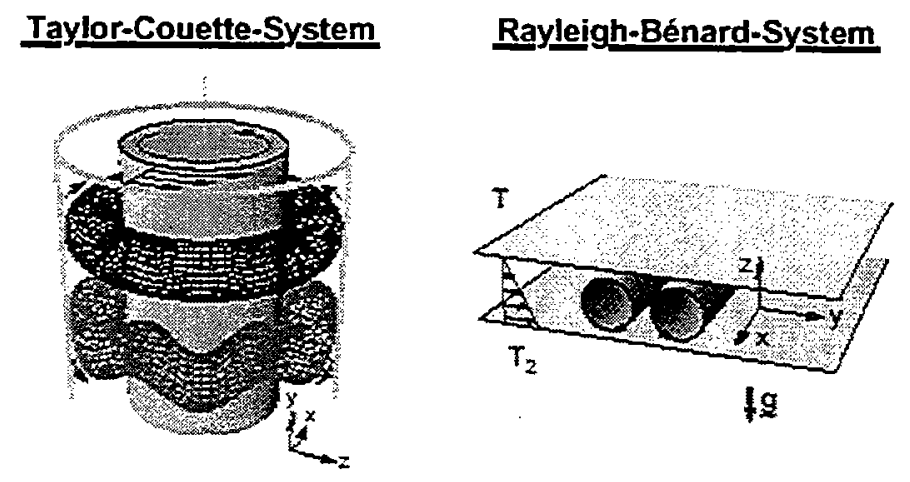

\section{Inclined Convection Layer}
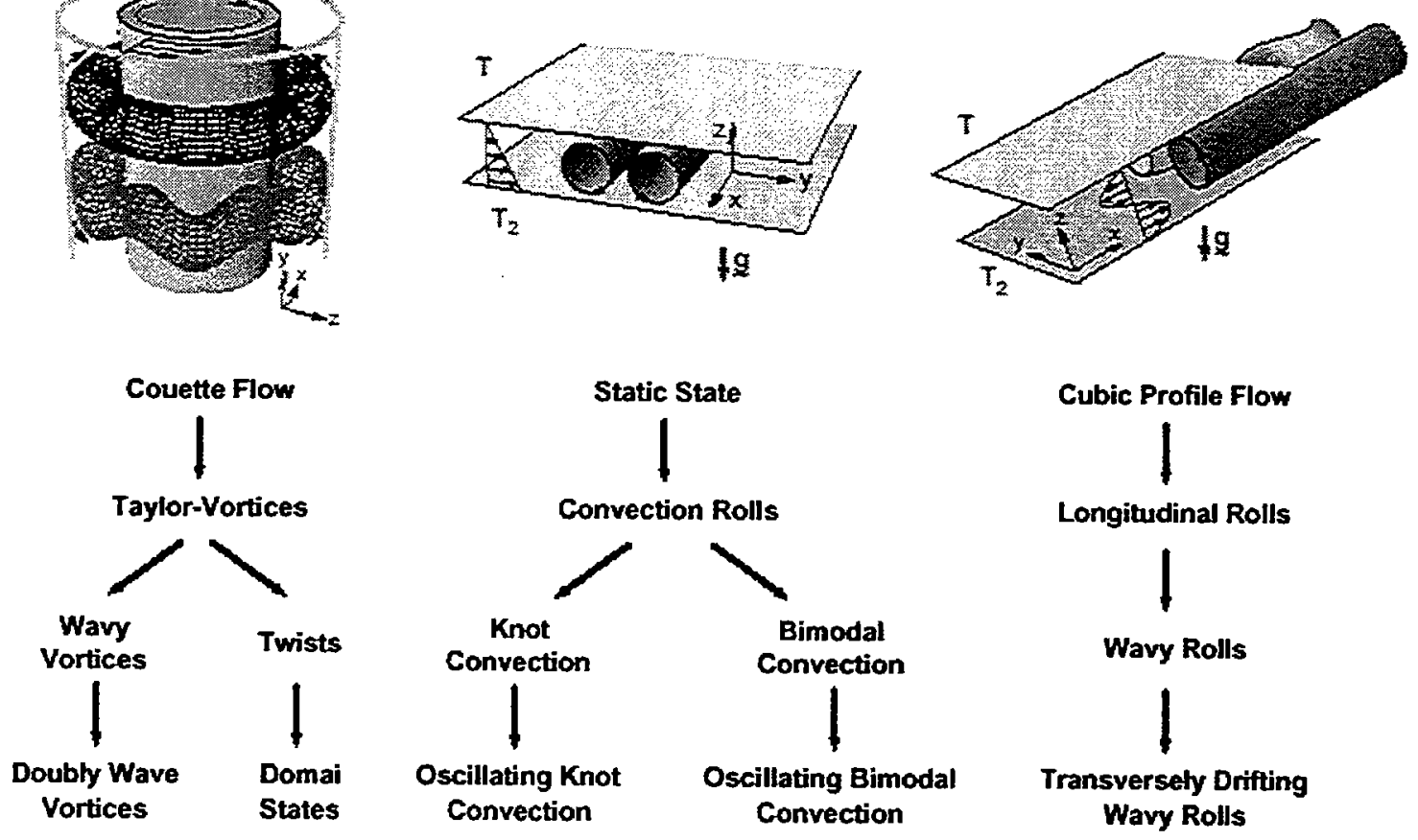

Figure 1: Examples of fluid systems with maximal symmetries and the typical sequence of bifurcations observed, starting with the basic roll solution.

control parameter and solutions describing more irregular spatio-temporal flow structures are typically observed in experimental realizations. Nevertheless, the regular, spatially periodic solutions usually exhibit most clearly the dynamical properties and transport mechanisms of the fluid system as a function of the control parameter.

\section{Secondary Solutions}

We now consider bifurcations which occur far from the critical point at which the laminar flow state becomes linearly unstable to roll patterns. We have seen in the previous lecture that the minimizing wave-vector of the most critical disturbance is infinitely degenerate when there is isotropy in the $x y$-plane. Here a weakly non-linear analysis is not suitable to detect bifurcations from secondary roll solutions because the non-linear terms in the Navier-Stokes equations play an equal role far from the linear stability point. Often isotropy manifests itself as phase turbulence for near critical parameter values, we therefore disregard isotropy so that we can analyze bifurcations from a roll solution with only one preferred direction. The basic equation can be written in the following canonical form 


\begin{tabular}{lll}
\hline A & translation in time & $\partial \varphi / \partial t=0$ \\
B & translation along roll axis & $\partial \varphi / \partial x=0$ \\
C & transverse periodicity & $\varphi(y+2 \pi / \alpha, z)=\varphi(y, z)$ \\
D transverse reflection & $\varphi(-y, z)=\varphi(y, z)$ or $a_{-m n}=a_{m n}$ \\
E inversion about roll axis & $\varphi(\pi / \alpha-y,-z)=-\varphi(y, z)$ or $a_{m n}=0$ for $m+n=$ odd \\
\hline
\end{tabular}

Table 1: Symmetry properties of two-dimensional rolls

$$
\mathbf{L} \varphi-R \mathbf{B} \varphi-\mathbf{V} \frac{\partial}{\partial t} \varphi=\mathbf{N} \varphi \varphi
$$

where $\mathbf{L}, \mathbf{B}$ and $\mathbf{V}$ are linear functionals and $\mathbf{N}$ represents the non-linear terms of the governing equations. The control parameter is now called $R$ and homogeneity in two spatial dimensions and in time is assumed, therefore the functionals above may only depend on $z$.

The stability of the basic state with respect to infinitesimal disturbances $\varphi_{0}$ is governed by equation (1) with vanishing right hand side. Without loss of generality a disturbance of the form

$$
\varphi_{0} \propto \exp \{i \mathbf{q} \cdot \mathbf{x}+\sigma t\}
$$

can be assumed, where $\mathbf{x}$ is the position vector and where the wave-vector $\mathbf{q}$ lies in the $x, y$-plane. The critical point $R_{c}$ is defined to be the point at which the real part $\sigma_{r}$ of the growth rate of the most unstable solution vanishes. In the case of no isotropy typically the minimizing solution is unique and the imaginary part $\sigma_{i}$ of the growth rate vanishes. Taking the $y$ direction to be parallel to the minimizing wave-vector $\mathbf{q}_{c}$ and $\alpha \equiv\left|\mathbf{q}_{c}\right|$ we can write the two dimensional solution bifurcating from the basic state as a Galerkin expansion

$$
\varphi=\sum_{m, n>0} a_{m n} \exp \{i m \alpha y\} f_{n}(z)
$$

where $f_{n}(z)=(-1)^{n-1} f_{n}(-z)$ for symmetry about midplane.

In table 1 we list the symmetry properties of rolls. They can be divided into the first three which are obeyed by all solutions of the form (3) and the remaining fourth and fifth which are satisfied in special cases. The fifth symmetry can occur only in problems such as Boussinesq Rayleigh-Bénard convection which have midplane symmetry.

The stability of secondary solutions can be studied through the superposition of infinitesimal disturbances of the form

$$
\tilde{\varphi}=\exp \{i b x+i d y+\sigma t\} \sum_{m, n>0} \tilde{a}_{m n} \exp \{i m \alpha y\} f_{n}(z) .
$$

When equation (1) is linearized in the disturbance $\tilde{\varphi}$ an homogeneous linear equation for the unknowns $\tilde{a}_{m n}$ is obtained with the growth rate $\sigma$ as eigenvalue. This linear eigenvalue problem for $\sigma$ is just

$$
\mathbf{L} \tilde{\varphi}-R \mathbf{B} \tilde{\varphi}-\mathbf{V} \sigma \tilde{\varphi}=\mathbf{N} \tilde{\varphi} \varphi+\mathbf{N} \varphi \tilde{\varphi}
$$




\begin{tabular}{lcccccc}
\hline $\begin{array}{l}\text { Properties } \\
\text { of disturbances }\end{array}$ & & $\sigma_{i} \neq 0$ & $b \neq 0$ & $d \neq 0$ & $\tilde{a}_{m n} \neq \tilde{a}_{-m n}$ & $\begin{array}{c}\tilde{a}_{m n} \neq 0 \text { for } \\
m+n=\text { odd }\end{array}$ \\
\hline $\begin{array}{l}\text { Symmetries } \\
\text { Broken }\end{array}$ & & $\begin{array}{c}\text { translation } \\
\text { in time }\end{array}$ & $\begin{array}{c}\text { longitudinal } \\
\text { translation }\end{array}$ & $\begin{array}{c}\text { transverse } \\
\text { periodicity }\end{array}$ & $\begin{array}{c}\text { transverse } \\
\text { reflection }\end{array}$ & $\begin{array}{c}\text { inversion } \\
\text { about axis }\end{array}$ \\
\hline Eckhaus & & & & $\mathrm{X}$ & $\mathrm{X}$ & \\
Crossroll & $\mathrm{CR}$ & & $\mathrm{X}$ & & & $\mathrm{X}$ \\
Knot & $\mathrm{KN}$ & & $\mathrm{X}$ & & & $\mathrm{X}$ \\
Even Blob & $\mathrm{EB}$ & $\mathrm{X}$ & $\mathrm{X}$ & & & $\mathrm{X}$ \\
Odd Blob & $\mathrm{OB}$ & $\mathrm{X}$ & $\mathrm{X}$ & & $\mathrm{X}$ & \\
Oscillatory & $\mathrm{OS}$ & $\mathrm{X}$ & $\mathrm{X}$ & & $\mathrm{X}$ & \\
Zig-Zag & $\mathrm{ZZ}$ & & $\mathrm{X}$ & & $\mathrm{X}$ & \\
Skewed Varicose & $\mathrm{SV}$ & & $\mathrm{X}$ & $\mathrm{X}$ & $\mathrm{X}$ & \\
Osc. Skewed Var. & & $\mathrm{X}$ & $\mathrm{X}$ & $\mathrm{X}$ & $\mathrm{X}$ & \\
\hline
\end{tabular}

Table 2: Symmetries Broken by Bifurcations from Steady Rolls

Of primary interest here are the growth rates $\sigma$ with largest real part $\sigma_{r}$ as a function of the horizontal wavenumbers $b$ and $d$. The growing disturbances correspond to a transition of the roll solution to tertiary solutions which exhibit more shapes and styles and which reflect the specific physical conditions to a higher degree. Table 2 characterizes each type of instability that can occur from steady rolls. The skewed varicose instability leads most quickly to turbulent convection. Each of these instabilities can be observed for some values of $\operatorname{Pr}-\alpha$. Figure 2 shows the enclosed domain of $R a-\alpha-\operatorname{Pr}$ space where roll solutions are stable. The Eckhaus instability usually causes rolls in an unstable region to be replaced by rolls in the stable region. Therefore the Eckhaus instability corresponds to a limitation of the available wavenumber for rolls and does not lead to a new type of solution.

\section{Tertiary Solutions}

Tertiary solution are twice spatially periodic solutions bifurcating from roll patterns. They can be described by expressions of the form

$$
\varphi=\sum_{l, m, n} a_{l m n} \exp \left\{i l \alpha_{x} x+i m \alpha_{y} y\right\} f_{n}(z)
$$

where we must have $a_{-l-m n}=a_{l m n}^{+}$for a real solution, where ()$^{+}$denotes complex conjugation. We have assumed that the instability of interest has $\sigma_{i}=0$. When an instability with a finite value of $\sigma_{i}=0$ occurs, it typically leads to traveling waves propagating in the $x$ direction which can be described by the representation above if $x$ is replaced by $\hat{x}=x-c t$. A partial list of tertiary solutions is given in Table 3.

The stability of these steady three-dimensional solutions can then be studied through the superposition of infinitesimal disturbances of the form

$$
\tilde{\varphi}=\exp \{i b x+i d y+\sigma t\} \sum_{l, m, n} \tilde{a}_{l m n} \exp \left\{i l \alpha_{x} x+i m \alpha_{y} y\right\} f_{n}(z)
$$




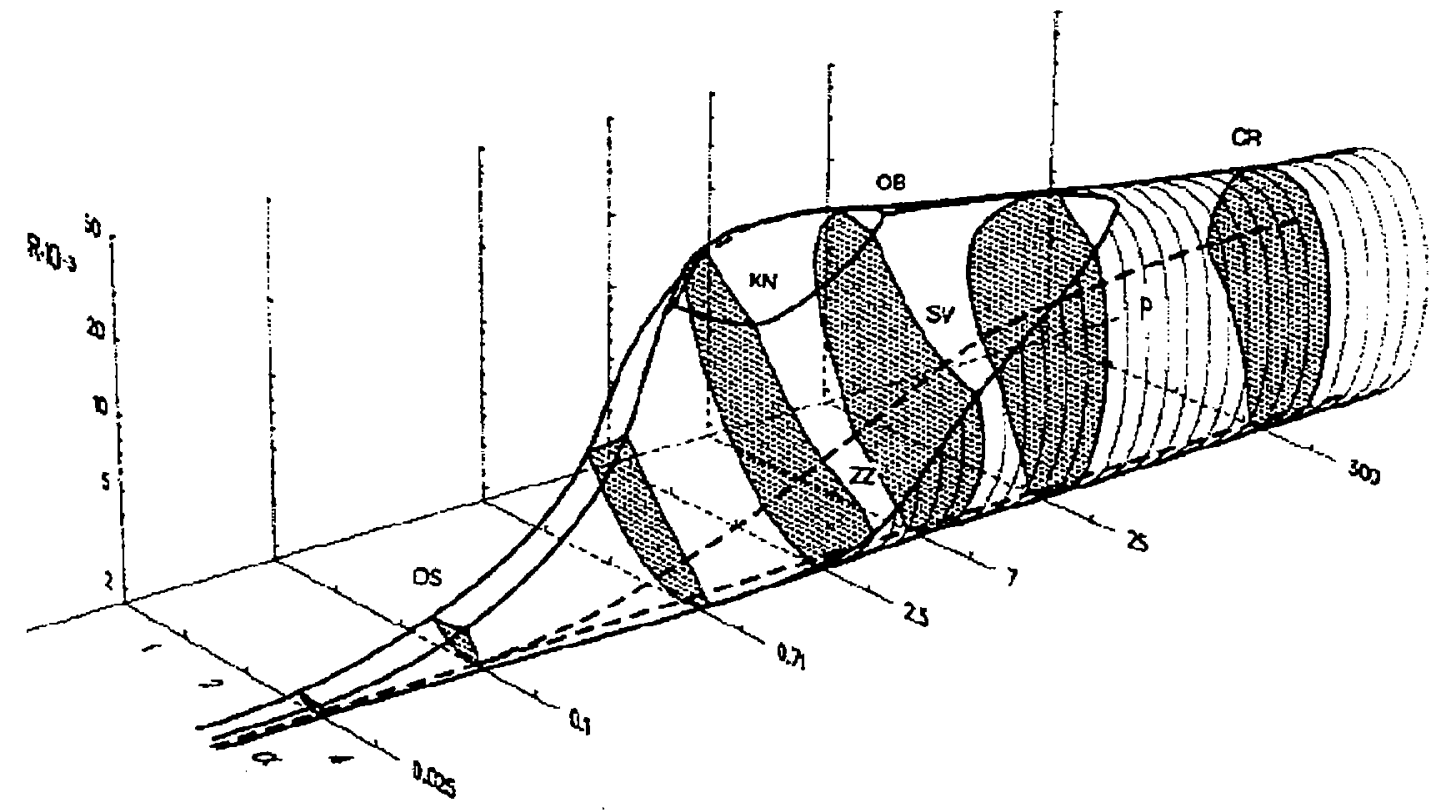

Figure 2: Region of stable convection rolls in the $R a-\alpha-\operatorname{Pr}$ parameter space. The region of stable rolls is bounded by surfaces corresponding to the onset of instabilities listed in Table 2. Note that $\operatorname{Pr}$ corresponds to $P$ in the figure and increases toward the right and $R$ corresponds to $R a$.

\begin{tabular}{l|l|l}
\hline Tertiary Solution & Reflection Symmetries & Inversion Symmetry \\
\hline knot-/bimodal convection & $a_{-l m n}=a_{l m n}=a_{l-m n}$ & $\begin{array}{l}a_{l m n}=0 \\
\text { for } l+m+n=\text { odd }\end{array}$ \\
\hline undulating rolls & $\begin{array}{l}a_{-l m n}=a_{l m n}, \\
a_{l-m n}=(-1)^{l} a_{l m n}\end{array}$ & $\begin{array}{l}a_{l m n}=0 \\
\text { for } m+n=\text { odd }\end{array}$ \\
\hline $\begin{array}{l}\text { Symmetric traveling wave } \\
\text { convection or wavy rolls } \\
\text { with Poiseuille flow }\end{array}$ & $a_{l-m n}=(-1)^{l} a_{l m n}$ & $\begin{array}{l}a_{l m n}=0 \\
\text { for } m+n=\text { odd }\end{array}$ \\
\hline $\begin{array}{l}\text { Wavy rolls with Couette } \\
\text { flow or wavy Taylor vortices } \\
\text { in small gap limit }\end{array}$ & $a_{l-m n}=(-1)^{l} a_{l m n}$ & $a_{l m n}=(-1)^{m+n} a_{-l m n}$ \\
\hline Traveling blob convection & $a_{-l m n}=a_{l m n}$ & $\begin{array}{l}a_{l m n}=0 \\
\text { for } l+m+n=\text { odd }\end{array}$ \\
\hline
\end{tabular}

Table 3: Examples of tertiary solutions and their symmetries listed in terms of the complex coefficients 
where the coefficients $\tilde{a}_{l m n}$ can be found by substituting $\varphi$ into equation (1), projecting onto the expansion functions, and solving the resulting non-linear equations using a Newton type method for some suitable spatial truncation size. The transition from tertiary to quarternary solutions is associated with the solution becoming time dependent. A much richer class of dynamical mechanisms for heat transport then become possible.

\section{Quarternary Solutions}

After the onset of three-dimensional tertiary solutions the continuous spatial symmetries such as the invariance with respect to the translation along the roll axis have been broken and have been replaced by reflection symmetries and inversion symmetries such as those shown in Table 3. The stability of the steady or traveling tertiary solutions can be investigated through the superposition of arbitrary infinitesimal disturbances. Using the general Floquet ansatz

$$
\tilde{\varphi}=\exp \{i b x+i d y+\sigma t\} \sum_{l, m, n} \tilde{a}_{l m n} \exp \left\{i l \alpha_{x} x+i m \alpha_{y} y\right\} f_{n}(z)
$$

we arrive at a linear homogeneous system of equations for the unknown coefficients $\tilde{a}_{l m n}$ with the growth rate $\sigma$ as eigenvalue. When the maximum real part of $\sigma$ as a function of $d$ and $b$ is less or equal to zero the tertiary solution is stable. Otherwise it is unstable.

The most strongly growing disturbances of tertiary stationary solutions are often those with non-vanishing imaginary part of $\sigma_{i}$. Since traveling wave type solutions are no longer possible after the translational invariance along the axis of the rolls has been broken, the time dependence must be taken into account explicitly. Time dependent three-dimensional solutions can be obtained through forward integration in time of the differential equations for the time dependent coefficients $a_{l m n}(t)$ in the representation for the quarternary solutions.

$$
\varphi=\sum_{l, m, n} a_{l m n}(t) \exp \left\{i l \alpha_{x} x+i m \alpha_{y} y\right\} f_{n}(z)
$$

The system of differential equations is obtained, just as in the case of the algebraic equations of tertiary solutions through projections of the equations of motion onto the space of the expansion functions. Examples of quarternary solutions, that is solutions in three-dimensions and the fourth dimension time, include oscillatory bimodal convection, oscillatory knot convection and pulsating traveling blob convection.

\section{Bimodal Convection}

\subsection{Steady Bimodal Convection: An example of a tertiary solution}

Steady bimodal convection is an example of a tertiary solution in Rayleigh-Bénard convection (see Figure 1). It corresponds to the superposition of a secondary roll pattern with smaller wavelength onto the given roll pattern as shown in the sketch in Figure 3. Through 


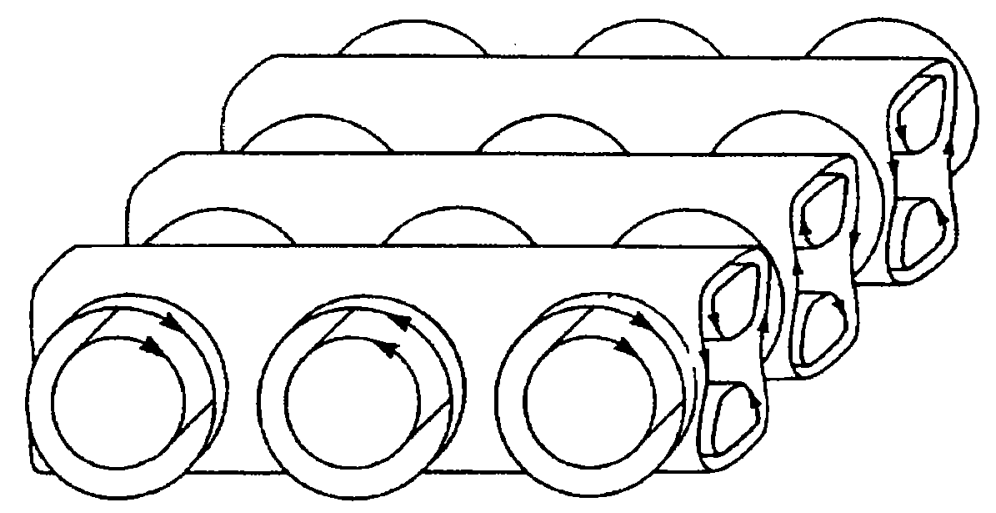

Figure 3: Schematic sketch of bimodal convection in a fluid heated from below. Note the superposition of a secondary roll pattern on the already existing roll pattern.

the onset of bimodal convection the convective heat transport becomes more efficient and the two roll patterns quickly reach comparable amplitudes as the Rayleigh number is increased beyond onset.

\subsection{Transition to Bimodal Convection: An heuristic argument}

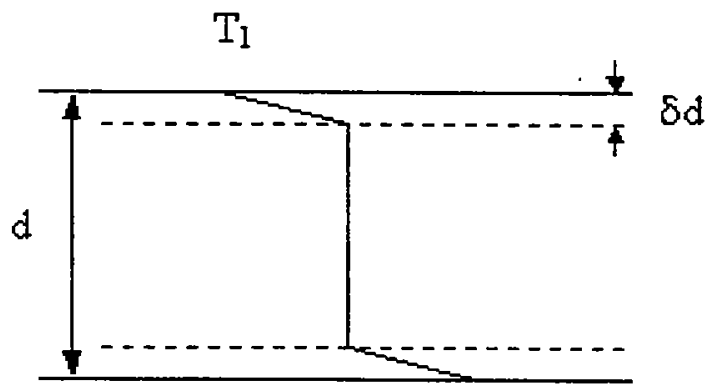

$\mathrm{T}_{2}$

Figure 4: The approximate form of the temperature profile for steady roll patterns

In Rayleigh-Bénard convection the mean temperature field for steady roll solutions has vanishing gradient in the interior of the flow and strong gradients near to the boundary. We can think of this thermal boundary layer as a subconvection layer with rescaled Rayleigh number $\frac{R}{2} \delta^{3}$, where $\delta$ is the non-dimensional thickness of the boundary layer. This situation is illustrated in Figure 4. The condition for convective instability in this layer is the Rayleigh condition for instability $\frac{R}{2} \delta^{3}>R_{c}$. If we assume that the gradient of temperature in the subconvection layer is approximately constant then the heat transport is $H \approx \frac{R}{2 \delta}$ and we can write the condition for instability of the boundary layer as

$$
H<\frac{R}{2}\left(\frac{R}{2 R_{c}}\right)^{1 / 3}
$$



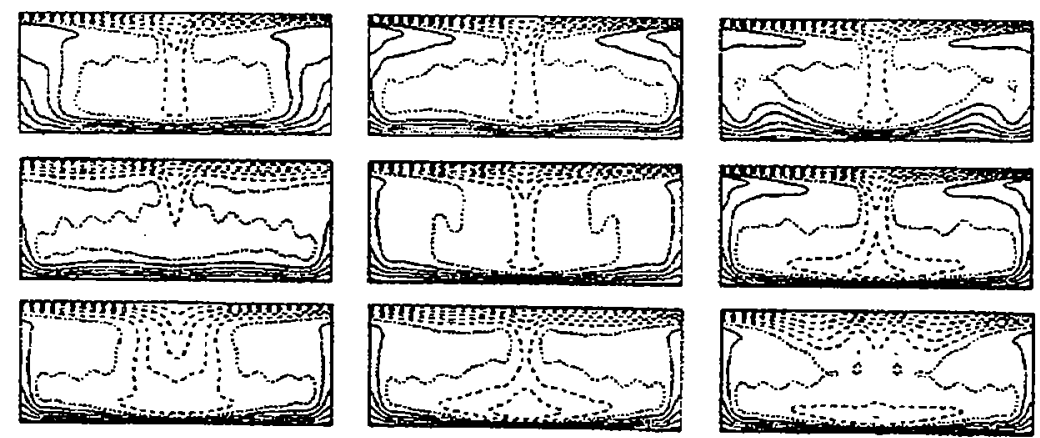

Figure 5: Isotherms at the $y, z$-planes $x=0, x=\pi / \alpha_{x}$ (from top to bottom) at the times $t=n \pi / 3 \omega$ for $n=0,1,2$. $y$ increases toward the right and $z$ increases upward. The parameter values $\operatorname{Pr}=30, R a=10^{5}, \alpha_{x}=4.5, \alpha_{y}=2.5$ have been used.

The most efficient configuration for heat transport to result from this type of instability is for tight boundary layer convection rolls to align perpendicular to the primary convection rolls as exemplified in the bimodal solution.

\subsection{Oscillatory Bimodal Convection: An example of a quarternary solu- tion}

An example of these types of convection patterns in nature could be the formation of bimodal convection patterns in clouds, which exhibit a very distinct rectangular pattern in the sky. They are typically high in Prandtl number and have a scale of the order of $100 \mathrm{~m}$ across. If the Prandtl number is in the range $10 \lesssim \operatorname{Pr} \lesssim 10^{2}$ the bifurcation from rolls to bimodal cells is followed by a further bifurcation to oscillatory bimodal convection. The thermal boundary layers periodically thicken and blobs of fluid hotter or cooler than average circulate through the convection cells. These oscillations are characterized to some extent by a resonance between the circulation time of the bimodal cell and the period of thickening and thinning of the thermal boundary layers.

There are two types of oscillatory bimodal convection, the symmetric one that does not change the spatial symmetry of steady bimodal convection and the other, called wavy oscillatory bimodal convection, which is characterized by the property that the set of coefficients $a_{l m n}(t)$ with

$$
-a_{-l m n}=a_{l m n}=a_{l-m n} \text { for } l+m+n=\text { odd and } a_{l m n}=0 \text { otherwise }
$$

are participating in the description of the solution in addition to those listed in Table 3 for bimodal convection. Figure 5 provides an impression of the time dependent structure of wavy oscillatory bimodal convection taken from numerical computations of [1] and in Figure 6 an experimental visualization is depicted. In the first we see a blob of cold fluid descending and impinging on the bottom of the layer while in the second figure we distinctly see the walls of the bimodal cells flexing back and forward. 


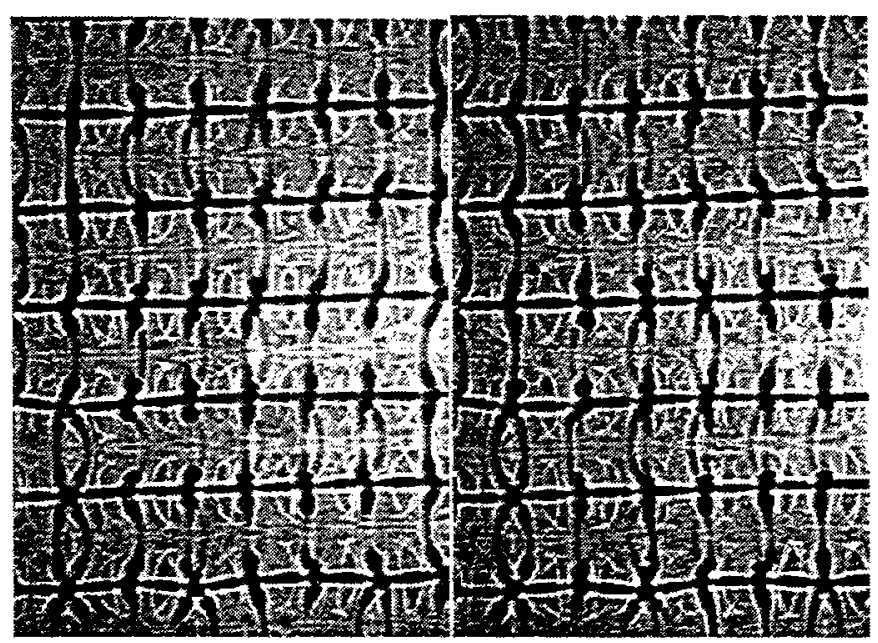

Figure 6: Shadowgraph observation of wavy oscillatory bimodal convection in a layer of silicon oil heated from below. The dark regions indicate hot rising fluid. The Prandtl and Rayleigh numbers are $\operatorname{Pr}=63, R a=1.5 \times 10^{5}$ and the wave numbers in the $x$-direction (toward the right) and $y$-direction are given by $\alpha_{x}=4.08, \alpha_{y}=2.04$. The right photograph was taken 25 seconds after the left one which corresponds to nearly half a period. Along the darker vertical lines small arches may be observed, pointing to the right or left, these are due to the oscillatory behaviour of the system.

It should be kept in mind that the realization of convection flows that are periodic in space and in time requires controlled initial conditions such that an approximately perfect roll pattern is realized after the onset of convection. The transition to bimodal attractors is sufficiently strong such that pattern imperfections can be eliminated in time except close to the sidewalls. The transition to oscillations usually occurs in a less homogeneous way and their phases tend to exhibit large scale variations. Without controlled initial conditions the convection flows at onset occur already in the form of patches of rolls with different horizontal orientations which tend to evolve in such a way that they ultimately reflect the geometrical configuration at the sidewalls of the layer, see the right column of shadowgraphs in Figure 8. As the Rayleigh number increases, the density of dislocations in the pattern increases rapidly and a chaotic structure of a kind of bimodal convection is realized when the Prandtl number is sufficiently high $(P r \gtrsim 10)$. The onset of oscillations in the form of hot and cold blobs emerging from the thermal boundary layer occurs initially at a few spots where the convection pattern deviates most strongly from the ideal periodic form. Laboratory experiments thus exhibit in general a more turbulent situation in qualitatively the same manner as in the case of the spatially and temporarily periodic solutions produced by the sequence of bifurcation approach. The latter method thus provides a sensible way toward an understanding of the processes occurring in turbulent convection as well as in other cases of fluid turbulence. 

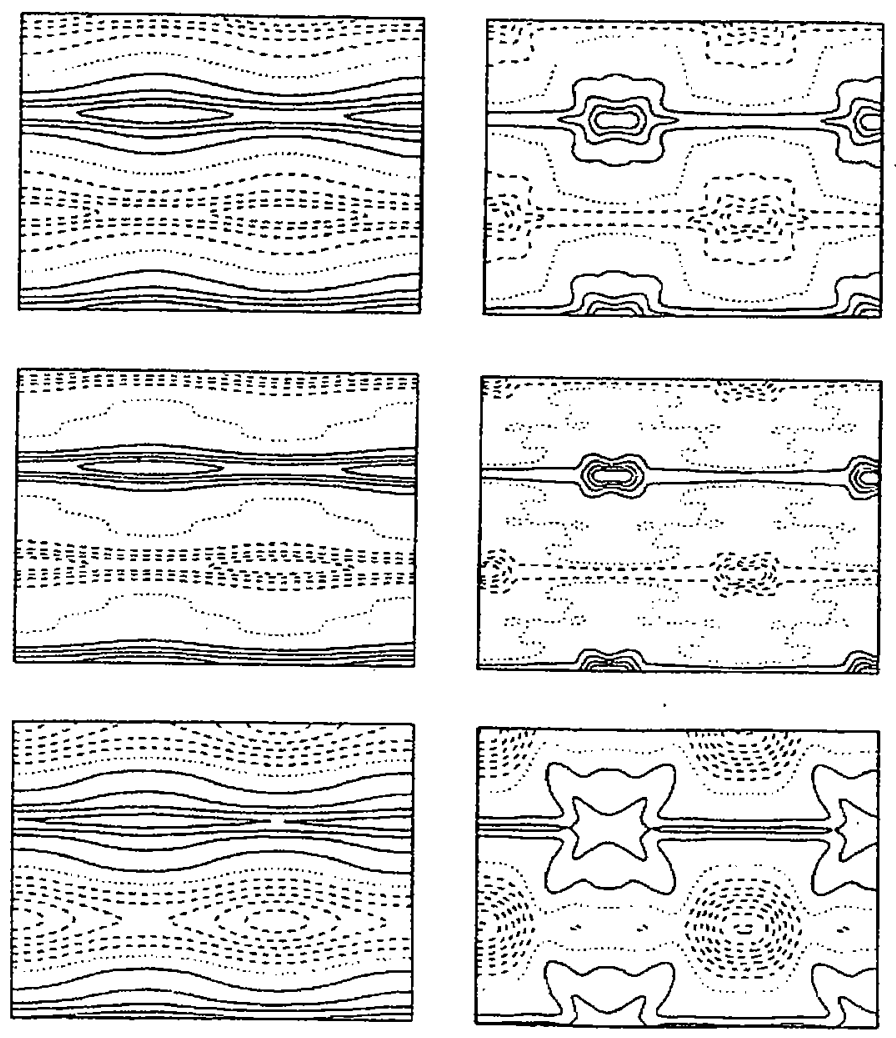

Figure 7: Numerical results for simulations of steady knot convection. The views are planar. The top two graphs show an average of the heat flux over the entire depth, $z$ of the convective layer, the two graphs in the middle show a section through the centre of the layer, $z=0$ and the bottom two graphs show a flux of the heat through the bottom boundary. Left hand column: $R=2.5 \cdot 10^{4}$, right hand column $R=8 \cdot 10^{4}$. 

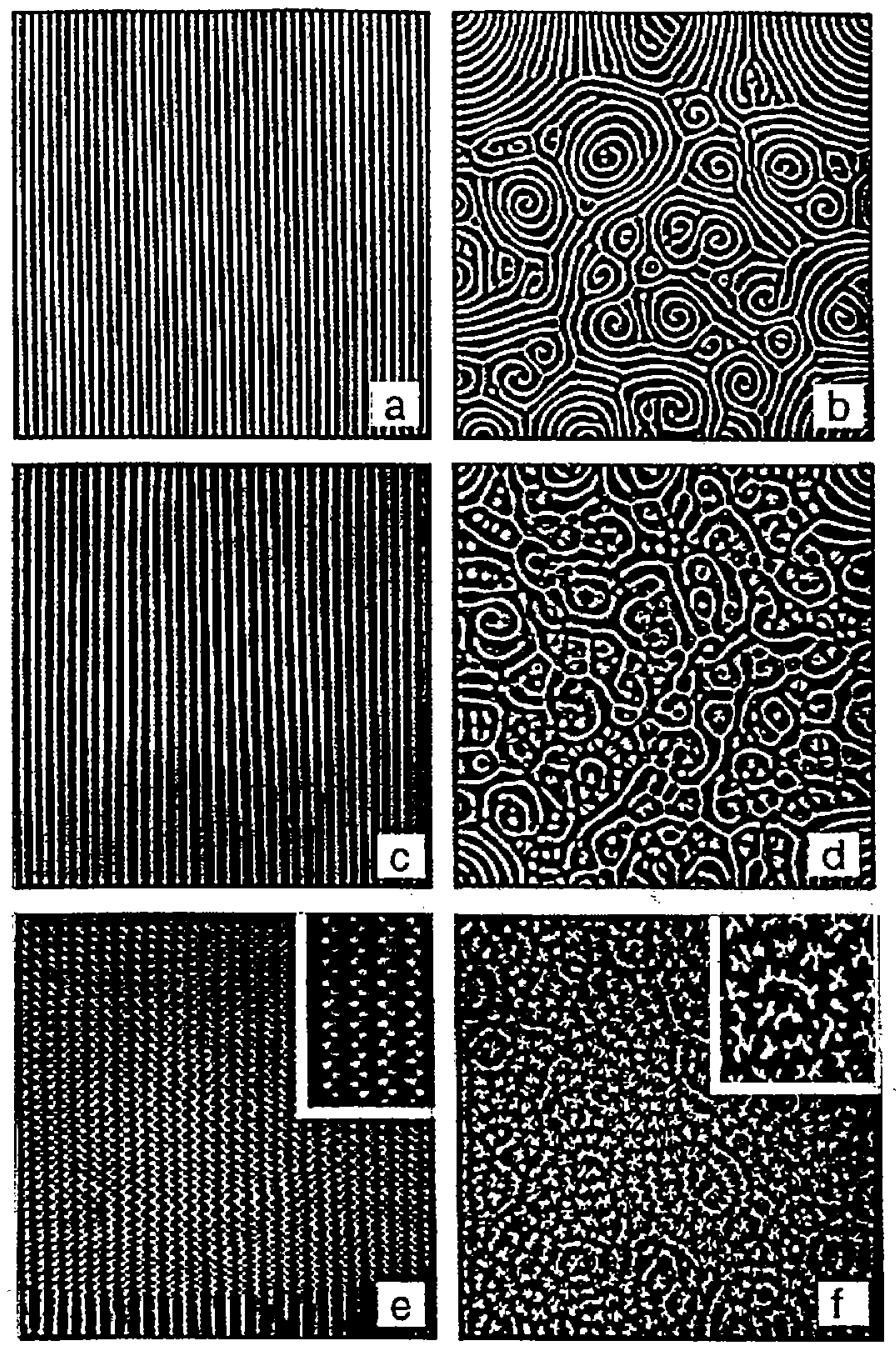

Figure 8: Digitally enhanced shadowgraph images of the convection patterns (taken from [2]). The distance from the critical point for onset of convection is measured as $\epsilon=(R-$ $\left.R_{c}\right) / R_{c}$ : (a) and (b) $\epsilon=0.920$; (c) $\epsilon=2.986$, (d) $\epsilon=3.000$, (e) and (f) $\epsilon=5.082$. The left column shows the effect of increasing the Rayleigh number on a field of rolls with uniform orientation, while the initial state in the right column contains patches of rolls oriented in arbitrary directions. As the Rayleigh number is increased the rolls undergo transition to wavy rolls. 


\section{References}

[1] R. Clever and F. Busse, "Steady and Oscillatory Bimodal Convection," J. Fluid Mech. 271, 103 (1994).

[2] R. Cakmur, D. Egolf, B. Plapp, and E. Bodenschatz, "Bistability and competition of spatiotemporal chaotic and fixed point attractors in Rayleigh-Benard convection," Phys. Rev. Lett. 79, 1853 (1997). 


\section{Lecture 4}

\section{Simple Approaches to Some Bounding \\ Louis Howard}

\section{On Some Properties of Good Chalk and People Working on Bounding Theory}

\section{Extremizing Functions and Functionals. Definitions and Simple Examples.}

We all know that if $\Phi\left(x_{i}\right)$ is a differentiable function, then the critical points $x_{i}$ that extremize the function can be determined from the conditions

$$
\frac{\partial \Phi\left(x_{i}\right)}{\partial x_{i}}=0 \text {. }
$$

But these simple conditions do not determine whether the critical values correspond to minima, maxima, or even guarantee an extremum. For example, consider the case shown in figure 1 of a horizontal inflection point and a monotonic function in a closed interval.
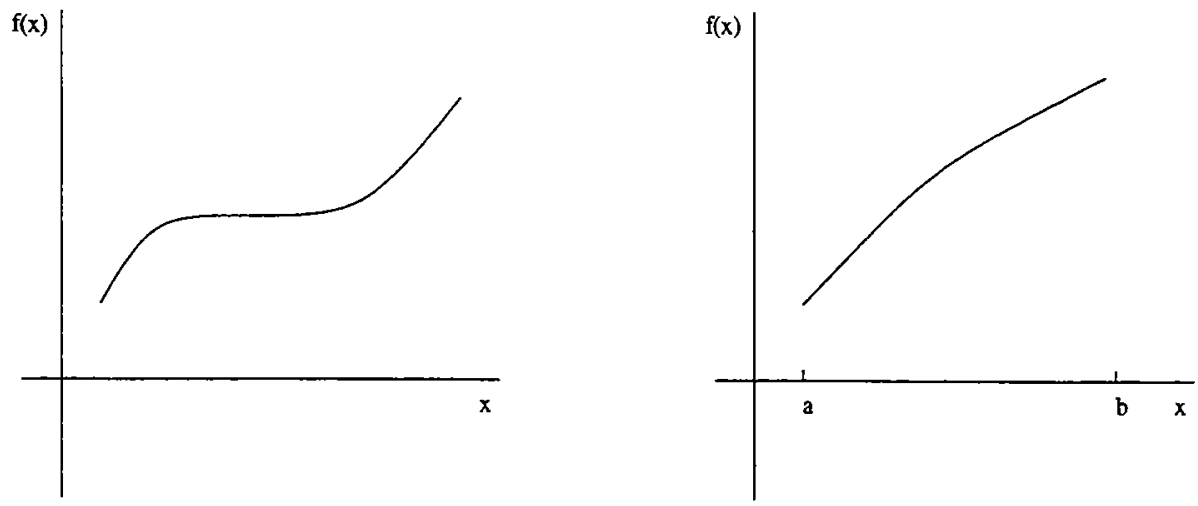

Figure 1: A horizontal inflection point and a monotonic function.

However, if the matrix of the second derivatives is positive definite at the critical point then there is indeed a (local) minimum, and if it is negative definite then we have a local maximum. But if this matrix is indefinite, it does not necessarily help us decide the character of the critical point. This is illustrated by the examples $f_{1}=x^{4}, f_{2}=-x^{4}, f_{3}=x^{3}$, all of which have $f^{\prime}(0)=f^{\prime \prime}(0)=0$, though $f_{1}$ has a minimum, $f_{2}$ a maximum and $f_{3}$ neither at $x=0$. (In the case of several variables, if the matrix of second derivatives has at least one positive and one negative eigenvalue, we can assert that the critical point is neither a minimum nor a maximum).

Another case where the above equations are not sufficient to determine the extrema of a function is when there is an imposed constraint. As an example consider the unit circle and 


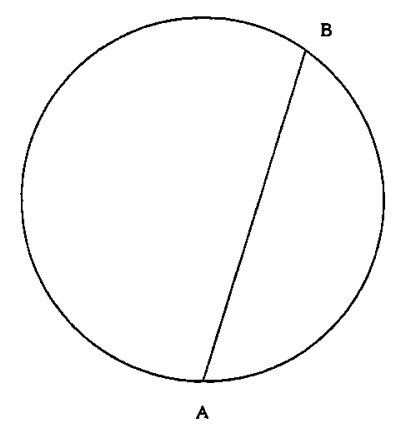

Figure 2: Points on a circle.

ask which two points $A=\left(x_{1}, y_{1}\right), B=\left(x_{2}, y_{2}\right)$ lying on it are furthest apart. The distance between them is given by

$$
D^{2}=\left(x_{1}-x_{2}\right)^{2}+\left(y_{1}-y_{2}\right)^{2}
$$

and the constraint takes the form

$$
x_{i}^{2}+y_{i}^{2}=1, \text { for } i=1,2 .
$$

The constraint can be automatically satisfied by letting $x_{i}=\cos \theta_{i}, y_{i}=\sin \theta_{i}$ and substituting into the expression for the distance. In this way there is no need to take into account the constraint explicitly. Then

$$
D^{2}=\left(\cos \theta_{1}-\cos \theta_{2}\right)^{2}+\left(\sin \theta_{1}-\sin \theta_{2}\right)^{2}=2-2 \cos \left(\theta_{1}-\theta_{2}\right),
$$

and maximum distance is obtained for $\theta_{1}-\theta_{2}=\pi$.

But when the constraint cannot be simply eliminated the method of Lagrange multipliers must be used. To find the extremum of $f\left(x_{1}, \ldots, x_{n}\right)$, subject to the constraints $g_{i}\left(x_{1}, \ldots, x_{n}\right)=0$ for $i=1,2, \ldots, m$, one forms the function

$$
\Phi(\mathbf{x}, \lambda)=f\left(x_{i}\right)-\sum_{i=1}^{m} \lambda_{i} g_{i},
$$

and solves

$$
\frac{\partial \Phi\left(x_{i}, \lambda_{j}\right)}{\partial x_{i}}=0, \quad \frac{\partial \Phi\left(x_{i}, \lambda_{j}\right)}{\partial \lambda_{i}}=g_{i}=0
$$

This seems to be a simple method but let us consider why it works. Suppose that the extremum of $f$ subject to the given constraints is at some point $\bar{x}_{i}$. If $g_{j}\left(x_{i}\right)$ is to remain zero then for small changes in the $x_{i}$ one must have,

$$
\nabla g_{j}\left(\bar{x}_{i}\right) \cdot d x_{i}=0
$$

in addition to

$$
d f=\nabla f\left(\bar{x}_{i}\right) \cdot d x_{i}=0
$$




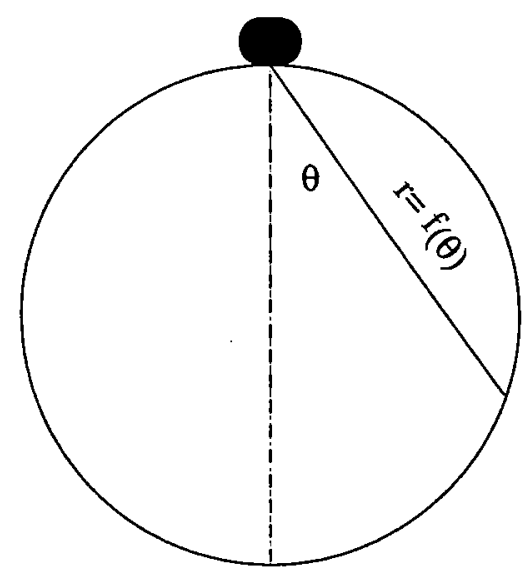

Figure 3: Shape of the Earth.

We may state this as " $\nabla f$ should be orthogonal to any vector $\mathbf{d x}$ which is orthogonal to all the $\nabla g_{i}$ ". In the language of linear algebra, $\nabla f$ should be in the orthogonal complement of the space $G^{\perp}$, which is itself the orthogonal complement of the space $G$ generated by the $\nabla g_{i}$. Since taking the orthogonal complement twice gets you back where you started (i.e. $\left.\left(G^{\perp}\right)^{\perp}=G\right), \nabla f$ must be in the space generated by the $\nabla g_{i}$, or $\nabla f=\sum_{i}^{m} \lambda_{i} \nabla g_{i}$ for some constants $\lambda_{i}$. This is the Lagrange multipliers rule.

More simply, if we maximize a function $f(x, y, z)$ subject to the constraint $g(x, y, z)=0$, then the admissible $\mathbf{d x}$ 's satisfy $\mathbf{d x} \cdot \nabla g=0$. That means $\mathbf{d x}$ can be any vector in the tangent plane to the surface $g=0$ at the critical point and $\nabla f$ must be orthogonal to this tangent plane. Thus $\nabla f$ must be parallel to $\nabla g$, i.e. $\nabla f=\lambda \nabla g$, yielding the Lagrange multipliers rule.

As an example let us consider the following problem. We ask to what shape one should transform the Earth in order to maximize his own weight, given that he cannot change his mass. Let us assume the Earth is incompressible, with an uniform density and search for an axisymmetric solution. Since it is incompressible, the Earth must have volume

$$
V=\frac{4 \pi a^{3}}{3}
$$

We introduce spherical polar coordinates with an origin at the position of the person, as shown in figure 3 . In this coordinate system the volume is given by

$$
V=\frac{2 \pi}{3} \int_{0}^{\frac{\pi}{2}} f^{3}(\theta) \sin \theta d \theta=\frac{4 \pi a^{3}}{3}
$$

and the person's weight is

$$
W=2 \pi \int_{0}^{\frac{\pi}{2}} \int_{0}^{f(r)}\left(\frac{G m \rho}{r^{2}} \cos \theta\right) r^{2} \sin \theta d r d \theta=2 \pi G m \rho \int_{0}^{\frac{\pi}{2}} f \sin \theta \cos \theta d \theta .
$$

Thus the relevant functional is

$$
\Phi=\int_{0}^{\frac{\pi}{2}}\left(f \sin \theta \cos \theta+\lambda f^{3} \sin \theta\right) d \theta .
$$


Setting its variation to zero gives

$$
\delta \Phi=\int_{0}^{\frac{\pi}{2}}\left(\delta f \cos \theta+3 \lambda f^{2} \delta f\right) \sin \theta d \theta=0,
$$

and so the minimum is obtained with

$$
f^{2}=-\frac{1}{3 \lambda} \cos \theta
$$

which is not too different from a sphere (which has $f=2 a \cos \theta$ ) and increases the weight of the person by $\left(\frac{27}{25}\right)^{\frac{1}{3}} \approx 1.02$.

If we extend our considerations to functionals we arrive at the Euler-Lagrange equations. For the simpler case when

$$
F(f)=\int L(f, \theta) d \theta
$$

the Euler-Lagrange equations following from $\delta F=0$ are $L_{1} \delta f=0$, (where the subscript denotes differentiation with respect to the corresponding argument), but if the functional is of the type,

$$
F(f)=\int L\left(f, f^{\prime}, \theta\right) d \theta
$$

then one has,

$$
\int\left(L_{1} \delta f+L_{2} \delta f^{\prime}\right) d \theta=0
$$

After integrating the second term by parts,

$$
\int\left(L_{1}-\frac{d L_{2}}{d \theta}\right) \delta f d \theta+\left[L_{2} \delta f\right]_{b s}=0,
$$

and assuming that $L_{2} \delta f$ vanishes on the bounding surface, one obtains the Euler-Lagrange equation,

$$
L_{1}-\frac{d L_{2}}{d \theta}=0
$$

In particular, if the functional is of the type

$$
F(f)=\int_{a}^{b} L\left(f, f^{\prime}, \theta\right) d \theta
$$

we get

$$
\delta F=\int_{a}^{b} L_{1} \delta f+L_{2} \delta f^{\prime}=\int_{a}^{b}\left(L_{1}-\frac{\partial}{\partial \theta} L_{2}\right) \delta f d \theta+\left[L_{2} \delta f\right]_{a}^{b} .
$$

This should be zero for all $\delta f$ 's that are admissible. In some cases the boundary term vanishes automatically, for example if $f$ is given at $x=a$ and $x=b$. If there are no 
such conditions we may first of all take $\delta f^{\prime}$ 's that are in fact zero at $\theta=a$ and $\theta=b$, but are otherwise arbitrary. Then, assuming that $\left(L_{1}-\frac{d}{d \theta} L_{2}\right)$ is continuous, we may conclude that $L_{1}-\frac{d}{d \theta} L_{2}=0$ at all interior points of $(a, b)$. Then, by taking $\delta f$ that is zero at, say $b$ but not zero at $a$, we conclude that $L_{2}\left(f(a), f^{\prime}(a), a\right)=0$. Similarly we may show that $L_{2}\left(f(b), f^{\prime}(b), b\right)=0$. In such cases the variational problem itself provides boundary conditions, so-called "natural boundary conditions", to supplement the Euler equation.

As an example, we minimize $\int_{0}^{1} f^{\prime 2}(x) d x$ subject to the two constraints $\int_{0}^{1} f^{2}(x) d x=$ 1, $\int_{0}^{1} f(x) d x=0$ (with no boundary conditions specified). To do this we consider the functional $\Phi=\int_{0}^{1}\left(f^{\prime 2}-\lambda_{1} f^{2}-\lambda_{2} f\right)$. Then,

$$
\delta \Phi=\int_{0}^{1}\left(2 f^{\prime} \delta f^{\prime}-2 \lambda_{1} f \delta f-\lambda_{2} \delta f\right) d x=2\left[f^{\prime} \delta f\right]_{0}^{1}-2 \int_{0}^{1}\left[f^{\prime \prime}+\lambda_{1} f+\frac{1}{2} \lambda_{2}\right] \delta f d x
$$

so the necessary conditions for a minimum are :

- Euler-Lagrange equation $f^{\prime \prime}+\lambda_{1} f+\frac{1}{2} \lambda_{2}=0$,

- the natural boundary conditions $f^{\prime}(0)=f^{\prime}(1)=0$,

- the constraints $\int_{0}^{1} f^{2} d x=1, \int_{0}^{1} f d x=0$.

One could write down the general solution of the differential equation and use the two boundary conditions and the two constraints to determine the two $\lambda$ 's and the two arbitrary constants in the general solution. It is a little neater to note that integrating the EulerLagrange equation from 0 to 1 and using the natural boundary condition and the second constraint gives $\frac{1}{2} \lambda_{2}=0$, hence $\lambda_{2}=0$. We then see that $f=\sqrt{2} \cos (n \pi x)$ and $\lambda_{1}=(n \pi)^{2}$, for some integer $n$ that cannot be zero because of the second constraint. $n=1$ gives the least-value of the integral - indeed if we integrate $f^{\prime}\left(f^{\prime \prime}+\lambda_{1} f\right)=0$ from 0 to 1 , using the first constraint and the natural boundary conditions, we see that $\lambda_{1}=\int_{0}^{1} f^{\prime 2} d x$, i.e. $\lambda_{1}$ itself is the required minimum value $\pi^{2}$. (Note that without the second constraint the minimum value would be zero, achieved by $f=1$.)

As a simple illustration the shortest path between two parabolas such as those shown in figure 4 , is a straight line perpendicular to both curves. In this example the Euler-Lagrange equation shows that the path must be straight, while the natural boundary conditions show that it should be orthogonal to each of the two parabolas at its endpoints, which is pretty obvious geometrically.

\section{Minimization of $\int f^{\prime 2}$ given $\int f^{2}$}

As an example, we consider the following problem:

$$
\text { Minimize } \int_{0}^{L} f^{\prime 2}(x) d x \text { subject to } \int_{0}^{L} f^{2}(x) d x=1 \text { and } f(0)=f(L)=0 .
$$

The technique of Lagrange multipliers gives $\Phi=\int_{0}^{L}\left(f^{\prime 2}-\lambda f^{2}\right) d x$, which has

$$
\delta \Phi=-2 \int_{0}^{L}\left(f^{\prime \prime}+\lambda f\right) \delta f d x
$$




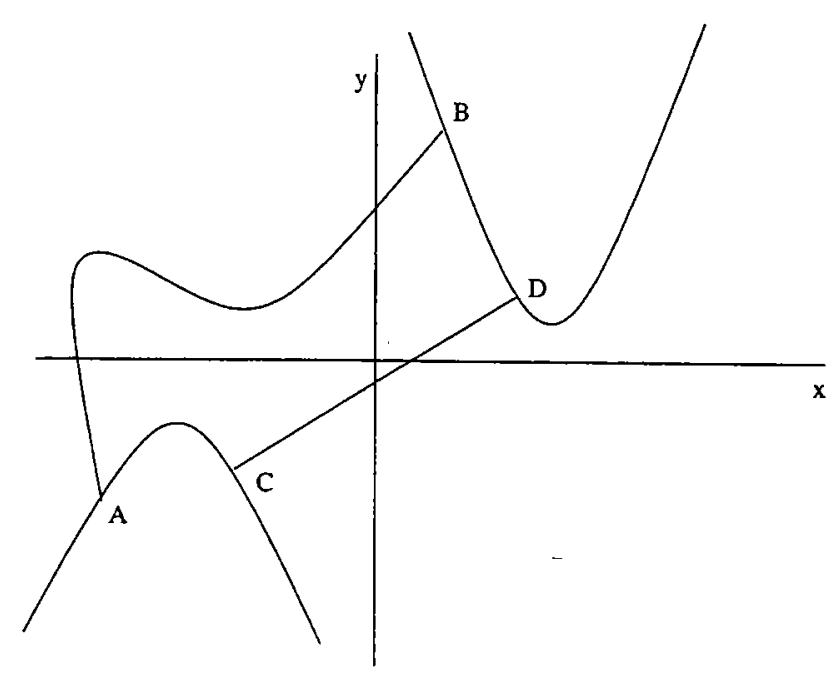

Figure 4: Distance between parabolas.

so the minimizing function satisfies $f^{\prime \prime}+\lambda f=0$, meaning that $f$ is proportional to $\sin (n \pi x / L)$ for some integer $n . n=1$ gives the minimal value. This yields the relation

$$
\int_{0}^{L} f^{\prime 2} d x \geq \frac{\pi^{2}}{L^{2}} \int_{0}^{L} f^{2}(x) d x \text { for all functions } f \text { with } f(0)=f(L)=0 .
$$

This derivation was not very rigorous, although it does give the correct answer.

The problem can also be tackled using Fourier series. Under rather mild restrictions, $f(x)$ has a Fourier sine series, $f(x) \sim \sum_{1}^{\infty} b_{n} \sin (n \pi x / L)$, and with a little more assumed about $f$, this series actually converges to $f$ in the interior of $(0, L)$ - for instance if $f$ is continuous and continuously differentiable there. Of course the sine series converges to 0 at $x=0$ and $L$, which need not be the values of $f$ at those points. For simplicity, however, we assume that $f$ vanishes at these endpoints, and that $f, f^{\prime}$ and $f^{\prime \prime}$ are all continuous on $[0, L]$. Then not only does the sine series of $f$ converge to $f$ on $[0, L]$, but the cosine series of $f^{\prime}$ converges to $f^{\prime}$ on this interval, and this cosine series is in fact the same as the formal term-by-term derivative of the sine series of $f$. (This would not be true unles $f(0)=f(L)=0$, however smooth $f$ might be on $[0, L]$.) Thus

$$
f(x)=\sum_{1}^{\infty} b_{n} \sin (n \pi x / L), \quad f^{\prime}(x)=\sum_{I}^{\infty} n \pi b_{n} \cos (n \pi x / L) / L,
$$

and

$$
\int_{0}^{L} f^{2}(x) d x=\frac{L}{2} \sum_{1}^{\infty} b_{n}^{2}, \quad \int_{0}^{L} f^{\prime 2}(x) d x=\frac{L}{2} \sum_{1}^{\infty}\left(\frac{n \pi}{L}\right)^{2} b_{n}^{2} .
$$

Thus $\int_{0}^{L} f^{\prime 2}(x) d x \geq(\pi / L)^{2} \int_{0}^{L} f^{2}(x) d x$, with equality only when all the $b_{n}$ beyond $b_{1}$ are zero. 
Another approach to this inequality that also avoids consideration of the Euler-Lagrange equation is the method of multiplicative variation: we set $f(x)=\sin (\pi x / L) g(x)$. Then we compute

$$
\begin{aligned}
\int_{0}^{L} f^{\prime 2} d x & =\int_{0}^{L}\left(\sin \frac{\pi x}{L} g^{\prime}(x)+\frac{\pi}{L} \cos \frac{\pi x}{L} g(x)\right)^{2} d x \\
& =\int_{0}^{L} \sin ^{2} \frac{\pi x}{L} g^{\prime 2} d x+\int_{0}^{L} 2 \frac{\pi}{L} \cos \frac{\pi x}{L} \sin \frac{\pi x}{L} g(x) g^{\prime}(x) d x+\int_{0}^{L}\left(\frac{\pi}{L}\right)^{2} \cos ^{2} \frac{\pi x}{L} g^{2} d x \\
& =\int_{0}^{L} \sin ^{2} \frac{\pi x}{L} g^{\prime 2} d x-\int_{0}^{L}\left(\frac{\pi}{L}\right)^{2}\left(\cos ^{2} \frac{\pi x}{L}-\sin ^{2} \frac{\pi x}{L}\right) g^{2} d x \\
& \quad+\int_{0}^{L}\left(\frac{\pi}{L}\right)^{2} \cos ^{2} \frac{\pi x}{L} g^{2} d x \\
& =\int_{0}^{L} \sin ^{2} \frac{\pi x}{L} g^{\prime 2}(x) d x+\frac{\pi^{2}}{L^{2}} \int_{0}^{L} f^{2}(x) d x \\
& \geq \frac{\pi^{2}}{L^{2}} \int_{0}^{L} f^{2} d x
\end{aligned}
$$

with equality only when $g^{\prime} \equiv 0$ everywhere on $(0, L)$ assuming it is continuous there.

A variation on this theme is the following little calculation:

$$
\begin{aligned}
0 & \leq \int_{0}^{L}\left(f^{\prime}(x)-\frac{\pi}{L} \cot \frac{\pi x}{L} f(x)\right)^{2} d x \\
& =\int_{0}^{L} f^{\prime 2} d x+\left(\frac{\pi}{L}\right)^{2} \int_{0}^{L} \cot ^{2} \frac{\pi x}{L} f^{2}(x) d x-\int_{0}^{L} 2 f(x) f^{\prime}(x) \frac{\pi}{L} \cot \frac{\pi x}{L} d x \\
& =\int_{0}^{L} f^{\prime 2} d x+\left(\frac{\pi}{L}\right)^{2} \int_{0}^{L} \cot ^{2} \frac{\pi x}{L} f^{2}(x) d x-\left[\frac{\pi}{L} f^{2}(x) \cot ^{2} \frac{\pi x}{L}\right]_{0}^{L} \\
& \quad-\frac{\pi}{L} \int_{0}^{L} f^{2}(x) \frac{\pi}{L} \operatorname{cosec} \frac{\pi x}{L} d x \\
& =\int_{0}^{L} f^{\prime 2} d x-\left(\frac{\pi}{L}\right)^{2} \int_{0}^{L} f^{2} d x
\end{aligned}
$$

In this argument we must assume that $f \rightarrow 0$ faster that $x^{1 / 2}$ as $x \rightarrow 0$ or than $(L-x)^{1 / 2}$ as $x \rightarrow L$. Some such hypothesis is needed to assume the existence of $\int_{0}^{L} f^{\prime 2}(x) d x$.

Claim: Assume that $f$ is continuous and differentiable on the interval $[-1,1], f(-1)=$ $f(1)=0, f(x)=\int_{-1}^{x} f^{\prime}(t) d t$ and that $\int_{-1}^{1} f^{\prime 2} d x$ exists. Then

$$
f^{2}(x) \leq\left(1-x^{2}\right)\left\langle f^{\prime 2}\right\rangle
$$

where $\langle g\rangle$ denotes $\int_{-1}^{1} g(x) d x / 2$ for any function $g$. 


\section{Proof:}

$$
\begin{aligned}
f^{2}(x) & =\left(\int_{-1}^{x} f^{\prime}(t) d t\right)^{2} \\
& \leq \int_{-1}^{x} f^{\prime 2}(t) d t \int_{-1}^{x} 1^{2} d t \\
& =(1+x) \int_{-1}^{x} f^{\prime 2}(t) d t
\end{aligned}
$$

Similarly

$$
f^{2}(x) \leq(1-x) \int_{x}^{1} f^{\prime 2} d t
$$

and combining the two gives

$$
f^{2}(x)\left(\frac{1}{1+x}+\frac{1}{1-x}\right) \leq 2\left\langle f^{2}\right\rangle
$$

leading to the required result.

Integrating both sides leads to

$$
\left\langle f^{2}\right\rangle \leq \frac{2}{3}\left\langle f^{\prime 2}\right\rangle
$$

However, we already know that the stronger relationship

$$
\left\langle f^{2}\right\rangle \leq \frac{4}{\pi^{2}}\left\langle f^{2}\right\rangle
$$

holds from the previous calculations, so the above method does not give the optimal estimate. Still, the pointwise estimate given by this result cannot be improved; it is only the integrated result that is less than optimal.

In two dimensions, if we wish to minimize $\int_{A}|\nabla f|^{2} d A$ subject to $\int_{A}|f|^{2} d A=1$, where $A$ is a region in $\mathbb{R}^{2}$, then the Lagrange method yields the relation $\nabla^{2} f+\lambda f=0$ for the minimizing function $f$. If $A$ is a circle centred on the origin with radius 1 then this has solutions $J_{m}\left(j_{m n} r\right) e^{\mathrm{i} m \theta}$, where $J_{m}$ is the $m$ th order Bessel function of the first kind and $j_{m n}$ is the $n$th positive root of $J_{m}$. Then $1=\int_{A}|f|^{2} d A=2 \pi \int_{0}^{1} r J_{m}^{2}\left(j_{m n} r\right) d r$ and so $\int_{A}|\nabla f|^{2} d A=2 \pi j_{m n} \int_{0}^{1} r J_{m}^{2}\left(j_{m n} r\right) d r=j_{m n}^{2}$. So the minimum value of $\int_{A}|\nabla f|^{2} d A$ is given by $j_{01}^{2} \approx 5.78$.

\section{The Dual Lagrange Problem}

We have

$$
L(\mathbf{x}, \boldsymbol{\lambda})=F(\mathbf{x})+\sum_{i=1}^{m} \lambda_{i} g_{i}
$$




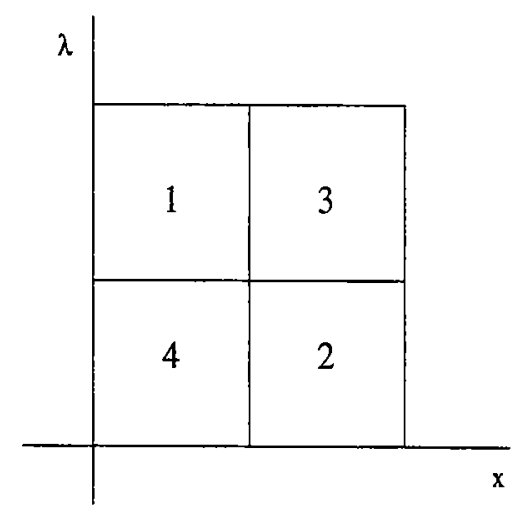

Figure 5: An example showing values of $L(\mathbf{x}, \boldsymbol{\lambda})$ for a case where $\sup _{\boldsymbol{\lambda}} \inf _{\mathbf{x}} L=2$ and $\inf _{\mathbf{x}} \sup _{\lambda} L=3$.

Suppose that $F$ has minimum value $F_{*}$, subject to the constraints $g_{i}=0$ for $i=1,2, \ldots, m$, which is attained when $\mathbf{x}=\mathbf{x}_{*}$.

Now let

$$
h(\boldsymbol{\lambda})=\inf _{\mathbf{x}} L(\mathbf{x}, \boldsymbol{\lambda}) \leq F_{*} \forall \boldsymbol{\lambda} .
$$

The dual problem is given by maximizing $h(\boldsymbol{\lambda})$. Say this has value $h_{*}$. Then $h_{*} \leq F_{*}$, sometimes with equality, though not always. An example of a case where there is not equality is shown in figure 5 .

\subsection{Further examples}

$$
F(x, y)=x^{2}+2 y^{2}, \text { with } g=1-x^{2}-y^{2} .
$$

Then $L(x, y, \lambda)=x^{2}+2 y^{2}+\lambda\left(1-x^{2}-y^{2}\right)$. Thus the Euler-Lagrange equations for seeking a minimum of $F$ with $g_{1}=0$ are $2 x-2 x \lambda=0,4 y-2 y \lambda=0$. Therefore either $\lambda=1$, $x= \pm 1$ and $y=0$ giving $F_{*}=1$, or $\lambda=2, x=0$ and $y= \pm 1$ giving $F_{*}=2$. Thus the minimum is $F_{*}=1$, attained at $x= \pm 1, y=0$.

Now consider

$$
h(\lambda)=\inf _{x} L(x, \lambda)= \begin{cases}-\infty & \text { if } \lambda>1 \\ 1 & \text { if } \lambda=1(\text { at } y=0) \\ 1 & \text { if } \lambda<1(\text { at } x=y=0)\end{cases}
$$

Thus $\max _{\lambda} h(\lambda)=1$ so that $h_{*}=1=F_{*}$. So for this example, $\max _{\lambda} h=\min _{\mathbf{x}, g_{1}(x, y)=0} F$. On the other hand consider again

$$
F=x^{2}+2 y^{2} \text {, but with } g=1-x^{4}-y^{4} \text {. }
$$

For this case, $L(x, y, \lambda)=x^{2}+2 y^{2}+\lambda\left(1-x^{4}-y^{4}\right)$. Thus using the Lagrange multiplier rule to seek a minimum of $F$ given $g=0$ we get

$$
2 x-4 \lambda x^{3}=0, \quad 4 y-4 \lambda y^{3}=0, \quad 1-x^{4}-y^{4}=0 .
$$


Therefore either

- $x \neq 0$. Then $\lambda=1 / 2 x^{2}$ and $y=0$ or $y= \pm \sqrt{2} x$. If $y=0$ then $x= \pm 1$ and $F_{*}=1$ Otherwise, $x= \pm 5^{-1 / 4}, y= \pm 5^{-1 / 4} \sqrt{2}$ and $F_{*}=5^{-1 / 2}+4 \cdot 5^{-1 / 2}=\sqrt{5}$, or

- $x=0$. Then the constraint requires $y= \pm 1$, (and hence $\lambda=1$ ) and then $F=2$, which is greater than 1 .

Thus the least value of $F$ is 1 , obtained at $x \pm 5^{-1 / 4}, y= \pm 5^{-1 / 4} \sqrt{2}$.

However, Lagrange multipliers are not really needed for this problem: considering only $x$ and $y \geq 0$ we could eliminate the constraint by setting $x=\sqrt{\cos \theta}, y=\sqrt{\sin \theta}$ and then $F=\cos \theta+2 \sin \theta, F^{\prime}=2 \cos \theta-\sin \theta$. At the minimum, $\tan \theta=2$ and hence $F=\sqrt{5}$. But at $\theta=0, F=1$ and at $\theta=\pi / 2, F=2$. Thus the minimum $F$ is 1 , attained at $x= \pm 1$, $y=0$.

But what is $h(\lambda)=\inf _{x, y}\left(x^{2}+2 y^{2}+\lambda\left(1-x^{4}-y^{4}\right)\right) ?$

$$
h(\lambda)= \begin{cases}-\infty & \text { if } \lambda>0 \\ 0 & \text { if } \lambda=0 \\ \lambda & \text { if } \lambda<0\end{cases}
$$

Therefore $\max _{\lambda} h(\lambda)=0$, so

$$
\max _{\lambda} h(\lambda)<\min _{x, y, x^{4}+y^{4}=1} F
$$

There is a "duality gap".

Remark: If our original problem had been to maximize $F(\mathbf{x})$ subject to the constraints $g_{1}=g_{2}=\cdots=g_{m}=0$ we would still have

$$
L(\mathbf{x}, \boldsymbol{\lambda})=F(\mathbf{x})+\sum_{k=1}^{m} \lambda_{k} g_{k}(\mathbf{x})
$$

and the same Lagrange multiplier rule: look for $x_{*}$ where

$$
\frac{\partial F}{\partial x_{i}}+\sum_{k=1}^{m} \lambda_{k} \frac{\partial g_{k}}{\partial x_{i}}=0, \text { and } g_{1}=g_{2}=\cdots=g_{m}=0
$$

If we have an $x_{*}$ that maximises $F$ subject to the constraint and consider

$$
H(\boldsymbol{\lambda})=\sup _{\mathbf{x}}\left(F(\mathbf{x})+\sum_{k=1}^{m} \lambda_{k} g_{k}(\mathbf{x})\right)
$$

then we have $\min _{\boldsymbol{\lambda}} H(\boldsymbol{\lambda}) \geq F\left(\mathbf{x}_{*}\right)$, since whatever $\boldsymbol{\lambda}$ might be, there is an $\mathbf{x}$ (namely $\mathbf{x}_{*}$ ) that makes $L(\mathbf{x}, \boldsymbol{\lambda})=F\left(\mathbf{x}_{*}\right)$, so $\sup _{\mathbf{x}} L(\mathbf{x}, \boldsymbol{\lambda}) \geq F\left(\mathbf{x}_{*}\right)$, and so $\min _{\lambda} H(\boldsymbol{\lambda}) \geq F\left(\mathbf{x}_{*}\right)$.

These maximum minimum dual problems are reminiscent of "Courant's maximum principle", a rather striking result about eigenvalues of symmetric or Hermitian matrices, Sturm-Liouville problems, etc. It will be recalled that the lowest eigenvalue $\lambda_{1}$ of a real symmetric matrix $A$ may be characterized as the minimum of $x^{T} A x / x T x$ for all non-zero 
vectors $x$, this minimum being achieved for $x=e$, the first eigenvector. Similarly the second eigenvalue $\lambda_{2}$ is the minimum of $x^{T} A x / x^{T} x$ for all vectors $x$ that are orthogonal to $e$, and the $k$ th eigenvalue is the same minimum over all vectors orthogonal to $e_{1}, e_{2}, \ldots, e_{k-1}$. Courant pointed out that the $k$ th eigenvalue can be described directly without explicit reference to the previous ones as follows: take an arbitrary set of $k-1$ vectors $v_{1}, \ldots, v_{k-1}$, and form $H\left(v_{1}, \ldots, v_{k-1}\right)=\min \left(x^{T} A x / x^{T} x\right)$ for all non-zero vectors $x$ that are orthogonal to $v_{1}, v_{2}, \ldots, v_{k-1}$. Then $\lambda_{k}=\max _{v_{1}, \ldots, v_{k-1}} H$. (See for instance Courant and Hilbert [1]).

\section{References}

[1] D. Hilbert and R. Courant, Methods of Mathematical Physics Volume I (John Wiley \& Sons, USA, 1989). 


\section{Lecture 5}

\section{Upper Bounds for Turbulent Transport}

F. H. Busse

Notes by Tomoki Tozuka and Huiquin Wang

\section{Introduction}

Malkus [1] observed kinks in $N u$ (Nusselt number) - $R a$ (Rayleigh number) relationship of turbulent Rayleigh-Benard convection and formulated a mean field theory for superposition of convective modes using hypothesis of maximum transport in 1954. In 1963, Howard [2] derived rigorous upper bound for Nusselt number, $N u \leq c R a^{\frac{1}{2}}$. Then, Busse $[3,4]$ improved bounds through incorporation of the continuity equation constraint, introduced multi-alpha solutions of variational problem, and derived upper bound $M \leq c R e^{2}$ (Re: Reynolds number) for an momentum transports in shear layers in 1969. On the other hand, Doering and Constantin [5] extended the method of Hopf to derive bounds on dissipation by turbulent flows in 1994 (see Lecture 6 for the detail). Nicodemus et al. [6] optimized DoeringConstantin approach in 1997 and Kerswell [7] proved the equivalence of Doering-Constantin and Howard-Busse methods in 1998 (see Lecture 10 for the proof). This lecture is focused on the Howard-Busse method.

The theory of upper bounds for functionals of turbulent flows provides rigorous bounds for transport properties. It also indicates characteristic properties of extremalizing vector fields, which are reflected in observations of turbulent flows and thus can provide some insights into properties of turbulence.

\section{Upper Bounds on Momentum Transport Between Two Moving Parallel Plates}

In this section, we consider a flow between two moving parallel plates as shown in Fig. 1. Using the distance $d$ between two plates as length scale, and $d^{2} / \mu$ as time scale, we write the Navier-Stokes equation for the incompressible fluid in the form

$$
\begin{gathered}
\frac{\partial}{\partial t} \mathbf{v}+\mathbf{v} \cdot \nabla \mathbf{v}+2 \Omega \times \mathbf{v}=-\nabla p+\nabla^{2} \mathbf{v} \\
\nabla \cdot \mathbf{v}=0 .
\end{gathered}
$$

We separate the velocity field $\mathbf{v}$ into a mean and a fluctuating part:

$$
\mathbf{v}=\mathbf{U}+\breve{\mathbf{v}} \text { with } \overline{\mathbf{v}}=0, \overline{\mathbf{v}} \equiv \mathbf{U}(z, t)
$$

where 


$$
\bar{\cdots} \equiv \lim _{L \rightarrow \infty} \frac{1}{4 L^{2}} \int_{-L}^{L} \int_{-L}^{L} \cdots d \mathbf{x} .
$$

We also separate the fluctuating part of the velocity field $\breve{v}$ into components perpendicular and parallel to the plates as

$$
\begin{gathered}
\breve{\mathbf{v}} \equiv \breve{\mathbf{u}}+\mathbf{k} \breve{w} \text { with } \breve{\mathbf{u}} \cdot \mathbf{k}=0 \text {. } \\
{[\text { width=]fig1.eps }}
\end{gathered}
$$

Figure 1: Schematic sketch of a flow between two moving parallel plates.

For $\Omega \cdot \mathbf{k}=0$ (e.g. Taylor-Couette case), since $\mathbf{U}$ does not have a $\mathbf{z}$-component because of the continuity equation, the average over planes $z=$ constant of (1) yields

$$
\begin{gathered}
\frac{\partial}{\partial t} \mathbf{U}+\overline{\breve{\mathbf{v}} \cdot \nabla \check{\mathbf{u}}}=\frac{\partial^{2}}{\partial z^{2}} \mathbf{U} \\
\overline{\breve{\mathbf{v}} \cdot \nabla \breve{w}}=-\frac{\partial}{\partial z} \bar{p}-2 \Omega \times \mathbf{U} .
\end{gathered}
$$

Subtracting (6) and (7) from the corresponding components of (1), we obtain the following equation for the fluctuating velocity field $\breve{\mathbf{v}}$ :

$$
\frac{\partial}{\partial t} \breve{\mathbf{v}}+\breve{\mathbf{v}} \cdot \nabla \breve{\mathbf{v}}-\overline{\breve{\mathbf{v}} \cdot \nabla \breve{\mathbf{v}}}+\mathbf{U} \cdot \nabla \breve{\mathbf{v}}+\breve{\mathbf{v}} \cdot \nabla \mathbf{U}+2 \Omega \times \breve{\mathbf{v}}=-\nabla \breve{p}+\nabla^{2} \breve{\mathbf{v}} .
$$

After multiplying the above equation with $\breve{v}$, taking the average over the entire fluid layer, and using the boundary conditions that $\breve{\mathbf{v}}$ vanishes at $z= \pm \frac{1}{2}$, we have the energy relationship

$$
\frac{1}{2} \frac{d}{d t}\left\langle|\breve{\mathbf{v}}|^{2}\right\rangle+\left\langle|\nabla \breve{\mathbf{v}}|^{2}\right\rangle+\left\langle\breve{\mathbf{u}} \cdot\left(\breve{w} \frac{\partial}{\partial z}\right) \mathbf{U}\right\rangle=0
$$

where

$$
\langle\cdots\rangle=\int_{-\frac{1}{2}}^{\frac{1}{2}} \cdots d z .
$$

The above energy relationship (9) can be further simplified if we restrict our attention to the fluid flow under stationary conditions:

$$
\frac{\partial}{\partial t} \mathbf{U}=0 ; \frac{d}{d t}\left\langle|\breve{\mathbf{v}}|^{2}\right\rangle=0
$$


The equation (6) under above condition yields

$$
\frac{d}{d z} \mathbf{U}=\overline{\breve{w} \breve{\mathbf{u}}}-\langle|\breve{w} \breve{\mathbf{u}}|\rangle-R e \cdot \mathbf{i} \text {. }
$$

Hence, using the above equation, we obtain the final form of the energy balance

$$
\left\langle|\nabla \breve{\mathbf{v}}|^{2}\right\rangle+\langle\breve{\mathbf{u}} \breve{w} \cdot(\breve{\mathbf{u}} \breve{w}-\langle\breve{\mathbf{u}} \breve{w}\rangle)\rangle-\operatorname{Re}\left\langle\breve{u_{x}} \breve{w}\right\rangle=0 .
$$

Here, the identity

$$
\left\langle\breve{\mathbf{u}}^{2}\right\rangle-\langle\breve{\mathbf{u}} \breve{w}\rangle^{2}=\left\langle|\overline{\mathbf{u} \breve{w}}-\langle\breve{\mathbf{u}} \breve{w}\rangle|^{2}\right\rangle
$$

has been used.

The momentum transport between two moving plates is obtained from its value at the boundary

$$
M \equiv-\left.\frac{\partial U_{x}}{\partial z}\right|_{z=\frac{1}{2}}=\left\langle\breve{w}_{x}\right\rangle+R e .
$$

Since $\left\langle\breve{w} \breve{u}_{x}\right\rangle \geq 0$, the momentum transport is bounded from below by the value of the laminar solution and increases by $\left\langle\breve{w} \breve{u}_{x}\right\rangle$ for turbulent flow. Thus, the goal here is to derive an upper bound for $\left\langle\breve{u}_{x} \breve{w}\right\rangle$ at a given value of $R e$ and this leads us to the formulation of the following variational problem. For a given $\mu$, find the minimum $R(\mu)$ of the functional

$$
R(\mathbf{v}, \mu) \equiv \frac{\left\langle|\nabla \mathbf{v}|^{2}\right\rangle}{\left\langle u_{x} w\right\rangle}+\mu \frac{\left\langle|\overline{\mathbf{u} w}-\langle\mathbf{u} w\rangle|^{2}\right\rangle}{\left\langle u_{x} w\right\rangle^{2}}
$$

among all vector fields with $\mathbf{v}=0$ at $z= \pm \frac{1}{2}$ where

$$
\mathbf{v} \equiv \mathbf{u}+\mathbf{k} w, \mathbf{u} \cdot \mathbf{k} \equiv \mathbf{0}
$$

Thus, the Euler-Lagrange equations for a stationary value of $R(\mathbf{v}, \mu)$ are

where

$$
\nabla^{2} \mathbf{v}-\nabla \pi=w \frac{d}{d z} \mathbf{U}^{*}+\mathbf{k} \mathbf{u} \cdot \frac{d}{d z} \mathbf{U}^{*}
$$

$$
\frac{d}{d z} \mathbf{U}^{*}=\overline{w \mathbf{u}}-\langle w \mathbf{u}\rangle-\left(R-\frac{\left\langle|\nabla \mathbf{v}|^{2}\right\rangle}{2\left\langle u_{x} w\right\rangle}\right) \mathbf{i}
$$

Since the functional is homogeneous, the normalization $\left\langle\breve{u}_{x} \breve{w}\right\rangle=\mu$ can be assumed.

$$
\begin{aligned}
& \text { The proof for } \frac{d R(\mu)}{d \mu}=\frac{\left\langle|\bar{w} \overline{\mathbf{u}}-\langle w \mathbf{u}\rangle|^{2}\right\rangle}{\left\langle w u_{x}\right\rangle^{2}} \text { is as follows: } \\
& \qquad \begin{aligned}
\left(\mu^{*}-\mu^{\prime}\right) \frac{\left\langle\overline{w^{*} \mathbf{u}^{*}}-\left.\left\langle w^{*} \mathbf{u}^{*}\right\rangle\right|^{2}\right\rangle}{\left\langle w^{*} u_{x}^{*}\right\rangle^{2}} & =R\left(\mathbf{v}^{*}, \mu^{*}\right)-R\left(\mathbf{v}^{*}, \mu^{\prime}\right) \\
& \leq R\left(\mu^{*}\right)-R\left(\mu^{\prime}\right) \\
& \leq R\left(\mathbf{v}^{\prime}, \mu^{*}\right)-R\left(\mathbf{v}^{\prime}, \mu^{\prime}\right) \\
& \leq\left(\mu^{*}-\mu^{\prime}\right) \frac{\left\langle\left|\overline{w^{\prime} \mathbf{u}^{\prime}}-\left\langle w^{\prime} \mathbf{u}^{\prime}\right\rangle\right|^{2}\right\rangle}{\left\langle w^{\prime} u_{x}^{\prime}\right\rangle^{2}}
\end{aligned}
\end{aligned}
$$

where $\mathbf{v}^{*}$ and $\mathbf{v}^{\prime}$ are the extremalizing vector fields for $\mu^{*}$ and $\mu^{\prime}$, respectively. For $\mu^{*} \rightarrow \mu^{\prime}$, the above result follows. 


$$
[\text { width }=] \text { fig2.eps }
$$

Figure 2: Schematic schetch of a thermal convection in a porous medium.

\section{Upper Bounds on the Heat Transport in a Porous Layer}

In this section, we consider a thermal convection in a porous medium as shown in Fig. 2. Using the distance $d$ between two plates as length scale, $d^{2} / \kappa$ as time scale, $\kappa / d$ as velocity scale, and $\left(T_{2}-T_{1}\right) / R$ as temperature scale, we write dimensionless equations based on Darcy-Law as

$$
\begin{gathered}
-\mathbf{u}+\mathbf{k} T-\nabla p=B\left(\frac{\partial}{\partial t} \mathbf{u}+\mathbf{u} \cdot \nabla \mathbf{u}\right) \approx 0 \\
\nabla \cdot \mathbf{u}=0 \\
\nabla^{2} T=\left(\frac{\partial}{\partial t}+\mathbf{u} \cdot \nabla\right) T
\end{gathered}
$$

where

$$
\begin{gathered}
B \equiv \frac{\kappa K}{d^{2} \nu} \\
R \equiv \frac{\gamma g K d\left(T_{2}-T_{1}\right)}{\nu \kappa} .
\end{gathered}
$$

and $K$ is the Darcy permeability coefficient.

We separate the temperature field $T$ into a mean and a fluctuating part

$$
T=\bar{T}+\theta, \text { with } \bar{\theta}=0
$$

By subtracting the horizontal average of (22) from (22), we obtain

$$
\begin{gathered}
\frac{\partial}{\partial t} \bar{T}+\overline{\mathbf{u} \cdot \nabla \bar{\theta}}=\frac{\partial^{2}}{\partial z^{2}} \bar{T} \\
\left(\frac{\partial}{\partial t}+\mathbf{u} \cdot \nabla \theta\right)+w \frac{\partial \bar{T}}{\partial z}-\frac{\partial}{\partial z} \overline{w \theta}=\nabla^{2} \theta
\end{gathered}
$$

Assuming the statistically stationary turbulence, we integrate (26) and obtain 


$$
\frac{\partial}{\partial z} \bar{T}=\overline{w \theta}-\langle w \theta\rangle-R
$$

By multiplying (20) by $\mathbf{u}$ and (27) by $\theta$, taking the average over the whole porous layer, and using (28), we obtain two dissipation integral relationships:

$$
\begin{gathered}
\left\langle|\mathbf{u}|^{2}\right\rangle=\langle w \theta\rangle \\
\left\langle|\nabla \theta|^{2}\right\rangle+\left\langle|\overline{w \theta}-\langle w \theta\rangle|^{2}\right\rangle=R\langle w \theta\rangle .
\end{gathered}
$$

The dimensionless heat transport across the porous layer can be obtained from its value at the boudary:

$$
H=-\left.\frac{\partial \bar{T}}{\partial z}\right|_{z= \pm \frac{1}{2}}=R+\langle w \theta\rangle \geq R
$$

Since $\langle w \theta\rangle$ is always positive from (29), the heat transport for the turbulent flow is always greater than that for the laminar flow, and it is bounded from below by the value of the laminar solution.

The goal here is to find an upper bound on the heat $\operatorname{transport}$ or $\langle w \theta\rangle$ at a given value of $\mathrm{R}$. We are thus led to the formulation of the following variational problem. For given $\mu>0$, find the minimum $P(\mu)$ of the functional

$$
P(\mathbf{u}, \theta, \mu) \equiv \frac{\left\langle|\mathbf{u}|^{2}\right\rangle\left\langle|\nabla \theta|^{2}\right\rangle+\mu\left\langle|\overline{w \theta}-\langle w \theta\rangle|^{2}\right\rangle}{\langle w \theta\rangle^{2}}
$$

for all fields $\mathbf{u}$ and $\theta$, which satisfy the constraint $\nabla \cdot \mathbf{u}=0$ and the boundary condition $w=\theta=0$ at $z= \pm \frac{1}{2}$. First, from the general form of the dissipation integral

$$
\left\langle|\mathbf{u}|^{2}\right\rangle \equiv\left\langle\nabla^{2} v \Delta_{2} v\right\rangle+\left\langle|\mathbf{k} \times \nabla \psi|^{2}\right\rangle
$$

and the property

$$
w=-\left(\frac{\partial^{2}}{\partial x^{2}}+\frac{\partial^{2}}{\partial y^{2}}\right) v \equiv-\Delta_{2} v
$$

it is clear that the minimum of the functional is obtained for $\nabla \times \mathbf{k} \psi=0$. Hence, the variational problem now depends only on the scalar variables $v$ and $\theta$.

The Euler-Lagrange equations for a stationary value can be thus written as

$$
\begin{aligned}
& \left\langle|\nabla \theta|^{2}\right\rangle \nabla^{2} w-[P\langle w \theta\rangle+\mu(\langle w \theta\rangle-\overline{w \theta})] \Delta_{2} \theta=0 \\
& \left\langle\nabla^{2} v \Delta_{2} v\right\rangle \nabla^{2} \theta+[P\langle w \theta\rangle+\mu(\langle w \theta\rangle-\overline{w \theta})] w=0
\end{aligned}
$$


Now, the nonlinearity is only through $z$-dependence and the equations are linear with respect to the $x, y$ dependence. This property allows us to write solutions in the form of superposition of waves. Because of the homogenity with respect to $\mathrm{x}$ and $\mathrm{y}$ in $\mathrm{w}$ and $\theta$, we can impose the following normalization conditions:

$$
\begin{gathered}
\left\langle|\nabla \theta|^{2}\right\rangle=1 \\
\left\langle\nabla^{2} v \Delta_{2} v\right\rangle=\left\langle|\mathbf{k} \times \nabla \nabla v|^{2}\right\rangle=1
\end{gathered}
$$

Then, we introduce the following general solutions for $w$ and $\theta$

$$
\begin{aligned}
& w=w^{(N)} \equiv \sum_{k=1}^{N} \alpha_{n}^{\frac{1}{2}} w_{n}(z) \Phi_{n}(x, y) \\
& \theta=\theta^{(N)} \equiv \sum_{k=1}^{N} \alpha_{n}^{-\frac{1}{2}} \theta_{n}(z) \Phi_{n}(x, y)
\end{aligned}
$$

where $\Phi_{n}$ satisfies the equation:

$$
\Delta_{2} \Phi_{n}=-\alpha_{n}^{2} \Phi_{n}
$$

and the orthonormalization condition

$$
\overline{\Phi_{n} \Phi_{m}}=\delta_{m n}
$$

Then, the Euler-Lagrangian equations can be reduced to

$$
\begin{aligned}
& \left(\frac{\partial^{2}}{\partial z^{2}}-\alpha_{n}^{2}\right) w_{n}+\alpha_{n} \Psi \theta_{n}=0 \\
& \left(\frac{\partial^{2}}{\partial z^{2}}-\alpha_{n}^{2}\right) \theta_{n}+\alpha_{n} \Psi w_{n}=0
\end{aligned}
$$

where

$$
\Psi \equiv P \sum_{n=1}^{N}\left\langle w_{n} \theta_{n}\right\rangle+\mu \sum_{n=1}^{N}\left(\left\langle w_{n} \theta_{n}\right\rangle-\overline{w_{n} \theta_{n}}\right)
$$

The above equations have the following properties [8]:

(1) By considering the equations for $w_{n}+\theta_{n}$ and $w_{n}-\theta_{n}$, we can obtain

$$
w_{n}=\theta_{n} .
$$


Thus, the problem can be reduced to

$$
\left(\frac{\partial^{2}}{\partial z^{2}}-\alpha_{n}^{2}\right) \theta_{n}+\alpha_{n} \Psi \theta_{n}=0
$$

(2) The functions $\theta_{n}(z)$ are either symmetric or antisymmetric in $z$.

(3) Since $\theta_{n} \equiv \theta_{m}$ follows from $\alpha_{n}=\alpha_{m}$, it can be assumed that all $\alpha_{n}$ are different.

(4) For $m \neq n$, by subtracting the $\mathrm{n}$-th equation of (47) multiplied by $\alpha_{n}^{-1} \theta_{m}$ from the m-th equation multiplied by $\alpha_{m}^{-1} \theta_{n}$, and averaging it using the partial integration, we obtain an important property

$$
\left\langle\theta_{m}^{\prime} \theta_{n}^{\prime}\right\rangle-\alpha_{m} \alpha_{n}\left\langle\theta_{m} \theta_{n}\right\rangle=0
$$

where $\theta_{m}^{\prime}$ denotes the z-derivative of $\theta_{m}$.

(5) Minimization of $P\left(\theta_{n}, \alpha_{n}, \mu\right)$ with respect to $\alpha_{n}$ yields

$$
\begin{gathered}
\frac{\partial}{\partial \alpha_{n}} I=0 \\
\alpha_{n}^{2}=\frac{\left\langle\theta_{n}^{\prime} \theta_{m}^{\prime}\right\rangle}{\left\langle\theta_{n} \theta_{m}\right\rangle} .
\end{gathered}
$$

\section{References}

[1] W. Malkus, "The heat transport and spectrum of thermal turbulence," Proc. Roy. Soc. London 225, 185 (1954).

[2] L. N. Howard, "Heat transport by turbulent transport," J. Fluid Mech. 17, 405 (1963).

[3] F. H. Busse, "Bounds on the transport of mass and momentum by turbulent flow beteen parallel plates," J. Applied Math. Phys. 20, 1 (1969).

[4] F. H. Busse, "On Howard's upper bound for heat transport by turbulent convection," J. Fluid Mech. 37, 457 (1969).

[5] C. R. Doering and P. Constantin, "Variational bounds on energy dissipation in incompressible flows; shear flow," Phys. Rev. E 49, 4087 (1994).

[6] S. G. R. Nicodemus and M. Holthaus, "Variational bound on energy dissipation in plate Couette flow," Phys. Rev. E 56, 6774 (1997).

[7] R. R. Kerswell, "Unification of variational principles for turbulent shear flows: The background method of Doering-Constantin and Howard-Busse's mean-fluctuation formulation," Physica D 121, 175 (1998).

[8] F. H. Busse and D. D. Joseph, "Bounds for heat transport in a porous layer," J. Fluid Mech. 54, 521 (1972). 


\section{References}

[1] W. Malkus, "The heat transport and spectrum of thermal turbulence," Proc. Roy. Soc. London 225, 185 (1954).

[2] L. N. Howard, "Heat transport by turbulent transport," J. Fluid Mech. 17, 405 (1963).

[3] F. H. Busse, "Bounds on the transport of mass and momentum by turbulent flow beteen parallel plates," J. Applied Math. Phys. 20, 1 (1969).

[4] F. H. Busse, "On Howard's upper bound for heat transport by turbulent convection," J. Fluid Mech. 37, 457 (1969).

[5] C. R. Doering and P. Constantin, "Variational bounds on energy dissipation in incompressible flows; shear flow," Phys. Rev. E 49, 4087 (1994).

[6] S. G. R. Nicodemus and M. Holthaus, "Variational bound on energy dissipation in plate Couette flow," Phys. Rev. E 56, 6774 (1997).

[7] R. R. Kerswell, "Unification of variational principles for turbulent shear flows: The background method of Doering-Constantin and Howard-Busse's mean-fluctuation formulation," Physica D 121, 175 (1998).

[8] F. H. Busse and D. D. Joseph, "Bounds for heat transport in a porous layer," J. Fluid Mech. 54, 521 (1972). 


\section{Lecture 6}

\section{Bounds on Turbulent Transport \\ C. Doering}

Notes by A. Alexakis \& E. Evstatiev

\section{Introduction to the Background Method}

The background method is a mathematical technique for deriving rigorous bounds on the energy dissipation rate in Navier-Stokes and similar problems.

\section{Momentum Transport Across a Shear Flow}

To introduce the general idea of the background method we are going to consider the example of momentum transport across a shear layer. Consider a flow between two two finite plates. The bottom plate is at rest while the top plate is moving with speed $U_{*}$, see Fig. 1. We introduce Cartesian coordinates with unit vectors $\mathbf{i}, \mathbf{j}$ and $\mathbf{k}$ so that the upper plate is moving in the $x$ direction, the lower plate is in the $y=0$ plane, the upper plate is in the $y=h$ plane. The boundary conditions for this problem are periodic in $x$ and $z$. The area of the plates is $A$; eventually we will take $A \rightarrow \infty$ to describe the problem in an infinite domain.

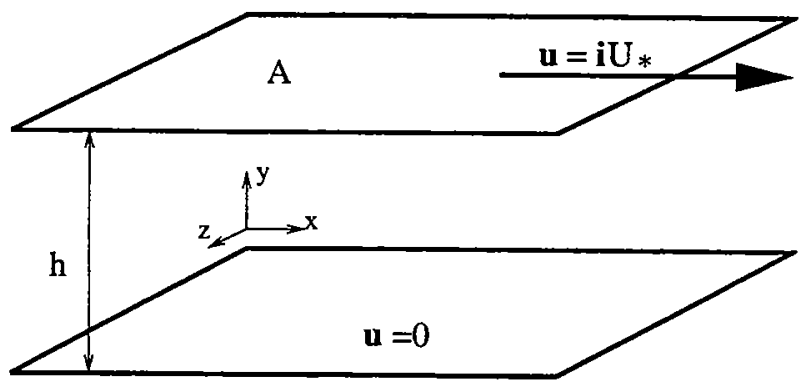

Figure 1: The Plane Couette flow.

The incompressible Navier-Stokes equations are

$$
\begin{gathered}
\partial_{t} \mathbf{u}+\mathbf{u} \cdot \nabla \mathbf{u}+\frac{1}{\rho} \nabla p=\nu \Delta \mathbf{u} \\
\nabla \cdot \mathbf{u}=0 .
\end{gathered}
$$

We start by asking the question: What is the vertical flux of horizontal momentum across the layer?

Dimensional analysis shows 


$$
\begin{aligned}
\text { Momentum flux } & =\frac{\text { horizontal momentum }}{\text { time } \times \text { area }} \\
& =\frac{\text { horizontal force }}{\text { area }}=\frac{F}{A} \\
& =\text { wall shear stress }=\tau .
\end{aligned}
$$

The dimensions of the quantities are

$$
[\tau]=\left[\frac{F}{A}\right]=\frac{M L}{T^{2}} \frac{1}{L^{2}}=M \frac{1}{T^{2}} \frac{1}{L}
$$

The system parameters' dimensions are $\left[U_{*}\right]=L / T,[h]=L,[A]=L^{2},[\nu]=L^{2} / T$, and $[\rho]=M / L^{3}$, where $M$ denotes the dimension of mass. With these we can express the space-time averaged momentum flux as

$$
\tau=\rho h A\left(\frac{U_{*}}{h}\right)^{2}\left(\frac{h}{A}\right) \times \beta\left(\frac{U_{*} h}{\nu}, \frac{A}{h^{2}}\right)=\rho U_{*}^{2} \times \beta(R e, a),
$$

where $R e=\frac{U_{*} h}{\nu}$ and $a=\frac{A}{h^{2}}$. We are interested in the function $\beta(R e, a)$, the dimesionless function of two dimensionless variables that fully describe the system.

An alternative version of the question could be posed in the following way. Define the time averaged dissipation rate per unit mass $\epsilon$, which is also equal to the time averaged power input required to maintain the flow. Quantitatively

$$
\epsilon=\frac{F U_{*}}{\rho A h}=\frac{\tau}{\rho} \frac{U_{*}}{h}
$$

Remembering the expression for $\tau$ in (4), we can write

$$
\epsilon=\frac{U_{*}^{3}}{h} \times \beta(R e, a)
$$

Therefore a bound on the energy dissipation rate gives also a bound on the momentum transport.

In what follows we derive bounds on $\epsilon$. First let us guess what we might find. For laminar flow we can expect the following dependence

$$
\tau \sim \rho \nu \frac{U_{*}}{h} \Rightarrow \beta \sim \frac{1}{R e} .
$$

For turbulent flow we do not expect dependence on the viscosity as we take $\nu \rightarrow 0$ (due to the cascade picture of energy transport across length scales), so we can write 


$$
\epsilon \sim U_{*}^{2} \frac{U_{*}}{h} \Rightarrow \beta \sim R e^{0}
$$

Further we consider the exact stationary solution of the Navier-Stokes equations for plane Couette flow, see Fig. 1:

$$
\mathbf{u}_{s t}=\mathrm{i} \frac{U_{*}}{h} y, \quad p_{s t}=\mathrm{const}, \quad \tau_{s t}=\rho \nu \frac{U_{*}}{h} \quad \beta_{s t}=\frac{1}{R e} .
$$

Now we set up a minimization problem. Let us multiply the Navier-Stokes equations (1) by $\rho \mathbf{u}$ and integrate over the volume. We have

$$
\frac{d}{d t} \int \frac{1}{2} \rho|\mathbf{u}|^{2} d^{3} x=-\int \rho \nu|\nabla \mathbf{u}|^{2} d^{3} x+U_{*} \int_{\text {top plate }} \rho \nu \frac{\partial u_{1}}{\partial y} d x d z
$$

The only surface integral that survives after we integrate by parts is over the top plate of the volume. The term on the left-hand side of (10) is the kinetic energy of the fluid. The first term on the right-hand side is the instantaneous bulk dissipation rate, and the second term on the right-hand side is the input power (equal to $U_{*} F(t)$, where $F(t)$ is the instantaneous force applied to sustain the motion of the upper plate.) Suppose the kinetic energy behaves as $o(t)$ for large times, then its long time average vanishes, and we arrive at the following definition of the space-time averaged dissipation energy

$$
\epsilon=\left\langle\nu|\nabla \mathbf{u}|^{2}\right\rangle
$$

Therefore it is obvious that

$$
\epsilon \geq \min _{\substack{\nabla \cdot \mathbf{u}=\left.0 \\ \mathbf{u}\right|_{y=0}=\left.0 \\ \mathbf{u}\right|_{y=h}=U_{*}}}\left\langle\nu|\nabla \mathbf{u}|^{2}\right\rangle
$$

To put this in a variational frame, we consider the functional

$$
\mathcal{F}[\mathbf{u}]=\int\left(\nu|\nabla \mathbf{u}|^{2}-2 q(x) \nabla \cdot \mathbf{u}\right) d^{3} x
$$

where $q(x)$ is a Lagrange multiplier, enforcing the divergence-free constraint, which plays the role of a pressure. Variation of the above functional with respect to $\mathbf{u}$ and $q$ and equating the results to zero yields

$$
\begin{aligned}
& 0=\frac{1}{2} \frac{\delta \mathcal{F}}{\delta \mathbf{u}}=-\nu \Delta \mathbf{u}+\nabla q \\
& 0=-\frac{1}{2} \frac{\delta \mathcal{F}}{\delta q}=\nabla \cdot \mathbf{u}
\end{aligned}
$$


Thus we obtain the stationary Stokes equations as the Euler-Lagrange equations. The solution is given by (9) where $p_{s t}$ is substituted by $q_{s t}$. We would like to know next if this solution really gives a minimum. The answer is affirmative and the proof follows from the sequence of realtions below.

Define

$$
\mathbf{u}=\mathbf{u}_{s t}+\mathbf{v}
$$

where $\mathbf{v}$ is the fluctuating deviation of $\mathbf{u}$ from plane Couette flow, satisfying

$$
\nabla \cdot \mathbf{v}=0,\left.\quad \mathbf{v}\right|_{y=0}=\left.\mathbf{v}\right|_{y=h}=0 .
$$

The gradient of $\mathbf{u}$ is given by

$$
\nabla \mathbf{u}=\mathbf{i j} \frac{U_{*}}{h}+\nabla \mathbf{v}
$$

from which follows

$$
|\nabla \mathbf{u}|^{2}=\frac{U_{*}^{2}}{h^{2}}+2 \frac{U_{*}}{h} \frac{\partial v_{1}}{\partial y}+|\nabla \mathbf{v}|^{2}
$$

If we find $\epsilon$ from formula (11), we obtain, after space-time averaging (noting that the cross term vanishes),

$$
\epsilon=\nu \frac{U_{*}^{2}}{h^{2}}+\left\langle\nu|\nabla \mathbf{v}|^{2}\right\rangle \geq \epsilon_{s t}
$$

which shows that indeed the solution (9) gives a minimum of $\epsilon$. Note that plane Couette flow is a solution for all $R e$ and $a$ so this lower bound is sharp. And sometimes this lower bound is also an upper bound.

We ask the question of when this solution is absolutely stable. Let us consider the equations for $\mathbf{v}$. They follow from the Navier-Stokes equations after the substitution of (15)

$$
\begin{aligned}
& \partial_{t} \mathbf{v}+\mathbf{v} \cdot \nabla \mathbf{u}_{s t}+\mathbf{u}_{s t} \cdot \nabla \mathbf{v}+\frac{1}{\rho} \nabla p=\nu \Delta \mathbf{v} \\
& \nabla \cdot \mathbf{v}=0
\end{aligned}
$$

We will prove the following statement: Plane Couette flow is absolutely stable-and hence the unique time asymptotic flow-for sufficiently low Re. To see that, multiply the first of Eqs. (20) by $\mathbf{v}$ and integrate over the volume

$$
\frac{d}{d t} \frac{1}{2} \int|\mathbf{v}|^{2} d^{3} x=-\int\left(\nu|\nabla \mathbf{v}|^{2}+\mathbf{v} \cdot\left(\nabla \mathbf{u}_{s t}\right)_{\mathrm{sym}} \cdot \mathbf{v}\right) d^{3} x
$$


and $\left(\nabla \mathbf{u}_{s t}\right)_{\text {sym }}=1 / 2\left(\left(\nabla \mathbf{u}_{s t}\right)+\left(\nabla \mathbf{u}_{s t}\right)^{t r}\right)$. On the left-hand side of this equation is time derivative of the perturbation energy $\mathcal{E}(t)=\int|\mathbf{v}|^{2} d^{3} x$. On the right-hand side we have a quadratic in the perturbation $\mathbf{v}$ form.

We note the inequality (sometimes referred to as Poincaré's inequality)

$$
\int|\nabla \mathbf{v}|^{2} \geq \frac{\pi^{2}}{h^{2}} \int|\mathbf{v}|^{2}
$$

Then we calculate

$$
\begin{aligned}
& \left|\int \mathbf{v} \cdot\left(\nabla \mathbf{u}_{s t}\right)_{\mathrm{sym}} \cdot \mathbf{v} d^{3} x\right|=\left|\int \frac{U_{*}}{h} v_{1} v_{2} d^{3} x\right| \leq \\
& \frac{U_{*}}{h} \int \frac{1}{2}\left(v_{1}^{2}+v_{2}^{2}\right) d^{3} x \leq \frac{U_{*}}{2 h} \int|\mathbf{v}|^{2} d^{3} x .
\end{aligned}
$$

So for the perturbation energy we have

$$
\begin{aligned}
\frac{d \mathcal{E}(t)}{d t} & \leq-2\left(\nu \frac{\pi^{2}}{h^{2}}-\frac{U_{*}}{2 h}\right) \mathcal{E}(t)= \\
& =-\frac{\nu}{h^{2}}\left(2 \pi^{2}-R e\right) \mathcal{E}(t)
\end{aligned}
$$

and finally using Gronwall's lemma we have

$$
\mathcal{E}(t) \leq \mathcal{E}(0) e^{-\frac{\nu}{h^{2}}\left(2 \pi^{2}-R e\right) t} \rightarrow 0 \quad \text { if } \quad R e<2 \pi^{2} \approx 20
$$

which proves the assertion.

A more precise calculation shows that the critical value of the Reynolds number for this kind of energy stability is $R e_{E} \approx 82.6$. To see how we can get a more precise value consider

$$
\frac{d \mathcal{E}(t)}{d t}=-2\left[\frac{\int\left(\nu|\nabla \mathbf{v}|^{2}+\mathbf{v} \cdot\left(\nabla \mathbf{u}_{s t}\right)_{\mathrm{sym}} \cdot \mathbf{v}\right) d^{3} x}{\int|\mathbf{v}|^{2} d^{3} x}\right] \mathcal{E}(t) \leq-2 \lambda_{0} \mathcal{E}(t)
$$

where

$$
\lambda_{0}=\min _{\substack{\nabla \cdot \mathbf{v}=0 \\ \mathbf{v}|\mathbf{v}|^{2} d^{3} x=1 \\ \mathbf{v}=0=\left.\mathbf{v}\right|_{y=h}=0}} \int\left(\nu|\nabla \mathbf{v}|^{2}+\mathbf{v} \cdot\left(\nabla \mathbf{u}_{s t}\right) \cdot \mathbf{v}\right) d^{3} x
$$

Define the functional

$$
\mathcal{F}[\mathbf{v}]=\int\left[\nu|\nabla \mathbf{v}|^{2}+\mathbf{v} \cdot\left(\nabla \mathbf{u}_{s t}\right) \cdot \mathbf{v}-2 p(x) \nabla \cdot \mathbf{v}-\lambda\left(|\mathbf{v}|^{2}-\frac{1}{A h}\right)\right] d^{3} x
$$


Upon variation with respect to $\mathbf{v}$ we obtain the eigenvalue problem

$$
\lambda \mathbf{v}=-\nu \Delta \mathbf{v}+\nabla p+\left(\nabla \mathbf{u}_{s t}\right) \cdot \mathbf{v}
$$

The region in the $R e-a$ phase plane where the lowes eigenvalue is positive defines the parameter region of energy stability of plane Couette flow. In applications to bound other solutions we need to generalize the method described in this section and this is done next.

\section{3 "Background" Method}

In the previous section we saw that the stationary solution (9) only exists as an absolutely stable solution for sufficiently low Reynolds numbers. For high Reynolds numbers we can not use it to put an upper bound on the energy dissipation rate. However, we are going to present a more general technique that mimics to some extent what we did in the previous section.

Decompose a general solution of the Navier-Stokes equation as

$$
\mathbf{u}=\mathbf{i} U(y)+\mathbf{v}(x, t)
$$

We call the vector field $\mathbf{i} U(y)$ a "background" field. The other part of the decomposition is a "fluctuating" field. The purpose of the background ${ }^{1}$ field is to "absorb" the boundary conditions, whereas the fluctuating part satisfies homogeneous boundary conditions:

$$
U(0)=0, \quad U(h)=U_{*},\left.\quad \mathbf{v}\right|_{y=0}=\left.\mathbf{v}\right|_{y=h}=0
$$

Next, from (1) and (30) we derive

$$
\partial_{t} \mathbf{v}+\mathbf{v} \cdot \nabla \mathbf{v}+U(y) \partial_{x} \mathbf{v}+\mathbf{i} v_{2} U^{\prime}(y)+\nabla p=\nu \Delta \mathbf{v}+\mathbf{i} \nu U^{\prime \prime}(y)
$$

and for the fluctuation energy evolution

$$
\frac{d}{d t} \frac{1}{2} \int|\mathbf{v}|^{2} d^{3} x=-\nu \int|\nabla \mathbf{v}|^{2} d^{3} x-\int U^{\prime}(y) v_{1} v_{2} d^{3} x-\nu \int U^{\prime}(y) \frac{\partial v_{1}}{\partial y} d^{3} x
$$

As before, note that $\nabla \mathbf{u}=\nabla \mathbf{v}+\mathbf{j i} U^{\prime}(y)$ and derive

$$
\frac{1}{2} \nu \int|\nabla \mathbf{u}|^{2} d^{3} x=\frac{1}{2} \nu \int|\nabla \mathbf{v}|^{2} d^{3} x+\frac{A}{2} \nu \int_{0}^{h} u^{\prime}(y)^{2} d y+\nu \int U^{\prime}(y) \frac{\partial v_{1}}{\partial y} d^{3} x
$$

Adding (33) and (34) we get

\footnotetext{
${ }^{I}$ From now on we drop the quotes on the words background and fluctuating but we should keep in mind that the background field is not (necessarily) a mean flow.
} 


$$
\frac{d}{d t} \int|\mathbf{v}|^{2} d^{3} x+\nu \int|\nabla \mathbf{u}|^{2} d^{3} x=-\int\left(\nu|\nabla \mathbf{v}|^{2}+2 U^{\prime}(y) v_{1} v_{2}\right) d^{3} x+A \nu \int_{0}^{h} U^{\prime}(y)^{2} d y
$$

The terms in the formula above can be identified as follows. The second term on the lefthand side is the total instantaneous dissipation rate, the second term on the right-hand side is the dissipation rate in the background flow, and the first term on the right-hand side is a quadratic form which we denote by $Q_{u}\{\mathbf{v}\}$.

The key point is: If we can find a background profile $U(y)$ so that $Q_{U}\{\mathbf{v}\}>0$, i.e., so that $Q_{U}\{\mathbf{v}\} \geq c \int|\mathbf{v}|^{2} d^{3} x$ with $c>0$, then

a) We are convinced that the kinetic energy is uniformly bounded in time (even as $t \rightarrow \infty$ ) because then

$$
\frac{d}{d t} \int|\mathbf{v}|^{2} d^{3} x \leq-c \int|\mathbf{v}|^{2} d^{3} x+A \nu \int_{0}^{h} U^{\prime}(y)^{2} d y
$$

from where after integrating, we deduce

$$
\int|\mathbf{v}|^{2} d^{3} x \leq e^{-c t} \int|\mathbf{v}(x, 0)|^{2} d^{3} x+\frac{1}{c}\left(1-e^{-c t}\right) A \nu \int_{0}^{h} U^{\prime}(y)^{2} d y
$$

b) The background flow produces an upper bound on $\epsilon$, for then the time averaged equation (35) gives

$$
\epsilon=\left\langle\nu|\nabla \mathbf{u}|^{2}\right\rangle \leq \frac{1}{h} \int_{0}^{h} U^{\prime}(y)^{2} d y
$$

\subsection{Trial background Method}

Lets take the background profile to be the piecewise-linear velocity profile given by the one shown in the Fig. 2. We can make the following estimate: using the fundamental theorem of calculus and the Cauchy-Schwarz inequality:

$$
\left|v_{i}\right|=\left|\int_{0}^{y} \frac{\partial v_{i}}{\partial y}\left(y^{\prime}\right) d y^{\prime}\right|=\left|\int_{0}^{y} 1 \cdot \frac{\partial v_{i}}{\partial y}\left(y^{\prime}\right) d y^{\prime}\right| \leq \sqrt{y}\left|\int_{0}^{y}\left(\frac{\partial v_{i}}{\partial y}\left(y^{\prime}\right)\right)^{2} d y^{\prime}\right|^{1 / 2}
$$

This implies

$$
\begin{gathered}
\left|\int_{0}^{h} U^{\prime}(y) v_{1} v_{2} d x^{3}\right| \leq \frac{U_{*}}{2 \delta} \int d x d z \int_{0}^{\delta} y\left(\int_{0}^{h / 2}\left(\frac{\partial v_{1}}{\partial y}\right)^{2} d y^{\prime}\right)^{1 / 2}\left(\int_{0}^{h / 2}\left(\frac{\partial v_{2}}{\partial y}\right)^{2} d y^{\prime}\right)^{1 / 2} d y+ \\
\frac{U_{*}}{2 \delta} \int d x d z \int_{h-\delta}^{h}(h-y)\left(\int_{h / 2}^{h}\left(\frac{\partial v_{1}}{\partial y}\right)^{2} d y^{\prime}\right)^{1 / 2}\left(\int_{h / 2}^{h}\left(\frac{\partial v_{2}}{\partial y}\right)^{2} d y^{\prime}\right)^{1 / 2} d y
\end{gathered}
$$




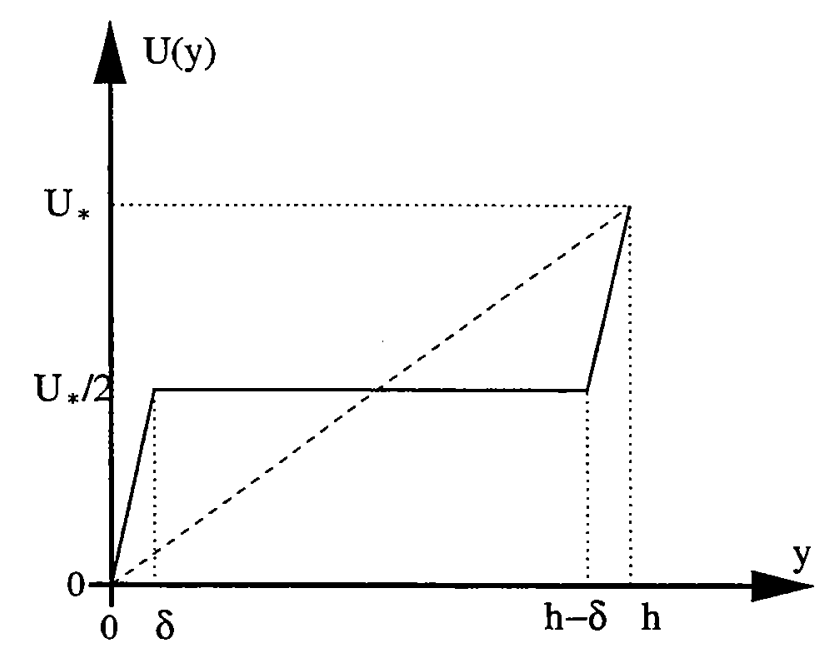

Figure 2: Trial function for the background profile.

Including all the other terms in $|\nabla \mathbf{v}|^{2}$ we obtain

$$
\left|\int U^{\prime}(y) v_{1} v_{2} d x^{3}\right| \leq \frac{U_{*}}{2 \delta} \frac{\delta^{2}}{2} \frac{1}{2} \int|\nabla \mathbf{v}|^{2} d x^{3}=\frac{U_{*} \delta}{8} \int|\nabla \mathbf{v}|^{2} d x^{3}
$$

This implies for $Q$ :

$$
\begin{gathered}
Q_{U}\{\mathbf{v}\}=\int\left(\nu|\nabla \mathbf{v}|^{2}+2 U^{\prime} v_{1} v_{2}\right) d x^{3} \geq \int \nu|\nabla \mathbf{v}|^{2} d x^{3}-2\left|\int U^{\prime} v_{1} v_{2} d x^{3}\right| \\
\geq \int \nu|\nabla \mathbf{v}|^{2} d x^{3}-\frac{U_{*} \delta}{4} \int|\nabla \mathbf{v}|^{2} d x^{3} \geq\left(\nu-\frac{U_{*} \delta}{4}\right) \int|\nabla \mathbf{v}|^{2} d x^{3} \geq \frac{\pi^{2}}{h^{2}}\left(\nu-\frac{U_{*} \delta}{4}\right) \int|\mathbf{v}|^{2} d x^{3} .
\end{gathered}
$$

So $Q_{U}\{v\} \geq 0$ if we choose $\delta \leq 4 \nu / U_{*}=4 h / R e$. This is the maximum value of $\delta$ that our estimates allow us to use, and gives a bound on the maximum possible energy dissipation rate for the set of background functions $U$ that we have chosen. Using this value of $\delta$ we obtain

$$
\epsilon \leq \frac{\nu}{h} \int_{0}^{h} U^{\prime}(y)^{2} d y=\frac{1}{8} \frac{U_{*}^{3}}{h} \quad \Rightarrow \quad \beta \leq \frac{1}{8}
$$

\section{Variational Problem for Optimal Background}

We can pose the following question: What is the optimal background velocity profile that gives the smallest possible bound

$$
\epsilon \leq \min _{U}\left\{\frac{1}{h} \int_{0}^{h} \nu U^{\prime}(y)^{2} d y\right\}
$$


under the constraints $Q_{U}\{\mathbf{v}\} \geq 0, U(0)=0$ and $U(h)=U_{*}$, where

$$
Q_{U}\{\mathbf{v}\}=\int\left[\nu|\nabla \mathbf{v}|^{2}+2 v_{1} v_{2} U^{\prime}(y)\right] d x^{3} .
$$

The constrain $Q_{U}\{\mathbf{v}\} \geq 0$ is equivalent to the spectral constraint $\lambda_{U} \geq 0$ where

$$
\lambda_{U}=\min _{\mathbf{v}} \frac{\int\left[\nu|\nabla \mathbf{v}|^{2}+2 v_{1} v_{2} U^{\prime}(y)\right] d x^{3}}{\int|\mathbf{v}|^{2} d x^{3}}
$$

under the constraints $\nabla \cdot \mathbf{v}=0$ and $\mathbf{v}=0$ at $y=0, h . \lambda$ is equal to the lowest eigenvalue of

$$
\begin{aligned}
\lambda \mathbf{v} & =-\nu \Delta \mathbf{v}+\nabla p+\mathbf{i} U^{\prime}(y) v_{2}+\mathbf{j} U^{\prime}(y) v_{1} \\
\nabla \cdot \mathbf{v} & =0 .
\end{aligned}
$$

We can then substitute the $Q_{U}(\mathbf{v}) \geq 0$ in (44) constraint with $\lambda_{U} \geq 0$

\subsection{The Geometry of the Spectral Constraint}

Let $U^{\prime}(y)=U_{*} / h+\phi(y)$ so that $\int_{0}^{h} \phi(y) d y=0$. Then equation (38) can be written as

$$
\epsilon \leq \min _{\phi}\left[\nu \frac{U_{*}^{2}}{h_{2}}+\frac{\nu}{h} \int_{0}^{h} \phi(y)^{2} d y\right]
$$

with the constraints $\int_{0}^{h} \phi d y=0$ and $\lambda_{\phi} \geq 0$ where we replace the label $U$ with $\phi$ in the spectral constraint. There is one remark we want to make for the above minimization problem:

The set of functions $\phi(y)$ with $\lambda_{\phi} \geq 0$ is convex Proof:

Concider two mean-zero functions $\phi_{1}(y)$ and $\phi_{2}(y)$. Then

$$
\lambda_{\phi_{1}} \geq 0 \Leftrightarrow \text { For every } \tilde{\mathbf{u}}, \quad \int\left(\frac{\nu}{2}|\nabla \tilde{\mathbf{u}}|^{2}+\left(\frac{U_{*}}{h}+\phi_{1}(y)\right) \tilde{u}_{1} \tilde{u}_{2}\right) d x^{3} \geq 0
$$

and

$$
\lambda_{\phi_{2}} \geq 0 \Leftrightarrow \text { For every } \tilde{\mathbf{u}}, \quad \int\left(\frac{\nu}{2}|\nabla \tilde{\mathbf{u}}|^{2}+\left(\frac{U_{*}}{h}+\phi_{2}(y)\right) \tilde{u}_{1} \tilde{u}_{2}\right) d x^{3} \geq 0 .
$$

Now let $0<a<1$. Using linearity in $\phi$ and the hypothesis that $\lambda_{\phi_{1}} \geq 0$ and $\lambda_{\phi_{2}} \geq 0$ we see that

$$
\int\left(\frac{\nu}{2}|\nabla \tilde{\mathbf{u}}|^{2}+\left(\frac{U_{*}}{h}+a \phi_{1}(y)+(1-a) \phi_{2}\right) \tilde{u}_{1} \tilde{u}_{2}\right) d x^{3} \geq 0 \Leftrightarrow \lambda_{a \phi_{1}+(1-a) \phi_{2}} \geq 0 .
$$

This proves that the set $\Phi=\left\{\phi \mid \lambda_{\phi}>0\right\}$ is convex. A sketch of the set $\Phi$ is shown in figure 3 , where the curve indicates the functions $\phi(y)$ that have $\lambda_{\phi}=0$. 


\subsection{Euler-Lagrange Equations for Optimal $\phi$}

It is clear from Fig. 3 that the condition for the unique minimizing $\phi$ (the $\phi$ closest in norm to $\phi=0)$ is

$$
\phi(y)=\gamma \mathcal{P}\left\{\frac{\delta \lambda_{\phi}}{\delta \phi}\right\}
$$

where $\mathcal{P}$ stands for projection onto mean zero function space. (The vector $\phi$ that is the minimum must be parallel to the gradient of $\lambda_{\phi}$ at $\lambda_{\phi}=0$.) The proportionality factor $\gamma$ is a Lagrange multiplier. To evaluate $\delta \lambda / \delta \phi$ we begin from (48):

$$
\lambda \mathbf{v}=-\nu \Delta \mathbf{v}+\nabla p+\mathbf{i}\left(U_{*}+\phi\right) v_{2}+\mathbf{j}\left(U_{*}+\phi\right) v_{3} .
$$

A change in $\phi$ to $\phi+\delta \phi$ implies a change in $\lambda$ to $\lambda+\delta \lambda$ and a change in the eigenfunction $\mathbf{v}$ to $\mathbf{v}+\delta \mathbf{v}$. To first order

$$
\delta \lambda \mathbf{v}+\lambda \delta \mathbf{v}=-\nu \Delta \delta \mathbf{v}+\nabla \delta p+\mathbf{i}\left(U_{*}+\phi\right) \delta v_{2}+\mathbf{j}\left(U_{*}+\phi\right) \delta v_{3}+\delta \phi\left(\mathbf{i} v_{2}+\mathbf{j} v_{3}\right)
$$
1 ,

Take the dot product with the original eigenfunction $\mathbf{v}$ and note that (using $\int|\mathbf{v}|^{2} d x^{3}=$

$$
\delta \lambda=\int \delta \phi 2 v_{1} v_{2} d x^{3}=A \int_{0}^{h} \delta \phi \overline{v_{1} v_{2}} d y
$$

so that

$$
\frac{\delta \lambda}{\delta \phi}(y)=2 A \overline{v_{1} v_{2}}(y)
$$

where we introduced the overbar for the horizontal average over $x$ and $z$. The projection then in (53) then gives

$$
\phi(y)=\gamma\left(\overline{v_{1} v_{2}}(y)-\left\langle v_{1} v_{2}\right\rangle\right)
$$

where the $2 A$ factor has been absorbed into the Lagrange multiplier.

The nonlinear equations we are therefore called to solve for the optimal bound are

$$
\begin{aligned}
0 & =-\nu \Delta v+\nabla p+\mathbf{i}\left(\frac{U^{*}}{h}+\phi\right) v_{2}+\mathbf{j}\left(\frac{U^{*}}{h}+\phi\right) v_{1} \\
0 & =\nabla \cdot v \\
\phi & =\gamma\left(\overline{v_{1} v_{2}}-\left\langle v_{1} v_{2}\right\rangle\right)
\end{aligned}
$$

where $\gamma$ is determined by the normalization condition $\int|\mathbf{v}|^{2} d x^{3}=1$.

We also have to note that $\gamma$ is a scalar only if the isospectral surface $\lambda_{\phi}=0$ is smooth. 


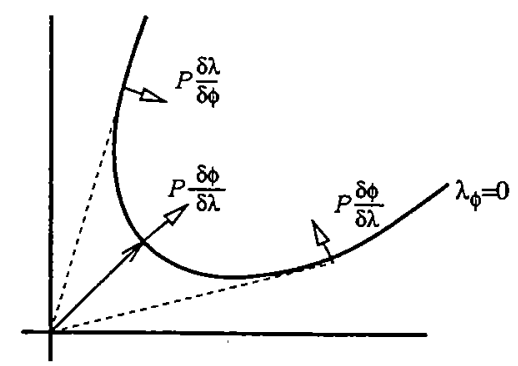

Figure 3: The space $\Phi$. The curve denotes where $\lambda_{\phi}=0$.

\subsection{Structure of the Optimal Bound}

Here we describe a general formulation of the Euler-Lagrange equations (61). The translation invariance in the $(x-z)$ plane allows us to write $\mathbf{v}$ as

$$
\mathbf{v}=\sum_{\alpha} \hat{\mathbf{v}}^{(\alpha)}(y) e^{i\left(\alpha_{x} x+\alpha_{z} z\right)}
$$

where $\alpha=\mathbf{i} \alpha_{1}+\mathbf{j} \alpha_{2}$ and the incompressibility condition now gives $\partial_{y} \hat{v}_{2}+i \alpha \cdot \hat{\mathbf{v}}=0$. We can write $Q_{\phi}$ as

$$
Q_{\phi}\{\mathbf{v}\}=\sum_{\alpha} Q_{\phi}^{(\alpha)}\left\{\hat{\mathbf{v}}^{(\alpha)}(y)\right\}
$$

where

$$
Q_{\phi}^{(\alpha)}\{\hat{\mathbf{v}}\}=A \int_{0}^{h}\left[\nu\left|\frac{d \hat{\mathbf{v}}}{d y}\right|^{2}+|\alpha|^{2}|\hat{\mathbf{v}}|^{2}+\left(\frac{U_{*}}{h}+\phi(y)\right)\left(\hat{v}_{1} \hat{v}_{2}^{*}+\hat{v}_{1}^{*} \hat{v}_{2}\right)\right] d y
$$

Since we want $Q_{\phi}$ to be positive we must demand

$$
Q_{\phi}^{(\alpha)} \geq 0 \quad \forall \alpha
$$

Note that if we drop the incompressibility condition $\left|\alpha_{1}\right|<\left|\alpha_{2}\right|$ would imply $Q^{\left(\alpha_{1}\right)}<Q^{\left(\alpha_{2}\right)}$ which does not generally hold for the incompressible case. The set of zero mean functions with positive $\lambda$ can now be written as

$$
\Phi=\left\{\phi \mid \lambda_{\phi} \geq 0\right\}=\bigcap_{\alpha}\left\{\phi \mid \lambda_{\phi}^{(\alpha)} \geq 0\right\}
$$

\subsubsection{Single Wavenumber Case (Smooth)}

First we examine the simplest possible case where the minimum bound comes from a single mode with wave number $\alpha$. (See Fig. 4) The optimal $\phi$ is given by

$$
\phi(y)=\gamma \Re\left\{\hat{v}_{1}^{(\alpha)}(y) \hat{v}_{2}^{(\alpha)}(y) *-\frac{1}{h} \int_{0}^{h} \hat{v}_{1}^{(\alpha)}\left(y^{\prime}\right) \hat{v}_{2}^{(\alpha)}\left(y^{\prime}\right)^{*} d y^{\prime}\right\}
$$

and the equations that $\hat{\mathbf{v}}$ must satisfy are: 


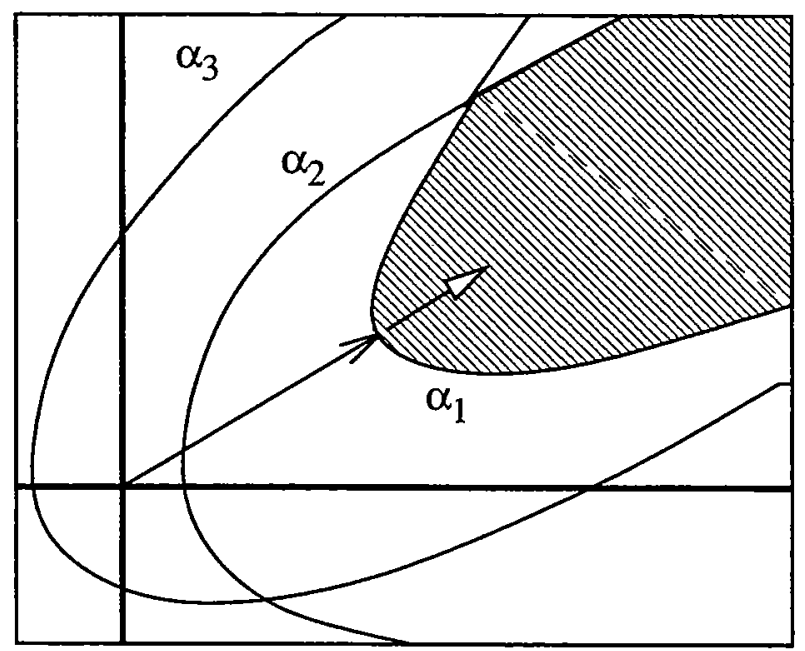

Figure 4: The space $\Phi$ in the case that the minimum $\phi$ is given by a single mode.

$$
\begin{aligned}
0 & =-\nu \Delta \hat{\mathbf{v}}^{(\alpha)}+\nabla p+\mathbf{i}\left(\frac{U^{*}}{h}+\phi\right) \hat{v}_{2}^{(\alpha)}+\mathbf{j}\left(\frac{U^{*}}{h}+\phi\right) \hat{v}_{1}^{(\alpha)} \\
0 & =\nabla \cdot \hat{\mathbf{v}}^{(\alpha)} \\
1 & =\int\left|\hat{\mathbf{v}}^{(\alpha)}\right|^{2} d^{3} x
\end{aligned}
$$

\subsubsection{Two Wavenumber Case}

The next case we examine is when the minimum is obtained at the intersection of the curves $\lambda_{\phi}^{\left(\alpha_{1}\right)}=0$ and $\lambda_{\phi}^{\left(\alpha_{2}\right)}=0$. (See figure 5.)

The optimal $\phi$ in this case is given as a linear combination of $\mathcal{P}\left(\delta \lambda^{1} / \delta \phi\right)$ and $\mathcal{P}\left(\delta \lambda^{2} / \delta \phi\right)$

$$
\begin{aligned}
\phi(y)= & \gamma_{1} \Re\left\{\hat{v}_{1}^{\left(\alpha_{1}\right)}(y) \hat{v}_{2}^{\left(\alpha_{1}\right)}(y) *-\frac{1}{h} \int_{0}^{h} \hat{v}_{1}^{\left(\alpha_{1}\right)}\left(y^{\prime}\right) \hat{v}_{2}^{\left(\alpha_{1}\right)}\left(y^{\prime}\right)^{*} d y^{\prime}\right\} \\
& +\gamma_{2} \Re\left\{\hat{v}_{1}^{\left(\alpha_{2}\right)}(y) \hat{v}_{2}^{\left(\alpha_{2}\right)}(y) *-\frac{1}{h} \int_{0}^{h} \hat{v}_{1}^{\left(\alpha_{2}\right)}\left(y^{\prime}\right) \hat{v}_{2}^{\left(\alpha_{2}\right)}\left(y^{\prime}\right)^{*} d y^{\prime}\right\}
\end{aligned}
$$

where $\gamma_{1}$ and $\gamma_{2}$ are to be determined from the normalization conditions and each $\hat{\mathbf{v}}^{\left(\alpha_{\mathbf{i}}\right)}$ must obey (70).

\subsubsection{General Situation}

For the more general case the solution will be given by

$$
\phi(y)=\sum_{n=1}^{N} \gamma_{n} \Re\left\{\hat{v}_{1}^{\left(\alpha_{\mathbf{n}}\right)}(y) \hat{v}_{2}^{\left(\alpha_{\mathbf{n}}\right)}(y) *-\frac{1}{h} \int_{0}^{h} \hat{v}_{1}^{\left(\alpha_{\mathbf{n}}\right)}\left(y^{\prime}\right) \hat{v}_{2}^{\left(\alpha_{\mathbf{n}}\right)}\left(y^{\prime}\right)^{*} d y^{\prime}\right\}
$$




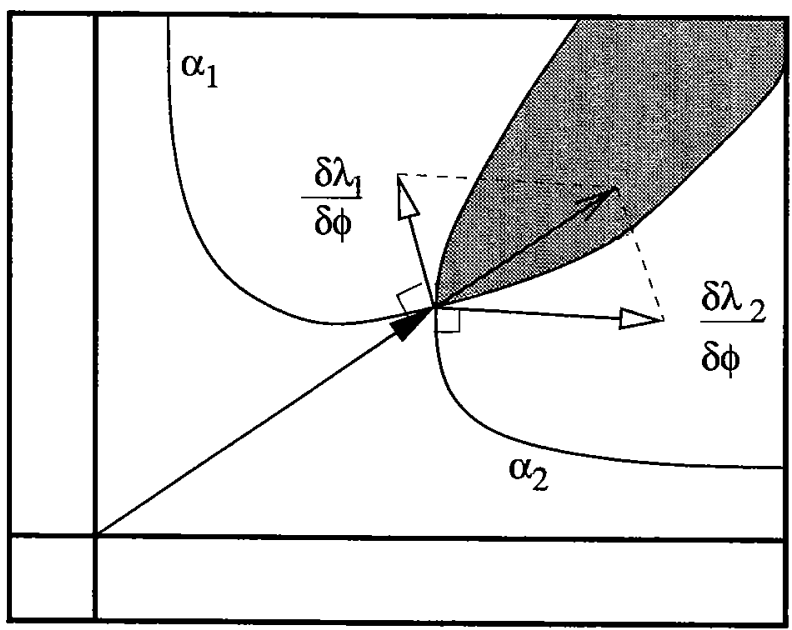

Figure 5: The space $\Phi$ in the case that the minimum $\phi$ is given by two modes.

where each $\hat{v}^{\left(\alpha_{\mathbf{n}}\right)}$ satisfies

$$
\begin{aligned}
0 & =-\nu \Delta \hat{\mathbf{v}}^{\left(\alpha_{\mathbf{n}}\right)}+\nabla p+\mathbf{i}\left(\frac{U^{*}}{h}+\phi\right) \hat{v}_{2}^{\left(\alpha_{\mathbf{n}}\right)}+\mathbf{j}\left(\frac{U^{*}}{h}+\phi\right) \hat{v}_{1}^{\left(\alpha_{\mathbf{n}}\right)} \\
0 & =\nabla \hat{\mathbf{v}}^{\left(\alpha_{\mathbf{n}}\right)} \\
1 & =\int\left|\hat{v}^{\left(\alpha_{\mathbf{n}}\right)}\right|^{2} d^{3} x
\end{aligned}
$$

and all the $\gamma_{n}$ are given by the normalization condition.

\subsection{Results and Reality}

Fig. (6) summarizes the results that have been obtained by solving the Euler Lagrange equations. The straight line $\sim R e^{-1}$ gives the results of the laminar flow which is an absolute minimum. For higher $R e$ the energy dissipation rate in the flow is bounded from above by the curve shown in the figure. The crosses represent experimental measurements on a turbulant shear layer. The experimental results still show a weak (logarithmic) dependence on the Reynolds number which is not captured by the bounding method. Perhaps further physical information given to the analysis would improve the bound. (We note that the graph is just a sketch.) 


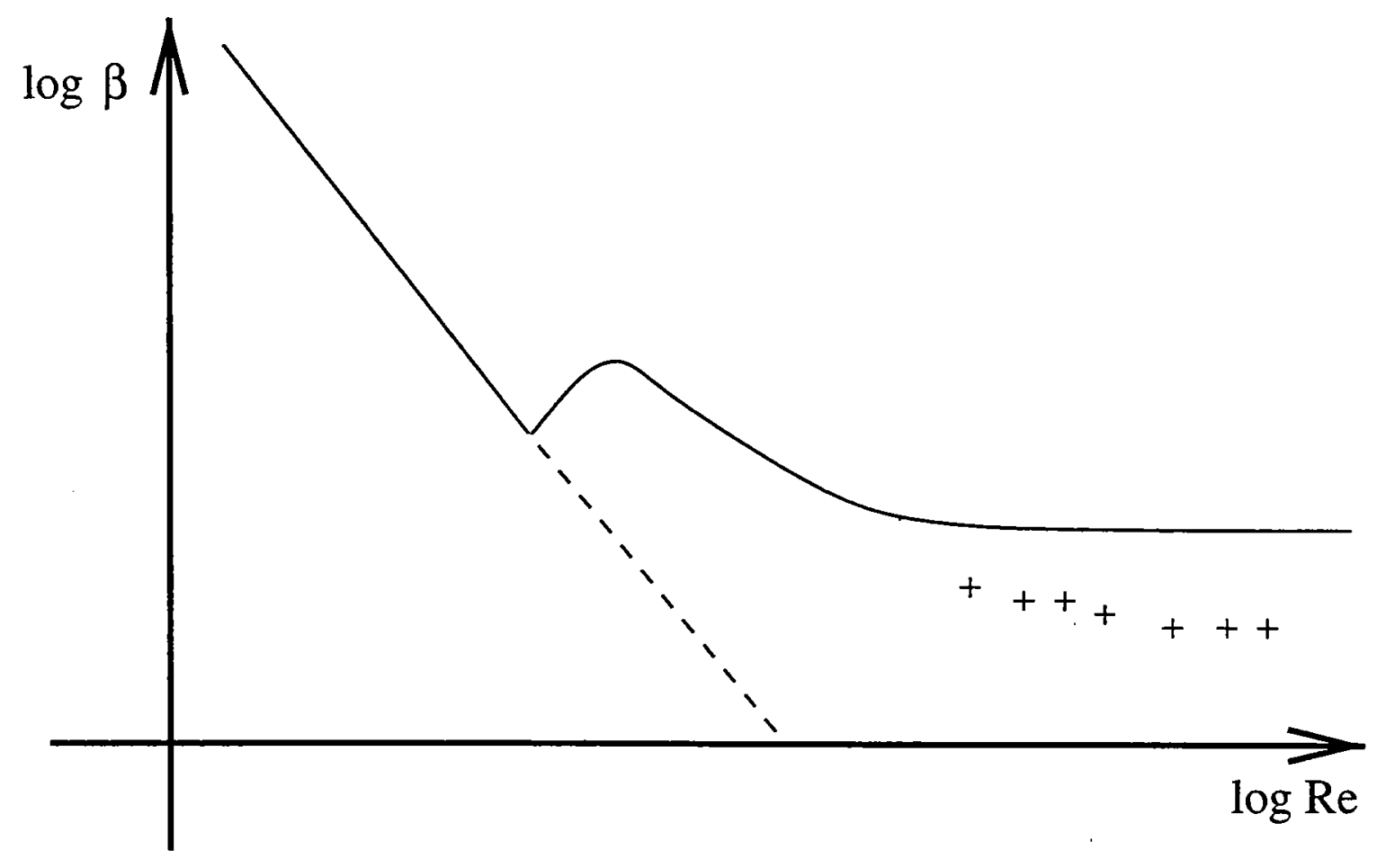

Figure 6: A sketch of the bound and the experimental data. 


\section{Lecture 7 \\ Multi- $\alpha$-Solutions \\ F. H. Busse \\ Notes by L. Lu and F. Petrelis}

\section{Introduction}

In the previous lecture, the Rayleigh-Bénard convection in a porous media is discussed and the problem of finding the upper bound of convective heat transport is formulated as a variational problem. In this lecture, we will try to solve this variational problem using the multi- $\alpha$-solution technique. An explicit bound of the Nusselt number will be obtained by the multi-boundary-layer approximation method. Then the extremalizing fields of the variational problem will be compared to those observed in turbulent flows. Finally, the convection in a rotating layer is studied in a similar way. The extremalizing fields of the corresponding variational problem are found by solving the Euler-Lagrange equations numerically.

\section{Multi- $\alpha$-Solutions}

From previous lecture, the variational problem is:

Given $\mu>0$ find the minimum of the functional

$$
\mathcal{P}(\mathbf{u}, \theta, \mu)=\frac{<|\mathbf{u}|^{2}><|\nabla \theta|^{2}>+\mu<|\overline{w \theta}-<w \theta>|^{2}>}{<w \theta>^{2}},
$$

among the $\mathbf{u}, \theta$ fields with

$$
\nabla \cdot \mathbf{u}=0,\left.\quad w\right|_{z= \pm \frac{1}{2}}=\left.\theta\right|_{z= \pm \frac{1}{2}}=0
$$

where

$$
w=\mathbf{u} \cdot \hat{\mathbf{k}}
$$

With the general representation for a solenoidal vector field,

$$
\mathbf{u}=\nabla \times(\nabla \times \hat{\mathbf{k}} \phi)+\nabla \times \hat{\mathbf{k}} \psi
$$

and the ansatz

$$
\begin{gathered}
w=w^{(N)} \equiv \sum_{n=1}^{N} \alpha_{n}^{\frac{1}{2}} w_{n}(z) \phi_{n}(x, y) \\
\theta=\theta^{(N)} \equiv \sum_{n=1}^{N} \alpha_{n}^{-\frac{1}{2}} \theta_{n}(z) \phi_{n}(x, y)
\end{gathered}
$$

where the functions $\phi_{n}(x, y)$ satisfy the equation

$$
\Delta_{2} \phi_{n}=-\alpha_{n}^{2} \phi_{n}
$$


the functional to be minimized becomes

$$
\mathcal{P}^{(N)}\left(\theta_{n} ; \alpha_{n}, \mu\right) \equiv \frac{I^{2}+\mu<\left(\sum_{\nu} \theta_{\nu}^{2}-\sum_{\nu}<\theta_{\nu}^{2}>\right)^{2}>}{\sum_{\nu}<\theta_{\nu}^{2}>^{2}} \quad n=1,2,3, \ldots, N
$$

where

$$
I \equiv \sum_{\nu=1}^{N} \frac{1}{\alpha_{\nu}}<{\theta_{\nu}}^{2}>+\alpha_{\nu}<\theta_{\nu}^{2}>
$$

See previous lecture notes for more details of the derivation of the functional and its physical meaning.

\subsection{Asymptotic Analysis of Minimum of $\mathcal{P}^{(N)}\left(\theta_{n} ; \alpha_{n}, \mu\right)$}

It is difficult to find an analytic solution of the Euler-Lagrange equations correponding to the variational problem (1) in closed form. Thus we attempt to seek its asymptotic solution as $\mu \rightarrow \infty$. To start, it is convenient now to change the normalization condition to

$$
\sum_{\nu=1}^{N}<\theta_{\nu}^{2}>=1
$$

And also we assume that the wave numbers $\alpha_{n}$ are ordered: $\alpha_{N}>\alpha_{N-1}>\cdots>\alpha_{1}$. In the asymptotic case of large $\mu$ it is obvious that in order to minimize the functional $\mathcal{P}$, the minimizing solution $\sum_{\nu} \theta_{\nu}^{2}$ must approach unity as closely as possible throughout the interval $-\frac{1}{2}<z<\frac{1}{2}$. Only near the boundaries $z= \pm \frac{1}{2}$ the boundary conditions (2) prevent a close approach. However a rapid increase from 0 to 1 near the boundary makes $\theta_{n}^{\prime}$ large, and consequently $I^{2}$ increases. But this growth can be moderated by assigning the sharpest growth rate to $\theta_{N}$, which is divided by the largest wave number $\alpha_{N}$. In the expression of $I^{2}, \theta_{N}$ is multiplied by $\alpha_{N}$. Thus $\theta_{N}$ has to decrease rapidly to 0 . Otherwise the large wavenumber would make $I^{2}$ grow even though the $\theta_{N}^{\prime}$ term is small. To satisfy the condition $\theta_{N}^{2}+\theta_{N-1}^{2} \approx 1$, the increasing part of $\theta_{N-1}$ must match the decreasing region of $\theta_{N}$. In summary, $\theta_{N}$ increases in a layer of thickness of order $O\left(\mu^{-r_{N}}\right)$, and decreases to 0 in the region of order $O\left(\mu^{-r_{N-1}}\right)$, which is the increasing part of $\theta_{N-1}$. This hierarchy (shown shematically in Fig (1)) continues until $\theta_{1}=1$ fills the region outside all the boundary layers of the rest $\theta_{n}$.

Thus we introduce two variables $\hat{\theta}$ and $\tilde{\theta}$ corresponding to the rising and falling regions respectively,

$$
\theta_{n}(z)= \begin{cases}\hat{\theta}\left(\zeta_{n}\right) & \text { for }\left|z \pm \frac{1}{2}\right| \approx O\left(\mu^{-r_{n}}\right) \\ \tilde{\theta}\left(\zeta_{n-1}\right) & \text { for }\left|z \pm \frac{1}{2}\right| \approx O\left(\mu^{-r_{n-1}}\right)\end{cases}
$$

where

$$
\zeta_{n} \equiv\left|z \pm \frac{1}{2}\right| \mu^{r_{n}}
$$




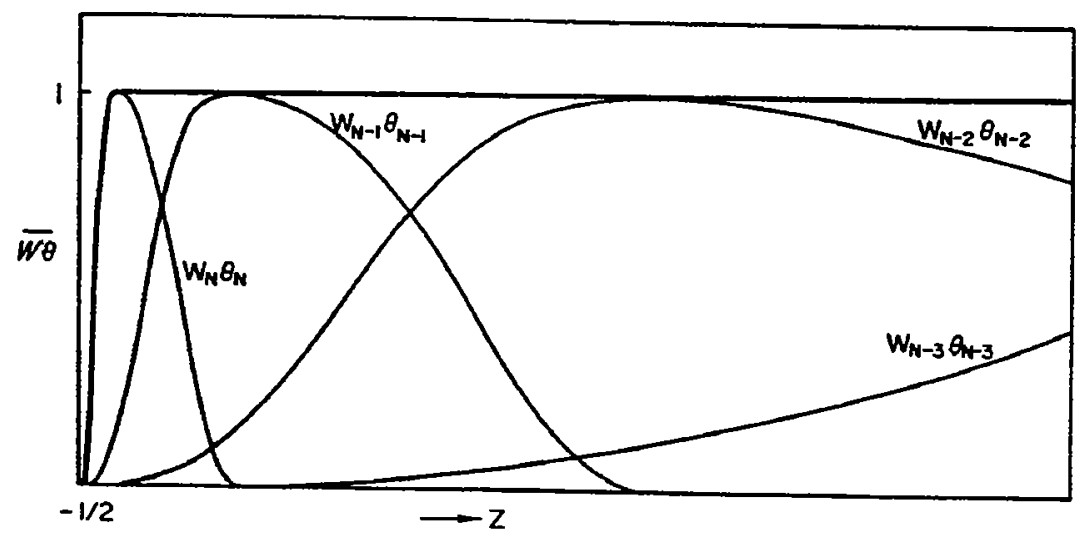

Figure 1: Qualitative sketch of the boundary layer structure of the extremalizing $\mathrm{N}-\alpha$ solution, in the case of convection in a porous layer, $w=\theta$.

near $z= \pm \frac{1}{2}$ and where

$$
\begin{array}{rlrl}
\hat{\theta}_{n}^{2}+\tilde{\theta}_{n+1}^{2} & \approx 1 & & \text { for } z \approx O\left(\mu^{-r_{n}}\right), \text { and } n=1,2, \ldots, N-1, \\
\tilde{\theta}_{1}^{2} \approx 1 & \text { for } z \approx O(1)=O\left(\mu^{-r_{0}}\right) .
\end{array}
$$

The matching condition is:

$$
\left.\hat{\theta}_{n}\left(\zeta_{n}\right)\right|_{\zeta_{n} \rightarrow \zeta_{n}^{(m)}}=\left.\tilde{\theta}_{n}\left(\zeta_{n-1}\right)\right|_{\zeta_{n-1} \rightarrow 0}=1,
$$

where the supcript $(m)$ means matching point. The boundary conditions for $\hat{\theta}_{n}$ and $\tilde{\theta}_{n}$ are:

$$
\hat{\theta}_{n}(0)=0, \quad \tilde{\theta}_{n}(\infty)=0 .
$$

The relations

$$
<\theta_{m}^{\prime} \theta_{n}^{\prime}>=\alpha_{m} \alpha_{n}<\theta_{m} \theta_{n}>
$$

yield

$$
\begin{aligned}
& \alpha_{n}^{2}=\frac{<\theta_{n}^{\prime 2}>}{<\theta_{n}^{2}>} \approx \mu^{r_{n}+r_{n-1}} \frac{\int_{0}^{\zeta_{n}^{(m)}} \tilde{\theta}_{n}^{\prime 2} d \zeta_{n}}{\int\left(1-\tilde{\theta}_{n}^{2}\right) d \zeta_{n-1}} \equiv \mu^{r_{n}+r_{n-1}} b_{n}^{2}, \quad \text { for } n=2,3, \ldots, N, \\
& \alpha_{1}^{2}=\mu^{r_{1}} 2 \int_{0}^{\zeta_{1}^{(m)}} \tilde{\theta}^{\prime 2} d \zeta_{1} \equiv \mu^{r_{1}} b_{1}^{2} .
\end{aligned}
$$

Thus the boundary-layer approximation of the functional $\mathcal{P}^{(N)}(\theta ; \mu)$ becomes

$$
\mathcal{P}^{(N)}(\theta ; \mu)=I^{2}+2 \mu^{1-r_{N}} \int_{0}^{\infty}\left(1-\hat{\theta}_{N}^{2}\right)^{2} d \zeta_{N}
$$


where

$$
I=\mu^{\frac{\tau_{1}}{2}} b_{1}+\sum_{n=1}^{N} \mu^{\frac{r_{n}-r_{n-1}}{2}}\left(\frac{1}{b_{n}} \int_{0}^{\zeta_{n}^{(m)}} \hat{\theta}_{n}^{\prime 2} d \zeta_{n}+b_{n} \int_{0}^{\infty}\left(1-\hat{\theta}_{n-1}^{2}\right)^{*} d \zeta_{n-1}\right)
$$

and where the term labelled with $\left(^{*}\right)$ vanishes when $n=1$. The minimum of the functional $\mathcal{P}^{(N)}$ as a function of $r_{1}, r_{2}, \ldots, r_{N}$ is reached when the partial derivatives with respect to each $r$ vanish. This yields:

$$
r_{1}=r_{2}-r_{1}=\cdots=r_{n}-r_{n-1}=\cdots=r_{N}-r_{N-1}=1-r_{N}
$$

Then

$$
r_{n}=\frac{n}{N+1} \quad \text { for } n=1,2, \ldots, N
$$

Accordingly,

$$
\mathcal{P}^{(N)}(\theta ; \mu)=\mu^{\frac{1}{N+1}}\left\{\hat{I}^{2}+2 \int_{0}^{\infty}\left(1-\hat{\theta}^{2}\right)^{2} d \zeta_{N}\right\}
$$

where

$$
\hat{I}=b_{1}+2 \sum_{n=1}^{N}\left\{\frac{1}{b_{n}} \int \hat{\theta}_{n}^{\prime 2} d \zeta_{n}+b_{n} \int\left(1-\hat{\theta}_{n-1}^{2}\right)^{*} d \zeta_{n-1}\right\} .
$$

The Euler-Lagrange equations corresponding to a stationary value of the functional above can be written as

$$
\begin{aligned}
\hat{\theta}_{n}^{\prime \prime}+b_{n} b_{n-1} \hat{\theta}_{n} & =0 \quad \text { for } n=1,2, \ldots, N-1, \\
\hat{I} \hat{\theta}_{N}^{\prime \prime}+b_{N}\left(1-\hat{\theta}_{N}^{2}\right) \hat{\theta}_{n} & =0 .
\end{aligned}
$$

The solutions of these equations satisfying the boundary conditions (13) and matching condition (12) are

$$
\begin{aligned}
& \hat{\theta}_{n}= \pm \sin \left(b_{n} b_{n+1}\right)^{\frac{1}{2}} \zeta_{n} \quad \text { for } 1 \leq \zeta_{n} \leq \frac{\pi}{2}\left(b_{n} b_{n+1}\right)^{\frac{1}{2}} \\
& \hat{\theta}_{N}= \pm \tanh \left(\frac{b_{N}}{2 \hat{I}}\right)^{\frac{1}{2}} \zeta_{N} .
\end{aligned}
$$

where the matching point has been chosen to be $\zeta_{n}^{(m)}=\frac{\pi}{2}\left(b_{n} b_{n+1}\right)^{\frac{1}{2}}$. Now the constants $b_{n}$ can be computed by using their definitions (15):

$$
\begin{gathered}
\int_{0}^{\zeta_{n}^{(m)}} \hat{\theta}_{n}^{\prime 2} d \zeta_{n}=\frac{\pi}{4} \sqrt{b_{n} b_{n+1}} \\
\int_{0}^{\zeta_{n}^{(m)}}\left(1-\hat{\theta}_{n}^{2}\right) d \zeta_{n}=\frac{\pi}{4}\left(b_{n} b_{n+1}\right)^{-\frac{1}{2}}
\end{gathered}
$$


And we obtain:

$$
\begin{aligned}
b_{n}^{2} & =b_{n}\left(b_{n+1} b_{n-1}\right)^{\frac{1}{2}} \quad \text { for } n=2,3, \ldots, N-1 \\
b_{1}^{2} & =\frac{\pi}{2} \sqrt{b_{1} b_{2}} \\
b_{N}^{2} & =\frac{8}{3 \pi} \sqrt{\frac{b_{N-1}}{2 \hat{I}}}
\end{aligned}
$$

Thus

$$
\begin{aligned}
b_{n} & =\frac{\pi}{2}\left(\frac{8}{3 \pi^{2} \sqrt{N}}\right)^{\frac{2 n-1}{N+1}} \quad \text { for } n=1,2, \ldots, N \\
\hat{I} & =2 N b_{1} .
\end{aligned}
$$

And finally

$$
\mathcal{P}^{(N)}(\mu) \equiv \min \mathcal{P}^{(N)}(\theta ; \mu)=\mu^{\frac{1}{N+1}} N(N+1) 4 b_{1}^{2}=N(N+1) \pi^{2}\left(\frac{64 \mu}{9 \pi^{4} N}\right)^{\frac{1}{N+1}}
$$

A comparison of the numerical computation of the extremalizing functions of the exact Euler-Lagrange equations and the asymptotic results is shown in Fig (2). They agree very well. The upper bound of $N u$ in Rayleigh-Benard convection by multi- $\alpha$-solution approach

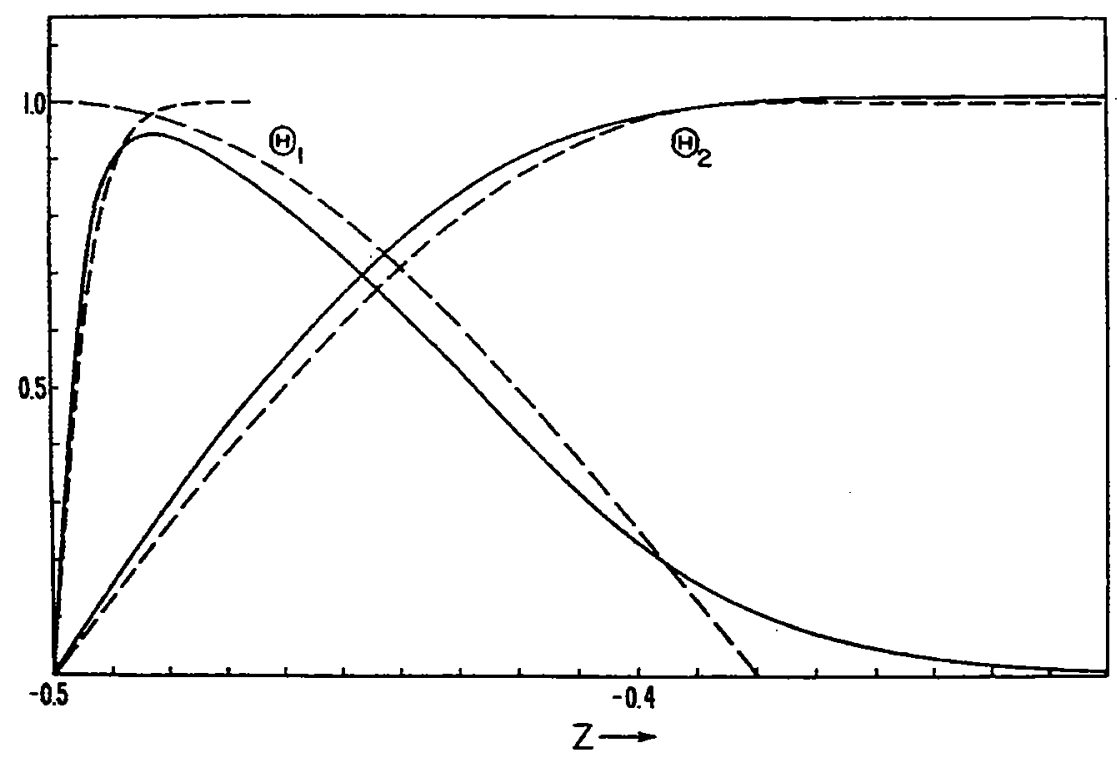

Figure 2: The two- $\alpha$-solution at $R=50 \pi^{2}$. Numerical computations(solid lines) are compared with the results (21) and for $\theta_{1}, \theta_{2}$ from the boundary layer theory.

is shown in Fig (1) in [1] and compared with experimental results. The result (23) shows that the minimum of $\mathcal{P}(\mu)$ among the class of functions $\left\{\mathcal{P}^{(N)}(\mu)\right\}$ is assumed sequentially by $N=1,2, \ldots$ as $\mu$ increases. The results of Busse and Joseph (1972) [2] indicate that the transition occurs in the form of a bifurcation in which the $(N+1)$ th component of the 
solution first appears as a small perturbation in the $N^{\text {th }}$ boundary layer of the $N$ - $\alpha$-solution. As a result the bound $P(\mu)$ appears as a smooth curve without kinks. This structure of the upper bounds is shown in Fig (3) where the upper bound of heat transfer is computed numerically for Rayleigh-Benard convection with stress-free boundary conditions [3]. Also

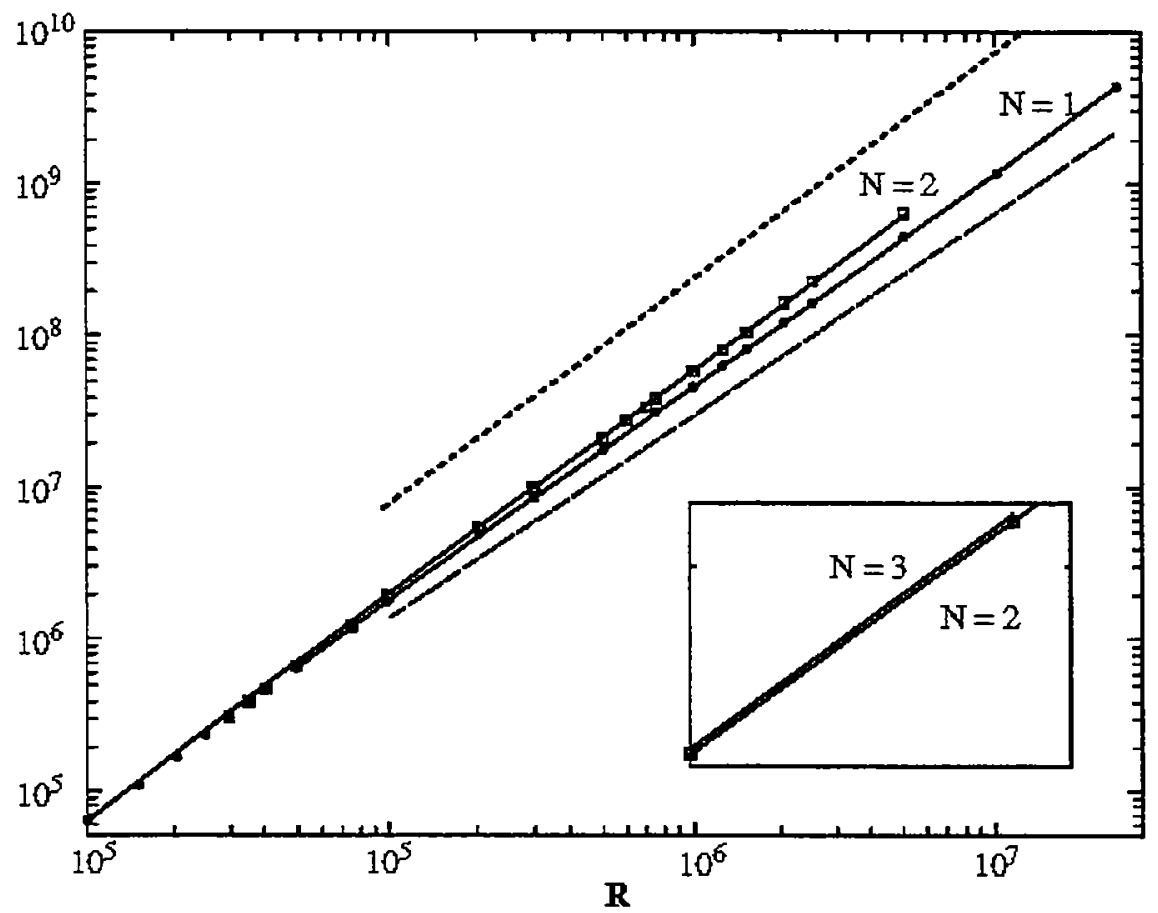

Figure 3: The upper bound $\mu$ as a function of $R$ in the case of 1- $\alpha$ - (filled circles), 2- $\alpha$ (open squares) and 3- $\alpha$-solutions (crosses). For comparison the upper bound obtained by Howard (1963) [4] without the constraint of continuity equation (dotted line) and numerical values obtained by Moore and Weiss (1973) [5] for 2-dimensional convection rolls with the Prandtl number $P=6.8$ (dashed line) are shown. The inset enlarges the part $2 \times 10^{6}<$ $R<2.6 \times 10^{6}, 1.5 \times 10^{8}<\mu<2.2 \times 10^{8}$ of the graph in order to indicate the small difference between results for $N=2$ and $N=3$. (From [3])

noticed is the boundary layer structure in this free-stress boundaries system as shown in Fig (4). The function $\overrightarrow{w \theta} /\langle w \theta\rangle$ for the $2-\alpha$ solution is close to unity over most of the interval, and only decreases sharply toward the $z= \pm \frac{1}{2}$ boundaries. The narrower boundary layer corresponds to larger wavenumber as can be seen from the curves for $\overline{w_{2} \theta_{2}} /\langle w \theta\rangle$ For this same system, Fig (5) shows the extremalizing $w_{1}, w_{2}, w_{3}$ functions corresponding to the 3 - $\alpha$-solution.

\subsection{Similarities Between Extremalizing Vector Fields and Observed Tur- bulence}

The extremalizing vector fields of the upper bound problems have in common with the observed turbulence that the wavenumber spectrum broadens as the $\mathrm{N}-\alpha$-solution is replaced by $(N+1)-\alpha$-solution. But the spectrum of the extremalizing field is discrete while that 


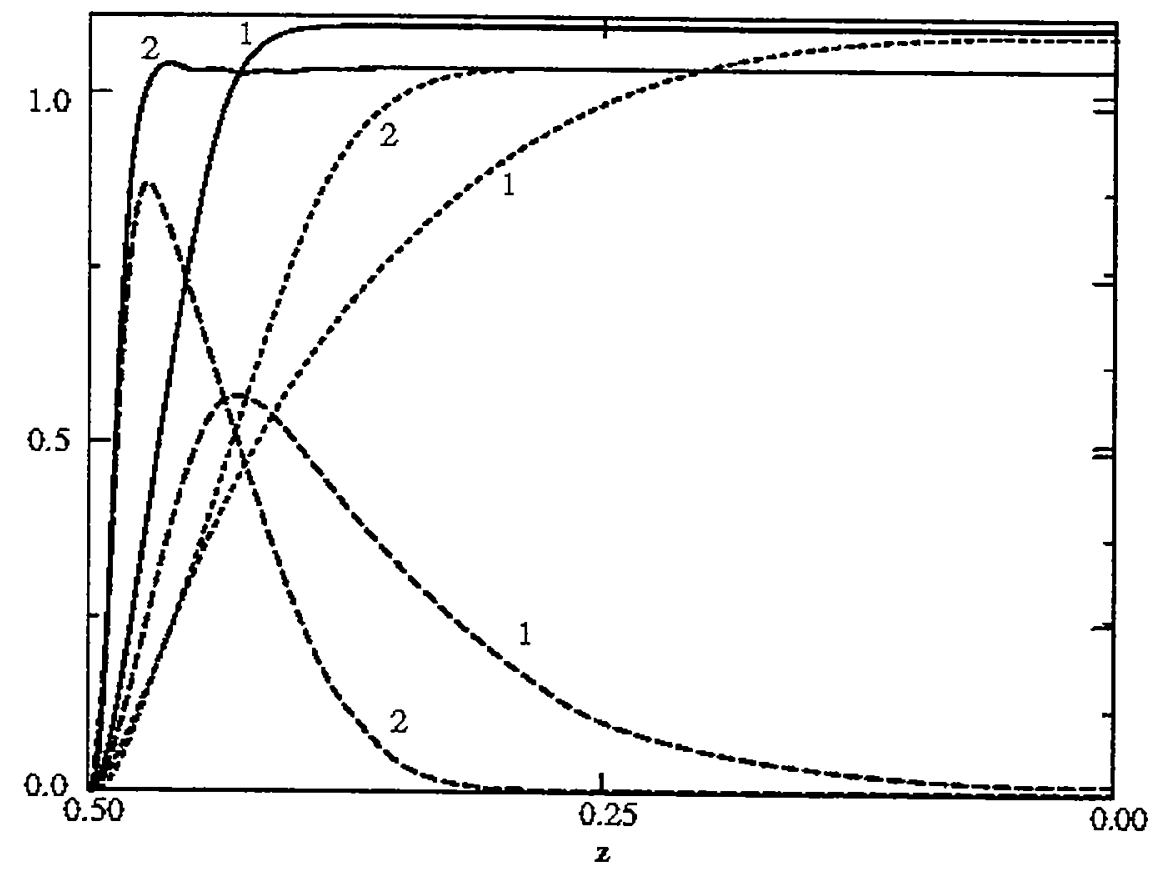

Figure 4: The functions $\overline{w \theta} /<w \theta>$ (solid lines), $\overline{w_{1} \theta_{1}} /<w \theta>$ (dotted lines) and $\overline{w_{2} \theta_{2}} /<$ $w \theta>$ (dashed lines) in the case of the $2-\alpha$-solution for $R=5 \times 10^{4}$ (labelled by 1) and $5 \times 10^{5}$ (labelled by 2 ). The slight wiggles exhibited by the function $\overline{w \theta}$ near the boundary for $R=5 \times 10^{5}$ are caused by the limited numerical resolution. (From [3])

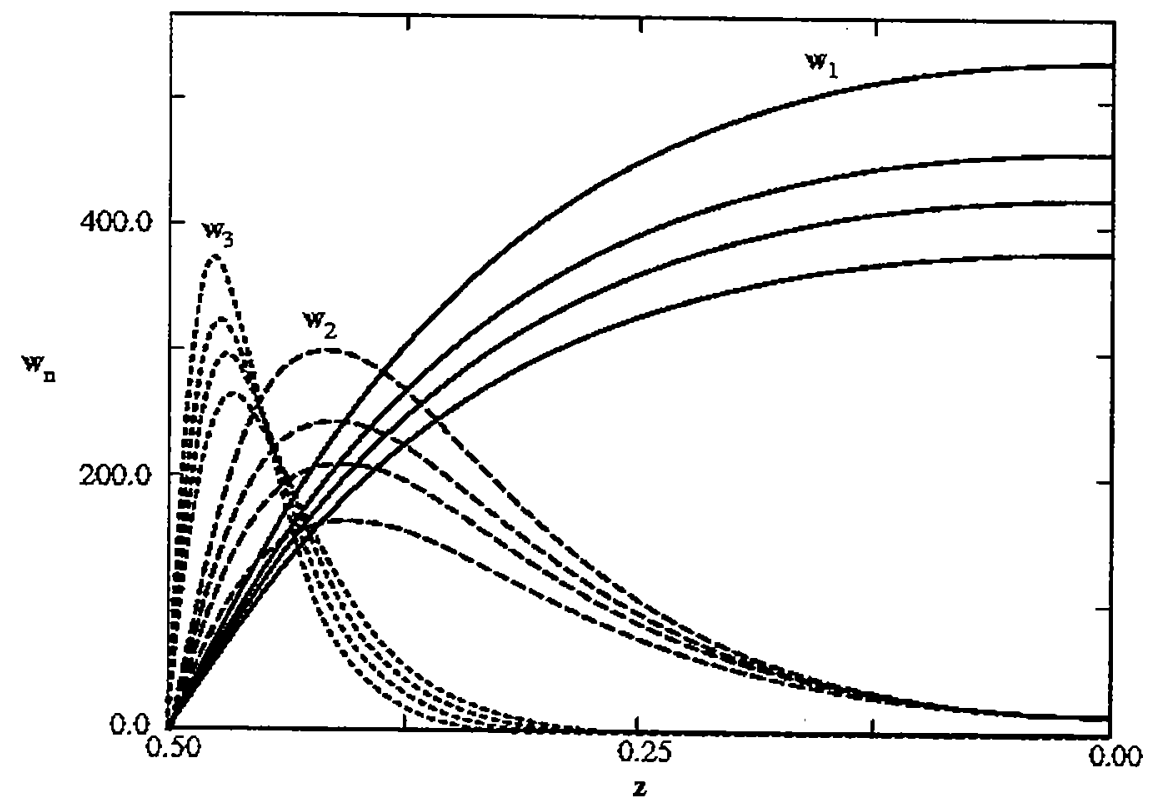

Figure 5: The function $w_{1}(z)$ (solid line), $w_{2}(z)$ (dashed line) and $w_{3}(z)$ (dotted line) of the $3-\alpha$-solutions for the Rayleigh numbers $R=10^{6}, 1.25 \times 10^{6}, 1.5 \times 10^{6}, 2 \times 10^{6}$ (from bottom to top). (From [3]) 
of the actual turbulence field is continuous. Since the N- $\alpha$ solution for the extremalizing vector field provides the upper bound only in a finite interval of the control parameters, the assumption of an infinite ratio between thicknesses of successesive boundary layers is not well satisfied. It turns out that this value assums $e^{2}$ in the case of solutions (23) for large $n, N$ and the value 4 for other cases of upper bound problems that have been studied. The profile of the extremalizing fields of turbulent shear flows thus have the form sketched in Fig (6).

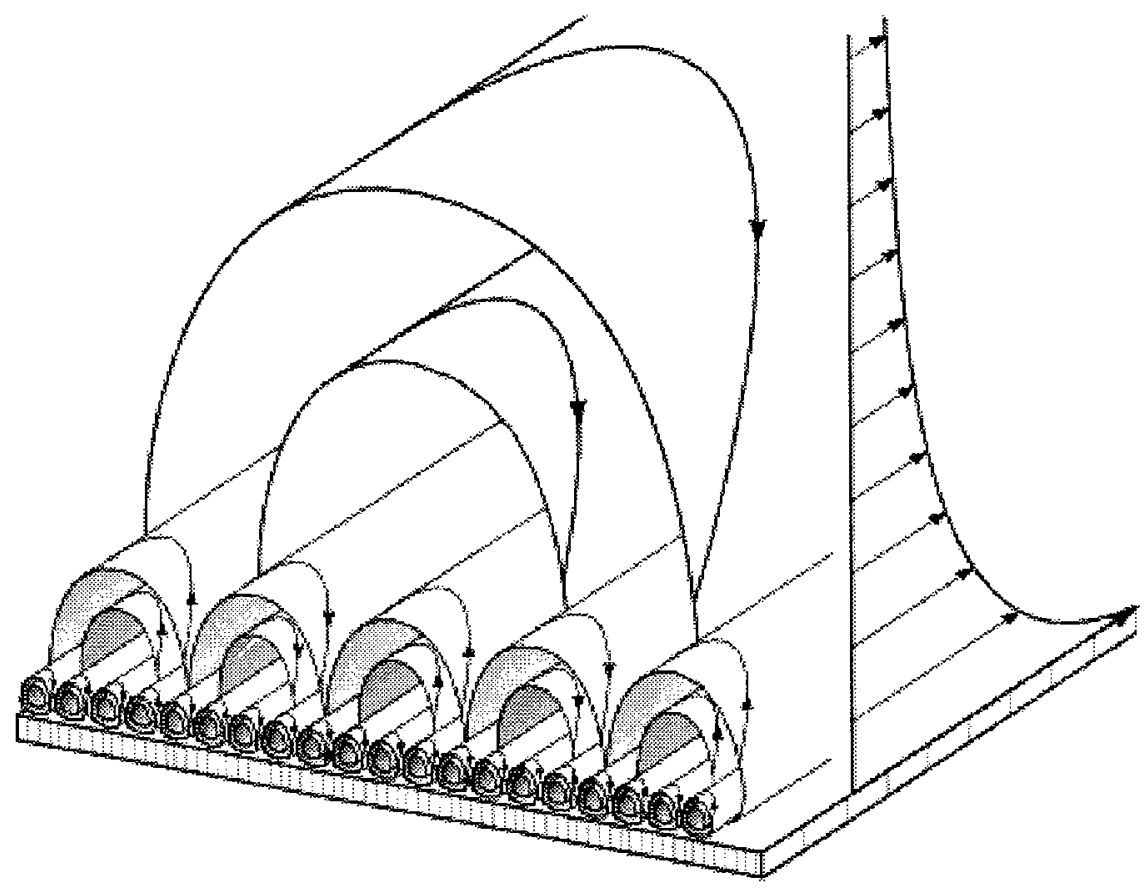

Figure 6: Qualitative sketch of the nested boundary layers which characterize the vector field of maximum transport. The profile of the mean shear flow is shown on the right side.

Generally, with increasing control parameter (e.g. $R a$ in Rayleigh-Bénard convection) the number of wavenumbers needed for the extremalizing multi- $\alpha$-solutions increases correspondingly. The transition of $N$ - $\alpha$-solutions to $(N+1)$ - $\alpha$-solutions exhibits a structure similar to bifurcation. In Rayleigh-Benard convection at high Prandtl number, the transition from convection rolls to bimodal convection occurs at the Rayleigh number of the order $2 \times 10^{4}$, the same as from the 1- $\alpha$-solution to the 2 - $\alpha$-solution. This bifurcation structure is also illustrated in Fig (7), which shows the transitions from the 1 - $\alpha$-solution to the 2 - $\alpha$-solution, and from 2 to 3 in a fluid layer heated from bellow with stress-free boundary conditions. This bifurcation structure of the extremalizing vector fields is a consequence of the property that eddies with an increasing number of length scales are needed to accomplish an optimal transport as the control parameter (Rayleigh number in convection) increases.

The profiles of averaged temperature and velocity fields are relatively easier to be measured experimentally in a turbulent flow. Thus it is of interest to compare the measuered profiles with the profiles corresponding to the extremalizing vector fields. The mean velocity 


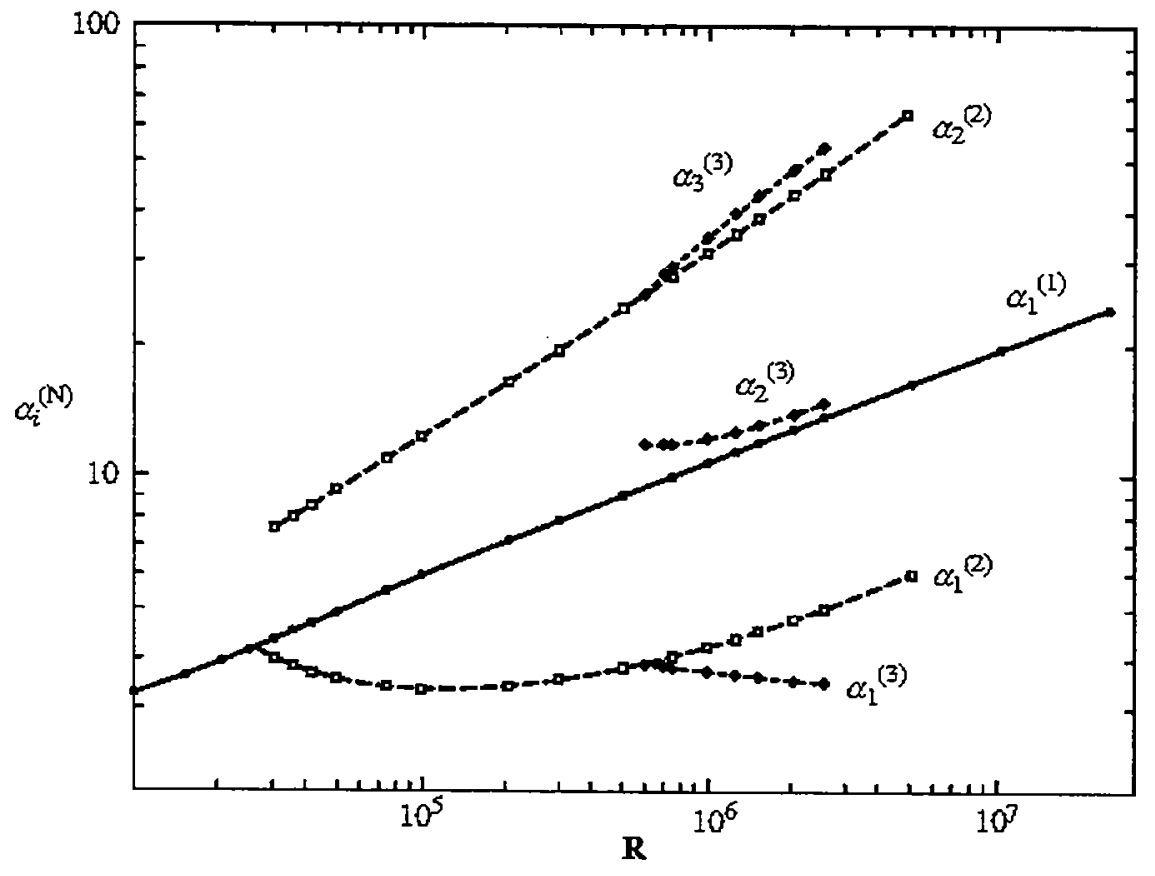

Figure 7: The wavenumbers $\alpha_{j}^{(i)}, j=1, \ldots, i, i=1,2,3$ of the extremalizing solutions as a function of $R$. (From [3])

profile in plane Couette flow is compared with the extremalizing field in Fig (8). The asymptotic profile of the extremalizing solution matches the velocity profile at high Reynolds number $(R=68000)$ very well in the middle of the interval except near the boundary. In fact the boundary layer thickness tends to zero only when $R \rightarrow \infty$. A finite $R=68000$ exhibits a finite boundary layer thickness as shown in the figure. Because of the turbulent mixing one tends to expect that the mean shear or mean temperature gradient is zero except around the boundaries where the velocity or temperature gradient is large. Indeed, experiments on turbulent convection in fluid layers heated from bellow as well as in the case of the vector field extremalizing the heat transport an isothermal interior is found when the averages over plane $z=$ constant are taken. Surprisingly this property does not hold true in the case of a shear layer as shown in Fig (8). The extremalizing field does not need the drop of half of the velocity difference between the plates across the boundary layers in order to accomplish an optimal transport. Only $3 / 8$ are required. Another example, the angular momentum transport by turbulent flow between differentially rotating coaxial cylinders, is shown in Fig (9). The extremalizing solution fits the experimental data even better than the logarithmic layer model [7].

The set of discrete wavenumbers characterizing the extremalizing vector field appears to be the most artificial feature when compared to the broad continuous wavenumber spectrum observed in turbulent flows. However, patterns of coherent structures in fully developed turbulences are very difficult to measure in laboratories. The fluctuations measured at a single point as a function of time which are interpreted as fluctuations in space via the Taylor hypothesis will usually generate a continuous spectrum even if, for instance, a perfect pattern 


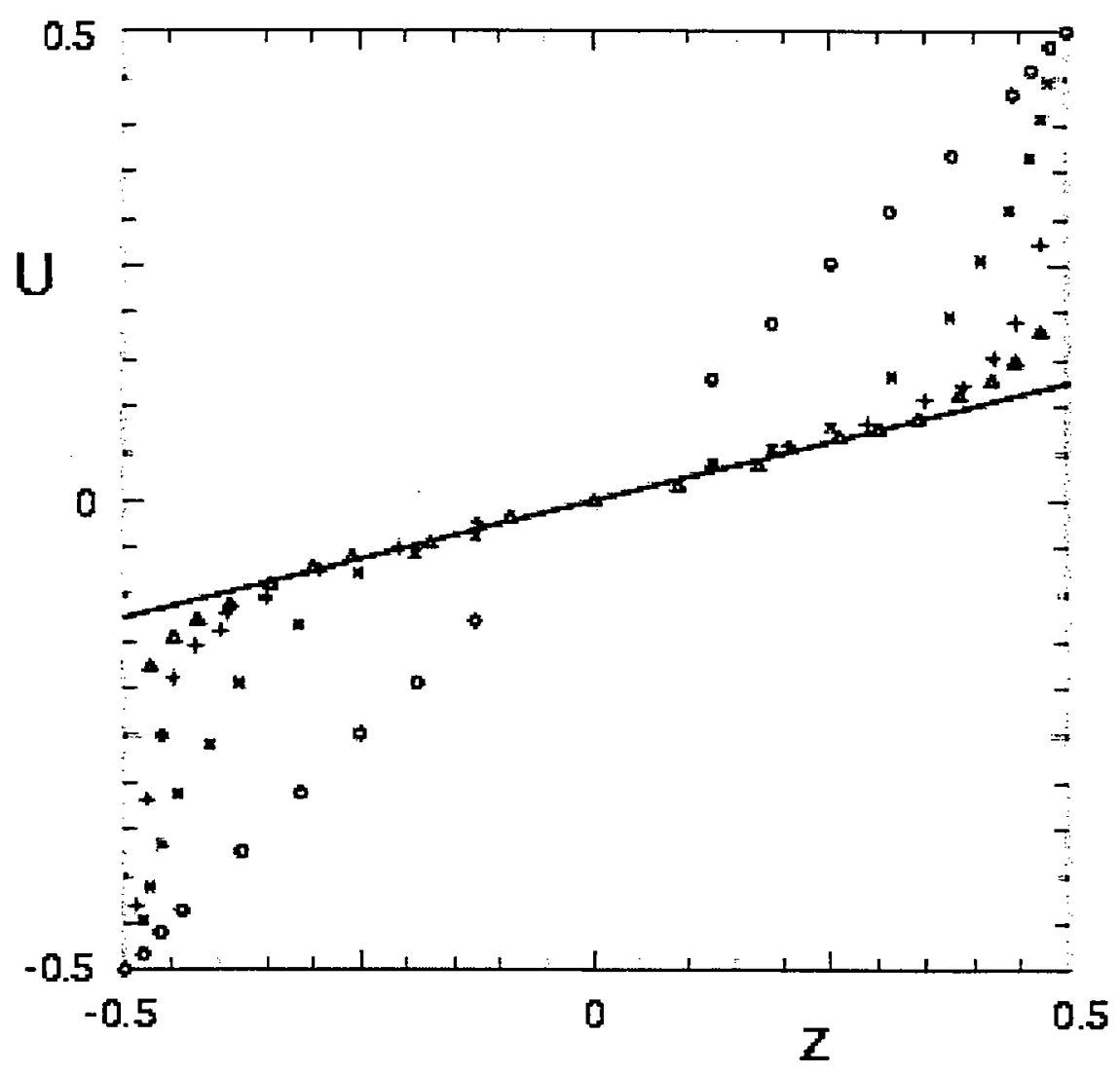

Figure 8: The mean velocity profile in plane Couette flow measured by Reichardt (1959) at $R e=2400(\circ), R e=5800(\times), R e=11800(+)$, and $R e=68000(\Delta)$. The straight line describes the asymptotic profile corresponding to the extremalizing solution of the variational problem [From [6]]. 


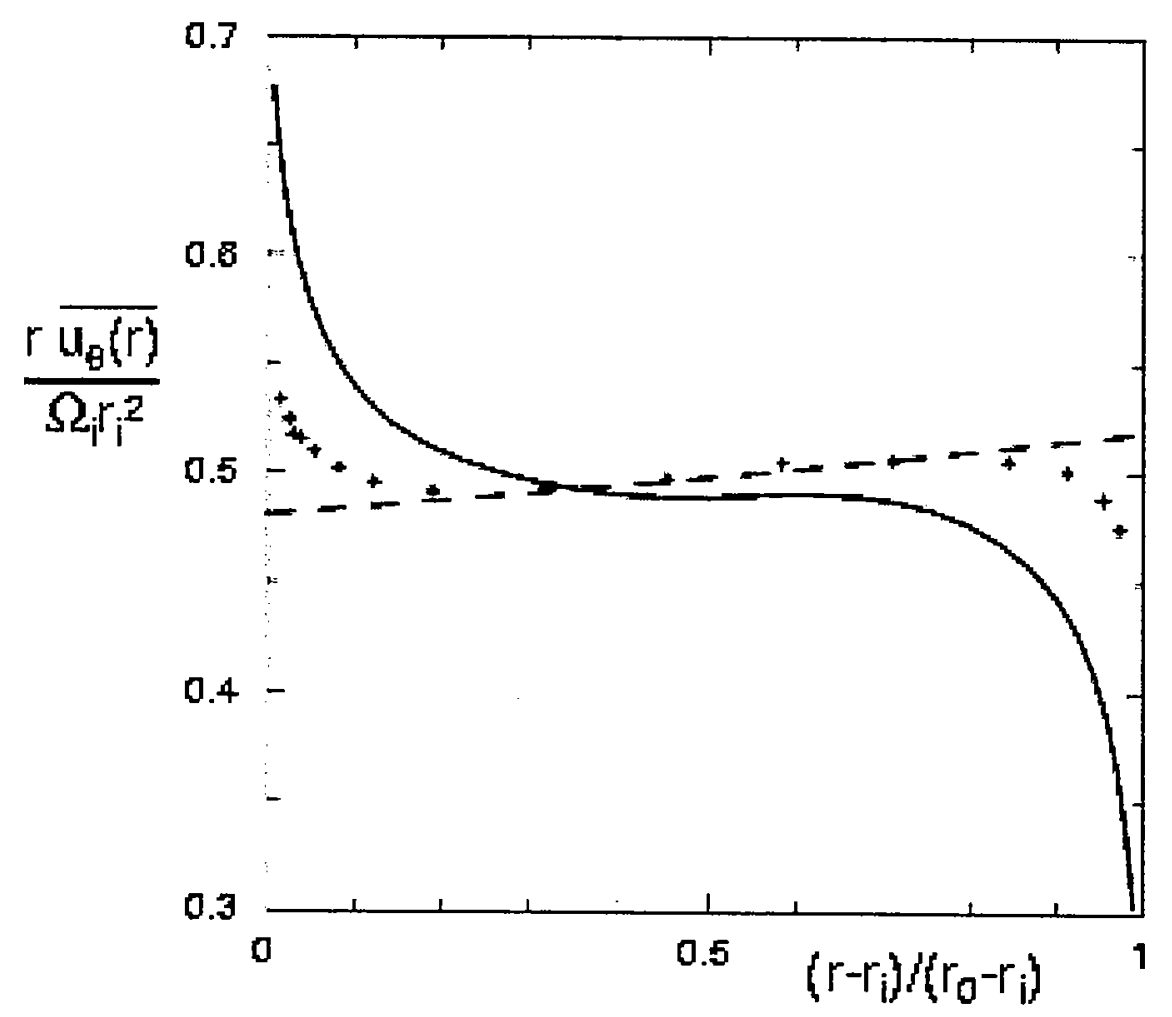

Figure 9: Measurements( + ) by Smith and Townsend (1982) [8] of the angular momentum density, $r U(r)$, normalized by the angular momentum of the inner cylinder, $\Omega_{i} r_{i}^{2}$, in comparison with the profile of a logarithmic layer model (solid line) (Lathrop et al, 1992 [7]) and with the profile of the extremalizing vector field in the limit of high Reynolds numbers (dashed line). A stationary outer cylinder with a radius ratio $\eta=0.667$ has been used (After Busse, 1996 [9]). 
of hexagons is advected by a mean flow. Few experiments can provide an instantaneous view of the two-dimensional structure of turbulence. Through the shadowgraph visualization technique [10], such a view can be obtained in the case of turbulent convection in a fluid layer heated from bellow. It is thus not surprising that a nearly stationary network of convection cells can be discerned in turbulent convection at a Rayleigh number of several $10^{6}$. Measurements of spectral peaks that can be compared with the discrete scales of the extremalizing fields have been obtained as shown in Fig (10). More detailed comparisons appear to be possible when numerical simulations of convection with sufficiently large horizontal periodicity intervals are carried out. Another property shared by extremalizing fields for different turbulent flows is shown in Fig 11, where the structures of shear flow boundary layers and of thermal boundary layers in convection are identical when scaled properly.

Finally, additional constraints will restrict the manifold of admissible vector fields in the variational problems and will lead to improved bounds.

\section{Convection in a Rotating System}

The geometry of this problem is shown in Fig (12). The length scale is $d$, time scale $\frac{d^{2}}{\kappa}$ and temperature $\frac{T_{2}-T_{1}}{R}$. The dimensionless governing equations are:

$$
\begin{gathered}
P^{-1}\left(\frac{\partial}{\partial t}+\mathbf{u} \cdot \nabla\right) \mathbf{u}=-\nabla \pi+\hat{\mathbf{k}} \Theta+\nabla^{2} \mathbf{u}-2 \Omega \times \mathbf{u} \\
\nabla \cdot \mathbf{u}=0 \\
\frac{\partial}{\partial t} \Theta+\mathbf{u} \cdot \nabla \Theta=R \mathbf{u} \cdot \hat{\mathbf{k}}+\nabla^{2} \Theta
\end{gathered}
$$

where

$$
\begin{gathered}
R=\frac{\gamma\left(T_{2}-T_{1}\right) g d^{3}}{\gamma \kappa}, \\
P=\frac{\nu}{\kappa}, \\
\Omega=\hat{\mathbf{k}} \Omega=\hat{\mathbf{k}} \frac{\Omega_{0} d^{2}}{\nu} .
\end{gathered}
$$

Assume the turbulence is stationary, and thus

$$
\Theta=\bar{\Theta}+\check{\Theta}, \quad \text { with } \overline{\hat{\Theta}}=0 \text {. }
$$

As usual, the over bar denotes a horizontal average over the plane $z=$ constant. By taking the horizontal average of the temperature equation (26), we have

$$
\frac{d}{d z} \bar{\Theta}=\overline{u_{z} \check{\Theta}}-\left\langle u_{z} \check{\Theta}>\right.
$$

Using the general representation of a solenoidal field,

$$
\mathbf{u}=\nabla \times(\nabla \Phi \times \hat{\mathbf{k}})+\nabla \Psi \times \hat{\mathbf{k}}=\delta \Phi+\epsilon \Psi
$$




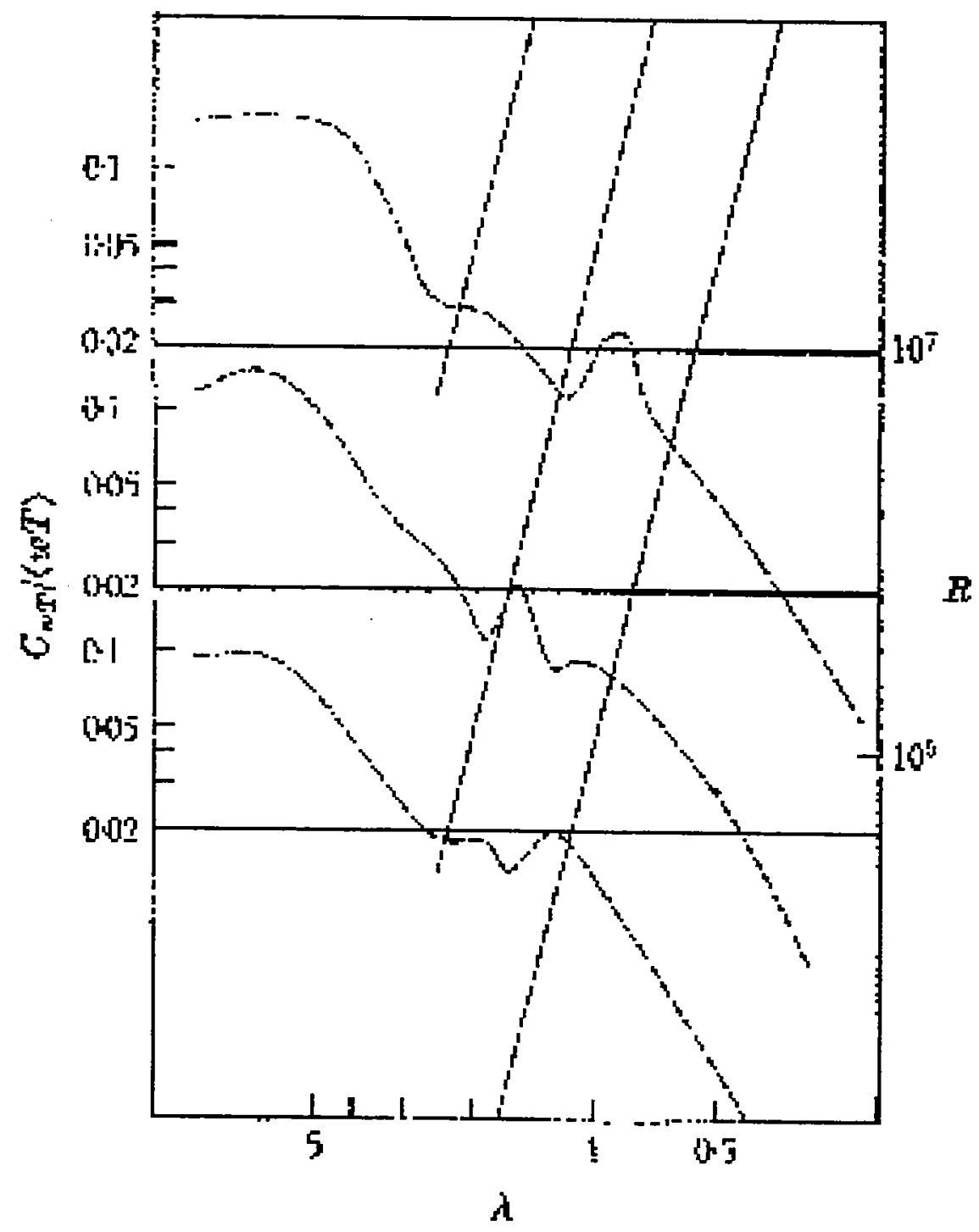

Figure 10: Three graphs of the normalized cospectra of $w$ and $\theta$, observed by Deardorff and Willis (1967) [11] at Rayleigh numbers $6 \times 10^{5}, 2.5 \times 10^{6}, 1.0 \times 10^{7}$ respectively, are plotted on top of a figure showing $l_{1}^{(N)} \equiv 2 \pi / \alpha_{1}^{(N)}$ as a function of the Rayleigh number for $N=2,3,4$. The three graphs have been arranged in such a way that the Rayleigh numbers of both plots coincide approximately at the level where the secondary maxima appear in the cospectra. 


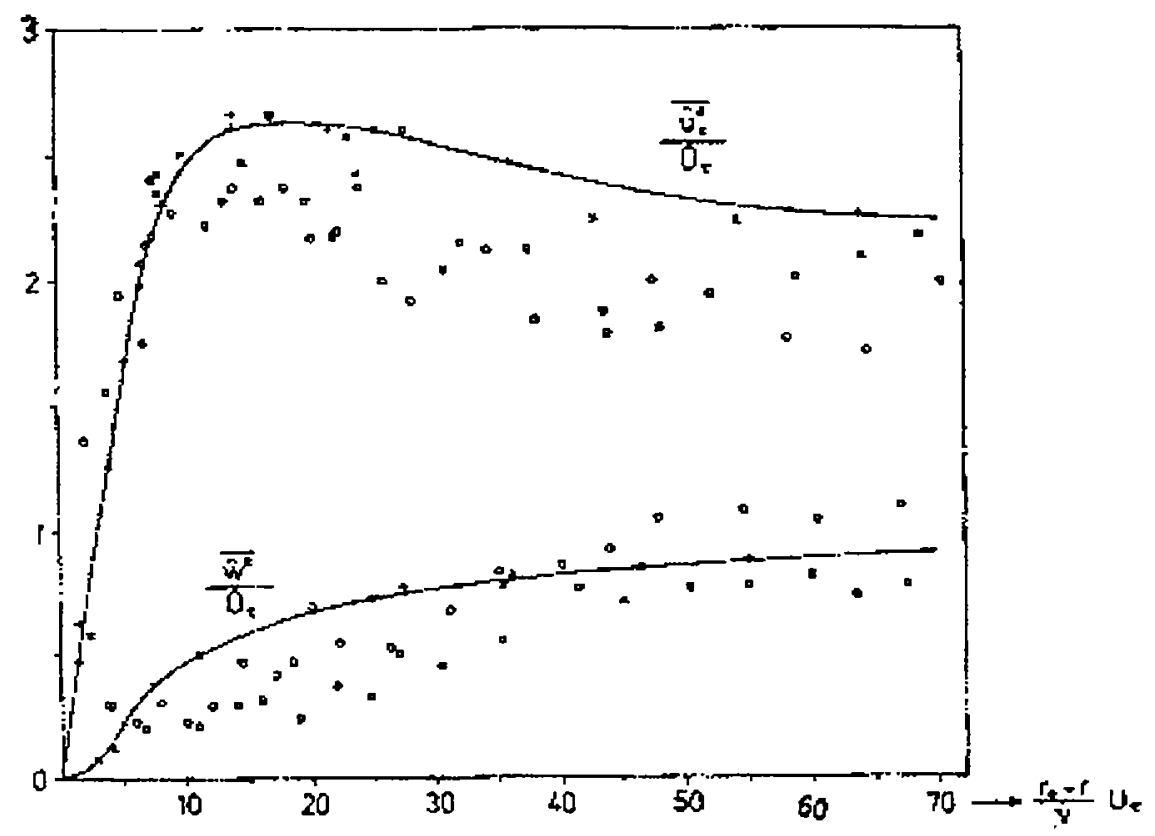

Figure 11: Root mean squre (r.m.s) values of the fluctuating components of the velocities in the streamwise direction, $\hat{u}_{x} / U_{\tau}$, and normal to the wall, $\hat{w} / U_{\tau}$, measured by Laufer (1954) [12] at $R e=2.5 \times 10^{5}(x)$ are compared with the r.m.s. values of the temperature flucuations $\hat{\theta}$ and of the vertical velocity $\hat{w}$ measuered in turbulent thermal convection by Deardorff and Willis(1967) [11]. The latter values have been obtained for $R a=2.5 \times$ $10^{6}(\circ)$ and $R a=2.5 \times 10^{7}(\square)$ are plotted in units resulting from the correspondence of the variational problems (after Busse, 1970)

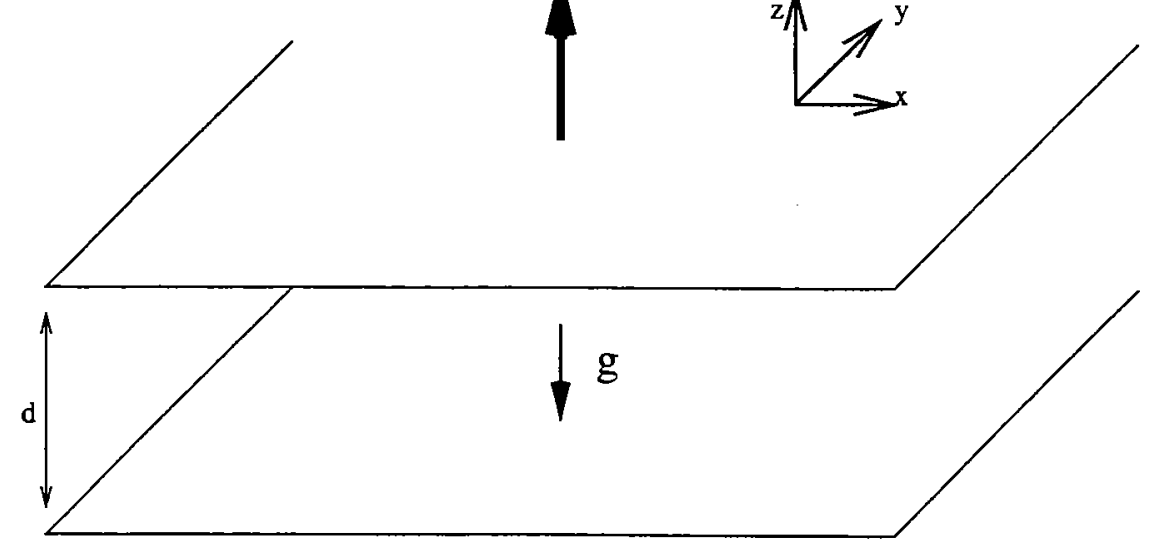

Figure 12: Geometry of convection in a rotating fluid layer. 
we obtain:

$$
\begin{aligned}
\nabla^{4} \Delta_{2} \Phi+2 \Omega \nabla \Delta_{2} \Psi-\Delta_{2} \check{\Theta} & =P^{-1}\left(\frac{\partial}{\partial t} \nabla^{2} \Delta_{2} \Phi+\delta \cdot(\mathbf{u} \cdot \nabla \mathbf{u})\right) \\
\nabla^{2} \Delta_{2} \Psi-2 \Omega \cdot \nabla \Delta_{2} \Phi & =P^{-1}\left(\frac{\partial}{\partial t} \Delta_{2} \Psi+\epsilon \cdot(\mathbf{u} \cdot \nabla \mathbf{u})\right) \\
\nabla^{2} \check{\Theta}-R \Delta_{2} \Phi+\Delta_{2} \Phi \frac{d}{d z} \bar{\Theta} & =\mathbf{u} \cdot \nabla \check{\Theta}-\overline{\mathbf{u} \cdot \nabla \check{\Theta}}+\frac{\partial}{\partial t} \check{\Theta}
\end{aligned}
$$

Then we are able to derive the following power integrals for stationary turbulent convection:

$$
\begin{gathered}
<\left|\hat{\mathbf{k}} \times \nabla \nabla^{2} \Phi\right|^{2}>+2 \Omega<\Delta_{2} \Phi \frac{\partial}{\partial z} \Psi>+<\check{\Theta} \Delta_{2} \Phi>=P^{-1}<\delta \Phi \cdot[(\delta \Phi+\epsilon \Psi) \cdot \nabla] \epsilon \Psi> \\
<\left|\hat{\mathbf{k}} \times \nabla \nabla^{2} \Psi\right|^{2}>-2 \Omega<\Delta_{2} \Phi \frac{\partial}{\partial t} \Psi>=P^{-1}<\epsilon \Psi[(\delta \Phi+\epsilon \Psi) \cdot \nabla] \delta \Phi> \\
<|\nabla \check{\Theta}|^{2}>+<\left|\overline{\Delta_{2} \Phi \check{\Theta}}-<\overline{\Delta_{2} \Phi \check{\Theta}}>\right|^{2}>=R<-\Delta_{2} \Phi \check{\Theta}>
\end{gathered}
$$

With these power integrals, the variational problem is formulated as follows:

For given values of the parameters $P, \tau$ and $\mu>0$ find the minimum $R(\mu, P, \tau)$ of the variational functional

$$
\mathcal{R}\left(\Phi^{*}, \Psi^{*}, \Theta^{*} ; \mu, P, \tau\right)=\mathcal{R}_{1}+\lambda\left(\mathcal{R}_{2}+\frac{\sqrt{\mu}}{P} \mathcal{R}_{3}\right)
$$

among all fields $\Phi^{*}, \Psi^{*}, \Theta^{*}$ satisfying the conditions

$$
\Phi^{*}=\frac{\partial^{2} \Phi^{*}}{\partial z^{2}}=\frac{\partial \Psi^{*}}{\partial z}=\Theta^{*}=0, \quad \text { at } \quad z= \pm \frac{1}{2}
$$

In the above expression

$$
\begin{aligned}
& \mathcal{R}_{1} \equiv \frac{\left(<\left|\hat{\mathbf{k}} \times \nabla \nabla \Psi^{*}\right|^{2}>+<\left|\hat{\mathbf{k}} \times \nabla \nabla^{2} \Phi^{*}\right|^{2}>\right)<\left|\nabla \Theta^{*}\right|^{2}>+\mu<\left(\overline{\Theta^{*} \Delta_{2} \Phi^{*}}-<\Theta^{*} \Delta_{2} \Phi^{*}>\right)^{2}>}{\left.<\Theta^{*} \Delta_{2} \Phi^{*}\right\rangle^{2}} \\
& \mathcal{R}_{2} \equiv \frac{<\left|\hat{\mathbf{k}} \times \nabla \nabla \Psi^{*}\right|^{2}>-\tau<\Delta_{2} \Phi^{*} \frac{\partial \Psi^{*}}{\partial z}>}{<\left|\hat{\mathbf{k}} \times \nabla \nabla \Psi^{*}\right|^{2}>+<\left|\hat{\mathbf{k}} \times \nabla \nabla^{2} \Phi^{*}\right|^{2}>} \\
& \mathcal{R}_{3} \equiv \frac{<\epsilon \Psi^{*} \cdot\left[\left(\epsilon \Psi^{*}+\delta \Phi^{*}\right) \cdot \nabla\right] \delta \Phi^{*}>}{\left[<\left|\hat{\mathbf{k}} \times \nabla \nabla \Psi^{*}\right|^{2}>+<\left|\hat{\mathbf{k}} \times \nabla \nabla^{2} \Phi^{*}\right|^{2}>\right]^{3 / 2}}
\end{aligned}
$$

The functional is homogeneous in $\Theta$ and in $(\Phi, \Psi)$. Hence the normalization

$$
\mu=-<\Theta \Delta_{2} \Phi>=<|\hat{\mathbf{k}} \times \nabla \nabla \Psi|^{2}>+<\left|\hat{\mathbf{k}} \times \nabla \nabla^{2} \Phi\right|^{2}>
$$


can be imposed. To solve this variational problem, the following ansatz is introduced:

$$
\begin{aligned}
& \Phi=\sum_{p=1}^{N} \phi_{p}(x, y) A_{p}(z), \\
& \Psi=\sum_{p=1}^{N} \phi_{p}(x, y) B_{p}(z), \\
& \Theta=\sum_{p=1}^{N} \phi_{p}(x, y) T_{p}(z),
\end{aligned}
$$

where the boundary conditions for $\Phi, \Psi$ and $\Theta$ can be satisfied by the choice

$$
\begin{gathered}
A_{p}=\sum_{q=1}^{M} a_{p q} \sin q \pi\left(z+\frac{1}{2}\right) \\
B_{p}=\sum_{q=1}^{M} b_{p q} \cos q \pi\left(z+\frac{1}{2}\right), \quad T_{p}=\sum_{q=1}^{M} t_{p q} \sin q \pi\left(z+\frac{1}{2}\right),
\end{gathered}
$$

for the $z$-dependence.

Then the time independent Euler-Lagrange equations are solved numerically to find the stationary state of the functional $R(\mu, P, \tau)$. Two types of solutions are tried. One of them is two-dimensional rolls, in which case a single wavenumber $(N=1$ in (43)) is assumed [13]. Since the $P$ dependence disappears in the Euler-Lagrange equations in the two dimensional case, to investigate the role of $P$ in determining the upper bounds, threedimensional hexagonal solutions are considered $(N \leq 5)[13]$. The Euler-Lagrange equations used here are time independent, which provide the upper bound of the heat transport by turbulent convection. Thus we don't observe the onset of convection in the form of coherent oscillation bellow the critical Rayleigh number when the Prandtl number $P$ is low enough.

The extremalizing fields of the two-dimensional roll and three-dimensional hexagon are shown in Fig (14) and Fig (13). The boundary layer can be seen to form in the roll solution (Fig (14) with increasing Rayleigh number with fixed $\tau^{2}$. In order to maximize the convective heat transport the function $\Pi=\overline{\theta \Delta_{2} \phi} \mid\left\langle\theta \Delta_{2} \phi\right\rangle$ must approach a constant value in the interior of the layer while keeping its rise from zero at the boundaries sufficiently smooth such that the dissipation of the flucuating variables does not contribute too much in the functional (3). This tendency is clearly seen in Fig (15) as the boundary layer forms. The three-dimensional hexagon solution has asymmetric components as is clearly evident in the $z$-dependences of $A_{1}(z)$ and $T_{1}(z)$ in Fig (13). The asymmetry increases with decreasing Prandtl number $P$ and with increasing $R$.

Finally, the upper bound on the heat transport is shown in Fig (16). The figure shows that the upper bound of convective heat transport by hexagon solutions extends to bellow the critical Rayleigh number $R a_{c}$, which is indicated by the vanishing of the maximum convective heat transport by the roll solutions. This point is particularly evident in for the $\tau^{2}=10^{4}$ and $P=0.0247$ case. But at $R a$ not far beyond the critical value, the heat transport by roll solution already exceeds the hexagon solution. This subcritical extent of the hexagon upper bound is not quite so dramatic for lower $\tau^{2}$ values. 


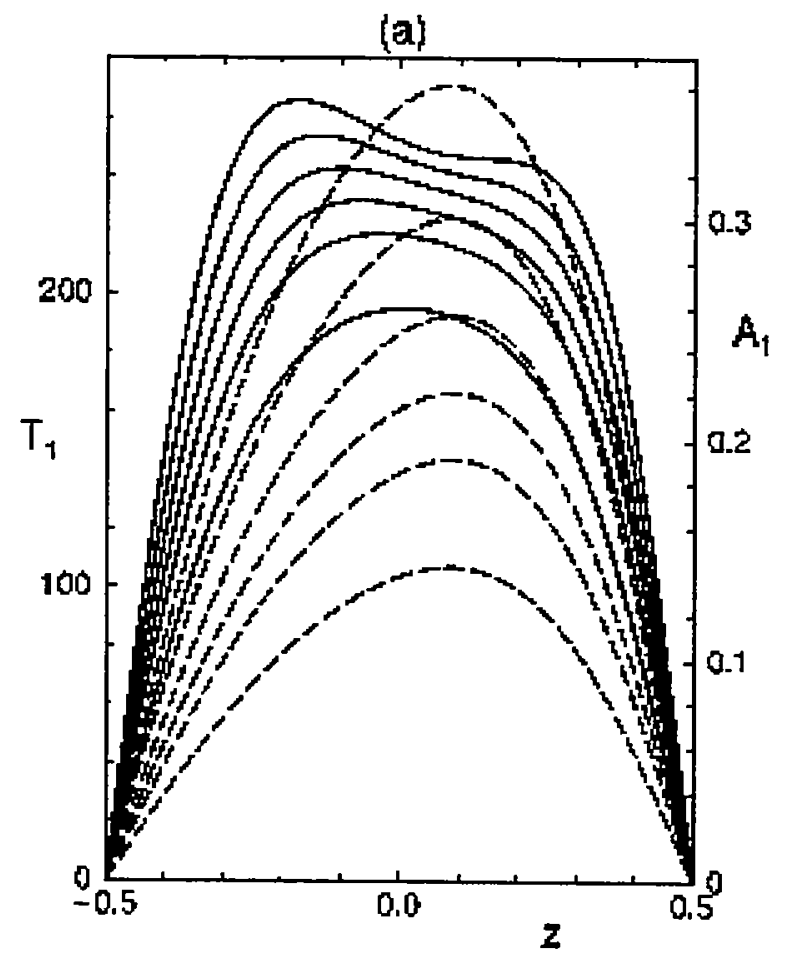

Figure 13: The $z$-dependences $T_{1}(z)$ (solid line, left ordinate) and $A_{1}(z)$ (dashed lines, right ordinate) for the etremalizing hexagon solution in the case $R=3000, P=1$ for $\tau^{2}=500,750,1000,1250,1500,2000$ (from top to bottom)

\section{References}

[1] F. Busse, "On Howard's upper bound for heat transport by turbulent convection," J. Fluid Mech. 37, 457 (1969).

[2] F. Busse and D. Joseph, "Bounds for heat transport in a porous layer," J. Fluid Mech. 54, 521 (1972).

[3] N. Vitanov and F. Busse, "Bounds on the heat transport in a horizontal fluid layer with stress-free boundaries," J. Applied Math. Phys. (ZAMP) 48, 310 (1997).

[4] L. Howard, "Heat transport by turbulent convecton," J. Fluid Mech. 17, 405 (1963).

[5] D. Moore and N. Weiss, "Two-dimensional Rayleigh-Bénard convection," J. Fluid Mech. 58, 289 (1973).

[6] F. Busse, "Bounds for turbulent shear flow," J. Fluid Mech. 41, 219 (1970).

[7] F. J. Lathrop, D.P. and H. Swinney, "Transition to shear-driven turbulence in CouetteTaylor flow," Phys. Rev. A 46, 6390 (1992).

[8] G. Smith and A. Townsend, "Turbulent Couette flow between concentric cylinders at large Taylor numbers," J. Fluid Mech. 123, 187 (1982). 
[9] F. Busse, in Nonlinear Physics of Complex Systems, Lecture Notes in Physics, edited by W. J.Parisi, S.C.Muller (Springer Verlag, New York, 1996).

[10] F. Busse and J. Whitehead, "Instabilities of convection rolls in a high Prandtl number convection," J. Fluid Mech. 66, 305 (1971).

[11] J. Deardroff and G. Willis, "Investigation of turbulent thermal convection between horizontal plates," J. Fluid Mech. 28, 675 (1967).

[12] J. Laufer, "The structure of turbulence in fully developed pipe flow," NACA Rep. 1174 (1954).

[13] N. Vitanov and F. Busse, "Bounds on the convective heat transport in a rotating layer," Phys. Rev. E 63, 16303 (2001). 


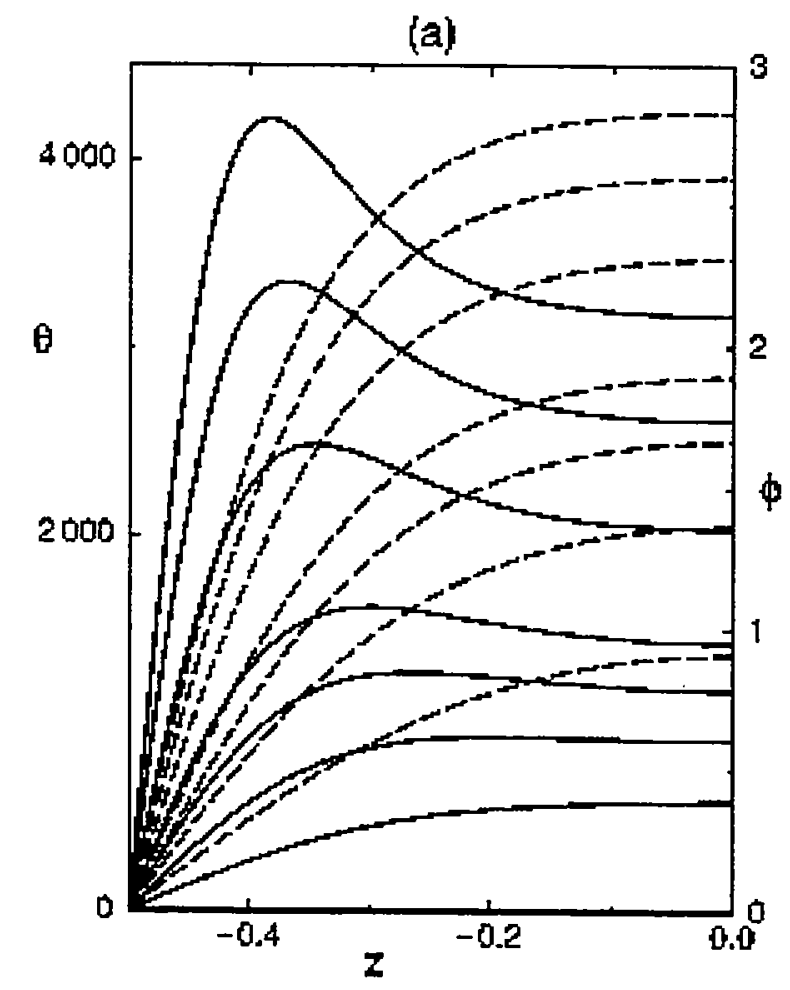

Figure 14: The $z$-dependence of the extremalizing fields $\theta$ (solid line, left ordinate) and $\psi$ (dashed lines, right ordinate) of the roll solution for $\tau^{2}=500$ in the cases $R=2 \times 10^{3}, 3 \times$ $10^{3}, 4 \times 10^{3}, 5 \times 10^{3}, 7.5 \times 10^{3}, 10^{4}, 1.25 \times 10^{4}$ (from top to bottom) 


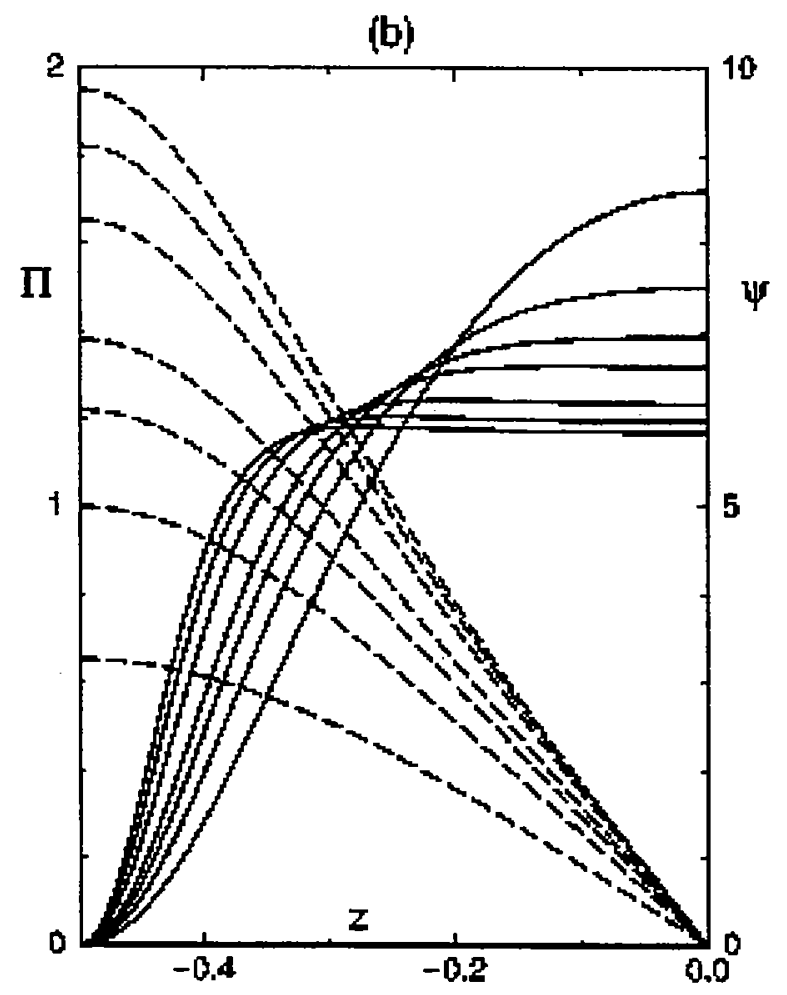

Figure 15: The function 


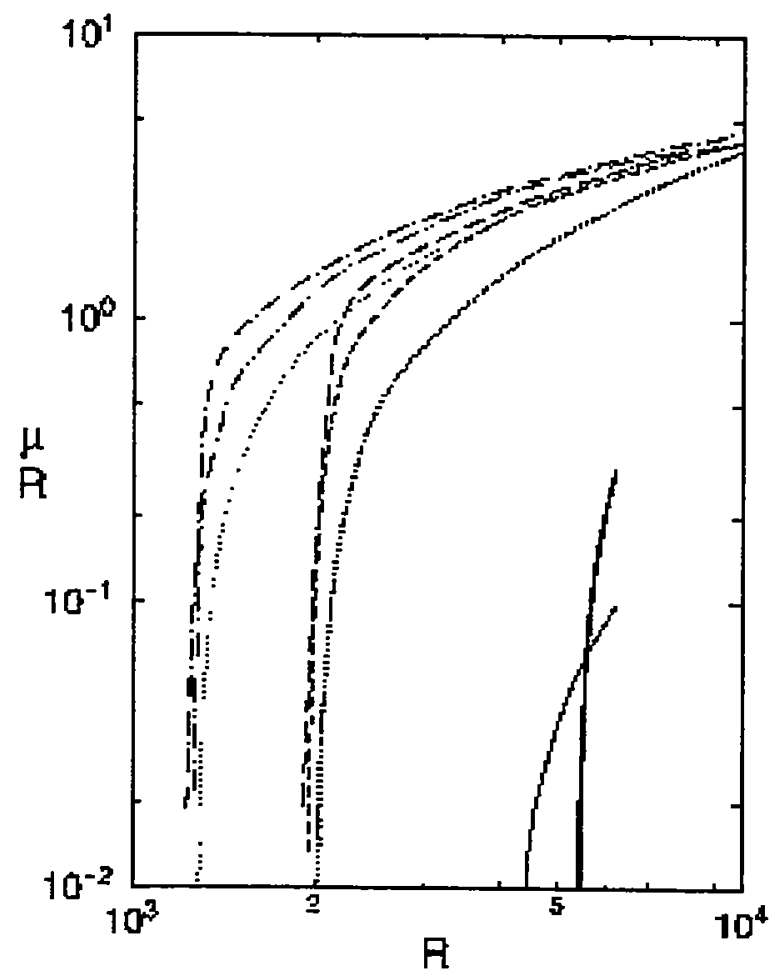

Figure 16: The upper bound $\mu$ for the convective heat transport by the hexagon solution for $P=0.1(0.0247)$ indicated by a dash-double-dotted (double-dotted) line for $\tau^{2}=1500$ ) and a short(long) dashed line for $\tau^{2}=1500$. The thin solid line indicates the upper bounds for $P=0.0247$ and $\tau^{2}=10^{4}$. For comparison the upper bounds given by rolls for $\tau^{2}=500,1500,10^{4}$ are indicated by the thin dotted line, the thick dotted line and the thick solid line, respectively 


\section{Lecture 8}

\section{Bounds for Rotating Fluids \\ P. Constantin}

Notes by U. Riemenschneider and S. Plasting

\section{Introduction}

Bounding problems in fluid turbulence have classically been concerned with finding bounds on one point quantities such as the time and space averaged dissipation rate $\mathcal{F}(R e)=$ $\nu\left\langle\|\nabla \mathbf{u}\|^{2}\right\rangle$. Another class of problem is to find bounds on two point quantities which depend both on the system control parameter and on a space- or time-like parameter. An example of such a quantity is the energy spectrum $E(k ; R e)=\frac{1}{t} \int_{0}^{t}|\hat{\mathbf{u}}(k)|^{2} \mathrm{~d} t$, where $k$ is the magnitude of the wave number, which is the density of the contributions to the kinetic energy on the wave-number magnitude axis. The total kinetic energy is $\frac{1}{2 t} \int_{0}^{t}\|\mathbf{u}\|^{2} \mathrm{~d} t=\int_{0}^{\infty} E(k) \mathrm{d} k$

This lecture deals with deriving rigorous upper bounds on transport quantities and energy spectra for rotating fluid systems. We present results for bounds on one and two point quantities which are derived by following the Constantin-Doering-Hopf bounding approach.

\section{Bounds for Rayleigh-Bénard Convection}

The effect of rotation on convective heat transport is an important issue in astrophysical and geophysical applications. Here we shall consider the heat transport through a fluid layer confined between two parallel plates heated from below with fixed temperature on both top and bottom plates, which is rotating with a constant rate around an axis of rotation perpendicular to the plates. No-slip boundary conditions will be assumed throughout. The non-dimensional equations for Boussinesq convection with rotation are

$$
\begin{gathered}
\frac{1}{P r}\left(\frac{\partial \mathbf{u}}{\partial t}+\mathbf{u} \cdot \nabla \mathbf{u}\right)+E^{-1} \hat{\mathbf{k}} \times \mathbf{u}+\nabla p=\Delta \mathbf{u}+R a \hat{\mathbf{k}} T \\
\boldsymbol{\nabla} \cdot \mathbf{u} \\
\frac{\partial T}{\partial t}+\mathbf{u} \cdot \nabla T=\Delta T
\end{gathered}
$$

where the Prandtl number is defined as $\operatorname{Pr}=\nu / \kappa$, the Ekman number is inversely proportional to the rotation rate, and the Rayleigh number is the standard non-dimensionalised temperature difference across the fluid layer.

In the limit of infinite Prandtl number one can neglect the inertial terms of the left hand side of Equation (1). In the remaining system of equations $T$ is the active scalar and the velocity vector $\mathbf{u}$ is linearly dependent on $T$. In the bounding analysis of this problem the full momentum equation can be utilised as a pointwise constraint due to its 
linearity. Following the derivation in [1] we are able to show that the following equations in the vertical component of velocity $w=\hat{\mathbf{k}} \cdot \mathbf{u}$ and the vertical component of vorticity $\xi=\hat{\mathbf{k}} \cdot(\boldsymbol{\nabla} \times \mathbf{u})$ fully determine the dynamics of the convective state

$$
\begin{gathered}
\Delta^{2} w-E^{-1} \frac{\partial \xi}{\partial z}=-R a \Delta_{H} T \\
-\Delta \xi-E^{-1} \frac{\partial w}{\partial z}=0
\end{gathered}
$$

subject to the boundary conditions

$$
w=\frac{\partial w}{\partial z}=0=\xi \quad \text { at } z=0,1 .
$$

Multiplying Equation (2) by $w$, Equation (3) by $\xi$, adding and integrating we deuce that the following $E$-independent bound holds pointwise in time

$$
\|\Delta w\|^{2}+2\|\Delta \xi\|^{2} \leq R a^{2}
$$

where we use a normalised $L^{2}$ norm

$$
\|f\|^{2}=\frac{1}{L^{2}} \int_{0}^{1} \int_{0}^{L} \int_{0}^{L}|f(x, y, z)|^{2} d x d y d z .
$$

Equation (3) can be rearranged to

$$
\frac{\partial w}{\partial z}=-E \Delta \xi
$$

The previous two expressions together imply that for strong rotation rates $(E \rightarrow 0)$ horizontal variations in $w$ are restricted and a stratification is set up such that a purely conductive state is realised.

The total non-dimensional heat transport is quantified by the Nusselt number which is defined as the long-time average of the vertical heat flux

$$
N=1+\left\langle\int_{0}^{1} b(z, t) d z\right\rangle
$$

where

$$
b(z, t):=\frac{1}{L^{2}} \int_{0}^{L} \int_{O}^{L} w(x, y, z, t) T(x, y, z, t) d x d y
$$

and $\langle\cdot\rangle$ is used to denote the long-time average

$$
\langle f\rangle=\limsup _{t=\rightarrow \infty} \frac{1}{t} \int_{0}^{t} f(s) d s .
$$




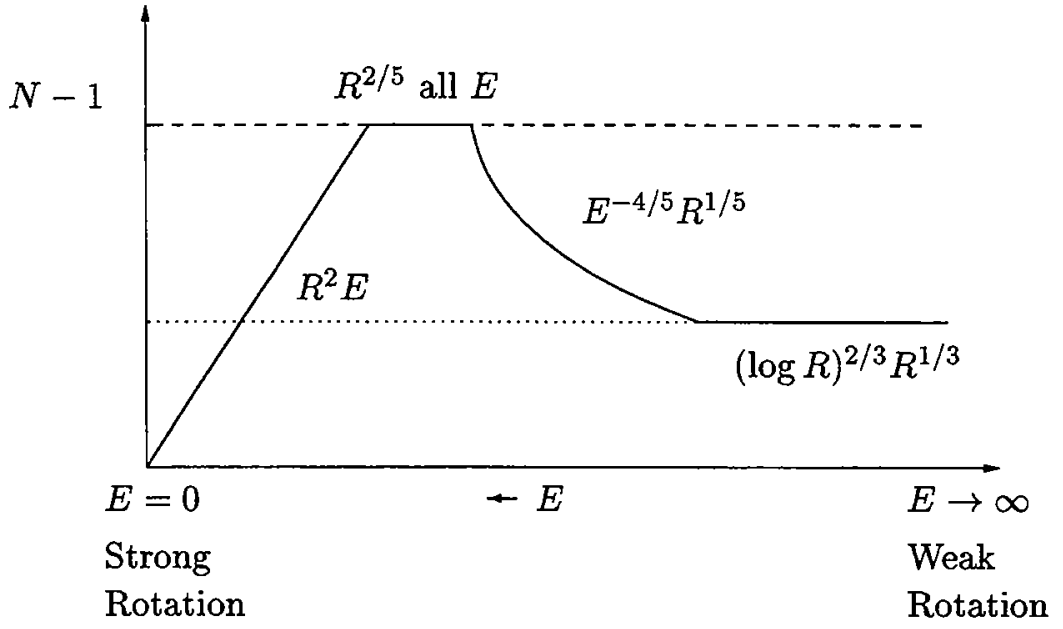

Figure 1: A plethora of upper bounds on the heat transport, $N$, in Rayleigh-Bénard convection for infinite Prandtl number.

Figure (1) shows the results of several upper bounding studies for the infinite Prandtl number problem. Upper bounds on $N-1$ are plotted against the Ekman number. The top most upper bound is a uniform bound in $E$ [2]. Intersecting this bound are two other upper bounds. The bound to the left has the proper qualitative dependence on rotation in that convection is suppressed in the limit of strong rotation $(E \rightarrow 0)$ which is suggested by the relations in (4) and (5) [3]. In the absence of rotation $(E=\infty)$ a logarithmic bound has been obtained [4], which is illustrated by the dotted line in the figure. Allowing for finite $E$ they find in [1] that there is a region in which the optimal bound is lowered from $R^{2 / 5}$ and connects to the logarithmic bound at some higher Ekman number.

\section{Bounds on the Energy Spectrum}

We now turn our attention to a problem for which an upper bound on the scaling of the energy spectrum in rotating turbulence has been caculated.

\subsection{Motivating Experiment of H. L. Swinney}

The motivation is a recent experiment by Baroud, Plapp, She and Swinney [5] for which Kolmogorov's theory for two-dimensional turbulence does not justify the scaling of the energy spectrum in the inverse cascade region. In the Experiment quasi-two-dimensional flow is studied in a rapidly rotating cylindrical annulus. The resulting velocity measurements yield a self-similar probability distribution function for longitudinal velocity differences, which are strongly non-Gaussian. The resulting energy spectrum is described by $E(k) \sim k^{-2}$ rather than the expected $E(k) \sim k^{-5 / 3}$ from Kolmogorov's theory. We shall outline a brief background to Kolmogorov's statistical study of turbulence, followed by a description of 


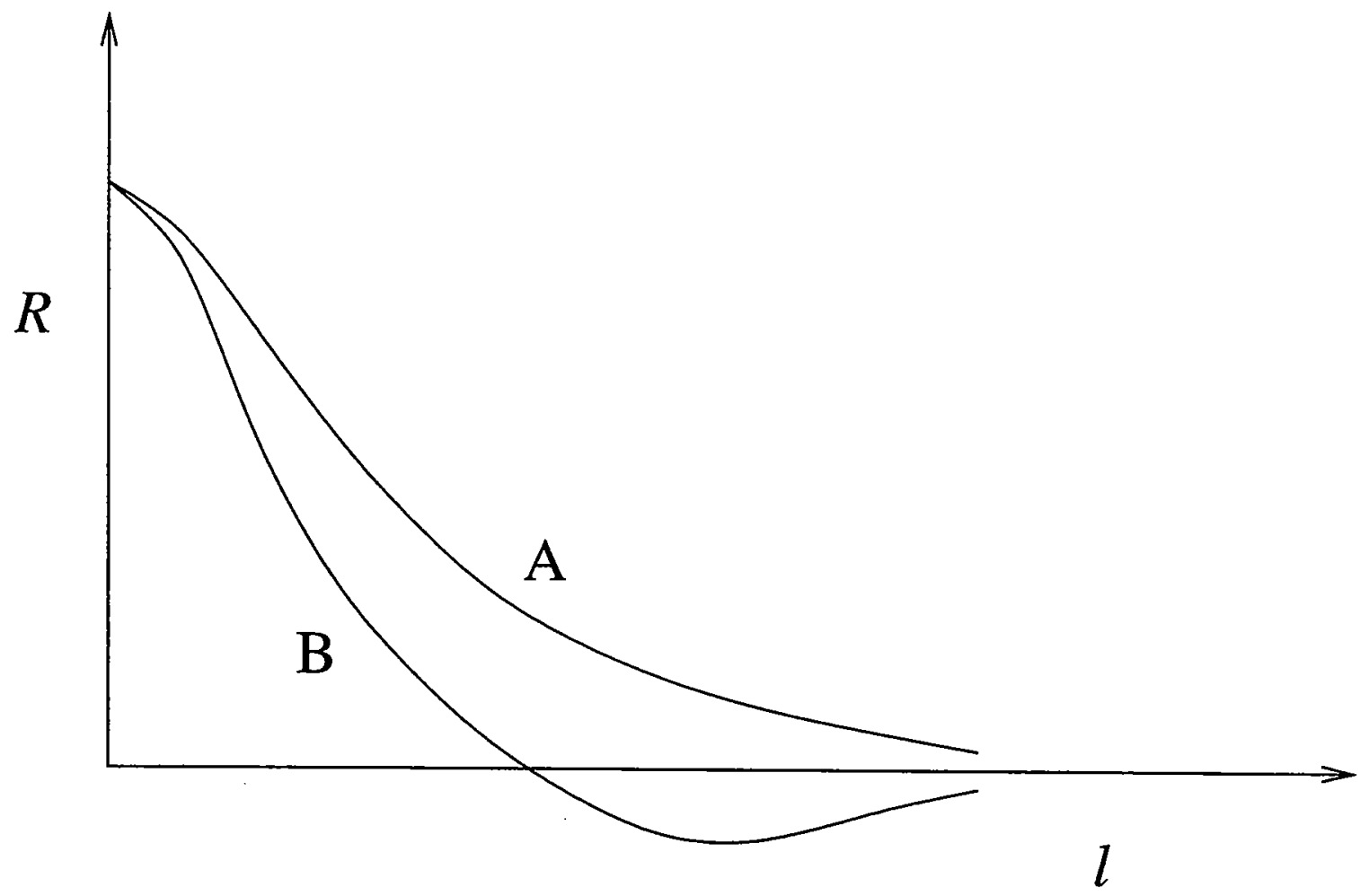

Figure 2: Typical correlation curves. $R$ is the correlation coefficient and $l$ the separation of sample points. For large separation $R$ tends to zero and for no separation $R=1$.

the experiment which. A rigorous upper bound for the energy spectrum is then presented under the assumption of quasi-geostophy.

\subsection{Background on Turbulence (See also [6])}

The most successful statistical theory of turbulence is that of Kolmogorov, which involves scaling laws for the structure function $S_{p}(l) \equiv\left\langle\delta v(l)^{p}\right\rangle \sim l^{\zeta_{p}}$ of velocity increments $\delta v(l)=$ $v(x+l)-v(x)$, where $l$ denotes the separation between two points. An often studied and very important question regarding turbulence is whether the statistics are self-similar across a wide range of spatial scales, or equivalently whether the probability distribution functions (PDFs) of the velocity increments have a functional form independent of the separation $l$.

Correlation curves such as in Figure 2 provide a method to study the scale and structure of turbulent motion. Supposing $u_{1}$ and $u_{2}$ are deviations from the mean flow at different positions but at the same instance, $\overline{u_{1} u_{2}}$ is known as a space correlation. Usually most attention is given to longitudinal or lateral correlations, i.e. to points separated parallel or perpendicular to the velocity components respectively. Correlations depend on both the direction and magnitude of $l$ and different behaviors in different directions may provide information about the structure of turbulence. When $l=0, u_{1}=u_{2}$ (provided they are in the same direction) and the correlation coefficient $R$ is by definition equal to 1 , where $R=\overline{u_{1} u_{2}} /\left(\bar{u}_{1}^{2} \bar{u}_{2}^{2}\right)^{1 / 2}$. As $l$ increases the velocity fluctuations become more and more 
independent of one another and $R$ asymptotes to 0 . A negative region in the correlation curve (figure $2 \mathrm{~B}$ ) implies that $u_{1}$ and $u_{2}$ are on average in opposite directions.

A correlation curve therefore gives an idea of the distances over which motions at different points significantly affect one another. This statistical analysis gives rise to the structure functions describing the spatial structure of the turbulent motion.

Using Fourier transforms an equation in terms of spectral functions may be obtained alternatively to the correlation functions. In the inertial range these depend only on the wave number $k$ and the energy dissipation $\epsilon, E=E(k, \epsilon)$.

$$
E(k)=\frac{1}{t} \int_{0}^{t}|\hat{u}(k)|^{2} d t
$$

and dimensional analysis then gives

$$
E(k)=A \epsilon^{2 / 3} k^{-5 / 3}
$$

where $A$ is a numerical constant. This is the famous 'Kolmogorov $-5 / 3$ law' which applies for flows of a high Reynold number under two hypothesis: 1) local isotropy and homogeneity, and 2) the existence of a wave number range independent of viscosity and large-scale properties at sufficiently large Reynolds numbers.

\subsection{The Experiment}

Kolmogorov's theory was developed without considering rotation, for planetary flows however, such as the Earth's atmosphere and ocean, this assumption may not apply since the Rossby number which measures the relative importance of the inertial and Coriolis forces in the Navier-Stokes equation is small,

$$
R=\frac{|\mathbf{u} \cdot \nabla \mathbf{u}|}{2|\Omega \times \mathbf{u}|}=\frac{L}{2 U \Omega} \ll 1 .
$$

The experiment carried out by Swinney using a rotating annular tank was the first to determine the statistical properties of turbulence in a low Rossby number flow. The experimental setup was as follows. An annular tank was filled with water and covered by a solid lid; the inner radius of the tank was $10.8 \mathrm{~cm}$ and the outer radius $43.2 \mathrm{~cm}$. The depth of the tank increased from $17.1 \mathrm{~cm}$ at the inner radius to $20.3 \mathrm{~cm}$ at the outer radius to simulate the $\beta$-effect of the earths' surface, for more details see [7]. A counter rotating jet was induced in the flow, by continuous pumping of water in to and out of the tank through two concentric rings at the bottom of the tank. A sketch of the setup is shown in Figure 3 and a more detailed description of it may be found in [5].

The purpose of the pumping at the bottom of the cylinder is to create a shear between the Ekman layer and the fluid in the tank and thus induce turbulence. The rapid rotation of the tank (11.0 rad/s) produces essentially $2 \mathrm{D}$ flow, except in the thin Ekman boundary layer at the top and bottom surfaces.

Time series measurements of the azimuthal velocity midway between the inner and outer wall of the tank were taken using hot film probes. In order to find a correlation of 

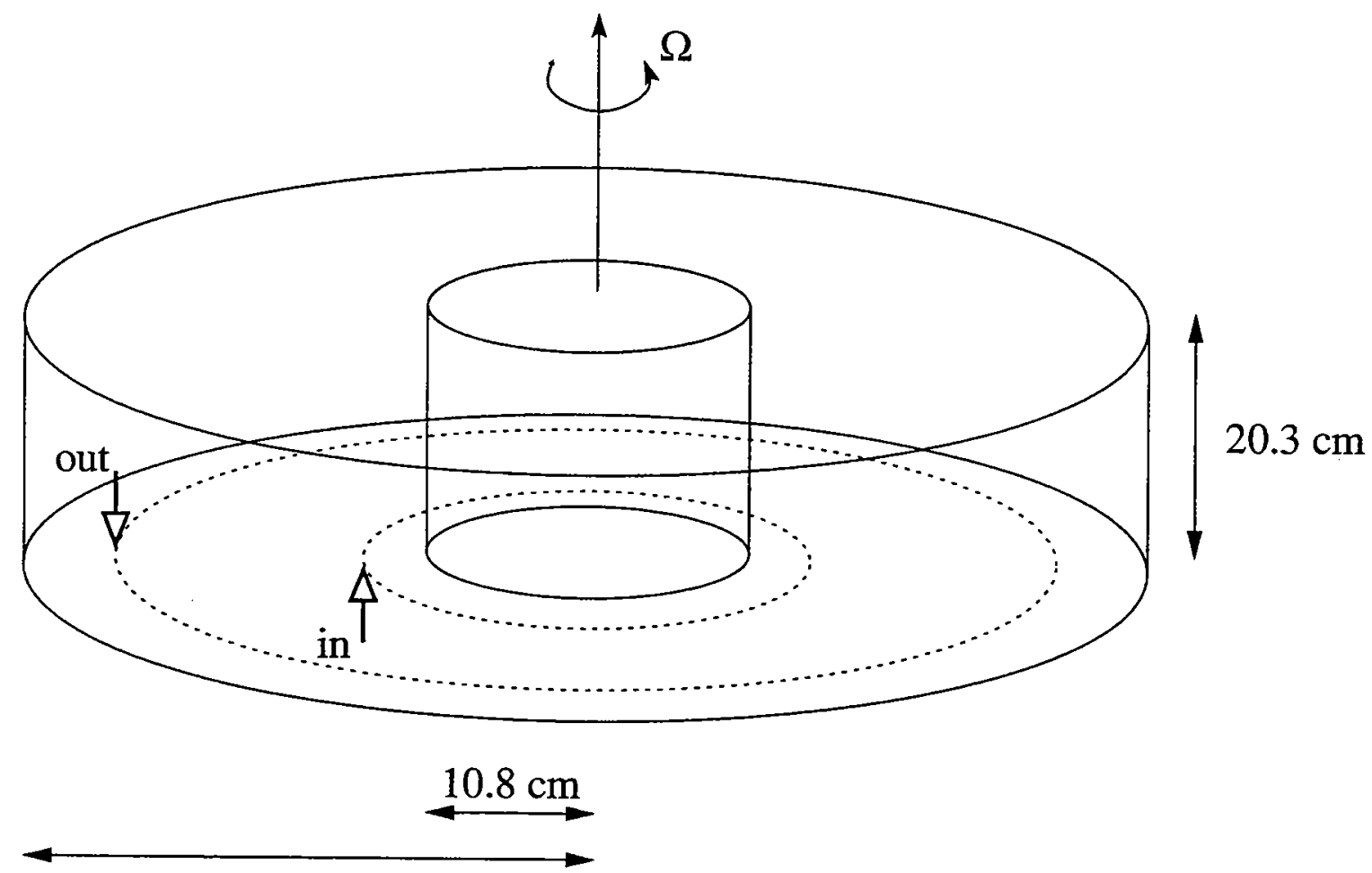

$43.2 \mathrm{~cm}$

Figure 3: Experimental apparatus. The dimensions of the tank are shown. Note that the tank is covered by a rigid lid. The dotted lines show the approximate positions of the two concentric rings pumping fluid in to and out of the tank, via the inner and outer ring respectively.

the velocity increments an autocorrelation is used, that is, the same velocity components at a single point (the hot film probes) at different instances are correlated. This depends on the time separation $s$ only, however, when the turbulent motion is occurring in a flow with a large mean velocity, as is the case in this experiment $\left(U_{\max } \simeq 22 \mathrm{~cm} / \mathrm{s}\right)$, it is possible for the turbulence to be advected past the point of observation more rapidly than the pattern of fluctuations is changing. An autocorrelation will then be directly related to the corresponding space correlation with separation in the mean flow direction, by just transforming the variables, $s=r / U$. This is referred to as Taylor's frozen in turbulence hypothesis.

The energy power spectrum is computed from the time series data obtained in the experiment and they find that $E(k) \sim k^{-2}$ for the inverse energy cascade.

\subsection{Inverse Energy Cascade}

In two-dimensional turbulence there are two conserved quantities, energy and enstrophy, which are candidates for cascades of the Kolmogorov type (see Figure 4(a)). However, to satisfy both conservation laws there must also be a reverse flow of kinetic energy, from 
(a)

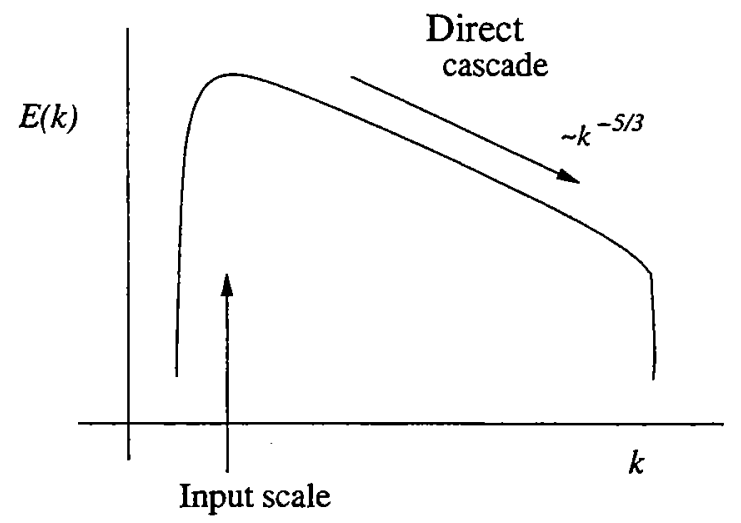

(b)

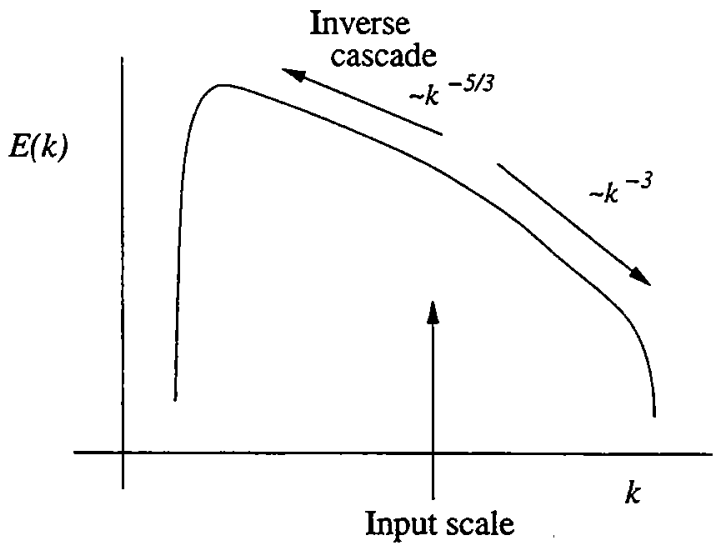

Figure 4: The energy cascade picture of fluid turbulence in (a). 3-dimensions and in (b) 2-dimensions. The input scale is the characteristic wave-number at which the fluid system is forced. In 3-dimensional turbulence energy is transfered inviscidly from the large scales (small $k$ ), associated with the energy input scale, to smaller scales where it is dissipated by viscous means at the Kolmogorov lengthscale. In 2-dimensional turbulence kinetic energy can transfer from the input scale up to larger scales. This phenomenon is known as the inverse energy cascade. For a review article on 2D turbulence see [8].

small scales to large scales, called the inverse energy cascade (Figure 4(b)). For strictly two-dimensional Navier-Stokes equations under homogeneous and isotropic conditions the Kolmogorov-Kraichnan theorem predicts a $k^{-5 / 3}$ inverse energy cascade spectrum at wavenumbers smaller than the forcing scale (for a review of two-dimensional turbulence [8]). In the Experiment which we assume is quasi-2D an inverse cascade is observed as small vortices, an array of vortex filaments are constantly injected at the boundaries of the outlets and inlets, merge to form larger vortices with maximum size limited only by the size of the experimental apparatus.

\subsection{Rotating Navier-Stokes Equations}

The equations of motion governing a body of fluid rotating at a constant rate about the $z$-axis are

$$
\begin{gathered}
\frac{\partial \mathbf{u}}{\partial t}+\mathbf{u} \cdot \nabla \mathbf{u}+\nabla \pi+2 \Omega \hat{\mathbf{k}} \times \mathbf{u}=\nu \Delta \mathbf{u} \\
\nabla \cdot \mathbf{u}=0 \\
\pi=\frac{p}{\rho}-\frac{1}{2}|\Omega(\hat{\mathbf{k}} \times \mathbf{r})|^{2}
\end{gathered}
$$


where $\mathbf{u}$ is the relative velocity. The Coriolis force $-2 \Omega \hat{\mathbf{k}} \times \mathbf{u}$ is always perpendicular to the velocity and hence does no work but tends to deflect moving fluid elements to the right (see [9] for a derivation of these equations). In the Experiment the pumping at the tank bottom produces a counter-rotating jet in the Ekman layer which generates the turbulence observed there.

\subsection{Is the Energy Dissipation Bounded in the Experiment?}

The Constantin-Doering variational approach can be used to prove the boundedness of the energy dissipation rate for the fluid system studied in the Experiment. The one technical issue is to develop a background field which is continuous, solenoidal and satisfies the necessary boundary conditions.

Natural boundary conditions for the Experiment are no-slip everywhere except at the bottom of the cylinder where fluid is injected through a ring of holes at a rate $W$ and sucked out at the same rate from a concentric ring of holes. The distance between the forcing rings, $l$, is defined as the integral length scale. We can thus define the boundary conditions as follows

$$
\begin{aligned}
& \mathbf{u}=W \varphi\left(\frac{x}{l}, \frac{y}{l}\right) \hat{\mathbf{k}} \quad \text { at } z=0 \\
& \mathbf{u}=\mathbf{0} \text { otherwise. }
\end{aligned}
$$

where $\varphi$ takes the values 1 at the input holes, -1 at the output holes and 0 everywhere else on the bottom boundary.

One can generate a smooth continuation of these boundary conditions in to an incompressible background field $U_{B}$ as follows: define $\chi(z)$ a smooth function satisfying $\chi(0)=1$, $\chi^{\prime}(0)=0$ and $\chi(H)=0, \chi^{\prime}(H)=0$, where $H$ is the height of the cylinder, which decreases rapidly over a small distance $\delta$ from $z=0$ (Figure 5). Now define $\psi(x, y)$ as the two-dimensional solution of

$$
\Delta_{H} \psi+\varphi=0
$$

where $\Delta_{H}=\frac{\partial^{2}}{\partial x^{2}}+\frac{\partial^{2}}{\partial y^{2}}$ is the horizontal Laplacian. Then it is easy to check that the following velocity profile is both incompressible and satisfies the boundary conditions of the experiment

$$
U_{B}(x, y, z)=W\left(\begin{array}{c}
l \chi^{\prime}(z)\left(\partial_{x} \psi\right)\left(\frac{x}{l}, \frac{y}{l}\right) \\
l \chi^{\prime}(z)\left(\partial_{y} \psi\right)\left(\frac{x}{l}, \frac{y}{l}\right) \\
\chi(z) \varphi\left(\frac{x}{l}, \frac{y}{l}\right)
\end{array}\right)
$$

Theorem 1: If $\frac{W l}{\nu}<c$ for some $c>0$ then $\forall$ initial conditions $\mathbf{u}_{\mathbf{0}}$

$$
\limsup _{t \rightarrow \infty} \frac{1}{t} \int_{0}^{t}\left\langle|\nabla \mathbf{u}|^{2}\right\rangle \leq \varepsilon_{B} \quad \text { where } \quad \varepsilon_{B} \leq c \frac{W^{3}}{H}
$$

Proof idea: This bound on the energy dissipation rate can be calculated using the Constantin-Doering background flow method [10] with background field $U_{B}$. 


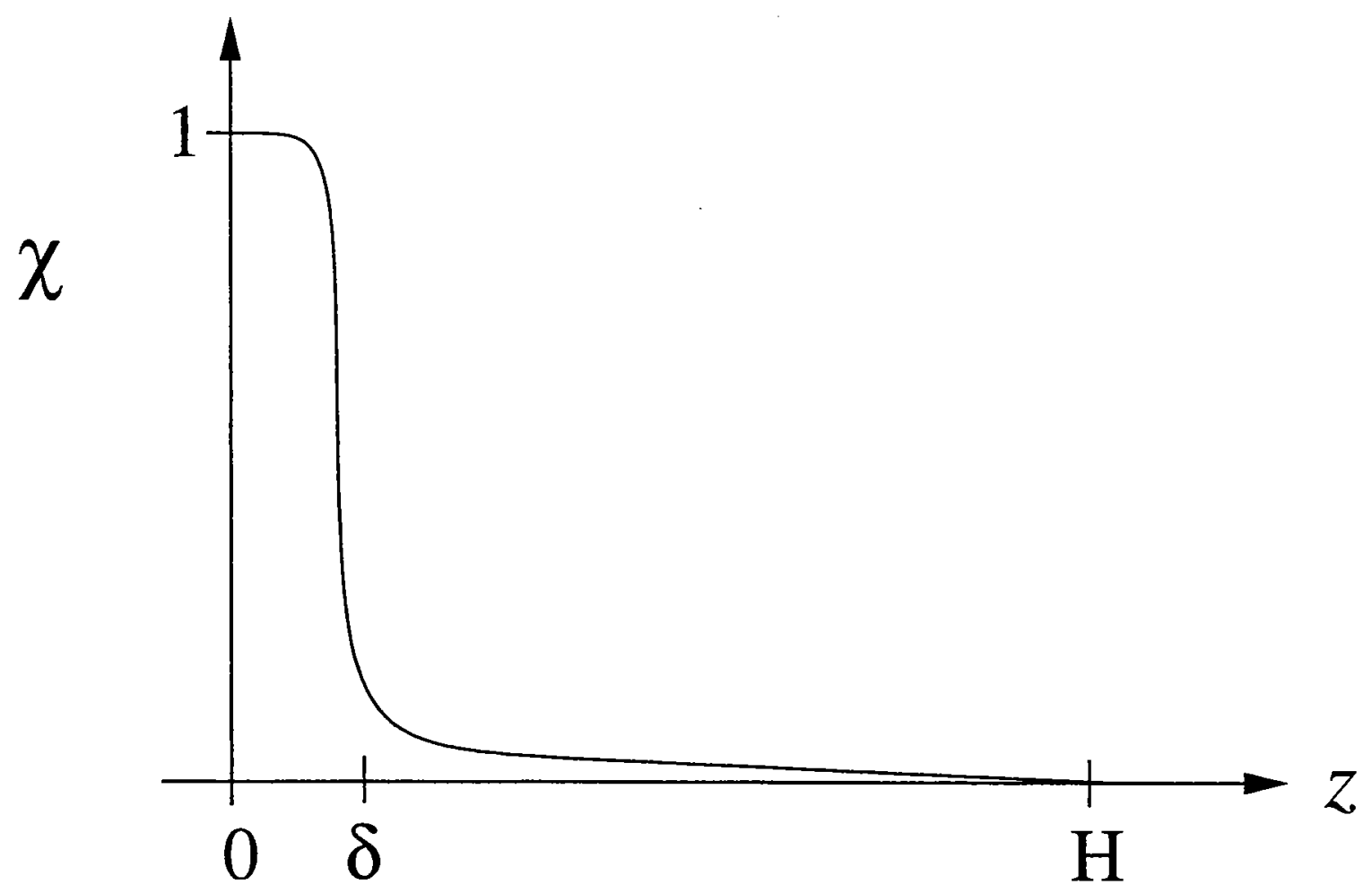

Figure 5: A sketch of the function $\chi$.

A stronger form of Theorem 1 can be proved if we assume that velocity variations in the vertical are much smaller than variations in the horizontal. So assuming that

$$
\left\langle|\delta w|^{2}\right\rangle \leq \gamma\left\langle|\delta u|^{2}+|\delta v|^{2}\right\rangle
$$

where $\gamma$ is a small number we can assert a stronger theorem.

Theorem 1a: If $\frac{W l}{\nu}<c \gamma^{-1 / 2}$ for some $c>0$ then $\forall$ initial conditions $\mathbf{u}_{0}$

$$
\limsup _{t \rightarrow \infty} \frac{1}{t} \int_{0}^{t}\left\langle|\nabla \mathbf{u}|^{2}\right\rangle \leq \varepsilon_{B} \quad \text { where } \quad \varepsilon_{B} \leq c \frac{W^{3}}{H}
$$

\subsection{Non-linear Taylor Proudman Theorem}

For flows with low values of the Rossby number $R o=L /(2 U \Omega)$, and the Ekman number $E=\nu /\left(2 L^{2} \Omega\right)$ a balance between the Coriolis force and the pressure gradient can be assumed in Equation (9), $\boldsymbol{\nabla} \pi+2 \Omega \hat{\mathbf{k}} \times \mathbf{u}=\mathbf{0}$. This balance is called the geostrophic balance. A simple consequence is that $\Omega \hat{\mathbf{k}} \cdot \nabla \mathbf{u}=\mathbf{0}$ or in words $\mathbf{u}$ has no vertical variation 
and is therefore two-dimensional. This property of geostrophic flows is known as the TaylorProudman Theorem.

A similar theorem has recently been proved for the three-dimensional Euler equations with large $\Omega$. This adds weight to the theoretical study of geostrophic flows for flow scenarios with low Rossby number and $E \ll R o$. (For example in the Experiment $R o=O\left(10^{-2}\right.$ ) while $E=O\left(10^{-6}\right)$.)

The momentum equation is now

$$
\frac{\partial \mathbf{u}}{\partial t}+\mathbf{u} \cdot \nabla \mathbf{u}+\nabla \pi+2 \Omega \hat{\mathbf{k}} \times \mathbf{u}=\mathbf{0}
$$

where $\mathbf{u}$ is again the relative velocity. In the following discussion the vorticity is denoted as $\omega=\nabla \times \mathbf{u}$.

Theorem 2: If we assume that the velocity field $\mathbf{u}$ is smooth and we measure time in units of the local eddy turnover time $|\nabla \mathbf{u}|_{\infty}^{-1}$, where ||$_{\infty}$ is the maximum norm, and define $a=\frac{\sup |\omega|}{\Omega}$, then

Two surfaces $z=z_{1}$ and $z=z_{2}$ initially separated by a distance

$L=z_{2}-z_{1}$ cannot get closer than $(1-3 a) L$ in each time step.

\subsection{Quasi-Geostrophy and $E(k) \sim k^{-2}$}

Using the simplest form of the quasi-geostrophic equations [11], which describe the departure from the geostrophic balance for strongly rotating fluids, to produce a rigorous upper bound $E(k) \leq C k^{-2}$ valid in the inverse cascade region for the energy spectrum can be derived [12]. This a priori result supports the inverse energy cascade observed in the Experiment.

Active scalar surface quasi-geostrophic equation

$$
\partial_{t} \theta+\mathbf{v} \cdot \nabla \theta+w_{E} \Lambda \theta=f
$$

with two-dimensional velocity $\mathbf{v}$ incompressible, $\boldsymbol{\nabla} \cdot \mathbf{v}=0$, and the dissipative term $w_{E} \Lambda \theta$ has a coefficient $w_{E}>0$ that comes from the Ekman pumping at the boundary.

The large forcing scale is defined by

$$
k_{j}^{-1}:=\frac{\left\langle k^{-1}|\hat{f}(k, t)|^{2}\right\rangle}{\left\langle|\hat{f}(k, t)|^{2}\right\rangle}
$$

and the theorem states that $E(k) \leq C k^{-2}$ for $k \leq k_{f}$ where $C$ is independent of the forcing scale $k_{f}$.

\section{References}

[1] P. Constantin, C. Hallstrom, and V. Poutkaradze, "Logarithmic bounds for infinite prandtl number rotating convection," Journal of Mathematical Physics 42, 773 (2001). 
[2] C. R. Doering and P. Constantin, "On upper bounds for infinite prandtl number convection with or without rotation," Journal of Mathematical Physics 42, 784 (2001).

[3] P. Constantin, C. Hallstrom, and V. Poutkaradze, "Heat transport in rotating convection," Physica D 125, 275 (1999).

[4] P. Constantin and C. R. Doering, "Infinite prandtl number convection," Journal of Statistical Physics 94, 159 (1999).

[5] C. Baroud, B. Plapp, Z. She, and H. Swinney, "Anomalous Self-Similarity in a Turbulent Rapidly Rotating Fluid," Phys. Rev. Lett. 88, (2002).

[6] D. J. Tritton, Physical Fluid Dynamics, 2nd ed. (Clarenden Press, Oxford, 1988).

[7] J. Pedlosky, Geophysical Fluid Dynamics, 2nd ed. (Springer Verlag, New York, 1987).

[8] R. H. Kraichnan and D. Montgomery, "Two-dimensional turbulence," Rep. Prog. Phys. 43, 547 (1980).

[9] S. Chandrasekhar, Hydrodynamic and hydromagnetic stability (Clarendon Press, Oxford, 1961).

[10] P. Constantin and C. Doering, "Variational bounds in dissipative systems," Physica D 82, 221 (1995).

[11] J. Pedlosky, Geophysical fluid dynamics, 2nd ed. (Springer, New York London, 1987).

[12] P. Constantin, "Energy Spectrum of Rotating Fluids," (unpublished, 2002). 


\title{
Lecture 9
}

\author{
Bounds on Mixing in Stratified Shear Flows \\ Colm-cille P. Caulfield \\ Notes by Jennifer Siggers
}

\section{Introduction and Motivation}

Mixing is a very common feature in the environmentally prevalent flows with both vertical velocity and density variation. Examples include the thermocline, the lutocline, planetary boundary layers, river mouths, etc. Such flows exhibit a characteristic life-cycle, where some external forcing intensifies the velocity shear, triggering a sequence of instabilities. These instabilities typically lead to a period of small-scale disordered turbulent motion, that is characterized by substantially enhanced mixing of fluid elements, and also dissipation. This dissipation inevitably extracts energy from the mean shear, which decreases in magnitude, leading ultimately to relaminarization of the underlying flow. Subsequent external forcing starts the cycle once again.

The problem is that crucial aspects of the life cycle are associated with motions that are inherently small scale, (of the order of millimetres) over time scales that are also short (of the order of seconds), but we wish to know what happens on much larger length and time scales, for example synoptic (i.e. of the order of hundreds to thousands of kilometres) and seasonal scales. For example we may want to know about the total global or atmospheric heat budget or pollutant transport within the entire system. Thus we might want to ask the deceptively simple question:

For a given kinetic energy input from the shear forcing, how much energy is lost to viscous dissipation and how much energy leads to mixing?

The objective of a significant amount of recent research has been to answer this question by identifying the mixing mechanisms. This has been done by finding the dependence of mixing events on bulk flow characteristics, their spatial localizations and their time dependence. Then it is possible to quantify the mixing appropriately, for example by distinguishing between reversible and irreversible processes and, more recently, by developing rigorous bounds. The ultimate aim that should always be remembered is the desire to generate robust parameterizations, useful to models of larger scale geophysical flows, that capture the essential characteristics of mixing within stratified sheared flow.

\section{Energetics of Stratified Shear Flows}

To identify some of the important aspects of the energetics of stratified shear flows, consider a simple flow that is infinite or periodic in the horizontal directions and has finite extent in the vertical direction. We use stress free boundary conditions with no normal flow through the boundary and assume an insulating temperature boundary condition. The velocity is assumed to vary from $-U_{0}$ to $+U_{0}$ over the length scale $d_{0}$, and the density varies from $\rho_{a}-\rho_{0}$ to $\rho_{a}+\rho_{0}$ over the length scale $\delta_{0}$, where $\rho_{0} \ll \rho_{a}$. Alternatively, 
$N^{2}(z)=-g / \rho_{a} \partial \bar{\rho} / \partial z$ where the variation in $\rho$ is "small" over the scale $d_{0}$. We therefore assume that the Boussinesq approximation is valid.

Richardson numbers are a useful tool for parameterizing mixing processes. There is a broad class of such numbers. Here, we define two of these; the bulk Richardson number:

$$
J=\frac{g \rho_{0} d_{0}}{\rho_{a} U_{0}^{2}}
$$

and the gradient Richardson number:

$$
R i(z)=\frac{-g d \bar{\rho} / d z}{\rho_{a}(d \bar{u} / d z)^{2}}=\frac{N^{2}}{(d \bar{u} / d z)^{2}},
$$

where the bar denotes averaging over the horizontal layer. Both the global $(J)$ and local $(R i)$ Richardson numbers are a measure of the relative importance of buoyancy force to inertia or alternatively the potential energy variations to kinetic energy variations.

In the Boussinesq approximation, the kinetic energy density of the flow is given by

$$
\mathcal{K}(t)=\frac{\left\langle|\mathbf{u}|^{2}\right\rangle}{2}
$$

where the angle brackets denote the average over the whole layer. We non-dimensionalize the equations with the scales $d_{0}, U_{0}$ and $\rho_{0}$. Dotting the Navier-Stokes equation with $\mathbf{u}$ and averaging over the domain yields the evolution equation for $\mathcal{K}$

$$
\begin{aligned}
\frac{d \mathcal{K}}{d t} & =-J\langle\rho w\rangle-\frac{1}{R e}\left\langle(\nabla \mathbf{u})^{2}\right\rangle \\
& \equiv \mathcal{H}-\mathcal{E}=-\mathcal{B}-\mathcal{E}
\end{aligned}
$$

where $\mathcal{H}$ is the heat flux, $\mathcal{B}=-\mathcal{H}$ is the buoyancy flux and $\mathcal{E}$ is the rate of dissipation.

The potential energy density is defined to be

$$
\mathcal{P}=J\langle\rho z\rangle=J\langle\bar{\rho} z\rangle_{z},
$$

where the subscript $z$ indicates averaging over the $z$-component only. The evolution equation for $\mathcal{P}$ is

$$
\frac{d \mathcal{P}}{d t}=\mathcal{B}+\mathcal{D}_{\mathcal{P}}
$$

where

$$
D_{\mathcal{P}}=\frac{2 J}{\sigma R e L_{z}}
$$

and $\mathcal{D}_{\mathcal{P}}$ is the inevtiable diffusion of the mean profile, which would occur in the absence of macroscopic fluid motion.

If the flow is statically stable $\mathcal{D}_{\mathcal{P}}>0$, denoting a continual conversion of internal energy into potential energy within the Boussinesq approximation. Energy is exchanged between $\mathcal{K}$ and $\mathcal{P}$ via $\mathcal{B}$, see figure 2 . Clearly, the buoyancy flux is intimately related to the process of mixing, but it is necessary to have a very clear view of what exactly we mean by mixing before quantitative advances can be made. 


\section{Concepts of Stirring and Mixing}

We consider mixing to be an irreversible change of the fluid properties that is inherently small scale. We wish to distinguish mixing from stirring, which we consider to be a large scale reversible motion of the fluid. Mixing, in our view, corresponds to an irreversible change of $\mathcal{P}$ caused by the motion of the fluid. However, the buoyancy flux $\mathcal{B}$ includes both mixing and stirring, so in order to quantify the amount of mixing taking place, we split the potential energy into two parts, the background potential energy, that is increased irreversibly by the mixing process and the available potential energy that may be reconverted back to kinetic energy, following the original conception of Lorenz. A particular algorithmic formulation, well-suited to numerical simulation was invented in [1], where the background potential energy is defined as

$$
\mathcal{P}_{B}=J\left\langle\rho_{B}(z) z\right\rangle_{z},
$$

where $\rho_{B}$ is the background density profile. The background density profile is the sorted statically stable profile of the fluid that has no horizontal variation, and is generated by adiabatic (within our Boussinesq incompressible framework this corresponds to volumepreserving) rearrangement or sorting of the fluid parcels into a state corresponding to the minimum possible potential energy that can be achieved by the flow. An example of the way this sorting is done is shown in figure 1. The remainder of $\mathcal{P}$ is the available potential energy $\mathcal{P}_{A}$ (i.e. available for reconversion into other forms of energy). We have,

$$
\begin{aligned}
\mathcal{P}_{A} & =\mathcal{P}-\mathcal{P}_{B} \\
\frac{d}{d t} \mathcal{P}_{\mathcal{A}} & =\mathcal{B}-\mathcal{M}=\mathcal{S} \\
\frac{d}{d t} \mathcal{P}_{\mathcal{B}} & =\mathcal{M}+\mathcal{D}_{\mathcal{P}} \\
\frac{d}{d t} \mathcal{K}(t) & =-\mathcal{S}-\mathcal{M}+\mathcal{D}
\end{aligned}
$$

where $\mathcal{S}$ and $\mathcal{M}$ are energy transfer rates defined by the above equations. A schematic view of the processes of energy transfer represented by these equations is shown in figure 2 . Figure 3 shows a schematic graph of possible values of $\mathcal{B}, \mathcal{M}$ and $d \mathcal{P}_{A} / d t$ for a typical fluid. The left hand half shows a situation where the fluid is moving upwards on average $(\mathcal{B}>0)$. The mixing rate $\mathcal{M}$ can actually be small during this stage, for example during the initial preturbulence roll-up of a Kelvin-Helmholtz billow. In the right hand half, the fluid is moving downwards on average $(\mathcal{B}<0)$, and this can correspond to a higher mixing rate. However, the averages of $\mathcal{B}$ and $\mathcal{M}$ for sufficiently long times are always equal, so that

$$
\lim _{t \rightarrow \infty} \int_{0}^{t} \mathcal{B} d t=\lim _{t \rightarrow \infty} \int_{0}^{t} \mathcal{M} d t, \quad \text { i.e. } \lim _{t \rightarrow \infty} \int_{0}^{t} \mathcal{S} d t=0 .
$$

\section{Mixing Efficiency}

Essentially the fundamental question posed in the introduction considers the efficiency of the mixing, i.e. the proportion of the kinetic energy lsot by the flow (or the driving 


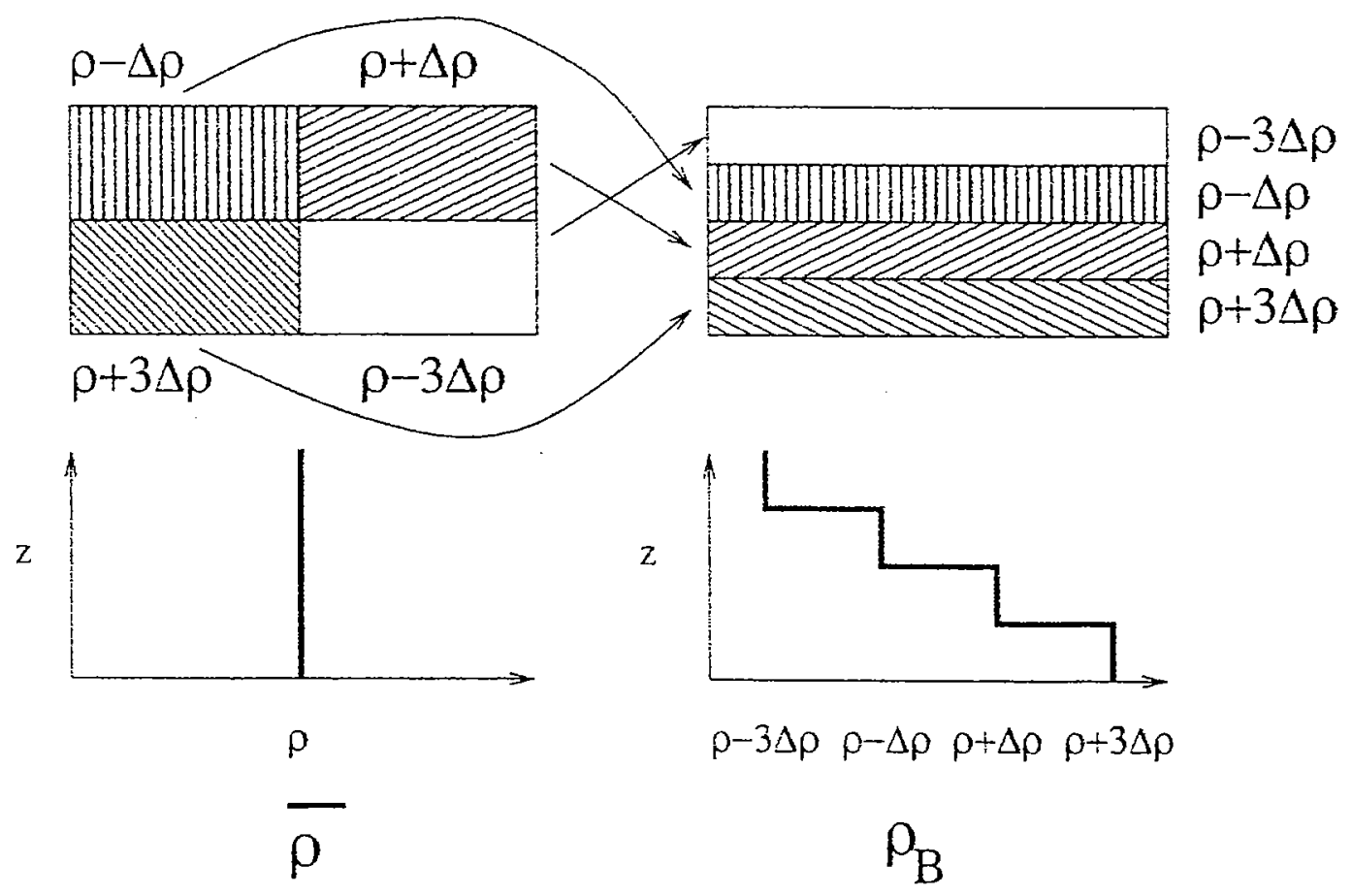

Figure 1: Diagrams showing an example of the actual state of the fluid (top left). The horizontally averaged density $\bar{\rho}$ is shown underneath, which is uniform in $z$ in this case. The sorted stable profile of the fluid for calculating the background density is shown (top right), with the heaviest fluid at the bottom, and the graph of background density is shown underneath. 


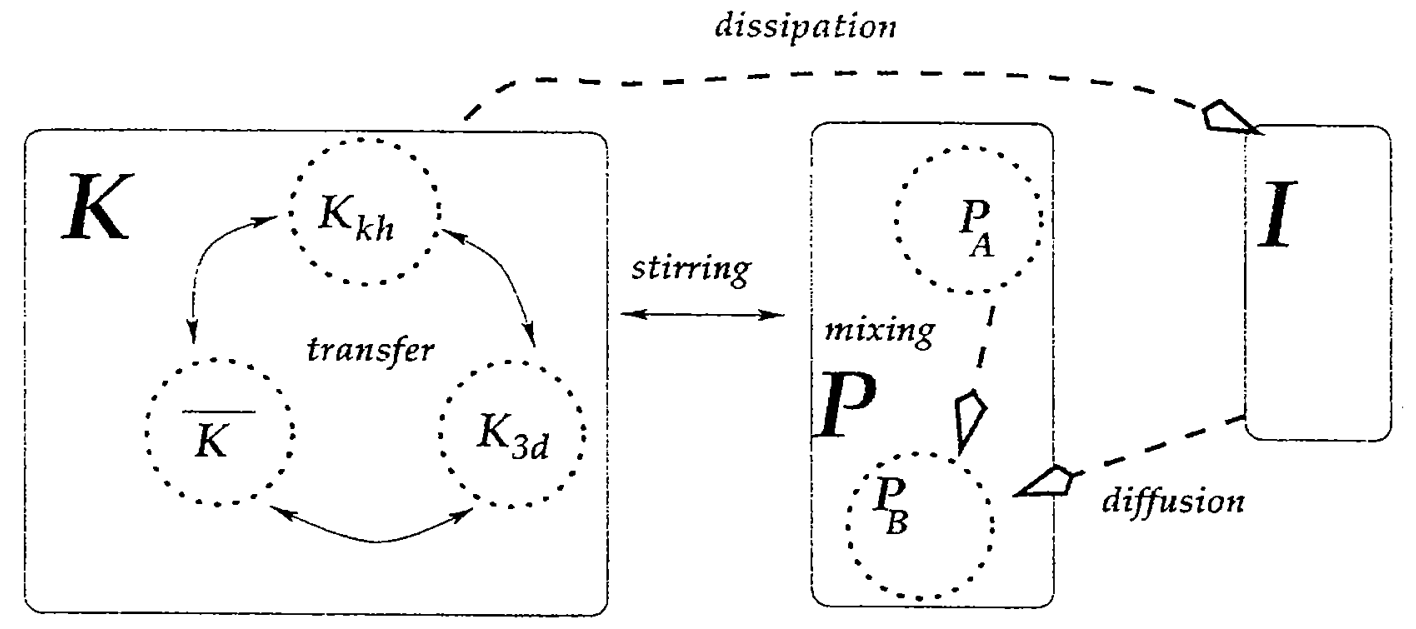

Figure 2: Diagram showing the mechanisms by which energy may be transferred in the fluid. $\mathcal{K}$ is the kinetic energy, $\mathcal{P}$ is the potential energy and $\mathcal{I}$ is the internal energy of the fluid (e.g. due to its temperature).

mechanism) that leads to mixing, or, equivalently, irreversible increases in potential energy. More formally, the mixing efficiency is usually (e.g. for grid-stirred experiments) defined as

$$
\frac{\triangle P E}{W O R K}
$$

(see $[2,3]$ etc.). This is the natural measure of the proportion of the kinetic energy input to the fluid that has led to irreversible mixing. Experimentally this is typically only determined at the very end of an experiment, once all reversible processes can be assumed to have died out. However, provided the background density profile can be determined explicitly, (as can be done straightforwardly in a numerical simulation) it is possible to define an instantaneous mixing efficiency:

$$
\mathcal{E}_{i} \equiv \frac{\mathcal{M}}{\mathcal{M}+\mathcal{E}}
$$

Naturally, it is also possible to define a long-time cumulative version

$$
\mathcal{E}_{c} \equiv \frac{\int_{0}^{t} \mathcal{M}(u) \mathrm{d} u}{\int_{0}^{t} \mathcal{M}(u) \mathrm{d} u+\int_{0}^{t} \mathcal{E}(u) \mathrm{d} u},
$$

that more closely approximates the experimental quantity.

The beautiful work of Winters [4] has shown that the diapycnal flux $\Phi_{d}$ is

$$
\Phi_{d}=\mathcal{M}+\mathcal{D}_{\mathcal{P}}=-\frac{J}{\operatorname{RePr}}\left\langle\frac{d z_{*}}{d \rho}|\nabla \rho|^{2}\right\rangle
$$




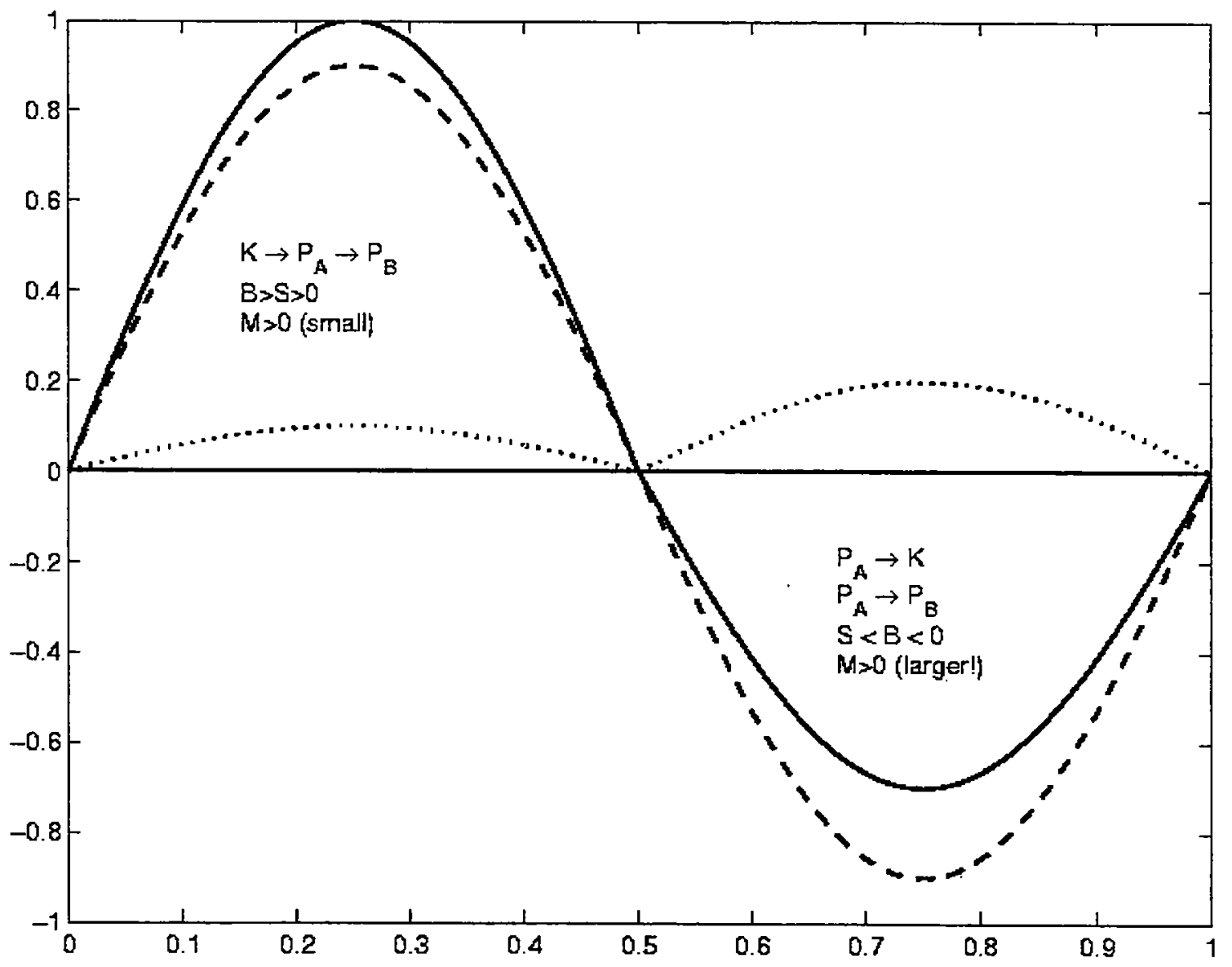

Figure 3: Diagram showing possible values of $\mathcal{B}$ (solid), $\mathcal{M}$ (dotted) and $d \mathcal{P}_{A} / d t$ (dashed) for a typical fluid as over a period of time. 
where $z_{*}$ is the coordinate associated with the rearanged fluid parcels that make up the background density profile $\rho_{B}$. A large $\Phi_{d}$ means that there is an enhanced irreversible transport of density, and hence an irreversible increase in potential energy. From the formula, it is apparent that this may occur if there is an enhanced density gradient and/or enhanced surface area of contact between fluids of different densities. $\Phi_{d}$ can also be related to the Cox number, or equivalently to the flux Richardson number.

The flux Richardson number is defined in sheared stratified turbulent flow as

$$
R_{f}=\frac{\mathcal{B}}{-\left\langle u^{\prime} w^{\prime}\right\rangle d \bar{u} / d z}
$$

where $\mathbf{u}^{\prime}=\mathbf{u}-\overline{\mathbf{u}}$. The long time average of $R_{f}$ always tends to the mixing efficiency $\mathcal{E}_{c}$. However, the denominator (essentially the shear production of turbulent kinetic energy, which corresponds to the kinetic energy lost by the mean, forcing flow) of the expression for $R_{f}$ is always positive in a steady state and so if $\mathcal{B}<0$, which often happens in the periods of most intense mixing then $R_{f}$ is negative! Hence it does not necessarily provide a good instantaneous estimate of the mixing efficiency.

\section{Previous Parameterizations}

Previous parametrizations of mixing within shear driven turbulence have focussed on appropriate descriptions of the flux Richardson number, since it is apparent that in a shear flow

$$
\frac{R_{f}}{R i}=\frac{k_{h}}{k_{m}}
$$

where

$$
k_{h}=\frac{\mathcal{B}}{N^{2}} \quad \text { and } \quad k_{m}=\frac{-\left\langle u^{\prime} w^{\prime}\right\rangle}{d \bar{u} / d z},
$$

are the eddy diffusivities of density and momentum respectively. Larger scale models often rely on sub-grid scale parameterizations based on eddy diffusivities. Although such models have many problems, they are commonly used, and so the determination of the flux Richardson number in terms of bulk properties of the flow has been the focus of much research.

For example, the Osborn-Cox Model [5] is a common oceanographic model that assumes that the flow is stationary and homogeneous. Also, both boundary effects and the effects of advection into and out of the domain are assumed to be unimportant. With these assumptions,

$$
k_{h}=\frac{R_{f}}{1-R_{f}} \frac{\mathcal{E}}{N^{2}}=\Gamma \frac{\mathcal{E}}{N^{2}}
$$

where $\Gamma$ is known as the flux coefficient. Historically, often $R_{f} \approx 0.15$ has been assumed, corresponding to $\Gamma \approx 0.2$ (based on oceanographic observations), although $0.05 \leq R_{f} \leq 0.3$ have been observed [6,7]. Rod-stirring experiments suggest $R_{f} \leq 0.8$ [3] and collated 


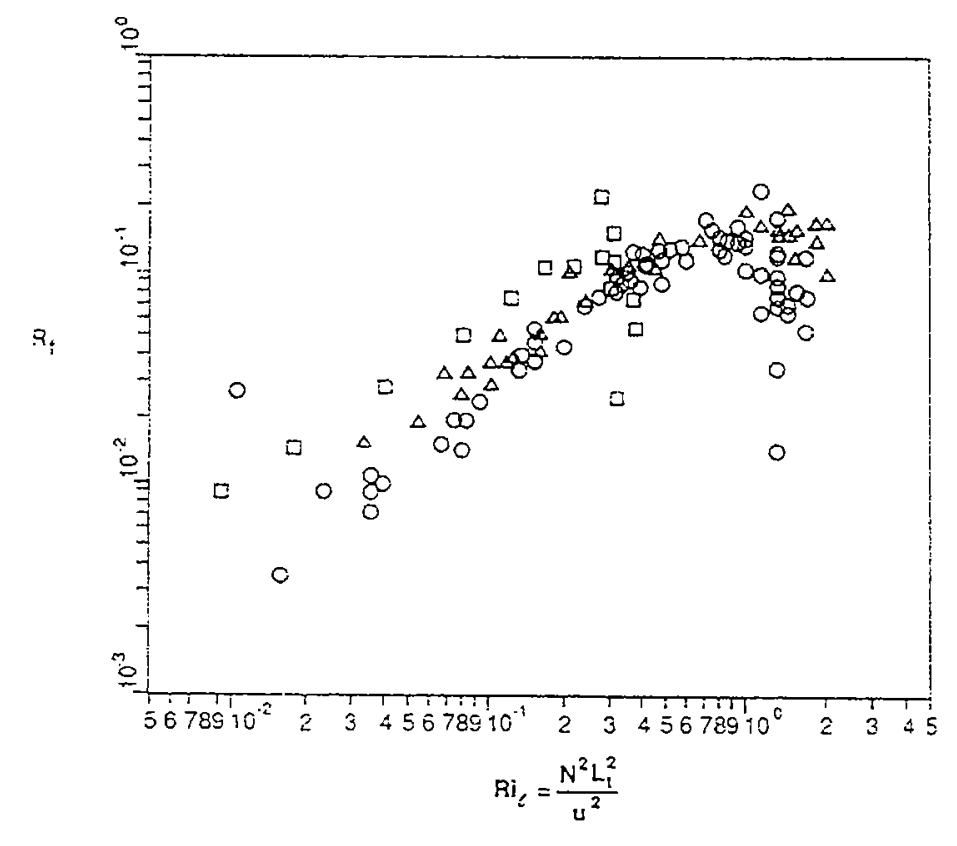

(b)

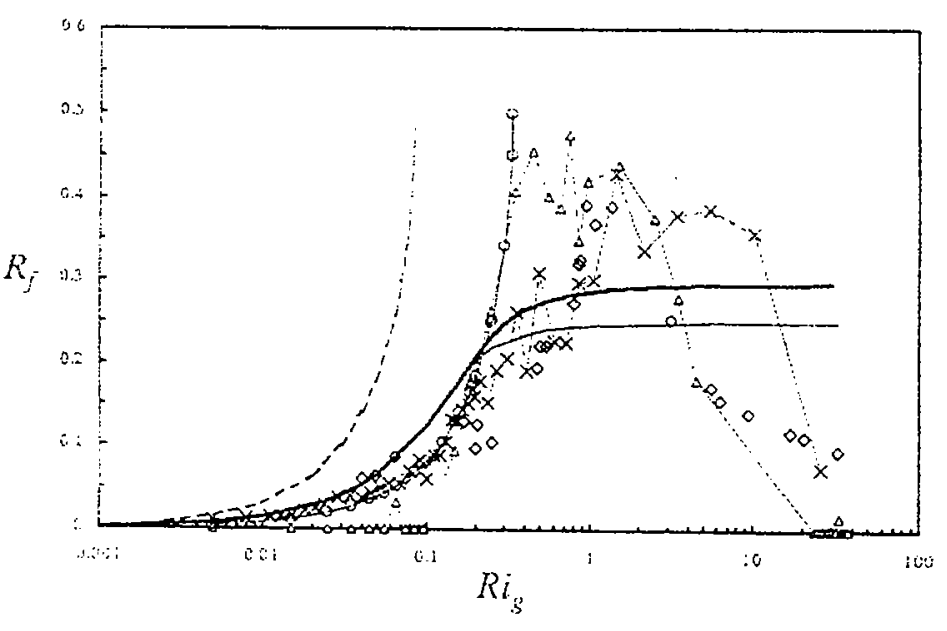




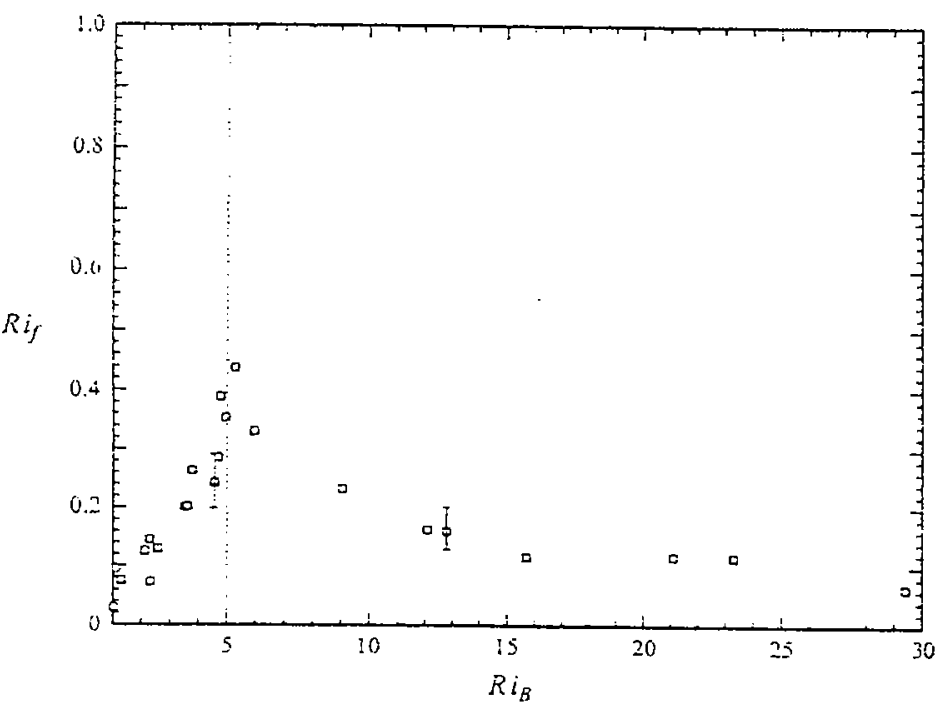

$(c)$

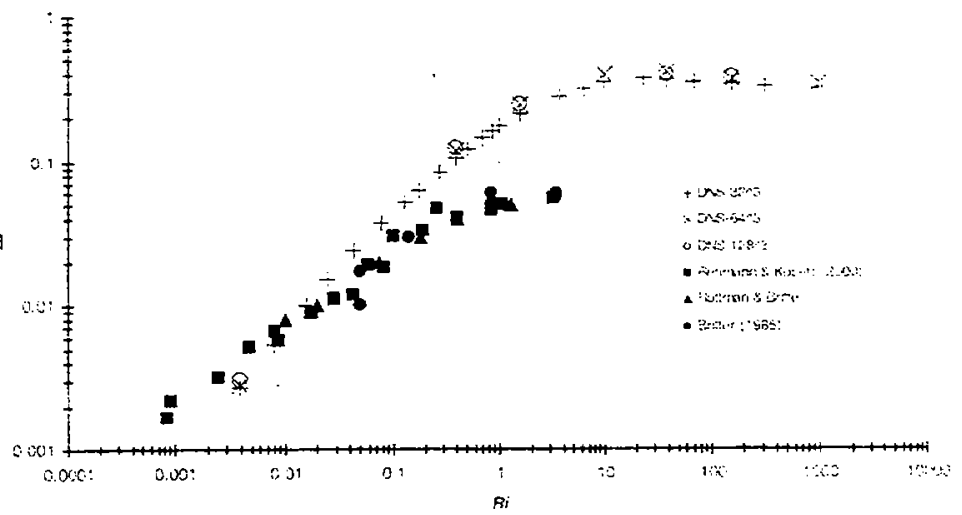

$(d)$

Figure 4: Four graphs showing values of the flux Richardson number $R_{f}$ as a function of gradient Richardson number $R i$ obtained by different sources. (a) Some experimentally measured values. The squares represent thermally stratified wind-tunnel data from [11], and the circles and triangles represent decaying and growing (shear-driven) stratified turbulence data in salinity-stratified fluids, as compiled in [12]. (b) Observed mixing efficiencies. The dashed curve is from [13], the thin solid curve is from [14], the bold solid curve is from [15], the triangles are from an experiment based in Salt Lake City and the crosses are from an experiment based at Los Alamos [16], the diamonds are from [17] and the circles are from a modified version of [13]. The graph is taken from [16]. (c) Experimental values from $[17] .(d)$ Values obtained by direct numerical simulation compared with experimental values (solid symbols). 
experiments suggest $R_{f} \leq 0.2$ [8]. The dependence of the mixing efficiency on $R i$ and $J$ was found in $[9,10]$ where is was also found that there is a tendency for the flows to form layers. A graph showing the relationship between $R i$ and $R_{f}$ is shown in figure $4(a)$.

However, some numerical calculations suggest the possibility of larger $\mathcal{E}_{c}$, and hence $R_{f}$, for example in pre-turbulent billows $[18,19,20]$. Also recent direct numerical simulations of homogeneous decaying turbulence suggest $R_{f} \approx 0.4$, which is consistent with rapid distortion theory calculations at high $J$ [21]. Stratified shear experiments have $R_{f} \approx 0.45$ and obesrvations have found values of $R_{f}$ between 0.4 and $0.45[22,17,16]$. The graphs in figure $4(b),(c)$ and $(d)$ show some results that have obtained higher values of $R_{f}$.

Some models have also produced high mixing efficiencies. For example, Pearson, Puttock \& Hunt [23] found that the mixing was related to local density perturbations and its efficiency was constant (and independent of stratification). There was also an apparent equipartition of $\mathcal{P}$ and $\mathcal{K}$. Weinstock [24] assumed that the dominant mixing processes occur at scales within the inertial subrange (i.e. those scales where there is homogeneous isotropic turbulence, that are much smaller than any characteristic forcing lengthscales and yet longer than the viscous Kolmogorov dissipation lengthscale). He showed, by manipulation of the Lagrangian velocity correlation function that $k_{h}$ is predicted to take a value consistent with $R_{f}=4 / 9$.

\section{Townsend's Model}

Townsend [25] developed an empirical model for the heat and momentum transport in turbulent stratified flow. His fundamental assumption was that the turbulence is little affected by the stratification of the fluid. This is obviously not the case in flows where the turbulence is driven on sufficiently large vertical length scales, for which the turbulent motions in the vertical direction are likely to be hindered by the stratification. However, if the dominant turbulent scales have sufficiently small scales, the turbulence can be assumed to be relatively independent of the stratification.

Townsend's empirical assumption is that all flow quantities can be described by characteristic scales of $u$ and the density fluctuations $\rho$. We define the r.m.s. turbulent kinetic energy intensity $q$

$$
q=\sqrt{\overline{|\mathbf{u}-\overline{\mathbf{u}}|^{2}}}
$$

and the r.m.s. density fluctuations $r$,

$$
r=\sqrt{\overline{|\rho-\bar{\rho}|^{2}}}
$$

The equations for the flow $\mathbf{v}$ and the density fluctuations $\rho$ in the Boussinesq approximation are

$$
\begin{aligned}
& \frac{\partial \mathbf{u}}{\partial t}+\mathbf{u} \cdot \nabla \mathbf{u}+\nabla p=-\rho \frac{\mathbf{g}}{\bar{\rho}}+\nu \nabla^{2} \mathbf{u} \\
& \frac{\partial \rho}{\partial t}+\mathbf{u} \cdot \nabla \rho=\kappa \nabla^{2} \rho \\
& \nabla \cdot \mathbf{u}=0
\end{aligned}
$$


Assuming that the dominant flow is horizontal, the flow field can be written as

$$
\mathbf{u}=U(z) \mathbf{x}+\mathbf{v}(\mathbf{r}) \text { where } U(z) \mathbf{x}=\overline{\mathbf{u}} \text { and } \overline{\mathbf{v}}=0 .
$$

Let $\mathbf{v}=(u, v, w)$; assuming a steady state and taking the dot product of $\mathbf{v}$ with equation (25) and integrating over the horizontal plane yields

$$
-\overline{(u w)} \frac{d U}{d z}-\frac{g}{\rho_{0}} \overline{(\rho w)}-\bar{\epsilon}=0 .
$$

Similarly, multiplying (26) by $\rho$ and using the same procedure we get

$$
\overline{(\rho w)} \frac{d \bar{\rho}}{d z}+\overline{\epsilon_{\rho}}=0
$$

where $\bar{\epsilon}$ is the horizontally averaged momentum dissipation rate and $\overline{\epsilon_{\rho}}$ is the horizontally averaged thermal dissipation rate.

The following parameterizations were proposed by Townsend and follow from a simple dimensional analysis:

$$
|\overline{u w}|=a_{1} q^{2}, \quad|\overline{\rho w}|=a_{2} r q, \quad \bar{\epsilon}=\frac{q^{3}}{L_{\epsilon}}, \quad \overline{\epsilon_{\rho}}=\frac{q r^{2}}{L_{\rho}}
$$

where $a_{1}, a_{2}$ are positive nondimensional constants and $L_{\epsilon}, L_{\rho}$ are the (constant) integral length scales of velocity and density fluctuations. Substituting equation (30) into (26) to eliminate $r$ yields a quadratic form for $q$ only.

Townsend [25] then proceeded to use these equations to derive a relation between the local fluxes and the local Richardson number, defined in equation (2). However, we shall assume that the vertical variation of the flow and fluid structure is small, so that through a vertical integration of equations (29) and (25) we can obtain a relation between the global Richardson number $J$ and the flux Richardson number $R_{f}$. The integration yields

$$
\langle q\rangle_{z}^{2}-\left(\frac{a_{1} L_{\epsilon} \Delta U}{2 d}\right)\langle q\rangle_{z}+\frac{g \Delta \rho L_{\rho} L_{\epsilon} a_{2}^{2}}{\rho_{0} d}=0
$$

where $\langle q\rangle_{z}$ results from the vertical integration of $q, \Delta U$ and $\Delta \rho$ are positive definite, and $d$ is the half-thickness of the layer. This quadratic form can be solved for $\langle q\rangle_{z}$, and thereby provide also an expression for $\langle r\rangle_{z}$. These can then be substituted into the expression for the flux Richardson number, defined in (19) to give

$$
R_{f}=\mathcal{E}_{c}=\frac{1}{2}\left(1-\sqrt{1-\frac{4\left\langle|\rho w|^{2}\right\rangle_{z}\langle\epsilon\rangle}{\left\langle|u w|^{2}\right\rangle_{z}\left\langle\epsilon_{\rho}\right\rangle_{z}}} J\right)
$$

It appears that if $J$ exceeds the critical value

$$
\frac{\left\langle|u w|^{2}\right\rangle_{z}\left\langle\epsilon_{\rho}\right\rangle_{z}}{4\left\langle|\rho w|^{2}\right\rangle_{z}\langle\epsilon\rangle}
$$




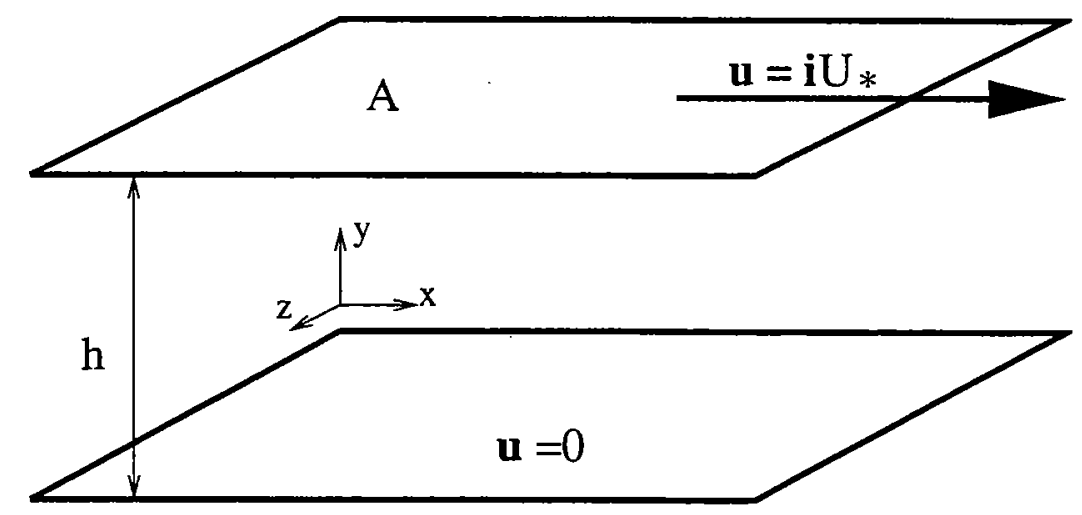

Figure 5: Schematic picture of set-up of numerical scheme

then there is no solution with physical meaning. As $J$ tends to this critical value from below, $\mathcal{E}_{c} \rightarrow 1 / 2$. Townsend interprets the critical value as the point above which "the energy supply is no longer sufficient and the motion collapses to almost laminar flow".

Heuristically, this theory appears to suggest an upper bound on mixing efficiency of $1 / 2$, consistently with the recent experimental and observational data.

We shall now try to apply the methods developed by Doering \& Constantin [26] to a model flow in order to derive rigorous upper bounds for the irreversible mixing rate $\mathcal{M}$ or equivalently the long-time average of the buoyancy flux, motivated by these suggestions that $R_{f}$ can be higher than is commonly assumed.

\section{Bounding Techniques for Stratified Shear Flows}

More specifically, for the problem of a stratified shear flow, the questions that we will ask are the following:

- Is it possible to generate a bound on mixing of heat?

- For a given forcing, how much energy is transferred into $\mathcal{P}$ the potential energy?

- Can we bound the long-time averaged buoyancy flux, i.e. can we bound the mixing rate $\mathcal{M}$ ? Does it depend on flow parameters? What is the associated mixing efficiency?

\subsection{Model Problem by C. P. Caulfield and R. R. Kerswell [27]: Stratified Couette Flow}

A simple model set-up that can be used for to investigate these issues is that of the stratified Couette Flow: two infinite bounding plates, placed at $z_{ \pm}= \pm 1 / 2$, and moving with velocities $-\Delta U / 2$ and $\Delta U / 2$ respectively within our non-dimensional scheme. The temperature imposed on these plates is constant and fixed in such a way as to ensure $\rho=\rho_{A} \mp \Delta \rho / 2$ on the upper and lower plates respectively. The set-up is illustrated in figure 5 . It is important to stress that this flow is statically stable, as distinct from more commonly considered convectively unstable flows. 
Using the following scales

- length: $d$,

- time: $d^{2} / \kappa$,

- density: $\Delta \rho$,

as well as the Boussinesq approximation, the governing equations (25)-(27) become

$$
\begin{aligned}
\frac{D \mathbf{u}}{D t}+\nabla p-\sigma \nabla^{2} \mathbf{u}+\sigma^{2} R e^{2} J \hat{\mathbf{z}} & =0, \quad(\mathcal{N S}) \\
\frac{\partial \rho}{\partial t}+\mathbf{u} \cdot \nabla \rho-\nabla^{2} \rho & =0, \quad(\mathcal{R}) \\
\nabla \cdot \mathbf{u} & =0,
\end{aligned}
$$

where the relevant parameters are

$$
R e=\frac{\Delta U d}{\nu}, \quad \sigma=\frac{\nu}{\kappa}, \quad J=\frac{g \Delta \rho d}{\rho_{0}(\Delta U)^{2}}
$$

and the boundary conditions are

$$
\begin{aligned}
& \mathbf{u}\left(z_{ \pm}\right)=\mp \sigma R e \hat{\mathbf{x}} \\
& \rho\left(z_{ \pm}\right)=\mp 1 / 2 .
\end{aligned}
$$

\subsection{Problem of Interest}

We will be particularly interested in long-time averages of the flow in order to define bounds on the states reached by the system under forcing.

We perform the standard manipulation of dotting $\mathcal{N S}$ with $\mathbf{u}$; the long time average of the result yields the energy balance equation

$$
\lim _{t \rightarrow \infty} \frac{1}{t} \int_{0}^{t}\left\langle|\nabla u|^{2}\right\rangle+\sigma R e^{2} J\left\langle u_{3} \rho\right\rangle+\frac{\sigma R e}{2}\left[\left.\frac{\partial \bar{u}}{\partial z}\right|_{z_{+}}+\left.\frac{\partial \bar{u}}{\partial z}\right|_{z_{-}}\right] d t^{\prime}=0
$$

where here a bar denotes the horizontal average of a quantity and angle brackets denote the average over all three space dimensions. Similar manipulations of the mass continuity equation give an entropy equation

$$
\lim _{t \rightarrow \infty} \frac{1}{t} \int_{0}^{t}\left\langle|\nabla \rho|^{2}\right\rangle+\frac{1}{2}\left[\left.\frac{\partial \bar{\rho}}{\partial z}\right|_{z_{+}}+\left.\frac{\partial \bar{\rho}}{\partial z}\right|_{z_{-}}\right] d t^{\prime}=0
$$

in the Boussinesq approximation, and finally multiplying the mass equation $\mathcal{R}$ by $z$ and averaging yields the potential energy equation

$$
\lim _{t \rightarrow \infty} \frac{1}{t} \int_{0}^{t} 1+\left\langle u_{3} \rho\right\rangle+\frac{1}{2}\left[\left.\frac{\partial \bar{\rho}}{\partial z}\right|_{z_{+}}+\left.\frac{\partial \bar{\rho}}{\partial z}\right|_{z+-}\right] d t^{\prime}=0
$$


Eliminating the boundary terms between these two equations provides a relation between the long-term averaged buoyancy flux $\mathcal{B}$ and diffusion terms, and shows that maximizing

$$
\mathcal{B}=\lim _{t \rightarrow \infty} \frac{1}{t} \int_{0}^{t} \sigma R e^{2} J\left\langle u_{3} \rho\right\rangle d t^{\prime}
$$

is equivalent to maximizing

$$
\lim _{t \rightarrow \infty} \frac{1}{t} \int_{0}^{t}\left(\sigma R e^{2} J\left\langle|\nabla \rho|^{2}\right\rangle-1\right) d t^{\prime}
$$

Note that the quadratic form in $\nabla \rho$ is more convenient to maximize, which is why it was chosen. To consider this problem, we use the Constantin-Doering-Hopf Method [26], also called the "background method".

\subsection{Constantin-Doering-Hopf Method}

We decompose $\mathbf{u}$ and $\rho$ in the following manner:

$$
\begin{aligned}
\mathbf{u}(\mathbf{x}, t) & =\phi(z) \hat{\mathbf{x}}+\mathbf{v}(\mathbf{x}, t), \\
\rho(\mathbf{x}, t) & =\tau(z)+\theta(\mathbf{x}, t) .
\end{aligned}
$$

Note that the background fields $\phi(z)$ and $\theta(z)$ are not the horizontal averages of the flow; this decomposition is certainly not unique, and allows us to chose the "background" fields $\phi(z)$ and $\theta(z)$ arbitrarily under the sole conditions that they satisfy the inhomogeneous boundary conditions with the fluctuations $\mathbf{v}$ and $\theta$ satisifying the homogeneous boundary conditions, i.e. $\phi=\mp \sigma R e, \tau=\mp 1 / 2, \mathbf{v}=\mathbf{0}$, and $\theta=0$ at $z_{ \pm}$.

The corresponding variational problem consists in maximizing the functional

$$
\mathcal{L}(\phi, \tau, a, b, \mathbf{v}, \theta)=\lim _{t \rightarrow \infty} \frac{1}{t} \int_{0}^{t}\left[\sigma R e^{2} J\left\langle\left|\frac{\mathrm{d} \tau}{\mathrm{d} z} \hat{\mathbf{z}}+\nabla \theta\right|^{2}\right\rangle-a\langle\mathbf{v} \cdot(\mathcal{N S})\rangle-b\langle\theta(\mathcal{R})\rangle\right] d t^{\prime},
$$

and where, formally $a \mathbf{v}$ is the Lagrange multiplier used to impose the condition that the flow should satisfy the Navier Stokes equation, and $b \theta$ is the Lagrange Multiplier used to impose $(\mathcal{R})$. This is actually equivalent to the statement that $-a \phi$ is the multiplier used to impose the mean momentum balance, $a$ is the multiplier used to impose the total power balance, $b$ the entropy flux balance and finally $-b \tau$ the mean heat balance, which can be shown from (48).

\subsection{Spectral Constraint}

Substituting the ansatz $(47)$ into the expressions for $(\mathcal{N S})$ and $(\mathcal{R})$ of the functional $\mathcal{L}$ yields

$$
\mathcal{L}=\sigma R e^{2} J\left\langle\tau^{\prime 2}\right\rangle-\lim _{t \rightarrow \infty} \frac{1}{t} \int_{0}^{t} \mathcal{G}(\tau, \phi, \mathbf{v}, \theta) d t^{\prime}
$$


where the prime denotes derivative with respect to $z$. Provided the infimum of $\mathcal{G}$ exists, then

$$
\mathcal{L} \leq \sigma R e^{2} J\left\langle{\tau^{\prime}}^{2}\right\rangle-\inf _{\mathbf{v}, \theta} \mathcal{G}
$$

where

$$
\begin{aligned}
\mathcal{G}= & \left\langle a \sigma|\nabla v|^{2}+\left(b-\sigma R e^{2} J\right)|\nabla \theta|^{2}+a v_{1} v_{3} \phi^{\prime}\right. \\
& \left.+\left(b \tau^{\prime}+a \sigma^{2} R e^{2} J\right) v_{3} \theta-\left(b-2 \sigma R e^{2} J\right) \theta \tau^{\prime \prime}-a \sigma \phi^{\prime \prime} v_{1}\right\rangle
\end{aligned}
$$

Convexity arguments show that the infimum exists only if:

$$
\left\langle a \sigma|\nabla v|^{2}+\left(b-\sigma R e^{2} J\right)|\nabla \theta|^{2}+a v_{1} v_{3} \phi^{\prime}+\left(b \tau^{\prime}+a \sigma^{2} R e^{2} J\right) v_{3} \theta\right\rangle \geq 0,\{\mathcal{S C}\}
$$

which represents the so-called spectral constraint. This implies straightforwardly that $a$ and $b$ must necessarily satisfy $a \sigma>0$ and $b>\sigma R e^{2} J$.

The Euler-Lagrange equations which must be satisfied to minimize $\mathcal{G}$ are given by

$$
\begin{aligned}
\frac{\delta \mathcal{G}}{\delta \mathbf{v}}=-2 a \nabla^{2} \mathbf{v}+a \phi^{\prime}+\left(b \tau^{\prime}+a \sigma^{2} \operatorname{Re}^{2} J\right) \theta \hat{\mathbf{z}}+\nabla p-a \sigma \phi^{\prime \prime} \hat{\mathbf{x}} & =0 \\
\frac{\delta \mathcal{G}}{\delta \theta}=-2\left(b-\sigma \operatorname{Re}^{2} J\right) \nabla^{2} \theta+\left(b \tau^{\prime}+a \sigma^{2} \operatorname{Re}^{2} J\right) v_{3}-\left(b-2 \sigma \operatorname{Re}^{2} J\right) \tau^{\prime \prime} & =0
\end{aligned}
$$

From these, the horizontally averaged part of these equations can be solved straightforwardly to provide the extremal mean parts:

$$
\begin{aligned}
& \bar{v}^{\star}=-\frac{1}{2}(\phi+\sigma R e z) \hat{\mathbf{x}} \\
& \bar{\theta}^{\star}=\frac{\left(2 \sigma R e^{2} J-b\right)}{2\left(b-\sigma R e^{2} J\right)}(\tau+z),
\end{aligned}
$$

where the background fields $\phi$ and $\tau$ are subject to the spectral constraint $\mathcal{S C}$. For these extremalising fields, the functional $\mathcal{F}$ has a conservative upper bound of

$$
\mathcal{L} \leq \mathcal{L}_{\max }=\frac{b^{2}}{4\left(b-\sigma R e^{2} J\right)}\left\langle\left(\tau^{\prime}+1\right)^{2}\right\rangle+\sigma R e^{2} J+\frac{\sigma}{4}\left\langle\left(\phi^{\prime}+\sigma R e\right)^{2}\right\rangle .
$$

\subsection{Distilled Variational Problem}

The remainder of the problem now consists in chosing the background fields $\tau$ and $\phi$ that satisfy the boundary conditions as well as the spectral constraints in order to make $\mathcal{L}_{\max }$ as small as possible. However, instead of optimizing the problem by spanning through all $\tau$ and $\phi$ possible, we will limit the study to a specific family of functions (with a boundary layer structure suggested by physical intuition) and minimize $\mathcal{L}_{\max }$ within that family. This restricted class of functions will undoubtedly lead us to an upper bound, but at this stage there is no way of knowing how conservative this bound will prove to be. 


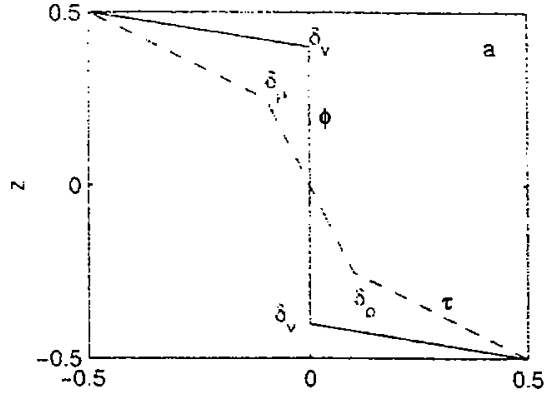

(a)

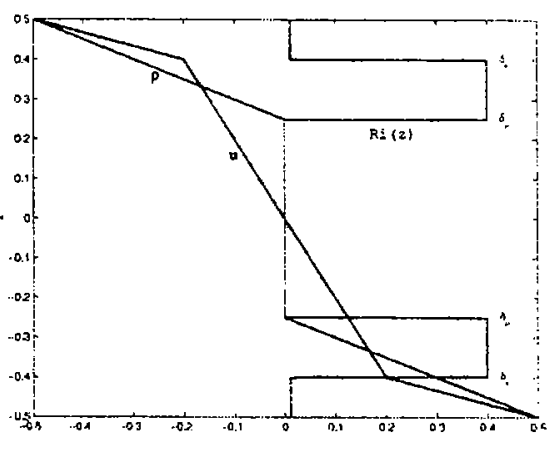

(b)

Figure 6: Form of the extremalizing solutions: (a) shows $\phi$ (solid) and $\tau$ (dashed) and (b) shows the $\mathbf{u}$ and $\rho$ for the same case with the Richardson number also shown.

The functions $\phi^{\prime}$ and $\tau^{\prime}$ are chosen to have a piece-wise linear structure with:

$$
\begin{aligned}
& \phi^{\prime}(z)=\left\{\begin{array}{cc}
-\frac{\sigma R e}{2 \delta_{v}} & \text { upper } \delta_{v} \\
0 & \text { interior, } \\
-\frac{\sigma R e}{2 \delta_{v}} & \text { lower } \delta_{v}
\end{array}\right. \\
& \tau^{\prime}(z)=\left\{\begin{array}{cc}
-\left(\frac{b-a \sigma^{2} R e^{2} J\left(1-2 \delta_{\rho}\right)}{2 b \delta_{\rho}}\right) & \text { upper } \delta_{\rho} \\
-\frac{a \sigma^{2} R e^{2} J}{b} & \text { interior } \\
-\left(\frac{b-a \sigma^{2} R e^{2} J\left(1-2 \delta_{\rho}\right)}{2 b \delta_{\rho}}\right) & \text { lower } \delta_{\rho} .
\end{array}\right.
\end{aligned}
$$

The graphs of the extremalizing solution are shown in figure 6 .

Substituting the extremalising fields into equation (47), and combining these with the ansatz for $\tau$ and $\phi$ into the energy, entropy and potential energy conservation equations $(41,42,43)$ yields a unique relation between the Lagrange multipliers $a$ and $b$ as well as conditions on the thicknesses of the boundary layers $\delta_{\rho}$ and $\delta_{v}$ :

$$
\begin{aligned}
b & =(2-a \sigma) \sigma R e^{2} J \\
\frac{1-2 \delta_{v}}{2 \delta v} & =\frac{4 J}{\sigma}\left[\frac{1-2 \delta_{\rho}}{2 \delta_{\rho}}\right]
\end{aligned}
$$

with $0<a \sigma<1$. Therefore

$$
\mathcal{L}_{\max }=\frac{\sigma R e^{2} J}{2 \delta_{\rho}}=\frac{\sigma^{2} R e^{2}}{4}\left[\frac{1-2 \delta_{v}}{2 \delta_{v}}+\frac{4 J}{\sigma}\right]
$$

still subject to the spectral constraints $\mathcal{S C}$.

\subsection{Simplified Spectral Constraint}

We shall again simplify the spectral constraints by using a conservative estimate, effectively separating the effects of velocity and density variation and requiring each to be satisfied 
independently. Using functional analysis together with the Cauchy-Schwartz inequality, it can be proven that the spectral constraints are satisfied provided that

$$
a \sigma-\frac{a \sigma R e \delta_{v}}{8 \sqrt{2}}-\frac{\sigma R e^{2} J^{3} \delta_{v}^{2}(1-a \sigma)}{\left(\sigma-2 \delta_{v}[\sigma-4 J]\right)^{2}} \geq 0 .
$$

In order to find a rigorous upper bound we must therefore minimize $\mathcal{L}_{\max }$ subject to the conditions $(60)$ and $0<a \sigma<1$. Additional manipulations show that $\mathcal{L}_{\max }$ is minimized when $\delta_{v}$ is maximized and for $R e>16 \sqrt{2}=22.6$ it attains the minimal value when

$$
\delta_{v}=\delta_{v}^{\star}=\frac{8 \sqrt{2}}{R e}
$$

It follows that

$$
\begin{aligned}
\delta_{\rho}^{\star} & =\frac{32 \sqrt{2} J}{\sigma(R e-16 \sqrt{2})+64 \sqrt{2} J} \\
a^{\star} \sigma & =1 \\
b^{\star} & =\sigma R e^{2} J \\
\mathcal{L}_{\max } & =\frac{\sigma^{2} R e^{3}}{64 \sqrt{2}}\left(1-\frac{16 \sqrt{2}}{R e}\right)+\sigma R e^{2} J \\
\mathcal{B} & \leq \mathcal{B}_{\max }=\frac{\sigma^{2} R e^{3}}{64 \sqrt{2}}\left(1-\frac{16 \sqrt{2}}{R e}\right) .
\end{aligned}
$$

\section{Implications}

Certain characteristics of the bounding flow are worthy of note. The total dissipation rate is given by

$$
\left\langle\left|\nabla u^{\star}\right|^{2}\right\rangle=\frac{\sigma^{2} R e^{2}}{4}\left(\frac{R e}{16 \sqrt{2}}+3\right),
$$

which is, perhaps surprisingly, independent of the bulk Richardson number $J$. However, we shall see that this result is consistent with the initial assumptions on the flow. The dimensional dissipation rate $\epsilon$ is given by

$$
\epsilon=\frac{U^{3}}{64 \sqrt{2} d}
$$

which has exactly the same scaling as that in the homogeneous Couette case. Again this result suggests that the flow stratification seems to have little influence on the global features of mixing in this problem, consistently with the underlying assumptions of Weinstock and Townsend. Similarly, it is found that both the long-time averaged buoyancy flux $\mathcal{B}_{\max } \&$ and the long-time averaged flux Richardson number (or equivalently the cumulative mixing efficiency $\mathcal{E}_{c}$ ) are independent of $J$, where

$$
\mathcal{E}_{c}=\frac{\mathcal{B}}{\mathcal{B}+\left\langle|\nabla u|^{2}\right\rangle}=\frac{1-\sigma^{2} R e^{2} /\left\langle|\nabla u|^{2}\right\rangle}{2-\sigma^{2} R e^{2} /\left\langle|\nabla u|^{2}\right\rangle} .
$$


When the Reynolds number $R e$ tends to infinity, $\mathcal{E}_{c}$ tends to the limit $1 / 2$ which suggests an equipartition of the total energy intput into the fluid between the viscous dissipation and the buoyancy flux (i.e. the irreversible changes to the potential energy) consistently with both recent observatons of Fernando and co-workers and the heuristic theoretical considerations of Weinstock and Townsend. The velocity boundary layer thickness is independant of the stratification and scales like $1 / R e$ as $R e$ increases.

However, the overall stratification still has an important role in the determination of the thickness of the density boundary, for example, or in the local gradient Richardson number $R i$; near the walls, it is indeed defined as

$$
\begin{aligned}
R i^{\dagger}( \pm 1 / 2) & =\frac{16 \sqrt{2}[\sigma(R e-16 \sqrt{2})+4 J]}{(R e+16 \sqrt{2})^{2}} \\
& \simeq \frac{16 \sqrt{2} \sigma}{R e}
\end{aligned}
$$

However, we see here again that $R i \rightarrow 16 \sqrt{2} \sigma / R e$ as $R e \rightarrow \infty$, suggesting that as the forcing is increased, the stratification is irrelevant to the flow in the boundary layers near the wall.

The interpretation of the results is that in the long-term averaged bounding flow, the middle layer is well-mixed and the mixing occurs principally in the boundary layers. In these thin layers the stratification does not dominate and the turbulence is driven through the shear on the walls; its characteristics depend principally on $R e$ and not on $R i$. We also note that the optimal shear in the bulk of the flow doesn't vanish completely, and is reduced by $50 \%$ from the laminar solution. This result is compatible with numerical experiments and observations, althoung not with the observed behaviour of usntratified Couette flows.

\section{Conclusions and Future Directions}

We have seen that mixing in stratified shear flows is an important problem. However, there is a wide variability in the estimates of mixing found so far, although the evidence suggests that the efficiency of mixing is a good way to describe the process.

Initial work with Bounding methods suggest that they can contribute greatly to our understanding of the problem, but there are still many open problems. For example, we would like to achieve a bound on mixing and to compare the conservative estimates of the flow with the actual flows obtained. Also we need to relate $\mathcal{E}_{c}$ to instantaneous values of $R_{f}$ and in particular develop rigorous bounds of both $\mathcal{E}_{c}$ and $R_{f}$. We also at the moment have no way of knowing how widely the results of bounding studies on highly simplified model problems can be applied to typical geophysical flows or indeed, whether our results can be embedded in an improved parameterization. There is clearly much more work to be done on this important problem.

\section{References}

[1] K. B. Winters, P. N. Lombard, J. J. Riley, and E. A. D'Asaro, "Available potential energy and mixing in density-stratified fluids," J. Fluid Mech. 289, 115 (1995). 
[2] Y. G. Park, J. A. Whitehead, and A. Gnanadeskian, "Turbulent mixing in stratified fluids - layer formation and energetics," J. Fluid Mech. 279, 279 (1994).

[3] L. H. Shih, J. R. Koseff, J. H. Ferziger, and C. R. Rehmann, "Scaling acid parameterization of stratified homogeneous turbulent shear flow," J. Fluid Mech. 412, 1 (2000).

[4] K. B. Winters and E. A. D'Asaro, "Diascalar flux and the rate of fluid mixing," J. Fluid Mech. 317, 179 (1996).

[5] T. R. Osborn, "Estimates of the local rate of vertical diffusion from dissipation measurements," J. Phys. Ocean. 10, 83 (1980).

[6] M. C. Gregg, "Diapycnal mixing in the thermocline - A Review," J. Geophys. Res. Oceans 92(c5), 5249 (1987).

[7] N. S. Oakey, "Determination of the rate of dissipation of turbulent energy from simultaneous temperature and velocity shear microstructure measurements," J. Phys. Ocean. 12, 256 (1982).

[8] H. J. S. Fernando, "Turbulent mixing in stratified fluids," Ann. Rev. Fluid Mech. 23, 35 (1991).

[9] O. M. Phillips, "Turbulence in a strongly stratified fluid - is it unstable?," Deep-Sea Res. 19, 79 (1972).

[10] E. S. Posmentier, "The generation of salinity fine structures by vertical diffusion," J. Phys. Ocean. 7, 298 (1977).

[11] J. H. Lienhard and C. W. Vanatta, "The decay of turbulence in thermally stratified flow," J. Fluid Mech. 210, 57 (1990).

[12] G. N. Ivey and J. Imberger, "On the nature of turbulence in a stratified fluid. Part I: The energetics of mixing," J. Phys. Ocean. 21, 650 (1990).

[13] A. A. Townsend, "The effects of radiative transfer on turbulent flow of a stratified fluid," J. Fluid Mech. 3, 361 (1958).

[14] G. L. Mellor and T. Yamada, "Development of a turbulence closure model for geophysical fluid problems," Rev. of Geophys. Space Phys. 20, 851 (1982).

[15] M. Nakanishi, "Improvement of the mellor-yamada turbulence closure model based on large eddy simulation data," Bound.-Layer Met. 99, 349 (2001).

[16] E. R. Pardyjak, P. Monti, and H. J. S. Fernando, "Flux richardson number measurements in stable atmospheric shear flows," J. Fluid Mech. 459, 307 (2002).

[17] E. J. Strang and H. J. S. Fernando, "Vertical mixing and transports through a stratified shear layer," J. Phys. Ocean. 31, 2026 (2001).

[18] C. P. Caulfield and W. R. Peltier, "The anatomy of the mixing transition in homogeneous and stratified shear layers," J. Fluid Mech. 413, 1 (2000). 
[19] W. D. Smyth and J. N. Moum, "Length scales of turbulence in stably stratified mixing layers," Phys. Fluids 12, 1327 (2000).

[20] W. D. Smyth and J. N. Moum, "Anisotropy of turbulence in stably stratified mixing layers," Phys. Fluids 12, 1343 (2000).

[21] Rottman and Stretch, (2002).

[22] W. J. Heck and H. A. Panofsky, "Stratospheric mixing estimates from heat flux measurements," The Natural Stratosphere of 1974, CIAP Monogr. No. 2, Dept. of Transportation TST-75-51, 6 (1975).

[23] H. J. Pearson, J. S. Puttock, and J. C. R. Hunt, "A statistical-model of fluid-element motions and vertical diffusion in a homogeneous stratified turbulent-flow," J. Fluid Mech. 129, 219 (1983).

[24] J. Weinstock, "Vertical turbulent diffusion in a stably stratified fluid," J. Atmos. Sci. 35, 1022 (1978).

[25] A. A. Townsend, The Structure of Turbulent Shear Flow, 2 ed. (Cambridge University Press, Cambridge, 1976).

[26] C. R. Doering and P. Constantin, "Energy-dissipation in shear driven turbulence," Phys. Rev. Lett. 69, 1648 (1992).

[27] C. P. Caulfield and R. R. Kerswell, "Maximal mixing rate in turbulent stably stratified Couette flow," Phys. Fluids 13, 894 (2001). 


\title{
Lecture 10
}

\section{Unification of Variational Principles for Turbulent Shear Flows: the Mean-fluctuation Formulation of Howard-Busse and the Background Method of Doering-Constantin}

\author{
Richard R. Kerswell \\ Notes by Huiqun Wang
}

\section{Introduction}

An upper bound on the energy dissipation rate in turbulent shear flows can be found either using Howard-Busse's mean-fluctuation method [1] or Doering-Constantin's background method[2]. Howard-Busse's method grew out of ideas put forward by Malkus [3], whereas the Doering-Constantin approach is based upon a mathematical device invented by Hopf [4]. Although the methods have very different origins and look unrelated, we show in this lecture that they are in fact intimately connected. They both seek to make stationary the same functional. However, the Howard-Busse method seeks to estimate this stationary (saddle) point from below as a maximization problem, whereas the Doering-Constantin method estimates this part from above as part of a minimization problem. We show this explicitly for the canonical problem of plane Couette flow.

\section{Couette Shear Flow}

We consider a homogeneous incompressible fluid with viscosity $\chi$ between two parallel, infinite plates . at $z= \pm \frac{1}{2} d$, which are sliding across each other with relative velocity $V_{0}$ in the $\underline{\hat{i}}$ direction. $\underline{\hat{i}}$ is the unit vector. The non-dimensionalized governing equations are:

$$
\frac{\partial \underline{V}}{\partial t}+\underline{V} \cdot \underline{\nabla V}+\underline{\nabla} p=\nabla^{2} \underline{V}
$$

$$
\underline{\nabla} \cdot \underline{V}=0
$$

with the boundary condition $\underline{V}=\mp \frac{1}{2} R e \underline{\hat{i}}$ at $z= \pm \frac{1}{2}$, where Reynolds number $R e=\frac{V_{0} d}{\chi}$. We will seek upper bounds on the momentum transport which equals the viscous dissipation rate $\left\langle|\nabla \underline{V}|^{2}\right\rangle$.

$$
\left\langle|\nabla \underline{V}|^{2}\right\rangle:=\lim _{L \rightarrow \infty} \frac{1}{4 L^{2}} \int_{-L}^{L} d x \int_{-L}^{L} d y \int_{-\frac{1}{2}}^{\frac{1}{2}} d z|\nabla \underline{V}|^{2} .
$$

\subsection{Howard-Busse Method}

The Howard-Busse variational formulation is based on a mean-fluctuation decomposition of the velocity field $\underline{V}(\underline{x}, t)=U(z) \hat{i}+\underline{v}(\underline{x}, t)$, and consists of solving the variational problem:

$$
\min \left(R_{e}\right)=\frac{\left\langle|\nabla \underline{v}|^{2}\right\rangle}{\left\langle v_{1} v_{3}\right\rangle}+\frac{\mu\left\langle\left(\overline{v_{1} v_{3}}-\left\langle v_{1} v_{3}\right\rangle\right)^{2}\right\rangle}{\left\langle v_{1} v_{3}\right\rangle^{2}}
$$


under the constraints $\nabla \cdot \underline{v}=0, \underline{v}\left(x, y, \pm \frac{1}{2}\right)=0,\left\langle v_{1} v_{3}\right\rangle=1$. where $\overline{(\bullet)}:=\lim _{L \rightarrow \infty} \frac{1}{4 L^{2}} \int_{-L}^{L} d x \int_{-L}^{L} d y(\bullet)$. This problem can be eqivalently formulated as the following. Substitute $\underline{V}(\underline{x}, t)$ into equation (1) and subtract the horizontal average, we obtain the power balance:

$$
D-R e^{2}=\left\langle|\nabla \underline{v}|^{2}\right\rangle+\left\langle\left(\overline{v_{1} v_{3}}-\left\langle v_{1} v_{3}\right\rangle\right)^{2}\right\rangle=\operatorname{Re}\left\langle v_{1} v_{3}\right\rangle
$$

where $D$ is the statistically averaged viscous dissipation. We maximize $R e\left\langle v_{1} v_{3}\right\rangle$ under the constraints of (2), the continuity equation, and the boundary conditions by considering the Lagrangian

$$
L=\operatorname{Re}\left\langle v_{1} v_{3}\right\rangle+\Lambda\left\{\left\langle|\nabla \underline{v}|^{2}\right\rangle+\left\langle\left(\overline{v_{1} v_{3}}-\left\langle v_{1} v_{3}\right\rangle\right)^{2}\right\rangle-\operatorname{Re}\left\langle v_{1} v_{3}\right\rangle\right\}-\langle 2 p(\underline{x}) \nabla \cdot \underline{v}\rangle
$$

where $\Lambda$ and $p(\underline{x})$ are Lagrange multipliers. The Euler-Lagrange equation for the velocity field is

$$
\left[\left(\overline{v_{1} v_{3}}-\left\langle v_{1} v_{3}\right\rangle\right)+\operatorname{Re}\left(\frac{1-\Lambda}{2 \Lambda}\right)\right]\left[\begin{array}{c}
v_{3} \\
0 \\
v_{1}
\end{array}\right]+\nabla p=\nabla^{2} \underline{v}
$$

Eliminating $\Lambda$ by using $\langle\underline{v} \cdot(3)\rangle$ and the constraint of equation (2), gives the optimization problem:

$$
\begin{gathered}
{\left[\left(\overline{v_{1} v_{3}}-\left\langle v_{1} v_{3}\right\rangle\right)-\frac{1}{2} R e-\frac{1}{2} \frac{\left\langle\left(\overline{v_{1} v_{3}}-\left\langle v_{1} v_{3}\right)\right)^{2}\right\rangle}{\left\langle v_{1} v_{3}\right\rangle}\right]\left[\begin{array}{c}
v_{3} \\
0 \\
v_{1}
\end{array}\right]+\nabla p=\nabla^{2} \underline{v}} \\
\nabla \cdot \underline{v}=0, \underline{v}\left(x, y, \pm \frac{1}{2}\right)=0
\end{gathered}
$$

from which the upper bound $D=\operatorname{Re}\left\langle v_{1} v_{3}\right\rangle+R e^{2}$ follows.

\subsection{Doering-Constantin Method}

The Doering-Constantin method decomposes the velocity into "background" and "fluctuation" fields $\underline{V}(\underline{x}, t)=\phi(z) \underline{\hat{i}}+\underline{\nu}(\underline{x}, t)$. The background flow $\phi(z)$ satisfies the boundary condition $\phi\left( \pm \frac{1}{2}\right)=\mp \frac{1}{2} R e$ so that the fluctuation field satisfies homogeneous boundary conditions.

Putting $\underline{V}(\underline{x}, t)=\phi(z) \underline{\hat{i}}+\underline{\nu}(\underline{x}, t)$ into $\partial \underline{V} / \partial t+\underline{V} \cdot \underline{\nabla V}+\underline{\nabla} p=\nabla^{2} \underline{V}$, we obtain

$$
\frac{\partial \underline{\nu}}{\partial t}+\underline{\nu} \cdot \underline{\nabla \nu}+\phi^{\prime} \nu_{3} \underline{\hat{i}}+\phi \frac{\partial \underline{\nu}}{\partial x}+\underline{\nabla} p=\nabla^{2} \underline{\nu}+\phi^{\prime \prime} \underline{\hat{i}}
$$

where $\phi^{\prime}:=d \phi / d z$ and $\phi^{\prime \prime}:=d^{2} \phi / d z^{2}$. Performing $\langle\underline{\underline{\nu}} \cdot(4)\rangle$, we obtain

$$
\frac{\partial}{\partial t}\left\langle\frac{1}{2} \underline{\nu}^{2}\right\rangle=\left\langle\phi^{\prime \prime} \nu_{1}\right\rangle-\left\langle|\nabla \underline{\nu}|^{2}\right\rangle-\left\langle\phi^{\prime} \nu_{1} \nu_{3}\right\rangle
$$

We also have the identity

$$
\left\langle|\nabla \underline{V}|^{2}\right\rangle=\left\langle\phi^{\prime} 2\right\rangle-2\left\langle\phi^{\prime \prime} \nu_{1}\right\rangle+\left\langle|\nabla \underline{\nu}|^{2}\right\rangle
$$

Performing $a \cdot(5)+(6)$ where a is some scalar gives 


$$
\left\langle|\nabla \underline{V}|^{2}\right\rangle+a \frac{\partial}{\partial t}\left\langle\frac{1}{2} \underline{\underline{\nu}}^{2}\right\rangle=\left\langle\phi^{\prime 2}\right\rangle-G(\phi, \underline{\nu} ; a)
$$

where $G(\phi, \underline{\nu} ; a)=\left\langle(a-1)|\nabla \underline{\nu}|^{2}+a \phi^{\prime} \nu_{1} \nu_{3}-(a-2) \phi^{\prime \prime} \nu_{1}\right\rangle$.

Taking long time averages leads to

$$
D=\lim _{T \rightarrow \infty} \frac{1}{T} \int_{0}^{T}\left\langle|\nabla \underline{V}|^{2}\right\rangle d t=\left\langle\phi^{\prime 2}\right\rangle-\lim _{T \rightarrow \infty} \frac{1}{T} \int_{0}^{T} G(\phi, \underline{\nu} ; a) d t
$$

If $\phi$ and $a$ are such that $\inf _{\underline{\nu}} G(\phi, \underline{\nu} ; a)>-\infty$ then there exists the bound

$$
D \leqslant\left\langle\phi^{\prime 2}\right\rangle-\inf _{\underline{\nu}} G(\phi, \underline{\nu} ; a)
$$

The Doering-Constantin problem is to minimize the background dissipation $\left\langle\phi^{\prime} 2\right\rangle$ subject to the spectral constraint inf $G>-\infty$ over all possible fluctuation fields $\underline{\nu}(\underline{x})$. Solving the Euler-Lagrange equation gives the stationary value of

$$
G\left(\phi, \underline{\nu}^{*} ; a\right)=-\frac{(a-2)^{2}}{4(a-1)}\left[\left\langle\phi^{\prime 2}\right\rangle-R e^{2}\right]
$$

This will be an infimum if and only if the dominant quadratic terms are positive definite, i.e.

$$
H(\phi, \underline{\nu}, a):=(a-1)\left\langle|\nabla \underline{\nu}|^{2}\right\rangle+a\left\langle\phi^{\prime} \nu_{1} \nu_{3}\right\rangle \geq 0
$$

for all allowable $\underline{\nu}$. This is called the spectral constraint.

The optimization problem is then to minimize the bound

$$
\lim _{T \rightarrow \infty} \frac{1}{T} \int_{0}^{T}\left\langle|\nabla \underline{\nu}|^{2}\right\rangle d t \leq \mathcal{D}:=\left\langle\phi^{\prime 2}\right\rangle-\inf _{\underline{\nu}} G=\frac{a^{2}}{4(a-1)}\left\langle\left(\phi^{\prime}+R e\right)^{2}\right\rangle+R e^{2}
$$

subject to the spectral constraint.

\section{Unification}

The Howard-Busse and Doering-Constantin methods can be unified by defining the following functional

$$
D(\underline{V}, \underline{\nu}, a):=\lim _{T \rightarrow \infty} \frac{1}{T} \int_{0}^{T}\left\langle|\nabla \underline{V}|^{2}\right\rangle-\left\langle a \underline{\nu} \cdot\left(\frac{\partial \underline{V}}{\partial t}+\underline{V} \cdot \underline{\nabla V}+\underline{\nabla p}-\nabla^{2} \underline{V}\right)\right\rangle d t
$$

where $\underline{V}=\phi(z) \underline{\hat{i}}+\underline{\nu}(\underline{x}, t)=\left[\phi(z)+\overline{\nu_{1}}(z)\right] \underline{\hat{i}}+\underline{v}(\underline{x}, t)$. Substitute $\underline{V}=\phi(z) \underline{\hat{i}}+\underline{\nu}(\underline{x}, t)$ into $D$, we obtain 


$$
\begin{gathered}
D(\underline{V}, \phi, a)=\lim _{T \rightarrow \infty} \frac{1}{T} \int_{0}^{T}\left\langle|\nabla \underline{V}|^{2}\right\rangle-\left\langle a(\underline{V}-\phi(z) \underline{\hat{i}}) \cdot\left(\frac{\partial \underline{V}}{\partial t}+\underline{V} \cdot \underline{\nabla V}+\underline{\nabla p}-\nabla^{2} \underline{V}\right)\right\rangle d t \\
=\lim _{T \rightarrow \infty} \frac{1}{T} \int_{0}^{T}\left\langle|\nabla \underline{V}|^{2}\right\rangle-a\left\langle\underline{V} \cdot\left(\frac{\partial V}{\partial t}+\underline{V} \cdot \underline{\nabla V}+\underline{\nabla p}-\nabla^{2} \underline{V}\right)\right\rangle \\
+a\left\langle\phi(z) \underline{\hat{i}} \cdot\left(\frac{\partial \underline{V}}{\partial t}+\underline{V} \cdot \underline{\nabla V}+\underline{\nabla p}-\nabla^{2} \underline{V}\right)\right\rangle d t
\end{gathered}
$$

This makes it clear that $a$ is acting as a Lagrange multiplier which imposes the total power balance and that $a \phi(z)$ is the Lagrange multiplier which imposes the mean momentum balance. Also

$$
D(\underline{\nu}, \phi, a)=\left\langle\phi^{\prime 2}\right\rangle-\lim _{T \rightarrow \infty} \frac{1}{T} \int_{0}^{T}\left\langle(a-1)|\nabla \underline{\nu}|^{2}+a \nu_{1} \nu_{3} \phi^{\prime}-(a-2) \phi^{\prime \prime} \nu_{1}\right\rangle d t
$$

and finally

$$
D\left(\underline{v}, \bar{\nu}_{1}, \phi, a\right)=\left\langle\phi^{\prime 2}\right\rangle-\lim _{T \rightarrow \infty} \frac{1}{T} \int_{0}^{T}\left\langle(a-1)|\nabla \underline{v}|^{2}+a v_{1} v_{3} \phi^{\prime}+(a-1) \bar{\nu}_{1}^{\prime 2}-(a-2) \phi^{\prime \prime} \bar{\nu}_{1}\right\rangle d t .
$$

The full variational problem is to solve the Euler-Lagrange equations $\frac{\delta D}{\delta \phi}=0 ; \frac{\delta D}{\delta \underline{v}}=\underline{0} ; \frac{\delta D}{\delta \bar{\nu}_{1}}=\underline{0} ; \frac{\delta D}{\delta a}=$ 0 . The Howard-Busse and Doering-Constantin methods consider complementary subsets of these equations.

\subsection{Howard-Busse Problem}

The Howard-Busse method solves $\frac{\delta D}{\delta \phi}=0, \frac{\delta D}{\delta \bar{\nu}_{1}}=0$ and $\frac{\delta D}{\delta a}=0$ leaving a maximization problem in $\underline{v}$.

$$
\begin{aligned}
& \frac{\delta D}{\delta \phi}=0 \quad \Rightarrow-2 \phi^{\prime \prime}+a{\overline{v_{1} v_{3}}}^{\prime}+(a-2) \bar{\nu}_{1}^{\prime \prime}=0 \\
& \Rightarrow \phi^{\prime}+R e=\frac{1}{2} a\left(\overline{v_{1} v_{3}}-\left\langle v_{1} v_{3}\right\rangle\right)+\frac{1}{2}(a-2) \bar{\nu}_{1}^{\prime} \\
& \frac{\delta D}{\delta \bar{\nu}_{1}}=0 \Rightarrow 2(a-1) \bar{\nu}_{1}^{\prime \prime}+(a-2) \phi^{\prime \prime}=0 \Rightarrow \bar{\nu}_{1}=-\frac{(a-2)}{2(a-1)}(\phi+R e z)
\end{aligned}
$$

Substituting these results into $D\left(\underline{v}, \bar{\nu}_{1}, \phi, a\right)$, we obtain

$$
D(\underline{v}, a)=R e^{2}+\operatorname{Re}\left\langle v_{1} v_{3}\right\rangle+(a-1)\left\{\operatorname{Re}\left\langle v_{1} v_{3}\right\rangle-\left\langle|\nabla \underline{v}|^{2}\right\rangle-\left\langle\left(\overline{v_{1} v_{3}}-\left\langle v_{1} v_{3}\right\rangle\right)^{2}\right\rangle\right\}
$$

This is equivalent to the problem of finding the maximum of $\left(R e^{2}+R e\left\langle v_{1} v_{3}\right\rangle\right)$ subject to the power constraint $\operatorname{Re}\langle u w\rangle=\left\langle|\nabla \underline{v}|^{2}\right\rangle+\left\langle\left(\overline{v_{1} v_{3}}-\left\langle v_{1} v_{3}\right)\right)^{2}\right\rangle$ with $(a-1)$ being the Lagrange multiplier and $\nabla \cdot \underline{v}=0$.

\subsection{Doering-Constantin problem}

The Doering-Constantin method solves $\frac{\delta D}{\delta \underline{v}}=\underline{0}$ and $\frac{\delta D}{\delta \bar{\nu}_{1}}=0$ leaving a minimization problem for $\phi, a$.

$$
\frac{\delta D}{\delta \bar{\nu}_{1}}=0 \Rightarrow \bar{\nu}_{1}=-\frac{(a-2)}{2(a-1)}(\phi+R e z) \text { as before. Now }
$$




$$
\frac{\delta D}{\delta \underline{v}}=\underline{0} \Rightarrow 2(a-1) \nabla^{2} \underline{v}-a \phi^{\prime}\left[\begin{array}{c}
v_{3} \\
0 \\
v_{1}
\end{array}\right]+\underline{\nabla} p=\underline{0}
$$

Taking $\langle\underline{v} \cdot()\rangle$ of this expression gwes

$$
\left\langle\underline{v} \cdot \frac{\delta D}{\delta \underline{v}}\right\rangle=0=\left\langle(a-1)|\nabla \underline{v}|^{2}+a \phi^{\prime} v_{1} v_{3}\right\rangle
$$

So, $D(\underline{v}, \phi, a)=\frac{a^{2}}{4(a-1)}\left\langle\left(\phi^{\prime}+R e\right)^{2}\right\rangle+R e^{2}-\left\langle(a-1)|\nabla \underline{v}|^{2}+a \phi^{\prime} v_{1} v_{3}\right\rangle$ is equivalent to

$$
D(\phi, a)=\frac{a^{2}}{4(a-1)}\left\langle\left(\phi^{\prime}+R e\right)^{2}\right\rangle+R e^{2}
$$

provided $\phi$ and $a$ satisfy the spectral constraint which ensures overestimation of the highest saddle point of $D$. This highest saddle point bounds the energy dissipation (see [5] for details).

\section{Discussion}

In this lecture, we have made a direct link between the Howard-Busse and Doering-Constantin variational methods for upper bounding turbulent transport in plane Couette shear flow. Similar arguments can be applied to turbulent heat transport for convection as well [6]. Both methods revolve around the same underlying functional. The Howard-Busse method seeks to find the highest saddle point of this functional by maximizing from below, while the Doering-Constantin method seeks to minimize from above. The consequence is that the ideal upper bounds derived from each method should coincide at the highest saddle point. Historically, this is seen in the results obtained in each approach. The original bound produced by Doering and Constantin [6] in 1992 was $\frac{1}{8 \sqrt{2}} \approx \frac{1}{11.3}$ as opposed to Busse's estimate [1] of $\simeq \frac{1}{99.7}$. Nicodemus et al. [8] improved the Doering-Constantin result down to $\sim \frac{1}{92.0}$ in 1998. Recently Plasting \& Kerswell [9] have solved the full problem to find the asymptotic result that $D \leq 0.008553$ in units of $\frac{V_{0}^{3}}{d}$ as $R e \rightarrow \infty$.

\section{References}

[1] F. H. Busse, Bounds for turbulent shear flow, J. Fluid Mech. 41 (1970) 219-240.

[2] C. R. Doering \& P. Constantin, Variational bounds on energy dissipation in incompressible flows: Shear flows, Phys. Rev. E49 (1994) 4087-4099.

[3] W. V. R. Malkus, The heat transport and spectrum of thermal turbulence, Proc. Roy. Soc. 225 (1954) 196-212.

[4] E. Hopf, Ein allgemeiner Endlichkeitssatz der Hydrodynamik, Math. Ann. 117 (1941) 764-775.

[5] R. R. Kerswell, Unification of variational principles for turbulent shear flows: the background method of Doering-Constantin and the mean-fluctuation formulation of Howard-Busse, Physica D 121 (1998) 175-192.

[6] R. R. Kerswell, New results in the variational approach to turbulent Boussinesq convection, Phys. Fluids, 13 (2001), 192-209. 
[7] C. R. Doering \& P. Constantin, Energy dissipation in shear driven turbulence, Phys. Rev. Lett. 69 (1992) 1648-1651.

[8] R. Nicodemus, S. Grossmann \& M. Holthaus, The background flow method. Part 1. Constructive approach to bounds on energy dissipation and the background flow mathod. Part 2. Asymptotic theory of dissipation bounds, J. Fluid Mech., 1998.

[9] S. C. Plasting \& R. R. Kerswell, Improved upper bound on the energy dissipation rate in plane Coutte flow: the full solution to Busse's problem and the Constantin-Doering-Hopf problem with 1-D background field, J. Fluid Mech, submitted, 2002. 


\title{
Horizontal Convection
}

\author{
Jennifer $\mathrm{H}$. Siggers \\ University of Cambridge, U.K.
}

\section{Introduction}

The surface temperature of the ocean is different at different points. Can this differential heating drive a large scale flow? If so how large can that flow be? In this report we analyze a simple model of the ocean and construct rigorous upper bounds on the heat transport that can be induced by a horizontal temperature gradient that is imposed on the top surface. We consider the model shown in figure 1, where the top surface has an imposed temperature distribution with a cosine profile $\Delta T \cos k x+T_{\mathrm{av}}$, and make a linear transformation of the true temperature to give the new non-dimensional temperature variable $T$, which is equal to $\cos k x$ on the top boundary. This set up is known as horizontal convection [1]. Notice

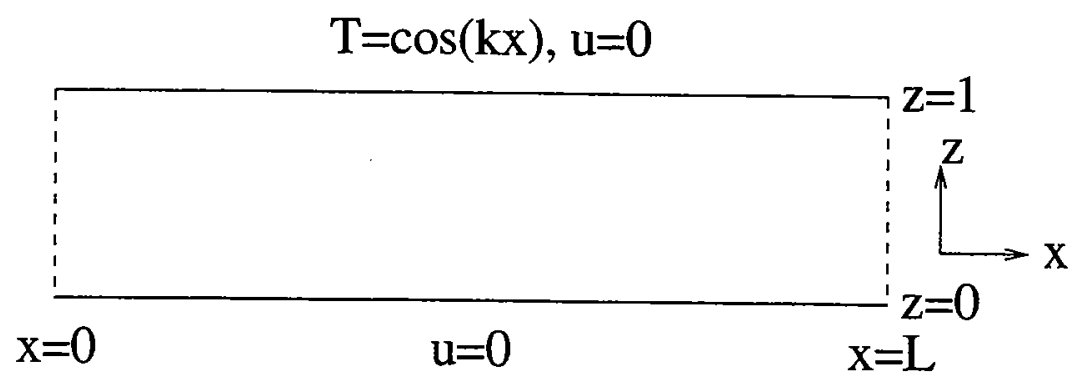

Figure 1: Set up of the horizontal convection problem

that the problem is in contrast to the usual Rayleigh-Bénard problem, where the motion is driven by vertical temperature gradients. In horizontal convection, it is the horizontal temperature gradient that drives the flow.

We use non-slip boundary conditions top and bottom and periodic side wall conditions, and we also need to specify a bottom boundary condition on the temperature. The box has dimensional width $W$ and depth $H$ and we non-dimensionalize these to give the new width $L=W / H$ and height 1 . For horizontal periodicity, we also require that $k=2 \pi n / L$ for some $n \in \mathbb{N}$.

We aim to construct rigorous bounds on the total heat transfer rate through the layer, which we measure using a horizontal Nusselt number. We do this for variety of different temperature boundary conditions on the bottom of the layer to investigate the dependence of the scaling of the horizontal Nusselt number on the conditions there. This is because since horizontal convection is driven by temperatures at the top surface only, we want to find a bound that is independent of what is happening at the lower boundary. Also we don't have a good idea of what is the true oceanographic boundary condition there.

We use the Boussinesq approximation to reduce the equations to the standard non- 


\begin{tabular}{|l|l|}
\hline Quantity & Approximate value \\
\hline$\nu$ & $1.52 \times 10^{-6} \mathrm{~m}^{2} \mathrm{~s}^{-1}$ \\
$\kappa$ & $1.4 \times 10^{-7} \mathrm{~m}^{2} \mathrm{~s}^{-1}$ \\
$g \alpha \Delta T$ & $10^{-2} \mathrm{~ms}^{-2}$ \\
$W$ & $2.0 \times 10^{7} \mathrm{~m}$ \\
$H$ & $4 \times 10^{3} \mathrm{~m}$ \\
$k$ & $1.25 \times 10^{-3}$ \\
$R_{H}$ & $3 \times 10^{21}$ \\
$\sigma$ & 10.9 \\
$L$ & $5.0 \times 10^{4}$ \\
\hline
\end{tabular}

Figure 2: Approximate oceanographic values of some parameters, from [2]

dimensional form:

$$
\begin{aligned}
& \dot{\mathbf{u}}+\mathbf{u} \cdot \nabla \mathbf{u}+\nabla p=\sigma R_{H} T \hat{\mathbf{z}}+\sigma \nabla^{2} \mathbf{u} \\
& \dot{T}+\mathbf{u} \cdot \nabla T=\nabla^{2} T \\
& \nabla \cdot \mathbf{u}=0
\end{aligned}
$$

where $\mathbf{u}$ is the non-dimensional velocity field, $T$ is the non-dimensional temperature and $p$ is the non-dimensional pressure. $\sigma=\nu / \kappa$ is the Prandtl number and $R_{H}=H^{3} g \alpha_{T} \Delta T / \kappa \nu$ is the horizontal Rayleigh number. $\nu$ is the kinematic viscosity, $\kappa$ is the thermal diffusivity and $g$ is the acceleration due to gravity. The table in figure 2 shows the approximate oceanographic values of some of these quantities. Note also that the governing equations do not possess a static solution, unlike the Rayleigh-Bénard problem, since from $(\mathcal{N S})$, we would need to satisfy $\nabla p=\sigma R_{H} T \hat{\mathbf{z}}$. Since $T$ must have some $x$-dependence in order to satisfy the boundary conditions, $T \hat{\mathbf{z}}$ cannot be gradient.

Thermal energy transport was considered by Sandström in the early 20th Century. He proposed the following theorem, (quoted from [3]):

Sandström's theorem: "A closed steady circulation can only be maintained in the ocean if the heat source is situated at a lower level than the cold source."

This implies that horizontal convection cannot induce a large-scale flow and is therefore unimportant in the oceanic context. However, the theorem as it stands is not strictly true. For example, Jeffreys [4] constructed a counter example to Sandström's theorem, the "hula hoop" model, shown in figure 3. The fluid is contained in an annulus and heat is applied on the right hand side and the fluid is cooled on the left. Jeffreys argued that this heating and cooling will set the fluid in motion, no matter at what height the heating and cooling are applied, and thus we can heat near the top and cool near the bottom, as shown, and still induce a flow in the fluid. In some ways, this counter example is a bit contrived, but it is certainly a rigorous case where Sandström's theorem breaks down.

A second counter example is provided by Rossby [5], who performed some experiments on horizontal convection, using a set up similar to that in figure 1 except that he imposed the differential heating on the bottom surface and had insulating temperature boundary 


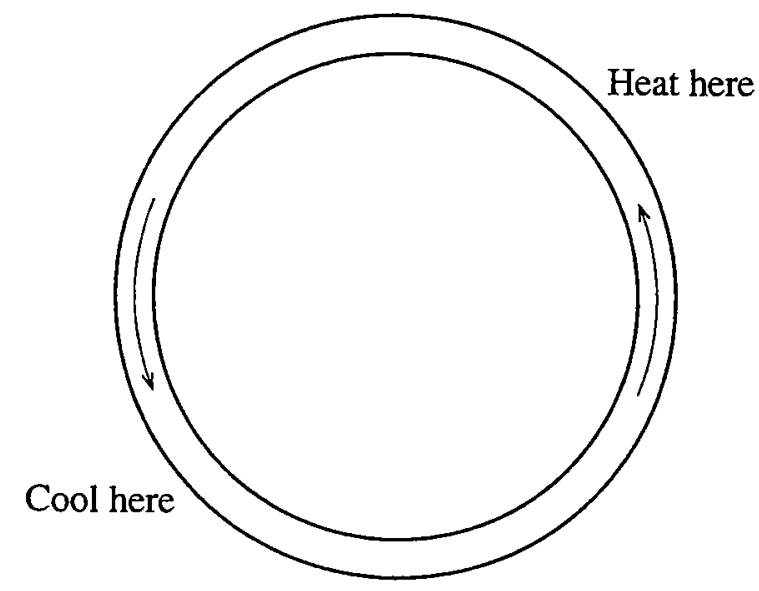

Figure 3: Schematic diagram of Jeffreys' hula hoop model

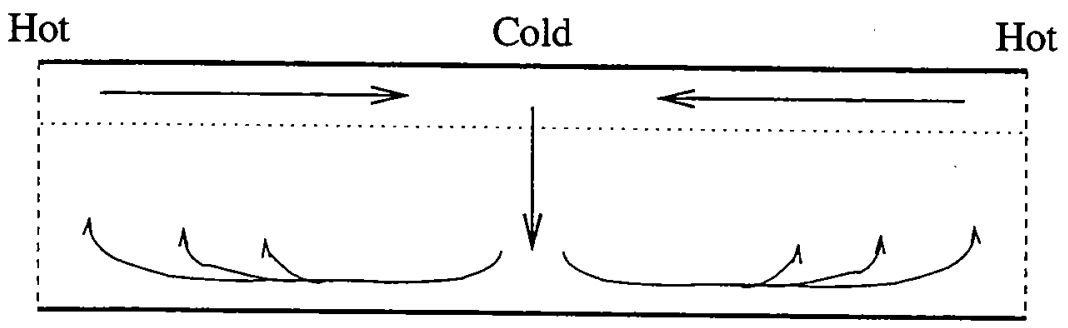

Figure 4: Schematic diagram showing the fluid motion. Note the strong downward motion in the central plume and large horizontal flows in the top boundary layer. There is also a slow recirculation in the rest of the layer.

conditions on the side and top walls. He found that there was a plume of hot rising water, over the hottest point on the bottom boundary. This rising motion induces a flow along the bottom of the box from the cold part to the hot part, and there is also a slow recirculation returning the fluid from the top of the box back down to the bottom.

However, even though Sandström's theorem is not completely true, in fact the main idea is correct: that thermal forcing at a single level as in the Rossby experiment is a relatively inefficient way to drive a flow when compared with Rayleigh-Bénard convection, for example, as we shall show in this report.

In the oceanic context the differential heating is at the top of the layer, which is why we consider this scenario rather than Rossby's though the two scenarios are linked via a reflection in the horizontal mid-plane, coupled with reversing the sign of the temperature field $T$. A schematic picture of the flow observed in numerical experiments (such as those in $[6,7,8,9]$ ) is shown in figure 4 , which is also the reverse of the flow that Rossby observed in his experiments. However, numerical simulations have only been performed for horizontal Rayleigh numbers $R_{H}$ up to about $10^{8}$, and it is not clear whether or not the flow structure in figure 4 persists into the oceanographic regime, in which $R_{H} \sim 10^{21}$.

Rossby [5] provided a consistent scaling argument for the width of the boundary layer 
in the flow. He assumed that there is a top boundary layer of width $\delta$ in which the vertical derivatives are of order $\delta^{-1}$ whilst the horizontal ones are of order unity. Then from $(\mathcal{H})$, balancing the advection term with the diffusion term, assuming temperature variations are of order 1 gives $\psi \sim \delta^{-1}$ and balancing the buoyancy term with the dissipation term in $(\mathcal{N S})$ yields $\delta \sim R_{H}^{-1 / 5}$.

Sandström [10] also proposed the following:

Sandström's conjecture: "If a viscous and diffusive fluid is non-uniformly heated from above then in the limit $\kappa \rightarrow 0$ with $\sigma=\nu / \kappa$ fixed, the motion in the fluid disappears."

To make this rigorous quantitatively, we need a measure of the "motion in the fluid". Such a measure is the maximum value of the streamfunction. However, the conjecture as stated has not been proven. Instead we can prove a weaker result for horizontal convection in the form of an anti-turbulence theorem. We need to define a notion of turbulence, used by Frisch [11]:

The law of finite energy dissipation: "If in an experiment on turbulent flow, all the control parameters are kept the same, except for the the viscosity, $\nu$, which is lowered as much as possible, the energy dissipation per unit mass behaves in a way consistent with a finite positive limit."

This law is also known as the zeroth law of turbulence. In fact, this definition does not exclude non-laminar flows in a boundary layer, but it does give a precise definition to work with. Then we may propose

The anti-turbulence theorem: If the only forcing is non-uniform heating applied at the surface of a Boussinesq fluid and if the viscosity, $\nu$, and thermal diffusivity, $\kappa$ are lowered to zero, with $\sigma=\nu / \kappa$ fixed, then in the limit the energy dissipation $\epsilon$ also vanishes.

This is finally a result that can be proved rigorously, which was done by Paparella and Young [9], who assumed a zero flux condition $\left(T_{z}=0\right.$ ) on the bottom boundary (where the subscript denotes differentiation with respect to $z$ ). It relies crucially on the following principle:

Boundedness principle for the temperature: For the set up shown in figure 1, with an imposed temperature distribution on the top surface and a no flux bottom temperature boundary condition, then at any time the temperature field is bounded by the maximum and minimum values imposed on the top surface or the maximum and minimum values of the initial temperature distribution.

This can be proved from $(\mathcal{H})$. The derivation for a similar (but slightly more complicated) case is given in section 4.1. If the system is allowed to relax for a sufficiently long time, then we expect that the temperature is everywhere bounded by the maximum and minimum values at the top surface, that is, it lies in the range $[-1,+1]$.

We shall use an overbar to denote the horizontal and time average and angle brackets to denote the space and time average:

$$
\left\ulcorner=\lim _{t_{0}, y_{0} \rightarrow \infty} \frac{1}{2 t_{0} y_{0}} \int_{0}^{t_{0}} \int_{-y_{0}}^{y_{0}} \int_{0}^{L} \cdot d x d y d t, \quad\langle\cdot\rangle=\int_{0}^{1}-d z\right.
$$


Now the energy dissipation per unit mass $\epsilon$ is given by $\nu\left\langle\left|\nabla_{d} \mathbf{u}_{d}\right|^{2}\right\rangle$ where $\nabla_{d}$ and $\mathbf{u}_{d}$ are the dimensional versions of $\nabla$ and $\mathbf{u}$. In non-dimensional units, this becomes $\epsilon=$ $\nu \kappa^{2}\left\langle|\nabla \mathbf{u}|^{2}\right\rangle / H^{4}$, and rearranging $\langle\mathbf{u} \cdot(\mathcal{N S})\rangle$ we have

$$
\epsilon=\frac{\nu \kappa^{2} R_{H}}{H^{4}}\langle w T\rangle
$$

Taking $\langle(1-z) \cdot(\mathcal{H})\rangle$ gives $\langle w T\rangle=-\left.\bar{T}\right|_{0}$ and so

$$
\epsilon=\frac{\nu \kappa^{2} R_{H}}{H^{4}}\left(-\left.\bar{T}\right|_{0}\right) \leq \frac{\nu \kappa^{2} R_{H}}{H^{4}}=\frac{\kappa g \alpha \Delta T}{H} \rightarrow 0 \quad \text { as } \kappa \rightarrow 0,
$$

where the inequality makes use of the lower bound on the temperature field, thus proving anti-turbulence.

In this report, we try to construct bounds on the strength of the convection for horizontal convection. Often the Nusselt number is used as a measure of the strength, but this measures the heat flux in the vertical direction, whereas for horizontal convection it is the horizontal heat flux that is of interest. Thus we need to define a horizontal Nusselt number $N u_{H}$. Ideally this would measure the total heat flux into (or equivalently out of) the top boundary, i.e.

$$
\overline{\chi(x, y, t) T_{z}(x, y, 1, t)}
$$

where $\chi(x, y, t)$ equals 1 if $T_{z}(x, y, 1, t)>0$ (corresponding to places where there is flux in) and 0 otherwise (corresponding to flux out). With a zero flux bottom boundary condition this equals $\left.\overline{\left|T_{z}\right|}\right|_{1} / 2$. However, we don't know which parts of the top boundary have heat fluxes into the layer and which have fluxes out and thus we don't know $\chi$. We might assume a symmetric arrangement, in which if $T>0$ at the top of the layer then there is a heat flux out of the layer (i.e. $T_{z}<0$ ), and if $T<0$ then the heat flux is into the layer (i.e. $T_{z}>0$ ). However, the solutions found in the numerics (see figure 4) are far from symmetric due to the cold plume, and so we might expect the area of the top surface where $\chi$ is 1 to be confined to a small areas around the points where $T$ takes its maximum value. Thus, this definition of the horizontal Nusselt number would be extremely hard to estimate mathematically, and instead we propose an alternative formulation.

In [9], which considered a zero flux bottom boundary condition, the form

$$
N u_{H}=\frac{\left\langle|\nabla T|^{2}\right\rangle}{\left\langle\left|\nabla T_{c}\right|^{2}\right\rangle}
$$

was used, where, since there is no static solution of the equations, we define the "conduction" solution $T_{c}$ to be the steady solution of the horizontal convection problem where the fluid is replaced by a solid (and thus we can neglect $(\mathcal{N S})$ and just solve $(\mathcal{H})$ with $\mathbf{u}=0$ ), so $T_{c}$ is the solution of $\nabla^{2} T_{c}=0$ together with the boundary conditions on $T$. The justification for the formula (1) can be seen if we take the time average of $(\mathcal{H})$, integrate over the vertical coordinate and take the average over the $y$-coordinate:

$$
\begin{aligned}
\lim _{t_{0} \rightarrow \infty, y_{0} \rightarrow \infty} \frac{1}{2 t_{0} y_{0}} \int_{0}^{t_{0}} \int_{-y_{0}}^{y_{0}} \int_{0}^{1}\left((u T)_{x}-\right. & \left.T_{x x}\right) d z d y d t \\
& =\lim _{t_{0} \rightarrow \infty, y_{0} \rightarrow \infty} \frac{1}{2 t_{0} y_{0}} \int_{0}^{t_{0}} \int_{-y_{0}}^{y_{0}}\left(\left.T_{z}\right|_{1}-\left.T_{z}\right|_{0}\right) d y d t
\end{aligned}
$$


Integrating with respect to $x$ gives

$$
\begin{aligned}
J_{H}(x):=\lim _{t_{0} \rightarrow \infty, y_{0} \rightarrow \infty} \frac{1}{2 t_{0} y_{0}} \int_{0}^{t_{0}} \int_{-y_{0}}^{y_{0}} \int_{0}^{1}\left(u T-T_{x}\right) d z d y d t \\
=\lim _{t_{0} \rightarrow \infty, y_{0} \rightarrow \infty} \frac{1}{2 t_{0} y_{0}} \int_{0}^{t_{0}} \int_{-y_{0}}^{y_{0}} \int^{x}\left(\left.T_{z}\right|_{1}-\left.T_{z}\right|_{0}\right) d x^{\prime} d y d t
\end{aligned}
$$

$J_{H}$ is the average heat flux through a plane of constant $x$, which, in general, is not constant as $x$ varies, so to obtain a formula for the horizontal Nusselt number, we must take a weighted average over $x$ of the form $\overline{f(x) J_{H}(x)}$. Looking at the form of the flow in figure 4, we want $f$ to be positive in the left half (where the heat transport is expected to be in the $+x$ direction) and negative in the right half. A simple weighting function $f$ satisfying these requirements is $-d T(x, y, 1, t) / d x=k \sin k x$. Taking the average and integrating by parts gives

$$
\overline{k \sin k x J_{H}}=\left.\overline{\cos k x T_{z}}\right|_{1}-\left.\overline{\cos k x T_{z}}\right|_{0},
$$

which equals $\left.\overline{\cos k x T_{z}}\right|_{1}$ with the zero flux bottom boundary condition. The horizontal Nusselt number is this quantity normalized by the corresponding value for the "conduction" state. Rearranging $\langle T \cdot(\mathcal{H})\rangle$ gives

$$
\left\langle|\nabla T|^{2}\right\rangle=\left.\overline{\cos k x T_{z}}\right|_{1}-\left.\overline{T T_{z}}\right|_{0}
$$

and for a zero flux bottom temperature boundary condition, $\left.\overline{T T_{z}}\right|_{0}$ vanishes, meaning that we obtain the form (1).

If instead we have a different bottom boundary condition for which the second equality in (2) does not hold identically (such as fixed temperature there) then the term $\left.\overline{\cos k x T_{z}}\right|_{0}$ is too difficult to estimate mathematically and so since we expect the fluxes through the top boundary to be much larger than those through the bottom, we neglect this term and in general we define the horizontal Nusselt number to be

$$
N u_{H}=\frac{\left.\overline{\cos k x T_{z}}\right|_{1}}{\left.\overline{\cos k x T_{c z}}\right|_{1}}=\frac{\left\langle|\nabla T|^{2}\right\rangle+\left.\overline{T T_{z}}\right|_{0}}{\left.\overline{\cos k x T_{c z}}\right|_{1}}
$$

where the second equality is derived from (3).

In this report, rigorous bounds on the horizontal Nusselt number, as defined by (4), will be sought for the problem of horizontal convection with the set up shown in figure 1 , using a variety of different bottom boundary conditions for the temperature. In section 2 we impose a fixed flux condition, and in section 3 a fixed temperature boundary condition. We obtain different scalings for the two cases and since the ocean floor is neither a perfect conductor nor a perfect insulator, in section 4 we use a boundary condition that can smoothly move between fixed flux and fixed temperature, and investigate how the scalings change as we move away from these two limits.

\section{Fixed Flux Bottom Boundary Condition}

We consider the horizontal convection set up shown in figure 1 with fixed positive heat flux $T_{z}=-F$ at the bottom of the layer. With this set up, the "conduction" solution $T_{c}$ is given 
by

$$
T_{c}=\frac{\cosh k z}{\cosh k} \cos k x+F(1-z) .
$$

Then the denominator of the horizontal Nusselt number (4) is

$$
\left.\overline{\cos k x T_{c z}}\right|_{1}=\frac{k}{2} \tanh k .
$$

If $F>0$, corresponding to a heat flux into the layer, then the temperature field is bounded from below for all time by the minimum of -1 and $\inf \left(\left.T\right|_{t=0}\right)$. Assuming that we have left the system to relax for long enough, then $T \geq-1$ everywhere. However, with this particular boundary condition, there is no analogous upper bound on the temperature field.

\subsection{Bound on the Horizontal Nusselt Number Using the Lower Bound on the Temperature}

We try to find the maximum value of the horizontal Nusselt number by using the DoeringConstantin background method [12]. We let $T(\mathrm{x}, t)=\tau(x, z)+\theta(\mathbf{x}, t)$, where the background field $\tau$ satisfies the boundary conditions on $T$ and therefore $\theta$ satisfies the homogeneous boundary conditions $\left(\theta=0\right.$ at $z=1$ and $\theta_{z}=0$ at $\left.z=0\right)$. Note that in contrast to [12], in which $\tau$ is a function of $z$ only, here $\tau$ must depend on the horizontal coordinate in order to satisfy the boundary conditions. We consider the variational formulation to bound the numerator of (4):

$$
\mathcal{L}=\left.\overline{\cos k x T_{z}}\right|_{1}-a\langle\mathbf{u} \cdot(\mathcal{N S})\rangle-b\langle\theta \cdot(\mathcal{H})\rangle
$$

where $a$ and $b$ are constant Lagrange multipliers. The first term in this expression is the term we are trying to bound and from this we subtract the constraints we wish to satisfy, multiplied by the Lagrange multipliers $a$ and $b$. Ideally we would require the full equations $(\mathcal{N S}, \mathcal{H}, \mathcal{C})$ to be satisfied at every point in the domain for all times, but this is too complicated to do analytically. Rearranging gives

$$
\begin{aligned}
\mathcal{L}=\left\langle|\nabla \tau|^{2}-a \sigma|\nabla \mathbf{u}|^{2}-(b-1)|\nabla \theta|^{2}+(b-2) \theta \nabla^{2} \tau\right. & -b \theta \mathbf{u} \cdot \nabla \tau\rangle \\
& +a \sigma R_{H}\langle w T\rangle-\left.F \bar{T}\right|_{0}+\left.2 F \bar{\theta}\right|_{0},
\end{aligned}
$$

and by taking $\langle(1-z) \cdot(\mathcal{H})\rangle$, we get $\langle w T\rangle=F-\left.\bar{T}\right|_{0}$. Using the fact that, as long as $F \geq 0$, the temperature field is bounded from below by -1 , and assuming that $a \sigma R_{H}-F \geq 0$ (to be checked a posteriori), we can bound the final three terms:

$a \sigma R_{H}\langle w T\rangle-\left.F \bar{T}\right|_{0}+\left.2 F \bar{\theta}\right|_{0}=a \sigma R_{H} F-\left.\left(a \sigma R_{H}-F\right) \bar{T}\right|_{0}-\left.2 F \bar{\tau}\right|_{0} \leq a \sigma R_{H}(F+1)-F-\left.2 F \bar{\tau}\right|_{0}$.

All the terms in this expression are either independent of $\theta$ and $\mathbf{u}$, or depend linearly on these quantities or are quadratic negative semi-definite terms, except for the term $\langle-b \theta \mathbf{u} \cdot \nabla \tau\rangle$. If this term is removed the whole expression is bounded above, and straightforward to maximize. Thus we first bound this this term by quadratic semi-definite quantities and then find and solve the Euler-Lagrange equations for the resulting functional to obtain a bound.

Our choice of background field $\tau$ is designed to minimize the worst case estimate of $\langle-b \theta \mathbf{u} \cdot \nabla \tau\rangle$. We should ideally like to set $\nabla \tau=0$ everywhere, but then we cannot satisfy 
the boundary conditions. Instead we choose $\nabla \tau=0$ everywhere except for a boundary layer top and bottom. We set $\tau=\tau_{0}(z)+\tau_{1}(z) \cos k x$ where

$$
\begin{aligned}
& \tau_{0}= \begin{cases}F\left(\delta_{0}-z\right), & \text { for } 0<z<\delta_{0}, \\
0, & \text { for } \delta_{0}<z<1,\end{cases} \\
& \tau_{1}= \begin{cases}0, & \text { for } 0<z<1-\delta_{1}, \\
\frac{z-1+\delta_{1}}{\delta_{1}}, & \text { for } 1-\delta_{1}<z<1,\end{cases}
\end{aligned}
$$

Now we can estimate $\langle-b \theta \mathbf{u} \cdot \nabla \tau\rangle$. This only has contributions from the top and bottom boundary layers, and using the estimates (27), (28) and (30) in appendix A, we obtain

$$
\langle-b \theta \mathbf{u} \cdot \nabla \tau\rangle \leq \alpha\left\langle|\nabla \mathbf{u}|^{2}\right\rangle+\beta\left\langle|\nabla \theta|^{2}\right\rangle
$$

where

$$
\begin{aligned}
& \alpha=b \max \left(\frac{F \delta_{0}^{2} c_{0}}{2 \pi^{2}}, \frac{\delta_{1} c_{1}}{2 \pi^{2}}\left(1+2 k \delta_{1}\right)\right), \\
& \beta=b\left(\frac{F \delta_{0}}{2 c_{0}}+\frac{2 \delta_{1}}{\pi^{2} c_{1}}\left(1+2 k \delta_{1}\right)\right) .
\end{aligned}
$$

So

$\mathcal{L} \leq\left\langle|\nabla \tau|^{2}-(a \sigma-\alpha)|\nabla \mathbf{u}|^{2}-(b-1-\beta)|\nabla \theta|^{2}+(b-2) \theta \nabla^{2} \tau\right\rangle+a \sigma R_{H}(1+F)-F\left(1+2 F \delta_{0}\right)$.

The Euler-Lagrange equations for an extremal value of the functional are

$$
\begin{aligned}
\nabla p-2(a \sigma-\alpha) \nabla^{2} \mathbf{u} & =0 \\
-2(b-1-\beta) \nabla^{2} \theta & =(b-2) \nabla^{2} \tau
\end{aligned}
$$

where the term $\nabla p$ has been added to ensure incompressibility, yielding the solution

$$
\theta^{*}=\frac{-(b-2)}{2(b-1-\beta)}\left(\tau-T_{c}\right), \quad \mathbf{u}^{*}=0
$$

which maximizes the functional as long as the spectral constraints $a \sigma \geq \alpha$ and $b-1 \geq \beta$ are satisfied. Substituting in the expressions for the extremalizing fields $\mathbf{u}^{*}$ and $\theta^{*}$, we get a bound on $\mathcal{L}$. Dotting (9) by $\theta^{*}$ and averaging, we obtain an equation that allows us to simplify the bound, giving

$\mathcal{L} \leq\left\langle|\nabla \tau|^{2}\right\rangle+\frac{(b-2)^{2}}{4(b-1-\beta)}\left(\left\langle\nabla \tau \cdot \nabla\left(\tau-T_{c}\right)\right\rangle-\left.\overline{F\left(\tau-T_{c}\right)}\right|_{0}\right)+a \sigma R_{H}(1+F)-F\left(1+2 F \delta_{0}\right)$

For our choice of $\tau$,

$$
\begin{aligned}
\left\langle|\nabla \tau|^{2}\right\rangle & =F^{2} \delta_{0}+\frac{1}{2 \delta_{1}}+\frac{k^{2} \delta_{1}}{6}, \\
\left\langle\nabla \tau \cdot \nabla T_{c}\right\rangle & =F^{2} \delta_{0}+\frac{k}{2} \tanh k
\end{aligned}
$$


meaning that

$$
\begin{aligned}
\mathcal{L} \leq \frac{1}{2 \delta_{1}}+\frac{k^{2} \delta_{1}}{6}+\frac{(b-2)^{2}}{4(b-1-\beta)}\left(\frac{1}{2 \delta_{1}}+\frac{k^{2} \delta_{1}}{6}-\frac{k}{2} \tanh k\right. & \left.+F^{2}\left(1-\delta_{0}\right)\right) \\
& +a \sigma R_{H}(1+F)-F-\delta_{0} F^{2} .
\end{aligned}
$$

To obtain the tightest bound we need to minimize (10) subject to the spectral constraints. These are of the form

$$
a \sigma \geq b \max \left(P c_{0}, Q c_{1}\right), \quad b-1 \geq b\left(\frac{R}{c_{0}}+\frac{S}{c_{1}}\right),
$$

where $P, Q, R$ and $S$ are independent of $a, b, c_{0}$ and $c_{1}$. Thus they are satisfied if and only if

$$
\frac{a \sigma(b-1)}{b^{2}} \lambda \geq P R+P S \frac{c_{0}}{c_{1}}, \quad \frac{a \sigma(b-1)}{b^{2}} \geq Q R \frac{c_{1}}{c_{0}}+Q S .
$$

A suitable value of $c_{0} / c_{1}$ can be chosen if and only if

$$
\frac{a \sigma(b-1)}{b^{2}}\left(\frac{a \sigma(b-1)}{b^{2}}-P R-Q S\right) \geq 0
$$

and since $a \sigma(b-1) / b^{2} \geq 0$, the spectral constraints are equivalent to $a \sigma \geq(P R+Q S) b^{2} /(b-$ 1).

Since both $b^{2} /(b-1)$ and $(b-2)^{2} / 4(b-1-\beta)$ are minimized at $b=2$ (and the quantity in the bracket multiplying $(b-2)^{2} / 4(b-1-\beta)$ in $(10)$ is positive) this means that $b=2$ is optimal in that it minimizes the right hand side of (10). We should also minimize $a \sigma$, so we set

$$
a \sigma=\left(\frac{F^{2} \delta_{0}^{3}}{\pi^{2}}+\frac{4 \delta_{1}^{2}}{\pi^{4}}\left(1+2 k \delta_{1}\right)^{2}\right) .
$$

For sufficiently large Rayleigh numbers, the value of $\delta_{0}$ is insignificant at leading order but we want $\delta_{1}$ to be as large as possible and so we set $\delta_{0}=0$ and $a \sigma=4 \delta_{1}^{2}\left(1+2 k \delta_{1}\right)^{2} / \pi^{4}$, leaving us with

$$
\mathcal{L} \leq \frac{1}{2 \delta_{1}}+\frac{k^{2} \delta_{1}}{6}+\frac{4 \delta_{1}^{2}}{\pi^{4}}\left(1+2 k \delta_{1}\right)^{2} R_{H}(1+F)-F .
$$

For sufficiently large $R_{H}$, the leading order terms will be $1 / 2 \delta_{1}+4 \delta_{1}^{2} R_{H}(1+F) / \pi^{4}$. These are minimized with the choice $\delta_{1}=(\pi / 2)^{4 / 3}\left(R_{H}(1+F)\right)^{-1 / 3}$, yielding the leading order bound $\mathcal{L} \lesssim 3\left(R_{H}(1+F)\right)^{1 / 3} / 2^{2 / 3} \pi^{4 / 3}$ and so

$$
N u_{H} \lesssim \frac{3 \cdot 2^{1 / 3}(1+F)^{1 / 3}}{\pi^{4 / 3} k \tanh k} R_{H}^{1 / 3},
$$

to leading order.

Note that we assumed $a \sigma R_{H}-F \geq 0$, which is always true for the given scalings as $R_{H} \rightarrow \infty$ with $F$ fixed. However, if $F$ is very large (i.e. if $\left.F^{3}(1+F)^{2} \geq\left(1+2 k \delta_{1}\right)^{6} R_{H} / 4 \pi^{4}\right)$ 
we cannot use the lower bound on $T$. We could choose $a \sigma R_{H}-F=0$ yielding the leading order bound

$$
N u_{H} \lesssim\left(\frac{1}{\pi^{2}} \sqrt{\frac{R_{H}}{F}}+F^{2}\right) / \frac{1}{2} k \tanh k
$$

if $R_{H}^{1 / 5} \lesssim F \lesssim R_{H}$ and

$$
N u_{H} \lesssim \frac{2 F^{2}}{k \tanh k}
$$

if $F \gtrsim R_{H}$. However, this is probably not optimal since we have been forced to choose $a \sigma R_{H}-F \geq 0$ in order to use the fact that $T \geq-1$ everywhere. For $F \gg 0$, we might expect that $T$ is well above -1 at the bottom boundary. Instead in the next section we bound the horizontal Nusselt number without using the bound on the temperature to see if we can get a better bound for $N u_{H}$ when $F$ is large.

\subsection{Bound on the Horizontal Nusselt Number Without Using the Lower Bound on the Temperature}

As $F$ becomes larger, since there are steep negative temperature gradients at the bottom boundary, we expect that the lower bound on the temperature there gives a poor estimate of the actual temperature. To attempt to find a better scaling, we do not use this lower bound and instead we must find an alternative way to bound the final three terms in (5). We have

$$
a \sigma R_{H}\langle w T\rangle-\left.F \bar{T}\right|_{0}+\left.2 F \bar{\theta}\right|_{0}=\left(a \sigma R_{H}-F\right)\langle w(\tau+\theta)\rangle+F^{2}-\left.2 F \bar{\tau}\right|_{0}
$$

and now the sign-indeterminate quadratic terms contributing to $\mathcal{L}$ are

$$
\left\langle\left(a \sigma R_{H}-F\right) w \theta-b \theta \mathbf{u} \cdot \nabla \tau\right\rangle
$$

which we bound by $\alpha\left\langle|\nabla \mathbf{u}|^{2}\right\rangle+\beta\left\langle|\nabla \theta|^{2}\right\rangle$ for suitable $\alpha$ and $\beta$. We choose the background field $\tau$ so that the integrand is zero over as much of the layer as possible. To do this we use $\tau=\tau_{0}(z)+\tau_{1}(z) \cos k x$ where $\tau_{1}$ is again given by (7) and

$$
\tau_{0}= \begin{cases}F\left(\delta_{0}-z\right)-\frac{\left(a \sigma R_{K}-F\right)}{b}\left(1-\delta_{0}\right), & \text { for } 0<z<\delta_{0}, \\ -\frac{\left(a \sigma R_{H}-F\right)}{b}(1-z), & \text { for } \delta_{0}<z<1,\end{cases}
$$

which means that the integrand is zero everywhere except in the boundary layers, and estimate $\alpha$ and $\beta$ using the bounds in appendix A.

Proceeding in the same way as for the small $F$ case, we obtain the Euler-Lagrange equations

$$
\begin{aligned}
\nabla p-2(a \sigma-\alpha) \nabla^{2} \mathbf{u} & =\left(a \sigma R_{H}-F\right) \tau \hat{\mathbf{z}} \\
-2(b-1-\beta) \nabla^{2} \theta & =(b-2) \nabla^{2} \tau
\end{aligned}
$$


which have the same solution for $\theta$, but now the solution for $\mathbf{u}$ is non-zero. Substituting in the extremal values $\mathbf{u}^{*}$ and $\theta^{*}$ gives

$$
\begin{aligned}
\mathcal{L} \leq\left\langle|\nabla \tau|^{2}\right\rangle+\frac{(b-2)^{2}}{4(b-1-\beta)}\left(\left\langle\nabla \tau \cdot \nabla\left(\tau-T_{c}\right)\right\rangle\right. & \left.-\left.F \overline{\left(\tau-T_{c}\right)}\right|_{0}\right)+\frac{1}{2}\left(a \sigma R_{H}-F\right)\left\langle w^{*} \tau\right\rangle \\
& +F^{2}-2 F\left(F \delta_{0}-\frac{a \sigma R_{H}-F}{b}\left(1-\delta_{0}\right)\right) .
\end{aligned}
$$

$\left\langle w^{*} \tau\right\rangle$ is estimated in equation (34) in appendix B, and we proceed in the same way as for small $F$. The best choice at leading order for $R_{H} \rightarrow \infty$ is $\delta_{0}=0$, a $=4 \delta_{1}^{2} / \pi^{4}$, $\delta_{1}=\pi^{8 / 5} / 2^{1 / 5} \cdot 3^{2 / 5} R_{H}^{2 / 5}$ and $b=8 / 3$ and we get

$$
N u_{H} \lesssim \frac{2^{11 / 5} R_{H}^{2 / 5}}{3^{3 / 5} \pi^{8 / 5} k \tanh k}
$$

and so this bound is not as good as (11). However, when $F \gg R_{H}^{1 / 5}$ we can show that $N u_{H} \lesssim\left(3 b^{3}-8 b^{2}+b+8\right) F^{2} / 2 b(b-1) k \tanh k$ at leading order. This bound is minimized when $b \approx 1.87$, giving

$$
N u_{H} \lesssim \frac{0.46 F^{2}}{k \tanh k}
$$

thus improving the prefactor of the corresponding results (12) and (13) in the previous section, but not the order of magnitude of the bound.

\subsection{Application to the Real Ocean!}

The total heat flux from the Earth's interior is $F_{E}=3 \times 10^{13} \mathrm{~W}$. For a large ocean, such as the Pacific or Atlantic, this means that the non-dimensional flux on the ocean floor is

$$
F=\frac{F_{E} H}{c \rho A \kappa \Delta T} \approx 60,
$$

where $H \sim 4000 \mathrm{~m}, c=4184 \mathrm{Jkg}^{-1} \mathrm{~K}^{-1}$ is the specific heat of the water, $\rho=1000 \mathrm{kgm}^{-3}$ is the density, $A=4 \pi\left(6.4 \times 10^{6}\right)^{2} \mathrm{~m}^{2}$ is the area of the surface of the Earth. Thus the $R_{H}^{1 / 3}$ scaling is appropriate here and with $k \sim 1.25 \times 10^{-3}$ we obtain $N u_{H} \lesssim 10^{13}$. The dimensionalized heat flux in an ocean covering the whole Earth would be approximately

$$
\frac{c \rho A \kappa \Delta T}{H} \overline{\sin k x J_{H}}=\left.\frac{c \rho A \kappa \Delta T}{k H} \overline{\cos k x T_{z}}\right|_{1} \sim 10^{22} \mathrm{~W},
$$

and thus for a large ocean, such as the Pacific or Atlantic, the heat flux due to horizontal convection is bounded by $10^{22} \gamma W$ where $\gamma$ is the proportion of the Earth's surface covered by the ocean.

\subsection{How does this Differ from the Rossby Scaling?}

Recall that Rossby [5] proposed a scaling for the boundary layer, in which $\partial / \partial z \sim \delta^{-1}$, $\partial / \partial x \sim 1$ and $\mathbf{u} \sim\left(\delta^{-2}, 0, \delta^{-1}\right)$. With this scaling $N u_{H} \sim\left\langle|\nabla \dot{T}|^{2}\right\rangle \sim R_{H}^{1 / 5}$, whereas our rigorous bound only gives $N u_{H} \lesssim C_{0} R_{H}^{1 / 3}$ for a constant $C_{0}$. 
It turns out that the difference in the scalings comes in the bound for $\langle-b \theta \mathbf{u} \cdot \nabla \tau\rangle$, specifically when we come to estimate $\int_{1-\delta_{1}}^{1}|w \theta| d z$. Our estimate (28) bounds this quantity by

$$
\frac{2 \delta_{1}^{2}}{\pi^{2}}\left(c \int_{1-\delta_{1}}^{1} w_{z}^{2} d z+\frac{1}{c} \int_{1-\delta_{1}}^{1} \theta_{z}^{2} d z\right)
$$

The problem comes in the next step when we estimate

$$
\int_{1-\delta_{1}}^{1} w_{z}^{2} d z \leq \frac{1}{4} \int_{1-\delta_{1}}^{1}|\nabla \mathbf{u}|^{2} d z
$$

In fact Rossby's scalings would have

$$
\int_{1-\delta_{1}}^{1} w_{z}^{2} d z \sim \delta_{1}^{2} \int_{1-\delta_{1}}^{1}|\nabla \mathbf{u}|^{2} d z
$$

since the term on the right hand side is dominated by $\int_{1-\delta_{1}}^{1} u_{z}^{2} d z$. If we could show that $\int_{1-\delta_{1}}^{1} w_{z}^{2} \leq K \delta_{1}^{2} \int_{1-\delta_{1}}^{1}|\nabla \mathbf{u}|^{2} d z$ (for some order 1 constant $K$ ), then we too would obtain a $R_{H}^{1 / 5}$ scaling of the horizontal Nusselt number. However, there is no obvious way to improve the estimate, and so the bound of order $R_{H}^{1 / 3}$ stands.

\section{Fixed Temperature on the Bottom Boundary}

We now consider the problem as shown in figure 1 with a fixed temperature $T=T_{0}$ at the bottom boundary. We try to bound the horizontal Nusselt number (4). The "conduction" solution $T_{c}$ is

$$
T_{c}=T_{0}(1-z)+\frac{\sinh k z \cos k x}{\sinh k}
$$

meaning that

$$
\left.\overline{\cos k x T_{c z}}\right|_{1}=\frac{k}{2} \operatorname{coth} k \text {. }
$$

We proceed in the same way as for the fixed flux case, letting $T=\tau(x, z)+\theta(\mathbf{x}, t)$ and constructing the functional

$$
\mathcal{L}=\left.\overline{\cos k x T_{z}}\right|_{1}-\langle a \mathbf{u} \cdot(\mathcal{N S})\rangle-\langle b \theta \cdot(\mathcal{H})\rangle
$$

We have $\langle w T\rangle=-T_{0}-\left.\bar{T}_{z}\right|_{0}$, but in this case, unlike the fixed flux, we cannot use a bounding principle on $T$ to bound this term, as in section 2.1 , because we need to know $\left.\bar{T}_{z}\right|_{0}$. So we must proceed in a similar way to section 2.2 and choose the background field to minimize the worst case estimate of

$$
\left\langle\left(a \sigma R_{H}-T_{0}\right) w \theta-b \theta \mathbf{u} \cdot \nabla \tau\right\rangle
$$

which we bound by $\alpha\left\langle|\nabla \mathbf{u}|^{2}\right\rangle+\beta\left\langle|\nabla \theta|^{2}\right\rangle$. Again, we let $T=\tau+\theta$ and choose $\tau=\tau_{0}(z)+$ $\tau_{1}(z) \cos k x$ that make the integrand zero over the bulk of the layer, and again $\tau_{1}$ is given by (7). However, in this case it is not clear whether or not it is best to have just a single 
boundary layer at the bottom for $\tau_{0}$ or to have boundary layers top and bottom, since with both options we can force the integrand to be zero over the bulk of the layer whilst satisfying the boundary conditions. However, we obtain the same scaling in each case, it is just the prefactor that may be improved. For simplicity and comparison with the fixed flux case, we just have a boundary layer at the bottom and let

$$
\tau_{0}= \begin{cases}\frac{1}{\delta_{0}}\left(T_{0}\left(\delta_{0}-z\right)-\frac{1}{b}\left(a \sigma R_{H}-T_{0}\right)\left(1-\delta_{0}\right) z\right), & \text { for } 0<z<\delta_{0}, \\ -\frac{1}{b}\left(a \sigma R_{H}-T_{0}\right)(1-z), & \text { for } \delta_{0}<z<1 .\end{cases}
$$

Proceeding as for the fixed flux case, solving the Euler-Lagrange equations we obtain the extremal bound

$$
\mathcal{L} \leq\left\langle|\nabla \tau|^{2}\right\rangle+\frac{(b-2)^{2}}{4(b-1-\beta)}\left\langle\nabla \tau \cdot \nabla\left(\tau-T_{c}\right)\right\rangle-T_{0}^{2}+\frac{\left(a \sigma R_{H}-T_{0}\right)^{2}}{4(a \sigma-\alpha)} \frac{k^{2} \delta_{1}^{5}}{504}\left(1+O\left(\delta_{1}\right)\right)
$$

where

$$
\begin{aligned}
& \alpha=\frac{1}{2 \pi^{2}} \max \left(\left|a \sigma R_{H}+(b-1) T_{0}\right| \delta_{0} c_{0}, b \delta_{1} c_{1}\left(1+2 k \delta_{1}\right)\right), \\
& \beta=\frac{2}{\pi^{2}} \max \left(\left|a \sigma R_{H}+(b-1) T_{0}\right| \frac{\delta_{0}}{c_{0}}, \frac{b \delta_{1}}{c_{1}}\left(1+2 k \delta_{1}\right)\right) .
\end{aligned}
$$

Making the simplifying assumption $b=2$ (though this is not optimal) yields

$$
\mathcal{L} \leq \frac{1}{4}\left(\frac{1}{\delta_{0}}-1\right)\left(a \sigma R_{H}+T_{0}\right)^{2}+\frac{1}{2 \delta_{1}}+\frac{k^{2} \delta_{1}}{6}+\frac{\left(a \sigma R_{H}-T_{0}\right)^{2}}{4(a \sigma-\alpha)} \frac{k^{2} \delta_{1}^{5}}{504}\left(1+O\left(\delta_{1}\right)\right)
$$

For moderate $T_{0}$, where we expect $\delta_{0} \ll 1$ and $\delta_{1} \ll 1$, it may be shown that the dominant contribution to the bound is given by $G$ where

$$
G=\frac{1}{4 \delta_{0}}\left(a \sigma R_{H}+T_{0}\right)^{2}+\frac{1}{2 \delta_{1}}
$$

For the bound to be as tight as possible, we need to choose $\delta_{0}$ and $\delta_{1}$ as large as possible. Subject to the spectral constraints, the best choice is $a \sigma=4 \delta_{1}^{2} / \pi^{4}$ and $\delta_{0}=2 \delta_{1} /\left|4 \delta_{1}^{2} R_{H} / \pi^{4}+T_{0}\right|$, meaning that

$$
G=\frac{1}{8 \delta_{1}}\left|\frac{4 \delta_{1}^{2} R_{H}}{\pi^{4}}+T_{0}\right|^{3}+\frac{1}{2 \delta_{1}} .
$$

Assuming that $x=4 \delta_{1}^{2} R_{H} / \pi^{4}+T_{0}>0$, (which can be checked) we have

$$
\frac{d G}{d \delta_{1}}=\frac{1}{8 \delta_{1}^{2}}\left(5 x^{3}-6 T_{0} x^{2}-4\right)
$$

It may be shown that there is only one positive root $x=x^{*}$ of $d G / d \delta_{1}=0$, which provides the minimum of $G$, giving the bound

$$
N u_{H} \lesssim R_{H}^{1 / 2} f\left(T_{0}\right)
$$




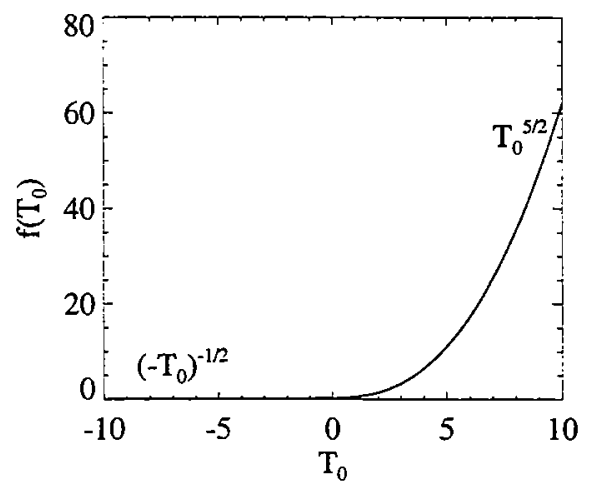

$(a)$

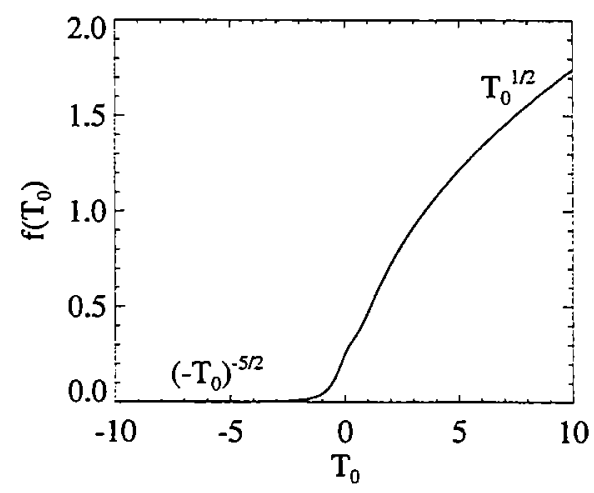

(b)

Figure 5: Prefactor for the bound on the Nusselt number with fixed temperature $T_{0}$ at the bottom of the layer and $k=1.25 \times 10^{-3}$. The bound is proportional to $R_{H}^{1 / 2}$ in each case. (a) shows the prefactor with the horizontal Nusselt number $N u_{H}=\left.\overline{\cos k x T_{z}}\right|_{1} /\left.\overline{\cos k x T_{c z}}\right|_{1}$, whilst (b) shows the prefactor with Nusselt number $N u=\left\langle|\nabla T|^{2}\right\rangle /\left\langle\left|\nabla T_{c}\right|^{2}\right\rangle$.

where

$$
f\left(T_{0}\right)=\frac{x^{* 3}+4}{2 \pi^{2} \sqrt{\left(x^{*}-T_{0}\right)} k \operatorname{coth} k}
$$

as long as $\left|T_{0}\right| \ll R_{H}$. A graph of $f$ is shown in figure $5(a)$.

For $T_{0} \gg 0$, the leading order contribution comes from the first term in (20) and we pick $a \sigma=T_{0} / 5 R_{H}$ and $\delta_{0}=\sqrt{5} \pi^{2} / 6 \sqrt{R_{H} T_{0}}$, giving

$$
N u_{H} \lesssim \frac{108 T_{0}^{5 / 2} R_{H}^{1 / 2}}{25 \sqrt{5} \pi^{2} k \operatorname{coth} k} .
$$

For $T_{0} \ll 0$, we may set $\delta_{0}=\delta_{1}=1$ and the dominant contribution is from the term $\left(1+O\left(\delta_{1}\right)\right)\left(a \sigma R_{H}-T_{0}\right)^{2} k^{2} \delta_{1}^{5} / 2016(a \sigma-\alpha)$. This is of order $-T_{0} R_{H} k^{2}$ multiplied by some prefactor, but to work out this prefactor we would have to solve (31) in appendix B to all orders. So the most we can say without doing the full calculation is that the bound on the horizontal Nusselt number is of order $-T_{0} R_{H}$.

\subsection{Connection to Rayleigh-Bénard Scaling}

For very large $T_{0}$ we would expect the motion to be dominated by the large vertical temperature gradient and look like Rayleigh-Bénard convection, and thus would expect the vertical Nusselt number to be bounded by $R_{V}^{1 / 2}$ multiplied by some prefactor, (where $R_{V}=T_{0} R_{H}$ is the vertical Rayleigh number). Similarly in the limit of small $T_{0}$, we would expect the Nusselt number to be bounded by something that tends to unity.

In order to check that the bounds match in the two limits, we define the Nusselt number to be

$$
N u=\frac{\left\langle|\nabla T|^{2}\right\rangle}{\left\langle\left|\nabla T_{c}\right|^{2}\right\rangle}
$$


and proceed to try to bound it. In this case

$$
\left\langle\left|\nabla T_{c}\right|^{2}\right\rangle=T_{0}^{2}+\frac{1}{2} k \operatorname{coth} k
$$

This time instead of $(20)$, we obtain the expression

$$
\begin{aligned}
\mathcal{L} \leq \frac{1}{\delta_{0}}\left(a \sigma R_{H}+T_{0}\right)^{2}-\frac{1}{4} a \sigma R_{H}\left(a \sigma R_{H}+4 T_{0}\right) & +\frac{1}{2 \delta_{1}}+\frac{k^{2} \delta_{1}}{6} \\
& +\frac{\left(a \sigma R_{H}-T_{0}\right)^{2}}{4(a \sigma-\alpha)} \frac{k^{2} \delta_{1}^{5}}{504}\left(1+O\left(\delta_{1}\right)\right) .
\end{aligned}
$$

For moderate $T_{0}$, we obtain (21) again, but this time $f\left(T_{0}\right)$ is given by the graph in figure $5(b)$. If $T_{0} \gg 1$ then

$$
N u \lesssim \frac{216}{25 \pi^{2}} \sqrt{\frac{2}{5}} \sqrt{T_{0} R_{H}}=\frac{216}{25 \pi^{2}} \sqrt{\frac{2}{5}} \sqrt{R_{V}} \approx 0.55 R_{V}^{1 / 2}
$$

to leading order and thus we recover the scaling of the Doering-Constantin result for Rayleigh-Bénard convection [12], although the prefactor is not optimal since we only used a bottom boundary layer and not a top one. If we use top and bottom boundary layers of equal depth, and optimize over the choices of constants $a, b, \delta_{0}, \delta_{1}$, then we get $N u_{H} \lesssim 3 \sqrt{3} R_{V}^{1 / 2} / 4 \pi^{2} \approx 0.13 R_{V}^{1 / 2}$ at leading order, and the prefactor agrees with the Rayleigh-Bénard result.

If $T_{0} \ll-R_{H}$ then we can choose $\delta_{0}=1$, and the leading order contribution is from the first two terms in the bound (22), which gives $\mathcal{L} \lesssim T_{0}^{2}$ and hence

$$
N u \lesssim 1
$$

to leading order, and so we also recover the result for Rayleigh-Bénard convection in the limit of small $T_{0}$.

\section{Intermediate Bottom Boundary Condition}

We now wish to see more clearly why the $R_{H}^{1 / 3}$ and $R_{H}^{1 / 2}$ scalings arise - what is the connection between them and what happens if we have a boundary condition that is not perfectly conducting or perfectly insulating?

We choose the bottom boundary condition $T-\lambda T_{z}=T_{0}$ at $z=0$, where $\lambda \geq 0$, smoothly moving from a perfectly insulating condition at $\lambda=\infty$ to a perfectly conducting condition for $\lambda=0$. This physically corresponds to the bottom of the layer being in contact with a thin imperfectly conducting sheet that is in contact with an infinite heat bath. For this boundary condition, it is not immediately obvious that the velocity and temperature fields stay bounded and thus we first prove their boundedness, which enables us to drop the averages of their time derivatives. 


\subsection{Bounds on the Temperature and Velocity Fields}

In this section, we prove that the fields are bounded in time, which is not completely obvious for the given boundary conditions. Thus for this section only (section 4.1$),\langle\cdot\rangle$ and - denote only spatial averages (and not long time average).

First of all we prove a boundedness principle for the temperature field, using ideas from [13]. We consider the solution of $(\mathcal{H})$ starting from some bounded initial temperature distribution at $t=0$, and solved on the time interval $t \in\left[0, t_{0}\right]$. We want to look for the point where $T$ attains its maximum value. Suppose the maximum occurs at a point where $z \neq 0,1$. At this point we must have $\nabla T=0, \nabla^{2} T \leq 0$ and so from $(\mathcal{H}), \partial T / \partial t \leq 0$, meaning that the maximum of $T$ is attained at $t=0$. If the maximum occurs at $z=0$, then we have $T_{z} \leq 0$ there, which implies, using the boundary condition, that $T \leq T_{0}$. Alternatively it can occur at $z=1$, in which case $T \leq 1$. A similar principle can be used to bound $T$ from below and thus $T$ is everywhere in the range

$$
\left[\min \left(-1, T_{0}, \inf \left(\left.T\right|_{t=0}\right)\right), \max \left(1, T_{0}, \sup \left(\left.T\right|_{t=0}\right)\right)\right] .
$$

If the system is allowed to relax for sufficiently long then $T$ will eventually be in the range

$$
\left[\min \left(-1, T_{0}\right), \max \left(1, T_{0}\right)\right]
$$

a result that we shall use when applying the background method.

To bound the velocity field, we first use Poincaré's inequality and obtain $\left\langle|\mathbf{u}|^{2}\right\rangle \leq$ $2\left\langle|\nabla \mathbf{u}|^{2}\right\rangle / \pi^{2}$. Rearranging $\langle\mathbf{u} \cdot(\mathcal{N S})\rangle$ yields averaging yields

$$
\begin{aligned}
\frac{1}{2} \frac{d}{d t}\left\langle|\mathbf{u}|^{2}\right\rangle & =R_{H}\langle w T\rangle-\left\langle|\nabla \mathbf{u}|^{2}\right\rangle \\
& \leq R_{H} \sqrt{\left\langle w^{2}\right\rangle\left\langle T^{2}\right\rangle}-\left\langle|\nabla \mathbf{u}|^{2}\right\rangle \\
& \leq R_{H} \sqrt{\left\langle|\mathbf{u}|^{2}\right\rangle\left\langle T^{2}\right\rangle}-\left\langle|\nabla \mathbf{u}|^{2}\right\rangle \\
\Rightarrow \quad \frac{d}{d t} \sqrt{\left\langle|\mathbf{u}|^{2}\right\rangle} & \leq R_{H} \sqrt{\left\langle T^{2}\right\rangle}-\frac{\left\langle|\nabla \mathbf{u}|^{2}\right\rangle}{\sqrt{\left\langle|\mathbf{u}|^{2}\right\rangle}} \\
& \leq R_{H} \sqrt{\left\langle T^{2}\right\rangle}-\frac{2}{\pi^{2}} \sqrt{\left\langle|\mathbf{u}|^{2}\right\rangle}
\end{aligned}
$$

meaning that $\left\langle|\mathbf{u}|^{2}\right\rangle$ is bounded above by its initial value and $\pi^{2} R_{H} \sqrt{\left\langle T^{2}\right\rangle} / 2$.

\subsection{The Set Up}

Having proved the boundedness of the fields, we can now begin to apply the DoeringConstantin method to bound the horizontal Nusselt number given by (4). With these boundary conditions, $T_{c}$ is given by

$$
T_{c}=T_{0}\left(\frac{1-z}{1+\lambda}\right)+\frac{\sinh k z+\lambda k \cosh k z}{\sinh k+\lambda k \cosh k} \cos k x
$$

giving

$$
\left.\overline{\cos k x T_{c z}}\right|_{1}=\frac{k}{2}\left(\frac{\cosh k+\lambda k \sinh k}{\sinh k+\lambda k \cosh k}\right)
$$


and so we try to find an upper bound on the numerator of $N u_{H}$

$$
\left.\overline{\cos k x \bar{T}_{z}}\right|_{1}=\left\langle|\nabla T|^{2}\right\rangle+\left.\overline{T\left(T-T_{0}\right)}\right|_{0} / \lambda .
$$

Letting $T=\tau+\theta$, where $\tau$ satisfies the boundary conditions on $T$ and $\theta$ satisfies the homogeneous boundary conditions, we have

$$
\begin{aligned}
\mathcal{L}= & \left.\overline{\cos k x T_{z}}\right|_{1}-\langle a \mathbf{u} \cdot(\mathcal{N S})\rangle-\langle b \theta \cdot(\mathcal{H})\rangle, \\
= & \left\langle|\nabla \tau|^{2}-a \sigma|\nabla \mathbf{u}|^{2}+(b-1) \theta \nabla^{2} \theta+(b-2) \theta \nabla^{2} \tau+a \sigma R_{H} w(\tau+\theta)\right. \\
& \quad-b \theta \mathbf{u} \cdot \nabla \tau\rangle-\left.\overline{\theta \theta_{z}}\right|_{0}-\left.2 \overline{\theta \tau_{z}}\right|_{0}+\left.\frac{1}{\lambda} \overline{T\left(T-T_{0}\right)}\right|_{0}, \\
= & \left\langle|\nabla \tau|^{2}-a \sigma|\nabla \mathbf{u}|^{2}+(b-1) \theta \nabla^{2} \theta+(b-2) \theta \nabla^{2} \tau+a \sigma R_{H} w(\tau+\theta)\right. \\
& \quad-b \theta \mathbf{u} \cdot \nabla \tau\rangle+\left.\frac{1}{\lambda} \overline{\left(\tau-T_{0}\right)^{2}}\right|_{0}+\frac{T_{0}}{\lambda}\left(\left.\bar{T}\right|_{0}-T_{0}\right), \\
= & \left\langle|\nabla \tau|^{2} \quad-a \sigma|\nabla \mathbf{u}|^{2}+(b-1) \theta \nabla^{2} \theta+(b-2) \theta \nabla^{2} \tau+\mu w(\tau+\theta)\right. \\
& \quad-b \theta \mathbf{u} \cdot \nabla \tau\rangle+\left.\frac{1}{\lambda} \overline{\left(\tau-T_{0}\right)^{2}}\right|_{0}-\frac{T_{0}^{2}}{1+\lambda} .
\end{aligned}
$$

where

$$
\mu=a \sigma R_{H}-\frac{T_{0}}{1+\lambda}
$$

and we have used the lower boundary conditions to obtain (23). Then to obtain (24), the final term in $(23)$ can be absorbed into the global average, using $(\mathcal{H})$ to derive an expression for $\left.\bar{T}\right|_{0}$ :

$$
\langle w T\rangle=-\left.\bar{T}\right|_{0}-\left.\overline{T_{z}}\right|_{0}=\frac{T_{0}}{\lambda}-\left.\left.\frac{1+\lambda}{\lambda} \bar{T}\right|_{0} \Rightarrow \bar{T}\right|_{0}=\frac{T_{0}-\lambda\langle w T\rangle}{1+\lambda} .
$$

Note also that in (24) we have chosen to rewrite any terms proportional to $|\nabla \theta|^{2}$ in terms of $\theta \nabla^{2} \theta$. This is because when the Euler-Lagrange equations are computed to minimize such terms, the former term would give some contributions from the boundary, which make the equation more difficult to solve, whereas the latter will not.

\subsection{Bound on the Horizontal Nusselt Number}

Starting from expression (24), we proceed to try to minimize $\mathcal{L}$ using the boundedness of the temperature. From (25) we can bound $\langle\mu w(\tau+\theta)\rangle=\mu\langle w T\rangle \leq M \mu$, where

$$
M= \begin{cases}\max \left(\frac{T_{0}+1+\lambda}{\lambda},-T_{0}\right), & \text { if } \mu \geq 0 \\ \max \left(\frac{T_{0}-1-\lambda}{\lambda},-T_{0}\right), & \text { if } \mu \leq 0\end{cases}
$$

We choose the background field $\tau$ to minimize the worst case estimate of $\langle-b \theta \mathbf{u} \cdot \nabla \tau\rangle$, choosing $\nabla \tau=0$ over as much as possible of the layer. In order to satisfy the boundary conditions we must again have top and bottom boundary layers. We choose $\tau_{1}$ to be given by (7) and

$$
\tau_{0}= \begin{cases}\frac{\delta_{0}-z}{\delta_{0}+\lambda} T_{0}, & \text { for } 0<z<\delta_{0} \\ 0, & \text { for } \delta_{0}<z<1\end{cases}
$$


With this choice,

$$
\langle-b \theta \mathbf{u} \cdot \nabla \tau\rangle \leq \alpha\left\langle|\nabla \mathbf{u}|^{2}\right\rangle+\beta\left\langle|\nabla \theta|^{2}\right\rangle,
$$

for all fields $\mathbf{u}$ and $\theta$ where, using the estimates in appendix $\mathrm{A}$,

$$
\begin{aligned}
& \alpha=b \max \left(\frac{\left|T_{0}\right|}{\delta_{0}+\lambda} \frac{\delta_{0}^{2} c_{0}}{2 \pi^{2}}, \frac{\delta_{1} c_{1}}{2 \pi^{2}}\left(1+2 k \delta_{1}\right)\right), \\
& \beta=b\left(\frac{\left|T_{0}\right|}{\delta_{0}+\lambda} \frac{\delta_{0}}{2 c_{0}}+\frac{2 \delta_{1}}{\pi^{2} c_{1}}\left(1+2 k \delta_{1}\right)\right) .
\end{aligned}
$$

Then

$$
\begin{aligned}
\mathcal{L} \leq\left\langle|\nabla \tau|^{2}-(a \sigma-\alpha)|\nabla \mathbf{u}|^{2}+(b-1-\beta) \theta \nabla^{2} \theta+(b-2) \theta \nabla^{2} \tau\right\rangle & \\
& +M \mu+\left.\frac{1}{\lambda} \overline{\left(\tau-T_{0}\right)^{2}}\right|_{0}-\frac{T_{0}^{2}}{1+\lambda},
\end{aligned}
$$

where the boundary term $-\left.\beta \overline{\theta \theta_{z}}\right|_{0}$ arising from the integration by parts has been neglected since it is negative semi-definite as long as $\beta, \lambda \geq 0$.

The Euler-Lagrange equations for minimization of the functional bound for $\mathcal{L}$ are

$$
\begin{aligned}
\nabla p-2(a \sigma-\alpha) \nabla^{2} \mathbf{u} & =0 \\
-2(b-1-\beta) \nabla^{2} \theta & =(b-2) \nabla^{2} \tau
\end{aligned}
$$

yielding the solution

$$
\mathbf{u}^{*}=0, \quad \theta^{*}=\frac{-(b-2)}{2(b-1-\beta)}\left(\tau-T_{c}\right),
$$

which minimizes the functional as long as the spectral constraints $a \sigma \geq \alpha$ and $b-1 \geq \beta$ are satisfied. The extremal bound is

$$
\begin{aligned}
\mathcal{L} \leq\left\langle|\nabla \tau|^{2}\right\rangle+\frac{(b-2)^{2}}{4(b-1-\beta)}\left(\left\langle\nabla \tau \cdot \nabla\left(\tau-T_{c}\right)\right\rangle\right. & \left.+\left.\frac{1}{\lambda} \overline{\left(\tau-T_{c}\right)\left(\tau-T_{0}\right)}\right|_{0}\right) \\
& +M \mu+\left.\frac{1}{\lambda} \overline{\left(\tau-T_{0}\right)^{2}}\right|_{0}-\frac{T_{0}^{2}}{1+\lambda},
\end{aligned}
$$

and similarly to section 2.1 we can show that $b=2$ is the value giving the tightest bound. We also have

$$
\begin{aligned}
\left\langle|\nabla \tau|^{2}\right\rangle & =\frac{\delta_{0} T_{0}^{2}}{\left(\delta_{0}+\lambda\right)^{2}}+\frac{1}{2 \delta_{1}}+\frac{k^{2} \delta_{1}}{6} \\
\left.\overline{\left(\tau-T_{0}\right)^{2}}\right|_{0} & =\frac{\lambda^{2} T_{0}^{2}}{\left(\delta_{0}+\lambda\right)^{2}} .
\end{aligned}
$$

In the following, we only consider the bounds as $R_{H} \rightarrow \infty$ with $T_{0}$ and $\lambda$ fixed; if $R_{H}$ is finite, it may be that a better bound can be obtained with a different scaling. As in section 2.1 , the choice $\delta_{0}=0$ does not affect the bound at leading order and subject to the spectral constraint, the optimal value of $a \sigma$ is $4 \delta_{1}^{2} / \pi^{4}$ to leading order, giving $\mathcal{L} \lesssim$ 
$1 / 2 \delta_{1}+4 M \delta_{1}^{2} R_{H} / \pi^{4}$. The tightest bound is obtained with $\delta_{1}=(\pi / 2)^{4 / 3}\left(M R_{H}\right)^{-1 / 3}$, giving $\mathcal{L} \leq 3\left(M R_{H}\right)^{1 / 3} / 2^{2 / 3} \pi^{4 / 3}$ and so the horizontal Nusselt number is bounded by

$$
N u_{H} \lesssim \begin{cases}3 \cdot 2^{1 / 3}\left(-T_{0} R_{H}\right)^{1 / 3} / \pi^{4 / 3} k\left(\frac{\cosh k+\lambda k \sinh k}{\sinh k+\lambda k \cosh k}\right), & \text { if } T_{0} \leq-1, \\ 3 \cdot 2^{1 / 3}\left(\frac{T_{0}+1+\lambda}{\lambda} R_{H}\right)^{1 / 3} / \pi^{4 / 3} k\left(\frac{\cosh k+\lambda k \sinh k}{\sinh k+\lambda k \cosh k}\right), & \text { if } T_{0} \geq-1\end{cases}
$$

If we proceed without utilising the boundedness of the temperature field, then as with the fixed flux case, the bound on $N u_{H}$ is proportional to $R_{H}^{2 / 5}$, and so the $R_{H}^{1 / 3}$ bound is always better as $R_{H} \rightarrow \infty$ with $T_{0}$ and $\lambda$ fixed.

As $\lambda \rightarrow \infty$ with $T_{0} / \lambda=F$ fixed, we immediately recover the bound for the fixed flux bottom boundary condition (11) in section 2.1. As $\lambda \rightarrow 0$, we might similarly hope to recover the bounds found in section 3 . However, things are not so simple as we might expect!

\subsection{Bound for Small $\lambda$ and Connection to Fixed Temperature Boundary Condition?}

As long as $\lambda>0$, then (26) shows that we have a bound of size $R_{H}^{1 / 3}$. However, if $\lambda=0$, then as shown in section 3 we can only get a bound of order $R_{H}^{1 / 2}$. Why do we have this difference?

In fact, as $\lambda \rightarrow 0$, both the bounds in (26) grow arbitrarily large (if $T_{0} \leq-1$ then this growth is in one of the omitted terms) and so, although the asymptotic behavior is $R_{H}^{1 / 3}$, . for any finite value of $R_{H}$, the prefactor is so huge that the bound will be larger than might be expected. Thus we may ask ourselves, whether there is some way to make the bounds connect in the limit of small $\lambda$ by using a different background field.

The bounds on $T$ at $z=0$ provide poor estimates for small $\lambda$ (unless $T_{0}=0$ ), and so we shall do better if we proceed without using this. Starting from the expression (24), we choose the background field $\tau$ to make the integrand of the unwanted terms $\langle\mu w \theta-b \theta \mathbf{u} \cdot \nabla \tau\rangle$ zero over as much of the layer as possible. Again we set $\tau=\tau_{0}(z)+\tau_{1}(z) \cos k x$ where $\tau_{1}$ is given by (7) and

$$
\tau_{0}= \begin{cases}\frac{1}{\delta_{0}+\lambda}\left(T_{0}\left(\delta_{0}-z\right)-\frac{\mu}{b}\left(1-\delta_{0}\right)(z+\lambda)\right), & \text { for } 0<z<\delta_{0} \\ -\frac{\mu}{b}(1-z), & \text { for } \delta_{0}<z<1\end{cases}
$$

which tends to the expression for fixed temperature (16) in the limit $\lambda \rightarrow 0$. Solving the Euler-Lagrange equations yields the bound

$$
\begin{aligned}
\mathcal{L} \leq\left\langle|\nabla \tau|^{2}\right\rangle+\frac{(b-2)^{2}}{4(b-1-\beta)}\left(\left\langle\nabla \tau \cdot \nabla\left(\tau-T_{c}\right)\right\rangle\right. & \left.+\left.\frac{1}{\lambda} \overline{\left(\tau-T_{c}\right)\left(\tau-T_{0}\right)}\right|_{0}\right)+\left.\frac{1}{\lambda} \overline{\left(\tau-T_{0}\right)^{2}}\right|_{0} \\
& -\frac{T_{0}^{2}}{1+\lambda}+\frac{\mu^{2}}{4(a \sigma-\alpha)} \frac{k^{2} \delta_{1}^{5}}{504}\left(1+O\left(\delta_{1}\right)\right)
\end{aligned}
$$


which tends to expression (17) as $\lambda \rightarrow 0$. Using appendix A, the estimates for $\alpha$ and $\beta$ are

$$
\begin{aligned}
& \alpha=\max \left(\frac{\left|\mu(\lambda+1)+b T_{0}\right|}{\delta_{0}+\lambda} \frac{\delta_{0}^{2} c_{0}}{2 \pi^{2}}, \frac{b \delta_{1} c_{1}}{2 \pi^{2}}\left(1+2 k \delta_{1}\right)\right), \\
& \beta=\frac{\left|\mu(\lambda+1)+b T_{0}\right|}{\delta_{0}+\lambda} \frac{\delta_{0}}{2 c_{0}}+\frac{2 b \delta_{1}}{\pi^{2} c_{1}}\left(1+2 k \delta_{1}\right) .
\end{aligned}
$$

Comparing these with (18) and (19) respectively, we see that as $\lambda \rightarrow 0$ we obtain the same limit for the second term in each expression, but not the first term. This is due to the fact that with the methods we have used, we cannot estimate the temperature on the lower boundary very well, and so we are forced to use the bound (30) rather than (29). This difference turns out to be crucial in the bounding procedure and thus we cannot obtain a continuous bound on the horizontal Nusselt number as $\lambda \rightarrow 0$.

\section{Conclusions and Discussion}

In summary, we have obtained upper bounds on the horizontal Nusselt number for horizontal convection using a variety of different boundary conditions on the bottom of the box. As long as the lower boundary is not perfectly conducting we found that the horizontal Nusselt number is always bounded by a constant prefactor times $R_{H}^{1 / 3}$, and if it is perfectly conducting then the bound increases to a prefactor times $R_{H}^{1 / 2}$.

In a similar way, we might ask if it is possible to use the analogous method to bound the dissipation $\epsilon$. However, it turns out that we cannot improve on the bound obtained using the method outlined in the introduction. For a fixed heat flux $F$ through the bottom, we get

$$
\epsilon \leq \frac{\kappa g \alpha \Delta T}{H}(1+F)
$$

and for the intermediate boundary conditions, we get

$$
\epsilon \leq \frac{\kappa g \alpha \Delta T}{H} \max \left(\frac{T_{0}+1+\lambda}{\lambda},-T_{0}\right)
$$

These bounds imply the anti-turbulence theorem in both cases. With the fixed temperature boundary condition, however, we can't easily relate the flux through the bottom to the temperature there, and in this case, using the Doering-Constantin method, the bound turns out to be $\left\langle|\nabla \mathbf{u}|^{2}\right\rangle \leq g\left(T_{0}\right) R^{3 / 2}$ for some function $g$, meaning that $\epsilon$ is bounded by a non-zero constant as $\kappa \rightarrow 0$ with $\sigma$ fixed, which does not prove anti-turbulence.

It would be interesting to have some idea of what the actual velocity and temperature fields look like in the asymptotic limit as $R_{H} \rightarrow \infty$, and see if figure 4 does indeed give the correct flow pattern in the limit. However, since we set the velocity to zero, the method we have used tells us nothing about the velocity field except perhaps that the velocities in the asymptotic solution are not very large in magnitude. It doesn't prove anything about the temperature field either, although the fact that we did consistently use the background field $\tau=\tau_{0}(z)+\tau_{1}(z) \cos k x$ where $\tau_{1}$ was given by (7), suggests that the real solution may have a top boundary layer and that the temperature field has little horizontal dependence deeper into the layer. Our choice of $\tau_{0}$ varied but we found that the depth of the bottom 
boundary layer did not matter at leading order, (except with the fixed bottom temperature condition), suggesting that the horizontally averaged temperature has no large gradients throughout the layer.

Possibly a more physically realistic set up in the oceanographic context would be to use a stress free velocity boundary condition at the top of the layer rather than the nonslip one, shown in 1, which was used throughout the report. Proceeding to bound the horizontal Nusselt number in a similar fashion, we encounter a problem. We cannot estimate $\int_{1-\delta}^{1} \overline{|u \theta|} d z$ in terms of $\left\langle|\nabla \mathbf{u}|^{2}\right\rangle$ and $\left\langle|\nabla \theta|^{2}\right\rangle$ as we have no control on the size of $u$ at the top boundary, and so we cannot easily find a bound on $N u_{H}$. Note that this problem does not occur for Rayleigh-Bénard convection, since the offending term only arises due to the horizontal dependence of $\tau$.

In summary the bound of $R_{H}^{1 / 3}$, (which holds asymptotically for all the boundary conditions investigated except for the fixed temperature condition), suggests that horizontal convection with an insulating or nearly insulating bottom boundary is much less efficient at transporting heat through a layer than Rayleigh-Bénard convection. In particular, since the bound less than order $R_{H}^{1 / 2}$, the scalings of the temperature and velocity fields in the boundary layers in horizontal convection cannot be independent of the molecular parameters $\nu$ and $\kappa[14,15]$.

So how relevant are these results to the ocean? We don't know the oceanographic bottom boundary conditions, and the bottom is certainly far from being flat! However, we have shown that there is only a weak dependence on these conditions, and so the results are probably still valid. However, possibly more significantly, there are many other processes going on in the ocean such as wind forcing, that can cause large amounts of mixing and these are probably much more significant factors in the circulation than horizontal convection.

I should like to thank Richard Kerswell for suggesting this project and for giving up a lot of time to discuss the problem, Neil Balmforth who provided many useful insights and Charles Doering for making some helpful suggestions. I am grateful to Woods Hole Oceanographic Institution for its funding and hospitality and to everyone on the GFD program for making my stay so enjoyable!

\section{A Estimates of Boundary Layer Integrals}

In this section we estimate the maximum possible size of some integrals that are needed to estimate the sign-indeterminate quadratic terms. The integrals that are needed are

$$
\int_{1-\delta}^{1} \overline{|w \theta|} d z, \quad \int_{1-\delta}^{1} \overline{|u \theta|} d z \text { and } \int_{0}^{\delta} \overline{|w \theta|} d z
$$

where $u$ and $w$ are zero on both the top and bottom boundaries and $\theta$ is zero at the top. At the bottom we have three possibilities: $\theta=0, \theta_{z}=0$ or $\theta-\lambda \theta_{z}=0$.

First we prove the result

If the functions $f$ and $g$ are both zero on the plane $z=z_{0}$ then

$$
\int_{z_{0}}^{z_{0}+\delta}|\overline{f g}| d z \leq \frac{2 \delta^{2}}{\pi^{2}}\left(c \int_{z_{0}}^{z_{0}+\delta} \overline{f_{z}^{2}} d z+\frac{1}{c} \int_{z_{0}}^{z_{0}+\delta} \overline{g_{z}^{2}} d z\right)
$$


The proof (thanks to Michael Proctor) is as follows:

$$
\int_{z_{0}}^{z_{0}+\delta}|\overline{f g}| d z \leq \overline{\left(\int_{z_{0}}^{z_{0}+\delta} f^{2} d z \int_{z_{0}}^{z_{0}+\delta} g^{2} d z\right)^{1 / 2}}
$$

(using the Cauchy-Schwarz inequality),

$$
\leq \frac{1}{2}\left(c \int_{z_{0}}^{z_{0}+\delta} \overline{f^{2}} d z+\frac{1}{c} \int_{z_{0}}^{z_{0}+\delta} \overline{g^{2}} d z\right)
$$

(using Young's inequality, $\sqrt{a b} \leq(c a+b / c) / 2$ for any $c>0$ ).

We can use the calculus of variations to minimize the ratio

$$
\int_{z_{0}}^{z_{0}+\delta} h_{z}^{2} d z / \int_{z_{0}}^{z_{0}+\delta} h^{2} d z
$$

subject to $h\left(z_{0}\right)=0$. The minimum value is $\pi^{2} / 4 \delta^{2}$, and hence the result follows.

Using this we have

$$
\begin{aligned}
& \int_{1-\delta}^{1} \overline{|u \theta|} d z \leq \frac{2 \delta^{2}}{\pi^{2}} \int_{1-\delta}^{1}\left(\overline{c u_{z}^{2}}+\frac{\overline{\theta_{z}^{2}}}{c}\right) d z \leq \frac{2 \delta^{2}}{\pi^{2}} \int_{1-\delta}^{1}\left(c \overline{|\nabla \mathbf{u}|^{2}}+\frac{1}{c} \overline{|\nabla \theta|^{2}}\right) d z \\
& \int_{1-\delta}^{1} \overline{|w \theta|} d z \leq \frac{2 \delta^{2}}{\pi^{2}} \int_{1-\delta}^{1}\left(\overline{c w_{z}^{2}}+\frac{\overline{\theta_{z}^{2}}}{c}\right) d z \leq \frac{2 \delta^{2}}{\pi^{2}} \int_{1-\delta}^{1}\left(\overline{c w_{z}^{2}}+\frac{1}{c} \overline{|\nabla \theta|^{2}}\right) d z
\end{aligned}
$$

and similarly if $\theta=0$ at $z=0$ then

$$
\int_{0}^{\delta} \overline{|w \theta|} d z \leq \frac{2 \delta^{2}}{\pi^{2}} \int_{0}^{\delta}\left(\overline{c w_{z}^{2}}+\frac{1}{c} \overline{|\nabla \theta|^{2}}\right) d z
$$

otherwise

$$
\begin{aligned}
\int_{0}^{\delta} \overline{|w \theta|} d z & \leq \overline{\left(\int_{0}^{\delta} w^{2} d z \int_{0}^{\delta} \theta^{2} d z\right)^{1 / 2}}, \text { (Cauchy-Schwartz) } \\
& \leq \frac{1}{2}\left(c \int_{0}^{\delta} \overline{w^{2}} d z+\frac{1}{c} \int_{0}^{\delta} \overline{\theta^{2}} d z\right), \text { (Young's inequality) } \\
& \leq \frac{1}{2}\left(\frac{4 \delta^{2} c}{\pi^{2}} \int_{0}^{\delta} \overline{w_{z}^{2}} d z+\frac{1}{c} \int_{0}^{\delta} \overline{\left(\int_{z}^{1} \theta_{z^{\prime}} d z^{\prime}\right)^{2}} d z\right) \\
& \leq \frac{2 \delta^{2} c}{\pi^{2}} \int_{0}^{\delta} \overline{w_{z}^{2}} d z+\frac{1}{2 c} \int_{0}^{\delta} \overline{\left((1-z) \int_{z}^{1} \theta_{z^{\prime}}^{2} d z^{\prime}\right)} d z, \text { (Cauchy-Schwartz) } \\
& \leq \frac{2 \delta^{2} c}{\pi^{2}} \int_{0}^{\delta} \overline{w_{z}^{2}} d z+\frac{\delta}{2 c}\left\langle|\nabla \theta|^{2}\right\rangle,
\end{aligned}
$$

where $c$ can take any positive value.

Rather than simply bounding $w_{z}^{2}$ by $|\nabla \mathbf{u}|^{2}$, we improve the bounds by using the following inequality, which is taken from [12]. Since $u_{x}+v_{y}+w_{z}=0$ then

$$
\begin{aligned}
& \left\langle u_{x} w_{z}+v_{y} w_{z}+w_{z}^{2}\right\rangle=0 \Rightarrow\left\langle u_{z} w_{x}+v_{z} w_{y}+w_{z}^{2}\right\rangle=0 \\
& \left\langle w_{z}^{2}\right\rangle=\left\langle\left(u_{x}+v_{y}\right)^{2}\right\rangle \Rightarrow\left\langle w_{z}^{2}-u_{x}^{2}-v_{y}^{2}-2 u_{y} v_{x}\right\rangle=0
\end{aligned}
$$


where the boundary conditions have been used to integrate by parts. Twice the first equation plus the second plus $\left\langle|\nabla \mathbf{u}|^{2}\right\rangle$ gives

$$
\left\langle 4 w_{z}^{2}+\left(u_{y}-v_{x}\right)^{2}+\left(u_{z}+w_{x}\right)^{2}+\left(v_{z}+w_{y}\right)^{2}\right\rangle=\left\langle|\nabla \mathbf{u}|^{2}\right\rangle \Rightarrow\left\langle w_{z}^{2}\right\rangle \leq \frac{1}{4}\left\langle|\nabla \mathbf{u}|^{2}\right\rangle .
$$

\section{B Estimate of the Size of $\left\langle w^{*} \tau\right\rangle$}

In section 2.2 , we obtained a term proportional to $\left\langle w^{*} \tau\right\rangle$ in the bounding procedure. This term must be estimated, which is done in this section.

In both cases $\mathbf{u}^{*}$ satisfies an equation of the form

$$
\nabla p-\nabla^{2} \mathbf{u}=P \tau \hat{\mathbf{z}}
$$

where $\tau=\tau_{0}(z)+\tau_{1}(z) \cos k x$ and

$$
\tau_{1}= \begin{cases}0, & \text { for } 0<z<1-\delta_{1}, \\ \frac{z-1+\delta_{1}}{\delta_{1}}, & \text { for } 1-\delta_{1}<z<1,\end{cases}
$$

Taking the curl gives

$$
\begin{aligned}
\nabla^{4} \psi & =-P \tau_{x}, \\
& = \begin{cases}\frac{P k}{\delta_{1}} y \sin k x & \text { for } y>0, \\
0 & \text { for } y<0,\end{cases}
\end{aligned}
$$

where $y=z-1+\delta_{1}$ and $\mathbf{u}=\left(-\psi_{z}, 0, \psi_{x}\right)$. The solution is of the form

$$
\psi=\left\{\begin{array}{c}
\frac{P}{k^{3} \delta_{1}}\left(y+\left(A^{\prime} y+B^{\prime}\right) \sinh k y+\left(C^{\prime} y+D^{\prime}\right) \cosh k y\right) \sin k x \\
\text { for } 0<y<\delta_{1} \\
\frac{P}{k^{3} \delta_{1}}((A y+B) \sinh k y+(C y+D) \cosh k y) \sin k x \\
\text { for }-1+\delta_{1}<y<0
\end{array}\right.
$$

for some constants $A, B, C, D, A^{\prime}, B^{\prime}, C^{\prime}$ and $D^{\prime}$ to be determined. Note that since $\psi$ is proportional to $\sin k x,\left\langle w^{*} \tau\right\rangle=\left\langle w^{*} \tau_{1} \cos k x\right\rangle$, with no contribution from $\tau_{0}$ and so the solution is only needed in the top boundary layer.

Matching $\psi, \psi_{y}, \psi_{y y}$ and $\psi_{y y y}$ at $\mathrm{y}=0$ gives

$$
A^{\prime}=A, \quad B^{\prime}=B-\frac{3}{2 k}, \quad C^{\prime}=C+\frac{1}{2}, \quad D^{\prime}=D .
$$

The boundary conditions at the top and bottom of the box imply that

$$
\begin{aligned}
\left(\begin{array}{cccc}
\delta_{1} s_{t} & s_{t} & \delta_{1} c_{t} & c_{t} \\
s_{t}+k \delta_{1} c_{t} & k c_{t} & k \delta_{1} s_{t}+c_{t} & k s_{t} \\
\left(\delta_{1}-1\right) s_{b} & s_{b} & \left(\delta_{1}-1\right) c_{b} & c_{b} \\
s_{b}+k\left(\delta_{1}-1\right) c_{b} & k c_{b} & k\left(\delta_{1}-1\right) s_{b}+c_{b} & k s_{b}
\end{array}\right) & \left(\begin{array}{c}
A \\
B \\
C \\
D
\end{array}\right) \\
& =\left(\begin{array}{c}
-\delta_{1}+\frac{3}{2 k} s_{t}-\frac{1}{2} \delta_{1} c_{t} \\
-1-\frac{1}{2} k \delta_{1} s_{t}+c_{t} \\
0 \\
0
\end{array}\right)
\end{aligned}
$$


where $c_{t}=\cosh k \delta_{1}, s_{t}=\sinh k \delta_{1}, c_{b}=\cosh k\left(\delta_{1}-1\right)$ and $s_{b}=\sinh k\left(\delta_{1}-1\right)$. Note that if $\delta_{1} \ll 1$ then the right hand side of this equation is $O\left(\delta_{1}^{4}\right)$, whilst the determinant of the matrix is $O(1)$, and thus $A, B, C$ and $D$ are $O\left(\delta_{1}^{4}\right)$ at the largest. In fact the first equation arising from this matrix equation is

$$
\delta_{1} s_{t} A+s_{t} B+\delta_{1} c_{t} C+c_{t} D=-\delta_{1}+\frac{3}{2 k} s_{t}-\frac{1}{2} \delta_{1} c_{t}
$$

The coefficients of the first three terms are at most $O\left(\delta_{1}\right)$, and so the terms must be $O\left(\delta_{1}^{5}\right)$. The right hand side is also $O\left(\delta_{1}^{5}\right)$ and $c_{t}$ is $O(1)$. Thus $D$ is $O\left(\delta_{1}^{5}\right)$. Therefore since $y$ is $O\left(\delta_{1}\right)$ in the top boundary layer,

$$
\begin{aligned}
\psi & =\frac{P}{k^{3} \delta_{1}}\left(y-\frac{3}{2 k} \sinh k y+\frac{1}{2} y \cosh k y+O\left(\delta_{1}^{5}\right)\right) \sin k x \\
& =O\left(P \delta_{1}^{4}\right)
\end{aligned}
$$

Therefore $w$ is also $O\left(P \delta_{1}^{4}\right)$ and so

$$
\left\langle w^{*} \tau\right\rangle=O\left(P \delta_{1}^{5}\right)
$$

In fact, by inverting the matrix in (32), we have, to leading order

$$
\begin{aligned}
\mathbf{u}^{*}=P\left(\left(\frac{1}{24}\left(1-\zeta^{4}\right) k \delta_{1}^{3}-\frac{\left(e^{4 k}-4 k e^{2 k}-1\right)(1-\zeta) k^{2} \delta_{1}^{4}}{12\left(\left(e^{2 k}-1\right)^{2}-4 k^{2} e^{2 k}\right)}+O\left(\delta_{1}^{5}\right)\right) \sin k x\right. \\
\left.0,\left(\frac{k^{2}}{120}\left(4-5 \zeta+\zeta^{5}\right) \delta_{1}^{4}+O\left(\delta_{1}^{5}\right)\right) \cos k x\right)
\end{aligned}
$$

in the top boundary layer, where $\zeta=y / \delta_{1}>0$, giving

$$
\left\langle w^{*} \tau\right\rangle=\frac{k^{2} P \delta_{1}^{5}}{504}\left(1+O\left(\delta_{1}\right)\right) \text {. }
$$

\section{References}

[1] M. E. Stern, Ocean Circulation Physics, International Geophysics Series, vol 19 (Academic Press, New York, 1975).

[2] W. R. Young, Notes on Rossby's problem, 2002, private communication.

[3] A. Defant, Physical Oceanography: Volume I (MacMillan, New York, 1961).

[4] H. Jeffreys, "On fluid motions produced by differences of temperature and humidity," Quart. J. Roy. Meteorol. Soc. 51, 347 (1925).

[5] T. Rossby, "On thermal convection driven by non-uniform heating from below: an experimental study," Deep-Sea Res. 12, 9 (1965).

[6] R. C. Somerville, "A non-linear spectral model of convection in a fluid unevenly heated from below," J. Atmos. Sci. 24, 665 (1967). 
[7] R. C. Beardsley and J. F. Festa, "A numerical model of convection driven by surface stress and non-uniform horizontal heating," J. Phys. Ocean. 2, 444 (1972).

[8] T. Rossby, "Numerical experiments with a fluid heated non-uniformly from below," Tellus 50A, 242 (1998).

[9] F. Paparella and W. R. Young, "Horizontal convection is non-turbulent (preprint)," J. Fluid Mech. (2002).

[10] J. W. Sandström, "Dynamische Versuche mit Meerwasser," Annals in Hydrodynamic Marine Meteorology (1908).

[11] U. Frisch, Turbulence: the legacy of A. N. Kolmogorov (Camb. Univ. Press, Cambridge, 1995).

[12] C. R. Doering and P. Constantin, "Variational bounds on energy dissipation in incompressible flows. III. Convection," Phys. Rev. E 53, 5957 (1996).

[13] M. H. Protter and H. F. Weinberger, Maximum Principles in Differential Equations (Springer-Verlag, New York, 1984).

[14] R. H. Kraichnan, "Turbulent thermal convection at arbitrary Prandtl number," Phys. Fluids 5, 1374 (1962).

[15] E. A. Spiegel, "Convection in stars," Annu. Rev. Astron. Astr. 9, 323 (1971). 


\title{
Inertial Wave Convection in Rotating Spherical Fluid Shells
}

\author{
Radostin D. Simitev ${ }^{1}$ \\ Institute of Physics, University of Bayreuth, D-95440 Bayreuth, Germany
}

\begin{abstract}
The report represents a study of the linear properties of thermal convection in rotating spheres and shells. An "inertial wave" analytical approximation based on the similarities of the convection flows and the solutions of the Poincare equation is developed for the region of small Prandtl numbers. Theoretical expression for the Rayleigh number of convection with insulating thermal boundary conditions is obtained and tested against full numerical solutions. A second part of the report describes the results of a numerical study of the convection regimes and transitions found in the region of small Prandtl numbers. The transition between retrograde and prograde drifting modes is examined in detail. A new multi-columnar solution is found to bound the region of wall-attached convection at very high rotation rates. Various predictions of the developed analytical theory are also tested and verified numerically. Finally convection patterns in the case of insulating thermal boundary conditions are observed and compared with the better studied case of perfectly conducting boundaries.
\end{abstract}

\section{Introduction}

In this report we study the linear problem of the onset of thermal convection in rotating spheres and spherical shells.

The interest in this problem is motivated mainly by geophysical and astrophysical applications and dates back at least half a century, during which period a vast amount of literature has been accumulated. The most comprehensive formulation of the problem and some early results were presented by Chandrasekhar(1961). The fundamental theoretical work was carried out by Roberts(1968) and Busse(1970a). It was predicted that convection would be in the form of slowly drifting columnar rolls with small azimuthal scale but the precise structure of the flow was not determined by the theories. Experimental studies (Carrigan and Busse(1983)) confirmed the qualitative features predicted by the theories for the range of Prandtl numbers of their working fluids. Later numerical studies (Zhang and Busse(1987), Ardes et al.(1997)) found that the form of convection pattern is strongly dependent on almost all parameters that enter the formulation of the problem and they identified various regimes of convection at onset. A number of excellent reviews on the subject exist and the reader is referred to Busse(2002) as one of the most recent ones.

However certain mathematical and numerical difficulties prevent the complete solution of the problem. The preferred mode of convection is usually non-axisymmetric and strongly time dependent even at the onset. Another analytical difficulty arises from the geometry of the system and more precisely from the fact that the role of the Coriolis force varies with the angle between gravity and the vector of angular velocity. On the numerical side the

\footnotetext{
${ }^{1}$ e-mail: radostin.simitev@uni-bayreuth.de
} 
investigation of the linear problem is hindered by the large number of parameters, including the Rayleigh and Prandtl numbers, the rotation rate parameter as well as the radius ratio of the spherical shell. In addition to that various choices of the boundary conditions, the heating model and the variation of gravity can be made at formulation. Having in mind all these difficulties, it is not surprising that many open questions still exist and that there are many possibilities for further investigations of the linear problem.

An efficient way of overcoming the lack of a complete solution has been to obtain analytical approximations in special cases. In the case of a thin shell the effects of rotation can be treated as a perturbation (Busse(1970b)). An analytical method for the description of low Prandtl number convection is based on the idea that the thermal convection can be treated as a perturbation of inertial oscillations, which on the other hand emerge as solutions of the Poincaré equation in rotating spheres (Zhang(1994)). Another method, applicable to the same parameter region, is the equatorial approximation described by Ardes et al.(1997). These analytical approximations need to be validated and tested against full numerical solutions. The numerics in addition reveal many new properties and phenomena not predicted by the theories.

Such an approach to the problem of convection in rotating spheres and shells has been adopted in the papers of Zhang and Busse(1987) and Ardes et al.(1997). Apart from testing various theoretical predictions, these papers report the most detailed numerical investigations of the parameter space so far. The preferred types of convection flow at relatively low and moderate Prandtl numbers are determined. Wall-attached regime is observed at lower values and columnar type flows at higher values of the Prandtl number. When the rotation parameter is varied, eigenmode competition is observed and transitions between several new modes and patterns are identified, including modes traveling in the retrograde or prograde direction. Many properties of these phenomena are investigated below in detail.

These two papers have provided useful ideas and starting points for the studies undertaken in the present report. Here we choose to focus our attention on the properties of inertial wave convection, which is observed in the region of small Prandtl numbers and intermediate to high values of the rotation parameter. This choice is motivated by several facts. Firstly, a convenient analytical approach is possible in this parameter region. The thermal convection can be considered to be a perturbation of inertial oscillations of the Poincare equation. Based on this idea we follow the method described by Zhang(1994) but show a different approach to obtain the results published by him. Furthermore we extend the analysis and solve the heat equation for a new case of perfectly insulating thermal boundary conditions. As a result complete analytical convection solutions are obtained and a theoretical expression for the critical Rayleigh number in the limit of small Prandtl numbers and high rotation rates is derived. The validity of the results is tested against numerical solutions of the full set of linear equations. Secondly, all previous studies agree that at low Prandtl numbers convection is much richer in dynamical behavior than at high Prandtl numbers of the order one or higher and that there can be regions where the preferred mode is still unknown. At the same time neither very high rotation rates nor very small Prandtl number cases have been reached so far. Several already known phenomena need to be studied in more detail as well. A particular goal is to outline the border between regions of retrograde and prograde drifting modes in the $P-\tau-\eta$ space. Furthermore, 
several analytical facts based on the inertial wave approximation developed in the first part of the report have to be tested. More precisely we wish to verify the prediction that in the regime of wall-attached convection the ratio between the frequency and the rotation parameter must remain constant for a broad range of rotation rates and at the same time have no radial dependence. Another prediction of the theory is the fact that the critical Rayleigh number of a particular mode does not depend on the rotation rate for small values of the Prandtl number and high rotation rates. Finally, a study of the dependences of the critical Rayleigh number and frequency on the radius ratio as well as a test of the validity of the analytical approximations for various thickness of the spherical shell has not yet been reported in previous studies.

A third part of the report, which deserves a study of its own, addresses the problem of the onset of thermal convection in rotating spherical shells, but in contrast with the second part in the case of perfectly insulating thermal boundary conditions. Since the case of insulating thermal boundary conditions has not been previously reported in the literature, we begin its exploration with a number of comparisons between the new case and the much better studied case of perfectly conducting thermal boundaries. We try to outline any differences or similarities in the Rayleigh number and frequency relationships as well as to observe whether regimes of patterns similar to the flows in the conducting case exist.

The report starts with a short description of the geometrical configuration and the formulation of the problem in section 2 . Section 3 describes the inertial wave approximation and extends the results of Zhang(1994) to the case of insulating thermal boundary conditions. The numerical methods used for solving the governing equations are introduced in section 4. Section 5 presents the main results emerging from the numerical study of low Prandtl number convection. In section 7 some preliminary results from the numerical study of convection with thermally insulating boundaries are reported and concluding remarks are given in section 8 .

\section{Mathematical Formulation of the Problem}

We consider the problem of convection in rotating spherical shells in its classical formulation with a uniform distribution of heat sources and a gravity force that increases with distance from the center of the sphere. Accordingly the distributions of temperature and gravity in the spherically symmetric basic state are given by

$$
T=T_{0}-\beta \tilde{r}^{2} / 2, \quad g=-\gamma \tilde{\boldsymbol{r}}
$$

where $\tilde{\boldsymbol{r}}$ is the position vector with respect to the center of the sphere and $\tilde{r}$ is its absolute value. The sphere is rotating with angular velocity $\Omega$ about a fixed axis given by the unit vector $k$. It will be convenient to introduce dimensionless variables. As length scale we use the difference $d$ between inner and outer radius of the spherical fluid shell. As time scale we use $d^{2} / \nu$ where $\nu$ is the kinematic viscosity of the fluid and as temperature scale we take $\beta d^{2} \nu / \kappa$ where $\kappa$ denotes the thermal diffusivity of the fluid. The dimensionless equations of motion for the velocity vector $\boldsymbol{u}$ and the heat equation for the deviation $\Theta$ of the temperature from the static distribution are given by

$$
\frac{\partial}{\partial t} u+u \cdot \nabla u+\tau k \times u=-\nabla \pi+r R \Theta+\nabla^{2} u
$$




$$
\begin{aligned}
\nabla \cdot \boldsymbol{u} & =0 \\
\left(\frac{\partial}{\partial t} \Theta+\boldsymbol{u} \cdot \nabla \Theta\right) P & =\boldsymbol{r} \cdot \boldsymbol{u}+\nabla^{2} \Theta
\end{aligned}
$$

where $\boldsymbol{r}$ is the dimensionless position vector and the Rayleigh number $R$, the Taylor number $\tau^{2}$, and the Prandtl number $P$ are defined by

$$
R=\frac{\alpha \gamma \beta d^{6}}{\nu \kappa}, \quad \tau=\frac{2 \Omega d^{2}}{\nu}, \quad P=\frac{\nu}{\kappa}
$$

The thermal expansivity has been denoted by $\alpha$ and the Boussinesq approximation has been assumed.

Stress-free velocity boundary conditions and two different types of temperature boundary conditions are imposed on the governing equations (2.2)

$$
\begin{array}{ccc}
\frac{\partial}{\partial r}\left(\frac{u_{\phi}}{r}\right) & = & \frac{\partial}{\partial r}\left(\frac{u_{\theta}}{r}\right)=0 \\
\text { Type A: } & \Theta=0 \\
\text { Type B: } & \frac{\partial}{\partial r} \Theta=0 \\
\text { at } r=r_{i} \equiv \frac{\eta}{1-\eta} & \text { and } & r=r_{0} \equiv \frac{1}{1-\eta},
\end{array}
$$

where $\eta$ denotes the radius ratio of the spherical shell.

This is a general formulation which is not restricted to the linear problem. In the present report we neglect the nonlinear terms in equations (2.2) and use them as a starting point for both the analytical and the numerical studies reported below.

\section{The Inertial Wave Approximation}

In this section we present an analytical approximation for the description of the linear properties of thermal convection in the region of small Parndtl numbers and intermediate to high values of the rotation parameter. We follow the perturbation analysis as described by Zhang(1994) and consider the thermal convection as a small perturbation of the inertial oscillations of the Poincaré equation, the solutions of which are known, but utilize a different approach in order to improve his results and extend the analysis to a new case of convection with insulating thermal boundary conditions.

The justification for such a perturbation analysis is based on the exceptionally good agreement between the numerical values of the frequencies of the preferred modes of convection and the frequencies of the Poincare inertial modes which will be demonstrated in the following section 5 of the report.

\section{The Perturbation Analysis}

We consider the linear problem in rotating fluid spheres and omit all nonlinear terms. Since we are interested in the small Prandtl number limit we neglect the time derivative in the 
heat equation (2.2c). After substituting the time derivative of the velocity field by its eigenvalue $\frac{\partial}{\partial t} \longrightarrow i \omega$ the basic equations (2.2) reduce to,

$$
\begin{gathered}
i \omega U+k \times U=-\nabla \pi+\frac{R}{\tau} r \Theta+\frac{1}{\tau} \nabla^{2} U \\
\nabla \cdot U=0 \\
\nabla^{2} \Theta=-r \cdot U
\end{gathered}
$$

In order to benefit from the results presented in Zhang(1994), for technical convenience, and because the approximation is valid for fluid spheres rather than shells, we need to rescale our basic equations. The connection with the dimensionless parameters (2.3) defined in the preceding section and used in the numerical analysis in the rest of the report is,

$$
R=R_{e q .(2.3)}(1-\eta)^{6}, \tau=\tau_{e q .(2.3)}(1-\eta)^{2}, \omega=-\omega_{e q .(2.3)} / \tau
$$

Stress-free velocity boundary conditions and conducting or insulating temperature boundary conditions, as given by equation (2.4) are assumed. We expand the dependent variables,

$$
\boldsymbol{U}=\boldsymbol{u}+\boldsymbol{u}_{1}, \pi=\pi_{\boldsymbol{o}}+\pi_{1}, \Theta=\Theta, \omega=\omega_{0}+\omega_{1},
$$

where $\boldsymbol{u}_{1}, \pi_{1}, \Theta$ and $\omega_{1}$ are small perturbations from the solutions of the Poincaré equation for the limit $1 / \tau \longrightarrow 0$, which are denoted by $\boldsymbol{u}, \pi$ and $\omega_{0}$. The above expansion is valid for

$$
\frac{1}{\tau} m^{\frac{5}{2}}<<1
$$

Substituting the expansions into (3.5)-(3.7), gives the zeroth order of the perturbation problem,

$$
\begin{gathered}
i \omega_{0} \boldsymbol{u}+\boldsymbol{k} \times \boldsymbol{u}=-\nabla \pi_{0} \\
\nabla \cdot \boldsymbol{u}=0 .
\end{gathered}
$$

This system is equivalent the the Poincare equation as shown in Greenspan(1968). The complete analytical solution of the Poincaré equation has been recently obtained by Zhang et al.(2001), but here we make use only of a particular class of solutions as will be described shortly. The next order of the perturbation analysis in the limit $\eta=0$ gives rise to,

$$
\begin{gathered}
i \omega_{0} \boldsymbol{u}_{1}+\boldsymbol{k} \times \boldsymbol{u}_{1}=-\nabla \pi_{1}+\frac{R}{\tau} \boldsymbol{r} \Theta+\frac{1}{\tau} \nabla^{2}\left(\boldsymbol{u}+\boldsymbol{u}_{b}\right)-i \omega_{1} \boldsymbol{u} \\
\nabla \cdot \boldsymbol{u}_{1}=0 \\
\nabla^{2} \Theta=-\boldsymbol{r} \cdot \boldsymbol{u},
\end{gathered}
$$


where $\boldsymbol{u}=\boldsymbol{u}_{i}+\boldsymbol{u}_{b}, \boldsymbol{u}_{i}$ is the perturbation of the interior flow and $\boldsymbol{u}_{b}$ is the boundary flow associated with the Ekman boundary layer, which is non-zero only in the vicinity of the outer spherical surface. While $\boldsymbol{u}_{i}$ is small compared to $\boldsymbol{u}, \boldsymbol{u}_{b}$ has to be large enough so that $\boldsymbol{u}_{b}+\boldsymbol{u}$ satisfies the stress-free boundary condition. It can be shown that the Ekman layer plays an essential role because the integral $H_{b}=\int_{v} \boldsymbol{u}^{*} \cdot \nabla^{2}\left(\boldsymbol{u}+\boldsymbol{u}_{\boldsymbol{b}}\right) d V$ appearing later on in the expression for the Rayleigh number is non-zero only if $\boldsymbol{u}_{b}$ is non-zero (Zhang(1994)). If we multiply eq. (3.12) by the complex conjugate of $\boldsymbol{u}, \boldsymbol{u}^{*}$, which also satisfies $\nabla \cdot \boldsymbol{u}^{*}=0$ and the boundary condition $u_{r}^{*}=0$ and integrate over the volume of the sphere, we obtain

$$
\int_{v} \boldsymbol{u}^{*} \cdot\left(i \omega_{0} \boldsymbol{u}_{1}+2 \boldsymbol{k} \times \boldsymbol{u}_{1}+\nabla \pi_{1}\right) d V=\int_{v} \boldsymbol{u}_{1} \cdot\left(i \omega_{0} \boldsymbol{u}^{*}-2 \boldsymbol{k} \times \boldsymbol{u}^{*}\right) d V
$$

We use,

$$
\int_{v} \boldsymbol{u}^{*} \cdot \nabla \pi_{1} d V=\int_{s} \pi_{1} u_{r}^{*} d S=0
$$

and the fact that $\boldsymbol{u}^{*}$ satisfies,

$$
i \omega_{0} \boldsymbol{u}^{*}+\boldsymbol{k} \times \boldsymbol{u}^{*}=\nabla \pi_{0}^{*}
$$

and observe that the integral given by (3.15) vanishes. Then the solvability conditions, where the real part gives the critical Rayleigh number and the imaginary part gives the correction of the frequency are,

$$
\begin{gathered}
\operatorname{Re}\left[R \int_{v} \boldsymbol{u}^{*} \cdot \boldsymbol{r} \Theta d V\right]=-\operatorname{Re}\left[\frac{1}{\tau} \int_{v} \boldsymbol{u}^{*} \cdot \nabla^{2}\left(\boldsymbol{u}+\boldsymbol{u}_{b}\right) d V\right] \\
\operatorname{Im}\left[R \int_{v} \boldsymbol{u}^{*} \cdot \boldsymbol{r} \Theta d V\right]=-\operatorname{Im}\left[\frac{1}{\tau} \int_{v} \boldsymbol{u}^{*} \cdot \nabla^{2}\left(\boldsymbol{u}+\boldsymbol{u}_{b}\right) d V\right]+\omega_{1} \int_{v} \boldsymbol{u}^{*} \cdot \boldsymbol{u} d V .
\end{gathered}
$$

Hence the critical Rayleigh number of a particular mode is given in the first order by,

$$
R=-\frac{\int_{v} \boldsymbol{u}^{*} \cdot \nabla^{2}\left(\boldsymbol{u}+\boldsymbol{u}_{b}\right) d V}{\int_{v} \boldsymbol{u}^{*} \cdot \boldsymbol{r} \Theta d V}=-\frac{H_{b}}{H_{\theta}}
$$

Now in order to evaluate (3.19) we need to obtain a solution $\Theta(r)$ of the heat equation (3.14).

\section{Solutions of the Zeroth Order Problem and the Heat Equation}

A general solution of the Poincaré equation is available (Zhang et al. (2001)), but for the purposes of the present report only a particular class of solutions, as suggested by the numerical analysis of the problem is relevant. In this subsection we demonstrate a convenient way to construct such solutions and using them solve the heat equation for both types of thermal boundary conditions. 
We first consider the zeroth order problem in the perturbation analysis (3.11). In order to eliminate the continuity equation we introduce the general representation for the solenoidal vector field $\boldsymbol{u}$

$$
\boldsymbol{u}=\nabla \times(\nabla \times \boldsymbol{r} v)+\nabla \times \boldsymbol{r} w
$$

where the scalar variables $v$ and $w$ are uniquely defined if the condition is imposed that their averages over surfaces $r=$ const. vanish. By acting with the operators $r \cdot \nabla \times \nabla \times$ and $r \cdot \nabla \times$ on the first equation of the system we obtain two equations for the poloidal and toroidal scalars $v$ and $w$

$$
\begin{gathered}
\left(-i \omega L_{2}+i m\right) \nabla^{2} v=-Q w \\
\left(-i \omega L_{2}+i m\right) w=Q v
\end{gathered}
$$

where $L_{2}$ is the negative Laplacian on the unit sphere and $Q$ is defined by

$$
Q \equiv k \cdot \nabla-\frac{1}{2}\left(L_{2} k \cdot \nabla+k \cdot \nabla L_{2}\right)
$$

The boundary conditions are also transformed to,

$$
v=\frac{\partial^{2}}{\partial r^{2}} v=\frac{\partial}{\partial r}\left(\frac{w}{r}\right)=0, \text { at } r=1
$$

The expressions,

$$
\begin{gathered}
v=\left(r^{m}-r^{m+2}\right) P_{m}^{m} \exp (i \omega t+i m \phi) \\
w=r^{m+1} 2 i \frac{(m+1)^{2}-1}{\omega(m+1)(m+2)-m} \frac{1}{(2 m+1)} P_{m+1}^{m} \exp (i \omega t+i m \phi),
\end{gathered}
$$

provide an exact solution of the zeroth order problem for

$$
\omega_{ \pm}=\frac{1}{m+2}\left(1 \pm \sqrt{\frac{m^{2}+4 m+3}{2 m+3}}\right)
$$

as can be easily verified by substituting (3.24) into (3.21) and using formula (7.41) of Appendix A.

Now the heat equation,

$$
\nabla^{2} \Theta=-\boldsymbol{r} \cdot \boldsymbol{u}=-L_{2} v=-\left(r^{m}-r^{m+2}\right) m(m+1) P_{m}^{m} \exp (i \omega t+i m \phi)
$$

suggests that $\Theta(r)$ contains a single spherical harmonic of the same order and degree as the poloidal scalar, and its radial dependence can be obtained as a solution of the radial part of $(3.26)$,

$$
-m(m+1)\left(r^{m}-r^{m+2}\right)=\nabla^{2} f(r)=\left(\frac{1}{r} \partial_{r}^{2} r-\frac{m(m+1)}{r^{2}}\right) f(r)
$$


Assuming a radial dependence of the type,

$$
f(r)=K_{1} r^{c_{1}}+K_{2} r^{c_{2}}
$$

plugging it into the radial equation and determining the constants $K_{1}, K_{2}, c_{1}, c_{2}$, we obtain the solution,

$$
\begin{aligned}
\Theta(r)= & -m(m+1) P_{m}^{m} \exp (i \omega t+i m \phi) * \\
& *\left[\frac{r^{m+2}}{(m+3)(m+2)-(m+1) m}-\frac{r^{m+4}}{(m+5)(m+4)-(m+1) m}+a r^{m}\right],
\end{aligned}
$$

where a harmonic term $a r^{m}$ has been added to the radial dependence. After using the insulating and the conducting thermal boundary conditions (2.4) of Types A and B at $r=1$ and determine the constant $a$ in both cases we finally obtain,

$$
\begin{aligned}
\Theta(r)= & -(m+1) P_{m}^{m} \exp (i \omega t+i m \phi) * \\
& *\left[\frac{m r^{m+2}-(m+2) r^{m}}{(m+3)(m+2)-(m+1) m}-\frac{m r^{m+4}-(m+4) r^{m}}{(m+5)(m+4)-(m+1) m}\right]
\end{aligned}
$$

for the insulating case and

$$
\begin{aligned}
\Theta(r)= & -m(m+1) P_{m}^{m} \exp (i \omega t+i m \phi) * \\
& *\left[\frac{r^{m+2}-r^{m}}{(m+3)(m+2)-(m+1) m}-\frac{r^{m+4}-r^{m}}{(m+5)(m+4)-(m+1) m}\right]
\end{aligned}
$$

for the conducting one.

\section{Evaluation of the Integrals}

After having obtained the solutions of the zeroth order problem and the heat equation the integrals $H_{b}$ and $H_{\theta}$, which appear in the solvability condition (3.18), need to be evaluated.

Making use of the formula for the components of a solenoidal vector in terms of poloidal and toroidal scalars,

$$
u=\left(\frac{L_{2}}{r} v, \frac{1}{r} \frac{\partial^{2}}{\partial \theta \partial r} r v+\frac{1}{\sin \theta} \frac{\partial}{\partial \phi} w, \frac{1}{r \sin \theta} \frac{\partial^{2}}{\partial \phi \partial r} r v-\frac{\partial}{\partial \theta} w\right)
$$

we proceed to the evaluation of the first integral.

$$
\begin{aligned}
H_{b}= & -\int_{v} \boldsymbol{u}^{*} \cdot \nabla^{2}\left(\boldsymbol{u}+\boldsymbol{u}_{b}\right) d V=-\int_{\boldsymbol{v}} \boldsymbol{u}^{*} \cdot \nabla^{2} \boldsymbol{u}_{b} d V=\int_{\boldsymbol{\sigma}} \boldsymbol{u}^{*} \cdot\left(\boldsymbol{r} \cdot \nabla \frac{\boldsymbol{u}_{b}}{r}\right) d S \\
= & \int_{\sigma}\left(u_{\theta}^{*} \frac{\partial}{\partial r} \frac{u_{\theta}}{r}+u_{\phi}^{*} \frac{\partial}{\partial r} \frac{u_{\phi}}{r}\right) d S \\
= & 2 \pi \int_{0}^{\pi}\left\{\left(\frac{1}{r} \frac{\partial^{2}}{\partial \theta \partial r} r v^{*}+\frac{1}{\sin \theta} \frac{\partial}{\partial \phi} w^{*}\right)\left(\frac{\partial^{2}}{\partial \theta \partial r} \frac{1}{r^{2}} \frac{\partial}{\partial r} r v+\frac{1}{\sin \theta} \frac{\partial^{2}}{\partial \phi \partial r} \frac{w}{r}\right)\right\} \sin \theta d \theta \\
& +2 \pi \int_{0}^{\pi}\left\{\left(\frac{1}{\sin \theta} \frac{\partial^{2}}{\partial \phi \partial r} \frac{1}{r^{2}} \frac{\partial}{\partial r} r v-\frac{\partial^{2}}{\partial \theta \partial r} \frac{1}{r^{2}} w\right)\left(\frac{1}{r \sin \theta} \frac{\partial^{2}}{\partial \phi \partial r} r v^{*}-\frac{\partial}{\partial \theta} w^{*}\right)\right\} \sin \theta d \theta \\
= & 2 \pi m(m+1)\left[2(4 m+2) \int_{0}^{1}\left|P_{m}^{m}\right|^{2} d(\cos \theta)+\right. \\
& \left.(m+2)\left|\frac{(m+1)^{2}-1}{\omega(m+1)(m+2)-m} \frac{2}{(2 m+1)}\right|^{2} \int_{0}^{1}\left|P_{m+1}^{m}\right|^{2} d(\cos \theta)\right] .
\end{aligned}
$$


The second integral may also be readily calculated. For the insulating thermal boundary case we have,

$$
\begin{aligned}
H_{\theta}= & \int_{v} \boldsymbol{u}^{*} \cdot \boldsymbol{r} \Theta d V=\int_{v} L_{2} v^{*} \Theta d V \\
= & 2 \pi m(m+1)^{2} \int_{0}^{1}\left|P_{m}^{m}\right|^{2} d(\cos \theta) * \\
& * \int_{0}^{1}\left(r^{m}-r^{m+2}\right)\left[\frac{m r^{m+4}-(m+4) r^{m}}{(m+5)(m+4)-(m+1) m}-\frac{m r^{m+2}-(m+2) r^{m}}{(m+3)(m+2)-(m+1) m}\right] r^{2} d r \\
= & \frac{8 \pi m(m+1)^{2}\left(14 m^{2}+59 m+63\right)}{(2 m+9)(2 m+7)(2 m+5)^{2}(2 m+3)^{2}} \int_{0}^{1}\left|P_{m}^{m}\right|^{2} d(\cos \theta),
\end{aligned}
$$

and in an analogous manner for the perfectly conducting case,

$$
\begin{aligned}
H_{\theta}= & 2 \pi m(m+1)^{2} \int_{0}^{1}\left|P_{m}^{m}\right|^{2} d(\cos \theta) * \\
& * \int_{0}^{1}\left(r^{m}-r^{m+2}\right)\left[\frac{r^{m+2}-r^{m}}{(m+3)(m+2)-(m+1) m}-\frac{r^{m+4}-r^{m}}{(m+5)(m+4)-(m+1) m}\right] r^{2} d r \\
= & \frac{2 \pi m^{2}(m+1)^{2}(40 m+108)}{(2 m+9)(2 m+7)(2 m+5)^{2}(2 m+3)^{2}} \int_{0}^{1}\left|P_{m}^{m}\right|^{2} d(\cos \theta) .
\end{aligned}
$$

\section{The Complete Analytical Solution}

After evaluating the integrals $H_{b}$ and $H_{\theta}$ we can readily write the expression for the critical Rayleigh number for a given $m$,

$$
\begin{aligned}
& R=m^{2}(m+2)^{3}\left\{\left[ \pm(m+1) \sqrt{\frac{m^{2}+4 m+3}{2 m+3}}+1\right]^{-2}(2 m+3)^{-1}+(2 m+1)\right\} *
\end{aligned}
$$

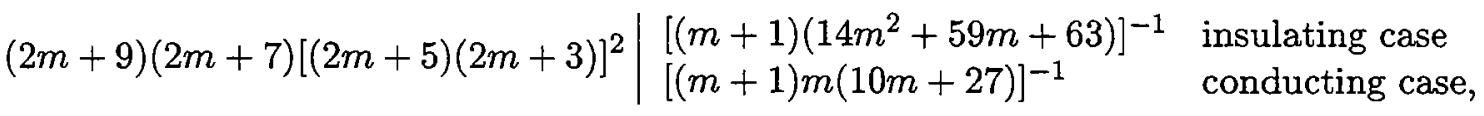

where the following property of the associated Legendre polynomials is used,

$$
\frac{\int_{0}^{1}\left|P_{m+1}^{m}\right|^{2} d(\cos \theta)}{\int_{0}^{1}\left|P_{m}^{m}\right|^{2} d(\cos \theta)}=\frac{(2 m+1)^{2}}{2 m+3}
$$

As a result of the perturbation analysis we obtained a complete solution for the convection in the limit of small Prandtl numbers and high rotation rates, which in the first order is represented by

$$
\left(R, m, \boldsymbol{u}, \omega_{0}, \Theta\right)
$$

where all state variables depend only on $m$. In order to obtain the critical Rayleigh number at onset, one has to loop through all values of $m$ and select the smallest possible $R$. Before proceeding to the numerical analysis several properties of the obtained solution must be 
outlined. Firstly, we notice that the frequency omega of the zeroth order solution has two values - a positive value corresponding to a retrograde drifting convection pattern and a negative one corresponding to a prograde drifting mode. These two modes are competing at onset and will be demonstrated in the numerical analysis in the following sections. Secondly, we observe that for a given $m$ the critical Rayleigh number (3.33) does not depend on the radial component as well as on the rotation rate in this approximation. These are particularly interesting features that we will address in the numerical simulations reported below.

\section{Numerical Test of the Analytical Solution}

Using the numerical method described in the following section we check the obtained analytical result (3.33) against numerical values. Since the approximation is derived for $\tau \longrightarrow \infty$, $P \longrightarrow 0$ and $\eta=0$ we use values of these parameters as close to the analytical assumptions as numerically reasonable. The comparison is represented in Fig. 1 with the numerical results for several different values of $m=2,4,8,12$ for parameters $P=10^{-6}, \eta=0.1$ and varying $\tau$. Naturally a strict independence of $R$ on $\tau$ as predicted by (3.33) can not be expected but an approximate independence is fulfilled over a large span of values of $\tau$. At very low and high values of $\tau$ the convection enters other regimes of flow as will be discussed in the following sections.

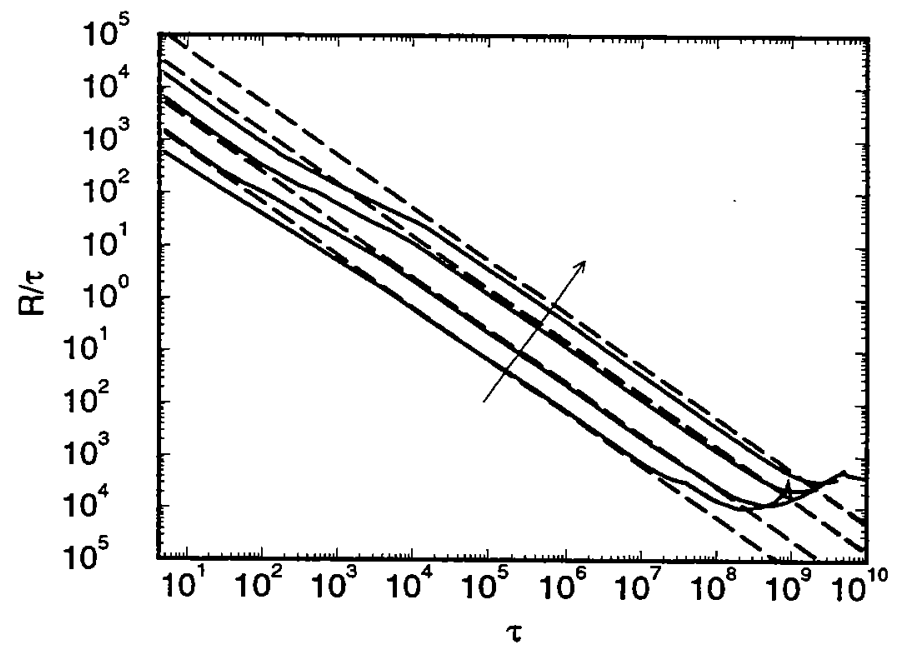

Figure 1: Comparison of numerical results (solid lines) with the analytical expression (dashed lines) for $P=10^{-6}, \eta=0.1$ and $m=2,4,8,12$ in direction of the arrow.

\section{Numerical Methods}

Following the earlier analyses by Zhang and Busse(1987) and Ardes et al.(1997), we use a Galerkin method for the numerical solution of equations (2.2).

In a manner similar to the described in the previous section we transform the full 
nonlinear vector equation (2.2a) to a couple of scalar equations for $v$ and $w$,

$$
\begin{gathered}
{\left[\left(\nabla^{2}-\frac{\partial}{\partial t}\right) L_{2}+\tau \boldsymbol{k} \times \boldsymbol{r} \cdot \nabla\right] \nabla^{2} v+\tau Q w-R L_{2} \Theta=-\boldsymbol{r} \cdot \nabla \times(\nabla \times(\boldsymbol{u} \cdot \nabla \boldsymbol{u}))} \\
{\left[\left(\nabla^{2}-\frac{\partial}{\partial t}\right) L_{2}+\tau \boldsymbol{k} \times \boldsymbol{r} \cdot \nabla\right] w-\tau Q v=r \cdot \nabla \times(\boldsymbol{u} \cdot \nabla \boldsymbol{u}) .}
\end{gathered}
$$

The boundary conditions are given by (2.4) and modified as in (3.24) at $r=r_{i} \equiv \frac{\eta}{1-\eta}$ and $r=r_{0} \equiv \frac{1}{1-\eta}$.

In order to solve equations (4.35) and (2.2c) by the Galerkin method, it is convenient to expand the dependent variables into complete systems of functions satisfying the boundary conditions,

$$
\begin{gathered}
v=\sum_{\nu, l, n} a_{\nu n l} \exp \{i \nu(m \varphi-\omega t)\} P_{l}^{|\nu| m}(\cos \theta) \sin n \pi\left(r-r_{i}\right) \\
w=\sum_{\nu, \hat{l}, n} c_{\nu n l} \exp \{i \nu(m \varphi-\omega t)\} P_{\hat{l}}^{|\nu| m}(\cos \theta) \cos (n-1) \pi\left(r-r_{i}\right)
\end{gathered}
$$

Type A:

$$
\Theta=\sum_{\nu, l, n} b_{\nu n l} \exp \{i \nu(m \varphi-\omega t)\} P_{l}^{|\nu| m}(\cos \theta) \sin n \pi\left(r-r_{i}\right)
$$

Type B:

$$
\Theta=\sum_{\nu, \hat{l}, n} b_{\nu n l} \exp \{i \nu(m \varphi-\omega t)\} P_{\hat{l}}^{|\nu| m}(\cos \theta) \cos (n-1) \pi\left(r-r_{i}\right)
$$

Note the difference in the sign of $\omega$ in (4.36) and (3.25). This representation has been chosen in such a way that solutions in the form of drifting waves which are $m$-periodic in the azimuthal direction are described by constant coefficients $a_{\nu l n}$ etc. More complex solutions can be described by time dependent coefficients $a_{\nu l n}$ etc. In both cases the conditions

$$
a_{\nu l n}=a_{-\nu l n}^{+}, b_{\nu l n}=b_{-\nu l n}^{+}, c_{\nu \hat{l n}}=c_{-\nu \hat{l n}}^{+}
$$

must be satisfied for real expressions (4.36) where the superscript + indicates the complex conjugate. Of particular interest are solutions that are symmetric with respect to the equatorial plane in which case the subscript $l$ runs through $|\nu| m+2 j$ for $j=0,1,2 \ldots$ while the subscript $\hat{l}$ runs through $|\nu| m+2 j+1$ for the same $j$. The associated Legendre polynomials will be assumed in such a form that the average of $\left[P_{l}^{\mu}\right]^{2}$ over the unit sphere is unity.

After the equations for the coefficients $a_{\nu l n}$ etc. have been obtained through a projection of the basic equations (4.35) and (2.2c) onto the space of the expansion functions used in (4.36) the system of equations for the coefficients must be truncated. We shall use the 
truncation condition that all coefficients and corresponding equations are neglected whose subscripts satisfy

$$
2 n+l-|\nu| m+2|\nu|>3+2 N_{T}
$$

The same condition applies for $\hat{l}$ instead of $l$. The condition is the same as used by Zhang and Busse (1987) and provides a triangular truncation such that as many functions in the radial as in the latitudinal direction are used for the representation of the solution.

For the linear problem of the onset of convection the right hand side of eqs. (4.35) and the term $u \cdot \nabla \Theta$ in eq. (2.2c) can be neglected and only terms with $\nu=1$ need to be kept in the representation (4.36). The linear homogeneous system of complex algebraic equations for the coefficients $a_{1 l n}$ represents an eigenvalue problem for $R$ and $\omega$ as the real and imaginary parts of the eigenvalue.

For the most cases presented in this report a truncation parameter of $N_{T}=18$, or a total of 513 coefficients have been used. In a few cases in the high rotation regime the resolution was increased to $N_{T}=30$, which already poses significant requirements on the computer capacity and computational times.

\section{Onset of Thermal Convection at Low Prandtl Numbers and High Rotation Rates}

In this section we present the basic results gained from the numerical investigation of the onset of convection at low Prandtl numbers in the case of conducting thermal boundary conditions of the type $\mathrm{A}$ and address the questions posed in the introductory section of the report.

The parameter exploration included a large number of points in the ranges of $10^{-5} \leq$ $P \leq 10,0<\tau$ and $0.1 \leq \eta \leq 0.8$. The computations with $P \geq 1$ do not actually belong in the low Prandtl number region and are only included for the sake of finding the border between the retrograde and prograde drifting modes, since these modes are found to exist even at Prandtl numbers of order unity and higher. Of course, the scenario of the evolution of the onset of convection with increasing rotation parameter $\tau$, as will be described below, is valid for a smaller region, which we believe to be approximately $10^{-5} \leq P \leq 10^{-2}$.

Ideally the proper way to investigate a given parameter region is to keep the values of all parameters of the problem fixed and vary in a continuous way only one of them. When the dependence on this parameter is well understood the process is repeated for all remaining parameters. In practice, of course, this is impossible. In an experimental situation for example, the variation of the Prandtl number is limited to the set of available working fluids. In a numerical study one has much weaker restrictions, but even then parameters such as the Prandtl number $P$ and the radius ratio $\eta$ are comparatively difficult to vary because of their relatively small domain and big changes of the properties of the flow with small changes of the values of these parameters. On the other hand the rotation rate can be varied comparatively easily in a wide range and as a result much smoother dependences are observed. Furthermore, any non-monotonic behavior guarantees a significant and welldefined transition between different states. 


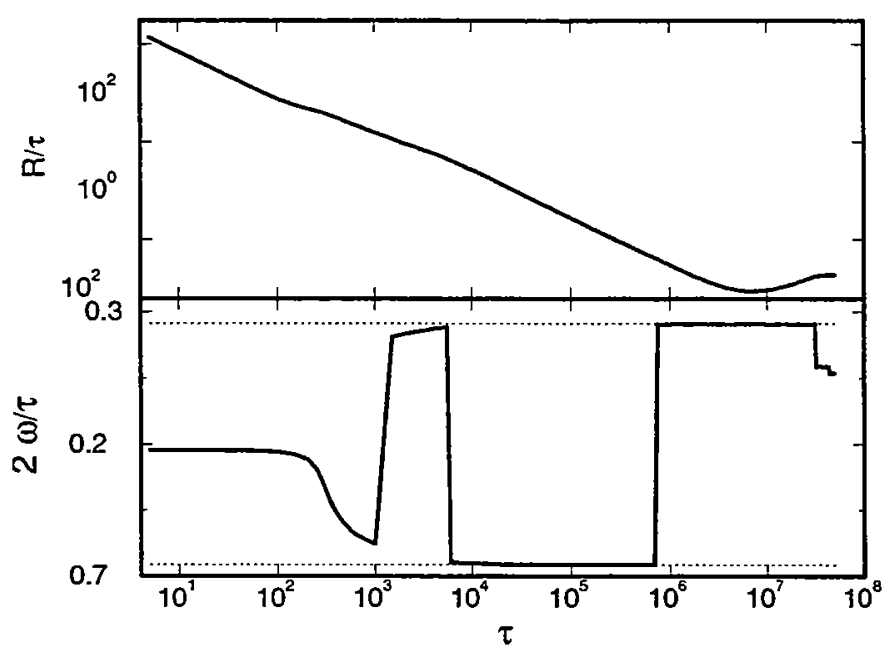

Figure 2: The Rayleigh number $R$ (upper plot) and the frequency $\omega$ (lower plot) as a function of $\tau$ in the case $P=10^{-4}, \eta=0.3, m=8$. The analytical predictions for $\omega$ based on inertial mode approximations are shown with dotted lines.

Following this approach, numerous cases in the low Prandtl number region were investigated. Fig. 2 represents a typical example of the results obtained in this region and provides an excellent overview and introduction to most of the various regimes of convection flow that can be expected here. Although all other parameters, $P=0.0001, \eta=0.3, m=8$, are fixed, this case is a typical example and is situated in the middle of the parameter region of interest. No substantial qualitative differences have been observed at other parameter values. The $\frac{\omega}{\tau}(\tau)$ curve is particularly instructive and several different states of the preferred mode can be immediately noticed.

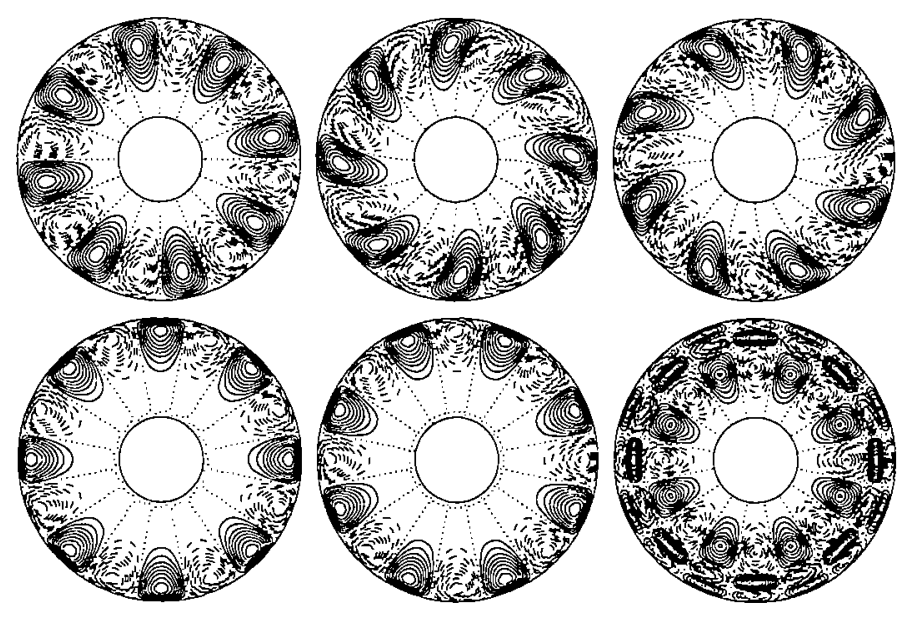

Figure 3: The streamfunctions $r \sin \theta \partial_{\phi} v$ in the equatorial plane illustrating the sequence of transitions for the same parameters as Fig. 2 and values of $\tau=5,950,1500,6 \cdot 10^{5}, 8 \cdot 10^{5}, 3.5 \cdot 10^{7}$.

At very low rotation parameters of order unity the convection cells form near the outer 

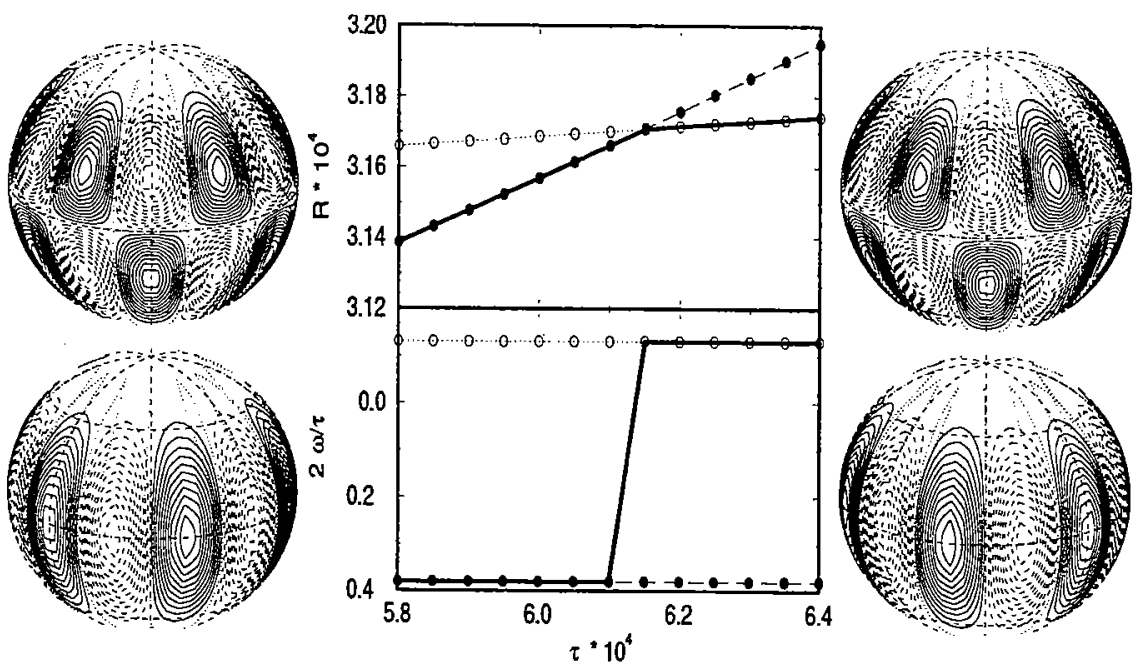

Figure 4: In the middle part: the Rayleigh numbers $R$ of the competing prograde(dotted line, empty circles) and retrograde mode(dashed line, filled circles) as well as its actual critical value(thick solid line) and the corresponding frequencies as a function of $\tau$ in the case $P=0.001, \eta=0.2, m=6$. On the left and right: Contours of constant radial velocity $u_{r}$ (down) and toroidal scalar $w$ on the spherical surface $r=0.9$ at both sides of the transition for $\tau=58000$ and 64000 .

surface in the equatorial region of the spherical shell as can be seen in Fig. 3, which illustrates the various states of the flow in an equatorial projection. At this values of the rotation parameter $\tau$ the critical Rayleigh number is still rather small. The preferred mode has a negative frequency which indicates a retrograde drift with time. This drift cannot, of course, be seen in the snapshots of Fig. 3. The solution has a relatively small toroidal component, which vanishes in the limit $\tau \longrightarrow 0$.

A new mode which does not exist at $\tau=0$ enters the picture and approaches the initial mode indicated above. As a result of the switch-over phenomenon described by Zhang and Busse(1987), the $R(\tau)$ and $\omega(\tau)$ curves of the two competing modes exhibit smooth bends, but for some more time the initial mode is still preferred. During this gradual transition the pattern of convection also changes gradually. The convection rolls are no longer straight and strictly radially oriented as they were near $\tau=0$, but change shape and become inclined as illustrated by the second plot of Fig. 3. The frequency still keeps its negative sign and the pattern exhibits a retrograde drift.

Past a particular value of the rotation parameter $\tau$, the value of the critical Rayleigh number $R$ of the competing mode becomes so much lower than the value of $R$ of the initially preferred mode that an abrupt jump occurs. This is especially obvious from the discontinuity of the $\frac{\omega}{\tau}(\tau)$ curve in Fig. 2. During this transition the frequency even changes sign. The change of sign indicates a change in the direction of the drift of the pattern. In the new state the preferred mode drifts in the prograde direction. Because the effects of the switch-over competition still act on the $\omega(\tau)$ curve of the new mode, it is also bend, which as before results in a pattern of spiraling rolls, as can be noticed in the third plot of Fig. 3 .

In a completely analogous way a second transition happens and the preferred mode 
changes back to retrograde drift. This next transition occurs at about $\tau=8000$ for the parameters of the case represented on Fig. 2 and brings the flow in the region described well by the inertial wave approximation.

We would like to draw the attention to the last two transitions seen in Fig. 2.

After the second transition and between values of about $8000<\tau<3.5 \cdot 10^{5}$, in the particular case of Fig. 2, the convection is strictly in the, so called, wall-attached regime which is well-described by the inertial wave as well as the equatorial approximation of Ardes et al.(1997). The theoretical values given by (3.25) have been plotted with dotted

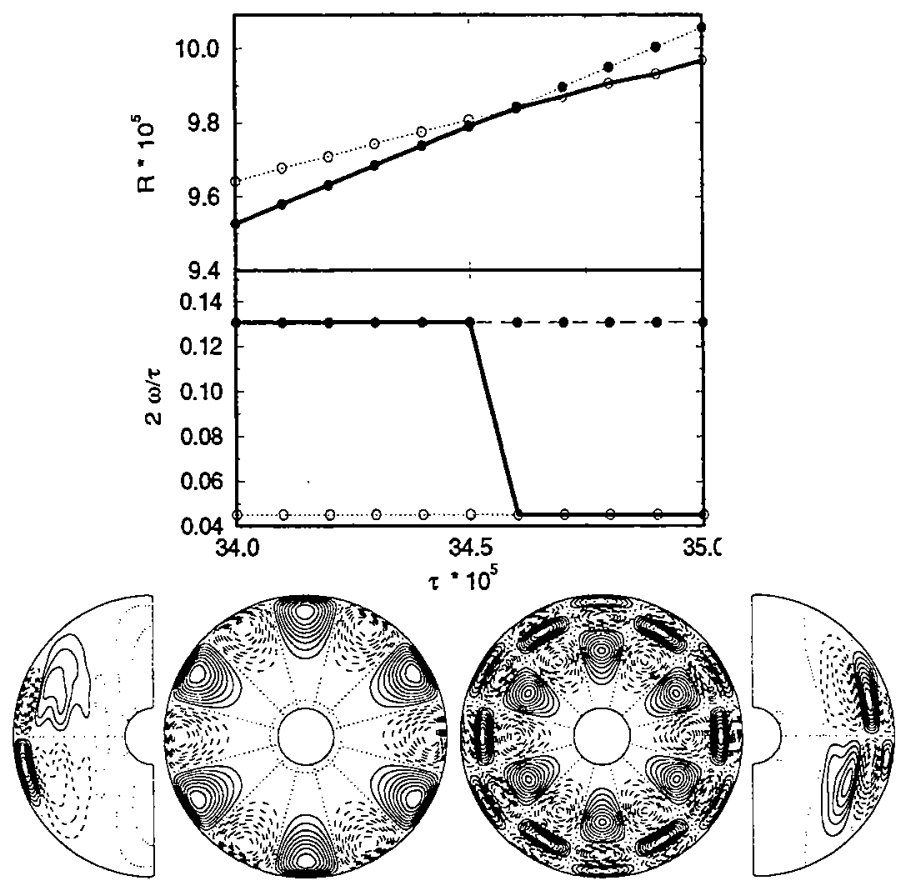

Figure 5: Same as Fig. 4. In the lower part the streamfunctions $r \sin \theta \partial_{\phi} v$ in the equatorial plane, as well as $\frac{\tau}{\sin \theta} \partial_{\theta} v$ in a meridional plane correspond to $\tau=3.45 \cdot 10^{6}$ and $\tau=3.48 \cdot 10^{6}$.

lines in Fig. 2. As can be seen they agree perfectly with the numerical values. It is worth noting that these values do not depend on $\tau$. What is more they do not depend on the aspect ratio as well and this will be a subject of further discussion in the present report. Since the expression (3.25) gives the frequencies of the inertial oscillations, found as solutions of the Poincaré equation in rotating spheres, this perfect agreement with the numerically computed frequency of the preferred mode of the convective flow is the basis for the argument that convection is small perturbation of inertial oscillations in this particular parameter regime. In the middle of the region the convection undergoes a new transition from a retrograde to prograde drifting modes, which is illustrated for somewhat different parameters in Fig. 4. These modes represent the generic case of wall-attached type of convection and the most notable difference between them is the direction of their drift. The two solutions differ mainly by a phase shift in the poloidal part of the velocity field and the corresponding amplitudes are quite close to each other. As can be seen in Fig. 4 at lower 


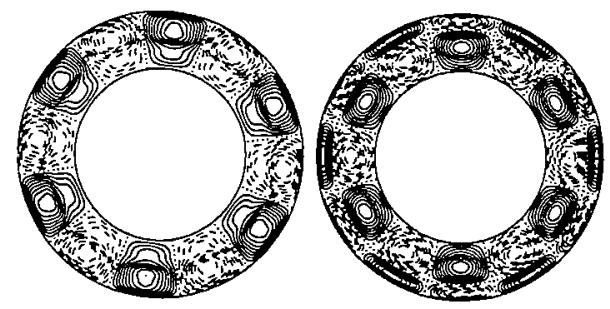

Figure 6: The streamfunctions $r \sin \theta \partial_{\theta} v$ in the equatorial plane illustrating the transition to multicolumnar state in the case $P=10^{-4}, \eta=0.6, m=6$ and values of $\tau=10^{7}$ for $2 \cdot 10^{7}$.

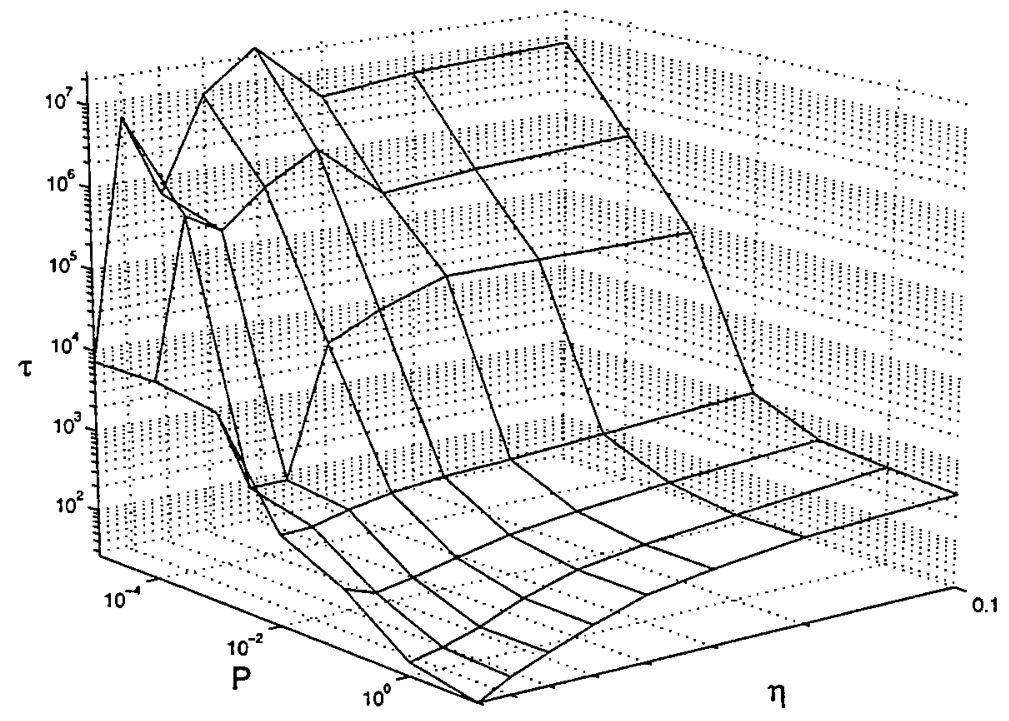

Figure 7: The boundary separating the retrograde (below the surface) and prograde mode(above the surface) for a fixed value of the wavenumber $m=6$.

value of the rotation parameter $\tau$ the retrograde mode has a lower critical Rayleigh number and therefore is preferred to the prograde mode. As $\tau$ increases the Rayleigh number $R$ of the retrograde mode grows faster than that of the competing prograde mode and at some critical value of $\tau$ eventually becomes larger. At this point the transition from retrograde to prograde mode occurs. The frequency exhibits a discontinuity, changes sign and the whole pattern starts drifting in the opposite direction. Apart from this obvious process no other physical reason for the transition is identified. Mathematically the two frequencies emerge as the two roots of a quadratic dispersion relation and the inertial wave approximation does not provide an explanation for the transition, since according to (3.33), the retrograde mode has a smaller Rayleigh number and thus is always preferred in contrast to the numerical results. Thus one may suggest that an explanation for the transition must be sought in higher orders of the perturbation analysis.

The last transition observed in Figs. 2 and 3 and shown in more detail in Fig. 5 is arguably the most interesting one of all. It was completely unexpected and represents a 
novel pattern observed for the first time at onset of convection. Examining the plots of the Rayleigh numbers and the frequencies of the competing modes, the same mechanism of transition as the one just described in connection with the retrograde - prograde switch is identified. But in all other aspects this transition is rather different from the previous one. Although the frequency again exhibits a finite discontinuity, it does not change sign this time and the pattern continues to drift in prograde direction. A major change in the structure of the convection solution can be observed. Besides the wall-attached mode which persists, several other concentric layers of convection columns appear. The whole pattern resembles a chess play-board in the sense that the streamlines of any given column are directed in the opposite direction with respect to the streamlines of all neighboring columns. This multicolumn solution seems to emerge as a result of the competition between the wall-attached type convection and the effects of the extremely high rotation which asymptotically favors columnar structures at a distance of about half the radius of the outer spherical shell (Busse(1970)). While in other parameter regions this competition leads to a multi-hump solutions and finally to spiraling columnar convection, here the tendency for wall-attachment

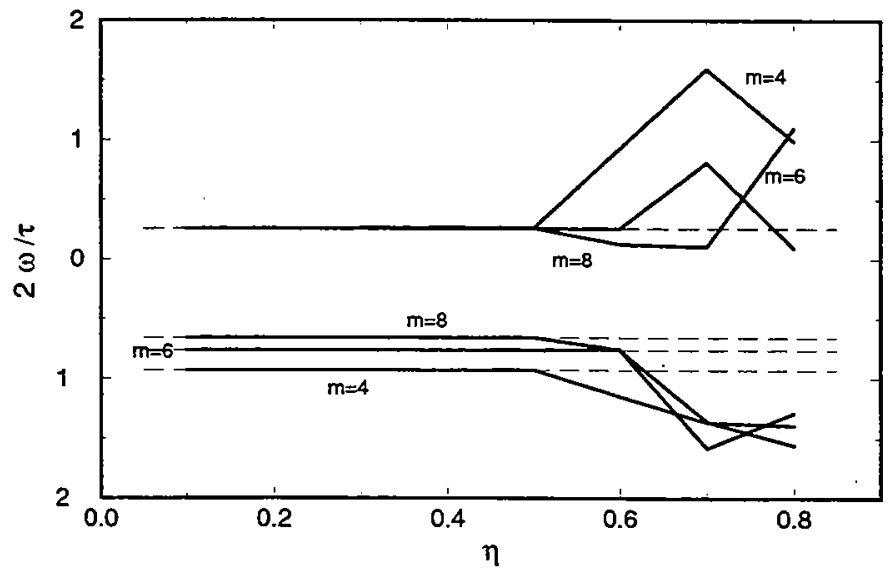

Figure 8: The ratio between the frequency $\omega$ and the rotation parameter $\tau$ of the retrograde(negative values) and prograde modes(positive values) as a function of $\eta$ in the case $P=10^{-5}$ for three different wavenumbers as indicated in the plot.

is compatible in strength to the effects of high rotation and leads to a breaking of the equatorially wall-attached cell into multiple columns that obey more closely the Taylor constraint of rotation. In contrast to the multi-hump and spiraling columnar solutions the multi-columns are strictly oriented in radial direction. As might be seen from the plots of $\frac{\omega}{\tau}$, this transition does not occur in a gradual manner but rather sharply at a well-determined value of $\tau$. The transition to multi-columnar convection is found to bound from above the regime of purely wall-attached convection at high rotation rates. Although the precise border between the two regimes is not investigated in the present report, the switch to multi-columnar convection has been found in all examined cases, regardless of the radius ratio $\eta$ and for all Prandtl numbers $P \leq 10^{-2}$. However at large $\eta$ the number of concentric layers diminishes because of the smaller size of the shell gap. As an illustration of this Fig. 6 is presented, for $\eta=0.6, m=6, P=0.0001$. Here an interesting way of conforming to the wall-attachment can be noticed in the first plot. 
Having examined in some detail the transitions between the various modes preferred at onset, we turn our attention to the other questions posed in the introductory section.

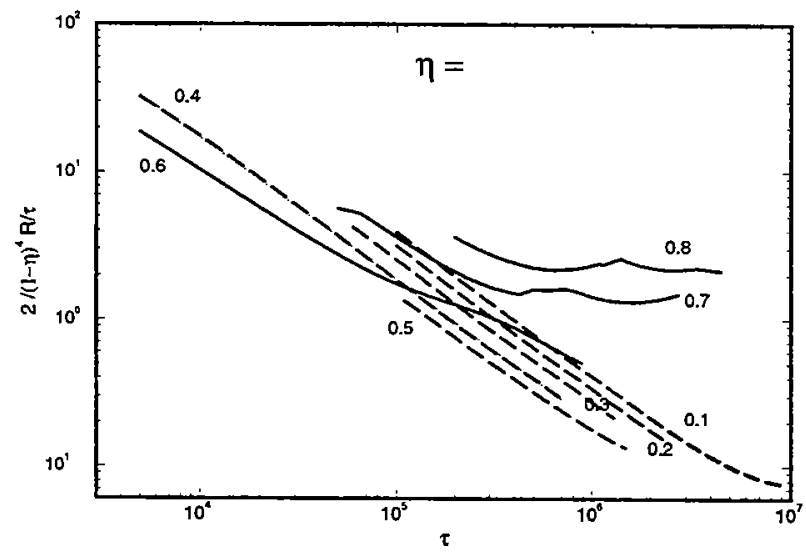

Figure 9: The Rayleigh number $R$ as a function of $\tau$ in the case $P=10^{-4}, m=8$ for different values of $\eta$ as indicated in the plot. A power law fit of the $\eta=0.4$ curve is plotted with a dashed line.

Fig. 7 represents an attempt of a precise determination of the border between the retrograde and prograde mode of the wall-attached convection in $P-\tau-\eta$ space. The construction of this border has been a major computational effort, since many of the points fall in the regions of very low Prandtl numbers and at the same time of very high rotation rates $\tau$, where increased numerical resolution and long computational times were required. As can be easily noticed, the surface is much smoother towards lower values of the radius ratio $\eta \leq 0.5$. This is not surprising since at higher values of $\eta$ the inner spherical boundary of the shell has a significant effect on the structures of the flow which start to show similarities to the case of plane layer convection and are much better described by the small-gap limit approximation. The availability of the border between the two modes opens a new possibility for tests of both, the equatorial and the inertial wave, approximations since they both pretend to be able to predict the preferred mode of convection through a selection of the lowest critical number of the various modes.

The next step towards numerical verification of the inertial mode approximation is shown on Fig. 8 , where the computed values of the $\frac{\omega}{\tau}$ ratio are plotted against the value predicted by the theory, for three different wavenumbers $m$. For values of the radius ratio $\eta \leq 0.5 \mathrm{a}$ perfect agreement can be observed. For values higher than that neither a good agreement nor a well-established dependency on the radius ratio $\eta$ is obvious. This is again no surprise since we do not expect the analytical approach to be valid for thin shells, since it is based on the assumption of a full fluid sphere.

Having studied the various preferred convection modes and verified some predictions of the theory, we would like to explore in more detail the dependence of the numerical values of the critical Rayleigh number on the radius ratio $\eta$ as well as on the rotation parameter $\tau$. In Fig. 9 the ratio $\frac{2}{(1-\eta)^{4}} \frac{R}{\tau}$ is plotted against $\tau$ for different values of the radius ratio $\eta$. For all values of $\eta \leq 0.5$ the Rayleigh number decreases with increasing $\eta$. As already discussed, this rule does not hold for $\eta \geq 0.5$. More interesting is the dependence on the 
rotation parameter $\tau$. For $\eta \leq 0.5$ the $R(\tau)$ obeys an power low as is obvious from the logarithmic plot of Fig. 9. A power law fit to the curve with $\eta=0.4$ is plotted with a dotted line and we can observe that it describes the $R(\tau)$ dependence very well. Fitting one can easily estimate the relationship,

$$
R \sim \tau^{\frac{1}{25}}=\tau^{0.04}
$$

This relationship suggests that for the investigated parameter region of very low Prandtl numbers and very high rotation rates and radius ratios less or equal to one half, the critical Rayleigh number $R$ is almost independent on the rotation parameter $\tau$. This confirms once again the predictions of the inertial mode approximation, since according to (3.33) the critical Rayleigh number does not depend on $\tau$. Of course, at very low $\tau$ the assumption $\frac{1}{\tau} \longrightarrow 0$ for the validity of the perturbation expansion is not satisfied, while at very high tau the convection leaves the wall-attached regime to enter the multi-columnar solution. This provides an explanation of why (5.39) holds only in the intermediate values of $\tau$ but deviates at very low and high rotation rates.

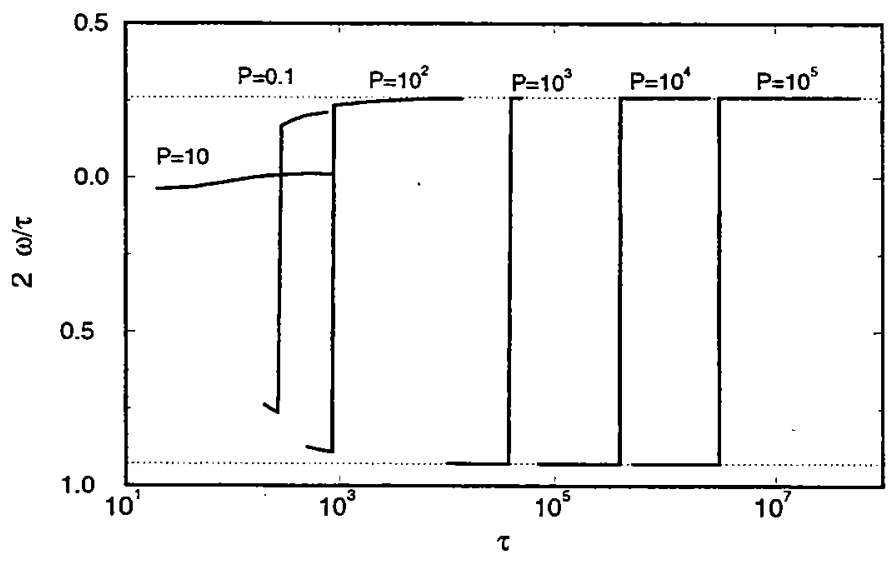

Figure 10: The frequency $\omega$ as a function of $\tau$ in the case $\eta=0.2, m=4$ for different values of $P$ as indicated in the plot. Theoretical values are plotted with dashed lines.

Finally, we would like to show how the wall-attached convection described by the equatorial and the inertial wave approximations is limited with respect to the variations of the Prandtl number. This question has, of course, been addressed in the earlier studies, but it is instructive to visit it again. Fig. 10 shows the $\frac{\omega}{\tau}$ ratio against $\tau$ for fixed values $m=4, \eta=0.2$ and for several Prandtl numbers. The theoretical prediction for $\frac{\omega}{\tau}$ has also been plotted with dotted lines. At $10^{-5} \leq P \leq 10^{-2}$ the theoretical prediction is exactly fulfilled, which indicates that these points are well in the regime of equatorially wall-attached convection. The higher values of $P \geq 10^{-1}$ are in the marginal region of the regime and the analytical prediction is only approached. Nevertheless, it is our belief that in the parameter region of the Prandtl numbers lower than 0.01 the qualitative scenario of the onset of convection outlined in this section of the report is followed. 


\section{The Onset of Convection with Insulating Thermal Bound- ary Conditions}

In this section we present some preliminary results emerging from the numerical investigation of the onset of convection in rotating spherical shells with insulating thermal boundary conditions of type B. Since we believe that convection in the case of insulating boundary conditions is not fundamentally different from convection with conducting boundary conditions, for which many results have been accumulated, the main purpose of this part of the report is to outline briefly the similarities and the differences between the two cases.

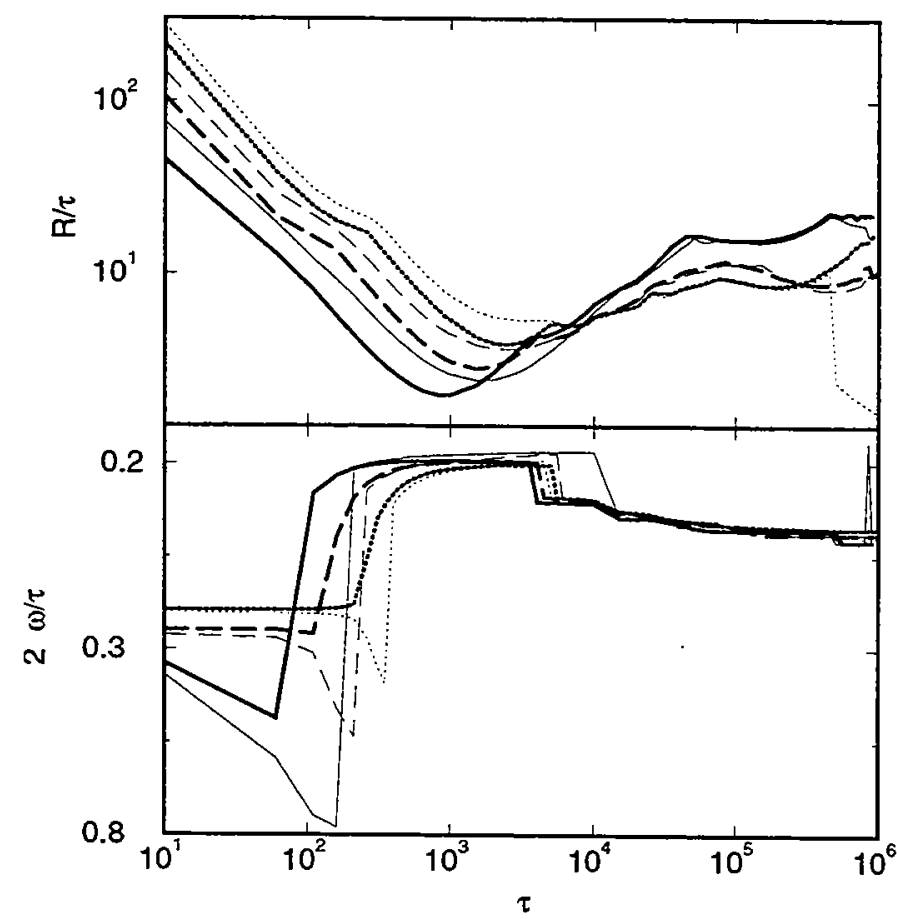

Figure 11: The Rayleigh number $R$ (upper plot) and the frequency $\omega$ (lower plot) as a function of $\tau$ in the case $P=0.1, \eta=0.4$ for values of the wavenumber $m=4$ (solid line), $m=6$ (long-dashed line) and $m=8$ (dotted line), in the case of insulating (thick lines) and conducting (thin lines) thermal boundary conditions.

Fig. 11 compares the critical Rayleigh numbers and frequencies of various modes of convection with different thermal boundary conditions. Towards low values of the rotation parameter, $\tau$, all modes of the flow with insulating boundary conditions have a lower value of the Rayleigh number than the corresponding modes with conducting temperature boundary conditions. At these low values of the rotation parameter the $R(\tau)$ curves of the two cases are well separated and it is easy to observe that they have roughly the same structure and form. This is apparent from the $\omega / \tau$ ratio plotted against $\tau$. At low values of the rotation parameter the two cases undergo the same types of transition from retrograde to prograde mode. A slight difference in the values of the $\omega / \tau$ may be noticed. A more important difference is that the transitions occur for much lower values of $\tau$ in the case of insulating 
boundary conditions. The value $P=0.1$ of the Prandtl number is on the border of the low Prandtl number region as it was described in section 4 . Thus as the rotation parameter increases when we cannot follow so well all the various transitions occurring at very low values of the Prandtl number. After a certain value of the rotation rate $\tau$ has been reached the $\omega / \tau$ ratio starts to change almost continuously since a large number of modes take part in the competition for providing a minimal $R$ and only a very small number of tiny jumps can be noticed. During the last big discontinuous transition the critical Rayleigh number of the modes with insulating thermal boundary conditions becomes larger than the Rayleigh number of the modes in the conducting case.But soon thereafter when the variation of $\omega / \tau$ becomes almost continuous, the Rayleigh numbers of the modes with the different boundary conditions become roughly equal and have also the same structure as can be seen in Fig. 11 .

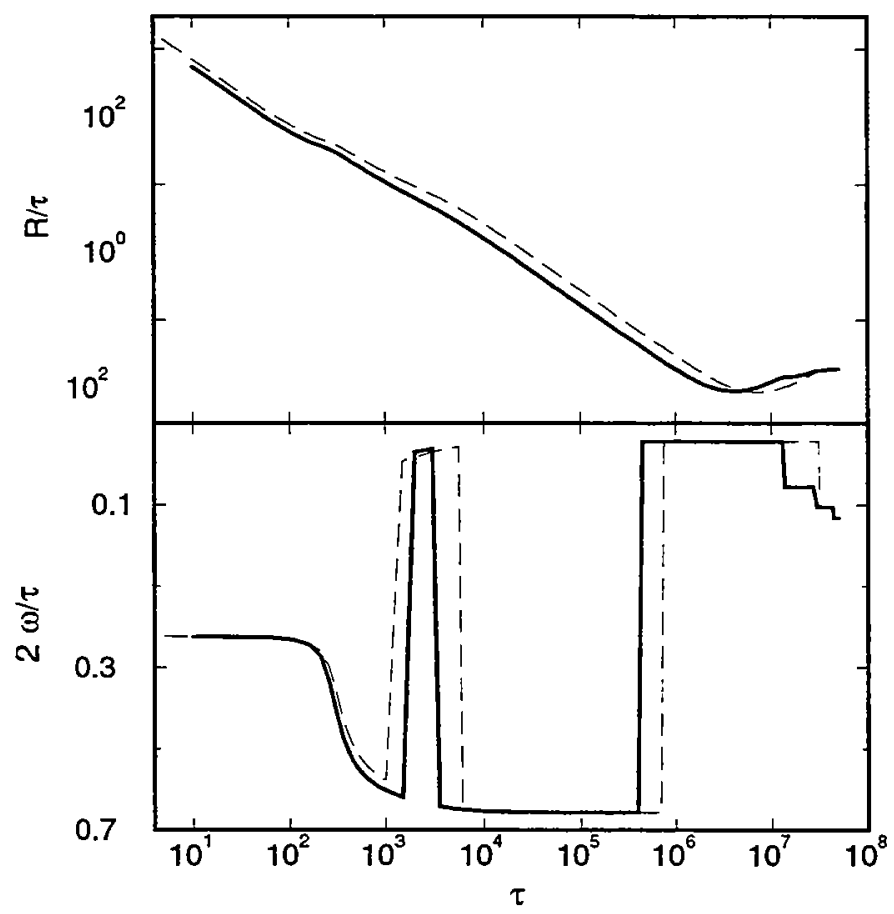

Figure 12: Same as Fig. 10 but in the case of $P=10^{-4}, \eta=0.3, m=8$

For low values of the Prandtl number the sequence of transitions with increasing rotation rate $\tau$ can be observed more clearly and in a more pure form. Fig. 12 shows again a comparison between convection obtained under the two different boundary conditions for the Prandtl number, $P=0.0001$. The same qualitative features as in Fig. 11 can be observed as well as the same number and type of transitions as described in section 4 where the same case but with the conducting boundary conditions was discussed in much detail.

This observation poses the question whether this is always the case and whether in addition to the similar transitions we may hope to observe the same regimes of convective flow at the various Prandtl number and rotation rate regions. The answer to this question requires an enormous computational task which we do not attempt to undertake here. Our 

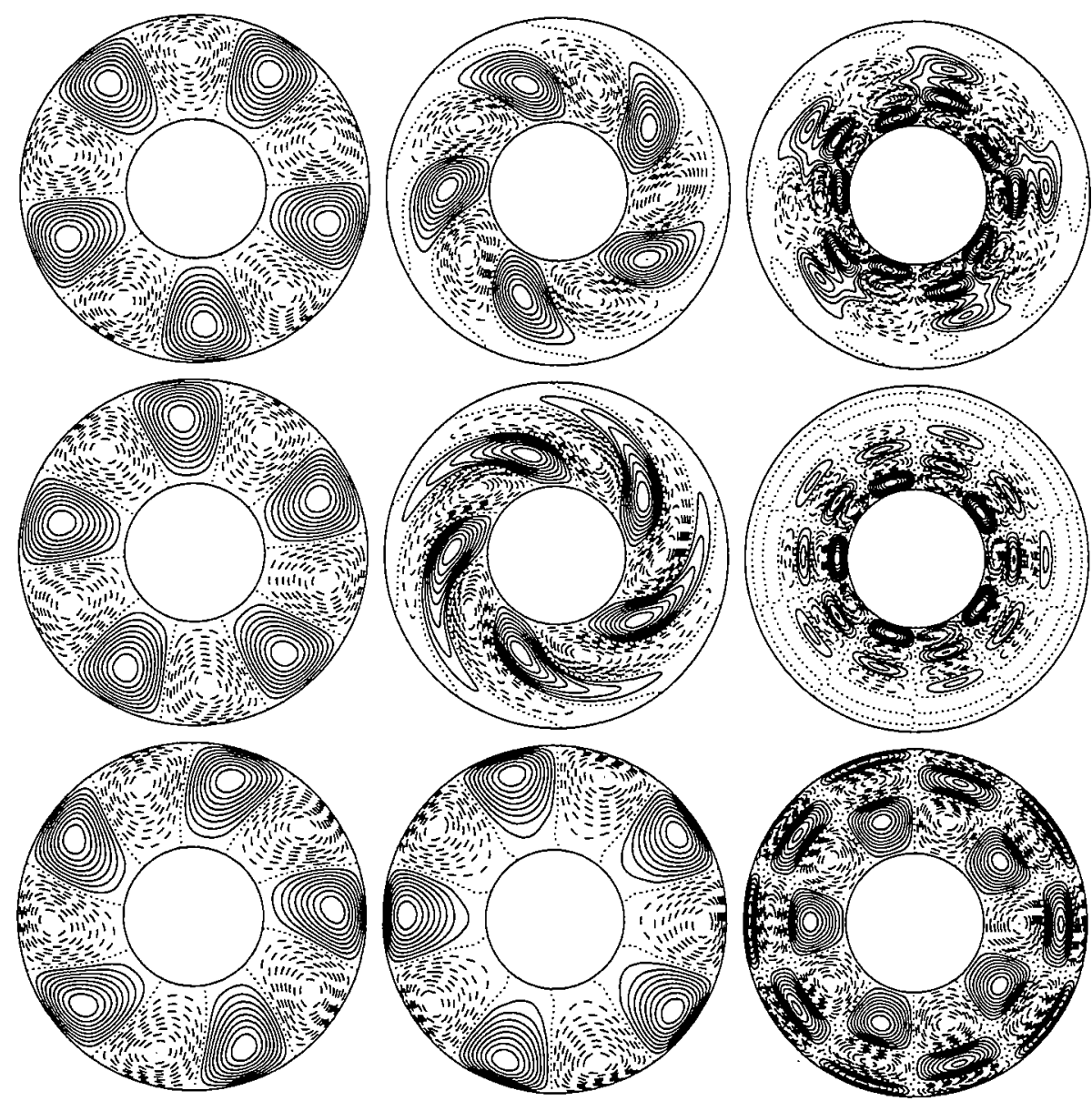

Figure 13: The streamfunctions $r \sin \theta \partial_{\theta} v$ in the equatorial plane illustrating various regimes of convection in the $P-\tau$ space in the case of insulating thermal boundary conditions. From left to right and top to bottom the parameters $(P, \tau)$ are: $(100,56.3),(100,5623.4),(100,562341.3)$, $(1,100),(1,10000),\left(1,10^{6}\right),(0.01,316.2),(0.01,31622.8),(0.01,3162278.1)$ for $\eta=0.4$ and $m=5$.

efforts are restricted to the investigation of a number of distant points in the $P-\tau$ space where we believed the various regimes of convection may manifest themselves. As shown in earlier studies (Ardes et al.(1997)) the border between the wall-attached and the columnar type of flow obeys the approximate relation,

$$
p^{1 / 4} \tau^{2}=\text { const. }
$$

Using this expression as a guideline we investigated several different cases with increasing Prandtl number, $P=0.001, P=1, P=100$. Indeed as Fig. 13 indicates, all major regimes of convection may be observed for the case of insulating thermal boundary conditions as well. At low Prandtl number, $P=0.001$ the wall-attached regime persist for a wide range of values of $\tau$. For high $P=1,100$ wall-attached pattern may also be found but only for a very limited range of low rotation rates. For moderate Prandtl numbers of the order of unity at high rotation rates the generic case of columnar convection takes place. 
It can also be found in a large region in the $P-\tau$ space and in the case of Fig. 13 we may notice how the pattern changes from elongated to shorter and thicker columns when the Prandtl number is increased from 1 to 100 . On the other end at very high values of the rotation parameter we find the newly observed pattern of multi-columnar convection. A very remarkable and surprising discovery is that it persists even at very high values of the Prandtl number if sufficiently high values of $\tau$ are reached.

\section{Concluding Remarks}

Rotating thermal convection has been a focus of intensive research for more than five decades now, but continues to be a constant source of open questions and possibilities for further developments. In the present report the linear properties of convection in rotating spherical shells have been studied from both analytical and numerical points of view. The most valuable result in this study seem to be the refinement and extension of the perturbation approach of Zhang(1994). Future efforts will be devoted to obtaining higher order analytical approximations that will enable us to explain some numerical results. On the numerical side many interesting features has been found and examined in considerable detail. Convection in the case of insulating thermal boundary conditions need to be further investigated.

\section{Acknowledgements}

I wish to express my deep gratitude and respect to our principal lecturer Friedrich Busse. I thank to him for his help, knowledge, ideas and a world of possibilities he has shared with me in the last two years being the adviser of my doctoral thesis project. He also supervised my GFD project this summer and many ideas presented here belong to him. His help with the analytical part of the report was invaluable.

I would like to thank the organizing committee of the GFD program for the opportunity to work in a challenging atmosphere, the members of the staff for sharing their knowledge and the rest of fellows for the nice time spent together.

It was a truly rewarding summer!

\section{Appendix A. A Formula Involving the $Q$ Operator}

For the solution of the zeroth order equations (3.21) and (3.22) we need to evaluate the expression $Q P_{n}^{m}(\cos \theta) f(r)$, where $P_{n}^{m}(\cos \theta)$ is the associated Legendre polynomial of degree $n$ and order $m$ 
and $f(r)$ is an arbitrary radial dependence.

$$
\begin{aligned}
& Q P_{n}^{m}(\cos \theta) f(r)=\left(k \cdot \nabla-\frac{1}{2}\left(L_{2} k \cdot \nabla+k \cdot \nabla L_{2}\right)\right) P_{n}^{m}(\cos \theta) f(r) \\
& =\left[\left(1-\frac{1}{2} L_{2}\right)\left(\cos \theta \frac{\partial}{\partial r}-\frac{\sin \theta}{r} \frac{\partial}{\partial \theta}\right)-\frac{1}{2}\left(\cos \theta \frac{\partial}{\partial r}-\frac{\sin \theta}{r} \frac{\partial}{\partial \theta}\right) L_{2}\right] P_{n}^{m}(\cos \theta) f(r) \\
& =\left[\left(1-\frac{1}{2} L_{2}\right)\left(\cos \theta \frac{\partial}{\partial r}-\frac{\sin ^{2} \theta}{r} \frac{\partial}{\partial(\cos \theta)}\right)-\frac{1}{2}\left(\cos \theta \frac{\partial}{\partial r}-\frac{\sin ^{2} \theta}{r} \frac{\partial}{\partial(\cos \theta)}\right) L_{2}\right] P_{n}^{m}(\cos \theta) f(r) \\
& =\left(1-\frac{1}{2} L_{2}\right)\left\{f^{\prime}\left(\frac{n+m}{2 n+1} P_{n-1}^{m}+\frac{n-m+1}{2 n+1} P_{n+1}^{m}\right)+\frac{f}{r}\left(\frac{(n+m)(n+1)}{2 n+1} P_{n-1}^{m}-\frac{n(n-m+1)}{2 n+1} P_{n+1}^{m}\right)\right\} \\
& -\frac{1}{2} n(n+1)\left\{f^{\prime}\left(\frac{n+m}{2 n+1} P_{n-1}^{m}+\frac{n-m+1}{2 n+1} P_{n+1}^{m}\right)+\frac{f}{r}\left(\frac{(n+m)(n+1)}{2 n+1} P_{n-1}^{m}-\frac{n(n-m+1)}{2 n+1} P_{n+1}^{m}\right)\right\} \\
& =\left(f^{\prime}+\frac{n+1}{r} f\right) \frac{\left(1-n^{2}\right)(n+m)}{2 n+1} P_{n-1}^{m}+\left(f^{\prime}-\frac{n}{r} f\right) \frac{\left(1-(n+1)^{2}\right)(n+1-m)}{2 n+1} P_{n+1}^{m},
\end{aligned}
$$

where formula (8.5.3) of Abramowitz and Stegun (1964) has been used.

\section{References}

Abramowitz, M., Stegun, I., Handbook of Mathematical Functions. National Bureau of Standards, 1964. (Reprint: Dover Publications Inc. 9th ed.)

Ardes, M., Busse, F.H.,Wicht, J.,Thermal convection in rotating spherical shells, Phys. Earth Plan. Int. $\underline{99}, 55-67,1997$

Busse, F.H., Thermal instabilities in rapidly rotating systems, J. Fluid Mech. $\underline{44}$, 441-460, 1970a

Busse, F.H., Differential Rotation in Stellar Convection Zones, Astrophys. J. 159, 629-639, $1970 \mathrm{~b}$

Busse, F.H., Convective flows in rapidly rotating spheres and their dynamo action, Phys. Fluids, 14, 1301-1314, 2002

Carrigan, C.R., Busse, F.H.,An experimental and theoretical investigation of the onset of convection in rotating spherical shells , J. Fluid Mech. 126,287-305, 1983

Chandrasekhar, S., Hydrodynamic and Hydromagnetic Stability, Oxford: Clarendon Press, 1961

Greenspan, H.P., The theory of rotating fluids, Cambridge University Press, 1968

Roberts, P.H., On the thermal instability of a rotating fluid sphere containing heat sources, Phil. Trans. Roy. Soc. London A 263, 93-117, 1968

Zhang, K., On coupling between the Poincaré equation and the heat equation, J. Fluid Mech. 26ㅇ, 211-229, 1994 
Zhang, K., and Busse, F.H., On the Onset of Convection in Rotating Spherical Shells, Geophys. Astrophys. Fluid Dyn. $\underline{39}, 119-147,1987$

Zhang, K., Earnshaw, P., Liao, X., Busse F.H., On inertial waves in a rotating fluid sphere, J. Fluid Mech. 년, 103-119, 2001 


\title{
Upper Bounds for Convection in an Internally Heated Fluid Layer
}

\author{
$\mathrm{Lu} \mathrm{Lu}$ \\ University of Michigan
}

\section{Introduction}

Finding the bounds of certain global quantities is an important approach in the theoretical study of turbulence. Particularly, maximum convective heat transport in Rayleigh-Bénard convection is a much-studied subject. The idea dates back to Malkus [1]. Following this idea, a rigorous upper bound of convective heat transport in Rayleigh-Bénard convection without continuity constraint was first derived by Howard [2], using a variational approach. In the same paper [2], a single-wavenumber boundary layer approximation is used to study the asymptotic solutions of the Euler-Lagrange equations. Busse [3] extended this asymptotic technique and introduced multi- $\alpha$-solutions, which has been proved fruitful in studying other fluid dynamics problems [4], [5], [6], and this approach is reviewed in [7]. A new approach to derive the rigorous bounds on turbulent flow quantities, the background method (DeoringContantin approach), appeared in 1992 [8], and subsequently applied to Rayleigh-Bénard convection [9], [10]. In this project report, these two above-mentioned techniques are applied to bound the minimum average temperature in a fluid layer with internal heating.

\section{Governing Equations}

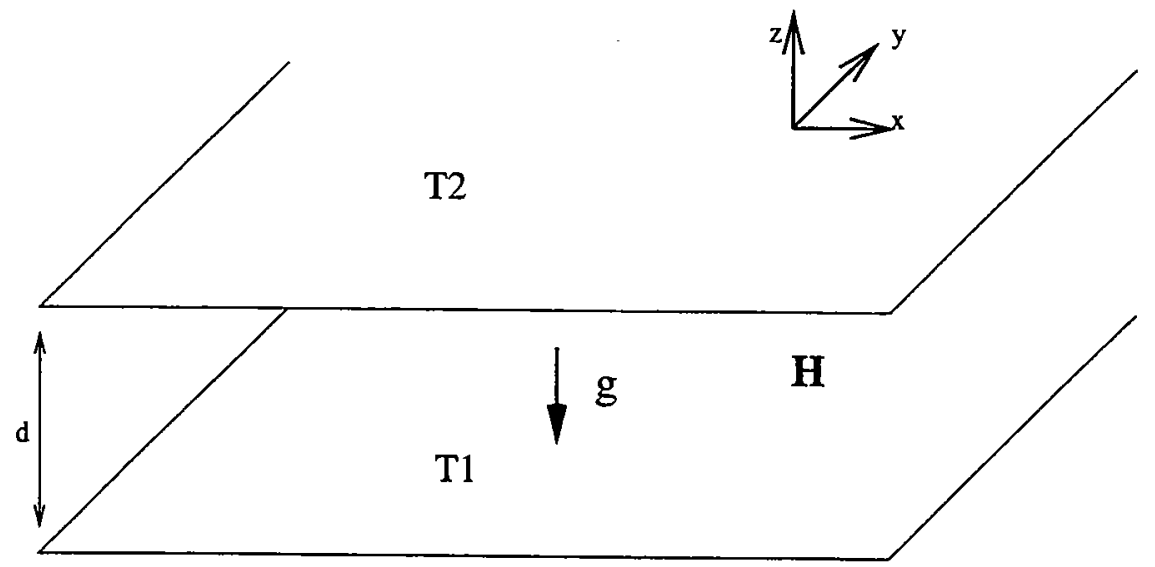

Figure 1: Geometry of convection with uniform internal heating.

The geometry is shown in Fig. (1). The setup is very similar to Rayleigh-Bénard convection. A fluid layer is confined between two parallel plates with a distance $d$. However,a uniform volumetric heat flux $H$ (with unit $\frac{J}{s \cdot m^{3}}$ ) is pumped into the fluid layer. The upper and lower plates are held at fixed temperatures $T_{0}$ and $T_{1}$ respectively, and there is no restriction on the temperature difference $\Delta T=T_{0}-T_{1}$. With internal heating, the governing equations are identical to Rayleigh-Bénard convection except for the additional term $\gamma$ in 
the heat equation:

$$
\begin{gathered}
\frac{\partial \mathbf{u}}{\partial t}+\mathbf{u} \cdot \nabla \mathbf{u}=-\nabla p+\nu \nabla^{2} \mathbf{u}+\hat{\mathbf{k}} g \alpha T \\
\frac{\partial T}{\partial t}+\mathbf{u} \cdot \nabla T=\kappa \nabla^{2} T+\gamma \\
\nabla \cdot \mathbf{u}=0
\end{gathered}
$$

with the boundary conditions

$$
\left.\mathbf{u}\right|_{z=0,1}=0,\left.\quad T\right|_{z=0}=T_{0},\left.\quad T\right|_{z=1}=T_{1}
$$

where

$$
\gamma=\frac{H}{\rho c}
$$

By introducing $\frac{d^{2}}{\kappa}$ as the unit of time, $d$ of length, $\frac{\kappa}{d}$ of velocity and $\frac{\kappa \nu}{g \alpha d^{3}}$ as the unit of temperature, the governing equations are put into the non-dimensional form

$$
\begin{gathered}
\operatorname{Pr}^{-1}\left(\frac{\partial \mathbf{u}}{\partial t}+\mathbf{u} \cdot \nabla \mathbf{u}\right)+\nabla p=\nabla^{2} \mathbf{u}+T \hat{\mathbf{k}} \\
\frac{\partial T}{\partial t}+\mathbf{u} \cdot \nabla T=\nabla^{2} T+R
\end{gathered}
$$

where

$$
\operatorname{Pr}=\frac{\nu}{\kappa}, \quad R=\frac{g \alpha d^{5} \gamma}{\kappa^{2} \nu}
$$

$R$ is the heat Rayleigh number, which is proportional to the internal heating rate. The boundary conditions in non-dimensional form are

$$
\left.\mathbf{u}\right|_{z=0,1}=0,\left.\quad T\right|_{z=0}=-T_{0},\left.\quad T\right|_{z=1}=0
$$

where $T_{0}=\frac{g \alpha d^{3}}{\kappa \nu}|\Delta T|$, which shows that the non-dimensional $T_{0}$ is equivalent to the role of the Rayleigh number $R a$. A negative non-dimensional temperature at the bottom plate means the upper plate is hotter. If $\left.T\right|_{z=0}$ is 0 , then two plates have the same temperature. The case when the bottom plate is hotter corresponds to a positive non-dimensional temperature at the bottom plate.

\section{$3 \quad$ Linear and Energy Stability}

A static solution can be found easily:

$$
T=-\frac{1}{2} R z^{2}+\left(\frac{1}{2} R+T_{0}\right) z-T_{0}
$$

where $\left.T\right|_{z=0}=-T_{0}$. Fig (2) shows several possibilities of the of the static solutions. When two plates have the same temperature, the profile is symmetric about $z=\frac{1}{2}$. The maximum 


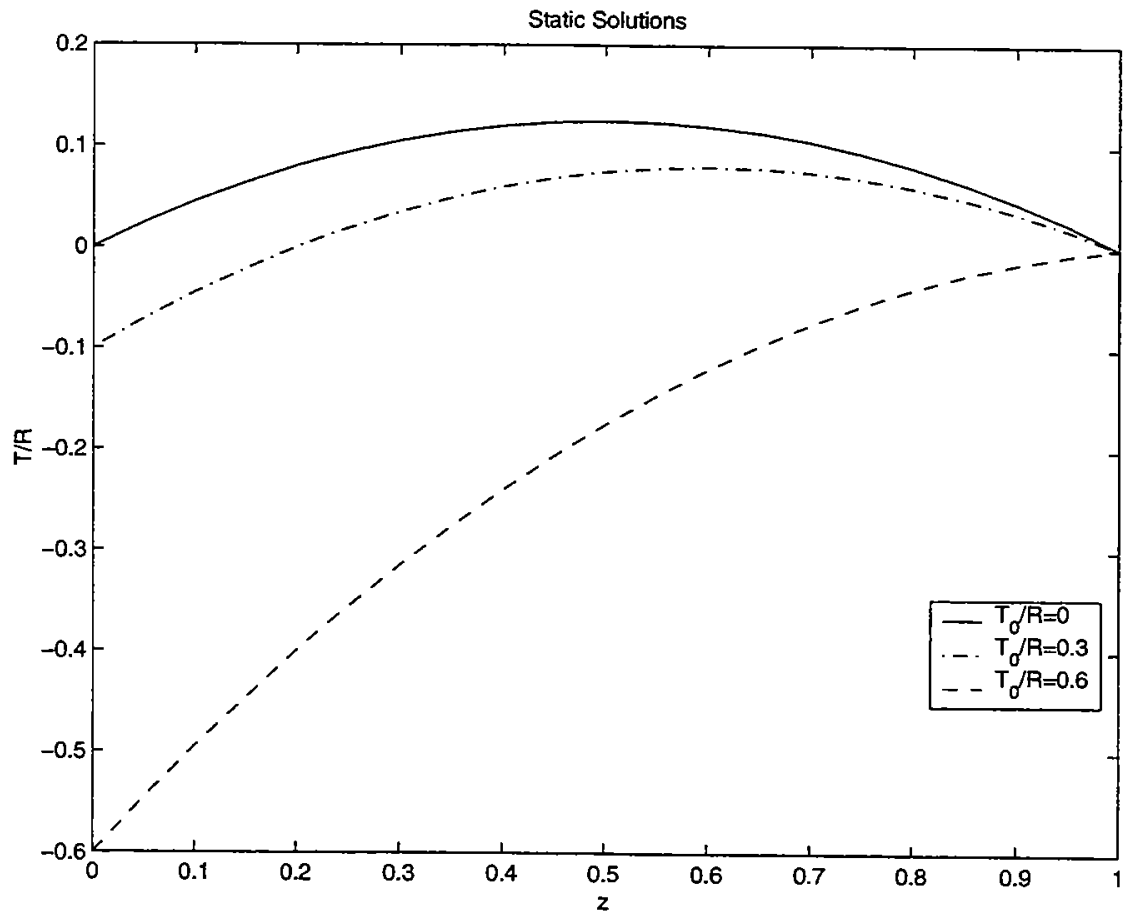

Figure 2: Static solutions of an internally heated fluid layer.

temperature occurs at $z=\frac{1}{2}$. Thus temperature gradient for the lower half of the fluid layer is positive, corresponding to a stable stratification. While in the upper half, the fluid layer is unstably stratified. This profile provides the possibility, when the internal heating rate $R$ is big enough, that convection starts in the upper half of the fluid layer. When $T_{0}$ increases, but not exceeding $\frac{1}{2} R$, the unstable stratification persists with the position of maximum temperature shifting toward the upper plate. Eventually, when $T_{0} \geq \frac{1}{2} R$, the temperature gradient is positive everywhere which suggests that the fluid layer is linearly stable, a fact that will be established in the analysis of linear stability bellow.

Let $T=-\frac{1}{2} R z^{2}+\left(\frac{1}{2} R+T_{0}\right) z-T_{0}+\theta$, where $\theta$ is the temperature disturbance. We can write down the linearized equations governing the growth of the disturbances $\theta$ and $\mathbf{u}$

$$
\begin{gathered}
\operatorname{Pr}^{-1}\left(\frac{\partial \mathbf{u}}{\partial t}\right)+\nabla p=\nabla^{2} \mathbf{u}+\theta \hat{\mathbf{k}} \\
\frac{\partial \theta}{\partial t}=\left(z-\frac{1}{2}-T_{0}\right) w+\nabla^{2} \theta \\
\nabla \cdot \mathbf{u}=0
\end{gathered}
$$

with the boundary conditions

$$
\left.\mathbf{u}\right|_{z=0,1}=0,\left.\quad \theta\right|_{z=0,1}=0 \text {. }
$$

Taking the $z$ component of the curl curl of the $\mathbf{u}$ equation leads to

$$
\operatorname{Pr}^{-1} \frac{\partial}{\partial t} \nabla^{2} w=\nabla^{4} w+\Delta_{2} \theta
$$


where $\Delta_{2}$ is the horizontal Laplacian, defined as

$$
\Delta_{2}=\frac{\partial^{2}}{\partial x^{2}}+\frac{\partial^{2}}{\partial y^{2}}
$$

Then we make the ansatz

$$
w=e^{s t} f(x, y) W(z), \quad \theta=e^{s t} f(x, y) \Theta(z),
$$

and

$$
\Delta_{2} f(x, y)=-a^{2} f(x, y)
$$

The exchange of stability holds in this problem ([11]). Hence to determine the critical $R$, we can set $s=0$ :

$$
\begin{gathered}
\left(D^{2}-a^{2}\right)^{2} W=a^{2} R \Theta \\
\left(D^{2}-a^{2}\right) \Theta=-\left(z-\frac{1}{2}-T_{0}\right) W
\end{gathered}
$$

with the boundary conditions

$$
W=D W=\theta=0 \quad \text { at } \quad z=0,1 .
$$

In Rayleigh-Bénard convection, the equations for linear stability turn out to be identical to those of energy stability. Thus it is of interest to investigate the energy stability of the internally heated fluid layer. The equations for the disturbances $\mathbf{u}$ and $\theta$ are

$$
\begin{gathered}
\operatorname{Pr}^{-1}\left(\frac{\partial \mathbf{u}}{\partial t}+\mathbf{u} \cdot \nabla \mathbf{u}+\nabla p\right)=\nabla^{2} \mathbf{u}+\theta \hat{\mathbf{k}} \\
\frac{\partial \theta}{\partial t}+\mathbf{u} \cdot \nabla \theta=\left(R z-\frac{1}{2} R-T_{0}\right) w+\nabla^{2} \theta \\
\nabla \cdot \mathbf{u}=0
\end{gathered}
$$

Using $\mathbf{u}$ - equation (9a), $\theta \times$ equation (9b), we integrate over the whole layer to get

$$
\begin{gathered}
\frac{1}{2} \frac{d}{d t} \frac{\left\langle\mathbf{u}^{2}\right\rangle}{P r}=-\left\langle|\nabla \mathbf{u}|^{2}\right\rangle+\langle w \theta\rangle \\
\frac{1}{2} \frac{d}{d t} \frac{\left\langle\theta^{2}\right\rangle}{R}=\left\langle\left(z-\frac{1}{2}-\frac{T_{0}}{R}\right) w \theta\right\rangle-\frac{\left\langle|\nabla \theta|^{2}\right\rangle}{R} .
\end{gathered}
$$

Introducing a balance parameter $s$ and adding the above equations yield

$$
\frac{d}{d t} \frac{1}{2}\left\{s \cdot \frac{\left\langle\mathbf{u}^{2}\right\rangle}{P r}+\frac{\left\langle\theta^{2}\right\rangle}{R}\right\}=-\left\{s \cdot\left\langle|\nabla \mathbf{u}|^{2}\right\rangle-\left\langle\left(z-\frac{1}{2}-\frac{T_{0}}{R}+s\right) w \theta\right\rangle+\frac{\left\langle|\nabla \theta|^{2}\right\rangle}{R}\right\}
$$

Let

$$
\lambda=s \cdot \frac{\left\langle|\nabla \mathbf{u}|^{2}\right\rangle-\left\langle\left(z-\frac{1}{2}-\frac{T_{0}}{R}+s\right) w \theta\right\rangle+\frac{\left\langle|\nabla \theta|^{2}\right\rangle}{R}}{\frac{1}{2}\left\{s \cdot \frac{\left\langle\mathbf{u}^{2}\right\rangle}{P r}+\frac{\left\langle\theta^{2}\right\rangle}{R}\right\}}
$$


then if $\min \lambda \leq 0$ among the fields with

$$
\nabla \cdot \mathbf{u}=0,\left.\quad \mathbf{u}\right|_{z=0,1}=0,\left.\quad \theta\right|_{z=0,1}=0
$$

the disturbances decay exponentially in time, which implies energy stability. To determine the critical value of $R$, we need the Euler-Lagrange equations:

$$
\begin{gathered}
s \cdot \nabla^{2} \mathbf{u}+\frac{1}{2}\left(z-\frac{1}{2}-\frac{T_{0}}{R}+s\right) \theta \hat{\mathbf{k}}+\nabla p+\lambda \frac{\mathbf{u}}{R}=0 \\
\nabla^{2} \theta+\frac{1}{2}\left(z+\frac{1}{2}-\frac{T_{0}}{R}\right) w+\lambda \theta=0 \\
\nabla \cdot \mathbf{u}=0
\end{gathered}
$$

Setting $\lambda=0$ and applying the ansatz (7) yields

$$
\begin{gathered}
s \cdot\left(D^{2}-a^{2}\right)^{2} W-\frac{1}{2}\left(z-\frac{1}{2}-\frac{T_{0}}{R}+s\right) a^{2} \Theta=0 \\
\left(D^{2}-a^{2}\right) \Theta+\frac{1}{2}\left(z-\frac{1}{2}-\frac{T_{0}}{R}+s\right) R W=0
\end{gathered}
$$

with the boundary conditions

$$
W=D W=\Theta=0 \quad \text { at } \quad z=0,1
$$

For each $s$, the critical $R_{E}(s)$ can be found by minimizing the eigenvalue $R$ with respect to the horizontal wave-number $a$. Then $s$ can be chosen such that it gives a maximal

$$
R_{E}\left(T_{0}\right)=\max _{s} \min _{a} R(a, s)
$$

The linear stability and energy stability equations are solved numerically, using a Chebyshev spectral method. The results are shown in Fig. (3) and Fig. (4). The first figure (with the bottom plate colder) shows the convergence of the linear stability to the straight line $T_{0}=\frac{1}{2} R$, which corresponds the temperature difference beyond which the entire fluid layer is stably stratified. However, the energy stability increases with $T_{0}$ but does not converge to the line $T_{0}=\frac{1}{2} R$. In the case of a hotter bottom plate, both the linear stability and energy stability lines converge to the critical Rayleigh number 1708 for Rayleigh-Bénard convection. This is expected since the fluid layer is linearly unstable even without any internal heating in that case. In both figures, the critical $R$ for energy stability is lower than that of linear stability. This suggests the possibility of subcritical bifurcations.

As shown above, even though the bottom plate is colder and the lower half of the fluid is stably stratified, the system can still be linearly unstable. Once the convection starts, it tries to lower the average temperature of the fluid layer. Thus it is of interest to investigate the scaling of the minimum average temperature. In the following two sections, this scaling will be studied with the background method and the multi- $\alpha$-solution approach. 


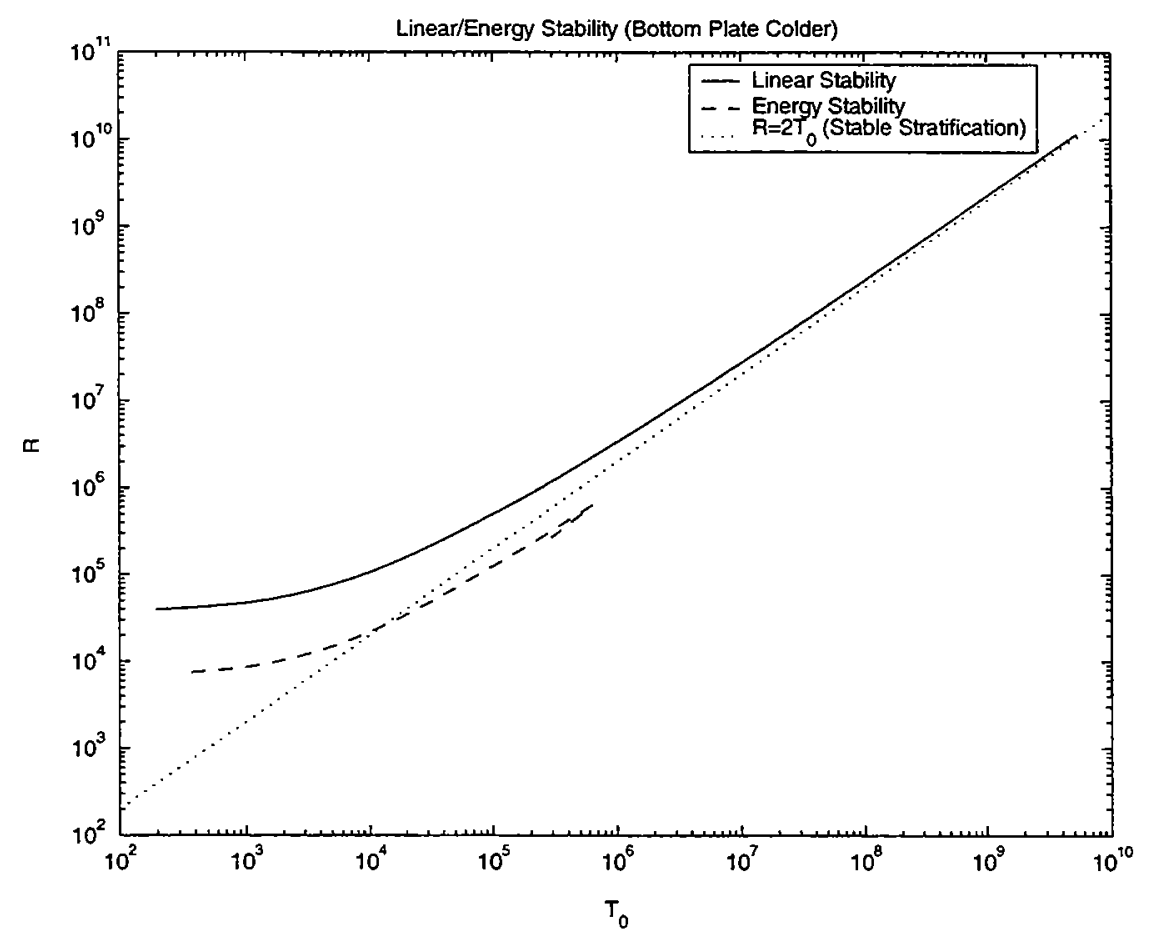

Figure 3: Linear and energy stability.

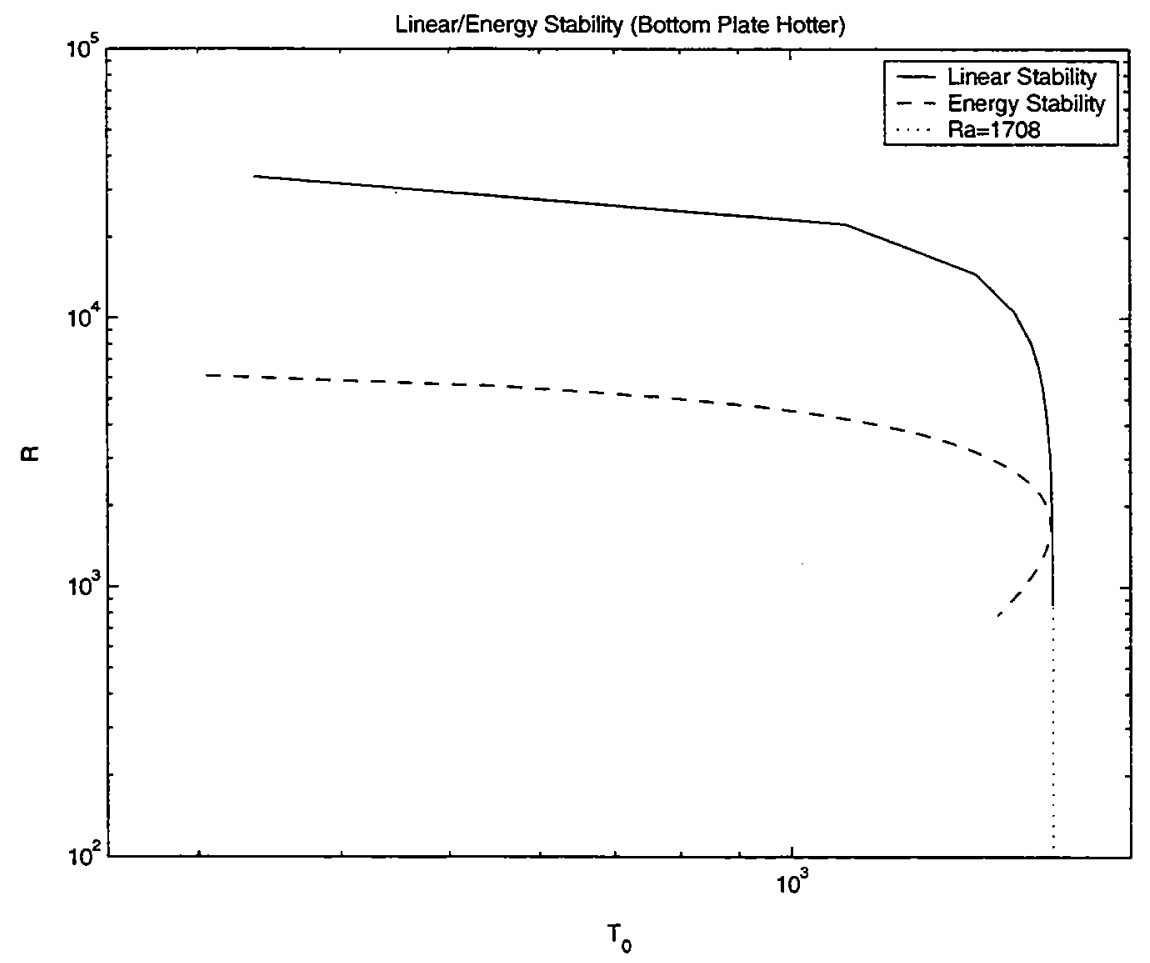

Figure 4: Linear and energy stability (Hotter lower plate). 


\section{Background method}

To apply the background method, first we decompose the temperature field $T$ into a background profile $\tau(z)$ and a fluctuating part $\theta(x, y, z, t)$ :

$$
T=\tau(z)+\theta(x, y, z, t) \text {. }
$$

The boundary conditions of $T$ are contained in $\tau(z)$ :

$$
\tau(0)=\tau(1)=0
$$

The velocity field $\mathbf{u}$ still satisfies divergence-free and no-slip boundary conditions:

$$
\nabla \cdot \mathbf{u}=0,\left.\quad \mathbf{u}\right|_{z=0,1}=0 .
$$

Then the governing equations (2) become

$$
\begin{gathered}
P r^{-1}\left(\frac{\partial \mathbf{u}}{\partial t}+\mathbf{u} \cdot \nabla \mathbf{u}\right)+\nabla p=\nabla^{2} \mathbf{u}+\tau \hat{\mathbf{k}}+T \hat{\mathbf{k}} \\
\frac{\partial \theta}{\partial t}+\mathbf{u} \cdot \nabla \theta=\nabla^{2} \theta+\tau^{\prime \prime}+R-w \tau^{\prime}
\end{gathered}
$$

Define $\langle\cdot\rangle=\lim _{t=1 \rightarrow \infty} \frac{1}{t} \int_{0}^{t} d t^{\prime} \frac{1}{L_{x} L_{y}} \int d x d y d z \cdot$ to be the average over both space and time. Then $\langle\mathbf{u} \cdot(18 a)\rangle$ yields

$$
\left\langle|\nabla \mathbf{u}|^{2}\right\rangle=\langle w \theta\rangle
$$

and $\langle\theta \cdot(18 b)\rangle,\langle\tau \cdot(18 b)\rangle$ yield, respectively,

$$
\begin{aligned}
& \left\langle w \theta \tau^{\prime}\right\rangle=-\left\langle|\nabla \theta|^{2}\right\rangle-\left\langle\theta^{\prime} \tau^{\prime}\right\rangle+R\langle\theta\rangle, \\
& -\left\langle w \theta \tau^{\prime}\right\rangle=-\left\langle\theta^{\prime} \tau^{\prime}\right\rangle+R\langle\tau\rangle-\left\langle\tau^{\prime 2}\right\rangle .
\end{aligned}
$$

The difference of the above two identities is

$$
R(\langle\theta\rangle-\langle\tau\rangle)=\left\langle|\nabla \theta|^{2}\right\rangle+2\left\langle w \theta \tau^{\prime}\right\rangle-\left\langle\tau^{\prime 2}\right\rangle
$$

Since $\langle T\rangle=\langle\tau\rangle+\langle\theta\rangle$, we have

$$
R\langle T\rangle=\left\langle|\nabla \theta|^{2}\right\rangle+2\left\langle w \theta \tau^{\prime}\right\rangle+2 R\langle\tau\rangle-\left\langle\tau^{2}\right\rangle
$$

The identity (23) can also be written as

$$
0=a\left\langle|\nabla \mathbf{u}|^{2}\right\rangle-a\langle w \theta\rangle
$$

where $a$ is a positive number used as an optimization parameter to be adjusted to yield the best prefactor. Adding equation (24) to equation (23) enables us to express the average temperature as follows:

$$
R\langle T\rangle=2 R\langle\tau\rangle-\left\langle\tau^{2}\right\rangle+H
$$


where

$$
H=\left\{\left\langle|\nabla \theta|^{2}\right\rangle+\left\langle\left(2 \tau^{\prime}-a\right) w \theta\right\rangle+a\left\langle|\nabla \mathbf{u}|^{2}\right\rangle\right\}
$$

We require the functional $H$ to be semi positive definite among the fields $\mathbf{u}$ and $\theta$ satisfying

$$
\nabla \cdot \mathbf{u}=0, \quad, \mathbf{u}|z=0,1=0, \quad \theta|_{z=0,1}=0 .
$$

A bound can readily be obtained by applying the following background profile

$$
\tau(z)=\left\{\begin{array}{lr}
\frac{a}{2} z, & 0 \leq z \leq 1-\delta \\
\frac{a(1-\delta)}{2 \delta}(1-z), & 1-\quad \delta<z \leq 1
\end{array}\right.
$$

With this background profile, $2 \tau^{\prime}-a$ vanishes in the interval $1 \leq z \leq 1-\delta$. Thus we only need to estimate $\left|\left\langle\left(2 \tau^{\prime}-a\right) w \theta\right\rangle\right|=\frac{a}{\delta}\langle w \theta\rangle$ in the region $1-\delta<z \leq 1$, and adjust $\delta$ and $a$ to make $H$ semi positive definite. Following ([9]), an estimate is given by

$$
\left|\left\langle\left(2 \tau^{\prime}-a\right) w \theta\right\rangle\right| \leq \frac{a}{\delta} \frac{\delta^{2}}{4}\left[\frac{c}{4}\left\langle|\nabla \mathbf{u}|^{2}\right\rangle+\frac{\delta^{2}}{4 c}\left\langle|\nabla \theta|^{2}\right\rangle\right]
$$

Then

$$
\left\{\left\langle|\nabla \theta|^{2}\right\rangle+\left\langle\left(2 \tau^{\prime}-a\right) w \theta\right\rangle+a\left\langle|\nabla \mathbf{u}|^{2}\right\rangle\right\} \geq\left(a-\frac{a}{\delta} \frac{\delta^{2} c}{16}\right)\left\langle|\nabla \mathbf{u}|^{2}\right\rangle+\left(1-\frac{a}{\delta} \frac{\delta^{2}}{4 c}\right)\left\langle|\nabla \theta|^{2}\right\rangle .
$$

The choice

$$
\frac{a \delta^{2}}{64}=1, \quad c=\frac{a \delta}{4}
$$

makes the right hand side equal to zero, which ensures $H \geq 0$. Then

$$
\begin{aligned}
R\langle T\rangle & \geq 2 R\langle\tau\rangle-\left\langle\tau^{2}\right\rangle \\
& =\frac{a R}{2}(1-\delta)-\frac{a^{2}}{4} \frac{1-\delta}{\delta} .
\end{aligned}
$$

Minimizing the right hand side with respect to $a$ gives the optimal choice of the parameter $a$

$$
a=\delta R \text {. }
$$

And $\delta$ can be solved from equation (30),

$$
\delta=\frac{4}{\sqrt[3]{R}}
$$

Finally,

$$
\begin{aligned}
\langle T\rangle & \geq 2\langle\tau\rangle-\frac{\left\langle\tau^{\prime 2}\right\rangle}{R} \\
& =\frac{1}{4} R \delta(1-\delta) \\
& =R^{2 / 3}-4 R^{1 / 3} .
\end{aligned}
$$

This shows that as $R \rightarrow \infty,\langle T\rangle \sim R^{2 / 3}$, which will be verified in the next section by using the multi- $\alpha$ solution approach. 


\section{Howard-Busse Approach}

\subsection{Formulation of the Functional}

The governing equations are repeated here again

$$
\begin{gathered}
\operatorname{Pr}^{-1}\left(\frac{\partial \mathbf{u}}{\partial t}+\mathbf{u} \cdot \nabla \mathbf{u}\right)+\nabla p=\nabla^{2} \mathbf{u}+T \hat{\mathbf{k}} \\
\frac{\partial T}{\partial t}+\mathbf{u} \cdot \nabla T=\nabla^{2} T+R
\end{gathered}
$$

with

$$
\left.\mathbf{u}\right|_{z=0,1}=0, \quad T(0)=-T_{0}, \quad T(1)=0 .
$$

Assume the turbulence is statistically stationary and the velocity field $\mathbf{v}$ and temperature $T$ can be decomposed into a time independent horizontal average and a meanless fluctuating part:

$$
T=\bar{T}+\theta, \quad \bar{\theta}=0 \quad \text { with } \quad \overline{\mathbf{u}}=0, \quad \bar{\theta}=0 . .
$$

After taking horizontal average of $(35 \mathrm{~b})$, we have

$$
\frac{d \overline{w \theta}}{d z}=\frac{d^{2} \bar{T}}{d^{2} z}+R
$$

Integrate once,

$$
\overline{w \theta}=\frac{d \bar{T}}{d z}+R z+c
$$

The integration constant $c$ is determined by integrating above equation over $[0,1]$. This yields

$$
\frac{d \bar{T}}{d z}=\overline{w \theta}-\langle w \theta\rangle-R\left(z-\frac{1}{2}\right)+T_{0}
$$

With the decomposition (36), equation (35b) can be written as

$$
\frac{\partial \theta}{\partial t}+w \frac{d \bar{T}}{d z}+\mathbf{u} \cdot \nabla \theta=\nabla^{2} \theta+\frac{d^{2} \bar{T}}{d^{2} z}+R=\nabla^{2} \theta+\frac{d \overline{w \theta}}{d z}
$$

where equation (37) has been used. Multiply both sides with $\theta$ and integrate over the bulk, we have

$$
\left\langle w \theta \frac{d \bar{T}}{d z}\right\rangle=-\left\langle|\nabla \theta|^{2}\right\rangle
$$

Together with equation (39), the above expression yields

$$
R\left\langle\left(z-\frac{1}{2}\right) w \theta\right\rangle=\left\langle|\nabla \theta|^{2}\right\rangle+\left\langle\left(\overline{w \theta}-\langle w \theta>)^{2}\right\rangle+T_{0}\langle w \theta\rangle\right.
$$


Another power integral is derived by multiplying equation (35a) by $\mathbf{u}$ and integrating over the bulk:

$$
\left\langle|\nabla \mathbf{u}|^{2}\right\rangle=\langle w \theta\rangle
$$

We can find an expression of the average temperature by multiplying equation (39) by $z$ and integrate over $[0,1]$ :

$$
\langle T\rangle=-\left\langle\left(z-\frac{1}{2}\right) w \theta\right\rangle+\frac{1}{12} R-\frac{1}{2} T_{0}
$$

In summary, we have the following expressions:

$$
\begin{gathered}
\left\langle|\nabla \mathbf{u}|^{2}\right\rangle=\langle w \theta\rangle \\
R\left\langle\left(z-\frac{1}{2}\right) w \theta\right\rangle=\left\langle|\nabla \theta|^{2}\right\rangle+\left\langle(\overline{w \theta}-\langle w \theta\rangle)^{2}\right\rangle+T_{0}\langle w \theta\rangle \\
\langle T\rangle=-\left\langle\left(z-\frac{1}{2}\right) w \theta\right\rangle+\frac{1}{12} R-\frac{1}{2} T_{0}
\end{gathered}
$$

From equation (46), $R$ can be expressed as

$$
R=\frac{\left\langle|\nabla \theta|^{2}\right\rangle+\left\langle(\overline{w \theta}-\langle w \theta\rangle)^{2}\right\rangle+T_{0}\langle w \theta\rangle}{\left\langle\left(z-\frac{1}{2}\right) w \theta\right\rangle}
$$

Substitute the above expression into equation (47),

$$
\begin{aligned}
\langle T\rangle & =-\left\langle\left(z-\frac{1}{2}\right) w \theta\right\rangle+\frac{1}{12} \frac{\left\langle|\nabla \theta|^{2}\right\rangle+\left\langle(\overline{w \theta}-\langle w \theta\rangle)^{2}\right\rangle+T_{0}\langle w \theta\rangle}{\left\langle\left(z-\frac{1}{2}\right) w \theta\right\rangle}-\frac{1}{2} T_{0} \\
& =\frac{\left\langle|\nabla \theta|^{2}\right\rangle+\left\langle(\overline{w \theta}-\langle w \theta\rangle)^{2}\right\rangle-12\left\langle\left(z-\frac{1}{2}\right) w \theta\right\rangle}{12\left\langle\left(z-\frac{1}{2}\right) w \theta\right\rangle^{2}}+T_{0}\left(\frac{\langle w \theta\rangle}{12\left\langle\left(z-\frac{1}{2}\right) w \theta\right\rangle}-\frac{1}{2}\right) .
\end{aligned}
$$

Let

$$
h(z)=\sqrt{12}\left(z-\frac{1}{2}\right)
$$

Notice that

$$
\langle h\rangle=0, \quad\left\langle h^{2}\right\rangle=1 .
$$

This property of $h(z)$ yields the following identity:

$$
\left\langle(\overline{w \theta}-h\langle h w \theta\rangle-\langle w \theta\rangle)^{2}\right\rangle=\left\langle\overline{w \theta}^{2}\right\rangle-\langle w \theta\rangle^{2}-\langle h w \theta\rangle^{2}
$$

Together with equation (45), the average temperature can be expressed as follows,

$$
\begin{aligned}
\langle\sqrt{12} T\rangle & =\frac{\left.\left\langle|\nabla \theta|^{2}\right\rangle+\langle\overline{w \theta}-\langle w \theta\rangle)^{2}\right\rangle-\langle h w \theta\rangle^{2}}{\langle h w \theta\rangle}+T_{0}\left(\frac{\langle w \theta\rangle}{\langle h w \theta\rangle}-\sqrt{3}\right) \\
& =\frac{\left\langle|\nabla \theta|^{2}\right\rangle\left\langle|\nabla \mathbf{u}|^{2}\right\rangle}{\langle w \theta\rangle\langle h w \theta\rangle}+\frac{\left\langle\overline{w \theta}-h\langle h w \theta\rangle-\langle w \theta>)^{2}\right\rangle}{\langle h w \theta\rangle}+T_{0}\left(\frac{\langle w \theta\rangle}{\langle h w \theta\rangle}-\sqrt{3}\right) .
\end{aligned}
$$


Thus the variational problem $\left(T_{0}=0\right.$ case $)$ can be formulated as Given $\mu=<h w \theta>$, find the minimum of the functional

$$
\mathcal{F}=\frac{\left\langle|\nabla \theta|^{2}\right\rangle\left\langle|\nabla \mathbf{u}|^{2}\right\rangle}{\langle h w \theta\rangle\langle w \theta\rangle}+\mu \frac{\left\langle(\overline{w \theta}-h\langle h w \theta\rangle-\langle w \theta\rangle)^{2}\right\rangle}{\langle h w \theta\rangle^{2}}
$$

among the $\mathbf{u}, \theta$ fields with

$$
\nabla \cdot \mathbf{u}=0,\left.\quad \mathbf{u}\right|_{z=0,1}=0,\left.\quad \theta\right|_{z=0,1}=0
$$

where

$$
w=\mathbf{u} \cdot \hat{\mathbf{k}}, \quad h(z)=\sqrt{12}\left(z-\frac{1}{2}\right) .
$$

Since the functional $\mathcal{F}$ is homogeneous in both $w$ and $\theta$, we can impose two normalization conditions

$$
\langle h w \theta\rangle=1, \quad\left\langle w^{2}\right\rangle=\left\langle\theta^{2}\right\rangle
$$

\subsection{Multi- $\alpha$ Solution}

We are seeking the minimum of the functional $\mathcal{F}$ as $\mu \rightarrow \infty$. This implies that $\overline{w \theta}=h+\langle w \theta\rangle$ (here and in the following discussion the normalization condtions (55) have been assumed.) in most of the interval $0<z<1$, which makes the second term in the functional vanish in this interval. Only near the boundary $z=0,1$ the boundary conditions prevent a close appoach of $\overline{w \theta}$ to $h+\langle w \theta\rangle$. And the contribution to the functional is thus from possible boundary layers at $z=0,1$. Since $h(1)+\langle w \theta\rangle=\sqrt{3}+\left\langle|\nabla \mathbf{u}|^{2}\right\rangle>0$ (equation (45 and definition (50)), there must be a boundary layer at $z=1$. At $z=0, h(0)+\langle w \theta\rangle=$ $-\sqrt{3}+\langle w \theta\rangle$ is indefinite. Thus the existence of a boundary layer at $z=0$ depends on whether $h(0)+\langle w \theta\rangle$ is zero. Without loss of generality, we assume there are two boundary layers at $z=0,1$ respectively, and make the ansatz

$$
w=\sum w_{n} \phi_{n}+w_{n}^{*} \phi_{n}^{*}, \quad \theta=\sum \theta_{n} \phi_{n}+\theta_{n}^{*} \phi_{n}^{*},
$$

where $\phi_{n}$ and $\phi_{n}^{*}$ satify

$$
\Delta_{2} \phi_{n}=-\alpha_{n}^{2} \phi_{n}, \quad \Delta_{2} \phi_{n}^{*}=-\alpha_{n}^{* 2} \phi_{n}^{*}
$$

We introduce the following boundary layer variables:

$$
\begin{gathered}
w= \begin{cases}\mu^{-p_{n}} \hat{w}\left(\zeta_{n}\right) & \text { for } 1-z=O\left(\mu^{-r_{n}}\right), \\
\mu^{-s_{n}} \tilde{w}\left(\zeta_{n-1}\right) & \text { for } 1-z=O\left(\mu^{-r_{n-1}}\right)\end{cases} \\
\theta= \begin{cases}\mu^{p_{n}} \hat{\theta}\left(\zeta_{n}\right) & \text { for } 1-z=O\left(\mu^{-r_{n}}\right), \\
\mu^{s_{n}} \tilde{\theta}\left(\zeta_{n-1}\right) & \text { for } 1-z=O\left(\mu^{-r_{n-1}}\right)\end{cases} \\
w^{*}= \begin{cases}\mu^{-p_{n}} \hat{w}^{*}\left(\zeta_{n}^{*}\right) & \text { for } z=O\left(\mu^{-r_{n}}\right), \\
\mu^{-s_{n}} \tilde{w}^{*}\left(\zeta_{n-1}^{*}\right) & \text { for } z=O\left(\mu^{-r_{n-1}}\right)\end{cases} \\
\theta^{*}= \begin{cases}\mu^{p_{n}} \hat{\theta}^{*}\left(\zeta_{n}^{*}\right) & \text { for } z=O\left(\mu^{-r_{n}}\right), \\
\mu^{s_{n}} \tilde{\theta}^{*}\left(\zeta_{n-1}^{*}\right) & \text { for } z=O\left(\mu^{-r_{n-1}}\right)\end{cases}
\end{gathered}
$$


where

$$
\zeta_{n}=(1-z) \mu^{r_{n}}, \quad \zeta_{n}^{*}=z \mu^{r_{n}}
$$

The boudnary layer structure is such that in the interior

$$
\tilde{w}_{1} \tilde{\theta}_{1}+\tilde{w}_{1}^{*} \tilde{\theta}_{1}^{*} \approx h+\langle w \theta\rangle
$$

and in the boundary layers

$\tilde{w}_{n} \tilde{\theta}_{n}+\hat{w}_{n-1} \hat{\theta}_{n-1} \approx h_{1}+\langle w \theta\rangle, \quad \tilde{w}_{n}^{*} \tilde{\theta}_{n}^{*}+\hat{w}_{n-1}^{*} \hat{\theta}_{n-1}^{*} \approx h_{0}+\langle w \theta\rangle, \quad$ for $\quad n=1, \ldots, N-1$,

where

$$
h_{0}=h(0)=-\sqrt{3}, \quad h_{1}=h(1)=\sqrt{3}
$$

With boundary layer approximations, the functional becomes:

$$
\begin{aligned}
& \hat{\mathcal{F}}_{N}=\frac{1}{\langle w \theta\rangle}\left\{\sum_{1}^{N} \mu^{2 p_{n}+r_{n}}\left(\int_{0}^{\infty} \hat{\theta}_{n}^{\prime 2} d \zeta_{n}+\int_{0}^{\infty} \hat{\theta}_{n}^{*^{\prime} 2} d \zeta_{n}^{*}\right)\right. \\
& \left.+\sum_{2}^{N} \mu^{q_{n}-r_{n}+2 s_{n}}\left(b_{n}^{2} \int_{0}^{\infty} \tilde{\theta}_{n}^{2} d \zeta_{n-1}+b_{n}^{* 2} \int_{0}^{\infty} \tilde{\theta}_{n}^{* 2} d \zeta_{n-1}^{*}\right)+\mu^{q_{1}}\left(b_{1}^{2}\left\langle\tilde{\theta}_{1}^{2}\right\rangle+b_{1}^{* 2}\left\langle\tilde{\theta}_{1}^{* 2}\right\rangle\right)\right\} \\
& \cdot\left\{\sum_{1}^{N} \mu^{3 r_{n}-2 p_{n}-q_{n}}\left(\frac{1}{b_{n}^{2}} \int_{0}^{\infty} \hat{w}_{n}^{\prime \prime 2} d \zeta_{n}+\frac{1}{b_{n}^{* 2}} \int_{0}^{\infty} \hat{w}_{n}^{*^{\prime \prime 2}} d \zeta_{n}^{*}\right)\right. \\
& \left.+\sum_{2}^{N} \mu^{q_{n}-r_{n-1}-2 s_{n}}\left(b_{n}^{2} \int_{0}^{\infty} \tilde{w}_{n}^{2} d \zeta_{n-1}+b_{n}^{* 2} \int_{0}^{\infty} \tilde{w}_{n}^{* 2} d \zeta_{n-1}^{*}\right) \mu^{q_{1}}\left(b_{1}^{2}<\tilde{w}_{1}^{2}>+b_{1}^{* 2}\left\langle\tilde{w}_{1}^{* 2}\right\rangle\right)\right\} \\
& +\left\{\mu^{1-r_{N}}\left(\int_{0}^{\infty}\left(\hat{w}_{N} \hat{\theta}_{N}-h_{1}-<w \theta>\right)^{2} d \zeta_{N}+\int_{0}^{\infty}\left(\hat{w}_{N}^{*} \hat{\theta}_{N}^{*}-h_{0}-<w \theta>\right)^{2} d \zeta_{N}\right)\right\}
\end{aligned}
$$

Balancing the exponents in the above exrepssion yields

$$
r_{n}=\frac{1-4^{-n}}{3-4^{-n}}, \quad q_{n}=\frac{2-4^{-n}}{3-4^{-n}}, \quad s_{n}=0, \quad 2 p_{n}=\frac{4^{-n}}{3-4^{-n}} .
$$

Then we have

$$
\hat{\mathcal{F}}_{N}=\mu^{\frac{2}{3-4^{-N}}} F_{N}
$$


where

$$
\begin{aligned}
F_{N}= & \frac{1}{\langle w \theta\rangle}\left\{\sum_{1}^{N}\left(\int_{0}^{\infty} \hat{\theta}_{n}^{\prime 2} d \zeta_{n}+\int_{0}^{\infty} \hat{\theta}_{n}^{*^{\prime} 2} d \zeta_{n}^{*}\right)\right. \\
& \left.\sum_{2}^{N}\left(b_{n}^{2} \int_{0}^{\infty} \tilde{\theta}_{n}^{2} d \zeta_{n-1}+b_{n}^{* 2} \int_{0}^{\infty} \tilde{\theta}_{n}^{* 2} d \zeta_{n-1}^{*}\right)+\left(b_{1}^{2}<\tilde{\theta}_{1}^{2}>+b_{1}^{* 2}<\tilde{\theta}_{1}^{* 2}>\right)\right\} \\
& \cdot\left\{\sum_{1}^{N}\left(\frac{1}{b_{n}^{2}} \int_{0}^{\infty} \hat{w}_{n}^{\prime \prime 2} d \zeta_{n}+\frac{1}{b_{n}^{* 2}} \int_{0}^{\infty} \hat{w}_{n}^{* \prime 2} d \zeta_{n}^{*}\right)\right. \\
& \left.\sum_{2}^{N}\left(b_{n}^{2} \int_{0}^{\infty} \tilde{w}_{n}^{2} d \zeta_{n-1}+b_{n}^{* 2} \int_{0}^{\infty} \tilde{w}_{n}^{* 2} d \zeta_{n-1}^{*}\right)+\left(b_{1}^{2}<\tilde{w}_{1}^{2}>+b_{1}^{* 2}<\tilde{w}_{1}^{* 2}>\right)\right\} \\
& +\left\{\int_{0}^{\infty}\left(\hat{w}_{N} \hat{\theta}_{N}-h_{1}-<w \theta>\right)^{2} d \zeta_{N}+\int_{0}^{\infty}\left(\hat{w}_{N}^{*} \hat{\theta}_{N}^{*}-h_{0}-<w \theta>\right)^{2} d \zeta_{N}^{*}\right\} .
\end{aligned}
$$

Now the Euler-Lagrange equatins for the functional $F_{N}$ can be written down:

$$
\begin{aligned}
& \frac{1}{\langle w \theta\rangle} \frac{D_{\theta}}{b_{n}^{2}} \hat{w}_{n}^{(4)}-\mu^{r_{N}-r_{n}} \hat{\theta}_{n}\left(h_{1}+\left\langle w \theta-\hat{w}_{n} \hat{\theta}_{n}-\tilde{w}_{n+1} \tilde{\theta}_{n+1}\right)=0,\right. \\
& \frac{1}{\langle w \theta\rangle} D_{w} \hat{\theta}_{n}^{\prime \prime}+\mu^{r_{N}-r_{n}} \hat{w}_{n}\left(h_{1}+\left\langle w \theta>-\hat{w}_{n} \hat{\theta}_{n}-\tilde{w}_{n+1} \tilde{\theta}_{n+1}\right)=0, \quad n=1, \ldots, N\right. \\
& \frac{b_{n+1}^{2}}{\langle w \theta\rangle} D_{\theta} \tilde{w}_{n+1}-\mu^{r_{N}-r_{n}} \tilde{\theta}_{n+1}\left(h_{1}+\left\langle w \theta>-\hat{w}_{n} \hat{\theta}_{n}-\tilde{w}_{n+1} \tilde{\theta}_{n+1}\right)=0\right. \\
& \frac{b_{n+1}^{2}}{\langle w \theta\rangle} D_{w} \tilde{\theta}_{n+1}-\mu^{r_{N}-r_{n}} \tilde{w}_{n+1}\left(h_{1}+\langle w \theta\rangle-\hat{w}_{n} \hat{\theta}_{n}-\tilde{w}_{n+1} \tilde{\theta}_{n+1}\right)=0, \quad n=1, \ldots, N-1
\end{aligned}
$$

And for $\tilde{w}_{1}, \tilde{\theta}_{1}$,

$$
\begin{aligned}
& \frac{D_{\theta}}{\langle w \theta\rangle} b_{1}^{2} \tilde{w}_{1}-\tilde{\theta}_{1}\left\{\frac{D_{\theta} D_{w}}{2\langle w \theta\rangle^{2}}(h\langle w \theta\rangle+1)+\mu^{r_{N}}\left(h+\langle w \theta\rangle-\tilde{w}_{1} \tilde{\theta}_{1}-\tilde{w}_{1}^{*} \tilde{\theta}_{1}^{*}\right)\right. \\
& \left.\quad+h\left(\int_{0}^{\infty}\left(\hat{w}_{N} \hat{\theta}_{N}-h_{1}-\langle w \theta\rangle\right)^{2} d \zeta_{N}+\int_{0}^{\infty}\left(\hat{w}_{N}^{*} \hat{\theta}_{N}^{*}-h_{0}-\langle w \theta\rangle\right)^{2} d \zeta_{N}^{*}\right)\right\}=0, \\
& \frac{D_{w}}{\langle w \theta\rangle} b_{1}^{2} \tilde{\theta}_{1}-\tilde{w}_{1}\left\{\frac{D_{\theta} D_{w}}{2\langle w \theta\rangle^{2}}(h\langle w \theta\rangle+1)+\mu^{\tau_{N}}\left(h+\langle w \theta\rangle-\tilde{w}_{1} \tilde{\theta}_{1}-\tilde{w}_{1}^{*} \tilde{\theta}_{1}^{*}\right)\right. \\
& \quad+h\left(\int_{0}^{\infty}\left(\hat{w}_{N} \hat{\theta}_{N}-h_{1}-\langle w \theta>)^{2} d \zeta_{N}+\int_{0}^{\infty}\left(\hat{w}_{N}^{*} \hat{\theta}_{N}^{*}-h_{0}-\langle w \theta\rangle\right)^{2} d \zeta_{N}^{*}\right)\right\}=0,
\end{aligned}
$$

The same set of equations are also satisfied by the starred quantities $\tilde{w}_{n}^{*}, \tilde{\theta}_{n}^{*}, \hat{w}_{n}^{*}, \hat{\theta}_{n}^{*}$.

From equation (75) and (76), we have

$$
\begin{aligned}
& D_{\theta} \tilde{w}_{1}^{2}=D_{w} \tilde{\theta}_{1}^{2}, \\
& D_{\theta} \tilde{w}_{1}^{* 2}=D_{w} \tilde{\theta}_{1}^{* 2} .
\end{aligned}
$$


Adding these two identities yields

$$
D_{\theta}\left\langle\tilde{w}_{1}^{2}+\tilde{w}_{1}^{* 2}\right\rangle=D_{w}\left\langle\tilde{\theta}_{1}^{2}+\tilde{\theta}_{1}^{* 2}\right\rangle .
$$

Hence the normalization condition $\left.\left\langle w^{2}\right\rangle=<\theta^{2}\right\rangle$ implies

$$
D_{\theta}=D_{w}=D \text {. }
$$

This identity together with equation (75) and (76) yields

$$
\tilde{w}_{1}^{2}=\tilde{\theta}_{1}^{2}, \quad b_{1}=b_{1}^{*} .
$$

Equation (73) together with equation (74) gives

$$
D_{\theta} \tilde{w}_{n+1}^{2}=D_{w} \tilde{\theta}_{n+1}^{2}
$$

Same identity holds for $\tilde{w}_{n+1}^{*}$ and $\tilde{\theta}_{n+1}^{*}$. Therefore

$$
\tilde{w}_{n+1}^{2}=\tilde{\theta}_{n+1}^{2}, \tilde{w}_{n+1}^{* 2}=\tilde{\theta}_{n+1}^{* 2} \quad \text { for } n=1, \ldots, N-1 .
$$

Substitute the above identity back into equation (73), we have

$$
\begin{array}{ll}
h_{1}+<w \theta>-\hat{w}_{n} \hat{\theta}_{n}-\tilde{w}_{n+1} \tilde{\theta}_{n+1}=\mu^{r_{n}-r_{N}} b_{n+1}^{2} \frac{D}{<w \theta>}, & n=1, \ldots, N-1, \\
h_{0}+<w \theta>-\hat{w}_{n}^{*} \hat{\theta}_{n}^{*}-\tilde{w}_{n+1}^{*} \tilde{\theta}_{n+1}^{*}=\mu^{r_{n}-r_{N}} b_{n+1}^{* 2} \frac{D}{<w \theta>}, \quad n=1, \ldots, N-1 .
\end{array}
$$

Then equation (71) and equation (72) become

$$
\begin{aligned}
\frac{1}{b_{n}^{2}} \hat{w}_{n}^{(4)}-b_{n+1}^{2} \hat{\theta}_{n} & =0, \\
\hat{\theta}_{n}^{\prime \prime}+b_{n+1}^{2} \hat{w}_{n} & =0, \quad n=1, \ldots, N-1 .
\end{aligned}
$$

The above equations hold in the region where $\hat{w}_{n} \hat{\theta}_{n} \neq h_{1}+\langle w \theta\rangle$. When the equality holds, then from equation (71) and equation (72) we can derive:

$$
\frac{\hat{w}_{n}^{(4)}}{b_{n}^{2}}=-\frac{\hat{\theta}_{n} \hat{\theta}_{n}^{\prime \prime}}{w_{n}}=\left(h_{1}+\langle w \theta\rangle\right)^{2} \frac{\hat{w}_{n}^{\prime \prime} \hat{w}_{n}-2 \hat{w}_{n}^{\prime 2}}{\hat{w}_{n}^{5}}
$$

With the following change of variables,

$$
\left\{\begin{array}{l}
\zeta=b_{n}^{1 / 3} b_{n+1}^{2 / 3} \zeta_{n} \\
\hat{\Omega}=b_{n}^{-1 / 3} b_{n+1}^{1 / 3}\left(h_{1}+\langle h w \theta\rangle\right)^{-1 / 2} \hat{w}_{n} \\
\hat{\Theta}=b_{n}^{1 / 3} b_{n+1}^{-1 / 3}\left(h_{1}+\langle h w \theta\rangle\right)^{-1 / 2} \hat{\theta}_{n}
\end{array}\right.
$$

equations (85), (86) and (87) become:

$$
\left\{\begin{array}{l}
\hat{\Omega}^{(4)}-\hat{\Theta}=0 \\
\hat{\Theta}^{\prime \prime}+\hat{\Omega}=0 \\
\hat{\Omega}^{(4)}=\frac{\hat{\Omega}^{\prime \prime} \hat{\Omega}-2 \hat{\Omega}^{\prime 2}}{\hat{\Omega}^{5}}
\end{array}\right.
$$


Starred quantities satisfy the same equations with $h_{1}$ replaced by $h_{0}$. This set of differential equations have been studied in $]$, which gives the constant $\beta$ :

$$
3 \beta=\int_{0}^{\infty} \hat{\Omega}^{\prime \prime 2} d \zeta+\int_{0}^{\infty}(1-\hat{\Omega} \hat{\Theta}) d \zeta
$$

And the integral

$$
\int_{0}^{\infty} \frac{w_{n}^{\prime \prime 2}}{b_{n}^{2}} d \zeta_{n}+\int_{0}^{\infty} b_{n+1}^{2} \tilde{w}_{n+1}^{2} d \zeta_{n}=3 \beta\left(h_{1}+\langle w \theta\rangle\right) b_{n}^{-1 / 3} b_{n+1}^{4 / 3}, \quad n=1, \ldots, N-1
$$

When $n=N$, the differential equations for $\hat{w}_{N}$ and $\hat{\theta}_{N}$ are

$$
\begin{aligned}
& \frac{D}{\left\langle w \theta>b_{N}^{2}\right.} \hat{w}_{N}^{(4)}-\left(h_{1}+\langle w \theta\rangle-\hat{w}_{N} \hat{\theta}_{N}\right) \hat{\theta}_{N}=0 \\
& \frac{D}{<w \theta>b_{N}^{2}} \hat{\theta}_{N}^{\prime \prime}+\left(h_{1}+\langle w \theta\rangle-\hat{w}_{N} \hat{\theta}_{N}\right) \hat{w}_{N}=0
\end{aligned}
$$

With the following change of variables,

$$
\left\{\begin{array}{l}
\zeta=b_{n}^{1 / 3}\left(h_{1}+\langle w \theta\rangle\right)^{1 / 3}\left(\frac{D}{\langle w \theta\rangle}\right)^{-1 / 3} \zeta_{N} \\
\Omega=b_{n}^{-1 / 3}\left(h_{1}+\langle h w \theta\rangle\right)^{-1 / 3}\left(\frac{D}{\langle w \theta\rangle}\right)^{-1 / 6} \hat{w}_{N} \\
\Theta=b_{n}^{1 / 3}\left(h_{1}+\langle h w \theta\rangle\right)^{-2 / 3}\left(\frac{D}{\langle w \theta\rangle}\right)^{1 / 6} \hat{\theta}_{N}
\end{array}\right.
$$

equation (92) and (93) become

$$
\begin{gathered}
\Omega^{(4)}-(1-\Omega \Theta) \Theta=0 \\
\Theta^{\prime \prime}+(1-\Omega \Theta) \Omega=0
\end{gathered}
$$

In ([2]) the following result is given:

$$
\sigma=\int_{0}^{\infty} \Omega^{\prime \prime 2} d \zeta=\int_{0}^{\infty} \Theta^{\prime 2} d \zeta=\frac{1}{4} \int_{0}^{\infty}(1-\Omega \Theta)^{2} d \zeta
$$

Thus the following integrals can be expressed in $\sigma$ :

$$
\begin{gathered}
\int_{0}^{\infty} \frac{\left(\hat{w}^{(4)}\right)^{2}}{b_{N}^{2}} d \zeta_{N}=\sigma\left(h_{1}+\langle w \theta\rangle\right)^{5 / 3}\left(\frac{D}{\langle w \theta\rangle}\right)^{-2 / 3} b_{N}^{-1 / 3}, \\
\int_{0}^{\infty} \hat{\theta}_{N}^{\prime} d \zeta_{N}=\sigma\left(h_{1}+\langle w \theta\rangle\right)^{5 / 3}\left(\frac{D}{\langle w \theta\rangle}\right)^{-2 / 3} b_{N}^{-1 / 3}, \\
\int_{0}^{\infty}\left(h_{1}+\langle h w \theta\rangle-\hat{w}_{N} \hat{\theta}_{N}\right)^{2} d \zeta_{N}=4 \sigma\left(h_{1}+\langle w \theta\rangle\right)^{5 / 3}\left(\frac{D}{\langle w \theta\rangle}\right)^{1 / 3} b_{N}^{-1 / 3} .
\end{gathered}
$$

Putting the above integrals together, the functional $F_{N}$ can be expressed as

$$
F_{N}=\frac{D^{2}}{\langle w \theta\rangle}+4 \sigma\left(h_{1}+\langle w \theta\rangle\right)^{5 / 3}\left(\frac{D}{\langle w \theta\rangle}\right)^{1 / 3} b_{N}^{-1 / 3}+4 \sigma\left(h_{0}+\langle w \theta\rangle\right)^{5 / 3}\left(\frac{D}{\langle w \theta\rangle}\right)^{1 / 3} b_{N}^{*-1 / 3}
$$


and

$$
\begin{aligned}
D= & \sum_{n=1}^{N-1} 3 \beta\left\{\left[\frac{b_{n+1}^{4}}{b_{n}}\right]^{1 / 3}\left(h_{1}+\langle w \theta\rangle\right)+\left[\frac{b_{n+1}^{* 4}}{b_{n}^{*}}\right]^{1 / 3}\left(h_{0}+\langle w \theta\rangle\right)\right\} \\
& +\sigma\left(\frac{D}{\langle w \theta\rangle}\right)^{-2 / 3}\left\{\left(h_{1}+\langle w \theta\rangle\right)^{5 / 3} b_{N}^{-1 / 3}+\left(h_{0}+\langle w \theta\rangle\right)^{5 / 3} b_{N}^{*-1 / 3}\right\}+b_{1}^{2}\langle w \theta\rangle .
\end{aligned}
$$

Minimizing $F_{N}$ with respect to $b_{n}$ and $b_{n}^{*}$ yields

$$
\begin{aligned}
& \frac{\partial D}{\partial b_{1}}=0 \Rightarrow 2 b_{1}\langle w \theta\rangle=\beta {\left[\left(h_{1}+\langle w \theta\rangle\right)\left(\frac{b_{2}}{b_{1}}\right)^{4 / 3}+\left(h_{0}+\langle w \theta\rangle\right)\left(\frac{b_{2}^{*}}{b_{1}}\right)^{4 / 3}\right], } \\
& \frac{\partial D}{\partial b_{n}}=0 \Rightarrow\left[\frac{b_{n+1}}{b_{n}}\right]^{4 / 3}=4\left[\frac{b_{n}}{b_{n-1}}\right]^{1 / 3}, \\
& \frac{\partial D}{\partial b_{n}^{*}}=0 \Rightarrow\left[\frac{b_{n+1}^{*}}{b_{n}^{*}}\right]^{4 / 3}=4\left[\frac{b_{n}^{*}}{b_{n-1}^{*}}\right]^{1 / 3}, \\
& \frac{\partial F_{N}}{\partial b_{N}}=0 \Rightarrow\left[\frac{b_{N+1}}{b_{N}}\right]^{4 / 3}=4\left[\frac{b_{N}}{b_{N-1}}\right]^{1 / 3}, \\
& \frac{\partial D}{\partial b_{N}^{*}}=0 \Rightarrow\left[\frac{b_{N+1}^{*}}{b_{N}^{*}}\right]^{4 / 3}=4\left[\frac{b_{N}^{*}}{b_{N-1}^{*}}\right]^{1 / 3},
\end{aligned}
$$

where

$$
\begin{aligned}
& b_{N+1}=\left(\frac{\sigma}{\beta}\right)^{4 / 3}\left(\frac{\left(h_{1}+\langle w \theta\rangle\right)\langle w \theta\rangle}{D}\right)^{1 / 2}, \\
& b_{N+1}^{*}=\left(\frac{\sigma}{\beta}\right)^{4 / 3}\left(\frac{\left(h_{0}+\langle w \theta\rangle\right)\langle w \theta\rangle}{D}\right)^{1 / 2} .
\end{aligned}
$$

From the above relations, $b_{n}$ can be solved

$$
b_{n+1}=4^{n-1}\left[\left(\frac{b_{N+1}}{4^{N-1}}\right)^{1-4^{-n}} \cdot\left(4 b_{1}\right)^{4^{-n}-4^{-N}}\right]^{\frac{1}{1-4^{-N}}} .
$$

$b_{n+1}^{*}$ has a similar form

$$
b_{n+1}^{*}=4^{n-1}\left[\left(\frac{b_{N+1}^{*}}{4^{N-1}}\right)^{1-4^{-n}} \cdot\left(4 b_{1}\right)^{4^{-n}-4^{-N}}\right]^{\frac{1}{1-4^{-N}}}
$$

It is clear from the above expressions that $b_{n} \neq b_{n}^{*}$ for $n \neq 1$ since $b_{N}$ (equation (108) is different from $b_{N}^{*}$ (equation (109). $b_{1}$ can be solved from equation (103) and the recursion relation

$$
b_{1}=\left\{\frac{\beta}{2^{5 / 3}\langle w \theta\rangle}\left(\frac{\sigma}{\beta}\right)^{\frac{3}{4\left(1-4^{-N}\right)}}\left[\left(h_{1}+\langle w \theta\rangle\right)^{\frac{4\left(1-4^{-N}\right)}{3-4^{-N}}}+\left(h_{0}+\langle w \theta\rangle\right)^{\frac{4\left(1-4^{-N}\right)}{3-4^{-N}}}\right]\right\}^{\frac{1-4^{-N}}{3-4^{-N}}}
$$


Putting all these together, the prefactor $F_{N}$ is a function of $\langle w \theta\rangle$ only:

$$
\begin{aligned}
F_{N} & =\frac{D^{2}}{<w \theta>} \frac{3-4^{-N}}{1-4^{-N}} \\
& =\left(3-4^{-N}\right)\left(1-4^{-N}\right) 2^{\frac{-4 N 4^{-N}}{3-4^{-N}}} \cdot\left(2^{5 / 3} \beta \times\left(\frac{\sigma}{\beta}\right)^{\frac{3}{4\left(1-4^{-N}\right)}}\right)^{\frac{4\left(1-4^{-N}\right)}{3-4^{-N}}} \\
& \times\left[\frac{\left(h_{1}+\langle w \theta\rangle\right)^{\frac{3-2 \cdot 4^{-N}}{2\left(1-4^{-N}\right)}}+\left(h_{0}+\langle w \theta\rangle\right)^{\frac{3-2 \cdot 4^{-N}}{2\left(1-4^{-N}\right)}}}{\langle w \theta\rangle^{\frac{1-3 \cdot 4^{-N}}{4\left(1-4^{-N}\right)}}}\right]^{\frac{4\left(1-4^{-N}\right)}{3-4^{-N}}}
\end{aligned}
$$

Now the value of $\langle w \theta\rangle$ can be determined by setting $\frac{F_{N}}{\langle w \theta\rangle}$ to zero. The resulting equation for $\langle w \theta\rangle$ is

$$
(\alpha-1) x^{\frac{3-2 c}{2(1-c)}}-\alpha x-\alpha x^{\frac{1}{1-c}}+(\alpha-1)=0
$$

where

$$
x=\frac{\sqrt{3}+<w \theta>}{\sqrt{3}-<w \theta>}, \quad \alpha=\frac{3-2 c}{1-3 c}, \quad c=4^{-N} .
$$

For general values of $N$, the above equation has to be solved numerically:

$$
\begin{array}{llrl}
N=1, & & \langle w \theta\rangle=0.4831 \\
N=2, & & \langle w \theta\rangle=0.9259 \\
N=3, & & \langle w \theta\rangle=1.0120
\end{array}
$$

When $N \rightarrow \infty$, the above equation can be solved exactly:

$$
\langle w \theta\rangle_{\infty}=\frac{3 \sqrt{3}}{5}=1.039
$$

This shows that there indeed is a boundary layer at $z=0$ since all $\langle w \theta\rangle$ 's are less than $h_{0}=\sqrt{3}$. Now we can write down the scaling of $\langle T\rangle$ as $N \rightarrow \infty$ :

$$
\langle T\rangle=\frac{1}{\sqrt{12}} F_{\infty} \mu^{2 / 3}=10.285 \mu^{2 / 3} .
$$

Recalling the identity (47) with $T_{0}=0$ :

$$
\langle T\rangle=-\left\langle\left(z-\frac{1}{2}\right) w \theta\right\rangle+\frac{1}{12} R,
$$

we know that as $\mu \rightarrow \infty$

$$
\mu \sim \frac{1}{\sqrt{12}} R
$$


This leads to the scaling of $\langle T\rangle$ with respect to $R$ :

$$
\langle T\rangle \sim 4.421 R^{2 / 3}
$$

The profiles of $\tilde{w}_{1}$ and $\tilde{\theta}_{1}$ can be determined from the fact that in the interior of the interval $0<z<1$

$$
\tilde{w}_{1} \tilde{\theta}_{1} \approx h+\langle w \theta\rangle, \quad \text { and } \quad \tilde{w}_{1}=\tilde{\theta}_{1} \text {. }
$$

In the case $N \rightarrow \infty, h=2 \sqrt{3} z-\frac{2 \sqrt{3}}{5}$. And then

$$
\tilde{w}_{1}=\sqrt{\left|2 \sqrt{3} z-\frac{2 \sqrt{3}}{5}\right|}, \quad \tilde{\theta}_{1}= \pm \sqrt{\left|2 \sqrt{3} z-\frac{2 \sqrt{3}}{5}\right|}
$$

However, whether $\theta$ changes sign in $0<z<1$ can not be inferred from the variational problem since only the product of $w$ and $\theta$ appears in the funcional $\mathcal{F}$. Thus the possibility of $w$ changing its sign can not be excluded.

\section{Conclusion}

In this project, the scaling of the min $\langle T\rangle$ has been studied for an internally heated fluid layer with both background method and multi- $\alpha$ solution approach. For the case when two plates are held at the same temperature these two methods yield the same scaling: $\langle T\rangle \sim R^{2 / 3}$. The prefactor given by the background method is about a quarter of that from the other appoach. By adjusting the background field we expect the prefactor to be closer to that predicted by the multi- $\alpha$ approach. However, The scaling of the minimum average temperature when two plates are at different temperatures is not clear yet. It is part of our future work to investicate the scaling in this case.

\section{References}

[1] W. Malkus, "The heat transport and spectrum of thermal turbulence," Proc. Roy. Soc. London 225, 196 (1954).

[2] L. Howard, "Heat transport by turbulent convecton," J. Fluid Mech. 17, 405 (1963).

[3] F. Busse, "On Howard's upper bound for heat transport by turbulent convection," J. Fluid Mech. 37, 457 (1969).

[4] F. Busse, "Bounds for turbulent shear flow," J. Fluid Mech. 41, 219 (1970).

[5] F. Busse and D. Joseph, "Bounds for heat transport in a porous layer," J. Fluid Mech. 54, 521 (1972).

[6] S.-K: Chan, "Infinite prandtl number turbulent convectoin," Stud. Appl. Maths 50, 13 (1971). 
[7] F. Busse, "The optimum theory of turbulence," Adv. Appl. Mech. 18, 77 (1978).

[8] C. Doering and P. Constantin, "Energy dissipation in shear driven turbulence," Phys. Rev. Lett. 69, 1648 (1992).

[9] C. Doering and P. Constantin, "Variatoinal bounds on energy dissipation in incompressible flows: Iii. convection," Phys. Rev. E 53, 5957 (1996).

[10] P. Constantin and C. Doering, "Infinite Prandtl number convection," J. Statist. Phys. 94, 159 (1999).

[11] A. Pellew and R. Southwell, "On maintained convective motion in a fluid heated from bellow," Proc. Roy. Soc. A 176, 312 (1940). 


\title{
Ball Release Experiment in a Centrifuge
}

\author{
Ulrike Riemenschneider \\ University of Southampton, U.K.
}

\section{Motivation}

The ball release experiments outlined in this work were motivated by laboratory experiments with tilted convective plumes on a centrifuge carried out by Sheremet (2002) [1]. These plume experiments were done to study the effect of the misalignment of gravity and Coriolis on small convective cells, which are argued to play an important role in the process of deep convection occurring in polar regions, such as the Labrador, Greenland and Weddell Seas. In these regions the stratification of the water column is often observed to be very weak, meaning that the underlying deep water is only marginally denser than the overlying surface waters. In the winter months, when wind surface cooling is very effective, the surface waters may actually become denser and large patches of water of up to hundreds of kilometers across may be vertically mixed to a depth of one to two kilometers in periods of only a few weeks. Field observations have suggested that most of the mixing actually happens in smaller convective cells, with horizontal scales of less than one kilometer. It is the effect of both components of rotation on these cells that was studied by Sheremet. The argument is that some of the convective motion maybe aligned along the axis of rotation, rather than along the axis parallel to the action of the gravitational acceleration, due to the TaylorProudman theorem (TP) constraining change in the horizontal velocities along the axis of rotation.

In these experiments the plumes were produced by injecting slightly dense coloured salty water into a tank of freshwater mounted on a centrifuge which was rotating at a constant angular velocity, and the formation of tilted Taylor columns ('Taylor ink-walls') was observed. Sheremet al.so found that for low Reynolds numbers the incoming jet of dyed fluid disintegrates into coherent blobs which descend neither in the direction of the buoyancy force $g_{e}$ nor in the direction of rotation $\Omega$. Instead they assume a trajectory at an angle which compares quite well with linear theory developed by Loper [2].

It is this blob structure of the plume that sparked the idea of studying the behavior of small spheres in the same experimental set up. In particular to see whether the behavior of plumes could be represented by the behavior of small solid spheres.

In this report, the experimental set up will be described in the section 2 , then the physics of flow around spheres will be briefly reviewed in section 3 . In section 4 the force balance on the particle will be outlined and following in section 5 will be the presentation of the experimental results, for the tilted experiments, as well as centre rotating and non-rotating experiments which were carried out for comparison. Finally we will mention the difficulties encountered in determining the density of the nylon spheres used in the experiment in section 6 and conclusions will be drawn in section 7. Appendix A contains tables of the experimental results. 


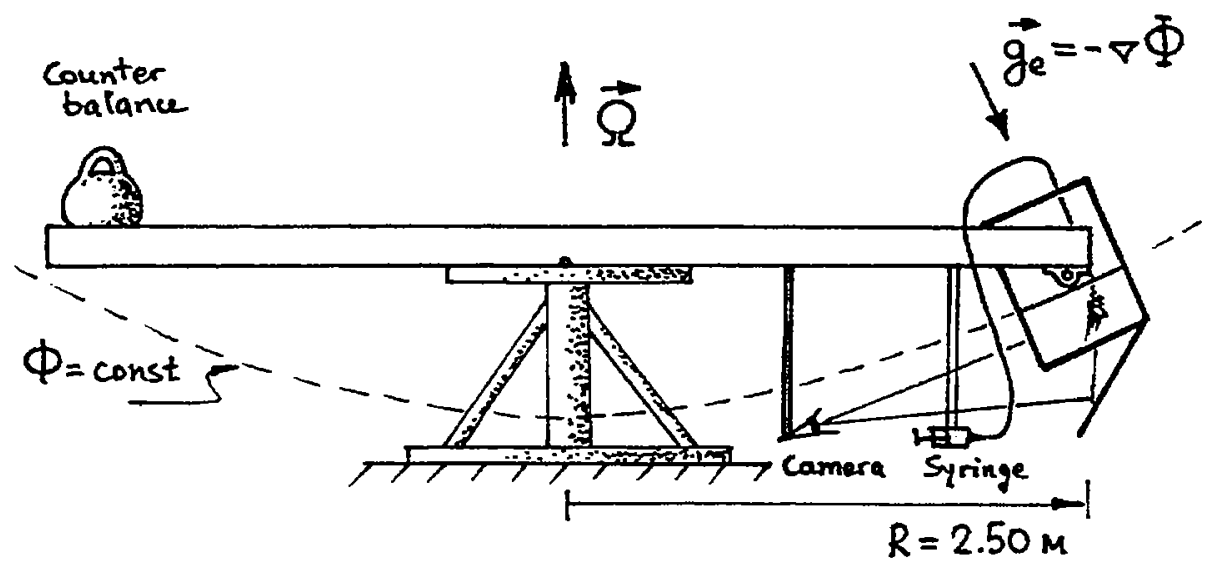

Figure 1: Sketch of the experimental set up as described in section 2

\section{Experimental Setup}

A sketch of the apparatus is shown in figure 1. The experiment was carried out on a centrifuge mounted on a rotating table and consisting of a wooden frame extending 2.5 meters in both directions away from the centre of the rotating table. A box-shaped Plexiglas tank of inner dimensions $29 \times 29 \times 50 \mathrm{~cm}$ (width $\mathrm{x}$ depth $\mathrm{x}$ height) was mounted on one end of the centrifuge at a distance of $246 \mathrm{~cm}$ from the centre of the table. Weights are attached to the opposite end to keep the centrifuge in balance. The tank was attached with two pivots so that it could be tilted inward at an angle of $30^{\circ}$ degrees and the rotation rate was adjusted to $2 \Omega=3.026 \mathrm{~s}^{-1}$ so that when the apparatus was spinning with the full angular velocity the surface of the water in the tank was parallel to the bottom of the tank. The tank was filled with enough water to ensure that the surface of the water was about $20 \mathrm{~cm}$ above the bottom when the tank was rotating. A lid was inserted into the tank such that the bottom $20 \mathrm{~cm}$ form a body of fluid that is bounded by solid walls on all sides. On top of the lid a remotely controlled release mechanism for the balls was attached, which allowed the nylon spheres to drop through a hole at the centre of the lid. The mechanism consisted of a metal tube containing up to 8 balls of $0.653 \mathrm{~cm}(1 / 2 \mathrm{inch})$ diameter, a Plexiglas slide transferring the balls from the tube to the hole in the lid and a solenoid pulling the slide. A sketch of the tank is shown in figure 2

The balls were marked with coloured lines around three geodesics perpendicular to each other in order to observe possible spinning motion.

Below the wooden arm a camera was mounted (digital or video) in order to record the experiment. Two mirrors were attached to the tank at an angle, one below it to provide a view from the bottom, and one on the right hand side, providing a view from the side onto the motion in the tank. A stop watch with $0.01 s$ resolution was also attached to the tank, to monitor the spin-up time, record the time of release of the spheres and measure their time of decent. The spin up time was 30 minutes and after a ball had reached the bottom we waited about 2 to 3 minutes before releasing the next ball in order to allow transient 


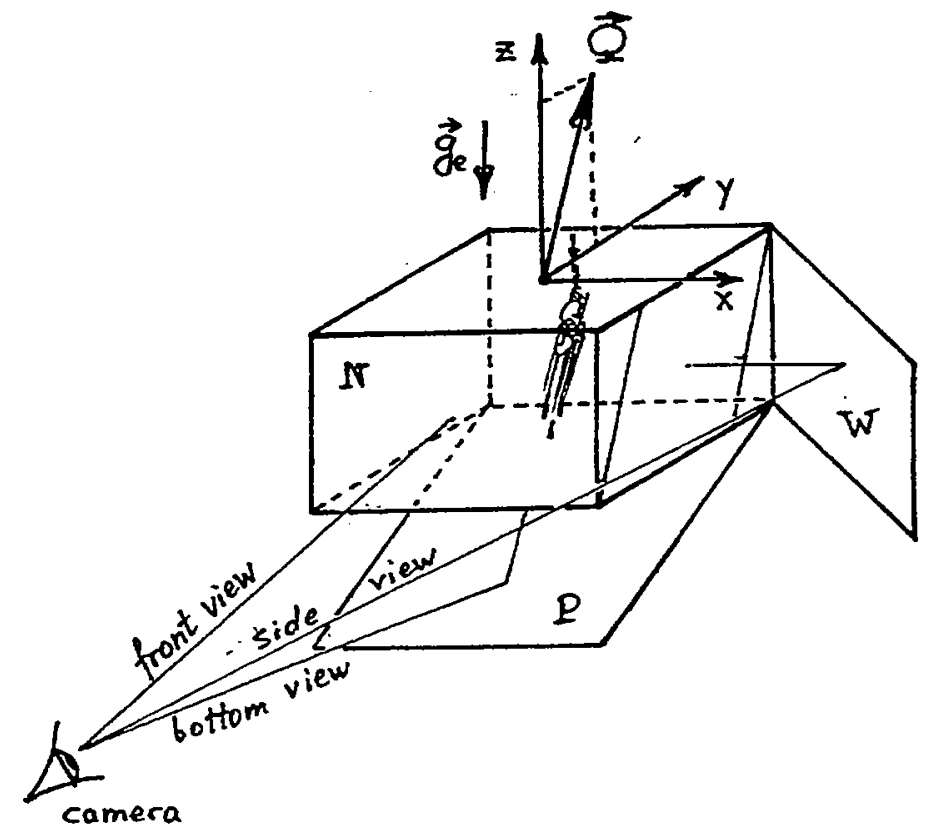

Figure 2: Schematic of the tank, showing the two mirrors below and on the right hand side of the tank to give a three dimensional view of the motion inside the tank.

motions to subside.

For experiments with the motion of the sphere co-axial along the axis of rotation, the tank is mounted at the centre of the rotating table.

\section{Taylor Clumn Formation and Wall Effects}

Taylor (1917) [3] discovered analytically that in a geostrophically balanced flow, for sufficiently low Rossby numbers, the vertical gradient of the horizontal velocity components is zero: $\frac{d u}{d z}=\frac{d v}{d z}=0$, this result is referred to as the Taylor-Proudman Theorem (TP). Later these findings were verified in laboratory experiments which showed the formation of Taylor columns as a result of the Taylor-Proudman constraint. The mechanism for the development of this column is that as the sphere is trying to move down through a water column, the fluid ahead of it has to move radially out of the way to allow the sphere to descent. The TP theorem states however, that fluid in solid body rotation that does not have any radial velocity initially cannot acquire any either. The only way in which the sphere can actually sink or rise, is due to a relaxation of the TP constraint in thin Ekman layers which develop on the surface of the sphere and at the top and bottom boundaries of the tank. If the boundaries of the tank are at a large distance away from the sphere, or at infinity in a theoretical consideration, the Taylor column will be gradually 'eroded' by viscous and inertial effects far away from the sphere and what is observed is a Taylor slug. Figure 3 shows both, the structure of a Taylor column as well as that of a Taylor slug. 

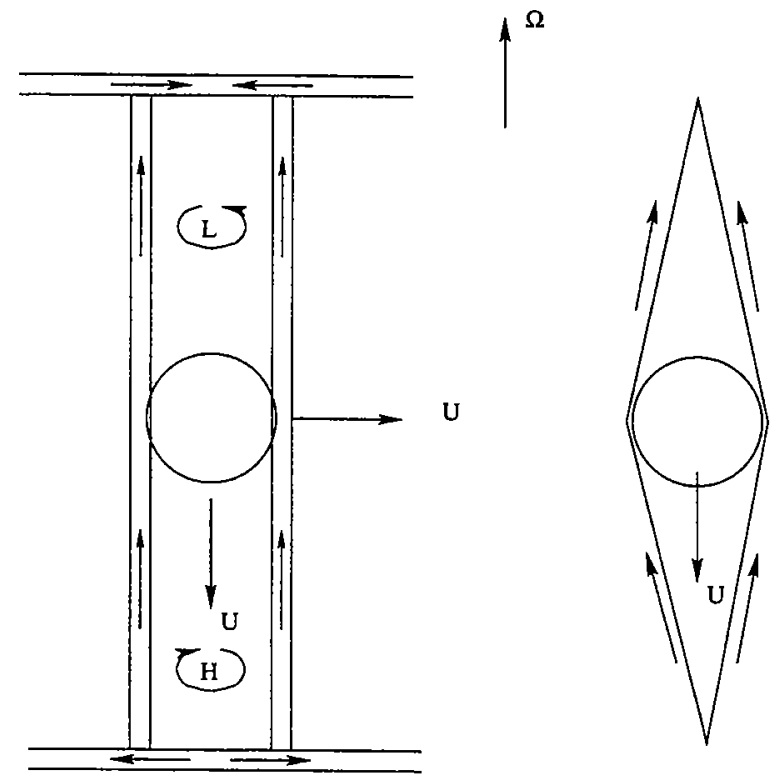

Figure 3: Sketch of the structure of a Taylor column interacting with the boundaries on the left and on the right a sketch of the appearance of the Taylor slug in the unbounded case. Both features may develop for motion of the sphere perpendicular, parallel or at some angle to the axis of rotation, however they will always be aligned with rotation.

There is a lot of uncertainty in determining the conditions under which Taylor columns from or do not form, the only sure way of knowing would be to try and visualise the flow around the sphere with dye, which we did not attempt in the experiments presented here.

\subsection{Motion Along the Axis of Rotation}

For coaxial motion of the particle Taylor observed that the Rossby number $R o=U / \Omega a$, where $a$ is the radius of the sphere, must be less than a critical value $1 / \pi$ for there to be a columnar structure accompanying the sphere. (Note: usually the Rossby number is defined to be $R o=U / 2 \Omega a$, but in Bush et al. [4] it is stated without the factor of $1 / 2$ ). Other experiments have suggested critical values as high as 0.7 Pritchard (1969) for substantial Taylor column formation. In the tilted experiments the Rossby number was typically about between 0.1 and 0.6 for motion parallel to the axis of rotation (we define $R o_{\|}=U_{\|} / \Omega a$ ). These numbers suggest that there may or may not be a column depending on which criterion you believe. For motion at negligibly small Rossby numbers, the vertical extent of the Taylor column is determined by the fluid viscosity. The theory predicts that a truncated Taylor column, or 'Taylor slug' will extend a characteristic distance $a T$ up- and downstream of a sphere of radius $a$. Maxworthy (1970) [5] however found experimentally, that the column is typically an order of magnitude less than that. Based on his findings we may expect a Taylor slug extending for about $3 \mathrm{~cm}$ ahead and behind of the sphere. 


\subsection{Motion Perpendicular to the Axis of Rotation}

For transverse motion the criteria are even less well understood. Unlike in the coaxial motion, where the sphere is actually trying to move up and down in the fluid and therefore defy Taylor-Proudman, here it simply translates through the fluid perpendicular to the axis of rotation taking a cylindrical body of fluid, extending above and below, with it. External fluid will stream past it as though it were a solid body. The Hide criterion for Taylor column formation states that such a column may only form if $h / D>R o$, where $h$ is the vertical length of the particle (its diameter in the case of a sphere) and $D$ the depth of the fluid layer. Ro is defined to be $U / 2 a \Omega$ in the case of a sphere. In our setup $h / D=0.0635$ where as a typical $R o_{\perp}=0.071$, so again we are on the boarder line. Experiments by Hide et al. (1966) however have shown that the Taylor columns only form for transverse motion whenever $h / D>0.5 R o$ in which case, our numbers would predict no column.

From the centre rotation experiments we have some evidence of wall effects on the velocity of descent of the sphere. It can be seen in all the plots of time versus horizontal position, that the sphere first accelerates slightly after it has been dropped and then slows down as it approaches the bottom. We are suggesting that this is due to a Taylor slug interacting with the lid as the ball is released and gradually losing its efficiency in slowing the ball down as it moves away from the wall, however as it starts interacting with the bottom boundary it again slows down the vertical motion of the sphere.

We are going to work with the assumption that we do have a small Taylor slug, but not a Taylor column associated with the spheres in the following experiments. This assumption is supported by the fact that Stewartson's theory, which we compare our results to, has been developed for the unbounded case and it agrees nicely with our experimental results as will be shown.

\section{Forces on a Spherical Particle in a Rotating Fluid}

To describe the forces acting on a particle which moves relative to fluid rotating as a solid body we adapt a local Cartesian coordinate system the origin of which is located where the sphere enters the tank through a small hole in the lid. The vertical axis is denoted by $\hat{h}$ and is parallel to the axis of rotation, $\hat{r}$ points radially away from the centre of rotation and $\hat{\lambda}$ denotes the azimuthal direction pointing in the direction of rotation. $(\hat{r}, \hat{\lambda}, \hat{h})$ form an orthogonal right-handed co-ordinate system.

\subsection{Newton's Law}

In such a setup, a particle would experience two types of buoyancy forces, the first one being the vertical buoyancy due to the gravitational acceleration: $-\left(m-m^{\prime}\right) g$ and the second one due to the centripetal acceleration radially outwards, which is expressed as: $\left(m-m^{\prime}\right) r \Omega^{2}$. Note that $m$ is the mass of the sphere and $m^{\prime}$ the mass of the fluid replaced by the sphere.

In addition to those forces, the particle experiences a hydrodynamic force, $\mathbf{F}_{h}$, due to the fluid motion around the particle. The hydrodynamic force can be decomposed into the drag and the lift on the particle. 

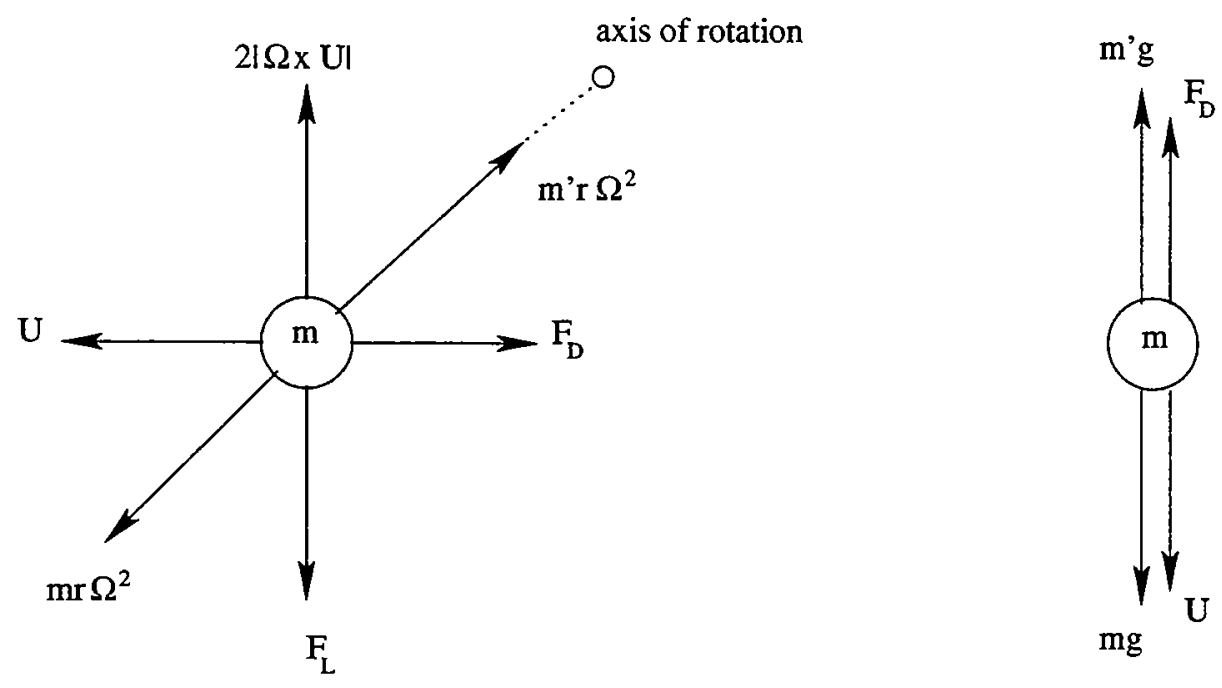

Figure 4: Schematic of the force acting on the ball. The horizontal forces are shown in the sketch on the left, and the vertical forces on the right.

The drag force, acts in the direction opposite to the motion of the particle, but it arises due to the friction and pressure between the particle and the surrounding fluid.

The lift on the other-hand acts perpendicular to the particles path of motion and the axis of rotation, and opposes the Coriolis force on the particle. Taylor (1922) [6] found that if the fluid motion about the particle is two-dimensional and sufficiently slow, then the lift force will be equal and opposite to Coriolis acting on a mass of fluid with the same volume as the object. This is not true however if the particle has three dimensional flow around it or there is an appreciable density difference between it and the surrounding fluid. In such a case the Coriolis force will exceed the lift and the particle will tend to be deflected to the right (see Bush [4] and references therein). Figure 4.1 shows an arrow diagram of the forces acting on the sphere.

According to Karanfilian et al. [7], the lift is due to the combined effect of the Coriolis acceleration of the fluid parcels surrounding the solid particle, the spin of the particle relative to the liquid, and the effect of the velocity gradient of of the liquid in the radial direction due to the solid body rotation.

The particle will move in accordance with Newton's laws, which may be expressed as follows:

$$
m \frac{d \mathbf{U}}{d t}=\mathbf{F}_{h}-2 m \Omega \times \mathbf{U}-\frac{4}{3} \pi a^{3} \Delta \rho \mathbf{g}_{\mathbf{e}}
$$

where

$$
\mathbf{F}_{h}=\int_{S} p_{d} \mathbf{n} d S+\int_{S} \mathbf{n} \cdot \tau d S
$$

is the hydrodynamic force acting on the particle. Here $\tau$ is the deviatoric stress tensor, and $p_{d}$ is the dynamic pressure. 

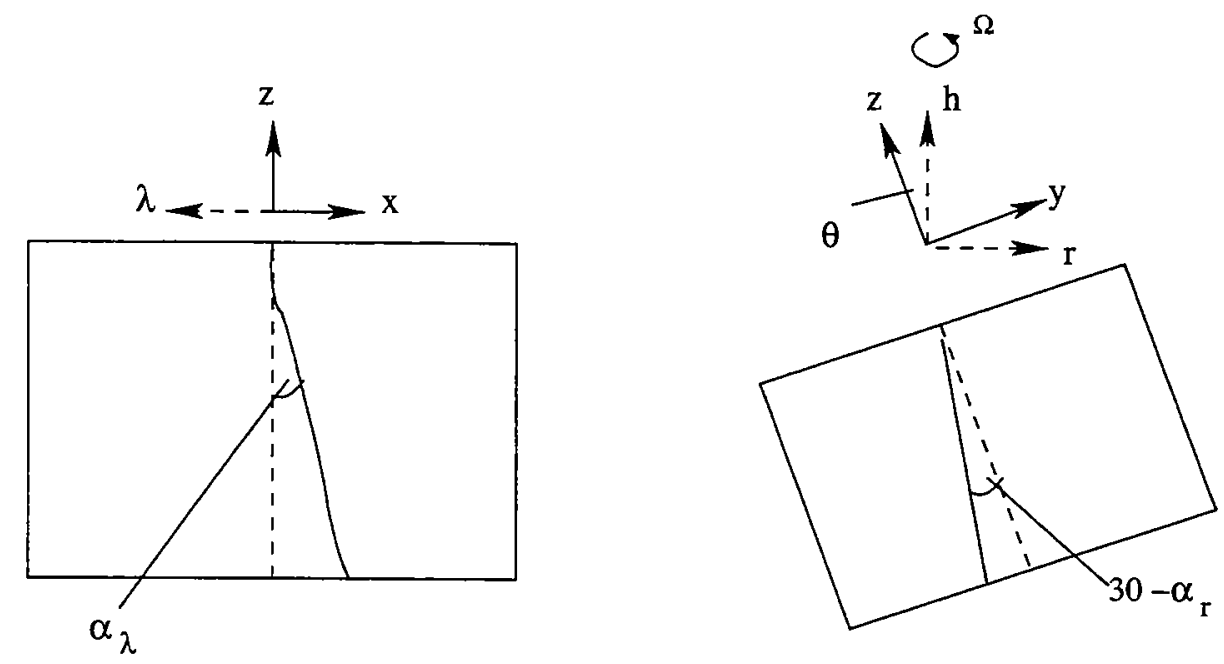

Figure 5: Sketch of the frontal (left sketch) and side (right sketch) view of the apparatus. The coordinate system shown with the dashed lines above the side view is the one we use consistently during the analysis, with the azimuthal unit vector pointing into the page. The dashed line is the vertical within the tank and the solid line shows the approximate trajectory of the particle, with $\alpha_{\lambda}$ and $\alpha_{r}$ denoting the angles of displacement in the azimuthal and radial direction respectively.

Newton's law in component form looks as follows:

$$
\begin{gathered}
0=F_{h r}-\frac{4}{3} \pi a^{3} \Delta \rho \Omega^{2} R+\frac{8}{3} \rho \pi a^{3} \Omega U_{r} \\
0=F_{h \lambda}-\frac{8}{3} \pi a^{3} \rho \Omega U_{\lambda} \\
0=F_{h h}+\frac{4}{3} \pi a^{3} \Delta \rho g
\end{gathered}
$$

if we assume that the motion of the particle is steady and $\Delta \rho \ll \rho$. Figure 4.1 gives an idea of the approximate path of the particle within the tank and explains the co-ordinate system we use.

\subsection{Stewartson's Drag Law}

Most existing theory for particles moving through a rotating fluid neglects the inertial terms and is therefore linear.

The most widely know description of flow around a sphere is that according to Stokes, which is linear and non-rotating. The drag for Stokes flow is $6 \pi \mu a U$, where $\mu$ is the viscosity of the fluid. In many problems involving centrifugal separation, the Reynolds number and Taylor numbers are small, meaning that viscous effects are dominating over inertial and rotating effects and under those conditions the motion may be treated as Stokes flow. If the motion is very slow however and the fluid has low viscosity, inertial and viscous forces are less important over rotational forces, leading to high Reynolds and Taylor numbers and 
relatively low Rossby numbers. In such a case Stokes law can not satisfactorily explain the particle motion. Stewartson $(1952,1953)[8,9]$ developed a linear theory for the high Taylor number and low Rossby number case, which we will adopt here. He found the drag parallel to the axis of rotation, for a sphere moving co-axially, to be

$$
F_{h_{\|}}=\frac{16}{3} \rho \Omega U_{\|} a^{3}
$$

In the case for a sphere moving normal to the axis of rotation he found the drag force to be

$$
F_{h_{\perp}}=-\frac{32 \pi^{2}}{3\left(16+\pi^{2}\right)} \rho \Omega U_{\perp} a^{3}
$$

where $\perp$ denotes that the motion is perpendicular to the axis of rotation, and a lateral force (lift), acting to the left of the motion,

$$
F_{L}=\frac{8 \pi^{3}}{3\left(16+\pi^{2}\right)} \rho \Omega U a^{3}
$$

Since this is a linear theory, we can superimpose these two different cases for a particle that moves through the fluid at an arbitrary angle. The resulting hydrodynamic force, maybe written in matrix form:

$$
\left(\begin{array}{l}
F_{r} \\
F_{\lambda} \\
F_{h}
\end{array}\right)=-\frac{16}{3} \rho \Omega a^{3}\left(\begin{array}{ccc}
\frac{2 \pi^{2}}{16+\pi^{2}} & -\frac{8 \pi}{16+\pi^{2}} & 0 \\
\frac{8 \pi}{16+\pi^{2}} & \frac{2 \pi^{2}}{16+\pi^{2}} & 0 \\
0 & 0 & 1
\end{array}\right)\left(\begin{array}{c}
U_{\tau} \\
U_{\lambda} \\
U_{h}
\end{array}\right)
$$

Note that the above matrix expression for $\mathbf{F}$ combines both the hydrodynamic and Coriolis forces proportional to $U_{\perp}$ from equation (1). It can therefore be balanced against the buoyancy forces

$$
\mathbf{F}=\mathbf{F}_{\mathbf{h}}+\text { Coriolis }=-\frac{4}{3} \pi \Delta \rho a^{3} \mathbf{g}_{e}
$$

Inverting the matrix (9) and combining it with (10) then gives us the following forcevelocity relationship for a rigid body:

$$
\left(\begin{array}{c}
U_{r} \\
U_{\lambda} \\
U_{h}
\end{array}\right)=\frac{\pi}{4 \Omega} \frac{\Delta \rho}{\rho}\left(\begin{array}{ccc}
1 / 2 & 2 / \pi & 0 \\
-2 / \pi & 1 / 2 & 0 \\
0 & 0 & 1
\end{array}\right)\left(\begin{array}{l}
g_{r} \\
g_{\lambda} \\
g_{h}
\end{array}\right)
$$

The vector for the effective gravity is given by $\mathbf{g}_{e}=\left(\Omega^{2} R, 0,-g\right)$. Writing (11) in component form we get: 


\begin{tabular}{lll}
\hline $\mathrm{a}$ & radius of the ball & $0.635 \mathrm{~cm}(1 / 4 \mathrm{in})$ \\
$\mathrm{m}$, & mass of the liquid displaced by the ball & $1.166 \mathrm{~g}$ \\
$\rho$ & density of the liquid & $1.11456 \mathrm{~g} / \mathrm{cm}^{3}$ \\
$\Delta \rho$ & density difference between fluid and sphere & $\sim 10^{-3} \mathrm{~g} / \mathrm{cm}^{3}$ \\
$\rho_{b}$ & density of the particle & $\rho+\Delta \rho$ \\
$\mathrm{g}$ & gravitational acceleration & $986 \mathrm{~cm} / \mathrm{s}^{2}$ \\
$\nu$ & kinematic viscosity of the salt solution & 0.0128 \\
$2 \Omega$ & rate of rotation & $3.027 \mathrm{~s}-1$ (or 2.621) \\
$\theta$ & tilt of the tank & $30^{\circ}$ \\
\hline
\end{tabular}

Table 1: Parameters applicable to all tilted tank experiments, and with some obvious exceptions to the centre-rotating and non-rotating experiments.

$$
\begin{gathered}
U_{r}=\frac{\pi}{4 \Omega} \frac{\Delta \rho}{\rho} \frac{1}{2} \Omega^{2} R \\
U_{\lambda}=-\frac{\pi}{4 \Omega} \frac{\Delta \rho}{\rho} \frac{2}{\pi} \Omega^{2} R \\
U_{h}=-\frac{\pi}{4 \Omega} \frac{\Delta \rho}{\rho} g
\end{gathered}
$$

The trajectory of the particle in the azimuthal direction may be found by noting that $\tan \alpha_{\lambda}=U_{\lambda} / U_{h} \approx 0.3638$ and therefore $\alpha_{\lambda} \approx 20^{\circ}$. Similarly the deflection of the particle in the radial direction is given by $\tan \alpha_{r}=U_{r} / U_{h} \approx 0.2875$ and so $\alpha_{r} \approx 16^{\circ}$. Note that the trajectory is independent of the density difference between the particle and the fluid, as it drops out on dividing one velocity component by another.

To predict actual velocities from the theory would mean one would need to know $\Delta \rho$, an indirect way of determining the absolute density difference is outlined in section 6 .

\section{Results}

\subsection{Tilted Tank Experiment}

Table 1 lists all the important parameters needed to describe all of the experiments.

\subsubsection{Observed Trajectories}

Figure 6 shows the trajectories of balls 1,2,5 and 6 from experiments N1 and N2. The solid line in the plots is the path as predicted by the linear theory from Stewartson. Both the azimuthal and the radial displacement of the sphere nicely fit the theoretical predictions. Another observation worthwhile noting is that the displacement in the radial direction seems to show a mild dependence on the individual characteristics of the ball, most probably the density difference. Table 5 (in the appendix) lists the predicted angles from the observed velocities. It seems that the faster balls (see table 4 for velocities) are deflected less in the 

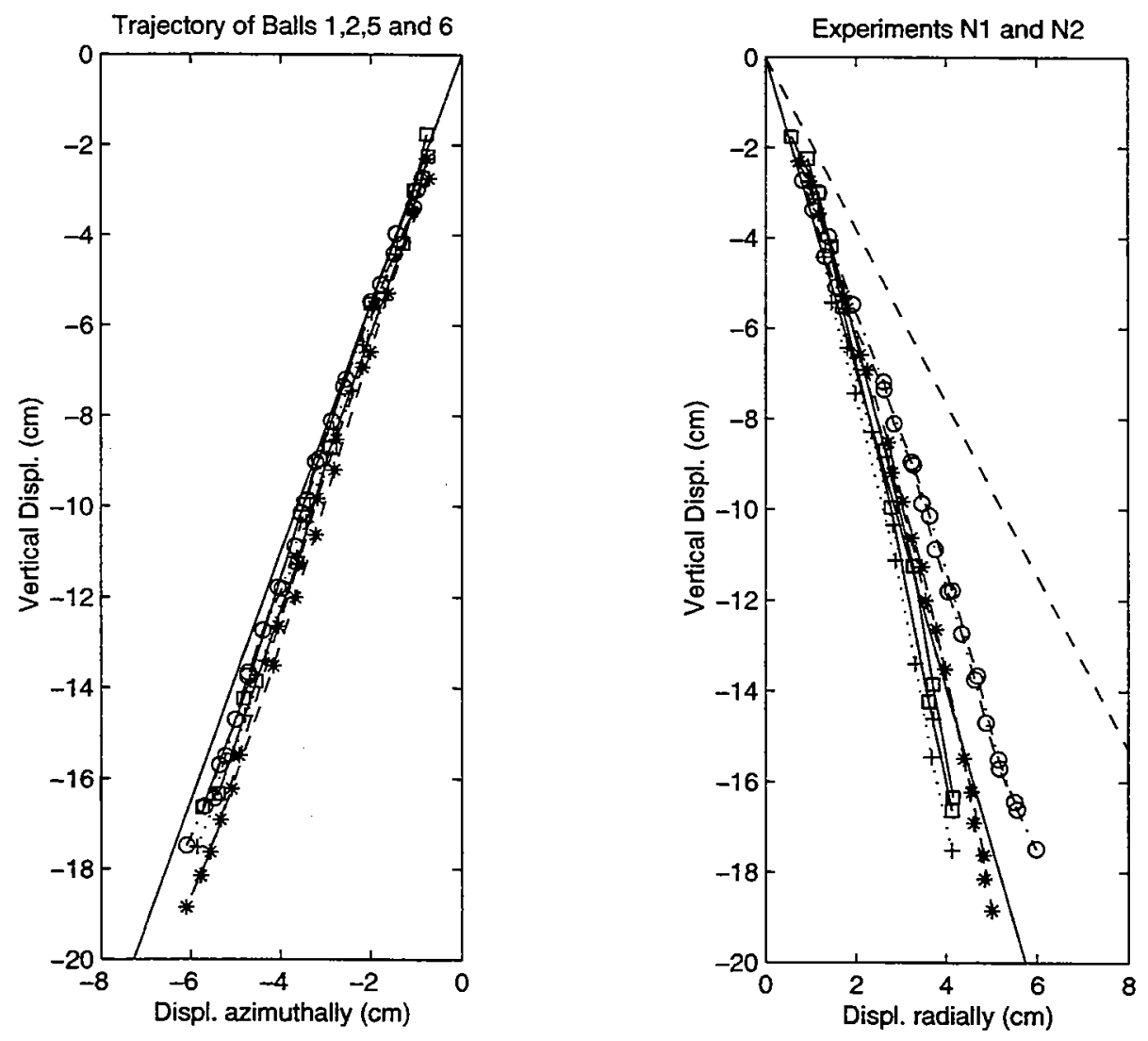

Figure 6: The plot on the left shows the azimuthal trajectories and the plot on the right the radial trajectories of balls $1,2,5$ and 6 in experiments $\mathrm{N} 1$ and N2. * - B1, + - B2, oB5 and $\square-$ B6. The solid line describes the angle predicted by linear theory and in both directions they agree nicely. Note that the axis of rotation is parallel to the vertical axis in the plots, the horizontal axis in the left plot is $\hat{\lambda}$ and in the right plot is $\hat{r}$. 
radial direction, or, if one looks at the trajectory as being a displacement from the vertical axis of the tank along which effective gravity is active, they are displaced further. This suggest some non-linear dependence of the radial trajectory.

\subsubsection{Resulting Velocities}

Table 4 (in the appendix) gives the velocity results obtained for each sphere in each of the three experiments. The main observation to take away is that in general the velocity in the azimuthal direction, $\hat{\lambda}$, was observed to be less than that in the radial direction $\hat{r}$. The table also lists the Rossby and Reynolds numbers for each of the spheres in the different experiments, which tell us that we are in a rotation dominated set up.

\subsection{Centre Rotation Experiments}

The fall velocity of a ball in a rotating fluid is substantially less than that of the same ball descending in the same, but non-rotating fluid. In order to estimate the difference of the effects of tilting on the speed of descent, a set of experiments was carried out with the the same tanks as that in the tilted case, but placed at the centre of the rotating table. In the first set of experiments (R1, R2, R3 and R4) the rotation rate was kept the same as in the tilted experiments $(2 \Omega=3.027)$ but in a second run of experiments ( $R 5, \mathrm{R} 6$ and $\mathrm{R} 7)$ the rate of rotation was lowered slightly so that, it would correspond to the 'vertical' component (here we mean the axis $z$ in figure 2 along which effective gravity acts) of the rotation in the tilted case, $2 \Omega=3.027 \cdot \cos \left(30^{\circ}\right)=2.621$. Figure 5.2 shows clearly the spread of the velocity results from the fast centre-rotating experiments indicated by the circles. The plot also shows the very consistent velocity data from the slower centre-rotating experiment. In the mean one can see that in all cases the velocity of descent is faster for the slow rotating experiment in comparison to the faster rotating experiment. This illustrates very nicely that TP becomes more effective with higher rotation. The exact velocity results for each ball are listed in table 7 .

\subsection{Non-rotating Experiments}

The experiments in the non-rotating tank were also carried out with the tank mounted at the centre of the table, but the table remained stationary. They were done in order to calibrate the spheres and determine their density difference to the water by measuring their terminal velocities (see section 6). The results of the velocity measurements are plotted in figure 8 (see table 8 for the actual numbers) along with the results from the rotating experiments for comparison. A very noticeable difference in descent velocities may be observed, between the rotating and the non-rotating experiments again pointing to the effects of TP, which are absent if the system is not rotating.

Table 2 lists average velocities for each ball for all sets of experiments. Ratios are computed for comparison and where known the theoretically predicted ratio is indicated. 


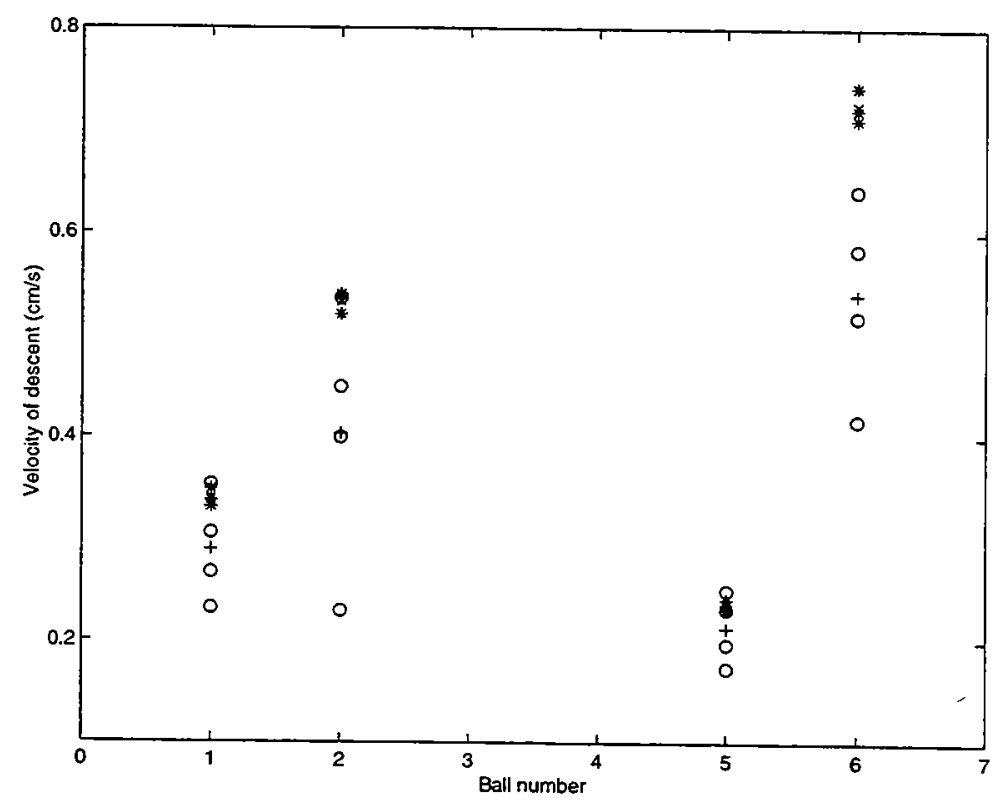

Figure 7: Plot of velocity spread for each of the balls from the fast and slow rotating experiments. $\circ$ - faster rotation, $2 \Omega=3.027$ ( + - mean), $*$ - slower rotation, $2 \Omega=2.621$ ( $\mathrm{x}$ - mean).

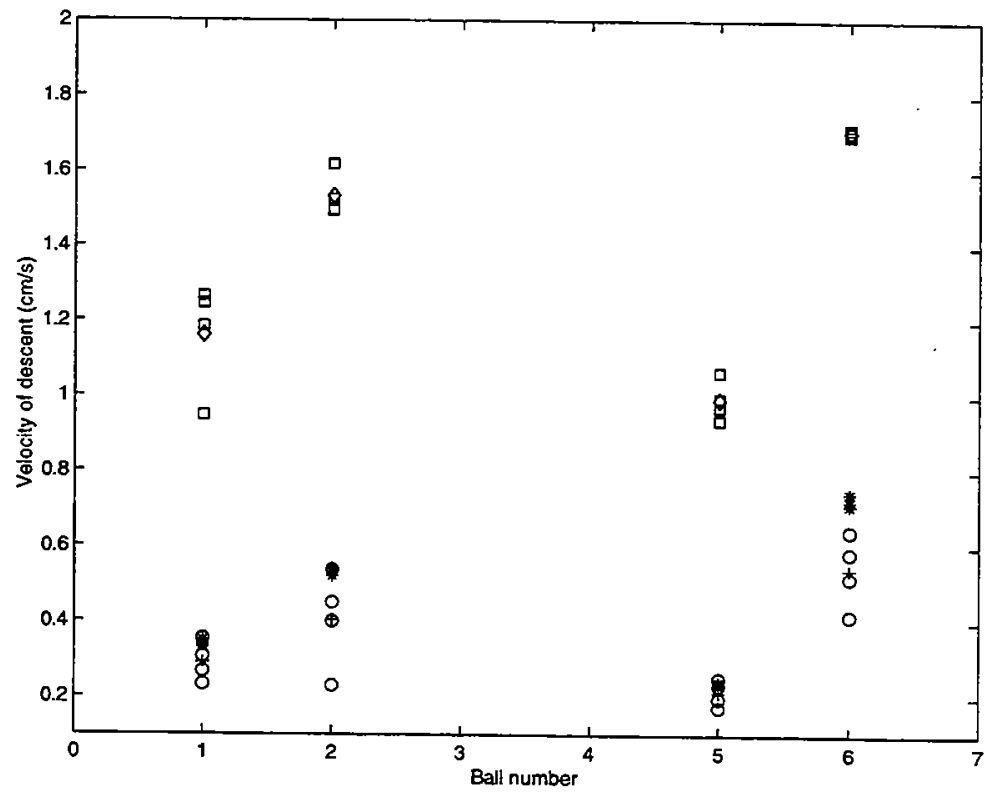

Figure 8: Plots of velocity spread for each of the balls from the rotating and non-rotating experiments: $\square$ - non-rotating ( $\diamond$ - mean), $\circ$ - faster rotation, $2 \Omega=3.027(+-$ mean), * slower rotation, $2 \Omega=2.621$ ( $\mathrm{x}-$ mean). 


\begin{tabular}{lcccc|c}
\hline & $\mathrm{B} 1$ & $\mathrm{~B} 2$ & $\mathrm{~B} 5$ & $\mathrm{~B} 6$ & Theory \\
\hline Non-rot. & 1.1605 & 1.5322 & 0.9914 & 1.7058 & \\
Slow rot. & 0.3389 & 0.5326 & 0.2361 & 0.7252 & \\
Fast rot. & 0.2891 & 0.4033 & 0.2126 & 0.5402 & \\
Tilted rot.(z) & 0.3250 & 0.4765 & 0.2139 & 0.5394 & \\
\hline $\mathrm{Sl} / \mathrm{Fa}$ & 1.1723 & 1.3206 & 1.1105 & 1.3425 & $1 / \cos 30^{\circ}=1.1547$ \\
$\mathrm{Nr} / \mathrm{Sl}$ & 3.4243 & 2.8768 & 4.1991 & 2.3522 & \\
Sl/Tilted & 1.0428 & 1.1177 & 1.0874 & 1.3445 & 1.0 \\
\hline
\end{tabular}

Table 2: Average velocities are listed for all sets of experiments. The last row, Tilted rot. (z), referes to the component of the velocity along the $z$-axis. Its magnitude should be comparable to that of the slow rotating velocity results. $\mathrm{Nr}$ - non-rotating, $\mathrm{Sl}$ - slow rotating, $\mathrm{Fa}$ - fast rotating.

\section{Estimation of Densities of the Spheres}

In order to achieve a small descent velocity and thus a small Rossby number flow the density difference $\Delta \rho$ between the ball of diameter $1.27 \mathrm{~cm}$ and water should be very small $O\left(10^{-3}\right)$. We were unable to calculate the density of each ball with such accuracy directly by weighting it and measuring its volume. Instead, we used an indirect method based on measuring the terminal descent velocity in non-rotating fluid. When a sphere descends in fluid at constant speed $V$, the Archimedes force (proportional to $\Delta \rho$ ) acting on it is balanced by the hydrodynamic drag

$$
\frac{4}{3} \pi a^{3} \Delta \rho g=\frac{1}{2} \rho V^{2} \pi a^{2} C_{D}\left(R_{e}\right)
$$

which is usually expressed in terms of the drag coefficient $C_{D}$ dependent only on a Reynolds number, $R_{e}=2 a V / \nu$. By fitting the well known measurements of the drag coefficient (Schlichting, BL Theory) in a range $0.1<R_{e}<1000$ we derived an empirical dependence

$$
C_{D}=\frac{24}{R_{e}}+\left(3 \frac{24}{R_{e}}\right)^{1 / 3}
$$

which not only approximates extremely well the data in this range (Fig. 6) but also has an exceptionally simple analytic form (stimulating a search for the underlying physical meaning). The first term in (16) represents the Stokes law with the drag proportional to $V$ in the linear viscous case $R_{e} \ll 1$. The second term describes the increase of the drag due to inertial reaction of the oncoming flow.

According to Taneda (1956) for $R_{e}>24$ the flow behind a sphere starts to separate, and a vortex ring forms in the wake. The length of the recirculation grows linearly with $R_{e}$ until the flow starts to oscillate gently for $R_{e}>130$. For $R_{e}>1000$ the flow in the wake is very turbulent, the drag coefficient approaches a constant value 0.47 , and hence the drag is proportional to $V^{2}$ in accordance with the Newton's law. Near $R_{e}=3 \cdot 10^{5}$ flow in the boundary layer becomes turbulent and the crisis of drag occurs. 


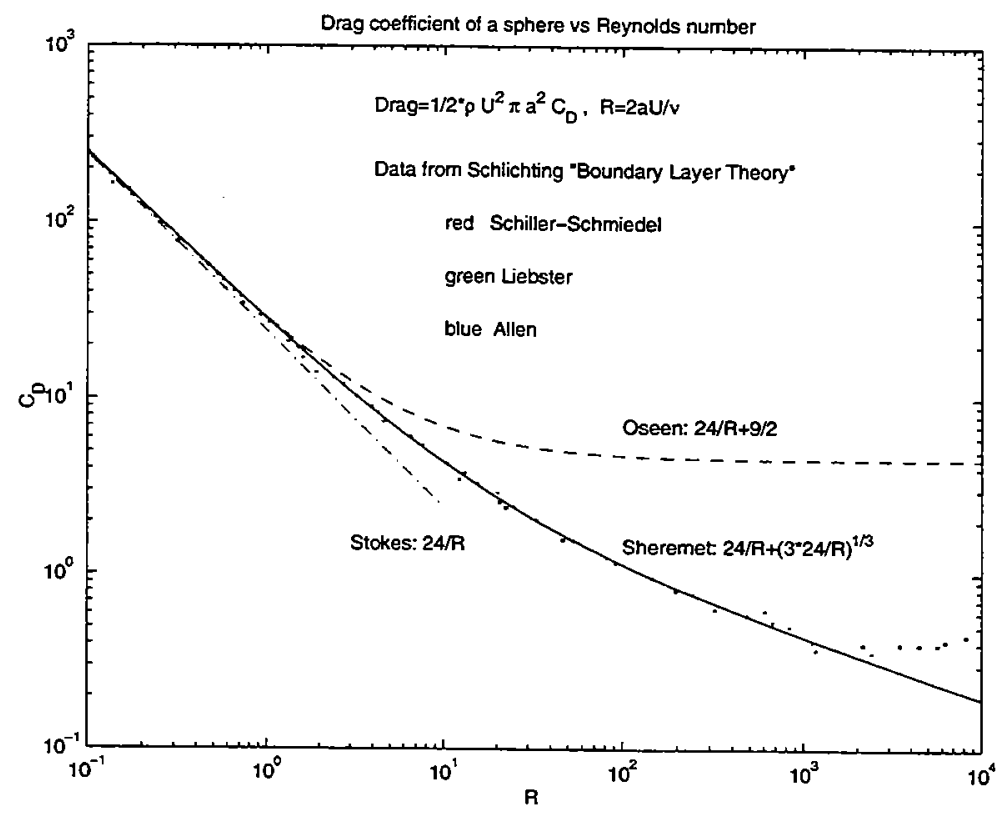

Figure 9: Plot of the drag coefficient versus the Reynolds number for motion of particles in non rotating fluids. Shown are the Oseen approximation (dashed) and Stokes law (dasheddotted). The solid line is a fit to the data by Sheremet.

In our non-rotating experiment the Reynolds number was $O(100)$ just in the middle of the validity range of (16), and we expect a steady wake with a recirculation of size about $2 a$. From the observed steady terminal velocities in the non-rotating experiments we calculated the density excess $\Delta \rho$ of each ball according to (15),(16), they are listed in the Table 3 . The same balls and salty water were used in all our rotating tank experiments.

For comparison we used Stewartson's drag law to estimate the density difference from the centre-rotating experiments at slow rotation rate. Since the agreement of velocities in the azimuthal direction is so good, we use the measured azimuthal velocity component $U_{\lambda}$ to estimate the difference in density between the ball and the surrounding salt solution. Solving the expression for the azimuthal component, we find the following expression for $\Delta \rho:$

$$
|\Delta \rho|=2 U_{\lambda} \frac{\rho}{\Omega R}
$$

In table 3 the estimated $\Delta \rho$ from both the non-rotating and slow centre-rotating experiments are listed. If one were to use the results from the non-rotating experiments, to estimate the velocities in the tilted case, one would get values which are 40 to $60 \%$ to high, (remember the linear dependence of the velocity components on $\Delta \rho$ ). 


\begin{tabular}{lccc}
\hline Ball & No-rot & Slow-rot & Ratio \\
\hline 1 & 1.0543 & 0.6430 & 1.6397 \\
\hline 2 & 1.4382 & 1.0113 & 1.4221 \\
\hline 5 & 0.7279 & 0.4481 & 1.6244 \\
\hline 6 & 1.7465 & 1.3761 & 1.2692 \\
\hline
\end{tabular}

Table 3: Table of the average estimates for $\Delta \rho$ from the non-rotating and the slow centrerotating experiments, and their ratios.

\section{Conclusion}

The experiments both in the tilted and in the centre-rotating case yielded some quite nice and consistent results, which in the case of the tilted case agree nicely with Stewartson's linear theory for motions of Rossby numbers less than 1 and Reynolds numbers between 10 to 60 .

Comparing the trajectories of the spheres to those of the blobs in the plume experiments it turns out that they have similar displacements in the radial direction, however the blobs descend at a much smaller angle in the azimuthal direction. We conclude that the path of the blobs in the plumes are in some aspects, but not all, consistent with the paths taken by solid spheres. This may be due to the presence of external circulation in the plume, and deformation of fluid parcels, which may influence its path.

In future it maybe interesting to repeat some of the experiment which gave less consistent results, such as the centre-rotating experiments and try to reconcile our initial intention of calibrating the balls and thus estimating their density difference to the fluid. In doing so more attention will have to be paid to the temperature of the surrounding fluid as this may well influence the density of both the saline solution and the nylon spheres through thermal expansion.

There are further observations we made, which have not been mentioned at all here. Firstly there were another four balls released in each of the experiments, which descended at considerably faster velocities then those we analyse here. They therefore involve flows of much larger Rossby numbers and it might be interesting to see whether Stewartson's drag law still applies for these spheres. Secondly, while descending in the rotating fluid, all spheres were observed to spin. We have had no time to look closer at the rate of spin of the spheres, at its origin or importance for determining the motion.

Finally, in order to have a more realistic comparison of the sphere trajectories to those of the blobs we are planning to carry out a few experiments studying the motion of single blobs in the same set up without them being surrounded by a plume.

\section{Acknowledgments}

I would like to express my thanks to Vitalii Sheremet for suggesting and supervising the project, for his help with setting up and running the experiments and giving me the 
opportunity to get an insight into what is involved in doing experimental work. My thanks also to Keith Bradley for building a lot of the equipment for the experiments and maintaining it throughout the summer.

George Veronis has been a great help in giving advise and contributing ideas to the project, and besides work, he has made life for all the fellows a lot easier by patiently taking us food-shopping almost every week. We also have to thank him for giving us some proper coaching on Softball which really captured all of us, and despite us (the fellows) loosing the staff versus fellows game, of which by the way you still owe us two innings, I believe we all enjoyed it tremendously!!

Finally thanks to all the staff and fellows for making this a summer of many new experiences. Good Eye! 


\section{A Tables}

\begin{tabular}{llcccccc}
\hline Ball & Exp. & $U_{r}$ & $U_{\lambda}$ & $U_{h}$ & $|\mathbf{U}|$ & $R_{e}$ & $R o$ \\
\hline 1 & $\mathrm{~N} 1$ & 0.0846 & -0.1022 & -0.3252 & 0.3512 & 34.85 & 0.3654 \\
& N2 & 0.0834 & -0.1071 & -0.3283 & 0.3553 & 35.25 & 0.3696 \\
& N3 & 0.0374 & -0.0388 & -0.1301 & 0.1408 & 13.97 & 0.1465 \\
\hline 2 & N1 & 0.1119 & -0.1698 & -0.5084 & 0.5476 & 54.33 & 0.5697 \\
& N2 & 0.1180 & -0.1588 & -0.4873 & 0.5259 & 52.18 & 0.5472 \\
& N3 & 0.1129 & -0.1480 & -0.4571 & 0.4935 & 48.97 & 0.5135 \\
\hline 5 & N1 & 0.0731 & -0.0710 & -0.2083 & 0.2319 & 23.01 & 0.2413 \\
& N2 & 0.0719 & -0.0748 & -0.2208 & 0.2440 & 24.21 & 0.2538 \\
& N3 & 0.0623 & -0.0579 & -0.1922 & 0.2102 & 20.85 & 0.2187 \\
\hline 6 & N1 & 0.1256 & -0.1807 & -0.5404 & 0.5835 & 57.89 & 0.6071 \\
& N2 & 0.1363 & -0.1930 & -0.5826 & 0.6287 & 62.38 & 0.6542 \\
& N3 & 0.1345 & -0.1704 & -0.5165 & 0.5603 & 55.59 & 0.5830 \\
\hline
\end{tabular}

Table 4: Table of the velocity results form the tilted tank experiments. We have the velocity radially outwards, $U_{r}$, the azimuthal component, $U_{\lambda}$, the vertical component, $U_{h}$, parallel to the axis of rotation, and the absolute velocity, $|\mathbf{u}|=\sqrt{U_{r}+U_{\lambda}+U_{h}}$. The last column specifies the Reynolds number of each sphere in the respective experiment. We define the Reynold number as $R_{e}=U d / \nu$, were $U$ - absolute velocity, $d$ - diameter of the ball and $\nu$ viscosity of the surrounding liquid. The Rossby number, which is defined by $R_{o}=U / 2 \Omega a$. 


\begin{tabular}{llcc}
\hline Ball & Exp. & ${ }_{r}$ & $\alpha_{\lambda}$ \\
\hline 1 & N1 & 14.58 & 17.45 \\
& N2 & 14.25 & 18.07 \\
& N3 & 16.04 & 16.61 \\
\hline 2 & N1 & 12.41 & 18.47 \\
& N2 & 13.61 & 18.05 \\
& N3 & 13.87 & 17.94 \\
\hline 5 & N1 & 19.34 & 18.82 \\
& N2 & 18.04 & 18.71 \\
& N3 & 17.96 & 16.76 \\
\hline 6 & N1 & 12.36 & 17.50 \\
& N2 & 13.16 & 18.33 \\
& N3 & 14.60 & 18.26 \\
\hline
\end{tabular}

Table 5: Angles of deflection derived from the velocity components. $\alpha_{r}$ is the angle at which the particle moves away from the $\hat{h}$-axis in the radial direction. $\alpha_{\lambda}$ is the angle at which the particle lags behind the rotation of the tank. All angles are in degrees.

\begin{tabular}{llc}
\hline Ball & Exp. & $U_{h}$ \\
\hline 1 & R1 & -0.2314 \\
& R2 & -0.3529 \\
& R3 & -0.3053 \\
& R4 & -0.2666 \\
\hline 2 & R1 & -0.2292 \\
& R2 & -0.5356 \\
& R3 & -0.4490 \\
& R4 & -0.3996 \\
\hline 5 & R1 & -0.1731 \\
& R2 & -0.2495 \\
& R3 & -0.2312 \\
& R4 & -0.1967 \\
\hline 6 & R1 & -0.4168 \\
& R2 & -0.6417 \\
& R3 & -0.5838 \\
& R4 & -0.5183 \\
\hline
\end{tabular}

Table 6: The velocity of descent is listed for each ball in the experiments R1, R2, R3a and R3b at a rotation rate of $2 \Omega=3.027$.

\begin{tabular}{llc}
\hline Ball & Exp. & $U_{h}$ \\
\hline 1 & R5 & -0.3372 \\
& R6 & -0.3309 \\
& R7 & -0.3485 \\
\hline 2 & R5 & -0.5199 \\
& R6 & -0.5396 \\
& R7 & -0.5382 \\
\hline 5 & R5 & -0.2412 \\
& R6 & -0.2313 \\
& R7 & -0.2359 \\
\hline 6 & R5 & -0.7433 \\
& R6 & -0.7112 \\
& R7 & -0.7210 \\
\hline
\end{tabular}

Table 7: The velocity of descent is listed for each ball in the three experiments R5, R6 and $\mathrm{R} 7$ at a rotation rate of $2 \Omega=2.621$. 


\begin{tabular}{clc}
\hline Ball & Exp. & $U_{h}$ \\
\hline 1 & S1 & -1.2647 \\
& S2 & -1.2458 \\
& S3 & -0.9469 \\
& S4 & -1.1845 \\
\hline 2 & S1 & -1.6173 \\
& S2 & -1.5237 \\
& S3 & -1.4638 \\
& S4 & -1.4939 \\
\hline 5 & S1 & -1.0645 \\
& S2 & -0.9941 \\
& S3 & -0.9705 \\
& S4 & -0.9365 \\
\hline 6 & S1 & $\mathrm{X}$ \\
& S2 & -1.7061 \\
& S3 & -1.6967 \\
& S4 & -1.7147 \\
\hline
\end{tabular}

Table 8: The velocity of descent is listed for each ball in the non-rotating experiments $\mathrm{S} 1$, S2, S3 and S4.

\section{References}

[1] V. Sheremet, "Laboratory experiments with tilted convective plumes on a centrifuge: a finite angle between the buoyancy force and the axis of rotation," submitted to JFM (2001).

[2] D. E. Loper, "On the structure of a taylor column driven by a buoyant parcel in an unbounded rotating fluid," J. Fluid Mech. 427, 131 (2001).

[3] G. Taylor, "Motion of sloids in fluids when the flow is not irrotational," Proc. Roy. Soc. London A 93, 99 (1917).

[4] J. Bush, H. Stone, and J. Tanzosh, "Particle motion in rotating viscous fluids: Historical survey and recent developments," Current Topics in The Physics of Fluids 1, 337 (1994).

[5] T. Maxworthy, "The flow created by a sphere moving along the axis of a rotating, lightly-viscous fluid," J. Fluid Mech. 40, 453 (1970).

[6] G. Taylor, "The motion of a sphere in a rotating liquid," Proc. Roy. Soc. London A 102, 180 (1922).

[7] S. K. Karanfilian and T. J. Kotas, "Motion of a spherical particle in a liquid rotating as a solid body," Proc. Roy. Soc. London A, 525 (1981).

[8] K. Stewartson, "On the slow motion of a sphere along the axis of rotation," Proc. Camb. Phil. Soc. 48, 168 (1952). 
[9] K. Stewartson, "On the slow motion of an ellipsoid in a rotating fluid," Quart. J. Mech. Appl. Math. 6, 141 (1953). 


\section{Rearrangement of Annular Rings of High Vorticity \\ Huiqun Wang \\ California Institute of Technology}

\section{Introduction}

Flight level measurements suggest that hurricanes have low vorticity eyes surrounded by high vorticity eyewalls (Kossin and Schubert 2001). The rearrangement of such high vorticity annular rings is an important factor in hurricane dynamics. In this study, I use a barotropic nondivergent model to investigate the evolution of a set of high vorticity annular rings with fixed circulation and scales similar to hurricanes.

The initial radial profile of vorticity is given by

$$
\zeta=\zeta_{0} \begin{cases}0, & 0 \leq r \leq r_{1} \\ S\left(\left(r_{2}-r\right) /\left(r_{2}-r_{1}\right)\right), & r_{1} \leq r \leq r_{2} \\ S\left(\left(r-r_{2}\right) /\left(r_{3}-r_{2}\right)\right), & r_{2} \leq r \leq r_{3} \\ 0, & r \geq r_{3}\end{cases}
$$

where $S(x)=1-3 x^{2}+2 x^{3}, r_{2}$ is fixed at $60 \mathrm{~km}, r_{3}-r_{1}$ ranges from $4 \mathrm{~km}$ to $116 \mathrm{~km}$ with an increment of $4 \mathrm{~km}$, and the constant $\zeta_{0}$ is chosen so that all the rings have the same circulation for $r \geq r_{3}$. Sample initial vorticity and aximuthal wind profiles are shown in Fig. 1.
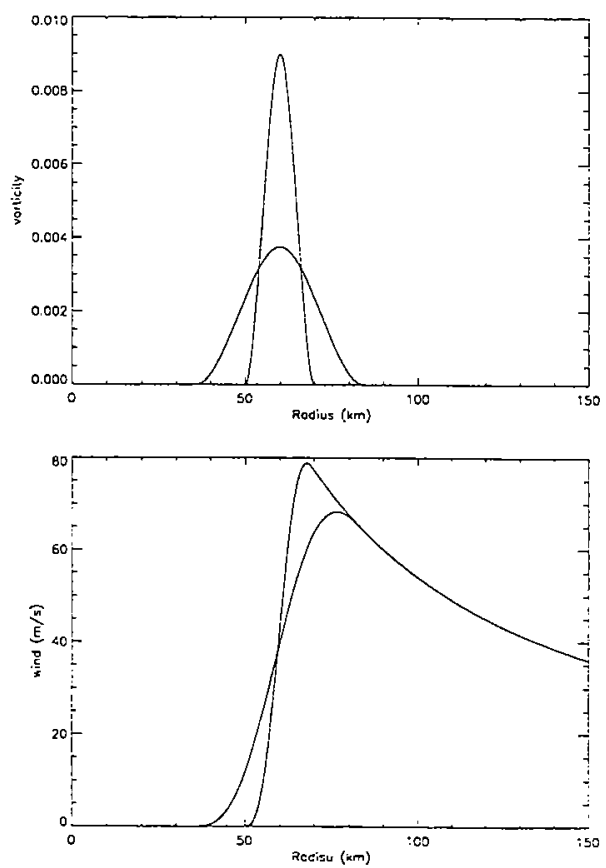

Figure 1: Sample radial profiles of the initial vorticity and tangential wind. 
After introducing the numerical model, I describe the simulated evolution of representative narrow, wide, and very wide annular rings. I then compare the initial wavenumber with the published results of linear stability analysis, and investigate the history of the domain-averaged enstrophy for different rings. I apply the minimum enstrophy theory to predict the final states, and investigate the advantages and disadvantages of the theory. I also compare the results for these rings with two sets of skewed rings.

\section{Numerical Model}

An adaptive multigrid barotropic nondivergent model (Fulton 2001) was used to simulate the evolution of the annular rings. This model solves the modified barotropic vorticity equation in Mercator coordinates

$$
\begin{gathered}
\frac{\partial q}{\partial t}+m^{2} \frac{\partial(\psi, q)}{\partial(x, y)}+\beta m \frac{\partial \psi}{\partial x}=\nu m^{2}\left(\frac{\partial^{2} q}{\partial x^{2}}+\frac{\partial^{2} q}{\partial y^{2}}\right), \\
m^{2}\left(\frac{\partial^{2} \psi}{\partial x^{2}}+\frac{\partial^{2} \psi}{\partial y^{2}}\right)-\gamma^{2} \psi=q
\end{gathered}
$$

where $q=\zeta-\gamma^{2} \psi$ is the potential vorticity anomaly, $\zeta$ is the relative vorticity, $f=2 \Omega \sin \phi$ is the Coriolis parameter, $\beta=d f / a d \phi=2 \Omega a^{-1} \cos \phi, m=\cos \phi_{0} / \cos \phi$ is the map factor, $\gamma^{-1}=\sqrt{g H} / f$ is the Rossby radius of deformation, $\psi$ is the stream function, and $\nu$ is the constant viscosity.

In this study, I ran the model in the pure barotropic, $f$-plane mode by setting $\gamma=0$, $m=1$, and $\beta=0$, so that the actual equations solved become

$$
\begin{gathered}
\frac{\partial \zeta}{\partial t}+\frac{\partial(\psi, \zeta)}{\partial(x, y)}=\nu\left(\frac{\partial^{2} \zeta}{\partial x^{2}}+\frac{\partial^{2} \zeta}{\partial y^{2}}\right) \\
\frac{\partial^{2} \psi}{\partial x^{2}}+\frac{\partial^{2} \psi}{\partial y^{2}}=\zeta .
\end{gathered}
$$

Associated with this model are the kinetic energy and enstrophy equations

$$
\begin{aligned}
& \frac{d \mathcal{E}}{d t}=-2 \nu \mathcal{Z} \\
& \frac{d \mathcal{Z}}{d t}=-2 \nu \mathcal{P}
\end{aligned}
$$

where $\mathcal{E}=\iint \frac{1}{2} \nabla \psi \cdot \nabla \psi d x d y$ is the energy, $\mathcal{Z}=\iint \frac{1}{2} \zeta^{2} d x d y$ is the enstrophy, $\mathcal{P}=\iint \frac{1}{2} \nabla \zeta$. $\nabla \zeta d x d y$ is the palinstrophy.

The numerical model uses the 4th order Runge-Kutta scheme to advance in time and has the option of 2 nd or 4 th order Arakawa Jacobian technique to approximate the advection terms. It has multiple movable or adaptive nests within the base grid. In this study, most of the simulations are run on a base domain of size $4096 \mathrm{~km} \times 4096 \mathrm{~km}$ with $128 \times 128$ grid points. There are 4 subsequent nests within the base domain, each of which has half the domain size and mesh size of its mother domain, so that the finest resolution is $2 \mathrm{~km}$. In a few runs, the resolution was increased to $256 \times 256$ grid points for the base domain and all the nests. 


\section{Ring Evolution}

Representative examples of the evolution of thin $(12 \mathrm{~km})$, wide $(60 \mathrm{~km})$, and very wide rings $(108 \mathrm{~km})$ are shown in Figs. 2-4. The rotational timescale $\tau=2 \pi r_{2} / v\left(r_{2}\right)$ for these three rings is about $2 \mathrm{hr}$.

Thin rings $\left(r_{3}-r_{1} \leq 20 \mathrm{~km}\right.$, Fig. 2$)$ initially break up into many vortices $(\geq 6)$ that rapidly merge into several vortices (4-5) as they rotate around. The resultant vortices persist for tens of rotational timescales before subsequent merger takes place. Such a configuration can be referred to as 'mesovortex' or 'vortex crystal' stage. Thin rings eventually evolve into monopoles.

For wide rings (24 km $\leq r_{3}-r_{1} \leq 104 \mathrm{~km}$, Fig. 3), initial instability takes longer to grow and shows lower wavenumbers. The few (2-5) resulting vortices gradually relax to a monopole.

Very wide rings $\left(r_{3}-r_{1} \geq 108 \mathrm{~km}\right.$, Fig. 4) show wavenumber two structures initially, but they never break up into individual vortices. The central low vorticity remains until the last timestep, which corresponds to about 80 rotational timescales.

\section{Initial Wavenumber}

Simulation results show that the initial instability wavenumber tends to decrease with increasing ring thickness (Fig. 5). For thin rings $\left(r_{3}-r_{1} \leq 20 \mathrm{~km}\right)$, this decrease is very sharp. For wide rings, there is usually a thickness range that corresponds to the same wavenumber, and the range appears to increase with decreasing wavenumber.

Schubert et al. (1999) performed a linear stability analysis for annular rings with piecewise constant radial profiles. Their Fig. 2 shows the unstable regions for wavenumbers $3-8$ in $\delta-\gamma$ space, where $\delta$ is the ratio of the inner and outer radii and $\gamma$ is the ratio of the inner vorticity and average vorticity. For the rings I study, $\gamma=0$. Converting into this notation, the initial wavenumber of my simulations agrees well with Schubert et al.'s linear stability results (Fig. 6).

\section{Enstrophy History}

The evolution of different rings is studied by plotting the normalized enstrophy as a function of time (Fig. 7). For thin rings $\left(r_{3}-r_{1} \leq 20 \mathrm{~km}\right)$, the rapid merger of initial vortices results in a sharp decrease of enstrophy early in the evolution. The enstrophy levels off with time during each mesovortex stage. Each subsequent merger leads to a rapid decrease of enstrophy and thus a stairstep pattern in the enstrophy history.

For wide rings ( $24 \mathrm{~km} \leq r_{3}-r_{1} \leq 104 \mathrm{~km}$ ), the early stage enstrophy decreases more slowly, consistent with the slower growth of initial instability. Subsequent relaxation to a monopole is gradual, without the transitional mesovortex stage. For very wide rings $\left(r_{3}-r_{1} \geq 108 \mathrm{~km}\right)$, consistent with the central low vorticity remaining unmixed during the entire simulation, the slope of the enstrophy curve does not change much with time. 


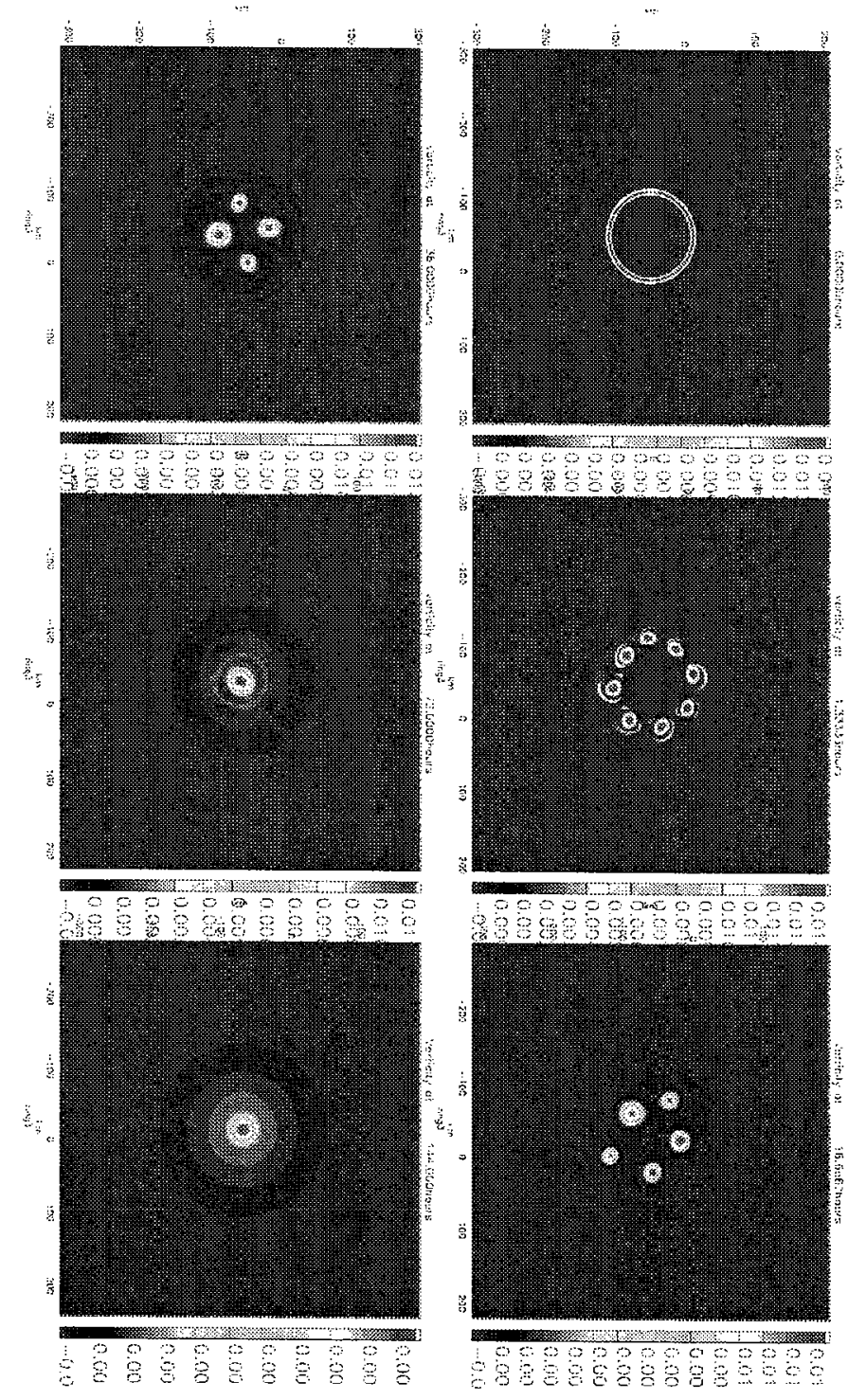

Figure 2: Evolution of thin ring: $r_{3}-r_{1}=12 \mathrm{~km}$. 

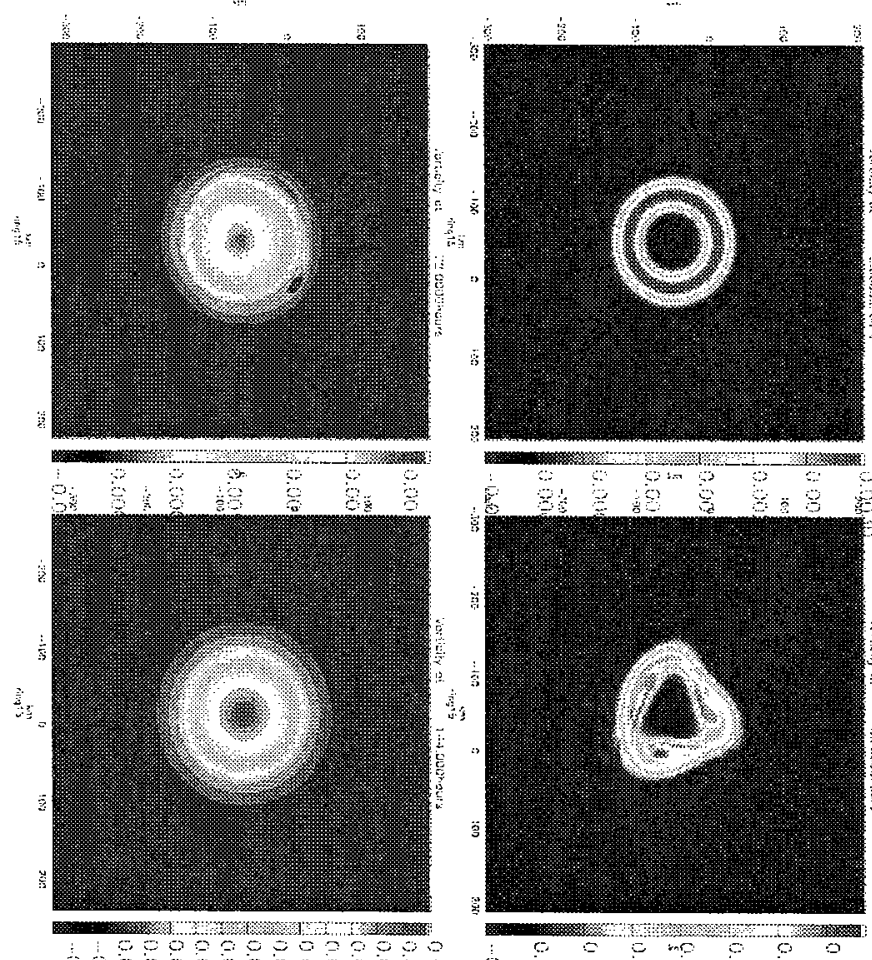

\&888985893850

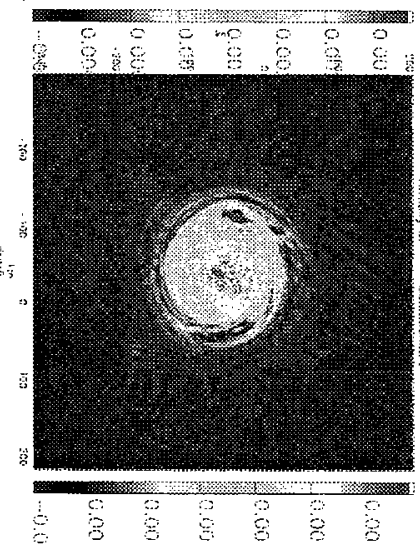

Figure 3: Evolution of wide ring: $r_{3}-r_{1}=60 \mathrm{~km}$. 

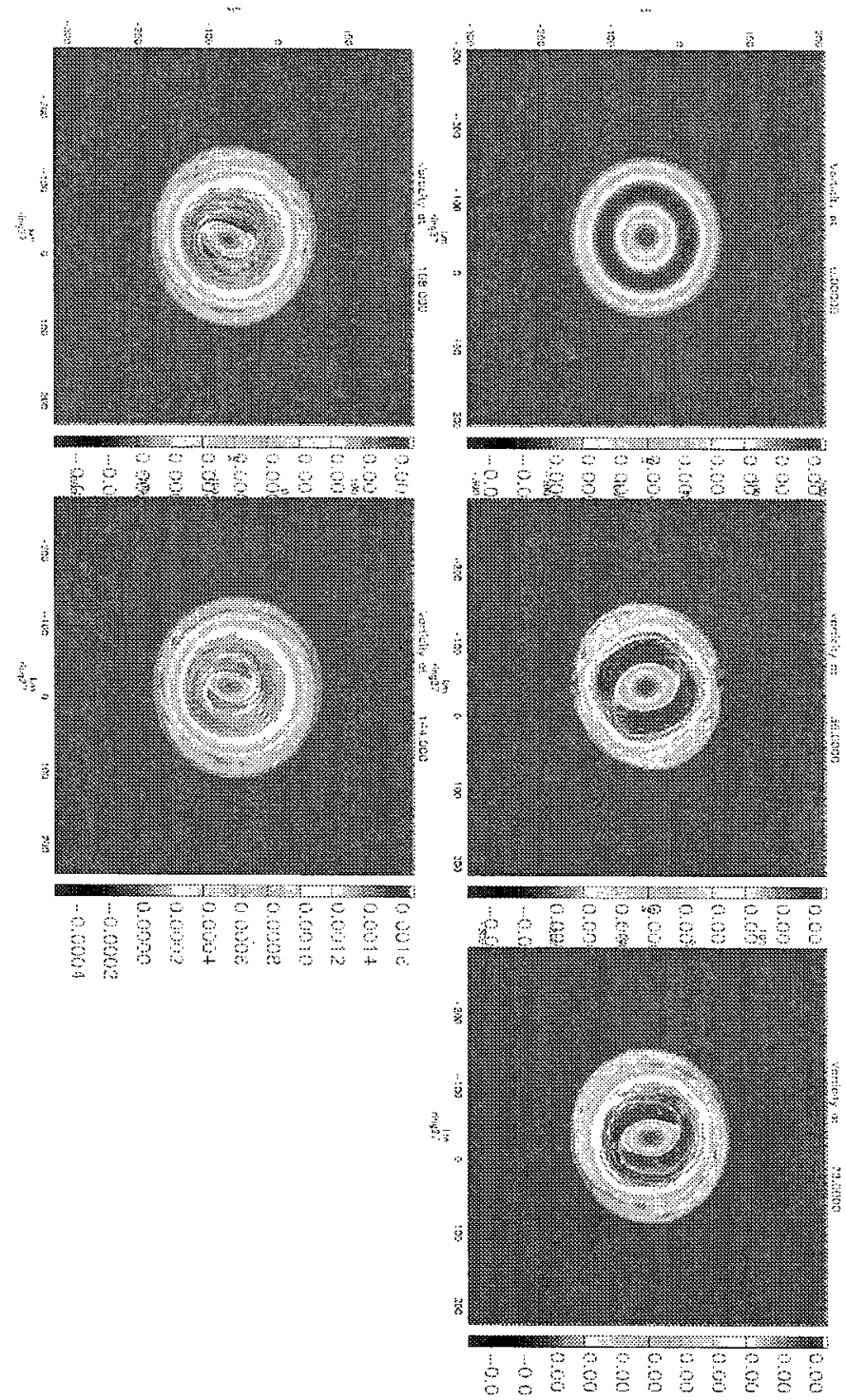

Figure 4: Evolution of very wide ring: $r_{3}-r_{1}=108 \mathrm{~km}$. 


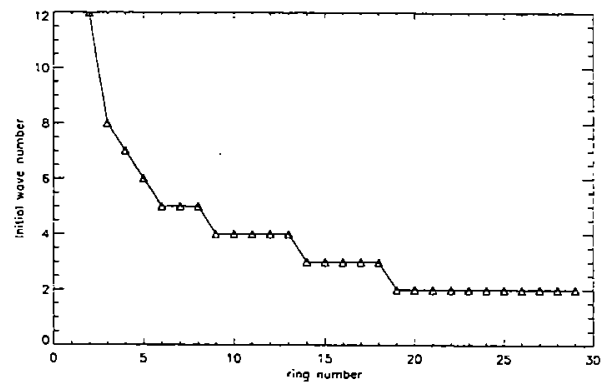

Figure 5: Initial wavenumber as a function of ring number. Ring width $=$ ring number $\times$ $4 \mathrm{~km}$.

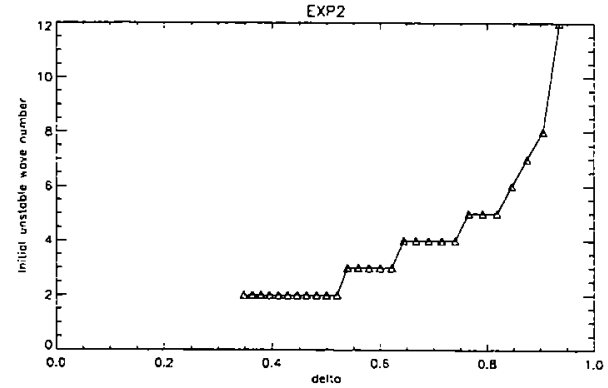

Figure 6: Initial wave number as a function of $\delta$ with $\gamma=0$ for comparison with the linear stability analysis of Schubert et al. (1999).

\section{The Minimum Enstrophy Theory and Final State}

\subsection{The Minimum Enstrophy Theory}

Consistent with Eq. (6), simulations show that the enstrophy decays much faster than the energy for small diffusivity $\nu$. Based on this result, a minimum enstrophy theory has been applied to predict the final states for annular rings (Schubert et al. 1999). The idea is to maximize the enstrophy deficit, i.e. to minimize the final enstrophy, under the constraint of constant energy or angular momentum.

In the case of minimum enstrophy with constrained energy and circulation, I vary the mixing radius $b$ and the wind profile $v(r)$ in the variational problem

$$
\begin{aligned}
0 & =\delta \int_{0}^{b}\left[\zeta_{0}^{2}-\zeta^{2}-\mu^{2}\left(v_{0}^{2}-v^{2}\right)\right] r d r \\
& =2 \int_{0}^{b}\left(-\zeta \delta \zeta+\mu^{2} v \delta v\right) r d r+\left[\zeta_{0}^{2}(b)-\zeta^{2}(b)\right] b \delta b \\
& =2 \int_{0}^{b}\left(\frac{d \zeta}{d r}+\mu^{2} v\right) \delta v r d r-2 b \zeta(b) \delta v(b)+\left[\zeta_{0}^{2}(b)-\zeta^{2}(b)\right] b \delta b \\
& =2 \int_{0}^{b}\left(\frac{d \zeta}{d r}+\mu^{2} v\right) \delta v r d r+\left[\zeta_{0}(b)-\zeta(b)\right]^{2} b \delta b
\end{aligned}
$$

where $\mu^{2}$ is the Lagrange multiplier, $v_{0}(r)$ and $\zeta_{0}(r)$ are the initial wind and vorticity 


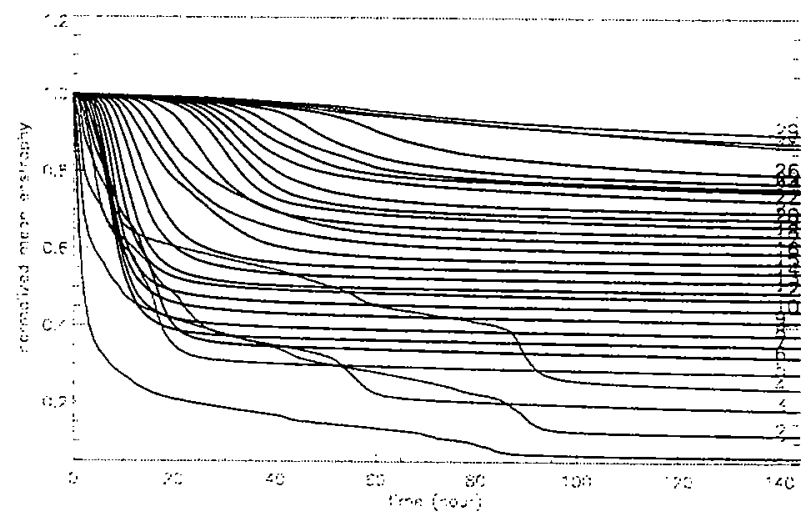

Figure 7: Simulated enstrophy normalized by initial enstrophy as a function of time for the set of rings.

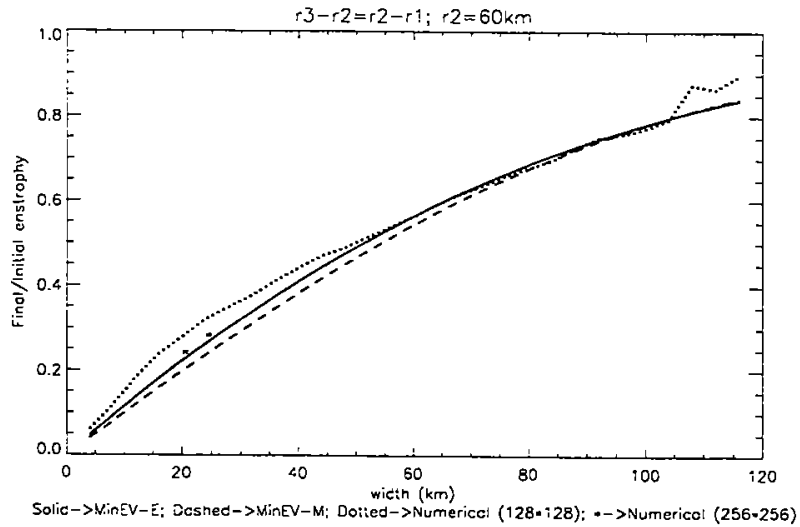

Figure 8: Comparison of theoretical and numerical values of the final enstrophy. Solid line is the prediction of a minimum enstrophy theory with constrained energy. Dashed line is the prediction of a minimum enstrophy theory with constrained angular momentum. Dotted line is the numerical result for runs with $128 \times 128$ resolution. Stars are the numerical results for runs with $256 \times 256$ resolution. 

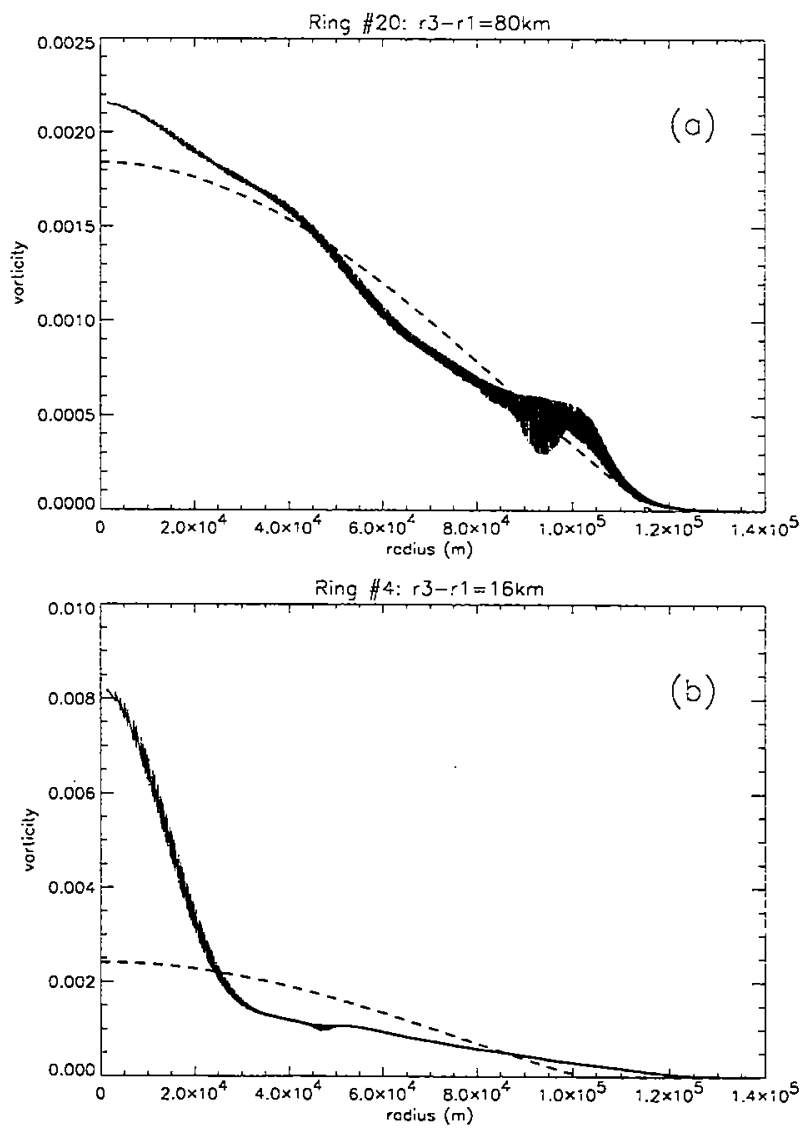

Figure 9: Theoretical and numerical final radial vorticity profile comparison. Numerical results are plotted as scatter plots, Predictions of the minimum enstrophy theory with constrained energy are plotted in dashed lines. 
profiles, $v(r)=v_{0}(r)$ for $r \geq b$. Upon solving the Euler-Lagrange equation resulting from the first term, I obtain the final wind and vorticity profiles

$$
\begin{gathered}
v(r)= \begin{cases}v_{0}(b) J_{1}(\mu r) / J_{1}(\mu b) & \text { if } 0 \leq r \leq b, \\
v_{0}(r) & \text { if } b \leq r<\infty .\end{cases} \\
\zeta(r)= \begin{cases}v_{0}(b) \mu J_{0}(\mu r) / J_{1}(\mu b) & \text { if } 0 \leq r \leq b, \\
0 & \text { if } b \leq r<\infty .\end{cases}
\end{gathered}
$$

Requiring $\zeta(b)=\zeta_{0}(b)=0$ yields

$$
J_{0}(\mu b)=0
$$

so that $\mu b$ must be a zero of the $J_{0}$ Bessel function. The first zero yields the lowest enstrophy, so that $\mu b \approx 2.4048$. Substituting Eq. (9) into the energy constraint $\int_{0}^{b} v_{0}^{2}(r) r d r=$ $\int_{0}^{b} v^{2}(r) r d r$ yields

$$
8 \pi^{2} \int_{0}^{b} v_{0}^{2}(r) r d r=C^{2}
$$

where $C=2 \pi b v_{0}(b)$ is the circulation. Given the initial tangential wind $v_{0}(r)$ and the associated initial vorticity $\zeta_{0}(r), \mu$, and $b$ can thus be determined from (11) and (12).

Similarly, the final enstrophy can be minimized under the constraint of conservation of angular momentum. This leads to the final wind and vorticity profiles

$$
\begin{gathered}
v(r)= \begin{cases}v_{0}(a)(r / a)\left[2-(r / a)^{2}\right], & \text { if } 0 \leq r \leq a, \\
v_{0}(r), & \text { if } a \leq r<\infty,\end{cases} \\
\zeta(r)= \begin{cases}{\left[4 v_{0}(a) / a\right]\left[1-(r / a)^{2}\right],} & \text { if } 0 \leq r \leq a, \\
0, & \text { if } a \leq r<\infty .\end{cases}
\end{gathered}
$$

\subsection{Final Enstrophy Comparison}

Integrating the square of the predicted final vorticity profile Eq. (10) and the square of the initial vorticity profile Eq. (1), I obtained the final and initial enstrophy for each ring. Comparing the ratio between the final and initial enstrophy with the enstrophy ratio between the last and first time step of the simulation (Fig. 8), I find that the predicted final enstrophy agrees well with numerical results for wide rings. The deviation for very wide rings is related to the persistence of the central low vorticity in the simulation and the relaxaition to monopole predicted by theory. The deviation for thin rings can be greatly improved by running the model at higher resolution, as shown by the stars in Fig. 8. A high-resolution simulation is expected to produce a larger differences for thin rings because more grid points are needed to resolve the initial vorticity profiles properly. So, the minimum enstrophy theory is able to predict the final enstrophy of both the thin and the wide rings in my study. 


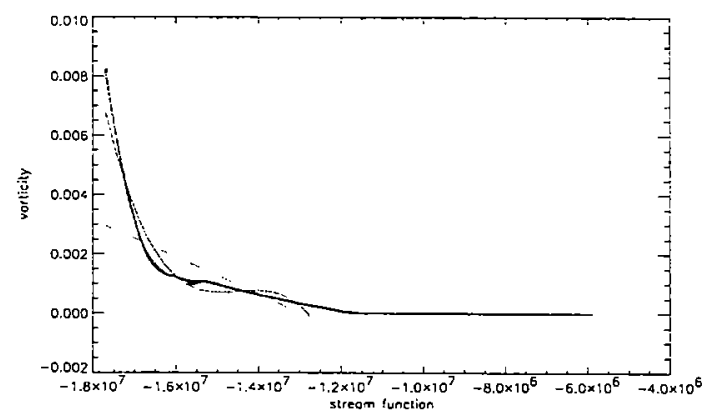

Figure 10: Final vorticity as a function of stream function for thin ring $\left(r_{3}-r_{1}=16\right.$ $\mathrm{km})$. Black line is scatter plot from the final time step. Green line is the prediction of the minimum enstrophy theory with constrained energy. Blue line is a 3rd order polynomial fit for the black scatter plot. Red line is a 5 th order polynomial fit for the black scatter plot.

\subsection{Final Vorticity Profile Comparison}

For wide rings, the vorticity profile predicted by theory, as expressed in Eq. (10) captures most of the features of the simulated final vorticity profile (Fig. 9a). However, for thin rings, the simulated final vorticity considerably overshoots the theoretical prediction at small radii (Fig. 9b). This deviation for thin rings can be viewed in the stream functionvorticity perspective, as shown in Fig. 10. The numerical curve is a scatter plot produced from the vorticity and stream function at every grid point in the output domain at the last time step. Such scatter plots at early times show many fat bands that collapse onto each other with time and eventually become the thin line in Fig. 10. Solving the stream function from (5) with the boundary condition that the final stream function matches the initial stream function at the mixing radius $b$, I obtain the green line in Fig. 10. This linear relationship is expected from a slight modification of the argument given in (8). If, when proceeding from the second line in (8), we integrate the $v \delta v$ term by parts instead of the $\zeta \delta \zeta$ term, we obtain

$$
0=2 \int_{0}^{b}\left(-\zeta-\mu^{2} \psi\right) \delta \zeta r d r+2 \mu^{2} b \psi(b) \delta v(b)+\left[\zeta_{0}^{2}(b)-\zeta^{2}(b)\right] b \delta b
$$

so that $\zeta=-\mu^{2} \psi$ for $0 \leq r \leq b$. Minimum enstrophy theory approximates the numerical curve by a line, though this curve can be better fit by a 5 th order polynomial. This suggests the possible existence of a better variational principle, but I will not pursue this further here.

\section{Comparison with Skewed Rings}

Two sets of experiments with skewed initial vorticity profiles have been performed to compare with the symmetric rings investigated before. One set has a sharper inner edge, while the other has a sharper outer edge. Sample initial conditions are shown in Fig. 11. All the rings have fixed $r_{2}=60 \mathrm{~km}$ and fixed circulation, as before. The evolution of the enstrophy for the thin and wide rings of the three sets of rings is similar, and the final enstrophy can 

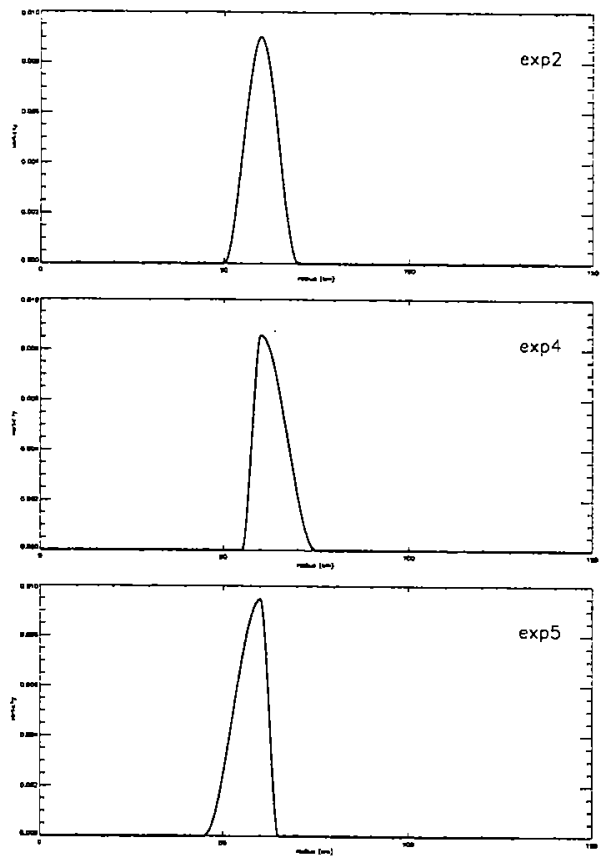

Figure 11: Example initial vorticity profiles for three sets of rings. Exp2: symmetric rings; Exp4: rings with sharper inner edge; Exp5: rings with sharper outer edge.

be predicted by the minimum enstrophy theory. Rings with a sharper inner edge usually have higher initial wave numbers than the others (Fig. 12), and even the widest such ring collapses into a monopole.

\section{Conclusion}

I have investigated the rearrangement of annular rings of high vorticity in this study. Thin rings initially break up into many vortices that subsequently merge. They often come into a configuration where several vortices rotate around for many rational timescales. Such "mesovortex states" correspond to the "stairs" in the enstrophy history plot. Wide rings have lower initial wavenumbers that take longer to grow, and they gradually evolve into monopoles. Very wide rings usually have low central vorticity throughout the simulation. However, all the rings with sharper inner edges evolve into monopoles. The minimum enstrophy theory is useful for predicting the final enstrophy for both thin and wide rings. Although it does an adequate job of predicting the final vorticity profile for wide rings, it fails for thin rings. Skewed rings with sharper inner edges usually have higher initial wave numbers than other rings with the same width, and this might be predicted by a more sophisticated linear theory than that of Schubert et al.

Acknowledgements I would like to thank Wayne Schubert for suggesting this project and guiding me through the summer, Ricardo Prieto for providing the numerical code, and Phil Morrison for help and advice. 


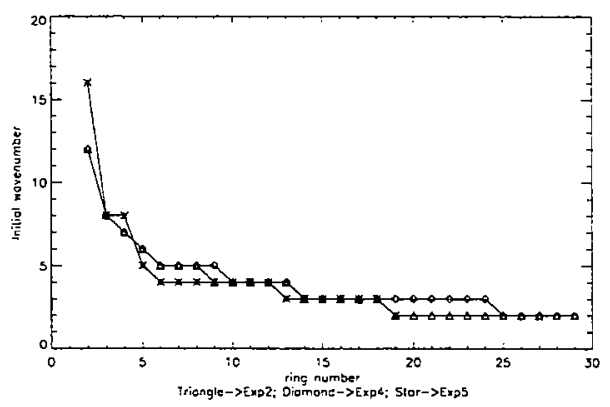

Figure 12: Initial wavenumber as a function of ring number for three sets of rings. $\triangle \operatorname{Exp} 2$ symmetric rings, $\diamond$ Exp4 rings with sharper inner edge, * Exp5 rings with sharper outer edge.

\section{References}

Fulton, S. R., 2001: An adaptive multigrid barotropic tropical cyclone track model. Mon. Wea. Rev., 129, 138-151.

Kossin, J. P., and W. H. Schubert, 2001: Mesovortices, polygonal flow patterns, and rapid pressure falls in hurricane-like vortices. J. Atmos. Sci., 58, 2196-2209.

Schubert, W. H., M. T. Montgomery, R. K. Taft, T. A. Guinn, S. R. Fulton, J. P. Kossin, and J. P. Edwards, 1999: Polygonal eyewalls, asymmetric eye contraction, and potential vorticity mixing in hurricanes. J. Atmos. Sci., 56, 1197-1223. 


\title{
Bounds on the Dissipation in MHD Problems in Plane Geometry with Magnetic and Velocity Shear
}

\author{
Francois Pétrélis \\ Ecole Normale Superieure de Paris, France
}

\begin{abstract}
We study the total dissipation for flows described by the magnetohydrodynamic (MHD) equations. For some boundary conditions a lower bound can be found that is achieved by the equivalent of Stokes flow for MHD. Using the Doering and Constantin method [1], also called "the background method," we derive an upper bound for the dissipation of some simple flows. In the case of a shear layer with both velocity and magnetic shear, the dependency of the upper bound as a function of the control parameters is determined. As a by-product of this calculation, an energy stability domain for this flow is calculated and a result that is bigger than that previously calculated by Tasso et al. [2] is obtained. We study a simple model of the sheet pinch and show that the upper bound tends to zero as the magnetic diffusivity tends to zero. In this sense, we obtain an antiturbulence result because there is no residual dissipation in the limit of infinite control parameter.
\end{abstract}

Plasma is said to be the state of matter which is the most widespread in the universe. Stars and interstellar medium are made of plasma. On Earth, there have been many experiments which attempt to use plasmas in order to achieve nuclear fusion. One of the properties of plasma is its ability to conduct electricity. Thus, the equations of motion are coupled to the equations of electromagnetism. Liquid metals share the same property. They are present in the interior of the Earth where they are responsible for the generation of magnetic fields by the dynamo effect. They are also used industrially for transporting heat, in nuclear fission devices for instance. Both liquid metals and plasmas can be described by the MHD equations [3].

In the framework of MHD, we focus on the total dissipation which is the sum of the viscous dissipation and the Joule dissipation integrated over the fluid volume. This quantity is important for various reasons. It is a global characteristic of the system and its time average is equal to the time average of the power injected into the system. Moreover, it is equal to the heat production in the system. For fusion experiments, it is of major importance because it produces the temperature increase that is hoped will lead to the onset of nuclear reactions. On the contrary, for dynamo experiments in liquid metals, the increase in temperature must be avoided because it increases the resistivity of the fluid and then increases the value of the critical velocity for dynamo action.

In hydrodynamics, viscous dissipation has been widely studied. One of the first results in this field was derived by Stokes, whose results were generalized by Keller et al. [4]. They proved that the solution of the Stokes equations minimizes the dissipation over all divergence-free fields satisfying boundary conditions of fixed velocity at the boundary. Note that the Stokes solution may only be a solution of the Navier-Stokes equations in the limit of zero control parameter (generally the Reynolds number). In most cases, for low values of the control parameter, a laminar solution exists, but upon increasing the control parameter, this solution may become unstable. From the evolution equation for the energy of a perturbation to the basic state, one finds that the dissipation gives rise to a decrease of this energy when 
the control parameter is smaller than a certain value. This value defines the domain of absolute stability in which the laminar solution is energy stable [5]. Outside this domain, the solution can be unstable and possibly turbulent. It is then impossible to explicitly calculate the solution of the Navier-Stokes equation and the value of the dissipation. Early studies by Howard [6] and Busse [7] tried to find upper bounds for the dissipation using a variational formulation with added constraints on the manifold of the fields over which the maximization is made. A very striking result is that the optimizing field seems to share some properties with the time average of the fields measured in experiments. More recently, Doering and Constantin [1] developed a different approach called "the background method" which allows for an easier evaluation of the upper bound on the dissipation.

In MHD, some results have been derived by Tasso et al. [2] concerning the energy stability for some flows. In some cases the energy domain that occurs is infinite, which insures stability of the laminar solution for all values of the control parameter [8]. Using the Howard-Busse method, Soward [9] derived an upper bound for the ohmic dissipation in a turbulent thermal layer permeated by a horizontal magnetic field maintained by dynamo action. With the same method, Wang et al. [10] obtained an upper bound for the dissipation in a cylindrical pinch.

In the present work, we derive a result on the lower bound for the dissipation in some MHD flows. For fixed values of the fields at the boundary, we prove that the Stokes-like solution of the MHD equations minimizes the total dissipation over all divergence-free fields satisfying the boundary conditions. We then apply the background method to two MHD problems. The first one is a shear layer with both magnetic and velocity shear. [13]. As a by-product of our calculation, we calculate a domain in which the basic state is energy stable. The result is bigger than previous results [2]. Both lower and upper bounds for the dissipation are derived, and their dependency on the parameters of the system are presented. The last part of this report deals with the study of a model of the plane sheet pinch. Using the background method, we prove that the upper bound for the dissipation tends to zero with the magnetic diffusivity, while all other parameters are held fixed. In this sense, this is an antiturbulence theorem because there is no residual dissipation in the limit of zero magnetic viscosity. A similar result was recently derived for horizontal convection [11] but it is the first time that the background method allows one to prove such a result. During this summer J. Siggers also applied the Doering-Constantin method to horizontal convection (see her report). In that case, at high value of the control parameter the flow is not stationary and develops boundary layers even if the dissipation tends to zero. According to numerical simulation [12], this is not the case for the plane sheet pinch in which the static solution seems to be always stable. Our result does not prove this stability, but is consistent with it because the dissipation of the static solution tends to zero with the magnetic viscosity.

\section{Stokes-like Solutions and Lower Bound}

Assuming that the flow is incompressible and that the electrical properties of the fluid are well described by the MHD equations, the velocity field $\mathbf{v}$ and the magnetic field $\mathbf{B}$ are 
solutions of

$$
\begin{array}{r}
\frac{\partial \mathbf{v}}{\partial t}+\mathbf{v} \cdot \nabla \mathbf{v}=\mathbf{f}-\nabla \tilde{P}+\nu \nabla^{2} \mathbf{v}+\mathbf{B} \cdot \nabla \mathbf{B} \text { and } \nabla \cdot \mathbf{v}=0 \\
\frac{\partial \mathbf{B}}{\partial t}+\mathbf{v} \cdot \nabla \mathbf{B}=\mathbf{B} \cdot \nabla \mathbf{v}+\eta \nabla^{2} \mathbf{B} \text { and } \nabla \cdot \mathbf{B}=0
\end{array}
$$

where $\mathbf{f}$ is a body force. Here $\tilde{P}=P / \rho+\mathbf{B}^{2} /(2 \rho)$ where $P$ is the hydrodynamic pressure, $\rho$ the density, and $\mathbf{B}^{2} / 2$ is the magnetic pressure. From now on, we drop the tilde. The magnetic and kinetic viscosity of the fluid are $\eta=\left(\mu_{0} \sigma\right)^{-1}$ and $\nu$, where $\sigma$ is its conductivity.

We have to be precise about the boundary conditions for the fields. If the fluid is in contact with a solid, it is natural to use no-slip boundary conditions for the velocity at the boundary, $\partial D$, and say $\left.\mathbf{v}\right|_{\partial D}=\mathbf{v}_{b}$ where $\mathbf{v}_{b}$ is the velocity of the boundary. The choice is not so simple for the magnetic field. We suppose that the solid at the boundary is a perfect conductor in which the magnetic field is frozen. Since the normal component of the magnetic field at the boundary is continuous, its value is then fixed at the boundary. By imposing the surface current at the boundary, we can fix the value of the discontinuity in the tangential component of the magnetic field and since its value is fixed in the solid, the tangential component of the magnetic field is also fixed at the boundary. This is a convenient but idealized boundary condition for the magnetic field. It has previously been used in studies of tearing instability and is sometimes called the "line-tied" boundary condition. Another boundary condition that is easier to impose experimentally than line tying, is to fix the tangential current at the boundary. In this case, some derivatives of the field are fixed while the normal component of the magnetic field must still be continuous. In this section, we restrict ourselves to line-tied boundary conditions.

The dissipation per unit mass is defined by

$$
D=\nu\left\langle|\nabla \times \mathbf{v}|^{2}\right\rangle+\eta\left\langle|\nabla \times \mathbf{B}|^{2}\right\rangle
$$

where $\langle f\rangle=\frac{1}{V} \int_{V} f(\vec{r}) d \vec{r}$ and $V$ is the volume of the fluid. Following Keller et al. [4], we define the excess dissipation rate by $D_{e}=\nu\left\langle|\nabla \times \mathbf{v}|^{2}\right\rangle+\eta\left\langle|\nabla \times \mathbf{B}|^{2}\right\rangle-2\langle\mathbf{f} \cdot \mathbf{v}\rangle$. This is the dissipation minus twice the power input by external body forces. It reduces to the total dissipation when the force does not input energy into the system. Without taking into account the MHD equations, we wonder which stationary velocity and magnetic fields are extremal values for the excess dissipation with the assumptions that the fields are divergence-free and have fixed values at the boundary. We introduce two Lagrange multipliers $-2 P(\vec{r})$ and $-2 Q(\vec{r})$ to insure that the fields are divergence-free. Taking the variational derivatives of $D_{e}-2 P \nabla \cdot \mathbf{v}-2 Q \nabla \cdot \mathbf{B}$ with respect to $\mathbf{v}$ and $\mathbf{B}$ gives

$$
\begin{aligned}
\nu \nabla^{2} \mathbf{v}+\mathbf{f}-\nabla P & =0, \\
\eta \nabla^{2} \mathbf{B}-\nabla Q & =0 .
\end{aligned}
$$

We call these equations, together with the divergence conditions, the Stokes-like equations for MHD. The two fields are not coupled in these equations, as one expects since $D_{e}$ is the sum of two functionals depending separately on the velocity and the magnetic field. The equations for the velocity field are the same as for Stokes flow without the magnetic field. This equation is the Navier-Stokes equation for a stationary velocity field in the low 
Reynolds number limit, where the inertia terms are neglected and where the Lorentz force is also neglected. The equation for the magnetic field is more surprising. The Lagrange multiplier used to enforce the divergence-free nature of the field appears as a pressure in equation (5). It is possible that the term $\mathbf{B} \cdot \nabla \mathbf{v}-\mathbf{v} \cdot \nabla \mathbf{B}$ of equation (2) reduces to the gradient of a scalar function. Then, the Stokes-like equation for the magnetic field is equivalent to the induction equation for a stationary magnetic field. Otherwise, choosing $Q=0$, equation (5) is the limit for low Reynolds number of the induction equation for a stationary magnetic field.

By taking the variational derivative of the functional, we proved that the Stokes-like solution of the MHD problem is a stationary value of the excess dissipation over all divergencefree magnetic and velocity fields with fixed values at the boundary. Indeed, it is possible to prove that this is a minimum over all continuous fields possessing piecewise continuous derivatives, satisfying the boundary conditions and being divergence-free. Let us write such a field as $\mathbf{v}_{t}=\mathbf{v}_{s}+\mathbf{u}$ (resp. $\mathbf{B}_{t}=\mathbf{B}_{s}+\mathbf{b}$ ) where the subscript $t$ stands for total field, $s$ stands for Stokes-like solution of equations (4) and (5). Note that $\mathbf{u}$ and $\mathbf{b}$ are zero at the boundary. We get

$$
\begin{aligned}
D_{e}\left(\mathbf{v}_{t}, \mathbf{B}_{t}\right) & =D_{e}\left(\mathbf{v}_{s}, \mathbf{b}_{s}\right)+D_{e}(\mathbf{u}, \mathbf{b})+2 \nu\left\langle\nabla \times \mathbf{v}_{s} \cdot \nabla \times \mathbf{v}\right\rangle+2 \eta\left\langle\nabla \times \mathbf{B}_{s} \cdot \nabla \times \mathbf{b}\right\rangle, \\
& =D_{e}\left(\mathbf{v}_{s}, \mathbf{b}_{s}\right)+D(\mathbf{u}, \mathbf{b})-2\left(\left\langle\mathbf{u} \cdot\left(\nu \nabla^{2} \mathbf{v}_{s}+\mathbf{f}\right)\right\rangle+\left\langle\eta \mathbf{b} \cdot \nabla^{2} \mathbf{B}_{s}\right\rangle\right) .
\end{aligned}
$$

Using the Stokes-like property of $\mathbf{v}_{s}$ and $\mathbf{b}_{\boldsymbol{s}}$, the term in parenthesis is seen to be zero and we are left with

$$
D_{e}\left(\mathbf{v}_{t}, \mathbf{B}_{t}\right)=D_{e}\left(\mathbf{v}_{s}, \mathbf{b}_{s}\right)+D(\mathbf{u}, \mathbf{b}) \geq D_{e}\left(\mathbf{v}_{s}, \mathbf{b}_{s}\right) .
$$

This result is a straightforward generalization to magnetohydrodynamic problems of the result of Keller et al. [4] for hydrodynamic flows. This calculation is valid for the particular boundary conditions of fixed velocity and magnetic field at the boundary, but this is not true for some boundary conditions, for instance if the tangential currents are fixed instead of the magnetic field.

The result is used in the next section where the stationary solution of the MHD equation is of Stokes-like form and is thus a minimum value for the excess dissipation of any realized solution, even not stationary. Another possible application is to estimate the lower bound on the excess dissipation rate by using trial functions for $\mathbf{v}$ and $\mathbf{b}$ rather than solving the MHD equations for stationary fields. In their paper, Keller et al. proved also a reciprocal principle: the Stokes solution of an hydrodynamic problem can be obtained as the maximizing field of another functional, the maximal value of which is the excess dissipation $D_{e}$. We think that the same result can be derived for MHD problems but the proof remains to be done.

\section{A Layer with Both Velocity and Magnetic Shear}

We now consider a layer of fluid that can support shear in both the velocity and magnetic fields. We assume a layer of height $d$ with two periodic boundary conditions in the horizontal directions $x, z$ as defined in Figure (1). The coordinates $(x, y, z)$ correspond to the unit vectors $\mathbf{i}, \mathbf{j}, \mathbf{k}$ respectively. It will turn out that our results are independent of any uniform horizontal magnetic field and thus only depend on the shear part of the applied field. We 


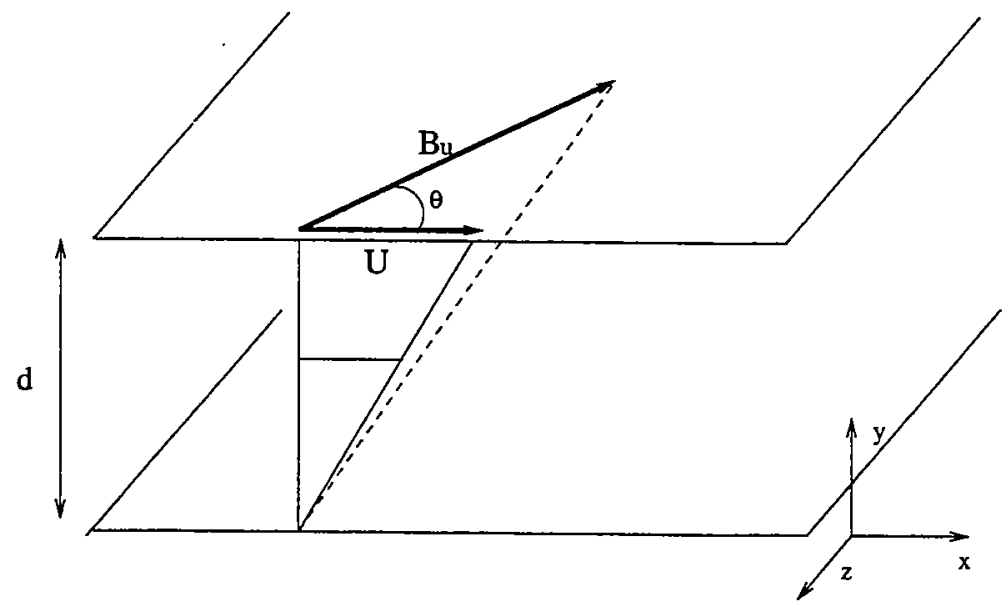

Figure 1: Sketch of a fluid layer submitted to a horizontal shear magnetic field and shear velocity field.

suppose that the value of the magnetic field is fixed at the boundary. The magnetic shear is imposed by setting $B_{u} \widehat{\rho}=B_{u}(\cos \theta \mathbf{i}+\sin \theta \mathbf{k})$ at the upper surface $y=d$ and $\mathbf{B}=0$ at the bottom surface $y=0$. The velocity shear is induced by moving the upper surface at $\mathbf{v}=U \mathbf{i}$, while making the bottom surface immobile. We use no-slip conditions for the velocity field, $\mathbf{v}=0$ at $y=0$ and $\mathbf{v}=U \mathbf{i}$ at $y=d$.

This is a simple model that can give rise to a variety of shear instabilities including the tearing mode instability. It occurs widely in plasma physics, for instance in Tokamak experiments [13]. Furth et al. [14] have studied the onset of tearing instability and the linear growth rate. Chen et al. [15] have studied the effect of a shear flow and took into account the kinetic viscosity. This simple model is also a plane-Taylor flow for a liquid metal subjected to a sheared magnetic field. Using energy methods Tasso et al. [2] calculated a domain in which the basic solution is energy stable. As a by-product of our calculation we improve Tasso's result. We focus on the dissipation as defined by equation (3). Note that even if we take gravity into account and if the layer is horizontal, the excess dissipation rate reduces to the dissipation rate. Moreover, for a quantity $\mathbf{g}$ that satisfies boundary conditions of fixed value at the boundary, we have $\left\langle|\nabla \mathbf{g}|^{2}\right\rangle=\left\langle|\nabla \times \mathbf{g}|^{2}\right\rangle$ where $|\nabla \mathbf{g}|^{2}=\sum_{\alpha, \beta}\left(\partial_{\alpha} g_{\beta}\right)^{2}$. Thus $D=\nu\left\langle|\nabla \mathbf{v}|^{2}\right\rangle+\eta\left\langle|\nabla \mathbf{B}|^{2}\right\rangle$.

For this problem we have 6 dimensional parameters $B_{u}, U, d, \nu, \eta, \theta$ that can be measured using 2 dimensions. We can then construct 4 dimensionless numbers which completely describe the system. The angle $\theta$ is one of them and three other possible numbers are

$$
P_{m}=\frac{\nu}{\eta}, R e=\frac{U d}{\nu} \text { and } M=\frac{B_{u} d}{\nu},
$$

respectively, the magnetic Prandtl number, the Reynolds number and the equivalent of the Reynolds number constructed with the magnetic field. We get, by dimensional analysis, that the dissipation is

$$
D=\frac{U^{3}}{d} \phi\left(P_{m}, R e, M, \theta\right)
$$


where $\phi$ is an unknown function of the dimensionless numbers.

At low value of $M$ and $R e$, one expects the velocity and magnetic fields to be independent. Indeed, a stationary laminar solution of the full set of equations is

$$
\mathbf{v}=\frac{U}{d} y \mathbf{i}, \text { and } \mathbf{B}=\frac{B_{u}}{d} y \widehat{\rho} .
$$

These are respectively a sheared velocity field and a sheared magnetic field. There is no power input by external forces, so the excess dissipation is equal to the dissipation $D_{l}$

$$
D_{l}=\nu\left(\frac{U}{d}\right)^{2}+\eta\left(\frac{B_{u}}{d}\right)^{2} \text {, or } \phi=\frac{1}{R e}\left(1+\frac{1}{P_{m}}\left(\frac{M}{R_{e}}\right)^{2}\right) \text {. }
$$

Note that the fields are solutions of the Stokes-like equations (4) and (5) with $P=Q=0$. Since the boundary conditions are that the values of the fields at the boundary are fixed, equation (6) proves that the dissipation for the laminar solution is a lower bound for the total dissipation of any solution of the MHD equations.

If we increase the value of $M$ and $R e$, we expect that the laminar stationary solution will become unstable and possibly turbulent. Using the energy equation for the perturbation to a basic state, we derive two results. The first one is obtained if we use the laminar stationary solution as a basic state. It gives values of $M$ and $R e$, below which all the fluctuations decrease to zero. We do not calculate the maximal values of $M$ and $R e$. Above these maximal values, the energy of some perturbations can increase. These maximal values define the energy stability domain in the $(M, R e)$ plane and is equivalent to the energy Reynolds number used for usual hydrodynamic instabilities [5]. Because we use crude estimates, the values of $M$ and $R e$ that we calculate are inside the true (i.e. optimal) energy stability domain. Even if they are not optimal, these are values below which the basic state is energy stable. For higher values of $M$ and $R e$, we use a well defined basic state and derive our second result which is an upper bound for the dissipation in the system.

\subsection{Energy Equation for Perturbations to a Basic State}

For generality, we derive the energy equation for perturbations to a basic state given by

$$
\mathbf{v}_{b}=U(y) \mathbf{i} \text { and } \mathbf{B}_{b}=B_{1}(y) \mathbf{i}+B_{2}(y) \mathbf{j}+B_{3}(y) \mathbf{k} .
$$

The total velocity and magnetic fields $\left(\mathbf{B}_{t}\right.$ and $\left.\mathbf{v}_{t}\right)$ are the sum of the basic state and the fluctuations ( $\mathbf{v}$ and $\mathbf{b}$ )

$$
\mathbf{v}_{t}=\mathbf{v}_{b}+\mathbf{v} \text { and } \mathbf{B}_{t}=\mathbf{B}_{b}+\mathbf{b} .
$$

The components of $\mathbf{v}$ (resp. b) are $v_{1}, v_{2}, v_{3}$ (resp. $b_{1}, b_{2}, b_{3}$ ). The direction of the velocity shear is $i$ and we have assumed that the basic state is independent of the horizontal coordinates. Upon taking the dot product of (2) with $\mathbf{b}$, integrating by parts, and applying the boundary conditions, we obtain

$$
\frac{\partial}{\partial t}\left\langle\frac{\mathbf{b}^{2}}{2}\right\rangle=-\left\langle v_{2} \mathbf{b} \cdot \mathbf{B}_{b}^{\prime}\right\rangle-\eta\left\langle|\nabla \mathbf{b}|^{2}\right\rangle+\left\langle\mathbf{b} \cdot\left(\mathbf{B}_{t} \cdot \nabla\right) \mathbf{v}\right\rangle+\left\langle U^{\prime} B_{t 2} b_{1}\right\rangle+\eta\left\langle\mathbf{b} \cdot \mathbf{B}_{b}^{\prime \prime}\right\rangle
$$


where $f^{\prime}$ means the derivative of $f$ with respect to $y$. Upon taking the dot product of equation (1) with $\mathbf{v}$, we obtain

$$
\frac{\partial}{\partial t}\left\langle\frac{\mathbf{v}^{2}}{2}\right\rangle=-\left\langle U^{\prime} v_{1} v_{2}\right\rangle-\nu\left\langle|\nabla \mathbf{v}|^{2}\right\rangle+\left\langle\mathbf{v} \cdot\left(\mathbf{B}_{t} \cdot \nabla\right) \mathbf{B}_{t}\right\rangle+\nu\left\langle v_{1} U^{\prime \prime}\right\rangle
$$

Because the fluctuations in velocity and magnetic field are zero at the boundaries, we have

$$
\left\langle\mathbf{v} \cdot\left(\mathbf{B}_{t} \cdot \nabla\right) \mathbf{B}_{t}\right\rangle=-\left\langle\mathbf{B}_{b} \cdot\left(\mathbf{B}_{t} \cdot \nabla\right) \mathbf{v}\right\rangle-\left\langle\mathbf{b} \cdot\left(\mathbf{B}_{t} \cdot \nabla\right) \mathbf{v}\right\rangle .
$$

This result is similar to the energy conservation in an electromechanical process which states that the mechanical power of the Lorentz force is opposite to the electrical power of the electromotive force. By integration by parts, one gets

$$
\left\langle\mathbf{B}_{b} \cdot\left(\mathbf{B}_{t} \cdot \nabla\right) \mathbf{v}\right\rangle=-\left\langle B_{t, 2} \mathbf{B}_{b}^{\prime} \cdot \mathbf{v}\right\rangle \text {. }
$$

Taking the sum of equations (12) and (13) and using the two former equations, we derive

$$
\begin{aligned}
\frac{\partial}{\partial t}\left\langle\epsilon_{p}\right\rangle= & -\left\langle U^{\prime} v_{1} v_{2}\right\rangle-\left\langle v_{2} \mathbf{b} \cdot \mathbf{B}_{b}^{\prime}\right\rangle-\nu\left\langle|\nabla \vec{v}|^{2}\right\rangle-\nu\left\langle U^{\prime} v_{1}^{\prime}\right\rangle+\left\langle U^{\prime} B_{b 2} b_{1}\right\rangle \\
& +\left\langle U^{\prime} b_{2} b_{1}\right\rangle-\eta\left\langle|\nabla \vec{b}|^{2}\right\rangle-\eta\left\langle\mathbf{B}_{b}^{\prime} \cdot \mathbf{b}^{\prime}\right\rangle+\left\langle B_{b, 2} \mathbf{B}_{b}^{\prime} \cdot \mathbf{v}\right\rangle+\left\langle b_{y} \mathbf{B}_{b}^{\prime} \cdot \mathbf{v}\right\rangle
\end{aligned}
$$

where $\epsilon_{p}=\frac{\mathbf{v}^{2}+\mathbf{b}^{2}}{2}$.

Using equation (16) for the case of the shear layer, we obtain two results. If the basic state is the stationary solution given by equations (8), we will show that up to some value of $M$, say $M_{E}$ which is function of $R e$ and $P_{m}$, the energy of any perturbation to the laminar solution tends to zero. Since this is a positive definite quantity, this means that asymptotically the perturbation tends to zero, and for values of $M$ and $\operatorname{Re}$ such that $M$ is smaller than $M_{E}$, the stationary basic state is the only stable solution of the problem. Using another well-defined background field, we also compute an upper bound for the dissipation.

Note that equation (16) is independent of any horizontal basic magnetic field which is independent of $y$. The results concerning the energy stability and the upper bound on dissipation are thus also independent of such fields. In the light of this remark, the simple example that we are dealing with appears to be more general. Any horizontal magnetic field applied at the boundaries can be decomposed into its value at the bottom surface plus a shear field, and only the latter part enters into our calculations of the energy stability and upper bounds on the dissipation.

\subsection{Energy Stability}

We use as background fields the laminar solution given by equation (8) and obtain the evolution equation for the energy of a perturbation

$$
\frac{\partial}{\partial t}\left\langle\epsilon_{p}\right\rangle=-\mathcal{Q}(\mathbf{v}, \mathbf{b})
$$

where $\mathcal{Q}$ is quadratic in the velocity and magnetic field

$$
\mathcal{Q}(\mathbf{v}, \mathbf{b})=\nu\left\langle|\nabla \mathbf{v}|^{2}\right\rangle+\eta\left\langle|\nabla \mathbf{b}|^{2}\right\rangle-\frac{B_{u}}{d}\left\langle b_{2}(\mathbf{v} \cdot \widehat{\rho})-v_{2}(\mathbf{b} \cdot \widehat{\rho})\right\rangle-\frac{U}{d}\left\langle v_{1} v_{2}-b_{1} b_{2}\right\rangle .
$$


If $\mathcal{Q}$ is positive for all $\mathbf{v}$ and $\mathbf{b}$ then the basic stationary state is energy stable. We rescale the variables and use $\tilde{\mathbf{v}}=\lambda \mathbf{v}$ and $\tilde{\mathbf{b}}=\mu \mathbf{v}$ with $\lambda$ and $\mu$ positive. This yields

$$
\begin{aligned}
\mathcal{Q}(\mathbf{v}, \mathbf{b})= & \tilde{\mathcal{Q}}(\tilde{\mathbf{v}}, \tilde{\mathbf{b}}) \\
= & \frac{\nu}{\lambda^{2}}\left\langle|\nabla \tilde{\mathbf{v}}|^{2}\right\rangle+\frac{\eta}{\mu^{2}}\left\langle|\nabla \tilde{\mathbf{b}}|^{2}\right\rangle \\
& -\frac{B_{u}}{d \mu \lambda}\left\langle\tilde{b}_{2}(\tilde{\mathbf{v}} \cdot \hat{\rho})-\tilde{v}_{2}(\tilde{\mathbf{b}} \cdot \widehat{\rho})\right\rangle-\frac{U}{d}\left\langle\frac{1}{\lambda^{2}} \tilde{v}_{1} \tilde{v}_{2}-\frac{1}{\mu^{2}} \tilde{b}_{1} \tilde{b}_{2}\right\rangle .
\end{aligned}
$$

Clearly, $\mathcal{Q}$ is positive definite if and only if $\tilde{\mathcal{Q}}$ is positive definite. We now consider $\tilde{\mathcal{Q}}$ and drop the tilde to relieve notation. We have the inequalities

$$
\begin{aligned}
\left|\left\langle b_{2}(\mathbf{v} \cdot \widehat{\rho})-v_{2}(\mathbf{b} \cdot \widehat{\rho})\right\rangle\right| & \leq \frac{1}{2}\left(\left\langle b_{2}^{2}\right\rangle+\left\langle(\mathbf{v} \cdot \widehat{\rho})^{2}\right\rangle+\left\langle(\mathbf{b} \cdot \widehat{\rho})^{2}\right\rangle+\left\langle v_{2}^{2}\right\rangle\right), \\
& \leq \frac{1}{2}\left(\left\langle\mathbf{b}^{2}\right\rangle+\left\langle\mathbf{v}^{2}\right\rangle\right) \\
\frac{\pi^{2}}{d^{2}}\left\langle\mathbf{v}^{2}\right\rangle & \leq\left\langle|\nabla \mathbf{v}|^{2}\right\rangle .
\end{aligned}
$$

The last one is true for a vector field periodic in the horizontal coordinates and zero at the top and bottom surface [1]. Thus it holds also for the magnetic field and we obtain that

$$
\mathcal{Q} \geq \frac{1}{\lambda^{2}}\left(\frac{\nu \pi^{2}}{d^{2}}-\frac{U}{2 d}-\frac{B_{u}}{2 d \alpha}\right)\left\langle\mathbf{v}^{2}\right\rangle+\frac{1}{\mu^{2}}\left(\frac{\eta \pi^{2}}{d^{2}}-\frac{U}{2 d}-\frac{B_{u} \alpha}{2 d}\right)\left\langle\mathbf{b}^{2}\right\rangle,
$$

where $\alpha=\frac{\mu}{\lambda}>0$. Note that $\theta$ does not enter into the expression of the bound of $\mathcal{Q}$, therefore the domain in which the basic state is energy stable will be independent of $\theta$. A sufficient condition for $\mathcal{Q}$ to be positive definite is that there exists an $\alpha>0$ such that

$$
2 \pi^{2}-\left(R e+\frac{M}{\alpha}\right)>0 \text { and } 2 \pi^{2}-P_{m}(R e+M \alpha)>0 .
$$

The maximal value of $M$ below which both inequalities are satisfied is

$$
M_{E}^{2}=\left(2 \pi^{2}-R e\right)\left(2 \pi^{2} / P_{m}-R e\right) .
$$

If $M \leq M_{E}$ the laminar flow is energy stable. Tasso et al. [2] determined the boundary of a domain in which the basic state is energy stable as $R e \max \left(1, P_{m}\right)+M_{E} \max \left(1, P_{m}\right)=2 \pi^{2}$, while our result can be written as

$\left(M_{E} \max \left(1, P_{m}\right)\right)^{2}=\left(2 \pi^{2} \max \left(1, P_{m}\right)-R e \max \left(1, P_{m}\right)\right)\left(2 \pi^{2} \frac{\max \left(1, P_{m}\right)}{P_{m}}-R e \max \left(1, P_{m}\right)\right)$. We plot in Figure (2) the domains for different values of $P_{m}$ in the

(Re $\left.\max \left(1, P_{m}\right), M_{E} \max \left(1, P_{m}\right)\right)$ plane. Note that with this choice of variables, the domains are the same for $P_{m}$ and $P_{m}^{-1}$. We therefore restrict ourselves to $P_{m} \geq 1$. For $P_{m}=1$ our result is the same as Tasso's. For $P_{m} \neq 1$ the domains are bigger than Tasso's. The gain in stability comes from the change of variables in equation (19) which gives a better estimate of $\mathcal{Q}$. Note that this procedure is equivalent to a less physical one consisting, in equation (20), of bounding any product $\langle a b\rangle$ by $\langle a b\rangle \leq \frac{1}{2}\left(\alpha\left\langle a^{2}\right\rangle+\frac{1}{\alpha}\left\langle b^{2}\right\rangle\right)$ where $a$ and $b$ are two fields and $\alpha$ is strictly positive. 


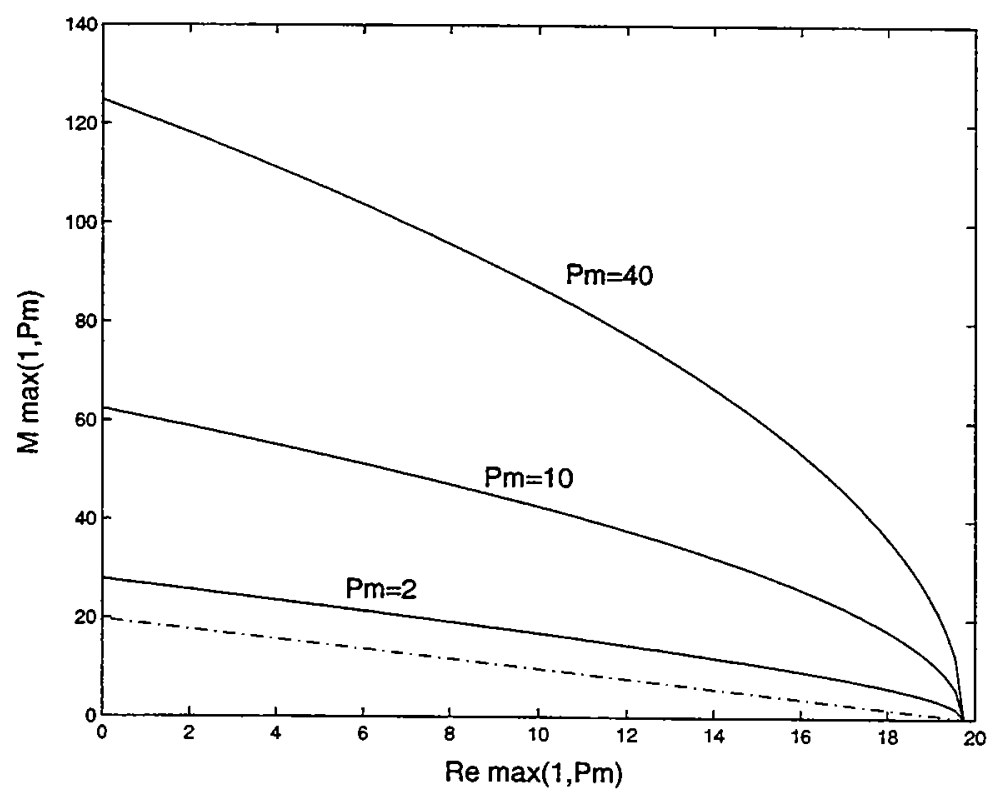

Figure 2: Boundary of domains in which the basic state is energy stable for a shear layer with both velocity and magnetic shear in the $\left(R_{e} \max \left(1, P_{m}\right), M_{E} \max \left(1, P_{m}\right)\right)$ plane. Below a curve, the laminar stationary solution is energy stable for the particular value of $P_{m}$. The dashed line is Tasso's result and our result for $P_{m}=1$. The continuous line are our results for increasing values of $P_{m}$. The curves are the same for $P_{m}$ and $P_{m}^{-1}$.

For $R e=0$, we get $M_{E}=2 \pi^{2} \sqrt{P_{m}}$. If we use the dimensionless parameter $M^{\prime}=\frac{B d}{\sqrt{\nu \eta}}$ instead of $M$, the boundary of the domain is independent of the magnetic Prandtl number, as the critical Rayleigh number is independent of the thermal Prandtl number in thermal convection. For $M=0$, we recover the plane-Couette instability for a conducting fluid. The value of $R e$ at the boundary of the domain depends on $P_{m}$ in a surprising way. Introducing the magnetic Reynolds number $R_{m}=R e / P_{m}$, the boundary of the domain is given by $\max \left(R e, R_{m}\right) \leq 2 \pi^{2}$. For $\nu \leq \eta, R e \geq R_{m}$, the domain is the same as for a nonconducting liquid flow. For $\nu \geq \eta, R e \leq R_{m}$, the boundary of the domain corresponds to a lower value of $R e$ than for a nonconducting liquid. It would be very interesting to understand if this effect is related to an underestimate of the energy stability domain due to our crude estimates of $\mathcal{Q}$, or if, for $P_{m} \geq 1$, not purely hydrodynamic modes are responsible for the lose of energy stability. Another possible explanation is related to the independence of this calculation on any uniform horizontal magnetic field. So the $P_{m}$ scaling for $R e$ at the boundary of the domain may be achieved for flows with a strong horizontal magnetic field. The domain that we have calculated is inside the energy stability domain which can be calculated by finding the maximal value of $M$ and $R e$ such that $\mathcal{Q}$ is positive definite. To conclude this section, note that using another positive definite quantity than the energy could give a bigger domain in which the basic state is energy stable. 


\subsection{Upper Bounds for the Dissipation}

Outside the optimal domain in which the basic solution is energy stable, the flow can be unstationary and possibly turbulent. Exact solutions can not be explicitly calculated but we are able to derive upper bounds for the dissipation using the energy equation (16). We do not use the stationary basic state as background fields. Instead we suppose that they satisfy the boundary conditions and depend only on $y$. We write $\mathbf{v}_{b}=U(y) \mathbf{i}$ and $\mathbf{B}_{b}=B_{b}(y) \widehat{\rho}$ with $U(0)=0, U(d)=U, B_{b}(0)=0$ and $B_{b}(d)=B_{u}$. The spatial average of the dissipation can be written as

$$
D=\nu\left\langle|\nabla \mathbf{v}|^{2}+2 U^{\prime} v_{1}^{\prime}+{U^{\prime 2}}^{2}\right\rangle+\eta\left(\left\langle|\nabla \mathbf{b}|^{2}+2 \mathbf{B}_{b}^{\prime} \cdot \mathbf{b}^{\prime}+\left(\mathbf{B}_{b}^{\prime}\right)^{2}\right\rangle\right) .
$$

Add half of the total dissipation to equation (16) and get

$$
\frac{\partial}{\partial t}\left\langle\epsilon_{p}\right\rangle+\frac{D}{2}=F\left(B_{b}, U\right)-\mathcal{Q}\left(\mathbf{v}, \mathbf{b}, B_{b}, U\right)
$$

where $F\left(B_{b}, U\right)$ is a function of the background field and $\mathcal{Q}\left(\mathbf{v}, \mathbf{b}, B_{b}, U\right)$ is a quadratic function in the velocity and magnetic perturbation fields, namely

$$
\begin{aligned}
F\left(B_{b}, U\right) & =\frac{\nu}{2}\left\langle\left(U^{\prime}\right)^{2}\right\rangle+\frac{\eta}{2}\left\langle\left(\mathbf{B}_{b}^{\prime}\right)^{2}\right\rangle \\
\mathcal{Q}\left(\mathbf{v}, \mathbf{b}, B_{b}, U\right) & =\frac{\nu}{2}\left\langle|\nabla \mathbf{v}|^{2}\right\rangle+\frac{\eta}{2}\left\langle|\nabla \mathbf{b}|^{2}\right\rangle-\left\langle U^{\prime}\left(b_{2} b_{1}-v_{2} v_{1}\right)\right\rangle-\left\langle\mathbf{B}_{b}^{\prime} \cdot\left(\mathbf{v} b_{2}-v_{2} \mathbf{b}\right)\right\rangle .
\end{aligned}
$$

Note that we have added half of the dissipation $D$ to equation (16) to derive equation (26) in order to get rid of the linear terms in the perturbation fields. Now, if we find background fields such that $\mathcal{Q}$ is positive definite, then we obtain an upper bound for the time average of the dissipation $\bar{D}$

$$
\bar{D} \leq F\left(B_{b}, U\right)
$$

For the magnetic (respectively velocity) background field, we use a piecewise linear function equal to $B_{u} / 2$ (respectively $U / 2$ ) for $\delta_{b} \leq y \leq d-\delta_{b}$ (respectively $\delta_{u} \leq y \leq d-\delta_{u}$ ) and of slope $B_{u} /\left(2 \delta_{b}\right)$ (respectively $U /\left(2 \delta_{u}\right)$ ) in the two boundary layers as sketched in Figure (3). Note that both heights of the boundary layers $\delta_{u}$ and $\delta_{b}$ must be smaller than $d / 2$. Using integration by parts and Hölder's inequality, it is easy to prove that (see for instance [1])

$$
\begin{gathered}
\left|\left\langle B_{b}^{\prime}\left((\mathbf{v} \cdot \widehat{\rho}) b_{2}-v_{2}(\mathbf{b} \cdot \widehat{\rho})\right)\right\rangle\right| \leq \frac{B_{u} \delta}{8}\left(\left\langle\alpha|\nabla \mathbf{v}|^{2}\right\rangle+\frac{1}{\alpha}\left\langle|\nabla \mathbf{b}|^{2}\right\rangle\right), \\
\left|\left\langle U^{\prime}\left(b_{1} b_{2}-v_{1} v_{2}\right)\right\rangle\right| \leq \frac{U \delta_{u}}{8}\left\langle|\nabla \mathbf{v}|^{2}+|\nabla \mathbf{b}|^{2}\right\rangle
\end{gathered}
$$

and hence show that

$$
\mathcal{Q} \geq\left(\frac{\nu}{2}-\frac{U \delta_{u}}{8}-\frac{B_{u} \delta_{b} \alpha}{8}\right)\left\langle|\nabla \mathbf{v}|^{2}\right\rangle+\left(\frac{\eta}{2}-\frac{U \delta_{u}}{8}-\frac{B_{u} \delta_{b}}{8 \alpha}\right)\left\langle|\nabla \mathbf{b}|^{2}\right\rangle
$$




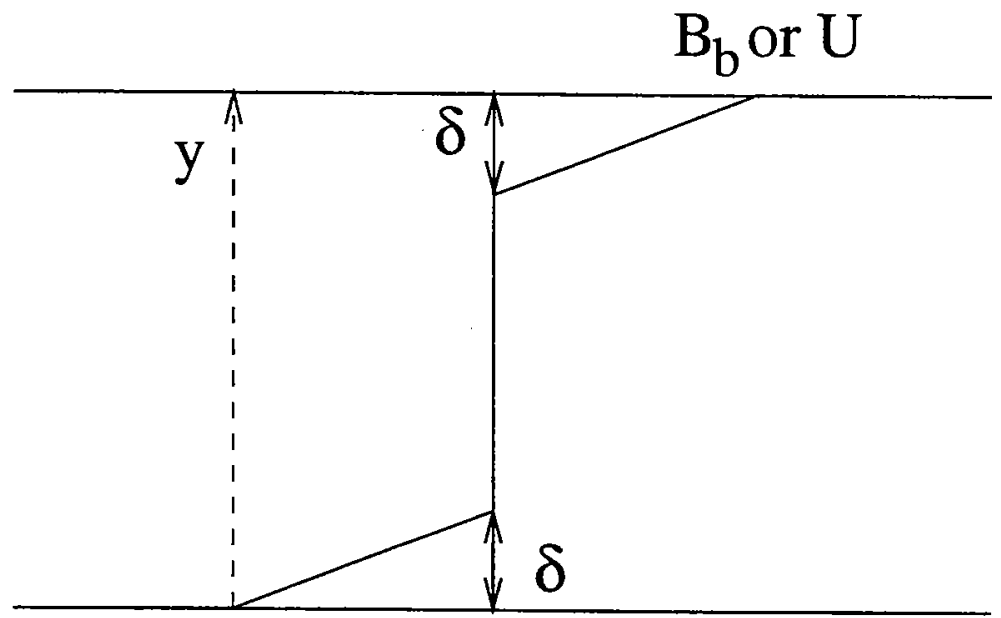

Figure 3: Piecewise linear background field. The height of the boundary layer is $\delta\left(\delta_{b}\right.$ for the magnetic field and $\delta_{u}$ for the velocity field).

Note that $\theta$ does not enter into the bound for $\mathcal{Q}$ and the upper bound for the dissipation will be independent of it. We first have to choose the height of the boundary layers such that $\mathcal{Q} \geq 0$ and then minimize $F=\frac{1}{2 d}\left(\frac{\nu U^{2}}{\delta_{u}}+\frac{\eta B_{u}^{2}}{\delta_{b}}\right)$ with the constraints that $\delta_{u}$ and $\delta_{b}$ are smaller than $d / 2$. If $\delta_{u}$ is fixed, the minimum of $F$ is obtained for the highest possible $\delta_{b}$. Introducing $y=\frac{U \delta_{u}}{\nu}$, we calculate the maximum $\delta_{b}$ that insures positivity of $\mathcal{Q}$. It leads to

$$
\frac{B_{u} \delta_{b}}{\nu}=g(y)=\sqrt{(4-y)\left(4 / P_{m}-y\right)} \text {. }
$$

Introducing $C=\frac{\eta B_{u}{ }^{3}}{\nu U^{3}}$, we get $D=\frac{U^{3}}{2 d}\left(\frac{1}{y}+\frac{C}{g(y)}\right)$. Minimizing $D$ over $y$, we find that the minimum is achieved for $y$ equal to $y_{0}$ which is the positive solution of

$$
\frac{\left((4-y)\left(4 / P_{m}-y\right)\right)^{3 / 2}}{y^{2}}=\frac{C}{2}\left(4+4 / P_{m}-2 y\right) \text {. }
$$

The two constraints on the height of the boundary layers in terms of $y$ are that $y \leq R e / 2$ and $g(y) \leq M / 2$. If $y_{0} \geq R e / 2$ then the boundary layer for the velocity is $d / 2$ and $y=R e / 2$. If $g(y) \geq M / 2$, the height of the magnetic boundary layer is $d / 2$ and $g(y)=M / 2$. Such values of the heights of the boundary layers are obtained for low values of $R e$ and $M$. In that case, the dissipation is that of the laminar solution. For higher values of $R e$ and $M$, the upper bound is obtained for $y=y_{0}$ and $g(y)=g\left(y_{0}\right)$, which can only be calculated numerically. In Figure (4) we plot $D d / U^{3}$ where $D$ is the upper bound for the dissipation as a function of $R e$ for $C=2$ and $P_{m}=1$.

At low values of $R e, D d / U^{3}$ is decreasing. This behavior occurs when the dissipation is equal to the laminar dissipation and the heights of the boundary layers are $d / 2$. For high $R e, D d / U^{3}$ is equal to a constant which depends on $C$ and $P_{m}$. We plot its value as a function of $P_{m}$ for different values of $C$ in Figure (5). Simple expansions of equation (32) give the asymptotic behaviors

$$
\lim _{P_{m} \rightarrow 0}\left(\lim _{R e \rightarrow \infty} \frac{D d}{U^{3}}\right)=\frac{1}{8} \text { and } \lim _{P_{m} \rightarrow \infty}\left(\lim _{R e \rightarrow \infty} \frac{D d}{U^{3}}\right)=\frac{P_{m}}{8},
$$




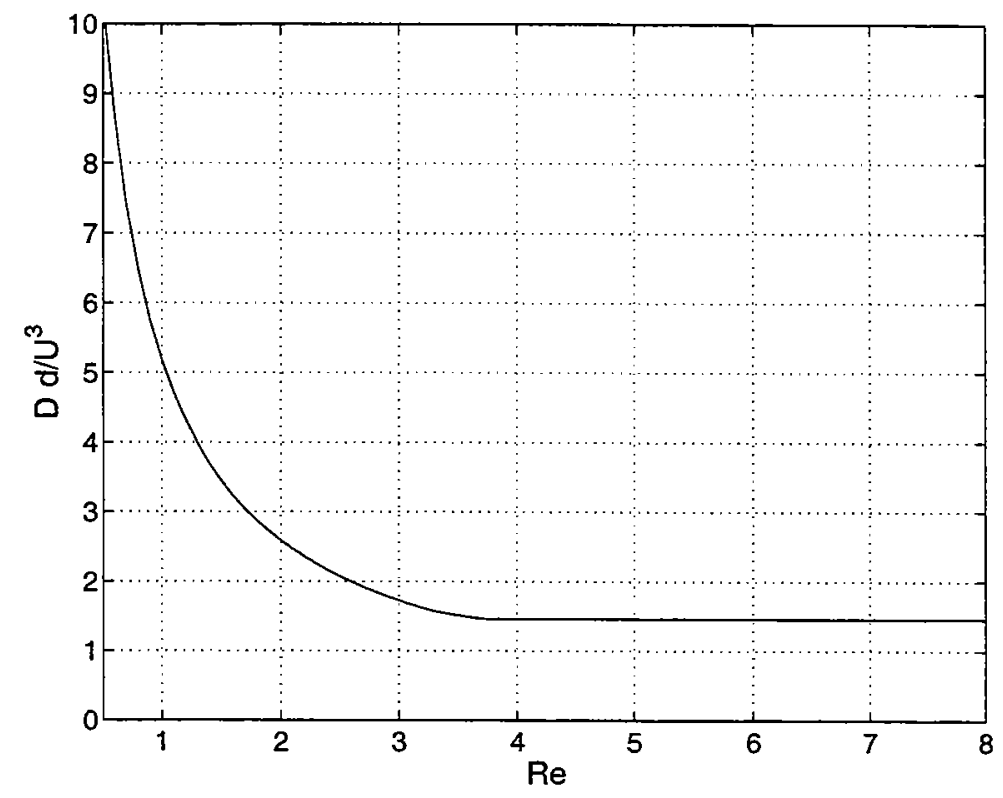

Figure 4: Value of the upper bound for the dissipation $D d / U^{3}$ as a function of $R e$ for $P_{m}=1$ and $C=2$.

which are plotted as a continuous line.

If we fix $M$ and $P_{m}, \phi$ decreases for low values of $R e$ and tends to a constant at high Re. We plot $\phi$ as a function of $R e$ for $M=2$ and different values of $P_{m}$ in Figure (6). The asymptotic behavior for $\phi$ is $\lim _{R e \rightarrow \infty} \phi=\frac{1}{8} \max \left(1, P_{m}\right)$. If we fix $R e$ and $P_{m}$ and vary $M$, $\phi$ decreases and tends to a constant. The asymptotic behavior of $D$ is then $D \simeq \frac{B^{3}}{d} \frac{1}{8 P_{m}^{1 / 2}}$ for $M \rightarrow \infty$.

The dependence of $\phi$ with $P_{m}$ for high $R e$ is similar to that of the boundary of the domain in which the basic state is energy stable. Here again a possible explanation of this scaling is that the bound does not depend on any uniform horizontal magnetic field. Therefore, if such a field is applied and if $\eta$ decreases, the total dissipation can increase because there is more resistive dissipation.

\section{Model of the Plane Sheet Pinch}

We study a simple model of the sheet pinch, similar to that studied numerically by Seehafer et al. [12]. A conducting fluid is located between two horizontal surfaces separated by a distance $d$. We use the same notations as in Figure (1). The problem is very similar to the shear layer. The only differences are in the values of the fields at the boundary. Both surfaces are at rest and we impose $\mathbf{v}=0$ at $y=0, d$. The normal component of the magnetic field is continuous and we supposed that $B_{2}=0$ at both surfaces. The horizontal current is fixed to be $J_{0} \mathbf{k}$. In terms of the magnetic field it gives $\frac{\partial B_{x}}{\partial y}=J_{0}$ and $\frac{\partial B_{z}}{\partial y}=0$.

A static solution is $\mathbf{v}=0, B_{x}=J_{0} y+C_{1}, B_{y}=0, B_{z}=C_{2}$ where $C_{1}$ and $C_{2}$ are constants. The dissipation is $D_{l}=\eta J_{0}^{2}$. Note that the result of equation (6) does not apply 


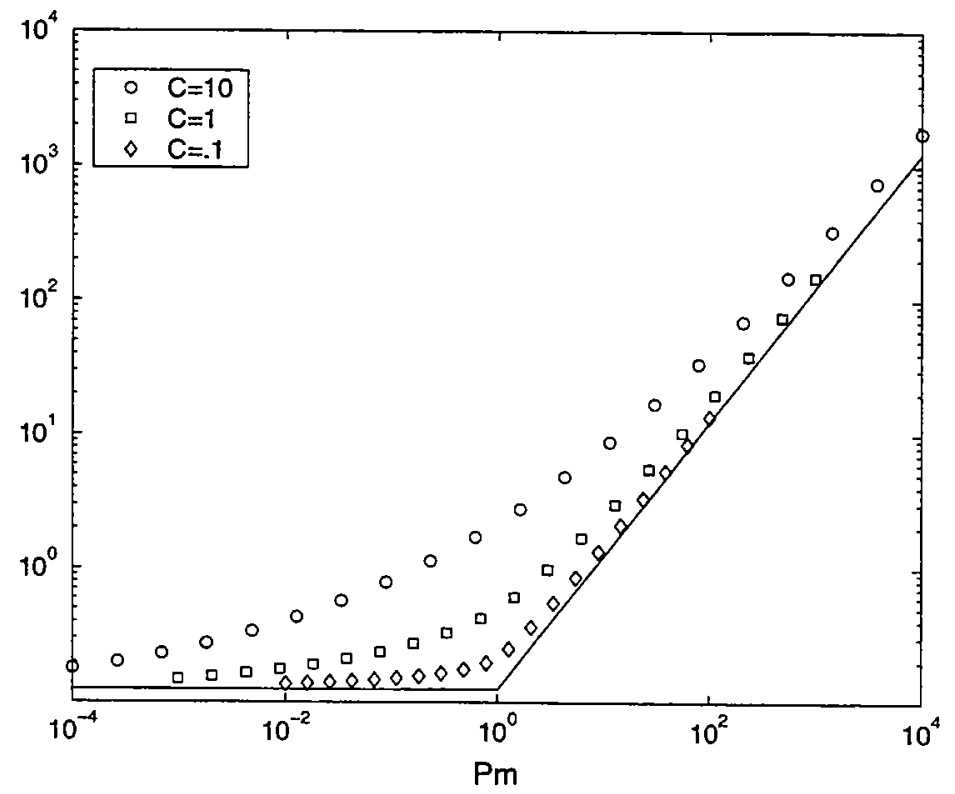

Figure 5: Value of the upper bound for the dissipation $D d / U^{3}$ for infinite $R e$ as a function of $P_{m}$ for some values of $C$. The continuous line is the asymptotic behavior (33).

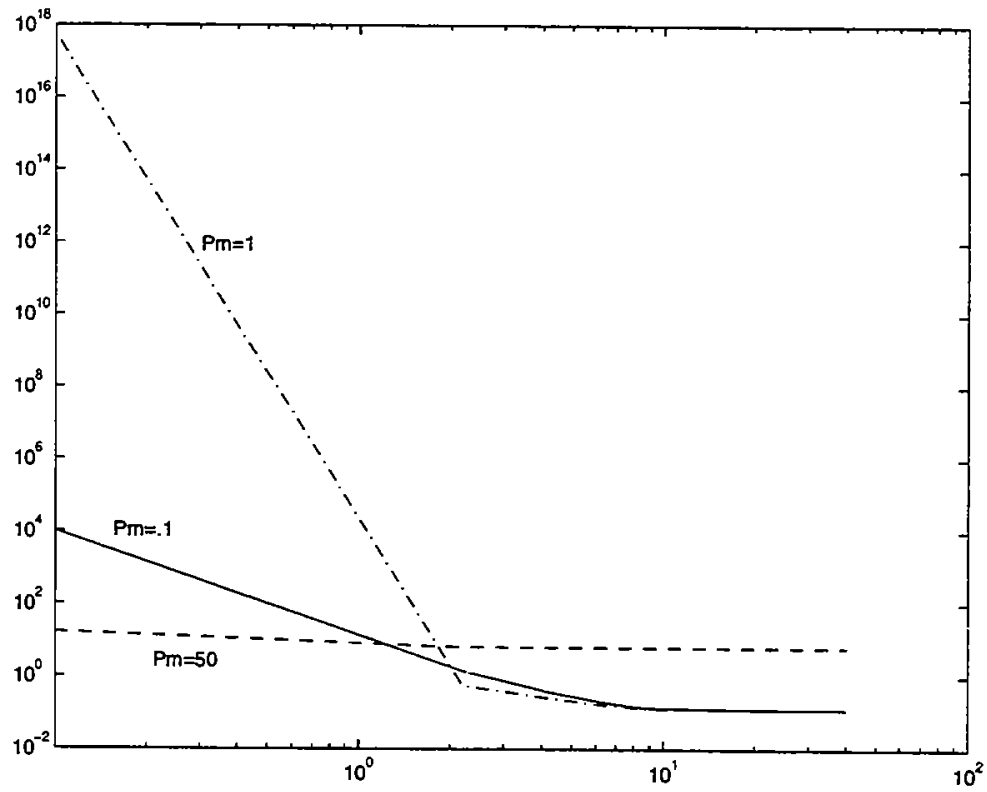

Figure 6: Value of the upper bound for the dissipation $\phi$ as a function of $\operatorname{Re}$ for $M=2$ and different values of $P_{m}$. 
here because the value of the magnetic field is not fixed at the boundaries, so that there is no reason for $D_{l}$ to be a minimum for the dissipation. Here again we use the expression $D=\nu\left\langle|\nabla \mathbf{v}|^{2}\right\rangle+\eta\left\langle|\nabla \mathbf{B}|^{2}\right\rangle$ which is equivalent to definition (3). Moreover, the evolution equation for the energy of a perturbation to a basic state is independent of any horizontal uniform magnetic field and we can set $C_{1}=C_{2}=0$. The background field that we use is of the form $\mathbf{B}_{b}=B_{1}(y) \mathbf{i}$ and the boundary conditions are $B_{1}^{\prime}(0)=B_{1}^{\prime}(d)=J_{0}$. The total magnetic field is written as $\mathbf{B}_{t}=\mathbf{B}_{b}+\mathbf{b}$ and the energy equation for the perturbation $\epsilon_{p}$ is

$$
\frac{\partial}{\partial t}\left\langle\epsilon_{p}\right\rangle+\frac{D}{2}=F\left(B_{1}\right)-\mathcal{Q}\left(\mathbf{v}, \mathbf{b}, B_{1}\right)
$$

with

$$
\begin{aligned}
F\left(B_{1}\right) & =\frac{\eta}{2}\left\langle\left(\vec{B}_{1}^{\prime}\right)^{2}\right\rangle \\
\mathcal{Q}\left(\mathbf{v}, \mathbf{b}, B_{b}\right) & =\frac{\nu}{2}\left\langle|\nabla \mathbf{v}|^{2}\right\rangle+\frac{\eta}{2}\left\langle|\nabla \mathbf{b}|^{2}\right\rangle-\left\langle B_{1}^{\prime}\left(v_{1} b_{2}-v_{2} b_{1}\right)\right\rangle-\frac{\eta J_{0}}{V} \int_{S} b_{1} d x d z .
\end{aligned}
$$

The surfaces of the layer are $S$ and the last term is a surface integral due to the boundary conditions. It is changed into a volume integral using $\frac{1}{V} \int_{S} b_{1} d x d z=\left\langle\frac{\partial b_{1}}{\partial y}\right\rangle$. To bound this term, we write

$$
\begin{aligned}
(\nabla \mathbf{b})^{2}+2 J_{0} \frac{\partial b_{1}}{\partial y} & =(1-c)|\nabla \mathbf{b}|^{2}+c|\nabla \mathbf{b}|^{2}+2 J_{0} \frac{\partial b_{1}}{\partial y} \\
& \geq(1-c)|\nabla \mathbf{b}|^{2}-\frac{J_{0}^{2}}{c},
\end{aligned}
$$

where $0<c<1$ and we have used $(\nabla \mathbf{b})^{2} \geq\left(\frac{\partial b_{1}}{\partial y}\right)^{2}$. We use a piecewise linear profile for $B_{1}^{\prime}$ of the form $B_{1}^{\prime}(y)=0$ if $\delta \leq y \leq d-\delta, B_{1}^{\prime}(y)=-J_{0}(y-\delta) / \delta$ if $y \leq \delta$ and $B_{1}^{\prime}(y)=J_{0}(y+\delta-d) / \delta$ if $y \geq d-\delta$. The value of $B_{1}$ can be deduced by trivial integrations but does not enter into the upper bound result. We get $\left\langle\left(B_{1}^{\prime}\right)^{2}\right\rangle=\frac{2 J_{0} \delta}{3 d}$. We then use two results

$$
\begin{array}{r}
\lim _{\delta \rightarrow 0}\left\langle\left(B_{1}^{\prime}\right)^{2}\right\rangle=0, \\
\lim _{\delta \rightarrow 0} Q\left(\mathbf{v}, \mathbf{b}, B_{b}\right) \geq-\eta \frac{J_{0}^{2}}{2 c} .
\end{array}
$$

We take the limit of $\delta=0$ in equation (26) and get

$$
\frac{\partial}{\partial t}\left\langle\epsilon_{p}\right\rangle+\frac{D}{2} \leq \eta \frac{J_{0}^{2}}{2 c}
$$

such that the upper bound for the time average of the dissipation is $\bar{D}=\eta \frac{J_{0}^{2}}{c}$ with $0<c<1$. Take the limit $c \rightarrow 1$ and get $\bar{D}=\eta J_{0}^{2}$. Note that if we choose stress-free boundary conditions for the velocity fields, we obtain the same results because equation (34) holds also in this case.

The upper bound for the dissipation is equal to the dissipation for the static solution. A first consequence is that this bound can not be improved if the manifold over which we 
maximize $D$ contains the static solution. Another consequence is that the dissipation tends to zero with $\eta$. As described by Paparella et al. [11], this is an antiturbulence theorem. The physical behaviors of the two systems are different. Whereas their simulations show instabilities of the laminar flow and formation of boundary layers, the numerical simulations of the voltage-driven sheet pinch done by Seehafer et al. [12] show that the static solution is always stable, which is consistent with our upper bound result.

\section{Conclusion}

We have reported various results on bounds on the dissipation in MHD flows. We defined the Stokes-like solution of the MHD problem and have shown that it minimizes the dissipation over any divergence-free velocity and magnetic fields that are fixed at the boundary. Using the background method, we calculated upper bounds for the dissipation in two examples of MHD flows. In the case of a shear layer with both velocity and magnetic shear, an upper bound was derived and its dependency on the parameters was calculated. As a by-product of this calculation, the energy stability domain was calculated with a result that is bigger than previous results. In the case of the plane sheet pinch, the upper bound for the dissipation was achieved by the static solution and tends to zero with the magnetic viscosity. This is an antiturbulence theorem in the sense that there is no residual dissipation in the limit of zero viscosity.

Note that the two examples differ only by the boundary conditions but their physical behaviors are completely different. Let us insist on the importance of the boundary conditions for MHD problems. So, a possible continuation of this work would be to modify the boundary conditions, mainly for the magnetic field. The effect of a non conducting external medium could be investigated. An inductive drive of the magnetic field would also be very relevant for Tokamak experiments.

Only crude estimates of the quadratic forms have been done in the case of the shear layer. The domain in which the basic state is energy stable and the upper bound for the dissipation can certainly be improved by using more accurate inequalities. They may also be improved by taking into account other evolution equations, for instance the one for the helicity or the cross-helicity.

This work has been done with another fellow, Alexandros Alexakis (see his report for other applications of bounds in MHD) and with the help of C. Doering, P. Morrison and J. Keller. I thank all of them for their enthusiasm and scientific advice. I also thank all of those who made the GFD program so pleasant and interesting.

\section{References}

[1] C. R. Doering and P. Constantin, "Variational bounds on energy dissipation in incompressible flows: Shear flow," PRE 49, 4087 (1994).

[2] H. Tasso and S. J. Camargo, "On the nonlinear stability of dissipative fluids," Nuovo Cimento 107 B, 733 (1992). 
[3] H. K. Moffatt, Magnetic field generation in electrically conducting fluids (Cambridge University Press, Cambridge, 1978).

[4] J. B. Keller, A. Rubenfeld, and J. E. Molyneux, "Extremum principles for slow viscous flows with applications to suspensions," JFM 97 (1967).

[5] B. Straughan, The Energy Method, Stability and Nonlinear Convection (SpringerVerlag, New-York, 1992).

[6] L. N. Howard, "Heat transport by turbulence convection," JFM 17, 405 (1963).

[7] F. H. Busse, "Bounds for turbulent shear flows," JFM 41, 219 (1970).

[8] H. Tasso, "Remarks on non-linear stability in dissipative magnetohydrodynamics," Nuovo Cimento 108 B, 827 (1993).

[9] A. Soward, "Bounds for turbulent convective dynamos," Geophys. Astrophys. Fluid Dynamics 15, 317 (1980).

[10] C. Y. Wang and A. Bhattacharjee, "Optimum theory for the energy dissipation in a turbulent pinch," Phys. Fluids B 3, 3462 (1991).

[11] F. Paparella and W. R. Young, to be published in J. Fluid Mech. (unpublished).

[12] N. Seehafer, E. Zienicke, and F. Feudel, "Absence of magnetohydrodynamic activity in the voltage-driven sheet pinch," PRE 54, 2863 (1996).

[13] G. Bateman, MHD Instabilities (MIT press, Cambridge, 1978).

[14] H. P. Furth, J. Killeen, and M. N. Rosenbluth, "Finite-resistivity instabilities of a sheet pinch," The Physics of Fluids 6, 459 (1963).

[15] X. L. Chen and P. Morrison, "The effect of viscosity on the resistive tearing mode with the presence of shear flow," Phys. Fluids B 11, 2575 (1990). 


\title{
Bounds on the Energy Dissipation on the Magnetic Couette and Poiseuille (Hartmann) Shear Flow
}

\author{
Alexandros Alexakis \\ University of Chicago
}

\section{Introduction}

We are going to study the stability and bounds on turbulent dissipation shear flows in a conducting fluid when a vertical (with respect to the flow) magnetic field is applied. More precisely we are going to investigate Couette flow and Poiseuile (Hartmann) flow in the presence of the magnetic field. First using integral inequalities we are going to estimate regions in the parameter space when the flow is energy stable. Then we are going to derive bounds on the dissipation valid even in the presence of turbulent flows.

\section{Couette Flow}

\subsection{Preliminaries}

First we consider we plane Couette flow. We consider two plates separated by a distance $d$ (from $-d / 2$ to $+d / 2$ ) that move with respect to each other with velocity $\mathrm{i} U^{*}$. The unit vector $\mathbf{i}$ is one of the horizontal directions and $\mathbf{j}$ is the vertical. Between the plates there is a conducting liquid of density $\rho \equiv 1$, magnetic diffusivity $\eta$ and viscosity $\nu$. For the top and bottom boundary we use no-slip boundary conditions for the velocity and "line-tied" for the magnetic field, e.g. $\left(B=\mathrm{j} B_{0}\right)$ where $B_{0}$ is an externally imposed field. We assume periodic boundary conditions for the other directions. The setup is shown in figure(1).

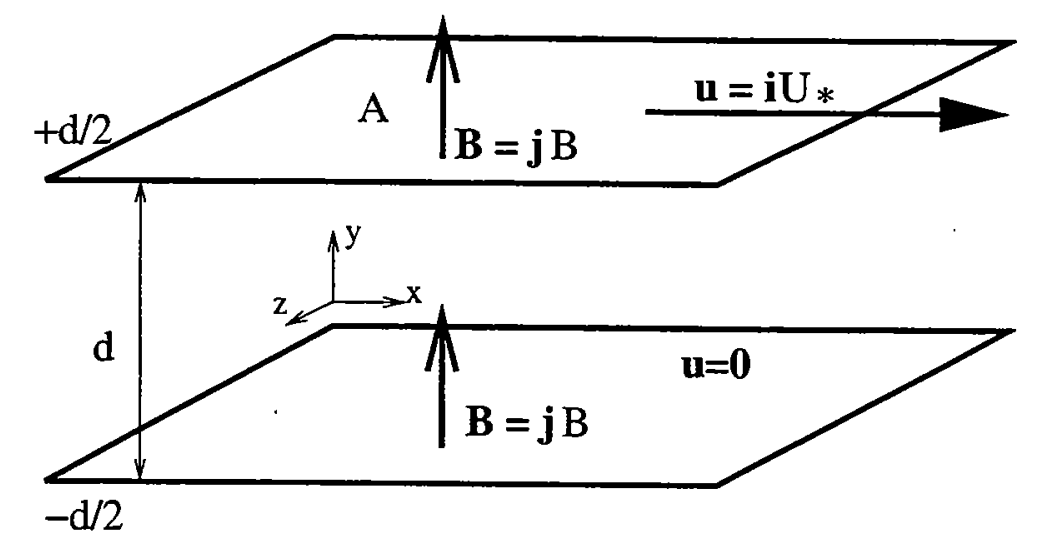

Figure 1: The setup for magnetic Couette flow

The equations of motion that govern this system are [1]

$$
\begin{aligned}
\partial_{t} u+u \cdot \nabla u & =-\nabla P+B \cdot \nabla B+\nu \nabla^{2} u \\
\partial_{t} B+u \cdot \nabla B & =B \cdot \nabla u+\eta \nabla^{2} B \\
\nabla \cdot u=0 & , \quad \nabla \cdot B=0 .
\end{aligned}
$$




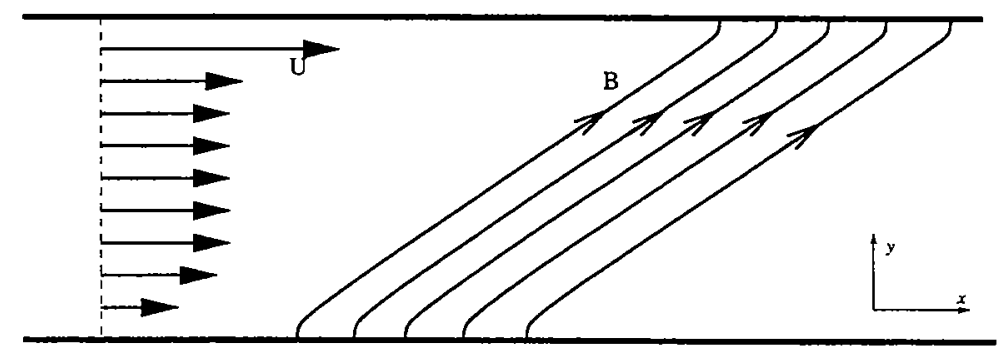

Figure 2: The laminar velocity profile and the magnetic field lines.

Where $B$ is the magnetic field and $u$ is the fluid velocity. There are three non-dimensional numbers that govern this system. Our choice is going to be the Reynolds number $\mathbf{R e}$, the Hartmann number $\mathbf{Q}$ and the Prandtl number $\mathbf{P}$ or alternatively the magnetic Reynolds number $\mathbf{R}_{\mathbf{M}}$. Their definition is given bellow:

$$
\mathbf{R e}=\frac{U^{*} d}{\nu}, \quad \mathbf{Q}=\frac{B_{2} d}{2 \sqrt{\nu \eta}}, \quad \mathbf{P r}=\frac{\nu}{\eta} \quad\left(\text { or } \mathbf{R}_{\mathbf{M}}=\mathbf{R e P r} .\right)
$$

The Hartmann number $\mathbf{Q}$ gives an estimate of how strong the magnetic field is when compared with the diffusive velocities $d / \sqrt{\nu \eta}$. In the limit $\mathbf{Q} \rightarrow 0$ we should obtain the non conductive fluid results. The energy dissipation of this system is given by

$$
\mathcal{D}=\nu\langle|\nabla u|\rangle+\eta\left\langle\left|\nabla B_{T}\right|\right\rangle=\frac{U^{* 3}}{d} \mathbf{D}
$$

$\mathbf{D}$ is a non-dimensional form of the dissipation and our principal aim is to estimate it as a function of the non-dimensional parameters mentioned before.

\subsection{The Laminar State}

The above set of equations allow for an exact laminar solution. Assuming homogeneity in the $x$ and $z$ direction and no time dependence we have $u=\mathbf{i} U(y), B=\mathbf{i} B_{1}(y)+\mathbf{j} B_{2}$ and

$$
\begin{aligned}
0 & =B_{2} \cdot \partial_{y} B_{1}+\nu \partial_{y}^{2} U \\
0 & =B_{2} \cdot \partial_{y} U+\eta \partial_{y}^{2} B_{1} \\
B_{2} & =\text { constant. }
\end{aligned}
$$

The last equation came from the solenoidal constraint on $B$. The above equations have the solution:

$$
B_{1}=\frac{1}{2} \sqrt{\frac{\nu}{\eta}} U^{*}\left[\frac{\cosh \left(\frac{B_{2} d}{2 \sqrt{\nu \eta}}\right)-\cosh \left(\frac{B_{2} y}{\sqrt{\nu \eta}}\right)}{\sinh \left(\frac{B_{2} d}{2 \sqrt{\nu \eta}}\right)}\right], \quad U=\frac{1}{2} U^{*}\left[1+\frac{\sinh \left(\frac{B_{2} y}{\sqrt{\nu \eta}}\right)}{\sinh \left(\frac{B_{2} d}{2 \sqrt{\nu \eta}}\right)}\right]
$$

In the limit $B_{2} d / \sqrt{\nu \eta} \rightarrow 0$ we return to plane Couette flow. The laminar solution for the velocity profile as well as the magnetic field lines is shown in figure (2). 
Next we examine the energy dissipation $\mathcal{D}_{l s}$ of the laminar solution. The dissipation can be easily calculated from (2) and it gives

$$
\begin{aligned}
\mathcal{D}_{l s}= & \mathcal{D}_{\text {visc }}+\mathcal{D}_{\text {magn }} \\
= & \sqrt{\frac{\nu}{\eta}} \frac{B_{2} U^{* 2}}{4 d}\left[\operatorname{coth}\left(\frac{B_{2} d}{2 \sqrt{\nu \eta}}\right)+\frac{\frac{B_{2} d}{2 \sqrt{\nu \eta}}}{\sinh ^{2}\left(\frac{B_{2} d}{2 \sqrt{\nu \eta}}\right)}\right]+ \\
& \sqrt{\frac{\nu}{\eta}} \frac{B_{2} U^{* 2}}{4 d}\left[\operatorname{coth}\left(\frac{B_{2} d}{2 \sqrt{\nu \eta}}\right)-\frac{\frac{B_{2} d}{2 \sqrt{\nu \eta}}}{\sinh ^{2}\left(\frac{B_{2} d}{2 \sqrt{\nu \eta}}\right)}\right] \\
= & \frac{1}{2} \sqrt{\frac{\nu}{\eta}} \frac{B_{2} U^{* 2}}{d} \operatorname{coth}\left(\frac{B_{2} d}{2 \sqrt{\nu \eta}}\right)
\end{aligned}
$$

or in a non-dimensional form

$$
\mathbf{D}=\mathbf{R e}^{-\mathbf{1}} \mathbf{Q} \operatorname{coth}(\mathbf{Q})
$$

There are a few points we have to make for the above equation. We note first that the viscous dissipation is always bigger than the resistive dissipation although the difference is exponentially small for large $\mathbf{Q}$. Moreover for fixed magnetic field $B_{2}$ and velocity $U$ the dissipation increases with the Prandtl number. In other words decreasing $\eta$ increases the dissipation. In the limit $\nu \rightarrow \infty, \eta \rightarrow \infty$ keeping the Prandtl number fixed the dissipation goes to the finite limit $\frac{1}{2} \operatorname{Pr} B_{2} U^{* 2} / d$. Taking the limit $\mathbf{Q}=B_{2} d / 2 \sqrt{\nu \eta} \rightarrow 0$ we obtain the plane Couette dissipation

$$
\mathcal{D}_{l s} \simeq \nu \frac{U^{* 2}}{d^{2}} \text { and for large } \mathbf{Q} \text { we obtain } \mathcal{D}_{l s} \simeq \frac{1}{2} \sqrt{\frac{\nu}{\eta}} \frac{B_{2} U^{* 2}}{d}
$$

\subsection{Stability}

Next we examine the energy stability of the above flow. Writing the magnetic and the velocity field as the laminar solution plus an arbitrary perturbation $u=U_{l s}+v$ and $B_{\text {total }}=$ $B_{l s}+b$ we obtain from (1):

$$
\begin{aligned}
\partial_{t} u+v \cdot \nabla v+U \cdot \nabla v+v \cdot \nabla U & =-\nabla P+B \cdot \nabla b+b \cdot \nabla B+b \cdot \nabla b+\nu \nabla^{2} v \\
\partial_{t} b+v \cdot \nabla B+b \cdot \nabla U+v \cdot \nabla b & =B \cdot \nabla v+b \cdot \nabla U+b \cdot \nabla v+\eta \nabla^{2} b \\
\nabla \cdot v=0 & , \nabla \cdot b=0 .
\end{aligned}
$$

where we dropped the index $l s$ for convenience. Multiplying the first one with $v$ and the second one with $b$ adding them and taking their space average we obtain

$$
\frac{1}{2} \partial_{t}\left\langle v^{2}+b^{2}\right\rangle=-\left\langle\left(v_{1} v_{2}-b_{1} b_{2}\right) U^{\prime}\right\rangle-\left\langle\left(b_{1} v_{2}-v_{1} b_{2}\right) B_{1}^{\prime}\right\rangle-\eta\left\langle|\nabla b|^{2}\right\rangle-\nu\left\langle|\nabla v|^{2}\right\rangle
$$

where the prime indicates a derivative with respect to $y$ and many terms dropped out due to the boundary conditions. Using the inequalities:

$$
\left\langle\left(v_{1} v_{2}-b_{1} b_{2}\right) U^{\prime}\right\rangle \leq \frac{1}{2}\left\langle\left(v_{1}^{2}+v_{2}^{2}+b_{1}^{2}+b_{2}^{2}\right)\right\rangle \max \left|U^{\prime}\right| \leq \frac{1}{2}\left\langle\left(v^{2}+b^{2}\right)\right\rangle \max \left|U^{\prime}\right|
$$




$$
\left\langle\left(b_{1} v_{2}-v_{1} b_{2}\right) B_{1}^{\prime}\right\rangle \leq \frac{1}{2}\left\langle\left(\tau v_{1}^{2}+\frac{1}{\tau} b_{2}^{2}+\tau v_{2}^{2}+\frac{1}{\tau} b_{1}^{2}\right)\right\rangle \max \left|B_{1}^{\prime}\right| \leq \frac{1}{2}\left\langle\left(\tau v^{2}+\frac{1}{\tau} b^{2}\right)\right\rangle \max \left|B_{1}^{\prime}\right|
$$

(where $\tau$ is a free parameter) and the Poincare inequality

$$
\left\langle|\nabla v|^{2}\right\rangle \geq \frac{\pi^{2}}{d^{2}}\left[\left\langle|v|^{2}\right\rangle\right.
$$

we end up with

$$
\begin{aligned}
\frac{1}{2} \partial_{t}\left\langle v^{2}+b^{2}\right\rangle & \leq-\left[\nu \frac{\pi^{2}}{d^{2}}-\frac{1}{2} \max \left|U^{\prime}\right|-\frac{1}{2} \tau \max \left|B_{1}^{\prime}\right|\right]\left\langle v^{2}\right\rangle \\
& -\left[\eta \frac{\pi^{2}}{d^{2}}-\frac{1}{2} \max \left|U^{\prime}\right|-\frac{1}{2 \tau} \max \left|B_{1}^{\prime}\right|\right]\left\langle b^{2}\right\rangle
\end{aligned}
$$

The energy of the perturbation is going to decrease if each term in the square brackets is greater than zero. Eliminating $\tau$ and recalling that $\max \left|U^{\prime}\right|=\frac{U^{*} B_{2}}{2 \sqrt{\nu \eta}} \operatorname{coth}\left(\frac{B_{2} d}{2 \sqrt{\nu \eta}}\right)$ and $\max \left|B_{1}^{\prime}\right|=\frac{B_{2} U^{*}}{2 \eta}$ We obtain that for stability:

$$
\frac{B_{2}^{2} U^{* 2}}{4 \eta^{2}}<\left[\frac{2 \pi^{2} \nu}{d^{2}}-\frac{U^{*} B_{2}}{2 \sqrt{\nu \eta}} \operatorname{coth}\left(\frac{B_{2} d}{2 \sqrt{\nu \eta}}\right)\right] \cdot\left[\frac{2 \pi^{2} \eta}{d^{2}}-\frac{U^{*} B_{2}}{2 \sqrt{\nu \eta}} \operatorname{coth}\left(\frac{B_{2} d}{2 \sqrt{\nu \eta}}\right)\right]
$$

where each term in the square brackets should be non-negative.

In dimensionless numbers

$$
\mathbf{Q}^{2} \mathbf{R e}^{2} \mathbf{P r}<\left[2 \pi^{2}-\mathbf{R e Q} \operatorname{coth}(\mathbf{Q})\right] \cdot\left[2 \pi^{2} \mathbf{P r}^{-1}-\mathbf{R e} \mathbf{Q} \operatorname{coth}(\mathbf{Q})\right]
$$

or

$$
\mathbf{Q}^{2} \mathbf{R}_{\mathbf{M}}^{\mathbf{2}}<\left[2 \pi^{2}-\mathbf{R e} \mathbf{Q} \operatorname{coth}(\mathbf{Q})\right] \cdot\left[2 \pi^{2}-\mathbf{R}_{\mathbf{M}} \mathbf{Q} \operatorname{coth}(\mathbf{Q})\right]
$$

For small $\mathbf{Q}$ we obtain that $\max \left\{\mathbf{R e}, \mathbf{R}_{\mathbf{M}}\right\} \leq 2 \pi^{2}$. For large $\mathbf{Q}$ the range of $\mathbf{R}_{\mathbf{M}}, \mathbf{R e}$ decreases inversely proportional to $\mathbf{Q}$ (e.g. $\max \left\{\mathbf{R e}, \mathbf{R}_{\mathbf{M}}\right\} \leq 2 \pi \mathbf{Q}^{-1}$ ). Figure (3) summarizes our results. We note that the conditions we derived are sufficient for energy stability but their violation does necessarily not imply energy instability. 


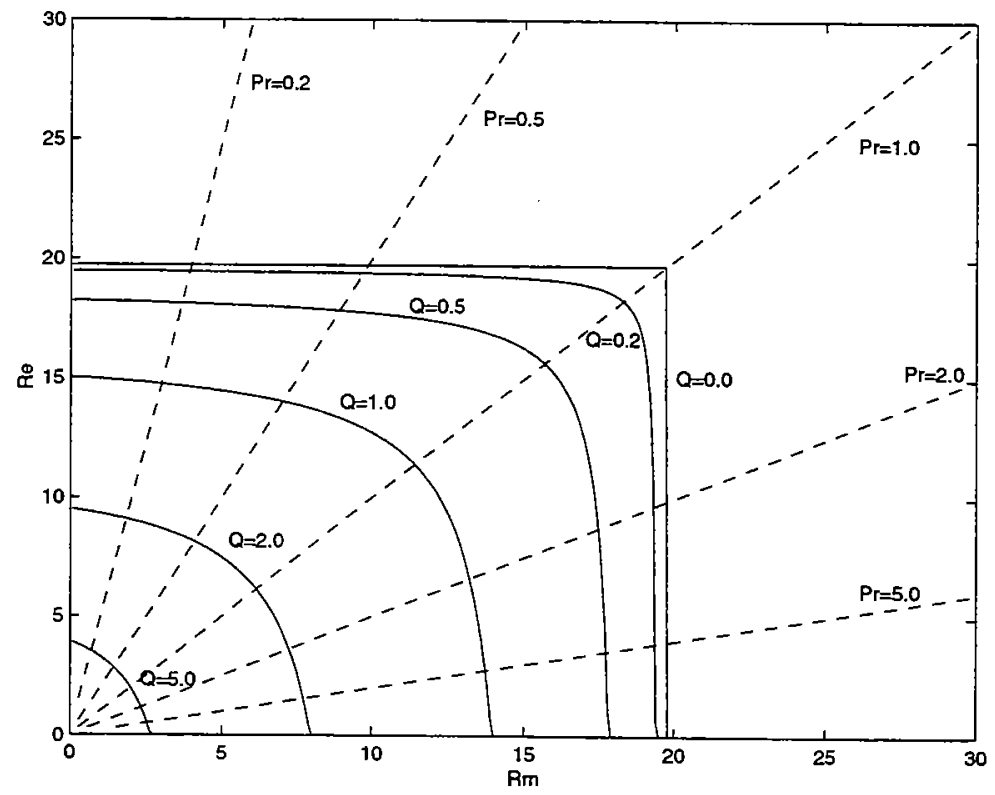

Figure 3: Energy stability reagions for magnetic Couette flow. The solid lines indicate the estimated stability boundaries for different values of $Q$. The dashed lines indicate constant Prandtl number

\subsection{Background Method}

Next we want to examine how the energy dissipation is modified when the flow is in a "turbulent" regime. We are going to use the Doering-Constantin background method [2] [3] to produce an upper bound on the dissipation. As in the energy stability method we are going to separate the flow to a background component $\mathbf{i} U(y), \mathbf{i} B_{1}(y)+\mathbf{j} B_{2}$ that we are going to leave undetermined and a fluctuating component $v, b$. Following the same procedure as in the energy method we obtain:

$$
\begin{gathered}
\frac{1}{2} \partial_{t}\left\langle v^{2}+b^{2}\right\rangle=\left\langle v_{1} B_{2} B_{1}^{\prime}\right\rangle+\left\langle b_{1} B_{2} U^{\prime}\right\rangle-\left\langle\left(v_{1} v_{2}-b_{1} b_{2}\right) U^{\prime}\right\rangle-\left\langle\left(b_{1} v_{2}-v_{1} b_{2}\right) B_{1}^{\prime}\right\rangle \\
-\nu\left\langle|\nabla v|^{2}\right\rangle-\eta\left\langle|\nabla b|^{2}\right\rangle+\nu\left\langle v_{1} U^{\prime \prime}\right\rangle+\eta\left\langle b_{1} B_{1}^{\prime \prime}\right\rangle
\end{gathered}
$$

where the linear and constant terms in $v$ and $b$ appeared because the background profile is no longer a solution of the MHD equations (1). To eliminate some of them we are going to add half of the total dissipation:

$$
\begin{gathered}
\frac{1}{2} \mathcal{D}=+\frac{1}{2} \nu\left\langle|\nabla(U+v)|^{2}\right\rangle+\frac{1}{2} \eta\left\langle|\nabla(B+b)|^{2}\right\rangle \\
=\frac{1}{2} \nu\left\langle|\nabla v|^{2}\right\rangle+\frac{1}{2} \eta\left\langle|\nabla b|^{2}\right\rangle+\nu\left\langle U^{\prime} \partial_{y} v_{1}\right\rangle+\eta\left\langle B_{1}^{\prime} \partial_{y} b_{1}\right\rangle+\frac{1}{2} \nu\left\langle U^{\prime 2}\right\rangle+\frac{1}{2} \eta\left\langle B_{1}^{\prime 2}\right\rangle
\end{gathered}
$$

by doing so, we obtain 


$$
\begin{aligned}
\frac{1}{2} \partial_{t}\left\langle v^{2}+b^{2}\right\rangle+\frac{1}{2} \mathcal{D} & =\left\langle v_{1} B_{2} B_{1}^{\prime}\right\rangle+\left\langle b_{1} B_{2} U^{\prime}\right\rangle-\left\langle\left(v_{1} v_{2}-b_{1} b_{2}\right) U^{\prime}\right\rangle-\left\langle\left(v_{1} b_{2}-b_{1} v_{2}\right) B_{1}^{\prime}\right\rangle \\
& -\frac{1}{2} \nu\left\langle|\nabla v|^{2}\right\rangle-\frac{1}{2} \eta\left\langle|\nabla b|^{2}\right\rangle+\frac{1}{2} \nu\left\langle U^{\prime 2}\right\rangle+\frac{1}{2} \eta\left\langle B_{1}^{\prime 2}\right\rangle
\end{aligned}
$$

To get rid of the remaining linear terms we will use the transformation $v=w-\mathbf{i} V(y)$ and $b=\beta-\mathbf{i} H(y)$ where

$$
\nu V^{\prime \prime}=B_{2} B_{1}^{\prime} \quad \text { and } \quad \eta H^{\prime \prime}=B_{2} U^{\prime}
$$

then

$$
\begin{aligned}
\partial_{t} \mathcal{E}+\frac{1}{2} \mathcal{D}= & -\left\langle\left(w_{1} w_{2}-\beta_{1} \beta_{2}\right) U^{\prime}\right\rangle-\left\langle\left(w_{1} \beta_{2}-\beta_{1} w_{2}\right) B_{1}^{\prime}\right\rangle+\frac{1}{2} \nu\left\langle|\nabla w|^{2}\right\rangle+\frac{1}{2} \eta\left\langle|\nabla \beta|^{2}\right\rangle \\
& +\frac{1}{2} \nu\left\langle U^{\prime 2}+V^{\prime 2}\right\rangle+\frac{1}{2} \eta\left\langle B_{1}^{\prime 2}+H^{\prime 2}\right\rangle
\end{aligned}
$$

where $\mathcal{E}=\frac{1}{2}\left\langle v^{2}+b^{2}\right\rangle$. We can write the above equation (22) in the form

$$
2 \partial_{t} \mathcal{E}+\mathcal{D}=-\mathcal{Q}_{U B_{1}}+\mathcal{D}_{b g}
$$

where $\mathcal{Q}_{U B_{1}}$ is a quadratic functional on $v$ and $b$ that depends on our choice of the background fields $U$ and $B_{1}$, and $\mathcal{D}_{b g}$ is the dissipation due to the background field. Our aim now is to choose an appropriate background field so that the quadratic term $\mathcal{Q}_{U B_{1}}$ is positive definite. If we succeed the we can prove by integrating over time that the the total energy is bounded in time. More by taking the time average of (23) that the total time averaged dissipation is $\mathcal{D} \leq \mathcal{D}_{b g}$.

From the form of $\mathcal{Q}_{U B_{1}}$ a natural choice for the background magnetic field is going to be $B_{1}=0$. For $U$ we are going to use the piece-wise linear profile

$$
U(y)=\left\{\begin{array}{lll}
\left(U^{*} / 2 \delta\right) y & \text { if }-d / 2 & \leq y \leq-d / 2+\delta \\
U^{*} / 2 & \text { if }-d / 2+\delta \leq y \leq d / 2-\delta \\
\left(U^{*} / 2 \delta\right)(d / 2-y) & \text { if } d / 2-\delta \leq y \leq d / 2
\end{array}\right.
$$

From (21) and the boundary conditions for $b$ we also have that

$$
H(y)=\frac{B_{2}}{\eta} \int_{0}^{y} U\left(y^{\prime}\right)-\langle U\rangle d y^{\prime} .
$$

The background fields $U$ and $H$ are shown in figure (4). We can easily now evaluate the dissipation of the background field and it is found to be

$$
\frac{1}{2} \mathcal{D}_{b g}=\frac{\nu U^{* 2}}{4 d} \frac{1}{\delta}+\frac{B_{2}^{2} U^{* 2}}{12 \eta d} \delta=\frac{\nu U^{* 2}}{4 d}\left(\frac{1}{\delta}+\frac{B_{2}^{2} \delta}{3 \nu \eta}\right)
$$

$\mathcal{D}_{b g}$ obtains its minimum value for $\delta_{\min }=\sqrt{3 \eta \nu / B_{2}^{2}}$, giving $\min \left\{\mathcal{D}_{b g}\right\}=\frac{1}{\sqrt{3}} \frac{U^{*} B_{2}}{d}$ 

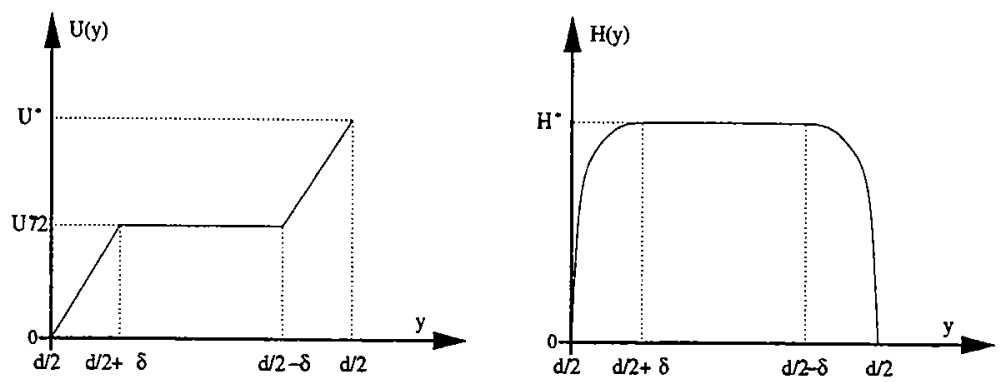

Figure 4: The two background fields $U(y)$ and $H(y)$. $H^{*}$ is equal to $B_{2} \delta / 4 \eta$.

Now we focus on the quadratic term $\mathcal{Q}_{U B_{1}}$ and try to determine the values of the free parameter $\delta$ that make it definite positive. Formally we would need to solve for the minimum of $\mathcal{Q}_{U B_{1}}$ that would lead to an eigenvalue problem that we would have to solve numerically. We are not going to follow this procedure here though but instead we are going to give rigorous estimates for the values of $\delta$ that guarantee the positivity of $\mathcal{Q}_{U B_{1}}$. Using the fundamental theorem of calculus and the Cauchy-Schwartz inequality we can show that

$$
\left|w_{i}\right|=\left|\int_{-d / 2}^{y} \frac{\partial w_{i}}{\partial y}\left(y^{\prime}\right) d y^{\prime}\right|=\left|\int_{-d / 2}^{y} 1 \cdot \frac{\partial w_{i}}{\partial y}\left(y^{\prime}\right) d y^{\prime}\right| \leq \sqrt{y+d / 2}\left|\int_{-d / 2}^{y}\left(\frac{\partial w_{i}}{\partial y}\left(y^{\prime}\right)\right)^{2} d y^{\prime}\right|^{1 / 2} .
$$

This implies

$$
\begin{gathered}
\left|\int_{-d / 2}^{d / 2} U^{\prime}(y) w_{1} w_{2} d x^{3}\right| \leq \frac{U_{*}}{2 \delta} \int d x d z \int_{0}^{\delta} y\left(\int_{-d / 2}^{0}\left(\frac{\partial w_{1}}{\partial y}\right)^{2} d y^{\prime}\right)^{1 / 2}\left(\int_{-d / 2}^{0}\left(\frac{\partial w_{2}}{\partial y}\right)^{2} d y^{\prime}\right)^{1 / 2} d y+ \\
\frac{U_{*}}{2 \delta} \int d x d z \int_{d / 2-\delta}^{d / 2}(d / 2-y)\left(\int_{0}^{d / 2}\left(\frac{\partial w_{1}}{\partial y}\right)^{2} d y^{\prime}\right)^{1 / 2}\left(\int_{0}^{d / 2}\left(\frac{\partial w_{2}}{\partial y}\right)^{2} d y^{\prime}\right)^{1 / 2} d y
\end{gathered}
$$

Including all the other terms in $|\nabla w|^{2}$ we obtain

$$
\left|\int U^{\prime}(y) w_{1} w_{2} d x^{3}\right| \leq \frac{U_{*}}{2 \delta} \frac{\delta^{2}}{2} \frac{1}{2} \int|\nabla w|^{2} d x^{3}=\frac{U_{*} \delta}{8} \int|\nabla w|^{2} d x^{3}
$$

and similarly for $\beta$

$$
\left|\int U^{\prime}(y) \beta_{1} \beta_{2} d x^{3}\right| \leq \frac{U_{*} \delta}{8} \int|\nabla \beta|^{2} d x^{3}
$$


This implies for $\mathcal{Q}_{U B_{1}}$ :

$$
\begin{aligned}
\mathcal{Q}_{U B_{1}}\{w, \beta\} & =\nu\left\langle|\nabla w|^{2}\right\rangle+\eta\left\langle|\nabla \beta|^{2}\right\rangle+2\left\langle U^{\prime}\left(w_{1} w_{2}-\beta_{1} \beta_{2}\right)\right\rangle \\
& \geq \nu\left\langle|\nabla w|^{2}\right\rangle+\eta\left\langle|\nabla \beta|^{2}\right\rangle-2\left|\left\langle U^{\prime} w_{1} w_{2}\right\rangle\right|-2\left|\left\langle U^{\prime} \beta_{1} \beta_{2}\right\rangle\right| \\
& \geq \nu\left\langle|\nabla w|^{2}\right\rangle+\eta\left\langle|\nabla \beta|^{2}\right\rangle-\frac{U_{*} \delta}{4}\left\langle|\nabla w|^{2}\right\rangle-\frac{U_{*} \delta}{4}\left\langle|\nabla \beta|^{2}\right\rangle \\
& \geq\left(\nu-\frac{U_{*} \delta}{4}\right)\left\langle|\nabla w|^{2}\right\rangle+\left(\eta-\frac{U_{*} \delta}{4}\right)\left\langle|\nabla \beta|^{2}\right\rangle \\
& \geq \frac{\pi^{2}}{d^{2}}\left(\min \{\nu, \eta\}-\frac{U_{*} \delta}{4}\right)\left\langle w^{2}+\beta^{2}\right\rangle .
\end{aligned}
$$

So $\mathcal{Q}_{U B_{1}}\{w, \beta\} \geq 0$ if we choose $\delta \leq 4 \nu / U_{*}=4 d / R e$. This is the maximum value of $\delta$ that our estimates allow us to use.

$$
\delta \leq \frac{4 \min \{\eta, \nu\}}{U^{*}}=\delta_{Q}
$$

The smallest value of $\mathcal{D}_{b g}$ (keeping $\mathcal{Q}_{U B_{1}}$ positive) is obtained for $\delta=\min \left\{\delta_{\min }, \delta_{Q}, d / 2\right\}$. So we end up with our final result on the Couette flow that if $\delta_{\min }<\delta_{Q}$ we are going to use $\delta_{\min }$ to evaluate the background dissipation, which means that if

$$
\frac{4 \min \{\nu, \eta\} B_{2}}{U^{*} \sqrt{3 \nu \eta}}>1 \quad \text { then } \quad \mathcal{D} \leq \frac{U^{* 2} B_{2}}{\sqrt{3} d} \sqrt{\frac{\nu}{\eta}}
$$

or in the non-dimensional form

$$
\text { if } \quad 8 \mathbf{Q}>\sqrt{3} \max \left\{\mathbf{R e}, \mathbf{R}_{\mathbf{M}}\right\} \quad \text { then } \quad \mathbf{D} \leq \frac{2 \mathbf{Q}}{\sqrt{3} \mathbf{R e}} .
$$

If on the other hand $\delta_{\min }>\delta_{Q}$ we are forced to use $\delta_{Q}$ in the evaluation of the background dissipation. So if

$$
\frac{4 \min \{\nu, \eta\} B_{2}}{U^{*} \sqrt{3 \nu \eta}}<1 \quad \text { then } \mathcal{D} \leq \frac{1}{8} \frac{\nu}{\min \{\nu, \eta\}} \frac{U^{* 3}}{d}+\frac{2}{3} \frac{\min \{\nu, \eta\}}{\eta} \frac{B_{2}^{2} U^{*}}{d}
$$

or in the non-dimensional form

$$
\text { if } 8 \mathbf{Q}<\sqrt{3} \max \left\{\mathbf{R e}, \mathbf{R}_{\mathbf{M}}\right\} \quad \text { then } \quad \mathbf{D} \leq \frac{1}{8} \max \{\mathbf{P r}, 1\}+\frac{8}{3} \frac{\mathbf{Q}^{\mathbf{2}}}{\mathbf{R e} \max \left\{\mathbf{R}_{\mathbf{M}}, \mathbf{R e}\right\}} .
$$

The first inequality (34) we have shows that for large enough magnetic field the dissipation is bounded by a function with the same dependence on $\mathbf{R e}$ and $\mathbf{Q}$ as the laminar. The prefactor has only a $15 \%$ difference. This gives an indication that the flow should be close to the laminar solution. If the magnetic field on the other hand is not strong enough then the dissipation becomes independent of the Reynolds number $\mathbf{R e}$ and has only a dependence on the Prandtl number Pr. The increase of the bound on the dissipation with Prandtl number is an interesting result that we cannot yet determine if it is the outcome of a bad estimate or it corresponds to a physical mechanism for increase of the dissipation. 


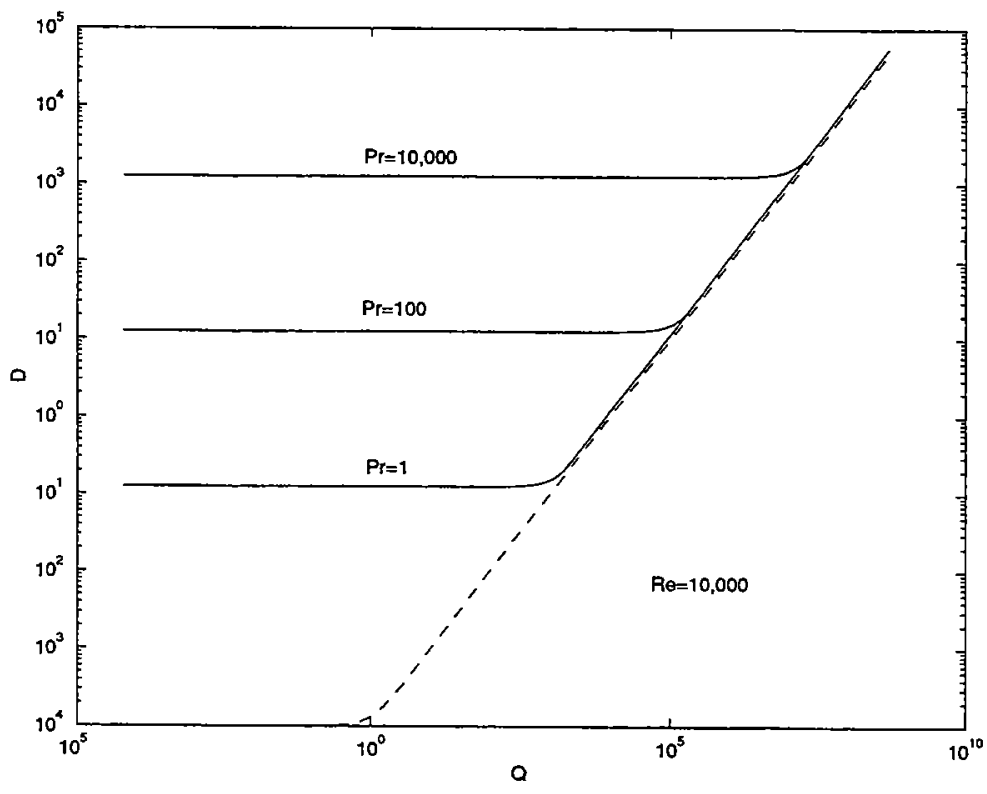

Figure 5: The dissipation as a function of $\mathbf{Q}$ for different Prandtl numbers. The dashed line shows the laminar solutions dissipation.

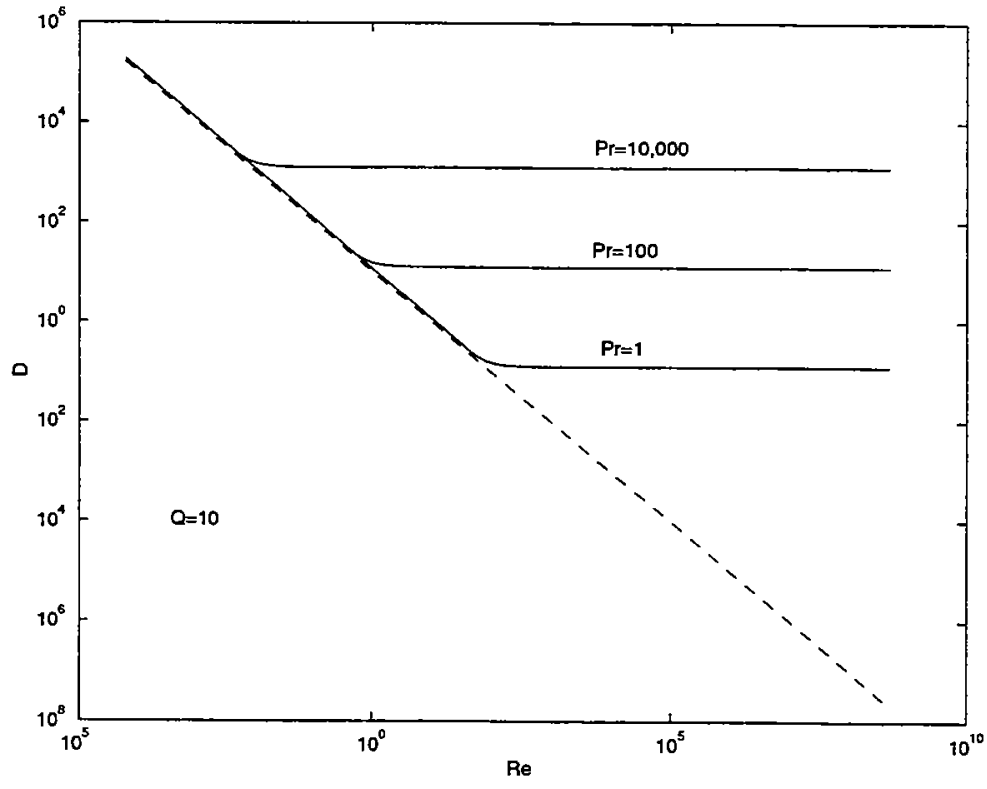

Figure 6: The dissipation as a function of Re for different Prandtl numbers. The dashed line shows the laminar solutions dissipation. 


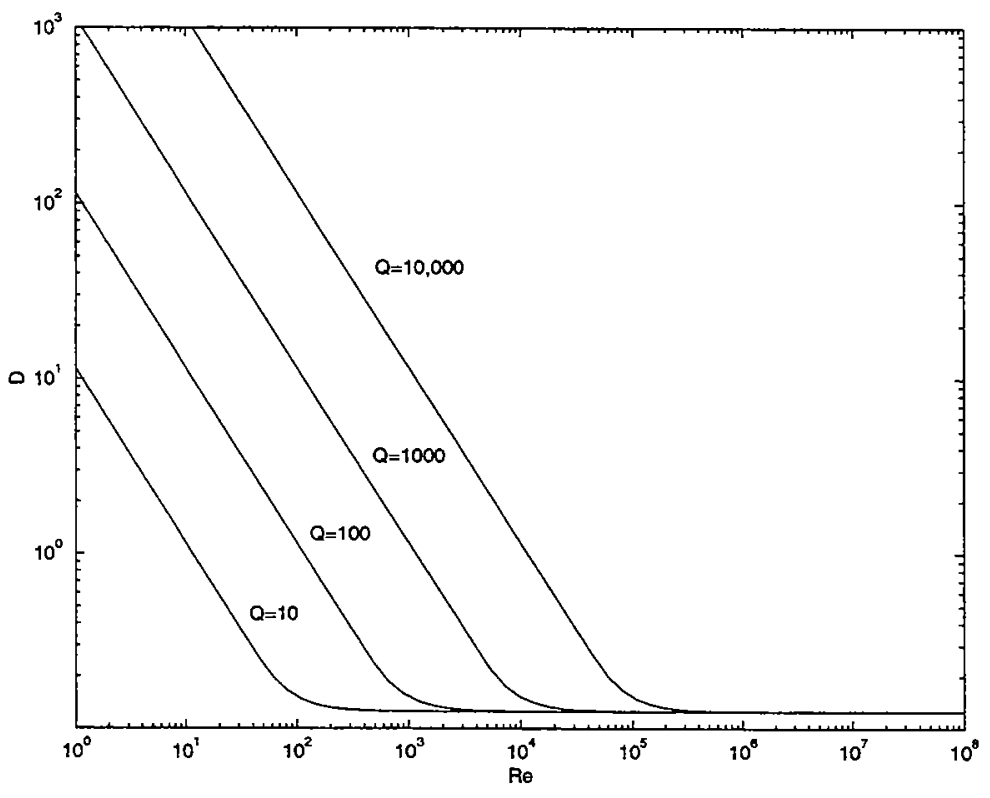

Figure 7: The dissipation as a function of $\mathbf{R e}$ for different values of $\mathbf{Q}$.

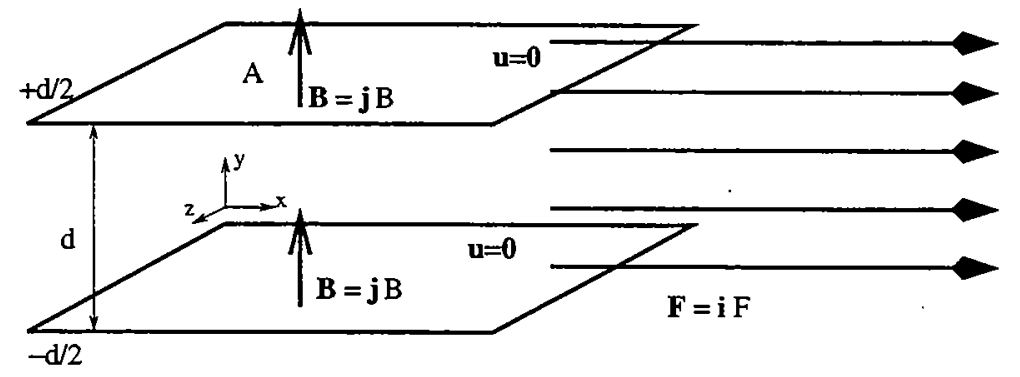

Figure 8: The setup for the Hartmann flow

\section{Magnetic Poiseuille (Hartmann) Flow}

\subsection{Preliminaries}

Next we turn to examine the magnetic Poiseuille or Hartmann flow named after Hartmann who first examined this kind of flow [4]. We consider the same set up as in $\S 2$, only this time both the top and bottom plate are held fixed and there is a constant pressure gradient or a uniform force field $F$ in the $\mathbf{i}$ direction. The same equations govern the current setup as in $\S 2$ with the addition of the force field in the momentum equation:

$$
\partial_{t} u+u \cdot \nabla u=-\nabla P+B \cdot \nabla B+\nu \nabla^{2} u+F .
$$

The non-dimensional numbers that parametrize our system are the Hartmann number defined as before, and the Grashoff number $\mathbf{G}$ and magnetic Grashoff $\mathbf{G}_{\mathbf{M}}$ number defined 


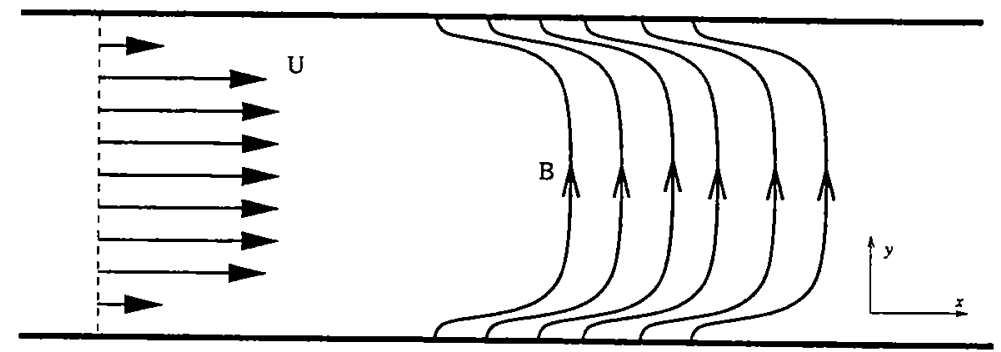

Figure 9: The laminar velocity profile and the magnetic field lines.

as

$$
\mathbf{G}=\frac{F d^{3}}{2 \nu^{2}}, \quad \mathbf{G}_{\mathbf{M}}=\mathbf{G P r}
$$

The energy dissipation is given by

$$
\mathcal{D}=\nu\left\langle|\nabla u|^{2}\right\rangle+\eta\left\langle|\nabla B|^{2}\right\rangle=F^{3 / 2} d^{1 / 2} \mathbf{D}
$$

where $\mathbf{D}$ is again the non-dimensional form of the dissipation we are going to use.

\subsection{Laminar Solution}

Assuming time and $x-z$ independence again we end up with the system of equations

$$
\begin{aligned}
0 & =B_{2} \cdot \partial_{y} B_{1}+\nu \partial_{y}^{2} U+F \\
0 & =B_{2} \cdot \partial_{y} U+\eta \partial_{y}^{2} B_{1} \\
B_{2} & =\text { constant. }
\end{aligned}
$$

They can be solved easily and the solution is given by:

$$
U=\frac{F d}{2 B_{2}} \sqrt{\frac{\eta}{\nu}}\left[\frac{\cosh \left(\frac{B_{2} d}{2 \sqrt{\nu \eta}}\right)-\cosh \left(\frac{B_{2} y}{\sqrt{\nu \eta}}\right)}{\sinh \left(\frac{B_{2} d}{2 \sqrt{\nu \eta}}\right)}\right], \quad B_{1}=\frac{F d}{2 B_{2}}\left[\frac{\sinh \left(\frac{B_{2} y}{\sqrt{\nu \eta}}\right)}{\sinh \left(\frac{B_{2} d}{2 \sqrt{\nu \eta}}\right)}-\frac{2 y}{d}\right] .
$$

The laminar velocity and the magnetic field lines are shown in figure (9). Again the limit $\mathbf{Q} \rightarrow 0$ brings us back to Poiseuille flow.

We evaluate the dissipation again and find it to be

$$
\mathcal{D}=\frac{F^{2} d}{2 B_{2}} \sqrt{\frac{\eta}{\nu}}\left[\operatorname{coth}\left(\frac{B_{2} d}{2 \sqrt{\nu \eta}}\right)-\frac{2 \sqrt{\nu \eta}}{B_{2} d}\right]
$$

or in the non-dimensional form

$$
\mathbf{D}=\frac{\mathbf{G}^{1 / 2}}{2 \sqrt{2} \mathbf{Q}}\left[\operatorname{coth}(\mathbf{Q})-\frac{1}{\mathbf{Q}}\right]
$$

$\mathbf{D}$ goes to $\frac{\mathbf{G}^{\mathbf{1} / \mathbf{2}}}{6 \sqrt{2}}$ for $\mathbf{Q}$ going to zero, and $\mathbf{D}$ goes to $\frac{\mathbf{G}^{\mathbf{1 / 2}}}{2 \sqrt{2} \mathbf{Q}}$ for $\mathbf{Q}$ going to infinity. Also as in Couette flow the dissipation goes to a finite limit as $\nu$ and $\eta$ go to zero, keeping their ratio (Prandtl number) fixed. 


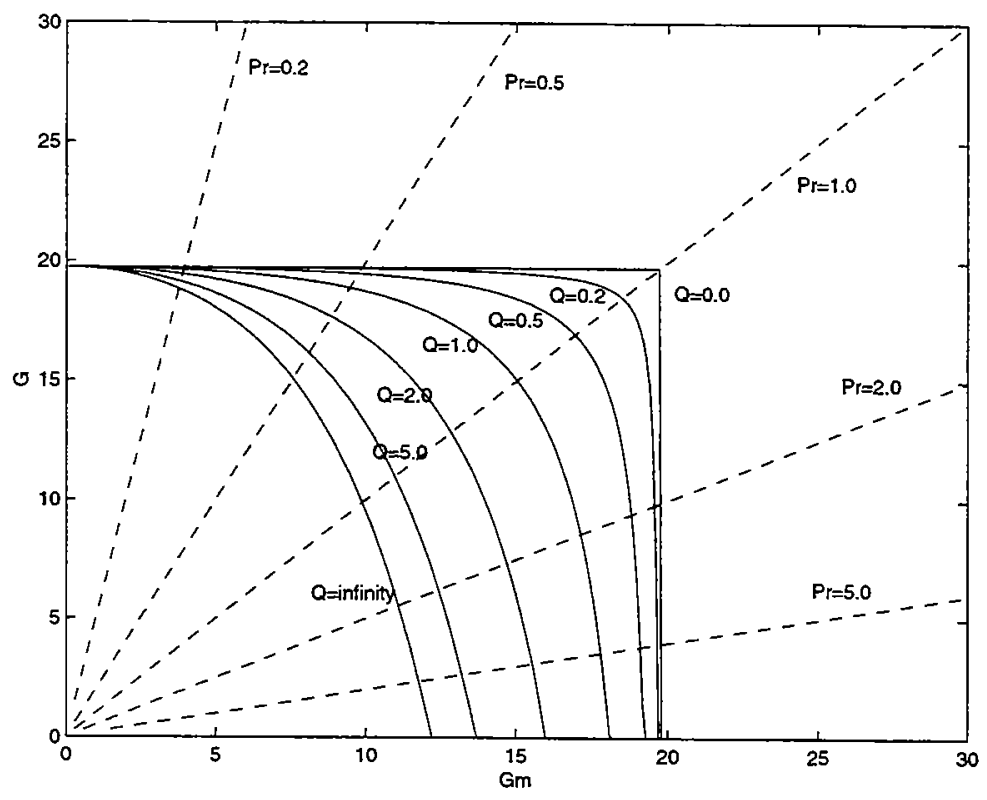

Figure 10: Energy stability reagions for Hartmann flow. The solid lines indicate the estimated stability boundaries for different values of $\mathbf{Q}$. The dashed lines indicate constant Prandtl number

\subsection{Stability}

Next we examine the energy stability of the Hartmann flow. The evolution of the energy is given by:

$$
\frac{1}{2} \partial_{t}\left\langle v^{2}+b^{2}\right\rangle=-\left\langle\left(v_{1} v_{2}-b_{1} b_{2}\right) U^{\prime}\right\rangle-\left\langle\left(v_{1} b_{2}-b_{1} v_{2}\right) B_{1}^{\prime}\right\rangle-\eta\left\langle|\nabla b|^{2}\right\rangle-\nu\left\langle|\nabla v|^{2}\right\rangle .
$$

Using the same inequalities as in the Couette flow we obtain

$$
B_{1 \max }^{\prime} \leq\left(\nu \frac{2 \pi^{2}}{d^{2}}-U_{\max }^{\prime}\right)\left(\eta \frac{2 \pi^{2}}{d^{2}}-U_{\max }^{\prime}\right)
$$

or

$$
\frac{F^{2}}{B_{2}^{2}}\left[\frac{B_{2} d}{2 \sqrt{\nu \eta}} \operatorname{coth}\left(\frac{B_{2} d}{2 \sqrt{\nu \eta}}\right)-1\right]^{2} \leq\left(\nu \frac{2 \pi^{2}}{d^{2}}-\frac{F d}{2 \nu}\right)\left(\eta \frac{2 \pi^{2}}{d^{2}}-\frac{F d}{2 \nu}\right)
$$

that gives in the non-dimensional form

$$
\mathbf{G}_{\mathbf{M}}^{\mathbf{2}}[\mathbf{Q} \operatorname{coth}(\mathbf{Q})-\mathbf{1}]^{2} \leq \mathbf{Q}^{\mathbf{2}}\left(2 \pi^{2}-\mathbf{G}\right)\left(2 \pi^{2}-\mathbf{G}_{\mathbf{M}}\right)
$$

As before we find that the energy stability is decreased as we increase $\mathbf{Q}$. Unlike the Couette flow though in the limit of large $Q$ the stability curve goes to the finite limit given by $\mathbf{G}_{\mathbf{M}}^{\mathbf{2}}<3\left(2 \pi^{2}-\mathbf{G}\right)\left(2 \pi^{2}-\mathbf{G}_{\mathbf{M}}\right)$ Our stability results are summarized in figure (10). 

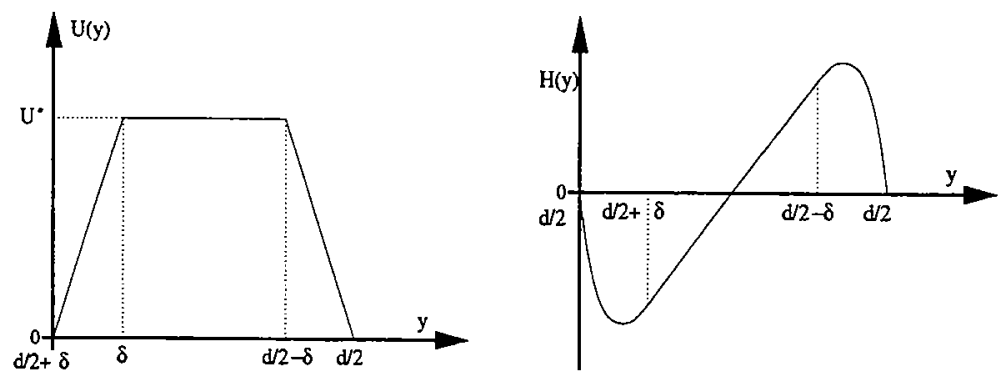

Figure 11: The two background fields $U(y)$ and $H^{*}(y)$

\subsection{Background Method}

Next examine the dissipation in the turbulent regime. Separating the flow to a background $U, B_{1}, B 2$ and a fluctuating component $b, v$ multiplying with $b, v$ and taking the spacial average as before we obtain

$$
\begin{aligned}
& \frac{1}{2} \partial_{t}\left\langle v^{2}+b^{2}\right\rangle=\left\langle v_{1} B_{2} B_{1}^{\prime}\right\rangle+\left\langle b_{1} B_{2} U^{\prime}\right\rangle-\left\langle\left(v_{1} v_{2}-b_{1} b_{2}\right) U^{\prime}\right\rangle-\left\langle\left(b_{1} v_{2}-v_{1} b_{2}\right) B_{1}^{\prime}\right\rangle \\
&-\nu\left\langle|\nabla v|^{2}\right\rangle-\eta\left\langle|\nabla b|^{2}\right\rangle+\nu\left\langle v_{1} U^{\prime \prime}\right\rangle+\eta\left\langle b_{1} B_{1}^{\prime \prime}\right\rangle+\langle F \cdot v\rangle .
\end{aligned}
$$

Adding half the dissipation we get

$$
\begin{gathered}
\partial_{t} \mathcal{E}+\frac{1}{2} \mathcal{D}=\left\langle v_{1} B_{2} B_{1}^{\prime}\right\rangle+\left\langle b_{1} B_{2} U^{\prime}\right\rangle+\left\langle F v_{1}\right\rangle-\left\langle\left(v_{1} v_{2}-b_{1} b_{2}\right) U^{\prime}\right\rangle-\left\langle\left(v_{1} b_{2}-b_{1} v_{2}\right) B_{1}^{\prime}\right\rangle \\
-\frac{1}{2} \nu\left\langle|\nabla v|^{2}\right\rangle-\frac{1}{2} \eta\left\langle|\nabla b|^{2}\right\rangle+\frac{1}{2} \nu\left\langle U^{\prime 2}\right\rangle+\frac{1}{2} \eta\left\langle B_{1}^{\prime 2}\right\rangle .
\end{gathered}
$$

Using $\mathcal{D}=\langle F \cdot u\rangle=\langle F U\rangle+\left\langle F v_{1}\right\rangle$ and $v=w-\mathbf{i} V(y)$ and $b=\beta-\mathbf{i} H(y)$ where $\nu V^{\prime \prime}=$ $B_{2} B_{1}^{\prime}$ and $\eta B_{1}^{\prime \prime}=B_{2} U^{\prime}$ we can write $(50)$ as

$$
2 \partial_{t} \mathcal{E}-\mathcal{D}=2 F\langle U\rangle-\mathcal{D}_{b g}+\mathcal{Q}_{U B_{1}}
$$

with $\mathcal{D}_{b g}=\nu\left\langle U^{\prime 2}\right\rangle+\eta\left\langle H^{\prime 2}\right\rangle$ and $H=-\frac{B_{2}}{\eta}(U-\langle U\rangle)$ and

$$
\mathcal{Q}_{U B_{1}}=\nu\left\langle|\nabla w|^{2}\right\rangle+\eta\left\langle|\nabla \beta|^{2}\right\rangle+2\left\langle\left(w_{1} w_{2}-\beta_{1} \beta_{2}\right) U^{\prime}\right\rangle+2\left\langle\left(w_{1} \beta_{2}-w_{2} \beta_{1}\right) U^{\prime}\right\rangle
$$

where we already picked $B_{1}=0$ for a background profile.

Contrary to the Couette flow case that the positivity of $Q_{U} B_{1}$ was leading to an upper bound on the dissipation, if $\mathcal{Q}_{U B_{1}} \geq 0$ then we have that $\mathcal{D} \geq F\langle U\rangle-\frac{1}{2} \mathcal{D}_{b g}$ which gives a lower bound on the dissipation.

The velocity back ground field we are going to choose is going to be

$$
U(y)=\left\{\begin{array}{lll}
\left(U^{*} / \delta\right) y & \text { if }-d / 2 & \leq y \leq-d / 2+\delta \\
\left.U^{*}\right) & \text { if }-d / 2+\delta \leq y \leq d / 2-\delta \\
\left(U^{*} / \delta\right)(d / 2-y) & \text { if } d / 2-\delta & \leq y \leq d / 2 .
\end{array}\right.
$$


with $U^{*}$ and $\delta$ undetermined parameters. $U(y)$ and $H(y)$ are shown in figure (11). Evaluating the background dissipation and $F\langle U\rangle$ we get:

$$
\begin{gathered}
F\langle U\rangle-\frac{1}{2} \mathcal{D}_{b g}=F U^{*}-F U^{*}\left(\frac{\delta}{d}\right)-\frac{\nu U^{* 2}}{d^{2}}\left(\frac{d}{\delta}\right)-\frac{B_{2}^{2} U^{* 2}}{2 \eta}\left[\frac{2}{3}\left(\frac{\delta}{d}\right)-\left(\frac{\delta}{d}\right)^{2}\right] \\
\simeq F U^{*}-\frac{\nu U^{* 2}}{d^{2}}\left(\frac{d}{\delta}\right)-\frac{B_{2}^{2} U^{* 2}}{2 \eta} \frac{2}{3}\left(\frac{\delta}{d}\right)
\end{gathered}
$$

where we dropped out terms of order $(\delta / d)^{2}$.

The above expression takes its minimum value when $\delta=\delta_{\min }=\sqrt{3 \nu \eta} / B_{2}$ and $U^{*}=$ $U_{\min }^{*}=(\sqrt{3} / 4)\left(F d / B_{2}\right) \sqrt{\eta / \nu}$

Now we turn to the quadratic term $\mathcal{Q}_{U B_{1}}$ and try to determine the constraint on on $\delta$ and $U^{*}$. The calculation is identical with the Couette flow and gives that for $\mathcal{Q}_{U B_{1}} \geq 0$ we have to have $U^{*} \delta \leq 2 \min \{\nu, \eta\}=\left(U^{*} \delta\right)_{Q}$. All we have to do now is to find the values of $U^{*}$ and $\delta$ that give the maximum possible of $2 F\langle U\rangle-\mathcal{D}_{b g}$ with out violating the constraint $\mathcal{Q}_{U B_{1}} \geq 0$. If $U_{\min }^{*} \delta_{\min } \leq\left(U^{*} \delta\right)_{Q}$ then the obvious choice for $U^{*}$ and $\delta$ is $U_{\min }^{*}$ and $\delta_{\min }$ that gives

$$
\text { If } U_{\min }^{*} \delta_{\min }=\frac{3 F d \eta}{4 B_{2}^{2}} \leq 2 \min \{\nu, \eta\} \text { then } \mathcal{D}_{b g} \geq \frac{\sqrt{3} F^{2} d}{4 B_{2}} \sqrt{\frac{\eta}{\nu}}
$$

or in dimensionless form

$$
\text { If } 3 \max \left\{\mathbf{G}, \mathbf{G}_{\mathbf{M}}\right\} \leq 16 \mathbf{Q}^{2} \text { then } \mathbf{D} \geq \frac{\sqrt{3}}{2}\left(\frac{\mathbf{G}^{\mathbf{1 / 2}}}{2 \sqrt{2} \mathbf{Q}}\right)
$$

If the condition $U_{\min }^{*} \delta_{\min } \leq\left(U^{*} \delta\right)_{Q}$ is violated then we have to evaluate the maximum of $2 F\langle U\rangle-\mathcal{D}_{b g}$ over $U^{*}$ and $\delta$ under the constraint that $U^{*} \delta=\left(U_{\delta}^{*}\right)_{Q}$ after some algebra we end up with

$$
\text { if } \frac{3 F \eta}{4 B_{2}^{2}} \geq 2 \min \{\nu, \eta\} \text { then } \mathcal{D}_{b g} \geq \frac{4 \sqrt{2 d}}{3 \sqrt{3}}\left(F-\frac{2 B^{2} \min \{\nu, \eta\}}{3 \eta}\right)^{3 / 2} \sqrt{\frac{\min \{\nu, \eta\}}{\nu}}
$$

or in dimensionless form

$$
\text { if } 3 \max \left\{\mathbf{G}, \mathbf{G}_{\mathbf{M}}\right\} \geq 16 \mathbf{Q}^{2} \text { then } \mathbf{D} \geq \frac{4 \sqrt{2}}{3 \sqrt{3}}\left(1-\frac{4 \mathbf{Q}^{\mathbf{2}}}{3 \max \left\{\mathbf{G}, \mathbf{G}_{\mathbf{M}}\right\}}\right)^{3 / 2} \min \left\{1, \mathbf{P r}^{-1 / 2}\right\}
$$

As in the Couette case for strong enough magnetic field the first inequality (55) indicates that the bound is very close ( $15 \%$ difference) to the laminar dissipation. On the other hand for small enough magnetic fields the bound on the dissipation becomes independent of $\mathbf{Q}$ and $\mathbf{R e}$ and decreases as the inverse square root of the Prandtl number for $\mathbf{P r}>1$. This 


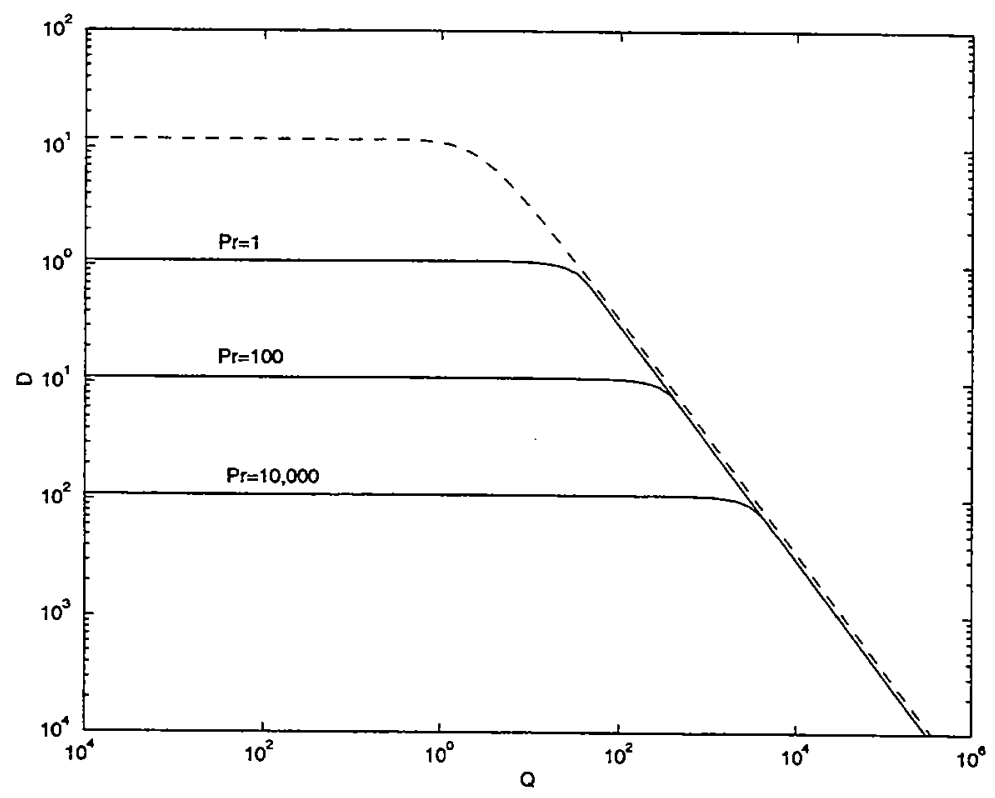

Figure 12: The dissipation as a function of $\mathbf{Q}$ for different Prandtl numbers. The dashed line shows the laminar solutions dissipation.

result is not in contradiction with the related result of the Couette flow that was giving a linear increase with the Prandtl number. The reason for the difference is the definition for the non-dimensional dissipation. If we had chosen $\tilde{D}=\mathcal{D} d /\langle u\rangle^{3}$ as our non dimensional dissipation we would have

$$
\tilde{D}=\frac{\mathcal{D} d}{\langle u\rangle^{3}}=\frac{\mathcal{D} d}{(\mathcal{D} / F)^{3}}=\frac{F^{3} d}{\mathcal{D}^{2}}=\frac{1}{\mathbf{D}^{2}}
$$

that gives the same scaling with Couette flow. The figures below $(12,13,14)$ summarize our results.

\section{Discussion}

We have examined the dissipation for two different kinds of flows in conducting fluids with an imposed vertical (to the flow) magnetic field, namely magnetic Couette flow and Hartmann flow. We have derived bounds on the dissipation and determined the bounds behavior at high Reynolds and magnetic Reynolds number. One of our basic results is that the dissipation is tending to the laminar value if the magnetic field is strong enough. If the magnetic field is not very strong and the Reynolds number is large the dissipation is independent of $\mathbf{R e}$ and $\mathbf{Q}$ and scales as the first power of the Prandtl number if $\operatorname{Pr}>1$ and is independent of it otherwise. The next figure (15) shows a quantitative comparison of experimental data [5] with our bound. The data show measurments of the drag coefficient $\mathbf{C}_{\mathbf{f}}$ as a function of $\mathbf{Q}$. The coefficient $\mathbf{C}_{\boldsymbol{f}}$ is defined as:

$$
\mathbf{C}_{\mathbf{f}}=\frac{F d}{\langle U\rangle^{2}}=\frac{d \mathcal{D}}{\langle U\rangle^{3}}=\tilde{\mathbf{D}}=\frac{1}{\bar{D}^{2}}
$$




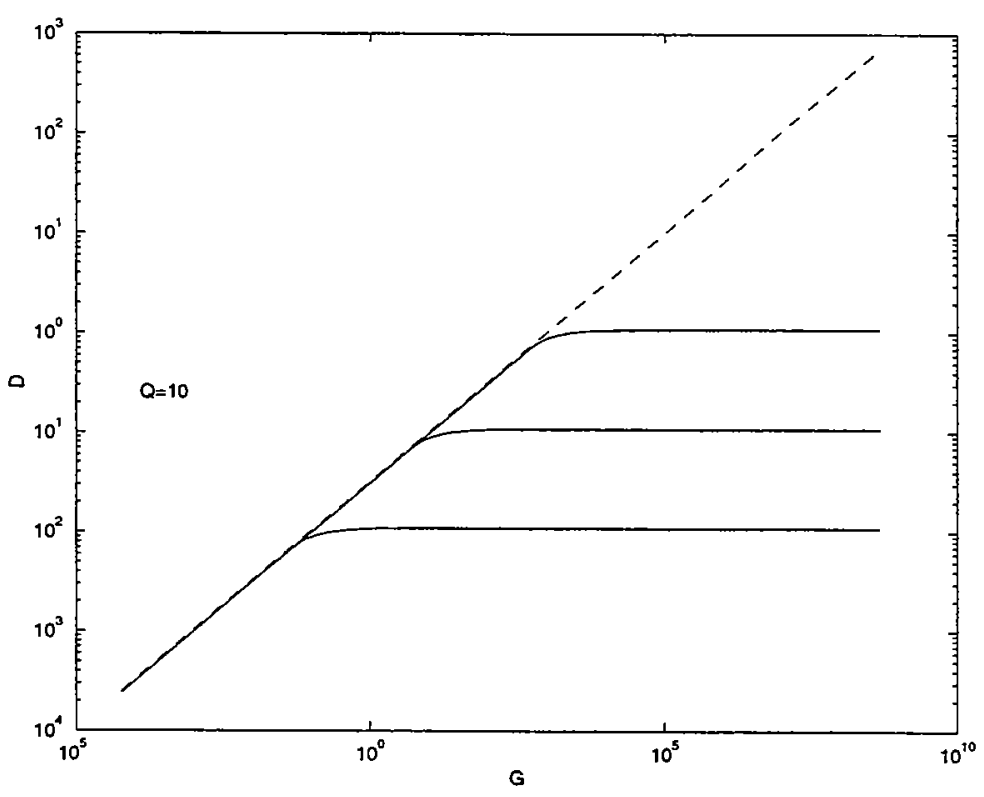

Figure 13: The dissipation as a function of Re for different Prandtl numbers. The dashed line shows the laminar solutions dissipation.

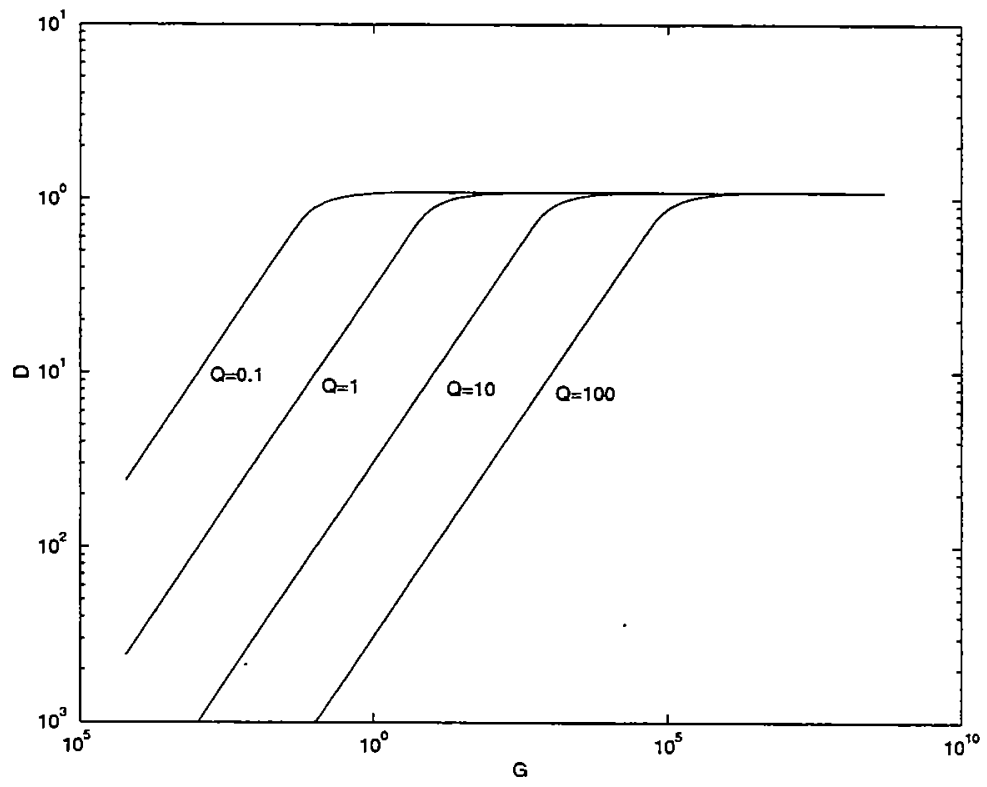

Figure 14: The dissipation as a function of $\mathbf{R e}$ for different values of $\mathbf{Q}$. 


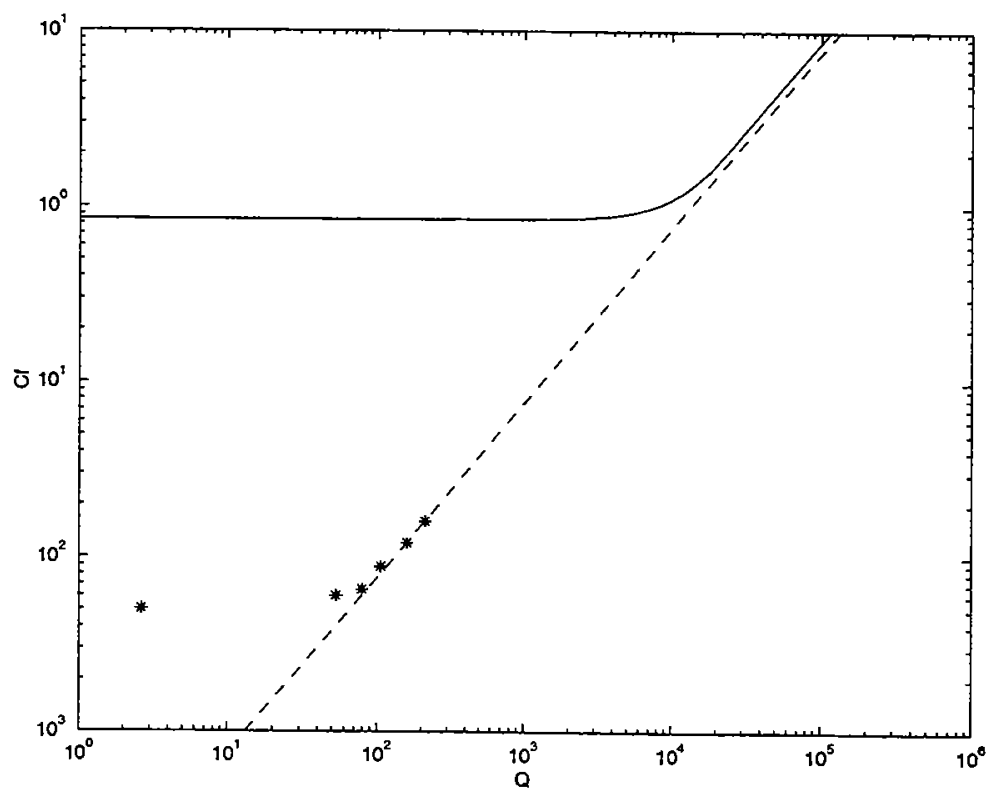

Figure 15: The drag coefficient $\mathbf{C}_{\mathbf{f}}$ as a function of $\mathbf{Q}$

Although there is a two orders of magnitude difference from our bound which is not suprizing for the rough estimates we used, the bound seems to capture the behavior of the dissipation up to a prefactor.

\section{References}

\section{References}

[1] H. K. Moffatt, Magnetic field generation in electrically conducting fluids (Cambridge University Press, Cambridge, 1978).

[2] C. R. Doering and P. Constantin, "Variational bounds on energy dissipation in incompressible flows: Shear flow," PRE 49, 4087 (1994).

[3] C. R. Doering, E. A. Speigel and R. A. Worthing, "Energy dissipation in a shear layer with suction," PRE 12, 1955 (2000).

[4] J. Hartmann, "Hg dynamics-i," Kgl. Danske Videnskab Selskab Mat.-Fys. Medd. 15, (1937).

[5] C.B.Reed and P. Lykoudis, "The effect of a tranverse magnetic field on shear turbulence," JFM 89, 147 (1978). 


\title{
On Cyclic and Oscillatory Convection in a Simplified Box Model with Entrainment
}

\author{
Tomoki Tozuka \\ University of Tokyo, Japan
}

\begin{abstract}
Fluctuating ventilation effect in a simplified box model with three tubes is studied theoretically. A small basin is cooled from above and connected to an infinitely large isothermal basin with a layer of fresh water at the surface. A necessary condition for a new layer to form after a convection event is derived, and the model can reproduce 'cyclic convections' observed in past laboratory experiments. A parameterization for interfacial entrainment is formulated based on a potential energy budget. This introduces two new regimes to the model. One is an equilibrium state with the interface located in between the middle tube and the bottom tube with inflows at the top and bottom tube and an outflow at the middle tube. The other is an 'oscillatory ventilation' where the upper layer thickness does not grow monotonously, but oscillates. This regime is a result of balances between entrainment, surface cooling, and flow through the three tubes. Comparisons with laboratory experiments are made.
\end{abstract}

\section{Introduction}

The thermohaline circulation has been studied extensively due to its importance to global climate variation. The deep convection branch of the thermohaline circulation occurs in very confined regions[1],[2], and the Nordic Sea is one of the important sites. Since the salinity is very low in the surface layer of the Nordic Sea, the convection caused by the intense surface cooling cannot reach to a great depth without an increase in salinity. Excluding the dense overflow, the most probable candidate for a source of salinity increase seems to be the salty water below the surface[3]. In an attempt to understand this process, Whitehead[4] analyzed a simple box model both analytically and numerically. The model consisted of a small basin cooled from above and a large isothermal basin with a surface fresh water layer maintained at constant thickness. The two basins were connected to each other by three tubes at top, middle, and bottom. Using a relaxation boundary condition for the temperature, multiple equilibria were obtained. As an extension of this study, te Raa[5] performed laboratory experiments and showed that two flow regimes exist; one is a selfsustained oscillation and the other is a steady-state with deep convection. However, the mechanism for this oscillation remains unexplained theoretically. Also, an interfacial mixing process was not considered in the box model theory.

In this study, we expand earlier studies of oscillatory behavior in the simplified box model. The paper is organized as follows. In the next section, a description of a threetube model and its behavior is presented. A necessary condition for the formation of a new layer after a convection event is derived. Also, a parameterization for the entrainment is formulated based on a discussion of potential energy budget. The theoretical model can then reproduce oscillations observed in the past laboratory experiments. In section 3 , theoretical results are compared to laboratory experiment data. Conclusions are given in the final section. 


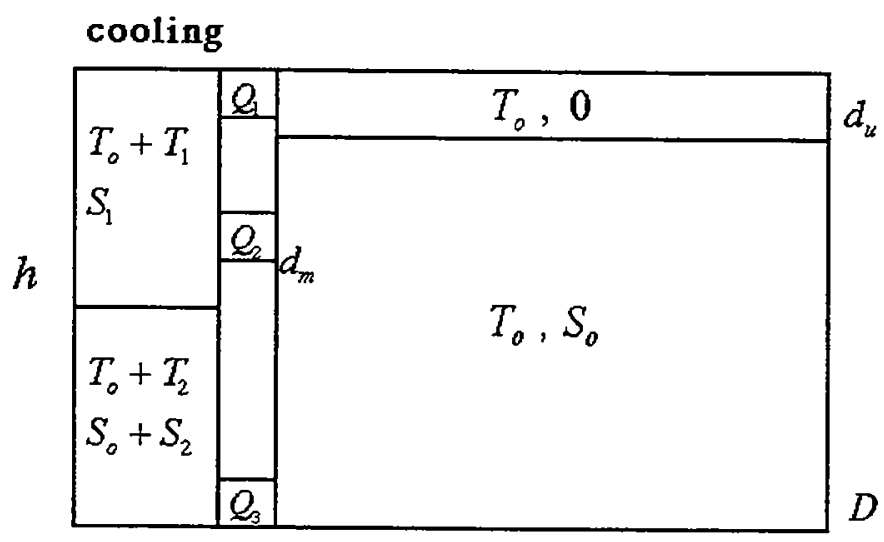

Figure 1: Schematic sketch of the three-tube model.

\section{Three-tube Model}

\subsection{Formulation}

Following Whitehead[4], a three-tube model is formulated here so that we can investigate the role played by the subsurface salty water. The importance of the middle tube may be further appreciated by comparing the result obtained here with two-tube model results presented in the Appendix.

A small basin, which represents a small region which contains a deep convection site, is cooled from above. This basin is connected to an infinitely large isothermal basin with three tubes, one at the surface, the second one at a depth of $d_{m}$, and the third one at the bottom. This differs from Whitehead[4], where the middle tube was placed at the depth of $D / 2$. The depth of both basins is equal at $D$. A shallow fresh water layer of depth $d_{u}$ with temperature $T_{o}$ are maintained on top with temperature $T_{o}$ and salinity $S_{o}$ in the larger basin. The parameters in the large basin $d_{u}, T_{o}$, and $S_{o}$ are kept fixed. In response to the surface cooling, the small basin contains a well-mixed surface layer of depth $h$, temperature $T_{o}+T_{1}$, and salinity $S_{1}$. The parameters in the small basin $h, T_{1}$, and $S_{1}$ can vary with time. A sketch of the box model is given in Fig.1.

We assume that a linear flow resistance in the tubes maintains a relation between the volume fluxes through the tubes $Q_{i}$ and the pressure difference between two basins of the form

$$
Q_{i}=C_{i}\left(p_{o i}-p_{i}\right) \text { for } i=1,2,3
$$

where we specify that

$$
C_{1}=\gamma C, C_{2}=C_{3}=C
$$

are hydraulic resistances of tube $i$, and $\gamma$ is a positive real number. Let $\eta$ denote the fluid surface elevation of the small basin relative to the large basin. Then, from the hydrostatic relation, the pressure at tube $i$ is given by

$$
p_{o 1}=0
$$




$$
\begin{aligned}
& p_{o 2}=\rho_{o} g\left[d_{u}+\left(1+\beta S_{o}\right)\left(d_{m}-d_{u}\right)\right] \\
& p_{o 3}=\rho_{o} g\left[d_{u}+\left(1+\beta S_{o}\right)\left(D-d_{u}\right)\right]
\end{aligned}
$$

in the large basin and

$$
p_{2}=\left\{\begin{array}{l}
p_{1}=\rho_{o} g \eta \\
\rho_{o} g\left[\eta+\left(1+\beta S_{1}-\alpha T_{1}\right) h+\left(1+\beta\left(S_{o}+S_{2}\right)-\alpha T_{2}\right)\left(d_{m}-h\right)\right] \text { for } 0<h \leq d_{m} \\
\rho_{o} g\left[\eta+\left(1+\beta S_{1}-\alpha T_{1}\right) d_{m}\right] \text { for } d_{m} \leq h \leq D
\end{array}\right\}
$$

$$
p_{3}=\rho_{o} g\left[\eta+(1+\beta S-\alpha T) h+\left(1+\beta\left(S_{o}+S_{2}\right)-\alpha T_{2}\right)(D-h)\right]
$$

in the small basin. Here, the density is calculated using a linear equation of state and $\rho_{o}$ is the density of fresh water at temperature $T_{o}$. The volume fluxes $Q_{i}$ obey

$$
\begin{gathered}
Q_{1}=-\gamma C \rho_{o} g \eta \\
Q_{2}=\left\{\begin{array}{l}
C \rho_{o} g\left[-\eta-\beta S_{o} d_{u}+\left(-\beta S_{2}+\alpha T_{2}\right) d_{m}\right. \\
\left.+\left(\beta\left(S_{o}+S_{2}-S_{1}\right)+\alpha\left(T_{1}-T_{2}\right)\right) h\right] \text { for } 0<h \leq d_{m} \\
C \rho_{o} g\left[-\eta-\beta S_{o} d_{u}+\left(\beta\left(S_{o}-S_{1}\right)+\alpha T_{1}\right) d_{m}\right] \text { for } d_{m} \leq h \leq D
\end{array}\right\} \\
Q_{3}=C \rho_{o} g\left[-\eta-\beta S_{o} d_{u}+\left(-\beta S_{2}+\alpha T_{2}\right) D+\left(\beta\left(S_{o}+S_{2}-S_{1}\right)+\alpha\left(T_{1}-T_{2}\right)\right) h\right] .
\end{gathered}
$$

Assuming that changes in the vertical acceleration with time are small, we obtain

$$
\eta=\frac{1}{2+\gamma}\left[-2 \beta S_{o} d_{u}+2\left(\beta\left(S_{o}+S_{2}-S_{1}\right)+\alpha\left(T_{1}-T_{2}\right)\right) h+\left(-\beta S_{2}+\alpha T_{2}\right)\left(D+d_{m}\right)\right]
$$

for $0<h \leq d_{m}$, and

$$
\begin{array}{r}
\eta=\frac{1}{2+\gamma}\left[-2 \beta S_{o} d_{u}+\left(\beta\left(S_{o}-S_{1}\right)+\alpha T_{1}\right) d_{m}+\left(-\beta S_{2}+\alpha T_{2}\right) D\right. \\
\left.+\left(\beta\left(S_{o}+S_{2}-S_{1}\right)+\alpha\left(T_{1}-T_{2}\right)\right) h\right]
\end{array}
$$

for $d_{m} \leq h \leq D$. Substituting (12) into (9), (10), and (11), we obtain

$$
\begin{array}{r}
Q_{1}=-\frac{\gamma C \rho_{o} g \beta S_{o} D}{2+\gamma}\left[-\frac{2 d_{u}}{D}+2\left(1+\frac{S_{2}}{S_{o}}-\frac{S_{1}}{S_{o}}+\frac{\alpha}{\beta S_{o}}\left(T_{1}-T_{2}\right)\right) \frac{h}{D}\right. \\
\left.+\left(-\frac{S_{2}}{S_{o}}+\frac{\alpha T_{2}}{\beta S_{o}}\right)\left(1+\frac{d_{m}}{D}\right)\right]
\end{array}
$$




$$
\begin{array}{r}
Q_{2}=\frac{C \rho_{o} g \beta S_{o} D}{2+\gamma}\left[-\gamma \frac{d_{u}}{D}+\left(-\frac{S_{2}}{S_{o}}+\frac{\alpha T_{2}}{\beta S_{o}}\right)\left((1+\gamma) \frac{d_{m}}{D}-1\right)\right. \\
\left.+\gamma\left(1+\frac{S_{2}}{S_{o}}-\frac{S_{1}}{S_{o}}+\frac{\alpha}{\beta S_{o}}\left(T_{1}-T_{2}\right)\right) \frac{h}{D}\right] \\
Q_{3}=\frac{C \rho_{o} g \beta S_{o} D}{2+\gamma}\left[-\gamma \frac{d_{u}}{D}+\left(-\frac{S_{2}}{S_{o}}+\frac{\alpha T_{2}}{\beta S_{o}}\right)\left(1+\gamma-\frac{d_{m}}{D}\right)\right. \\
\left.+\gamma\left(1+\frac{S_{2}}{S_{o}}-\frac{S_{1}}{S_{o}}+\frac{\alpha}{\beta S_{o}}\left(T_{1}-T_{2}\right)\right) \frac{h}{D}\right]
\end{array}
$$

for $0<h \leq d_{m}$, and substituting (13) into (9), (10), and (11), we obtain

$$
\begin{array}{r}
Q_{1}=-\frac{\gamma C \rho_{o} g \beta S_{o} D}{2+\gamma}\left[-\frac{2 d_{u}}{D}+(\beta\right. \\
\left.\left.+1-\frac{S_{1}}{S_{o}}\right)+\frac{\alpha T_{1}}{\beta S_{o}}\right) \frac{d_{m}}{D}+\left(-\frac{S_{2}}{S_{o}}+\frac{\alpha T_{2}}{\beta S_{o}}\right) \\
\left.+\left(1+\frac{S_{2}}{S_{o}}-\frac{S_{1}}{S_{o}}+\frac{\alpha}{\beta S_{o}}\left(T_{1}-T_{2}\right)\right) \frac{h}{D}\right] \\
Q_{2}=\frac{C \rho_{o} g \beta S_{o} D}{2+\gamma}\left[-\gamma \frac{d_{u}}{D}+\left(1-\frac{S_{1}}{S_{o}}+\frac{\alpha T_{1}}{\beta S_{o}}\right)(1+\gamma) \frac{d_{m}}{D}-\left(-\frac{S_{2}}{S_{o}}+\frac{\alpha T_{2}}{\beta S_{o}}\right)\right. \\
\left.-\left(1+\frac{S_{2}}{S_{o}}-\frac{S_{1}}{S_{o}}+\frac{\alpha}{\beta S_{o}}\left(T_{1}-T_{2}\right)\right) \frac{h}{D}\right] \\
Q_{3}=\frac{C \rho_{o} g \beta S_{o} D}{2+\gamma}\left[-\gamma \frac{d_{u}}{D}-\left(1-\frac{S_{1}}{S_{o}}+\frac{\alpha T_{1}}{\beta S_{o}}\right) \frac{d_{m}}{D}+(1+\gamma)\left(-\frac{S_{2}}{S_{o}}+\frac{\alpha T_{2}}{\beta S_{o}}\right)\right. \\
\left.+(1+\gamma)\left(1+\frac{S_{2}}{S_{o}}-\frac{S_{1}}{S_{o}}+\frac{\alpha}{\beta S_{o}}\left(T_{1}-T_{2}\right)\right) \frac{h}{D}\right]
\end{array}
$$

for $d_{m} \leq h \leq D$.

The upper layer mass conservation equation is

$$
A \frac{d h}{d t}=Q_{1}
$$

for $0<h \leq d_{m}$, and

$$
A \frac{d h}{d t}=Q_{1}+Q_{2}
$$

for $d_{m} \leq h \leq D$. The heat and salt balance equations are

$$
\begin{gathered}
A h \frac{d T_{1}}{d t}=\frac{K_{a}}{\rho_{o} c_{p}}\left(T^{*}-T_{1}\right)-T_{1} Q_{1} \Gamma\left(+Q_{1}\right) \\
A h \frac{d S_{1}}{d t}=-S_{1} Q_{1} \Gamma\left(+Q_{1}\right)
\end{gathered}
$$




$$
\begin{aligned}
& A(D-h) \frac{d T_{2}}{d t}=-T_{2} Q_{2} \Gamma\left(+Q_{2}\right)-T_{2} Q_{3} \Gamma\left(+Q_{3}\right) \\
& A(D-h) \frac{d S_{2}}{d t}=-S_{2} Q_{2} \Gamma\left(+Q_{2}\right)-S_{2} Q_{3} \Gamma\left(+Q_{3}\right)
\end{aligned}
$$

for $0<h \leq d_{m}$, and

$$
\begin{gathered}
A h \frac{d T_{1}}{d t}=\frac{K_{a}}{\rho_{o} c_{p}}\left(T^{*}-T_{1}\right)-T_{1} Q_{1} \Gamma\left(+Q_{1}\right)-T_{1} Q_{2} \Gamma\left(+Q_{2}\right) \\
A h \frac{d S_{1}}{d t}=-S_{1} Q_{1} \Gamma\left(+Q_{1}\right)+\left(S_{o}-S_{1}\right) Q_{2} \Gamma\left(+Q_{2}\right) \\
A(D-h) \frac{d T_{2}}{d t}=-T_{2} Q_{3} \Gamma\left(+Q_{3}\right) \\
A(D-h) \frac{d S_{2}}{d t}=-S_{2} Q_{3} \Gamma\left(+Q_{3}\right)
\end{gathered}
$$

for $d_{m} \leq h \leq D$. Here, we have taken that heat flux is proportional to $T^{*}-T_{1}$. This is called a restoring boundary condition for the temperature, and is also known as Haney boundary condition[6]. A zero salt-flux boundary condition is used.

When the density stratification in the small basin becomes unstable:

$$
\rho\left(T_{o}+T_{1}, S_{1}\right)>\rho\left(T_{o}+T_{2}, S_{o}+S_{2}\right)
$$

a convective adjustment occurs.

\subsection{Parameterization of Entrainment}

It is well known that entrainment plays an important role in upper ocean dynamics. In addition, te Raa[5] observed a strong interfacial entrainment in laboratory experiments. Thus, we here formulate an one-dimensional mixed layer model, which parameterizes the entrainment process at the interface, following Kraus and Turner[7] and Davis et al.[8]. We expect that an inclusion of entrainment should lead to more realistic representation of situations in the ocean and the laboratory experiment of te Raa[5]. The model results without the entrainment are provided in the Appendix.

The upper-layer potential energy is defined as

$$
P=g \int_{-D}^{0}\left(z-z_{o}\right) \rho d z
$$

where $z=z_{o}$ is a reference level, and $z=-D$ is a level below the mixed layer at which turbulent and radiative fluxes of heat is assumed to be negligible. The density conservation equation is

$$
\frac{\partial \rho}{\partial t}+\frac{\partial}{\partial z} \overline{w^{\prime} \rho^{\prime}}=0
$$


where $\overline{w^{\prime} \rho^{\prime}}$ is the vertical turbulent flux of mass. Here, advective terms have been neglected, because we cannot incorporate their effect in simple models such as the one considered in this study. Multiplying Eq.(32) by $g\left(z-z_{o}\right)$ and integrating it from $z=-D$ to $z=0$, we obtain

$$
\frac{\partial P}{\partial t}=g \int_{-D}^{0} \overline{w^{\prime} \rho^{\prime}} d z+\frac{\alpha g}{c_{p}} Q_{o} z_{o}
$$

where

$$
Q_{o}=\frac{c_{p}}{\alpha} \overline{w^{\prime} \rho^{\prime}}(0)=-c_{p} \rho \overline{w^{\prime} T^{\prime}}(0)
$$

is the net downward heat flux at the surface. Note that the heat fluxes are assumed to vanish at $z=-D$.

From Tennekes and Lumley[9], the turbulent kinetic energy budget equation is

$$
\left(\frac{\partial}{\partial t}+\mathbf{u} \cdot \nabla+w \frac{\partial}{\partial z}\right) \bar{e}_{k}=-g \overline{w^{\prime} \rho^{\prime}}-\rho \overline{\mathbf{u} w^{\prime}} \cdot \frac{\partial}{\partial z} \mathbf{u}-\frac{\partial}{\partial z} \overline{w^{\prime}\left(p^{\prime}+e_{k}\right)}-\rho \epsilon
$$

where

$$
e_{k}=\frac{\rho}{2}\left(\mathbf{u} \cdot \mathbf{u}+w^{2}\right)
$$

Here, the first term is the production of turbulent kinetic energy by the vertical buoyancy flux, the second term is the production of turbulent kinetic energy by shear, the third term is the vertical divergence of the turbulent flux of turbulent kinetic energy, and the final term is the viscous dissipation term. Again, the second term and the third term are beyond the framework of box model and are neglected. Also, based on the laboratory experiment, Deardorff et al. [10] showed that a fixed fraction $m_{c}(=0.83)$ of potential energy gained by the surface cooling is dissipated. Thus, the potential energy equation (33) can be rewritten as

$$
\frac{\partial P}{\partial t}=-m_{c} \frac{\alpha g D}{2 c} Q_{o}+\frac{\alpha g}{c} Q_{o} z_{o}
$$

For the bulk mixed layer with thickness $h$, the potential energy equation is

$$
\frac{g h}{2} \Delta \rho \frac{\partial h}{\partial t}=\frac{\alpha g}{c}\left[-\frac{h}{2} Q_{o}+\frac{h}{2} m_{c} Q_{o}\right]
$$

Therefore, the entrainment velocity in the three-tube model is parameterized by

$$
w_{e}=-\frac{\alpha}{c_{p} \Delta \rho}\left(1-m_{c}\right) Q_{o}
$$

where $\Delta \rho$ is the density difference between the upper and lower layer. Since we are using the restoring boundary condition for heat, the entrainment velocity is

$$
w_{e}=-\frac{\alpha\left(1-m_{c}\right) K_{a}\left(T^{*}-T_{1}\right)}{c_{p} A \Delta \rho}
$$


Including the entrainment process, the upper layer mass conservation equation can be rewritten as

$$
A \frac{d h}{d t}=Q_{1}+A w_{e}
$$

for $0<h \leq d_{m}$, and

$$
A \frac{d h}{d t}=Q_{1}+Q_{2}+A w_{e}
$$

for $d_{m} \leq h \leq D$. The upper layer heat and salt balance equations are rewritten as

$$
\begin{gathered}
A h \frac{d T_{1}}{d t}=\frac{K_{a}}{\rho_{o} c_{p}}\left(T^{*}-T_{1}\right)-T_{1} Q_{1} \Gamma\left(+Q_{1}\right)+A\left(T_{2}-T_{1}\right) w_{e} \\
A h \frac{d S_{1}}{d t}=-S_{1} Q_{1} \Gamma\left(+Q_{1}\right)+A\left(S_{o}+S_{2}-S_{1}\right) w_{e}
\end{gathered}
$$

for $0<h \leq d_{m}$, and

$$
\begin{gathered}
A h \frac{d T_{1}}{d t}=\frac{K_{a}}{\rho_{o} c_{p}}\left(T^{*}-T_{1}\right)-T_{1} Q_{1} \Gamma\left(+Q_{1}\right)-T_{1} Q_{2} \Gamma\left(+Q_{2}\right)+A\left(T_{2}-T_{1}\right) w_{e} \\
A h \frac{d S_{1}}{d t}=-S_{1} Q_{1} \Gamma\left(+Q_{1}\right)+\left(S_{o}-S_{1}\right) Q_{2} \Gamma\left(+Q_{2}\right)+A\left(S_{o}+S_{2}-S_{1}\right) w_{e}
\end{gathered}
$$

for $d_{m} \leq h \leq D$.

\subsection{Non-dimensionalized Equations}

Using the transformations

$$
\begin{gathered}
\tilde{Q}_{i}=\frac{Q_{i}}{Q_{s s}}, \tilde{T}_{i}=\frac{\alpha T_{i}}{\beta S_{o}}, \tilde{d}_{u}=\frac{d_{u}}{D}, \tilde{d}_{m}=\frac{d_{m}}{D} \\
\tilde{S}_{i}=\frac{S_{i}}{S_{o}}, \tilde{T}^{*}=\frac{\alpha T^{*}}{\beta S_{o}}, \tilde{t}=\frac{A D}{Q_{s s}} t, \tilde{K}_{a}=\frac{K_{a}}{\rho_{o} c_{p} Q_{s s}}
\end{gathered}
$$

where

$$
Q_{s s}=\frac{\gamma C \rho_{o} g \beta S_{o} D}{2+\gamma}
$$

the model equations are non-dimensionalized as

$$
\begin{gathered}
\tilde{Q}_{1}=2 \tilde{d_{u}}-2\left(1+\tilde{S_{2}}-\tilde{S_{1}}+\tilde{T}_{1}-\tilde{T}_{2}\right) \tilde{h}-\left(-\tilde{S_{2}}+\tilde{T}_{2}\right)\left(1+\tilde{d_{m}}\right) \\
\tilde{Q}_{2}=-\tilde{d}_{u}+\left(-\tilde{S_{2}}+\tilde{T}_{2}\right)\left(\left(\gamma^{-1}+1\right) \tilde{d_{m}}-\gamma^{-1}\right)+\left(1+\tilde{S}_{2}-\tilde{S}_{1}+\tilde{T}_{1}-\tilde{T_{2}}\right) \tilde{h}
\end{gathered}
$$




$$
\begin{aligned}
& \tilde{Q_{3}}=-\tilde{d_{u}}+\left(-\tilde{S_{2}}+\tilde{T_{2}}\right)\left(\gamma^{-1}+1-\gamma^{-1} \tilde{d_{m}}\right)+\left(1+\tilde{S_{2}}-\tilde{S_{1}}+\tilde{T_{1}}-\tilde{T_{2}}\right) \tilde{h} \\
& \frac{d \tilde{h}}{d \tilde{t}}=\tilde{Q}_{1}+\tilde{w}_{e} \\
& \tilde{h} \frac{d \tilde{T}_{1}}{d \tilde{t}}=\tilde{K}_{a}\left(\tilde{T}^{*}-\tilde{T}_{1}\right)-\tilde{T}_{1} \tilde{Q}_{1} \Gamma\left(+\tilde{Q}_{1}\right)+\left(\tilde{T}_{2}-\tilde{T}_{1}\right) \tilde{w}_{e} \\
& \tilde{h} \frac{d \tilde{S}_{1}}{d \tilde{t}}=-\tilde{S}_{1} \tilde{Q}_{1} \Gamma\left(+\tilde{Q}_{1}\right)+\left(1+\tilde{S}_{2}-\tilde{S}_{1}\right) \tilde{w}_{e} \\
& (1-\tilde{h}) \frac{d \tilde{T}_{2}}{d \tilde{t}}=-\tilde{T}_{2} \tilde{Q}_{2} \Gamma\left(+\tilde{Q}_{2}\right)-\tilde{T}_{2} \tilde{Q}_{3} \Gamma\left(+\tilde{Q}_{3}\right) \\
& (1-\tilde{h}) \frac{d \tilde{S}_{2}}{d \tilde{t}}=-\tilde{S}_{2} \tilde{Q}_{2} \Gamma\left(+\tilde{Q}_{2}\right)-\tilde{S}_{2} \tilde{Q}_{3} \Gamma\left(+\tilde{Q}_{3}\right)
\end{aligned}
$$

for $0<\tilde{h} \leq \tilde{d_{m}}$, and

$$
\begin{gathered}
\tilde{Q_{1}}=2 \tilde{d}_{u}-\left(1-\tilde{S}_{1}+\tilde{T}_{1}\right) \tilde{d_{m}}-\left(-\tilde{S}_{2}+\tilde{T}_{2}\right)-\left(1+\tilde{S}_{2}-\tilde{S}_{1}+\tilde{T}_{1}-\tilde{T}_{2}\right) \tilde{h} \\
\tilde{Q}_{2}=-\tilde{d}_{u}+\left(1-\tilde{S}_{1}+\tilde{T}_{1}\right)\left(1+\gamma^{-1}\right) \tilde{d_{m}}-\gamma^{-1}\left(-\tilde{S}_{2}+\tilde{T}_{2}\right)-\gamma^{-1}\left(1+\tilde{S}_{2}-\tilde{S}_{1}+\tilde{T}_{1}-\tilde{T_{2}}\right) \tilde{h} \\
\tilde{Q}_{3}=-\tilde{d}_{u}-\gamma^{-1}\left(1-\tilde{S}_{1}+\tilde{T}_{1}\right) \tilde{d_{m}}+\left(1+\gamma^{-1}\right)\left(-\tilde{S}_{2}+\tilde{T}_{2}\right)+\left(1+\gamma^{-1}\right)\left(1+\tilde{S}_{2}-\tilde{S}_{1}+\tilde{T}_{1}-\tilde{T}_{2}\right) \tilde{h} \\
\frac{d \tilde{h}}{d \tilde{t}}=\tilde{Q}_{1}+\tilde{Q}_{2}+\tilde{w}_{e} \\
\tilde{h} \frac{d \tilde{T}_{1}}{d \tilde{t}}=\tilde{K}_{a}\left(\tilde{T}^{*}-\tilde{T}_{1}\right)-\tilde{T_{1}} \tilde{Q}_{1} \Gamma\left(+\tilde{Q}_{1}\right)-\tilde{T}_{1} \tilde{Q}_{2} \Gamma\left(+\tilde{Q}_{2}\right)+\left(\tilde{T}_{2}-\tilde{T}_{1}\right) \tilde{w}_{e} \\
\tilde{h} \frac{d \tilde{S}_{1}}{d \tilde{t}}=-\tilde{S}_{1} \tilde{Q}_{1} \Gamma\left(+\tilde{Q}_{1}\right)+\left(1-\tilde{S}_{1}\right) \tilde{Q}_{2} \Gamma\left(+\tilde{Q}_{2}\right)+\left(1+\tilde{S}_{2}-\tilde{S}_{1}\right) \tilde{w}_{e} \\
(1-\tilde{h}) \frac{d \tilde{T}_{2}}{d \tilde{t}}=-\tilde{T}_{2} \tilde{Q}_{3} \Gamma\left(+\tilde{Q}_{3}\right) \\
(1-\tilde{h}) \frac{d \tilde{S}_{2}}{d \tilde{t}}=-\tilde{S}_{2} \tilde{Q}_{3} \Gamma\left(+\tilde{Q}_{3}\right)
\end{gathered}
$$

for $\tilde{d_{m}} \leq \tilde{h} \leq 1$. Here,

$$
\tilde{w}_{e}=-\left(1-m_{c}\right) \frac{\tilde{K}_{a}\left(\tilde{T}^{*}-\tilde{T}_{1}\right)}{\left(1+\tilde{S}_{2}-\tilde{T}_{2}\right)-\left(\tilde{S}_{1}-\tilde{T}_{1}\right)} .
$$




\subsection{New Layer Formation}

In laboratory experiments, it was observed that a new layer of low salinity water formed on top after a convective overturning (Whitehead, pers. comm.). In order to reproduce this phenomenon and resulting oscillations, we derive a necessary condition for this process to occur.

Fresh water flowing in from the top tube tries to form a new layer above the well-mixed thick layer of temperature $\tilde{T}_{2}$ and salinity $1+\tilde{S}_{2}$ after a convective adjustment, or the interface reaching the bottom. Since the upper layer thickness is $\tilde{h}=0$ then, the volume flux $\tilde{Q}_{1}$ is

$$
\tilde{Q}_{1}=\tilde{d}_{u}-\tilde{T}_{2}+\tilde{S_{2}}
$$

A new layer is formed on top with a thickness of

$$
\tilde{h}_{o}=\tilde{Q}_{1} \Delta \tilde{t}
$$

after one time step $\Delta \tilde{t}$. However, the new layer is quickly cooled by the surface heat loss, $\tilde{K}_{a} \tilde{T}^{*}$, and becomes denser. From the heat balance equation, an increase in the density of the new layer after one time step is

$$
\Delta \tilde{\rho}_{1}=-\frac{\tilde{K}_{a} \tilde{T}^{*} \Delta \tilde{t}}{\tilde{Q}_{1} \Delta \tilde{t}}=-\frac{\tilde{K}_{a} \tilde{T}^{*}}{\tilde{Q}_{1}} .
$$

To maintain static stability after the cooling,

$$
\Delta \tilde{\rho}_{1}=-\frac{\tilde{K}_{a} \tilde{T}^{*}}{\tilde{Q}_{1}}<1+\left(\tilde{S}_{2}-\tilde{T}_{2}\right)
$$

Thus, the necessary condition for $\tilde{Q}_{1}$ to prevent convective overturning is

$$
\tilde{Q}_{1}>-\frac{\tilde{K}_{a} \tilde{T}^{*}}{1+\left(\tilde{S}_{2}-\tilde{T}_{2}\right)} .
$$

Note that this condition is independent of the size of the time step. If this is not satisfied, the new layer becomes denser than the thick layer below, leading to another convective overturning. However, as the volume flux $\tilde{Q}_{1}$ progressively increases due to an increase in the density of the whole layer, the cooling of the thicker new layer decelerates. At some point, the above condition may be satisfied and the upper layer starts to grow again.

\subsection{Steady-state Solutions}

Numerous calculations were performed over varieties of parameter ranges and sensitivity of the model to dimensionless parameters were investigated. Calculations were initiated from $\tilde{T}_{2}=\tilde{S}_{2}=\tilde{S}_{1}=\tilde{T}_{1}=0$ and $\tilde{h}=\tilde{d}_{u}$ with no flow at each tube.

Figure 2 shows the sensitivity of the model to the depth $\tilde{d_{m}}$. The inclusion of entrainment results in drastic changes in the equilibrium states. This can be compared with no 
(a) $\gamma=0.05$

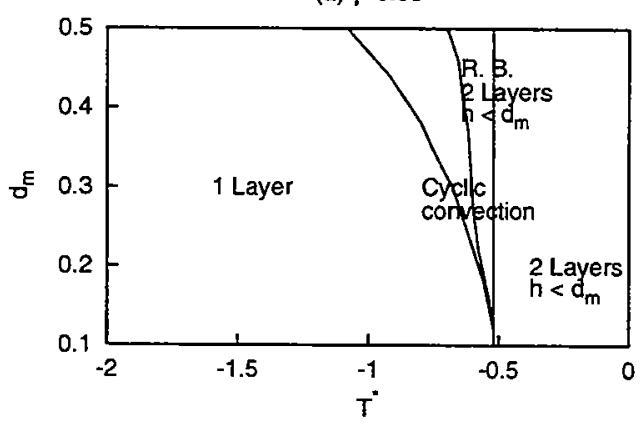

(b) $\gamma=1.0$

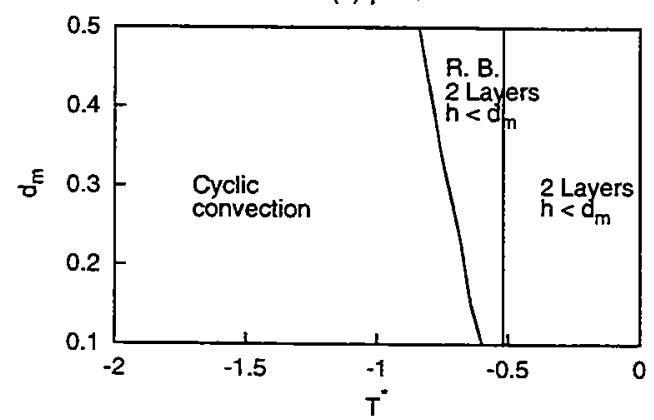

Figure 2: Regime diagrams for the three-tube model with entrainment at $\tilde{K}_{a}=0.5$ and $\tilde{d}_{u}=0.03$ for (a) $\gamma=0.05$ and (b) $\gamma=1.0$.

entrainment case illustrated in Fig. 11. When the cooling is weak, an equilibrium state with the interface above the middle-tube exists independent of $\gamma$ (indicated by '2 Layers $\left.h<d_{m}{ }^{\prime}\right)$. The upper layer temperature becomes $\tilde{T}^{*}$ and all flow stops.

Since the entrainment leads to a faster upper layer deepening and faster increase in upper layer density due to the salinity, the upper layer either reaches the bottom or becomes statically unstable at much weaker cooling than without entrainment. The interface reaches the bottom with forcing temperature $\tilde{T}^{*}$ as high as -0.52 with entrainment, whereas it reaches the bottom only after $\tilde{T}^{*}$ is decreased below -1.06 without entrainment. For a small $\gamma$, an equilibrium state with only one layer emerges as the cooling is enhanced (indicated by '1 Layer'). For this regime, the upper layer either becomes statically unstable or reaches the bottom, but the volume flux $\tilde{Q}_{1}$ never accelerates enough to satisfy the necessary condition. The small basin has inflows through the top and middle tube, and outflow through the bottom tube. This corresponds to 'deep convection' state in te Raa[5].

Equilibrium states depend upon the depth of the middle tube for a large $\gamma$. When $\tilde{d}_{m}$ is deep, the interface reaches the bottom once, but the model reaches an equilibrium state with the interface above the middle tube (indicated by 'R.B. 2 Layer $h<d_{m}{ }^{\prime}$ ). On the other hand, an 'oscillatory ventilation' mode exists for shallower $\tilde{d}_{m}$. This regime is a result of subtle interplay between entrainment, surface cooling, and flow through the three tubes. The upper layer thickness does not grow monotonously, but oscillates in the 'oscillatory ventilation'. This is contrasted with 'cyclic convection', where the convection has a cyclic nature, but the upper layer grows monotonously. This regime appears as the cooling temperature is further decreased. They are discussed more in detail in the next section.

The model is also very sensitive to the upper layer depth of the large basin (Fig. 3). Another interesting equilibrium state exists for relatively deep $\tilde{d}_{u}$ with forcing temperature of $-0.58<\tilde{T}^{*}<-0.46$ for large $\gamma$ and $-0.76<\tilde{T}^{*}<-0.46$ for small $\gamma$. The model reaches equilibrium with the interface located in between the middle tube and the bottom tube. Since the pressure difference at the bottom tube remains even after the model reaches the equilibrium, the inflow through the bottom tube persists. Thus, the water flows out from the small basin only through the middle tube and no deep water is formed in this 
(a) $\gamma=0.05$

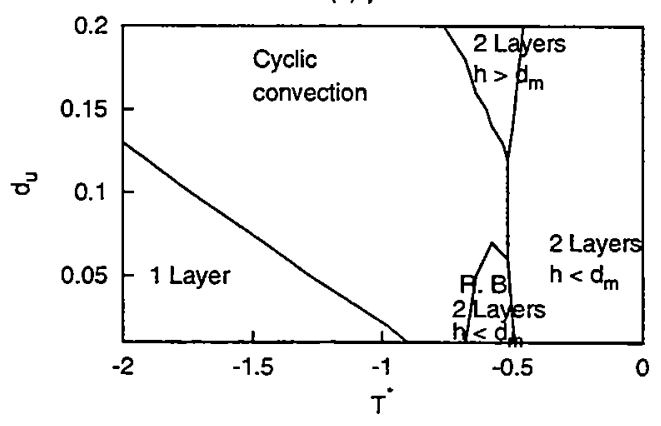

(a) $\gamma=1.00$

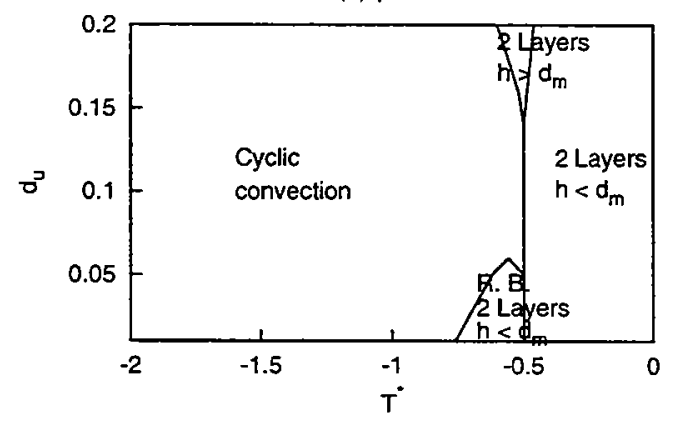

Figure 3: Regime diagrams for the three-tube model with entrainment at $\tilde{K}_{a}=0.5$ and $\tilde{d}_{m}=0.5$ for (a) $\gamma=0.05$ and (b) $\gamma=1.0$.

regime. A similar equilibrium state exists for three-tube model without entrainment, but the pressure difference at the bottom vanishes completely and the flow through the bottom tube stops $\left(\tilde{Q}_{3}=0\right)$ when the equilibrium is reached. The time evolution of this regime is also discussed in the next section.

\subsection{Time-dependent Solutions}

For certain parameter range, very interesting oscillations are obtained (Fig.4), which do not exist in the model without entrainment (upper layer variables and thickness are set to zero in figures, when there is only one active layer after reaching the bottom or the convective adjustment). At $\tilde{t}=40$, the interface reaches the bottom. As the volume flux $\tilde{Q}_{1}$ becomes large and satisfies the necessary condition (at $\tilde{t}=41$ ), the upper layer starts to grow again. Although $\tilde{\rho}_{1}$ initially increases, it begins to decrease after $\tilde{Q}_{2}$ becomes negative, and the salinity source at the mid-depth is lost. Then, $\tilde{Q}_{3}(>0)$ becomes larger than $\tilde{w}_{e}$, and the upper layer starts to become shallower. This is possible because the upper layer grows rapidly without increasing its density much, and the integrated mass above the bottom tube; the entrainment leads to faster deepening but only redistributes the mass within the small basin.

The inflow at the bottom tube $\left(\tilde{Q}_{3}>0\right)$ causes both $\tilde{T}_{2}$ and $\tilde{S}_{2}$ to increase, and since the temperature increase is faster, $\tilde{\rho}_{2}$ decreases. This in turn makes the density difference $\tilde{\rho}_{2}-\tilde{\rho}_{1}$ smaller, leading to an acceleration of the entrainment. The increased rate of entrainment results in an increase in $\tilde{S}_{1}$ and $\tilde{\rho}_{1}$, which leads to further decrease in the density difference and acceleration of the entrainment process. When $\tilde{w}_{e}$ becomes larger than $\tilde{Q}_{3}$ (at $\tilde{t}=142$ ), the upper layer starts to grow again. Then, it reaches the bottom $($ at $\tilde{t}=168)$ and the whole cycle repeats itself.

A self-sustained 'cyclic convection' is also possible (Fig. 5). We start our description of this oscillation from $\tilde{t}=4.3$, when the upper layer starts to grow. The upper layer temperature $\tilde{T}_{1}$ decreases rapidly due to the surface cooling, while the salinity $\tilde{S}_{1}$ increases slowly due to the entrainment. When the interface descends below the middle tube at $\tilde{t}=5$, the warm and salty water flows into the upper layer of the small basin $\left(\tilde{Q}_{2}>0\right)$, causing $\tilde{T}_{1}$ 

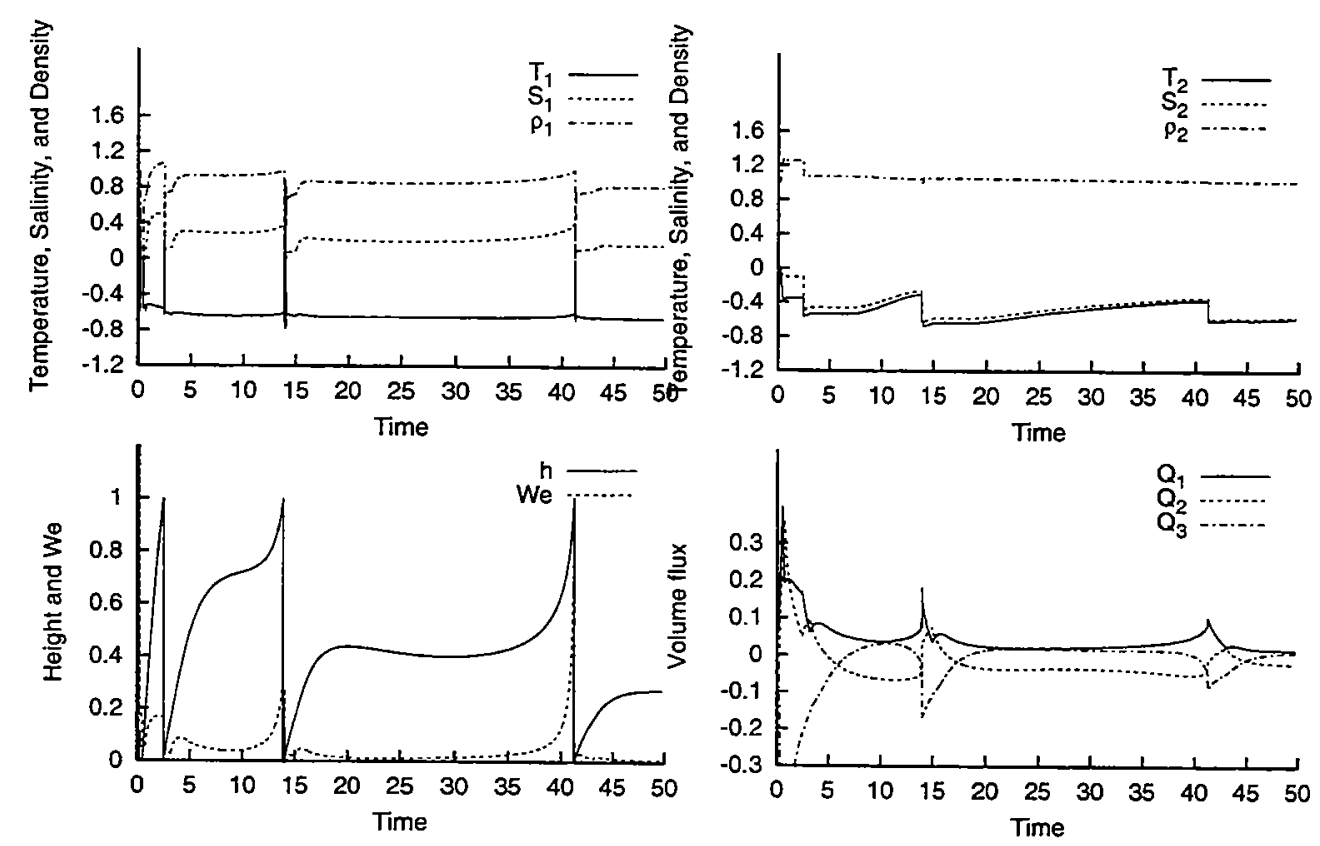

Figure 4: Time evolution of temperature, salinity, density, upper layer thickness, entrainment velocity, and flow rate at $\tilde{K}_{a}=0.5, \tilde{d}_{u}=0.03, \tilde{d}_{m}=0.25, \tilde{T}^{*}=-0.54$, and $\gamma=1.0$.

to initially increase and $\tilde{S}_{1}$ to increase steadily. At $\tilde{t}=7.8$, the interface reaches the bottom and we now have one layer state in the small basin. Although the necessary condition for the new layer formation is not satisfied in the beginning, the density of the whole layer and the flow rate $\tilde{Q}_{1}$ gradually increase. Finally, the necessary condition for stable layer initiation is satisfied and the newly formed layer starts to grow from $\tilde{t}=8.1$. The 'cyclic convection' is also seen in the three-tube model without entrainment and the two-tube mode (see Appendeces). However, the salinity plays no role in the 'cyclic convection' of the two-tube model.

Although it shows no oscillatory behavior, the equilibrium state with the interface in between the middle tube and the bottom tube shows very interesting features (Fig. 6), which cannot be obtained without the entrainment process. Until the interface reaches the middle tube (at $\tilde{t}=6$ ), the outflow at the middle tube and the bottom tube have the same magnitude. After the interface descends below the middle tube, the outflow through the middle tube accelerates, while the flow at the bottom tube reverses (at $\tilde{t}=8$ ). As the model approaches the equilibrium, the inflow through the bottom tube $\tilde{Q}_{3}$ and the entrainment velocity $\tilde{w}_{e}$ balance each other.

\section{Comparison with Laboratory Experiments}

The number of laboratory experiments is still limited, but we believe that it is worthwhile to make some comparison with the theoretical results obtained in the present study. The experimental set up is identical to the box model used in this study. The middle tube was placed at $\tilde{d_{m}}=0.5$, and the surface fresh water layer of thickness $\tilde{d_{u}}=0.05$ was maintained 

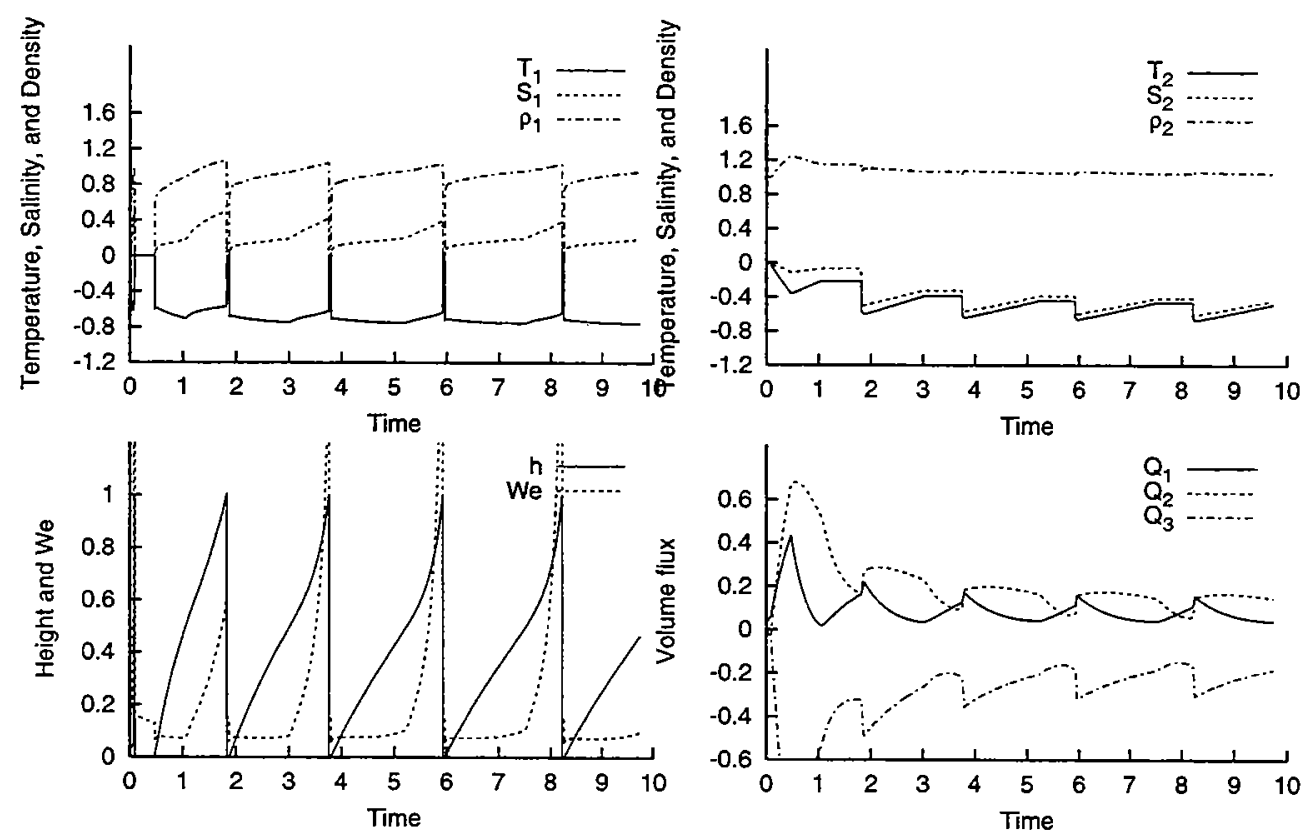

Figure 5: Time evolution of temperature, salinity, density, upper layer thickness, entrainment velocity, and flow rate at $\tilde{K}_{a}=0.5, \tilde{d}_{u}=0.03, \tilde{d}_{m}=0.3, \tilde{T}^{*}=-1.0$, and $\gamma=1.0$.
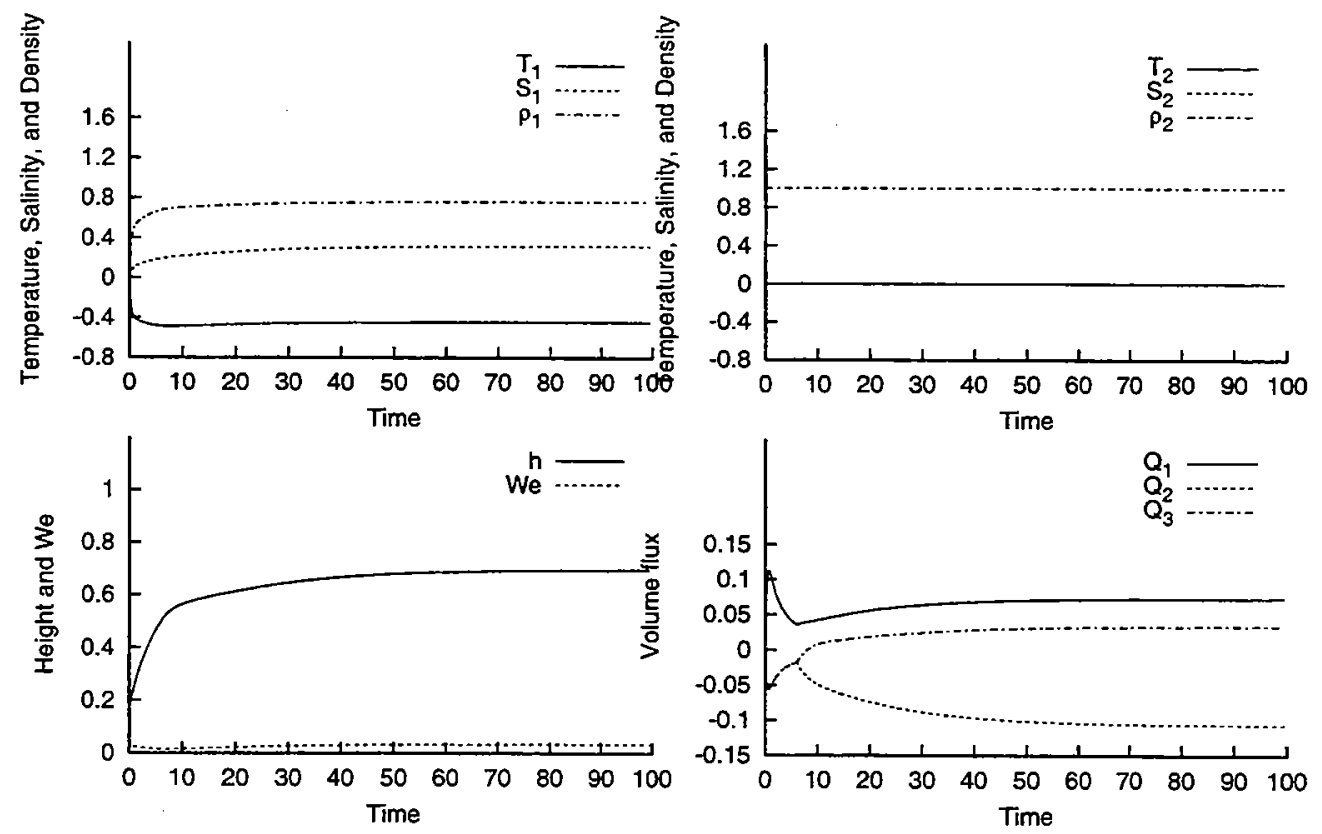

Figure 6: Time evolution of temperature, salinity, density, upper layer thickness, entrainment velocity, and flow rate at $\tilde{K}_{a}=0.5, \tilde{d}_{u}=0.18, \tilde{d}_{m}=0.5, \tilde{T}^{*}=-0.54$, and $\gamma=1.0$. 

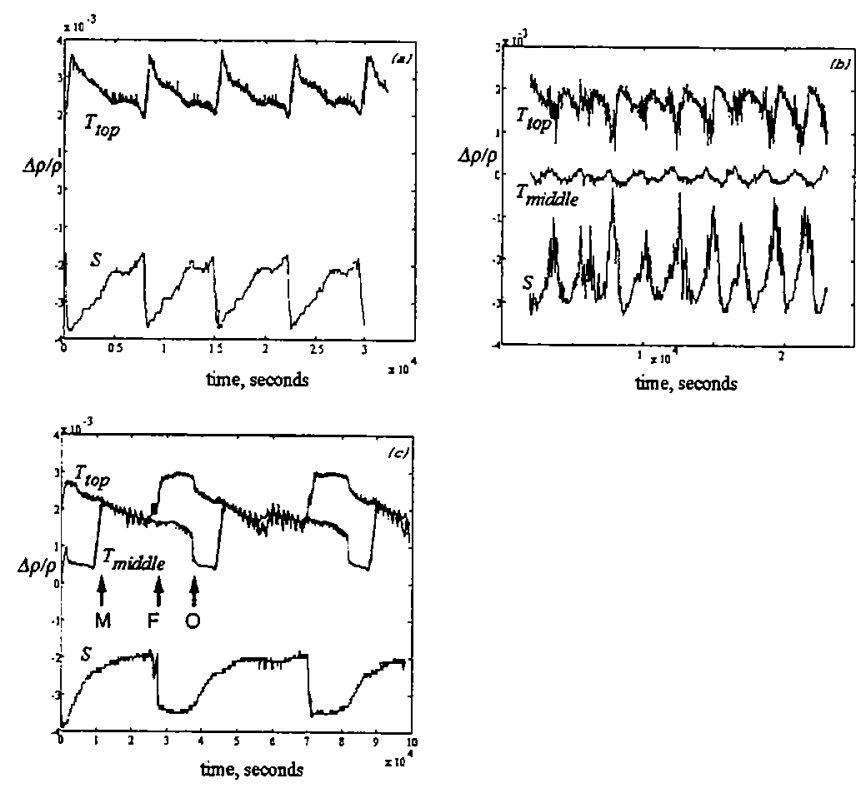

Figure 7: Time evolution of the temperature and salinity obtained in laboratory experiments with (a) $\tilde{T}^{*}=-22^{\circ} \mathrm{C}$, (b) $\tilde{T}^{*}=-30^{\circ} \mathrm{C}$, and $\tilde{T}^{*}=-18^{\circ} \mathrm{C}$ (provided by Whitehead).

in the large basin.

Figure 7 shows time evolution of the density contributions of temperature and salinity at different forcing temperature. All three experiments are similar to the behavior of 'cyclic convection'. For each cycle, the temperature at the top initially decreases due to the surface cooling. However, the temperature and salinity increase afterward due to the entrainment. When the density stratification becomes unstable, a convective overturning occurs and another cycle starts.

The experimental results further suggest that the period becomes shorter as the surface forcing is enhanced; the period is 40000 seconds for $\tilde{T}^{*}=-18^{\circ} \mathrm{C}, 7500$ seconds for $\tilde{T}^{*}=$ $-22^{\circ} \mathrm{C}$, and 2200 seconds for $\tilde{T}^{*}=-30^{\circ} \mathrm{C}$. This is qualitatively consistent with our box model result (Fig. 8). The period becomes shorter, because the inflow through the top tube is larger and the entrainment velocity is faster when the surface forcing is enhanced.

Although an upside-down version of the three-tube model was used (heating is at the bottom and a layer of salty water is maintained at the bottom of the large basin) for practical reasons, experiments in te Raa[5] contain the same physics. Hence, more comparison are made with the present theory. In her experiments, the middle tube was also placed at $d_{m}=0.5$, and the bottom salty water layer of thickness $d_{u}=0.033$ was maintained in the large basin. From experiments, it was determined that $\tilde{K}_{a}=1.2$ and $\gamma=0.004$.

Our box model successfully explains some of the unexplained phenomena in the experiment. First, the mechanism for the shift in flow regime as the surface forcing is strengthened was unknown. In order to clarify this transition, we made a regime diagram of $\tilde{T}^{*}$ and $\gamma$ (Fig. 9). We decided to vary $\gamma$ in the regime diagram since $\gamma$ seems to be the most uncertain value derived from the laboratory experiments. When $\gamma$ is about 0.15 , the present model successfully reproduces this shift at $\tilde{T}^{*}=-1.3$. The shift itself occurs, because it becomes 


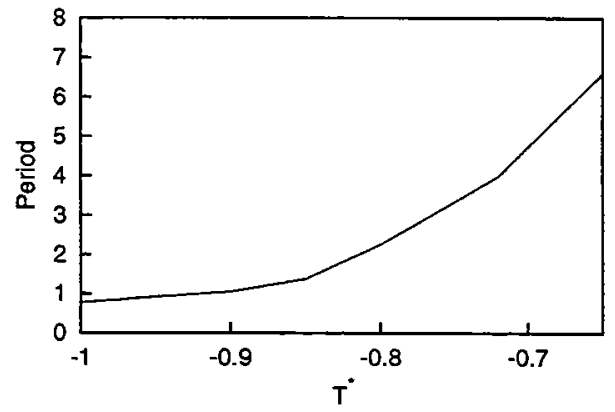

Figure 8: Period of 'cyclic convection' for $\tilde{K}_{a}=0.5$ and $\tilde{K}_{a}=1.0$, when $\tilde{d}_{u}=0.05$ and $\tilde{d_{m}}=0.5$.

(a) with entrainment

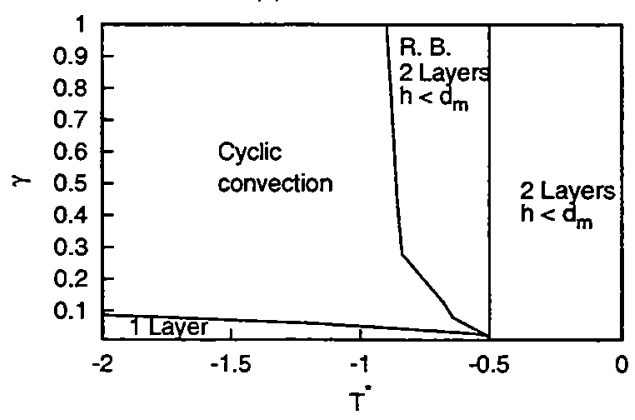

(b) without entrainment

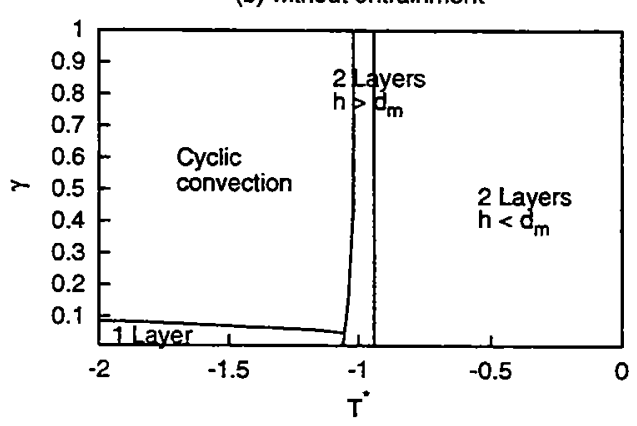

Figure 9: Regime diagrams for the three-tube model (a) with entrainment and (b) without entrainment at $\tilde{K}_{a}=1.2, \tilde{d}_{u}=0.033$, and $\tilde{d}_{m}=0.5$. 
more difficult to satisfy the necessary condition (70) as the surface forcing is enhanced. The volume flux $\tilde{Q}_{1}$ slowly increases with the strengthening forcing for small $\gamma$.

In addition, a reason for not being able to find the shallow convection state, or '2 Layers $h<d_{m}$ ' mode, is also clear from Fig. 9. The shallow convection state exists only when $\tilde{T}^{*}>-0.50$ in the model with entrainment, whereas in the laboratory experiments of te Raa[5], $\left|\tilde{T}^{*}\right|$ was set between 0.8 and 1.7. If the entrainment is excluded from the box model, the existence of the shallow convection state is predicted in this forcing temperature range. Therefore, this gives an additional evidence that the entrainment is a crutial process here.

However, the period of oscillation in laboratory experiments, especially when the cooling is weak, is much longer in the experiments than in the box model theory. Also, the value of $\gamma$ suggested from this study is much larger than the experimentally determined value of te Raa[5]. One possible reason for these inconsistencies is the linear flow relation we used in our box model; it may not correctly explain the flow through the three tubes in the laboratory experiments. Although the linear equation of state is used in this study, nonlinearity certainly becomes important as the temperature and salinity varies over a large range. Also, the double diffusive processes may play an important role, since the small basin is in the 'diffusive-layering' regime (cold and fresh water over warm and salty water).

\section{Conclusions}

We have found two distinct modes for the oscillatory behaviors of the simplified threetube box model. Two new processes are included in the box model in the present study comapared with the past studies[4],[5].

First, the necessary condition for a new layer formation has been found. It applies after the convective adjustment occurs or the interface reaches the bottom. This allows the model to have 'cyclic convection'. Then, the entrainment process is parameterized, where a fixed percentage of potential energy input by the surface cooling is used to entrain water from the lower layer. This introduces two new equilibrium states to the model. One is the equilibrium state with the interface located in between the middle tube and the bottom tube. This has inflows at the top and bottom tube and an outflow at the middle tube. The other is an 'oscillatory' mode where the upper layer thickness does not grow monotonically, but oscillates. This regime is a result of subtle interplay between entrainment, surface cooling, and flow through the three tubes.

The current result may represent some aspects of the thermohaline circulation in the real ocean. As suggested by Fig. 3, a thickening in the surface fresh water layer outside the deep convection site may shut down the deep water formation without changing the heat flux (for small $\gamma$ at about $-0.7<\tilde{T}^{*}<-0.5$ ). For thick fresh water layer, a ' 2 Layers $h>d_{m}$ mode with inflows at the top and bottom tube and an outflow at the middle tube exists. On the other hand, we have a ' 1 Layer' mode with inflows at the top and middle tube and an outflow at the bottom tube for thin fresh water layer. This feature is also simulated in the past coupled GCM studies[11]. When freshwater was released to the North Atlantic Ocean (between $50^{\circ} \mathrm{N}$ and $70^{\circ} \mathrm{N}$ ), the thermohaline circulation weakened and became shallower, allowing deep inflow of Antarctic bottom water.

Although the box model presented in this paper is very simple, this study suggests the 
important roles played by the freshwater layer above the halocline and salty water below it. Also, it was shown that it is important to take entrainment process into account even in simple box models. Future studies should shed light on the role played by the double diffusive process and nonlinearity in equation of state.

\section{Acknowledgements}

The author is very grateful to the supervisor, Jack Whitehead for his guidance of the whole summer project and his kindness in providing his laboratory experiment data. The author would like to express his sincere thanks to Claes Rooth for many fruitful discussions and valuable suggestions during the course of this study. Discussions with Joe Keller and Takamitsu Ito were both very helpful. Finally, I would like to thank all the fellows and GFD staffs for the great summer.

\section{References}

[1] H. Stommel, "On the smallness of sinking regions in the ocean," Proc. Nat. Aca. Sci. 48, 766 (1962).

[2] J. A. Whitehead, "Thermohaline ocean processes and models," Ann. Rev. Fluid Mech. 27, 89 (1995).

[3] Lab Sea Group, "The Labrador Sea deep convection experiment," Bull. Am. Met. Soc. 10, 2033 (1998).

[4] J. A. Whitehead, "Stratified convection with multiple states," Ocean Modelling 2, 109 (2000).

[5] L. te Raa, "Convective oscillations in a laboratory model," GFD Fellow Report 43, 237 (2001).

[6] R. Haney, "Surface thermal condition for ocean circulation models," J. Phys. Oceanogr. 1, 241 (1971).

[7] E. B. Kraus and J. S. Turner, "A one-dimensional model of the seasonal thermocline: II, The general theory and its consequences," Tellus 19, 98 (1967).

[8] R. E. Davis, R. deSzoeke, and P. Niiler, "Variability in the upper ocean during MILE. Part II: Modeling the mixed layer response," Deep-Sea Res. 28, 1453 (1981).

[9] H. Tennekes and J. L. Lumley, A first course in turbulence (M.I.T. Press, Cambridge, Massachusetts, 1972).

[10] J. W. Deardorff, G. E. Willis, and D. K. Lilly, "Laboratory investigation of non-steady penetrative convection," J. Fluid Mech. 35, 7 (1969).

[11] S. Manabe and R. J. Stouffer, "Simulation of abrupt climate change induced by freshwater input to the north atlantic ocean," Nature 378, 165 (1995). 


\section{Appendix A: Two-tube Model without Entrainment}

\section{Formulation}

In this Appendix, a two-tube model is constructed by removing the middle tube. By comparing reslts obtained here with results in section 2 , the importance of the middle tube will become more clear. Since we want to concentrate on the role played by the middle tube, the entraninment process is omitted from the two-tube model.

Using same assumptions, the volume fluxes are

$$
Q_{1}=-Q_{2}=-\frac{\gamma C \rho_{o} g D}{\gamma+1}\left[\left(\beta\left(S_{o}+S_{2}-S_{1}\right)+\alpha\left(T_{1}-T_{2}\right)\right) \frac{h}{D}-\beta S_{o} \frac{d_{u}}{D}+\left(\alpha T_{2}-\beta S_{2}\right)\right]
$$

the upper layer mass conservation equation is

$$
A \frac{d h}{d t}=Q_{1}
$$

and the heat and salt balance equations are

$$
\begin{gathered}
A h \frac{d T_{1}}{d t}=\frac{K_{a}}{\rho_{o} c_{p}}\left(T^{*}-T_{1}\right)-T_{1} Q_{1} \Gamma\left(+Q_{1}\right) \\
A h \frac{d S_{1}}{d t}=-S_{1} Q_{1} \Gamma\left(+Q_{1}\right) \\
A(D-h) \frac{d T_{2}}{d t}=-T_{2} Q_{2} \Gamma\left(+Q_{2}\right) \\
A(D-h) \frac{d S_{2}}{d t}=-S_{2} Q_{2} \Gamma\left(+Q_{2}\right) .
\end{gathered}
$$

Using the following transformations

$$
\tilde{Q}_{i}=\frac{Q_{i}}{Q_{s}}, \tilde{T}_{i}=\frac{\alpha T_{i}}{\beta S_{o}}, \tilde{d}_{u}=\frac{d_{u}}{D}, \tilde{S}_{i}=\frac{S_{i}}{S_{o}}, \tilde{T}^{*}=\frac{\alpha T^{*}}{\beta S_{\circ}}, \tilde{t}=\frac{A D}{Q_{s}} t, \tilde{K}_{a}=\frac{K_{a}}{\rho_{o} c_{p} Q_{s}}
$$

where

$$
Q_{s}=\frac{\gamma C \rho_{o} g \beta S_{o} D}{\gamma+1}
$$

the nondimensionalized equations are

$$
\tilde{Q}_{1}=-\tilde{Q_{2}}=-\left[\left(1+\tilde{S_{2}}-\tilde{S_{1}}+\tilde{T}_{1}-\tilde{T_{2}}\right) \tilde{h}-\tilde{d}_{u}+\left(\tilde{T}_{2}-\tilde{S_{2}}\right)\right]
$$




$$
\begin{gathered}
\frac{d \tilde{h}}{d \tilde{t}}=\tilde{Q}_{1} \\
\tilde{h} \frac{d \tilde{T}_{1}}{d \tilde{t}}=\tilde{K}_{a}\left(\tilde{T}^{*}-\tilde{T}_{1}\right)-\tilde{T}_{1} \tilde{Q}_{1} \Gamma\left(+\tilde{Q}_{1}\right) \\
\tilde{h} \frac{d \tilde{S}_{1}}{d \tilde{t}}=-\tilde{S}_{1} \tilde{Q}_{1} \Gamma\left(+\tilde{Q}_{1}\right) \\
(1-\tilde{h}) \frac{d \tilde{T}_{2}}{d \tilde{t}}=-\tilde{T}_{2} \tilde{Q}_{2} \Gamma\left(+\tilde{Q}_{2}\right) \\
(1-\tilde{h}) \frac{d \tilde{S}_{2}}{d \tilde{t}}=-\tilde{S}_{2} \tilde{Q}_{2} \Gamma\left(+\tilde{Q}_{2}\right) .
\end{gathered}
$$

Thus, we have three dimensionless parameters for this simple model.

The necessary condition for a new layer formation is

$$
\tilde{Q}_{1}>-\frac{\tilde{K}_{a} \tilde{T}^{*}}{1+\left(\tilde{S}_{2}-\tilde{T}_{2}\right)} .
$$

However, there is an upper bound for the volume flux $\tilde{Q}_{1}$, which depends on the value of $\tilde{T}^{*}$ and $\tilde{d}_{u}$ :

$$
\tilde{Q}_{1}<\tilde{d}_{u}-\tilde{T}^{*}
$$

or

$$
\tilde{Q}_{1}<\tilde{d}_{u}-\tilde{T}^{*}-1
$$

in case the small basin loses all of its salinity. This is possible when the upper layer reaches the bottom or after an infinite number of convective adjustments takes place. Hence, the new layer cannot form when

$$
\tilde{d}_{u}-\tilde{T}^{*}<-\frac{\tilde{K}_{a} \tilde{T}^{*}}{1-\tilde{T}^{*}+\tilde{S}_{2}}
$$

or

$$
\tilde{d}_{u}-\tilde{T}^{*}-1<\tilde{K}_{a}
$$

for no salinity. 


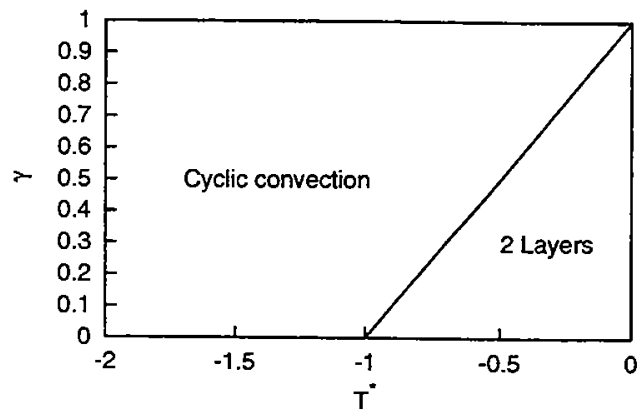

Figure 10: Regime diagrams for the the two-tube model with $\tilde{K}_{a}=0.5$.

\section{Steady-state Solutions}

Equilibrium states of the model is discussed for progressively stronger cooling. When the cooling is weak, the model reaches equilibrium before the interface reaches the bottom. The upper layer temperature $\tilde{T}_{1}$ is quickly cooled and becomes $\tilde{T}^{*}$. The volume flux $Q_{1}$ and $Q_{2}$ become zero and the equilibrium depth of the upper layer is

$$
\tilde{h}_{e q}=\frac{\tilde{d_{u}}}{\tilde{T}^{*}+1}
$$

Since there is no interfacial mixing in this model, we have assumed that $\tilde{T}_{2}=\tilde{S}_{2}=\tilde{S}_{1}=0$.

In order to reach equilibrium before reaching the bottom $\left(\tilde{h}_{e q}<1\right)$,

$$
\tilde{T^{*}}{ }_{c}+1>\tilde{d_{u}}
$$

For the forcing temperature below $\tilde{T}^{*}{ }_{c}$, the interface reaches the bottom before reaching the equilibrium state. However, if the cooling is not strong enough to strengthen the flow to satisfy necessary condition, it is not possible to form a stable new layer. Now, we obtain a new equilibrium state with one fresh layer of temperature $\tilde{T}^{*}$, and the volume flux as predicted from Eq.(87). Finally, a third 'cyclic convection' regime can exist as the forcing temperature is decreased further. The above mentioned three regimes in the two-tube model can be summarized by Fig. 10 .

\section{Appendix B: Three-tube Model without Entrainment}

Equilibrium states with different cooling temperature are investigated using different values of middle-tube depth $\tilde{d}_{m}$ (Fig.11). Equilibrium states have no $\gamma$-dependence at forcing temperature $\tilde{T}^{*}$ below -1.06 . At weak cooling $\left(\tilde{T}^{*}<-0.72\right)$, the model has an equilibrium state with the interface above the middle tube, but when the cooling is enhanced, the model reaches an equilibrium state with the interface between the middle tube and the bottom tube. For small $\gamma$, an equilibrium state with 1 layer emerges, when cooling is further 

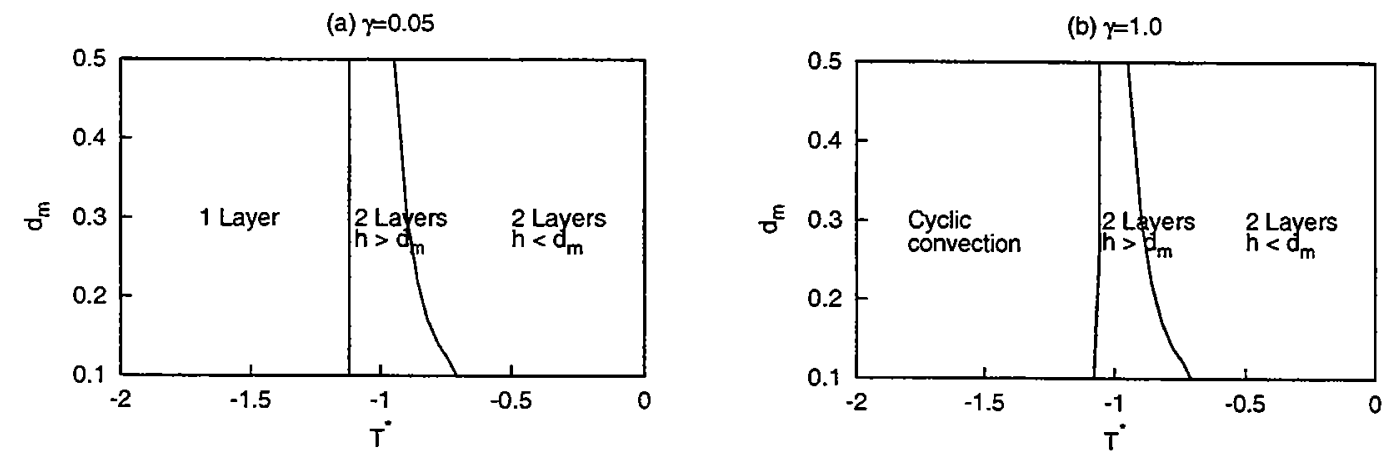

Figure 11: Regime diagrams for the three-tube model without entrainment at $\tilde{K}_{a}=0.5$ and $\tilde{d}_{u}=0.03$ for (a) $\gamma=0.05$ and (b) $\gamma=1.0$.
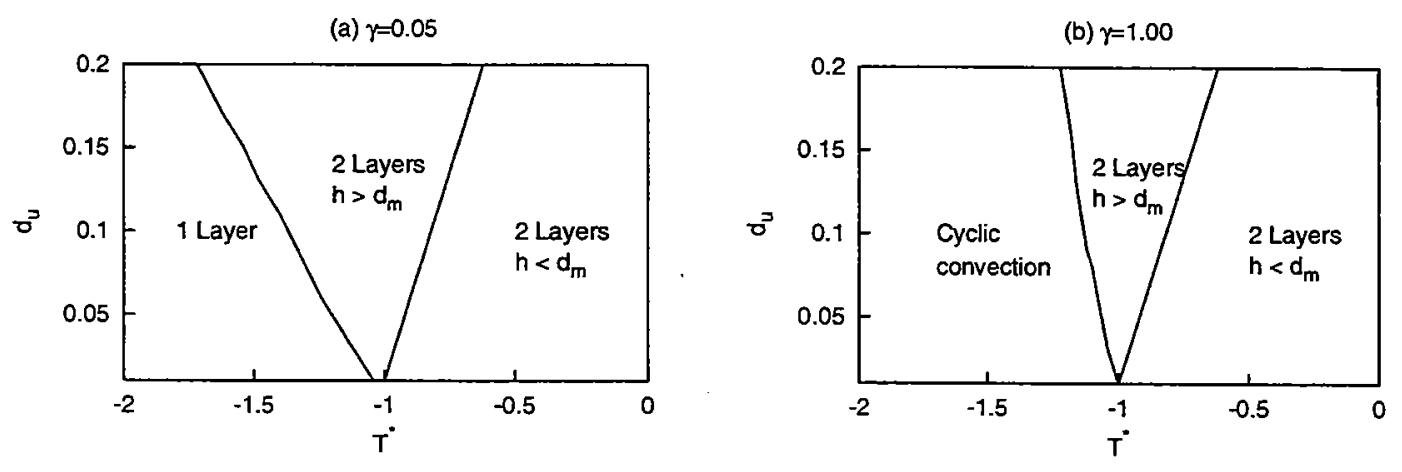

Figure 12: Regime diagrams for the three-tube model without entrainment at $\tilde{K}_{a}=0.5$ and $\tilde{d}_{m}=0.5$ for (a) $\gamma=0.05$ and (b) $\gamma=1.0$.

enhanced. On the other hand, 'cyclic convection' regime appears for large $\gamma$; with larger $\gamma$ values, the volume flux at the top tube becomes larger, so that it is easier to satisfy the necessary condition (70).

Similarly, three-tube model is sensitive to the fresh water layer thickness of the large basin (Fig. 12). Compared with shallow $\tilde{d}_{u}$, the interface reaches the middle tube at warmer $\tilde{T}^{*}$, but the equilibrium states of 'cyclic convection' or '1 Layer' emerges at colder $\tilde{T}^{*}$ for deeper $\tilde{d_{u}}$. 


\title{
Bound on the Heat Transport through a Layer from the Boundary Layer Theory Perspective: Fixed Heat Flux
}

\author{
Evstati G. Evstatiev \\ University of Texas, Austin
}

\section{Introduction}

Bounds on the heat transport in the Rayleigh-Bénard convection problem is a fundamental problem. The first mathematical formulation of the problem was given in the pioneer paper by Howard[1]. The problem was formulated as an optimization problem. The motivation was that nature might "choose" to realize a process that maximizes the heat transport when the flow is turbulent. This is only a hypothesis, but in certain cases the features of the solution to the so formulated problem has qualitative agreement with real physical flows. In any case, the solution of the maximization problem gives a bound on the quantity of transport of heat flux.

The problem of thermal convection can be realized in different experimental settings. In some, the boundaries can have infinitely bigger thermal conductivity that the fluid in the layer. This is the so called "fixed temperature" problem. In other experimental settings we might have the opposite case: The fluid's thermal conductivity might be much larger than the thermal conductivity of the boundaries; the latter is "fixed heat flux" problem. The physical basis for this nomination is that in the former case the fluid on the boundary has the temperature of the boundary, whereas in the latter, this is not required. Instead, the heat flux through the boundary, which is proportional to the gradient of the temperature on the boundary, is fixed. In this study we are concerned with the fixed heat flux problem.

Despite the different boundary conditions arising from different experimental settings, the physics behind both phenomena is similar. When the temperature difference between the upper and lower plates (or analogously, the temperature gradient) is small, the fluid is in a pure conductive state so that the velocity throughout the layer is zero. As we start increasing the temperature difference (or the heat flux), the system becomes unstable and the fluid starts to move. There is a critical parameter that describes when this first happens-a control parameter. This is the Rayleigh number. As we keep increasing the Rayleigh number, the fluid sets into turbulent motion.

In turbulent regime it is believed that quantities reach asymptotic behavior and have certain scaling determined by the Rayleigh number. For example, the quantity that describes how much bigger the heat flux in a turbulent regime is, compared to that in a pure conductive state, is the Nusselt number $N u$. In the fixed temperature problem the scaling derived in the paper by Howard[1] - maximizing over fields with one horizontal wavenumber-is $N u \sim R a^{\frac{3}{8}}$, while the scaling derived by Busse[5]-maximizing over multiple horizontal wavenumbers-is $N u \sim R a^{\frac{1}{2}}$. In a recent paper by Otero et al. [3], using a different method, it is shown that the scaling for the fixed heat flux problem is $N u \sim R a^{\frac{1}{2}}$. However, for a single horizontal wave number C. Doering and J. Otero[4], following a method by Howard[2], have derived an estimate that leads to a scaling $N u \sim R a^{\frac{5}{12}}$. We will try to follow the Howard-Busse approach and derive a scaling for the Nusselt number in the fixed heat flux problem, using a single wave number approximation. We will comment on 
applying the multi-alpha approach developed by Busse[5].

\section{Formulation of the Problem}

We describe the physical setup in this section and then formulate the problem. Consider a fluid between two horizontal infinite plates. The lower plate is heated so the fluid at the bottom is hotter than the fluid at the top. There are two opposing forces that act on the fluid-the buoyancy force and the gravity. The equations that describe this convection problem are the Boussinesq equations

$$
\begin{gathered}
\frac{\partial \mathbf{u}}{\partial t}+\mathbf{u} \cdot \nabla \mathbf{u}+1 \rho \nabla p-\alpha g T \hat{\mathbf{k}}=\nu \nabla^{2} \mathbf{u} \\
\nabla \cdot \mathbf{u}=0 \\
\frac{\partial T^{*}}{\partial t}+\mathbf{u} \cdot \nabla T^{*}=\kappa \nabla^{2} T^{*}
\end{gathered}
$$

The meaning of the quantities in the above equations is the following. $\mathbf{u}=(u, v, w)$ is the velocity vector, $p$ is the deviation of the pressure from the hydrostatic pressure, corresponding to the horizontal average of the temperature, $\alpha$ is the coefficient of thermal expansion, $g$ is the acceleration of gravity, $T^{*}$ is the temperature, $\kappa$ is the thermal conductivity of the fluid. The fluid occupies the space in the direction of $z$ from 0 to $d$. The boundary conditions are

$$
\mathbf{u}(0)=\mathbf{u}(d)=0,\left.\quad \kappa \frac{\partial \overline{T^{*}}}{\partial z}\right|_{0}=\left.\kappa \frac{\partial \overline{T^{*}}}{\partial z}\right|_{d}=-\kappa \beta=\text { const. }
$$

We split the temperature in a horizontal averaged part $\overline{T^{*}}$, and a deviating part $T$, so that

$$
T^{*}=\overline{T^{*}}+T
$$

Our notation is: An over-bar denotes horizontal average, and angle brackets-volume average. Then we can write

$$
\langle w T\rangle=\frac{1}{d} \int_{0}^{d} \overline{w T} d z
$$

If we multiply Eq. (1) by $\mathbf{u}$ and average over the volume, we get 


$$
\alpha g\langle w T\rangle=\nu\left\langle|\nabla \mathbf{u}|^{2}\right\rangle
$$

This equation expresses the balance between the rate of generation of energy motion in the field of the buoyancy force $\alpha g T \mathbf{k}$, and the rate of dissipation of energy by viscosity. If we average Eq. (3) horizontally, using the boundary conditions for $\mathbf{u}$ and $T^{*}$, we obtain

$$
\frac{d \overline{w T^{*}}}{d z}=\kappa \frac{d^{2} \overline{T^{*}}}{d z^{2}}
$$

Since $\bar{w}=0, \overline{w T^{*}}=\overline{w T}$. From Eq. (8) we see that

$$
\frac{d}{d z}\left(\overline{w T}-\kappa \frac{d \overline{T^{*}}}{d z}\right)=0
$$

and therefore the sum in the braces in Eq. (9) is constant equal to the its average over the volume

$$
\begin{aligned}
\overline{w T}-\kappa \frac{d \overline{T^{*}}}{d z} & =\langle w T\rangle-\kappa \frac{1}{d} \int_{0}^{d} \kappa \frac{d \overline{T^{*}}}{d z} \\
& =\langle w T\rangle+\kappa \frac{\Delta T}{d}
\end{aligned}
$$

where by definition

$$
-\Delta T=\overline{T^{*}}(d)-\overline{T^{*}}(0)
$$

Finally from (10) we obtain

$$
-\kappa \frac{d \overline{T^{*}}}{d z}=\kappa \frac{\Delta T}{d}+\langle w T\rangle-\overline{w T}
$$

Multiplying Eq. (3) by $T$, averaging and using (12) we obtain

$$
\kappa^{-1}\left[\langle w T\rangle^{2}-\left\langle\overline{w T}^{2}\right\rangle\right]+\frac{\Delta T}{d}\langle w T\rangle=\kappa\left\langle|\nabla T|^{2}\right\rangle .
$$

Putting (7) and (13) into a dimensionless form with $d$ as a length scale, $\kappa / d$ as velocity scale, and $\beta d$ as a temperature scale have the two "power integrals" (called so in the paper by Howard[1])

$$
R\langle w T\rangle=\left\langle|\nabla \mathbf{v}|^{2}\right\rangle
$$




$$
\Delta T\langle w T\rangle+\langle w T\rangle^{2}-\langle\overline{w T}\rangle=\left\langle|\nabla T|^{2}\right\rangle,
$$

where $R=\alpha g \beta d^{4} / \kappa \nu$ is the Rayleigh number based on the given constant heat flux at the boundary. Its relation to the Rayleigh number based on the temperature difference between the plates is

$$
R a=R \Delta T
$$

Define the Nusselt number as the ratio of the total heat flux and the conductive heat flux through the layer. The total heat flux is given by $-\kappa \beta$, whereas the conductive heat flux by $-\kappa \Delta T$. In dimensionless form we have

$$
N u=\frac{1}{\Delta T}
$$

where now $\Delta T$ is dimensionless temperature difference. The problem we will try to solve is to find a bound on the Nusslet number (17) i.e., we will try to find a relation between $N u$ and the Rayleigh number $R$ (or, $R a$.) of the form $N u \sim R^{p}$ for some $p$. We do this in the following sections.

\section{Bounding as a Minimization Problem}

Multiplying Eq.(8) by $z$, integrating by parts and using the boundary conditions for $T$ we obtain for the left-hand and the right-hand sides

$$
\begin{aligned}
& \kappa \int_{0}^{d} z \frac{d^{2} \overline{T^{*}}}{d z^{2}} d z=-\kappa d \beta+\kappa \Delta T \\
& \int_{0}^{d} z \frac{d \overline{w T^{*}}}{d z} d z=-d\langle w T\rangle
\end{aligned}
$$

and after putting those in a dimensionless form we have

$$
\Delta T=1-\langle w T\rangle
$$

Using (18), we rewrite the power integrals (14) and (15) in the form

$$
\begin{gathered}
R\langle w T\rangle=\left\langle|\nabla \mathbf{v}|^{2}\right\rangle, \\
\langle w T\rangle-\left\langle\overline{w T}^{2}\right\rangle=\left\langle|\nabla T|^{2}\right\rangle .
\end{gathered}
$$


Using (19) in the right-hand side of $(21)$ by $\langle w T\rangle$ and regrouping we get

$$
\langle w T\rangle=\frac{\langle w T\rangle^{2}-\frac{1}{R}\left\langle|\nabla \mathbf{v}|^{2}\right\rangle\left\langle|\nabla T|^{2}\right\rangle}{\left\langle\overline{w T}^{2}\right\rangle}
$$

Substituting (21) into (17) and using (18) we can write

$$
\frac{1}{N}=\frac{\left\langle\overline{w T}^{2}\right\rangle-\langle w T\rangle^{2}+\frac{1}{R}\left\langle|\nabla \mathbf{u}|^{2}\right\rangle\left\langle|\nabla T|^{2}\right\rangle}{\left\langle\overline{w T}^{2}\right\rangle}
$$

Maximizing the Nusselt number is equivalent to minimizing (22). The maximal $N u$ Will provide a bound on the total heat transport throughout the layer of fluid. Therefore, we will look for a minimum of the functional

$$
\mathcal{F}[\mathbf{v}, T]=\frac{\left\langle\overline{w T}^{2}\right\rangle-\langle w T\rangle^{2}+\lambda\left\langle|\nabla \mathbf{u}|^{2}\right\rangle\left\langle|\nabla T|^{2}\right\rangle}{\left\langle\overline{w T}^{2}\right\rangle}
$$

where $\lambda=1 / R$.

The so derived functional is to be minimized among functions that satisfy the boundary conditions

$$
\mathbf{v}(0)=\mathbf{v}(1)=d T /\left.d z\right|_{z=0}=d T /\left.d z\right|_{z=1}=0,
$$

the continuity equation

$$
\nabla \cdot \mathbf{v}=0
$$

and the power integrals (19) and (20).

We continue the analysis in the following section by making a certain simplification. We will assume a single wave number horizontal dependence of the test functions.

\section{Bound with a Single Wave Number}

We assume the following form of the functions $w$ and $T$

$$
\begin{aligned}
& w(x, y, z)=\omega(z) \phi(x, y) \\
& T(x, y, z)=\theta(z) \phi(x, y)
\end{aligned}
$$

and the function $\phi(x, y)$ having the properties 


$$
\left(\frac{\partial^{2}}{\partial x^{2}}+\frac{\partial^{2}}{\partial y^{2}}\right) \phi(x, y)=-a^{2} \phi(x, y), \quad \overline{\phi^{2}}=1
$$

Now we can write

$$
\left\langle|\nabla T|^{2}\right\rangle=\left\langle\theta^{\prime 2}+a^{2} \theta^{2}\right\rangle
$$

We note that the continuity equation alone is not enough to determine the relation between $\omega$ and $\left\langle|\nabla \mathbf{v}|^{2}\right\rangle$, but if only the minimum of the latter is requested, we can write

$$
\left\langle|\nabla \mathbf{v}|^{2}\right\rangle=\left\langle a^{-2} \omega^{\prime \prime 2}+2 \omega^{\prime 2}+a^{2} \omega^{2}\right\rangle
$$

With (27) and (28) we can express the functional (23) in terms of only $\omega$ and $\theta$ only

$$
\mathcal{F}[\omega, \theta]=\frac{\left\langle\omega^{2} \theta^{2}\right\rangle-\langle\omega \theta\rangle^{2}+\lambda\left\langle\theta^{\prime 2}+a^{2} \theta^{2}\right\rangle\left\langle a^{-2} \omega^{\prime \prime 2}+2 \omega^{2}+a^{2} \omega^{2}\right\rangle}{\left\langle\omega^{2} \theta^{2}\right\rangle}
$$

The boundary conditions for the functions $\omega$ and $\theta$ are

$$
\omega=\omega^{\prime}=\theta^{\prime}=0 \quad \text { at } \quad z=0,1 .
$$

Since the functional (29) is homogeneous of degree zero in $\omega$ and $\theta$, we can choose the amplitudes of the test functions so that they satisfy two conditions

$$
\begin{aligned}
& \langle\omega \theta\rangle=1 \\
& \left\langle\omega^{2}\right\rangle=\left\langle\theta^{2}\right\rangle .
\end{aligned}
$$

The Euler-Lagrange equations following from the functional (29) are

$$
\begin{gathered}
E q 1 \omega \theta^{2}(1-\mathcal{F})-\langle\omega \theta\rangle \theta+\lambda\left\langle\theta^{\prime 2}+a^{2} \theta^{2}\right\rangle\left[a^{-2} \omega^{i v}+2 \omega^{\prime \prime}+a^{2} \omega\right]=0 \\
E q 2 \omega^{2} \theta(1-\mathcal{F})-\langle\omega \theta\rangle \omega+\lambda\left[-\theta^{\prime \prime}+a^{2} \theta\right]\left\langle a^{-2} \omega^{\prime \prime 2}+2 \omega^{\prime 2}+a^{2} \omega^{2}\right\rangle=0
\end{gathered}
$$

Since these equations are difficult to solve analytically, we resort to numerical methods to solve them. The results are given in the next section.

In the rest of this section we apply the boundary layer approximation. It consists of the following. The form of the functional (29) suggests that the minimizing functions should be nearly constant throughout a large portion of the interval $[0,1]$. To satisfy the boundary conditions, $\omega$ will need to drop to zero together with its derivative, and so will the derivative of $\theta$. Therefore we expect that there will be a narrow interval around the two boundaries where the derivatives of the functions will have large values-boundary layers. The values 
of the functions will change fast within these boundary layers and the thickness of the latter will determine the magnitude of their growth. The contributions to the integrals are expected to come predominantly from the boundary layers. Therefore, we will derive equations that describe the functions $\omega, \theta$ only in the boundary layer-the boundary layer equations-and will approximate the full interval of integration by integration over the thin boundary layers (there are two of them.)

Note that the first two terms in (29) are of order one, see (31). To minimize the functional and comply with (31), both of them will approach the value of 1 so that their difference approaches zero. The two tendencies-the terms with the derivatives approaching zero and the difference between the first two terms approaching zero-must occur simultaneously and have the same order of magnitude. In mathematical form, the above reasoning can be formulated as follows. Assume the following scaling of the boundary layer thickness (as a small parameter we choose $\lambda=1 / R$ )

$$
\omega=\lambda^{p} \omega_{1}, \quad \theta=\lambda^{-p} \theta_{1}, \quad z=\lambda^{r} \zeta, \quad a^{2}=\lambda^{-q} b^{2},
$$

where the functions $\omega_{1}, \theta_{1}$ are of order one inside the boundary layers. From the argument above

$$
r e l\left\langle\omega^{2} \theta^{2}\right\rangle \rightarrow 1 \quad \text { as } \lambda \rightarrow 0
$$

Because of the relation $\left\langle\omega^{2} \theta^{2}\right\rangle=\left\langle(1+(1-\omega \theta))^{2}\right\rangle$ we see that as $\omega \theta \rightarrow 1$ we must have (35). Substitution of (34) into (29), and taking into account (35), we obtain

$$
\begin{aligned}
F F \mathcal{F}= & 2 \lambda^{r} \int_{0}^{\infty}\left(1-\omega_{1} \theta_{1}\right)^{2} d \zeta+\lambda\left[2 \lambda^{-2 p-r} \int_{0}^{\infty}\left(\frac{d \theta_{1}}{d \zeta}\right)^{2} d \zeta+b^{2} \lambda^{-q}\right] \\
& \times\left[2 b^{-2} \lambda^{q+2 p-3 r} \int_{0}^{\infty}\left(\frac{d^{2} \omega_{1}}{d \zeta^{2}}\right)^{2} d \zeta+4 \lambda^{2 p-r} \int_{0}^{\infty}\left(\frac{d \omega_{1}}{d \zeta}\right)^{2} d \zeta+b^{2} \lambda^{-q}\right]
\end{aligned}
$$

After expansion of the terms in the square brackets we obtain the following exponents

$$
r, 1+q-4 r, 1-2 r, 1-2 p-r-q, 1+2 p-3 r, 1-q+2 p-r, 1-2 q .
$$

We need to maximize the minimal possible exponent among (37). Let $e$ be the minimal of all exponents. Of all 7 inequalities, consider

$$
\begin{aligned}
r & \geq e \\
1+q-4 r & \geq e \\
1-2 q & \geq e .
\end{aligned}
$$

Multiplying (38) by $8,(39)$ by 2 , and adding to (40) we get $3 \geq 11$ from which we deduce that $e=3 / 11$. By adding 4 times (38) to (39), and using (40) we get $4 / 11=5 e-1 \leq$ $q \leq 1 / 2(1-e)=4 / 11$, so that $q=4 / 11$. Similarly, from (38) and (39) we get $3 / 11=e \leq$ $r \leq 1 / 4(1+q-e)=3 / 11$, and thus $q=3 / 11$. From the fourth and fifth of $(37)$ we find $1 / 11=e-1+3 r \leq 2 p \leq 1-r-q-e=1 / 11$ which shows that $p=1 / 22$. For these values of $p, q, r$ all exponents in (37) take the same maximal value of $3 / 11$ except the third 
and sixth, which become $5 / 11$ (we neglect those.) Hence the maximum value is uniquely determined. If we set $\mathcal{F}=\lambda^{\frac{3}{11}} \mathcal{F}_{1}$, we obtain within the boundary layer approximation

$$
\mathcal{F}_{1}=2 \int_{0}^{\infty}\left(1-\omega_{1} \theta_{1}\right)^{2} d \zeta+\left[2 \int_{0}^{\infty}\left(\frac{d \theta_{1}}{d \zeta}\right)^{2} d \zeta+b^{2}\right]\left[2 b^{-2} \int_{0}^{\infty}\left(\frac{d^{2} \omega_{1}}{d \zeta^{2}}\right)^{2} d \zeta+b^{2}\right] .
$$

We need to minimize $\mathcal{F}_{1}$ among functions $\omega_{1}$ and $\theta_{1}$ that satisfy the boundary conditions

$$
\begin{gathered}
\omega_{1}(0)=\omega^{\prime}(0)=\theta^{\prime}(0)=0, \\
\theta_{1} \rightarrow 0, \quad \omega_{1} \theta_{1} \rightarrow \quad \text { as } \zeta \rightarrow \infty .
\end{gathered}
$$

Varying $\mathcal{F}_{1}$ with respect to $\omega_{1}, \theta_{1}$, and $b^{2}$ we find the following equations

$$
\begin{aligned}
& b^{-2}\left[2 \int_{0}^{\infty} \theta_{1}^{2} d \zeta+b^{2}\right] \frac{d^{4} \omega_{1}}{d \zeta^{4}}-\left(1-\omega_{1} \theta_{1}\right) \theta_{1}=0, \\
& {\left[2 b^{-2} \int_{0}^{\infty} \omega^{\prime \prime 2} d \zeta+b^{2}\right] \frac{d^{2} \theta_{1}}{d \zeta^{2}}+\left(1-\omega_{1} \theta_{1}\right) \omega_{1}=0,} \\
& \frac{2}{b^{2}} \int_{0}^{\infty} \omega^{\prime \prime 2} d \zeta+b^{2}+\left[2 \int_{0}^{\infty} \theta_{1}^{\prime 2} d \zeta+b^{2}\right]\left[-2 b^{-4} \int_{0}^{\infty} \omega^{\prime \prime 2} d \zeta+1\right]=0 .
\end{aligned}
$$

From (42) and (43) we obtain

$$
\begin{aligned}
b^{-2}\left[2 \int_{0}^{\infty} \theta_{1}^{\prime 2} d \zeta+b^{2}\right] \int_{0}^{\infty} \omega^{\prime \prime 2} d \zeta & =\int_{0}^{\infty}\left(1-\omega_{1} \theta_{1}\right) \omega_{1} \theta_{1} d \zeta= \\
& =\left[2 b^{-2} \int_{0}^{\infty} \omega^{\prime 2} d \zeta+b^{2}\right] \int_{0}^{\infty} \theta_{1}^{\prime 2} d \zeta
\end{aligned}
$$

and therefore

$$
\int_{0}^{\infty} \omega^{\prime \prime 2} d \zeta=b^{2} \int_{0}^{\infty} \theta_{1}^{2} d \zeta \equiv b^{2} \mu
$$

a which defines $\mu$. Substituting these into (44) we find

$$
2 \mu+b^{2}+\left(2 \mu+b^{2}\right)\left(-2 \mu b^{-2}+1\right) \equiv 2\left(2 \mu+b^{2}\right)\left(1-\mu b^{-2}\right)=0
$$

which shows that $\mu=b^{2}$. Using this in (42) and (43) we find

$$
\begin{gathered}
3\left(d^{4} \omega_{1} / d \zeta^{4}\right)-\left(1-\omega_{1} \theta_{1}\right) \theta_{1}=0 \\
3 b^{2}\left(d^{2} \theta_{1} / d \zeta^{2}\right)+\left(1-\omega_{1} \theta_{1}\right) \omega_{1}=0
\end{gathered}
$$

Setting

$$
\omega_{1}=\left(3 b^{4}\right)^{\frac{1}{6}} \Omega, \quad \theta_{1}=\left(3 b^{4}\right)^{-\frac{1}{6} \Theta}, \quad \zeta=(3 b)^{\frac{1}{3}} \xi,
$$

equations(46) and (47) become

$$
\begin{aligned}
& d^{4} \Omega / d \xi^{4}-(1-\Omega \Theta) \Theta=0 \\
& d^{2} \Theta / d \xi^{2}+(1-\Omega \Theta) \Omega=0
\end{aligned}
$$


These equations have boundary conditions $\Omega(0)=\Omega^{\prime}(0)=\Theta^{\prime}(0)=0, \Theta \rightarrow 0$ and $\Omega \Theta \rightarrow 1$ as $\xi \rightarrow \infty$. The functions $\Omega$ and $\Theta$ can be determined independently of knowing $b$. Once we know the solutions of (49), (50), we can determine $b$

$$
\text { relation } \quad b^{2}=\mu=\int_{0}^{\infty} \theta_{1}^{\prime 2} d \zeta=\left(3 b^{4}\right)^{-\frac{1}{3}}(3 b)^{-\frac{1}{3}} \int_{0}^{\infty}\left(\frac{d \Theta}{d \xi}\right)^{2} d \xi
$$

from which

$$
b^{\frac{11}{3}}=3^{-\frac{2}{3}} \int_{0}^{\infty}\left(\frac{d \Theta}{d \xi}\right)^{2} d \xi
$$

We can see from (49) and (50) that

$$
\int_{0}^{\infty} \Omega^{\prime 2} d \xi=\int_{0}^{\infty} \Theta^{\prime 2} d \xi
$$

Using the renormalization (eq:renorm) and the relation (51) in the functional (23) we find for the minimal value of $F_{1}$

$$
\mathcal{F}_{1}=33 b^{4}
$$

herefore, the minimal value of the functional (??) becomes $\mathcal{F}=33 b^{4} \lambda^{\frac{3}{11}}$ or

$$
\mathcal{F}=33 b^{4} R^{-\frac{3}{11}}
$$

We note that the equations (1) and (2) follow from the following functional

$$
\mathcal{J}=\frac{1}{6} \int_{0}^{\infty}\left(\Theta^{\prime 2}+\Omega^{\prime \prime 2}+(1-\Omega \Theta)^{2}\right) d \xi
$$

Relations between different quantities are given below

$$
\begin{aligned}
N u & =\left(33 b^{4}\right)^{-1} R^{\frac{3}{11}}=\left(33 b^{4}\right)^{-\frac{11}{8}} R a^{\frac{3}{8}} \\
R & =\left(33 b^{4}\right)^{-\frac{11}{8}} R a^{\frac{11}{8}} \\
a & =b R^{\frac{2}{11}}=\left(\frac{R a}{33}\right)^{\frac{1}{4}} \\
z & =(3 b)^{\frac{1}{3}} R^{-\frac{3}{11}} \\
\omega(z) & =\left(33 b^{4}\right)^{\frac{1}{6}} R^{-\frac{1}{22}}, \Omega \\
\theta(z) & =\left(33 b^{4}\right)^{-\frac{1}{6}} R^{\frac{1}{22}} \Theta .
\end{aligned}
$$

As seen from (56), from the boundary layer theory we have the scaling $N u \sim R a^{\frac{3}{8}}$, the same as in the fixed temperature problem. To further test this scaling, we do some numerical computations. The results are given in the next section. 


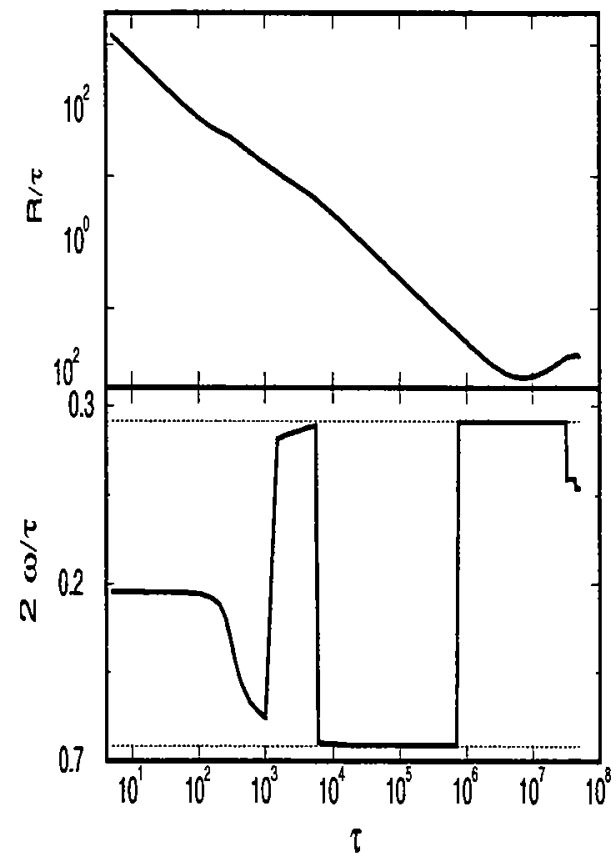

Figure 1: Velocity and temperature deviation: Fixed flux. $R=10^{7}, a=8.5456$.

\section{$5 \quad$ Numerical Results and Discussion}

In the previous section we used asymptotic methods to derive a particular scaling of the Nusselt number in the limit of large $R$. In this section we solve the complete equations (46), (47)(the single alpha approximation) for finite $R$. We will try to verify as much as we can the asymptotic theory's prediction of the $3 / 8$ scaling.

First, in Figure 1 we present the plots of the velocity and the temperature profiles for $R=10^{7}$. The minimizing wave number has value $a=8.5456$. For comparison, we give the similar plot for the fixed temperature problem in Fig. 2. We note the following difference.

In the rising and falling parts of the velocity profile there is a slight bend which is absent in the analogous plot for the velocity profile for fixed temperature. Our investigation showed that this reflects the different boundary conditions of the fixed heat flux problem (to see that, we solved the the Euler-Lagrange equations (46), (47) with zero boundary condition for the temperature deviation $\theta$; also, we solved the equations for the fixed temperature problem with boundary condition $\theta^{\prime}=0$ and we observed the bend appear.) As we increase the Rayleigh number $R$, this bend becomes more and more pronounced: In Fig. 3 we present a plot of the velocity for $R=10^{9}$.

The difference between the fixed heat flux and the fixed temperature problems is also shown in Fig. 4which is compared with its fixed temperature analogue shown in Fig. 5.

In Fig. 6 we show the product of $\omega$ and $\theta$ and, again, compare that with its fixed temperature analogue in Fig. 7. The two curves have very similar behavior.

Next we show our results for the dependence of the Nusselt number on $R$ on a $\log -\log$ plot, Fig. 8.

Our data (pluses) is compared to the data points (dotted line) kindly provided by 


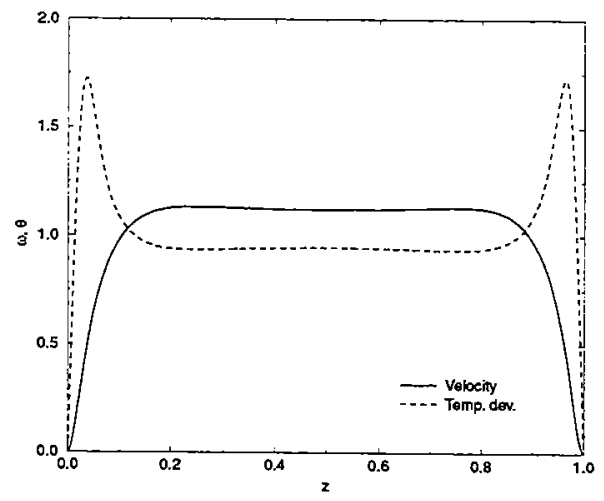

Figure 2: Velocity and temperature deviation: Fixed temperature. $R=10^{7}, a=11.1778$.

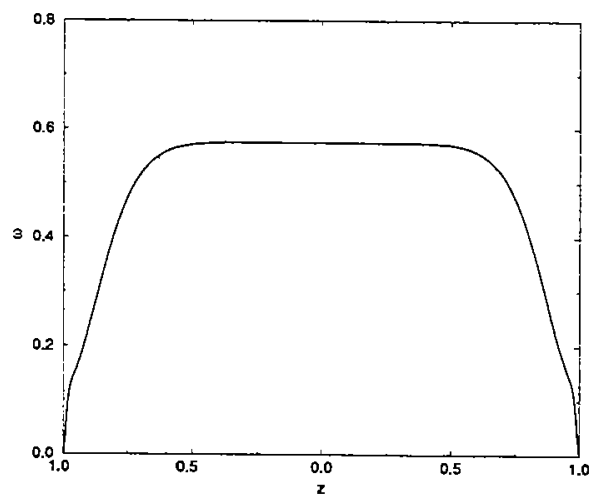

Figure 3: Velocity (this run was made with a different normalization of the velocity and temperature deviation.) $R=10^{9}, a=19.3072$.

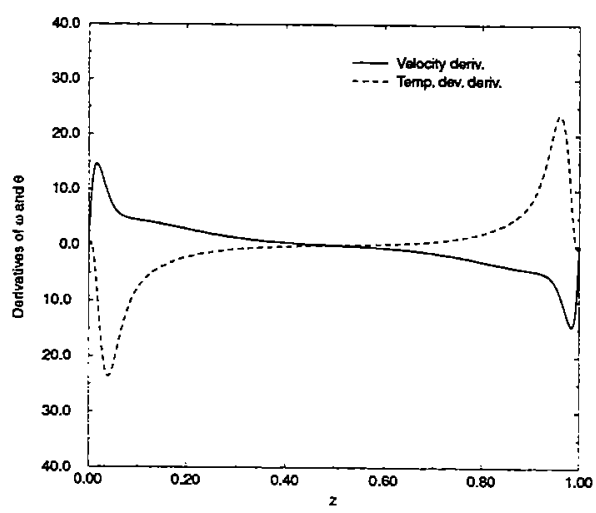

Figure 4: Derivatives of $\omega$ and $\theta$ : Fixed flux. $R=10^{7}, a=8.5456$. 


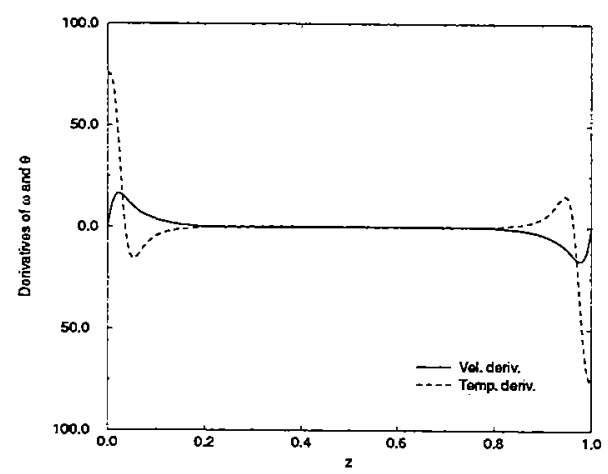

Figure 5: Derivatives of $\omega$ and $\theta$ : Fixed temperature. $R=10^{7}, a=11.1778$.

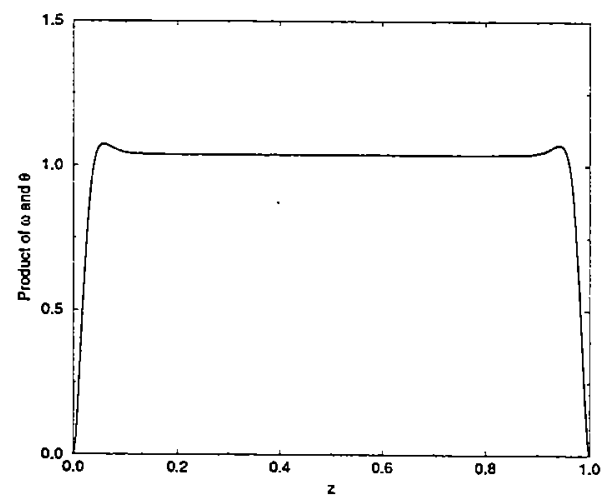

Figure 6: Product of $\omega$ and $\theta$ : Fixed flux. $R=10^{7}, a=8.5456$.

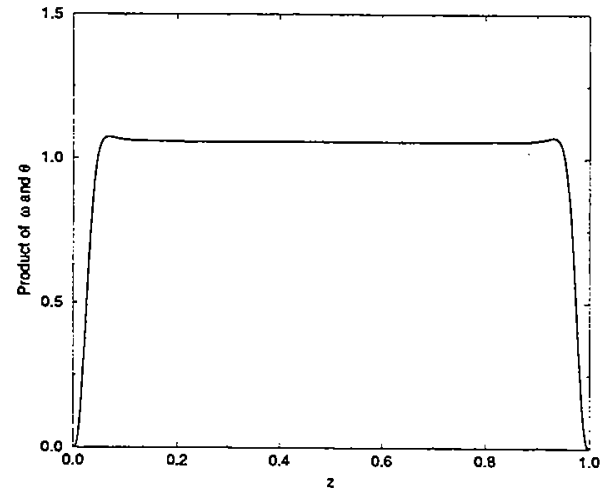

Figure 7: Product of $\omega$ and $\theta$ : Fixed temperature. $R=10^{7}, a=11.1778$. 


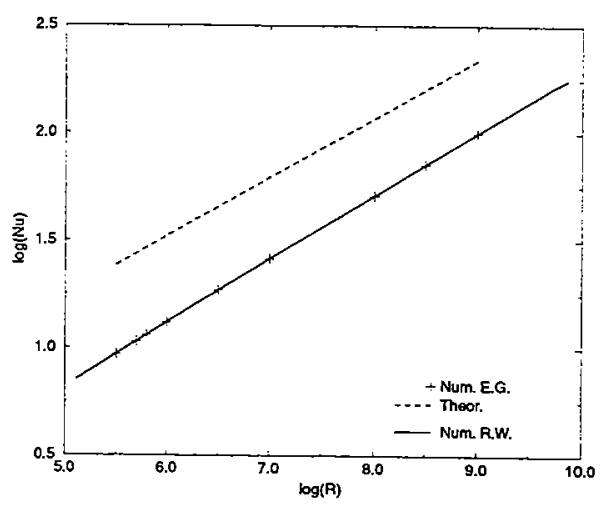

Figure 8: $\log (N u)$ vs. $\log (R)$.

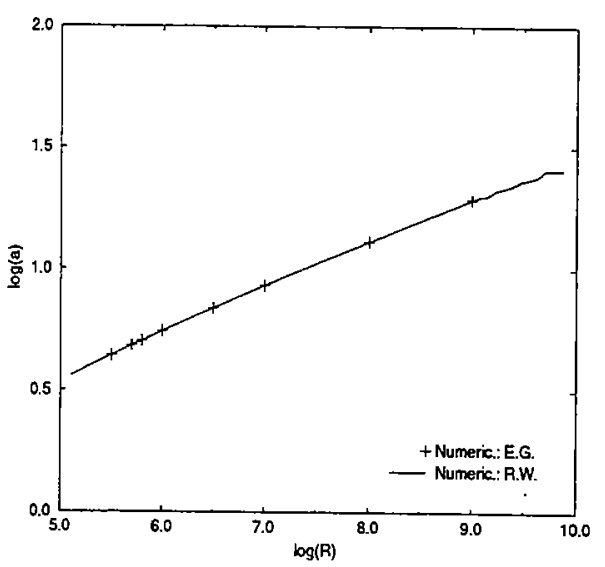

Figure 9: Optimal wave number $a$.

Rodney Worthing. Having found the minimizing values for the wave number $a$ numerically, from relation (58) we can determine the value of $b$. For $R=10^{9}$ it is $b=0.446$. With this value and with relation (56) we plot the bounding curve suggested by the theory in the preceding section (dashed line.)

Finally we give plots for the dependence of the wave number on $R$. First we compare our data points to those of Rodney Worthing in Fig. 9.

Then in Fig. 10 we compare that with the theoretical prediction of C. Doering and J. Otero[4] who derived the scaling $N u \sim R a^{\frac{5}{12}}$. They predict the dependence $a \sim R^{\frac{3}{17}}$, whereas we deduced in the preceding section the dependence $a \sim R \frac{2}{11}$. We have transformed the curves so that our theoretical curve be a horizontal line. From this plot we see that the Doering-Otero theory agrees somewhat better than what follows from the prediction of the preceding section.

We wanted to see if we really capture the asymptotic behavior-and the scaling-with our numerical data that extends up to $R=10^{9}$. We calculated the slope for the analogous dependence $\log (N u)$ vs. $\log (R)$ for the fixed temperature problem. We ended up with similar values for the slope at $R=10^{7.5}: 0.41$. It has been proved that the single wave 


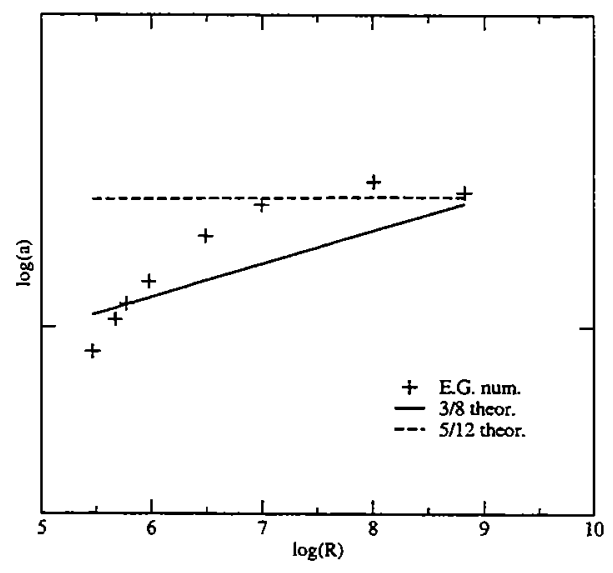

Figure 10: Optimal wave number $a$.

number bound gives a scaling $N u \sim R^{\frac{3}{8}}(3 / 8=0.375$.) This leads us to the thought that we would have to go to much higher values of the Rayleigh number $R$ than $10^{9}$.

Another way of estimating the value of $b$ is by solving the boundary layer equations 49) and (50). We approached this problem in a couple of different ways. In one way we tried to minimize the functional (55) by truncating the upper boundary to some finite value (say 4), solving (49) and (50) up to this value and evaluating the functional (55). We considered the boundary conditions for $\Theta, \Omega^{\prime \prime}$ and $\Omega^{\prime \prime \prime}$ at the upper limit of (55) as parameters and so tried to minimize $\mathcal{J}$ with respect to those parameters. In the other way we used a boundary value problem solver provided by Matlab, again, assuming that we have reached asymptotic behavior of the solutions for some finite value of the independent variable $\xi$. Then we changed this value and solved the problem again. In both ways we encountered some problems. In the first approach we were able to do several iterations (using gradient methods) in the course of minimizing $\mathcal{J}$. However our results were very sensitive to the initial point we chose and were not very consistent. In the second approach we observed extreme sensitivity on the truncation limit. Generally we would expect that increasing the the truncation value would lead to convergence of the solution. Unfortunately that was not the case: The solution changed dramatically even for small changes of the truncation limit (e.g. from 4 to 4.5.) In fact, beyond some point we were not able to find a solution at all. Our second approach worked very successfully for the fixed temperature case which differs only by the boundary condition for the temperature deviation $\Theta$.

The difference in the velocity profile suggests the possibility of different structure of the boundary layer in the fixed heat flux problem. It may be the reason for our difficulties in solving the boundary layer equations. It also suggests the possibility of two boundary layers, or signifies of the importance of an intermediate region (between the boundary layer and the interior part where the functions are predominantly constant.) This question could be clarified by a successful attempt in solving the boundary layer equations and comparing their solution to the solution of the complete Euler-Lagrange equations in the single alpha approximation.

Finally we comment on applying to the problem of bounding the heat transport the multi-alpha approach developed by Busse[5]. If we assume that the scaling $3 / 8$ is correct, 
we find that in this general case the Nusselt number scales as $N u \sim R^{\frac{1}{3}}$ or, equivalently $N u \sim R a^{\frac{1}{2}}$. The latter result has also been found by Otero et al.[3] by applying the background method developed by Doering and Constantin.

\section{Acknowledgments}

I would like to thank Louis Howard, Charles Doering and Friedrich Busse for their help and advice during the work on this project. I thank J. Otero for useful conversations, Glenn Ierley for indispensable help with the numerical computations at high values of the Rayleigh number, Neil Balmforth, also for helping me with the numerical computations. I thank Rodney Worthing for sharing data from his computations. I would like to thank the Woods Hole Oceanographic Institution for giving me the opportunity to participate in this summer program.

\section{References}

[1] Howard, L. N. Heat transport by turbulent convection. J. Fluid Mech. 17, pp. 405-432 (1963)

[2] Howard, L. N. Bounds on flow quantities. Annual Review of Fluid Mech. 4, pp. 473-494 (1972)

[3] Otero, J., Wittenberg, R. W., Worthing, R. A., Doering, C. R. Bounds on RayleighBénard convection with an imposed heat flux. to appear in J. Fluid Mech. (2002)

[4] Otero, J., Doering, C. R. Private communication.

[5] Busse, F. H. On Howard's upper bound for heat transport by turbulent convection. $J$. Fluid Mech. 37, pp. 457-477 (1969) 


\title{
Upper Bounds on the Heat Transport in Infinite Prandtl Number Convection
}

\author{
S. C. Plasting \\ University of Bristol, U.K.
}

\begin{abstract}
We study the asymptotic scaling with Rayleigh number, $R a$, of the vertical heat transport through a layer of fluid of infinite Prandtl number confined between two horizontal plates. The plates are at fixed temperature with heating from below. Previous work based on Howard's optimum theory yields an upper bound on the Nusselt number, $N u$, that scales as $R a^{1 / 3}$. Using the Background method a rigorous upper bound of $R a^{2 / 5}$ has been deduced and with additional information derived from the governing equations an improved bound of the form $R a^{1 / 3}(\log R a)^{2 / 3}$ can be derived. In this report we investigate why the Background method falls short of the earlier result obtained using Howard's optimum theory. We show that these two methods seek to optimise the same functional within a min-max scheme. We compute the optimal piecewise linear solution to the Background method and examine the associated eigenfunctions.
\end{abstract}

\section{Introduction}

The theoretical study of variational bounds on turbulent transport quantities began in 1963 with Howard's Optimum Theory [1]. In the absence of an incompressibility constraint Howard solved Euler-Lagrange equations analytically to obtain an upper bound on the heat transport in turbulent Boussinesq convection which scaled like $R a^{1 / 2}$. Later the constraint of incompressibility was utilised and boundary layer methods were developed to solve Howard's Euler-Lagrange equations for the maximum heat transport problem. Two theoretical tools emerged. The first was the single- $\alpha$ [2], or single horizontal wave-number, test function method. The solutions to the boundary layer equations in this analysis yield a lower bound on the true optimal solution. The second method, due to Busse [3], was the multi- $\alpha$ solutions, a multiple boundary layer solution of the underlying Euler-Lagrange equations, with an arbitrary number of horizontal wave-numbers. Busse's multi- $\alpha$ solution was indeed the optimal solution to Howard's variational problem.

The multi- $\alpha$ solutions of Busse were later used by Chan [4] to calculate an upper bound on the heat transport for the closely related problem of infinite Prandtl number convection. Chan found an improvement to the asymptotic scaling of the upper bound on the heat transport by imposing the momentum equation directly as a point-wise constraint. $\mathrm{He}$ calculated an upper bound on the heat transport with an asymptotic scaling of $R a^{1 / 3}$.

In the nineties a complementary variational problem for bounding the heat transport in turbulent convection was developed by Doering and Constatin [5]. The so-called Background method seeks to estimate the optimal solution to the maximisation problem from above, therefore any test function satisfying certain well-defined constraints will yield a rigorous upper bound on the heat transport. The duality of the Optimum Theory and the Background method was proved for the problem of arbitrary Prandtl number convection by Kerswell [6]. The Background method has recently been applied to the problem of infinite Prandtl number convection in two distinct ways. First, using piecewise linear test functions and standard functional inequalities an upper bound of $R a^{2 / 5}$ was calculated which is 
uniform in rotation rate for rotation perpendicular to the fluid layer [7]. Second, using the Background method and the extra information that the temperature at any point may not exceed the maximum temperature on the boundary an upper bound of $R a^{1 / 3}(\log R a)^{2 / 3}$ was deduced [8]. Though not uniform with respect to rotation this upper bound captures the form of Chan's result with a logarithmic correction.

In a number of other fluid problems, namely plane Couette flow, pipe flow or Poisuille flow, and arbitrary Prandtl number convection, piecewise linear test profiles for the Background method have been able to achieve optimal scaling in these variational problems. Since Chan's result implies that the optimal scaling for infinite Prandtl number convection is $R a^{1 / 3}$, it is interesting to ask why piecewise linear test profiles do not capture the optimal scaling in this problem. Otero [9] also found a $2 / 5$ scaling by numerically optimising the upper bound over piecewise linear test profiles and hence showed that the functional estimates used to calculate the upper bound in [7] are tight.

The structure of this report is as follows. We first introduce the basic equations for infinite Prandtl number Boussinesq convection and define quantities and derive identities which will be frequently referred to in the rest of our presentation. Secondly we will study the seemingly disparate variational methods of Doering-Otero and of Howard-Chan. We will show that both of these methods can be derived from a single specified functional. We will verify the numerical calculation of the optimal piecewise linear test profiles due to Otero and produce trial functions for Chan's dual problem which will be used to construct lower bounds on the optimal upper bound.

\section{Basic Equation and Derived Quantities}

We consider convection between two infinitely extended parallel plates with fixed temperature on the plates. We impose no-slip boundary conditions on the plates and periodic boundary conditions for all variables in the $x, y$-plane. Gravity is perpendicular to the impenetrable plates and the fluid sandwiched between the plates is incompressible.

\subsection{Basic equations}

The basic first order equations of motion for this system are the Rayleigh-Bénard equations. In non-dimensionalised form these are as follows

$$
\begin{gathered}
\frac{1}{\sigma}\left(\frac{\partial \mathbf{u}}{\partial t}+\mathbf{u} \cdot \nabla \mathbf{u}\right)+\nabla p=\operatorname{Ra} T \hat{\mathbf{z}}+\Delta \mathbf{u} \\
\frac{\partial T}{\partial t}+\mathbf{u} \cdot \nabla T=\Delta T
\end{gathered}
$$

where the control parameters are the non-dimensionalised temperature difference across the layer Ra, the Rayleigh number, and the ratio of kinematic viscosity to thermal diffusivity $\sigma$, the Prandtl number. In the limit of infinite Prandtl number the inertial terms in the momentum equation drop and we are left with a linear dependence of the velocity field on temperature

$$
\Delta \mathbf{u}+\operatorname{Ra} T \hat{\mathbf{z}}=\nabla p
$$


We can dispense of the incompressibility constraint $\nabla \cdot \mathbf{u}=0$ and the pressure in the following manner. Let $\mathcal{N}:=\Delta \mathbf{u}+\operatorname{RaT} \hat{\mathbf{z}}-\nabla p=0$ and denote by $\mathcal{N}_{3}$ the third component of $\mathcal{N}$. The components of Equation (3) are

$$
\begin{aligned}
\Delta u & =p_{x} \\
\Delta v & =p_{y} \\
\Delta w+\operatorname{Ra} T & =p_{z}
\end{aligned}
$$

where $\mathbf{u}=(u, v, w)$. With the help of incompressibility taking $\boldsymbol{\nabla} \cdot(\mathcal{N})$ yields

$$
\Delta p=\operatorname{Ra} T_{z}
$$

and taking $\Delta\left(\mathcal{N}_{3}\right)$ gives us

$$
\Delta^{2} w+\operatorname{Ra} \Delta T=\Delta p_{z}
$$

substituting for $p$ we form the only dynamical constraint for this problem

$$
\Delta^{2} w+\operatorname{Ra} \Delta_{H} T=0
$$

in which the horizontal Laplacian applied to $T$ is defined as $\Delta_{H}=\frac{\partial^{2}}{\partial x^{2}}+\frac{\partial^{2}}{\partial y^{2}}$. So we have seen that the horizontal velocity components $u, v$ are purely depending on the diagnostic pressure variable. In Figure (1) we show how the problem is entirely reduced to this pointwise constraint and boundary conditions for $w$ only.

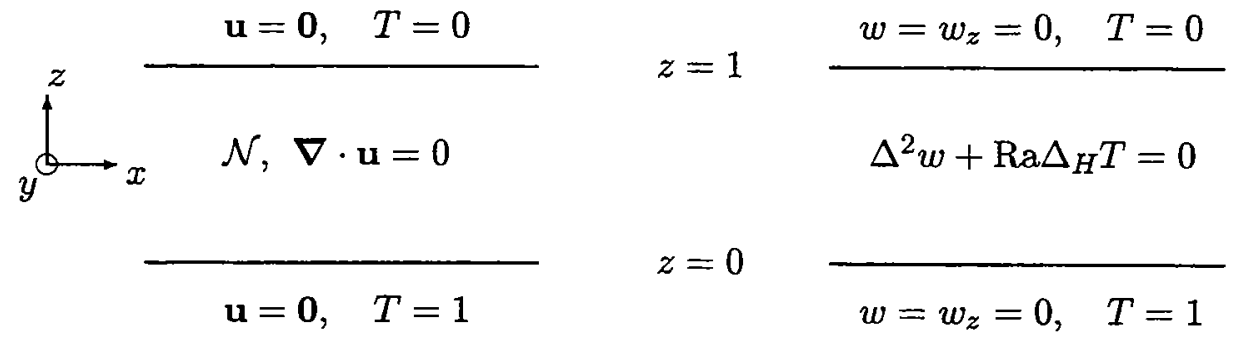

(a)

(b)

Figure 1: Comparison of the point-wise constraints and boundary conditions in (a) finite Prandtl number Rayleigh-Bénard convection and (b) infinite Prandtl number convection.

\subsection{Notation}

The periodic domain in $x, y$ is defined as $\left[0, L_{x}\right] \times\left[0, L_{y}\right]$. Horizontal and global space averages can be defined:

$$
\overline{(\cdot)}:=\frac{1}{L_{x} L_{y}} \int_{0}^{L_{x}} \mathrm{~d} x \int_{0}^{L_{y}} \mathrm{~d} y(\cdot), \quad\langle(\cdot)\rangle:=\int_{0}^{1} \mathrm{~d} z \overline{(\cdot)}
$$


Long-time average:

$$
\langle(\cdot)\rangle_{\infty}=\lim _{\mathcal{T} \rightarrow \infty}\langle(\cdot)\rangle_{\mathcal{T}}
$$

and the $L_{2}$-norm:

$$
\|(\cdot)\|=\left\langle(\cdot)^{2}\right\rangle^{1 / 2}
$$

For functions depending on $z$ only we use a prime to denote the $z$-derivative of that function, so

$$
f^{\prime}(z):=\frac{\mathrm{d} f}{\mathrm{~d} z}
$$

\subsection{Definitions}

Equation (2) can be rewritten as

$$
\frac{\partial T}{\partial t}=-\nabla \cdot(\mathbf{j}+\mathbf{J})
$$

where $\mathbf{j}$ is the conductive heat flow, $\mathbf{j}:=-\nabla T$, and $\mathbf{J}$ is the convective heat flow, $\mathbf{J}:=\mathbf{u} T$. In the purely conductive state the average heat transport between the plates is

$$
\langle\hat{\mathbf{z}} \cdot(-\nabla T)\rangle=-\left.T\right|_{0} ^{1}=1
$$

The total average heat transport between the plates is

$$
\langle\hat{\mathbf{z}} \cdot(\mathbf{j}+\mathbf{J})\rangle=1+\langle w T\rangle
$$

We define the Nusselt number, $\mathrm{Nu}$, as the ratio of the long-time averaged total heat transport to conductive heat transport across the plates. This is simply the ratio of the expressions in Equation (6) and Equation (5), therefore we have

$$
\mathrm{Nu}=1+\langle w T\rangle_{\infty}
$$

Using the global entropy flux balance, $\langle T \mathcal{H}\rangle=0$,

$$
\frac{\mathrm{d}}{\mathrm{d} t} \frac{1}{2}\|T\|^{2}+\|\nabla T\|^{2}=1+\langle w T\rangle
$$

and appealing to the temperature maximum principle, we find the following equivalent definitions of $\mathrm{Nu}$ as a simple consequence

$$
\mathrm{Nu}=\left\langle\|\nabla T\|^{2}\right\rangle_{\infty}
$$

We will see below that the point-wise constraint in Equation (4) and the global entropy flux balance constraint (7) are at the centre of both of the bounding problems. 


\section{Doering-Otero Approach}

The background decomposition of the temperature field is

$$
T(\mathbf{x}, t)=\tau(z)+\theta(\mathbf{x}, t)
$$

where $\tau(z)$ takes up the fixed temperature boundary conditions, $\tau(0)=1$ and $\tau(1)=0$, and $\theta$ must therefore satisfy homogeneous boundary conditions. Substituting this change of variables into the heat equation yields

$$
\frac{\partial \theta}{\partial t}+\mathbf{u} \cdot \nabla \theta=\Delta \theta+\tau^{\prime \prime}-w \tau^{\prime}
$$

We have the following identity

$$
\|\nabla T\|^{2}=\|\nabla \theta\|^{2}+\left\|\tau^{\prime}\right\|^{2}-2\left\langle\theta \tau^{\prime \prime}\right\rangle
$$

and multiplying Equation (10) by $\theta$ and taking the global average produces

$$
\frac{1}{2} \frac{\mathrm{d}}{\mathrm{d} t}\|\theta\|^{2}=-\|\nabla \theta\|^{2}+\left\langle\theta \tau^{\prime \prime}-w \theta \tau^{\prime}\right\rangle
$$

Adding $b \times(12)$ to $(11)$ gives

$$
\frac{b}{2} \frac{\mathrm{d}}{\mathrm{d} t}\|\theta\|+\|\nabla T\|^{2}=\left\|\tau^{\prime}\right\|^{2}-\mathcal{G}(\tau, w, \theta, b)
$$

where $\mathcal{G}=\left\langle(b-1)\|\nabla \theta\|^{2}-(b-2) \theta \tau^{\prime \prime}+b w \theta \tau^{\prime}\right\rangle$. By adding a balance parameter, $b$, we are generalising the work of Otero, who takes $b=2$ to remove the centre term in $\mathcal{G}$.

Taking a long-time average we have the following upper bound on the Nusselt number

$$
\mathrm{Nu} \leq\left\|\tau^{\prime}\right\|^{2}-\inf \langle\mathcal{G}(\tau, w, \theta, b)\rangle_{\infty}
$$

provided that inf $\mathcal{G}$ exists. We can drop time averages here because the infimum will be achieved by steady fields.

To minimise $\mathcal{G}$ we set up the following Lagrangian

$$
L=\mathcal{G}-\left\langle q(\mathbf{x})\left(\Delta w+R a \Delta_{H} \theta\right)\right\rangle
$$

where $q(\mathbf{x})$ is a Lagrange multiplier with natural boundary conditions which imposes the point-wise constraint in (4). By taking the horizontal average of the $\theta$ variation of $L$ we uncover the mean of the optimal fluctuation in terms of $\tau$

$$
\frac{\delta L}{\delta \theta}=-2(b-1) \Delta \theta-(b-2) \tau^{\prime \prime}+b w \tau^{\prime}-\operatorname{Ra} \Delta_{H} q=0
$$

The $q$ term drops when we take a horizontal average $\frac{\overline{\delta L}}{\delta \theta}=0$, and we find that

$$
2(b-1) \bar{\theta}^{\prime \prime}+(b-2) \tau^{\prime \prime}=0 .
$$


Two integrations and consideration of the boundary conditions reveal that

$$
\bar{\theta}=-\frac{(b-2)}{2(b-1)}[\tau+z-1]
$$

If we subtract off the mean part from the fluctuation field by setting $\hat{\theta}=\theta-\bar{\theta}$ and substitute this into Expression (14) we yield the following:

If $\tau(z)$ satisfies the spectral constraint $\mathcal{Q}(\tau, w, \hat{\theta}, b)=\left\{(b-1)\|\nabla \hat{\theta}\|^{2}+b\left\langle\hat{\theta} w \tau^{\prime}\right\rangle\right\} \geq 0$ over all fields $(w, \hat{\theta})$ which satisfy $\Delta^{2} w+\operatorname{Ra} \Delta_{H} \hat{\theta}=0$ and the relavent boundary conditions (Figure 1(b)), then the following upper bound on $N u$ holds

$$
\mathrm{Nu}-1 \leq \frac{b^{2}}{4(b-1)}\left(\left\|\tau^{\prime}\right\|^{2}-1\right) \text {. }
$$

We must also have $b>1$ in order that the quadratic functional $\mathcal{Q}$ has a minimum value.

\section{Howard-Chan Approach} We begin by assuming statistical stationarity for all horizontal averages, then $\langle w T\rangle_{\infty}=$
$\langle w T\rangle$ and moreover

$$
\mathrm{Nu}=1+\langle w T\rangle
$$

We make the mean-fluctuation decomposition of the temperature field $T=\bar{T}+\hat{\theta}$. Where now $\bar{T}$ is the time independent horizontal mean and $\overline{\hat{\theta}}=0$.

A horizontal average of Equation (2) after integration gives

$$
\frac{\mathrm{d} \bar{T}}{\mathrm{~d} z}=\overline{w \hat{\theta}}-\langle w \hat{\theta}\rangle-1
$$

and multiplying Equation (2) by $T$ and global averaging yields

$$
\left\langle\bar{T} \frac{\mathrm{d}^{2} \bar{T}}{\mathrm{~d} z^{2}}\right\rangle=-\|\nabla \hat{\theta}\|^{2}
$$

then inserting (16) into (17) we deduce the so-called second power integral

$$
\left.\|\nabla \hat{\theta}\|^{2}+\|\overline{w \hat{\theta}}-\langle w \hat{\theta}\rangle\|^{2}\right\rangle=\langle w \hat{\theta}\rangle .
$$

We can form unity by taking the ratio of terms in the previous balance

$$
1=\frac{\langle w \hat{\theta}\rangle-\|\nabla \hat{\theta}\|^{2}}{\|\overline{\hat{\hat{\theta}}}-\langle w \hat{\theta}\rangle\|^{2}}
$$

and subsequently we can define the homogeneous functional which Chan [4] seeks to maximise. Let $F:=\mathrm{Nu}-1$ and multiply the above representation of unity by $\langle w \hat{\theta}\rangle$ to form a
homogeneous functional

$$
F=\frac{\langle w \hat{\theta}\rangle^{2}-\langle w \hat{\theta}\rangle\|\nabla \hat{\theta}\|^{2}}{\|\overline{\hat{\theta}}-\langle w \hat{\theta}\rangle\|^{2}}
$$


the supremum of which is an upper bound on $\mathrm{Nu}-1$. The maximisation of $F$ is performed over the competitor fields and the constraint in Equation (18) is imposed post facto by normalising $\langle w \hat{\theta}\rangle=F$.

Chan studied the following Lagrangian form

$$
G=F-\left\langle q(\mathbf{x})\left(\Delta^{2} w+\operatorname{Ra} \Delta_{H} \hat{\theta}\right)\right\rangle
$$

where $q$ is a Lagrange multiplier satisfying natural boundary conditions which imposes the point-wise constraint in Equation (4). This is exactly the functional Eq. (25) in [4] without the normalisation $\langle w \hat{\theta}\rangle=1$. Taking variations of this functional with respect to $w$ and then $\hat{\theta}$ one finds that

$$
\begin{gathered}
\frac{\delta G}{\delta w}=\frac{\hat{\theta}\left(2\langle w \hat{\theta}\rangle-\|\nabla \hat{\theta}\|^{2}\right)}{\|\overline{w \hat{\theta}}-\langle w \hat{\theta}\rangle\|^{2}}-\frac{2 F \hat{\theta}(\overline{w \hat{\theta}}-\langle w \hat{\theta}\rangle)}{\|\overline{w \hat{\theta}}-\langle w \hat{\theta}\rangle\|^{2}}-\Delta^{2} q=0 \\
\frac{\delta G}{\delta \hat{\theta}}=\frac{2\langle w \hat{\theta}\rangle(w+\Delta \hat{\theta})-w\|\nabla \hat{\theta}\|^{2}}{\|\overline{w \hat{\theta}}-\langle w \hat{\theta}\rangle\|^{2}}-\frac{2 F w \overline{(w \hat{\theta}}-\langle w \hat{\theta}\rangle)}{\|\overline{w \hat{\theta}}-\langle w \hat{\theta}\rangle\|^{2}}-\operatorname{Ra} \Delta_{H} q=0
\end{gathered}
$$

Multiplying these equations through by $\|\overline{w \hat{\theta}}-\langle w \hat{\theta}\rangle\|^{2}$ and substituting in

$$
\|\nabla \hat{\theta}\|^{2}=\langle w \hat{\theta}\rangle-\|\overline{w \hat{\theta}}-\langle w \hat{\theta}\rangle\|^{2}
$$

we deduce the following Euler-Lagrange equations for $w$ and $\hat{\theta}$

$$
\begin{aligned}
\hat{\theta}\left(\langle w \hat{\theta}\rangle+\|\overline{w \hat{\theta}}-\langle w \hat{\theta}\rangle\|^{2}\right)-2 F \hat{\theta}(\overline{w \hat{\theta}}-\langle w \hat{\theta}\rangle)-\left(\Delta^{2} q\right)\|\overline{w \hat{\theta}}-\langle w \hat{\theta}\rangle\|^{2}=0 \\
\begin{aligned}
2 \Delta \hat{\theta}\langle w \hat{\theta}\rangle & +w\left(\langle w \hat{\theta}\rangle+\|\overline{w \hat{\theta}}-\langle w \hat{\theta}\rangle\|^{2}\right) \\
& -2 F w(\overline{w \hat{\theta}}-\langle w \hat{\theta}\rangle)-\operatorname{Ra}\left(\Delta_{H} q\right)\|\overline{w \hat{\theta}}-\langle w \hat{\theta}\rangle\|^{2}=0 .
\end{aligned}
\end{aligned}
$$

It can be shown that if we normalise $w$ and $\theta$ as Chan does, namely $w \rightarrow\langle w \hat{\theta}\rangle^{-\frac{1}{2}} R^{-\frac{1}{2}} w$ and $\hat{\theta} \rightarrow\langle w \hat{\theta}\rangle^{-\frac{1}{2}} R^{\frac{1}{2}} \hat{\theta}$ so that $\langle w \theta\rangle \rightarrow 1$ then equations (22) and (21) become exactly the Euler-Lagrange equations (27) in [4] which Chan solves using Busse's multi- $\alpha$ solution.

Having derived the Doering-Otero and Howard-Chan approaches in the previous two sections we now turn to proving the duality between the two methods. 


\section{A Unifying Functional}

Claim: The Doering-Otero principle and the Howard-Chan principle both seek to optimise the following functional

$$
N:=\|\nabla T\|^{2}-b\langle\theta \mathcal{H}\rangle-\left\langle q(\mathbf{x})\left(\Delta^{2} w+\operatorname{Ra} \Delta_{H} \theta\right)\right\rangle
$$

where $\theta$ is defined as in (9) and $\mathcal{H}$ is the heat equation

$$
\mathcal{H}:=\frac{\partial \theta}{\partial t}+\mathbf{u} \cdot \nabla \theta-\Delta \theta+w \tau^{\prime}-\tau^{\prime \prime}=0
$$

Proof:

We start by deriving all of the variational derivatives of $N$. In terms of $\tau$ and $\theta$ we have

$$
\begin{array}{r}
N(\tau, w, \theta, b, q)=\left\|\tau^{\prime}\right\|^{2}-\left\langle(b-1)|\nabla \theta|^{2}-(b-2) \theta \tau^{\prime \prime}+b \theta w \tau^{\prime}\right\rangle \\
-\left\langle q(\mathbf{x})\left(\Delta^{2} w+\operatorname{Ra} \Delta_{H} \theta\right)\right\rangle
\end{array}
$$

Variations are taken in $\tau, \theta, w, q$ and then variational equation for the mean and fluctuating part of $\theta$ are deduced.

$$
\begin{gathered}
\frac{\delta N}{\delta \tau}=-2 \tau^{\prime \prime}+(b-2) \bar{\theta}^{\prime \prime}+b(\overline{w \theta})^{\prime}=0 \\
\frac{\delta N}{\delta \theta}=2(b-1) \Delta \theta+(b-2) \tau^{\prime \prime}-b w \tau^{\prime}-\operatorname{Ra} \Delta_{H} q=0 \\
\frac{\delta N}{\delta w}=-b \theta \tau^{\prime}-\Delta^{2} q=0 \\
\frac{\delta N}{\delta q}=\Delta^{2} w+\operatorname{Ra} \Delta_{H} \theta=0 \\
\frac{\delta N}{\delta \theta}=0 \quad\left\{\begin{array}{l}
\frac{\delta N}{\delta \bar{\theta}}=2(b-1) \bar{\theta}^{\prime \prime}+(b-2) \tau^{\prime \prime}=0 \\
\frac{\delta N}{\delta \hat{\theta}}=2(b-1) \Delta \hat{\theta}-b w \tau^{\prime}-\operatorname{Ra} \Delta_{H} q=0
\end{array}\right.
\end{gathered}
$$

\section{Part 1: Doering-Otero Principle}

Solve $\frac{\delta N}{\delta \bar{\theta}}=0$ 
Two integrations give

$$
\bar{\theta}=\frac{b-2}{2(b-1)}[\tau+z-1]
$$

Plugging this into $N$ we have

$N(\tau, w, \hat{\theta}, b, q)-1=\frac{b^{2}}{4(b-1)}\left(\left\|\tau^{\prime}\right\|^{2}-1\right)-\left\langle(b-1)|\nabla \hat{\theta}|^{2}+b \hat{\theta} w \tau^{\prime}\right\rangle-\left\langle q(\mathbf{x})\left(\Delta^{2} w+\operatorname{Ra} \Delta_{H} \hat{\theta}\right)\right\rangle$

Compare this with the functional Otero studies. Setting $b=2$ we have $N=\left\|\tau^{\prime}\right\|^{2}-$ $\left\langle|\nabla \hat{\theta}|^{2}+2 \hat{\theta} w \tau^{\prime}\right\rangle-\left\langle q(\mathbf{x})\left(\Delta^{2} w+\operatorname{Ra} \Delta_{H} \hat{\theta}\right)\right\rangle$ and the fluctuation field has no mean part. It is clear that we must require $b>1$ to ensure that a minimum of the right hand side exists.

\section{Part 2: Howard-Chan Principle}

Solve $\frac{\delta N}{\delta \bar{\theta}}=0$ and $\frac{\delta N}{\delta \tau}=0$ simultaneously to deduce equations for the background field and the mean of the fluctuation field in terms of the mean-less fluctuation field $\hat{\theta}$.

$$
\left\{\begin{array}{l}
\tau^{\prime}=\frac{2(b-1)}{b}\{\overline{w \theta}-\langle w \theta\rangle\}-1 \\
\bar{\theta}^{\prime}=-\frac{b-2}{b}\{\overline{w \theta}-\langle w \theta\rangle\}
\end{array}\right\}
$$

Plug these expressions into $N$, of Equation (24), noticing that $\overline{w \theta}=\overline{w \hat{\theta}}$, after some algebra we have

$$
\begin{array}{r}
N(w, \hat{\theta}, b, q)=1+\langle w \hat{\theta}\rangle+(b-1)\left\{\langle w \hat{\theta}\rangle-\|\overline{w \hat{\theta}}-\langle w \hat{\theta}\rangle\|^{2}-\|\nabla \hat{\theta}\|^{2}\right\} \\
-\left\langle q(\mathbf{x})\left(\Delta^{2} w+\operatorname{Ra} \Delta_{H} \hat{\theta}\right)\right\rangle
\end{array}
$$

Now we see that the Lagrange multiplier $b$ is imposing the global entropy flux balance (Equation 18) and $q$ is imposing the point-wise constraint in Equation (4). The remaining variational equations for $w$ and $\hat{\theta}$ are

$$
\begin{gathered}
\frac{\delta N}{\delta \hat{\theta}}=w+(b-1)\{w-2 w(\overline{w \hat{\theta}}-\langle w \hat{\theta}\rangle)+2 \Delta \hat{\theta}\}-\mathrm{Ra} \Delta_{H} q=0 \\
\frac{\delta N}{\delta w}=\hat{\theta}+(b-1)\{\hat{\theta}-2 \hat{\theta}(\overline{w \hat{\theta}}-\langle w \hat{\theta}\rangle)\}-\Delta^{2} q=0
\end{gathered}
$$

We can calculate $\left\langle\hat{\theta} \frac{\delta N}{\delta \hat{\theta}}\right\rangle=0$ and $\left\langle w \frac{\delta N}{\delta w}\right\rangle=0$ in order to obtain a value for $b$. The equations to be solved are

$$
(2-b)\langle w \hat{\theta}\rangle-\operatorname{Ra}\left\langle\left(\Delta_{H} q\right) \hat{\theta}\right\rangle=0
$$


and

$$
(2-b)\langle w \hat{\theta}\rangle+2(b-1)\|\nabla \hat{\theta}\|^{2}-\left\langle\left(\Delta^{2} q\right) w\right\rangle=0 .
$$

Given that $\left\langle\left(\Delta^{2} q\right) w\right\rangle=\left\langle q\left(\Delta^{2} w\right)\right\rangle=\left\langle q\left(-\operatorname{Ra} \Delta_{H} \hat{\theta}\right\rangle\right.$ we can add these two equations and solve for $b$ to find that

$$
b=\frac{\|\nabla \hat{\theta}\|^{2}-2\langle w \hat{\theta}\rangle}{\|\nabla \hat{\theta}\|^{2}-\langle w \hat{\theta}\rangle}
$$

With the use of the second power integral we can rearrange this expression to give

$$
b-1=\frac{\langle w \hat{\theta}\rangle}{\|\overline{w \hat{\theta}}-\langle w \hat{\theta}\rangle\|^{2}}
$$

for easy insertion back into Equations (26) and (27). Inserting and multiplying the resulting expressions through by $\|\overline{w \hat{\theta}}-\langle w \hat{\theta}\rangle\|^{2}$ we arrive at the expressions

$$
\begin{gathered}
\left.\hat{\theta}\left(\langle w \hat{\theta}\rangle+\|\overline{w \hat{\theta}}-\langle w \hat{\theta}\rangle\|^{2}\right)-2 \hat{\theta}\langle w \hat{\theta}\rangle \overline{w \hat{\theta}}-\langle w \hat{\theta}\rangle\right)-\left(\Delta^{2} q\right)\|\overline{w \hat{\theta}}-\langle w \hat{\theta}\rangle\|^{2}=0 \\
\left.2 \Delta \hat{\theta}\langle w \hat{\theta}\rangle+w\left(\langle w \hat{\theta}\rangle+\|\overline{w \hat{\theta}}-\langle w \hat{\theta}\rangle\|^{2}\right)-2 w\langle w \hat{\theta}\rangle \overline{(w \hat{\theta}}-\langle w \hat{\theta}\rangle\right) \\
-\operatorname{Ra}\left(\Delta_{H} q\right)\|\overline{w \hat{\theta}}-\langle w \hat{\theta}\rangle\|^{2}=0
\end{gathered}
$$

Replacing the $\langle w \hat{\theta}\rangle$ which multiply $(w \hat{\theta}-\langle w \hat{\theta}\rangle)$ in both equations by the functional $F$ we have exactly the Euler-Lagrange equations that were derived from Chan's homogeneous ratio (Equations 21-22).

As a final comment we note that for the problems to intersect the Howard-Chan problem must also satisfy the spectral constraint so that the top maximum is selected. This means that $(b-1)>0$, which is consistent with Equation (29) since $\langle w \hat{\theta}\rangle$ is positive due to the second power integral.

\section{Piecewise Linear Background Profiles}

In the framework of the Background method rigorous upper bounds on $\mathrm{Nu}$ are easily calculated by using piecewise linear test profiles for $\tau$ (see Figure 2). These functions are odd functions about the channel midplane, they take the value of $1 / 2$ in the interior of the channel and change linearly over two boundary layers of thickness $\delta$, such that they satisfy the boundary conditions on $\tau$, and the derivative of $\tau$ is:

$$
\tau^{\prime}=\left\{\begin{array}{cc}
0 & \text { for } z \in(\delta, 1-\delta) \\
\frac{1}{2 \delta} & \text { otherwise. }
\end{array}\right\}
$$




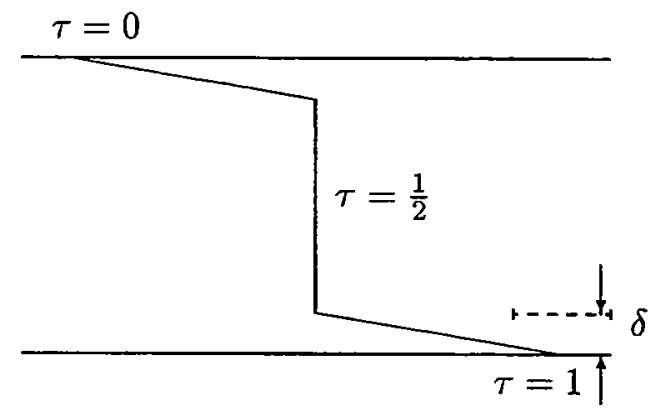

Figure 2: Piecewise linear $\tau$.

We take $b=2$ in keeping with Otero [9], in which case $N u \leq\left\langle\tau^{\prime 2}\right\rangle$. Simple integration shows that the upper bound on the heat transport is $N u \leq \frac{1}{2 \delta}$ if $\tau$ satisfies the following condition

$$
\tau \text { is an admissible test function if } \inf Q \geq 0
$$

for all $w$ and $\theta$ with $\Delta^{2} w+R a \Delta_{H} \theta=0$ and $w=D w=\theta=0$ at $z=0,1$.

The piecewise linear profile which produces the lowest upper bound is found by minimising the following functional

$$
\mathcal{G}=Q-\left\langle q(\mathbf{x})\left(\Delta w+R a \Delta_{H} \theta\right)\right\rangle+2 \lambda\left(\left\langle\theta^{2}\right\rangle-1\right)
$$

where $q$ is a point-wise Lagrange Multiplier imposing the momentum constraint, and $\lambda$ is used to normalise $\theta$. The Euler-Lagrange equations for this functional are

$$
\begin{gathered}
\lambda \theta=\left(D^{2}-k^{2}\right) \theta-w \tau^{\prime}+\frac{R a}{2} k^{2} q, \\
0=2 \theta \tau^{\prime}+\left(D^{2}-k^{2}\right)^{2} q, \\
0=\left(D^{2}-k^{2}\right)^{2} w-R a k^{2} \theta,
\end{gathered}
$$

where all of the fields have been Fourier expanded as $f=f(z) e^{i k x}$, and each variable $q, w$, and $\theta$ now only depend on $z$ and must satisfy the following boundary conditions

$$
\theta=w=D w=q=D q=0 \quad \text { at } z=0,1 .
$$

Given that only $\tau^{\prime}$ is discontinuous at $z=\delta$ and $z=1-\delta$ we must solve for $w, \theta$ and $q$ inside three regions $[0, \delta],[\delta, 1-\delta]$ and $[1-\delta, \delta]$, and impose matching conditions between the regions. Since equations (33-35) are second order in $\theta$ and fourth order in $w$ and $g$, the natural matching conditions are

$$
\begin{gathered}
{[\theta]=[D \theta]=0} \\
{\left[D^{i} w\right]=\left[D^{i} g\right]=0 \quad \text { for } i=0,1,2,3}
\end{gathered}
$$

where $[f]$ denotes the jump in the value of $f$ at either $z=\delta$ or $z=1-\delta$, and the superscript denotes the $n$th $z$-derivative. 


\subsection{Solution Using Complex Eigenfunctions}

In Otero's thesis he successfully applies the method of finding complex analytic eigenfunctions to many variational problems in turbulent convection. The assumption is made, and later accounted for, that the most critical eigenfunctions are even about the mid-plane. Thus the following symmetry conditions are also imposed:

$$
D \theta=D w=D^{3} w=D g=D^{3} g=0 \quad \text { at } z=1 / 2 .
$$

The solutions is then required in only two regions:

$$
\text { Region I: }[0, \delta] \quad \text { Region 2: }[\delta, 1 / 2] \text {. }
$$

$\lambda$ is set to zero and equations (33- 35) are solved in complex eigenfunctions on each region. The boundary conditions at $z=0$ are built in to the solution in Region I and the symmetry conditions at $z=\frac{1}{2}$ are built in to the solution in Region II. The 10 matching condition are used to specify 10 unknown coefficients in the two solutions. These conditions can be collected in to a $10 \times 10$ linear homogeneous system, say $M x=0$, where $x$ represents a vector of 10 unknown coefficients. Non-trivial solutions exist if $\operatorname{det} M=0$. The following numerical recipe is used to optimise the upper bound:

\section{Technique:}

- Fix $R a$.

- For fixed $k$ graph $\operatorname{det} M$ versus $\delta$ and find the minimum $\delta$ such that $\operatorname{det} M=0$, label this $\delta_{0}$.

- Select the minimum $\delta_{0}$ over all $k$, label this $\delta_{c}$.

Then $\delta_{c}$ corresponds to the largest $\delta$ for which condition(32) holds and hence the lowest upper bound for piecewise linear profiles is $N u \leq \frac{1}{2 \delta_{c}}$.

\subsection{Calculating the Eigenvalue Spectrum of Constraint (32)}

It is easily shown by multiplying Equation (33) by $\theta$, globally averaging and using constraints (34) and (35), that $Q=-\lambda$. Condition (32) is thus equivalent to requiring that the highest eigenvalue, say $\lambda_{0}$, of eigenvalue problem (33) subject to (34) and (35), over all $k$-space are negative semi-definite. We can therefore repose condition $(32)$ as

$$
\begin{gathered}
\tau \text { is an admissible test function if } \\
\lambda_{0}^{*}:=\max _{k} \lambda_{0} \text { satisfies } \lambda_{0}^{*} \leq 0
\end{gathered}
$$

We calculate the spectrum $\lambda_{0}(k)$ of Equations (33-35) using finite difference methods together with the shooting technique to match the solutions on each region at the point $z=\delta . \lambda_{0}^{*}$ is found to be a monotone increasing function of $\delta$. We are able to find the critical $\delta$, say $\delta_{c}$, for which $\lambda_{0}^{*}=0$ as illustrated below.

\section{Technique:}




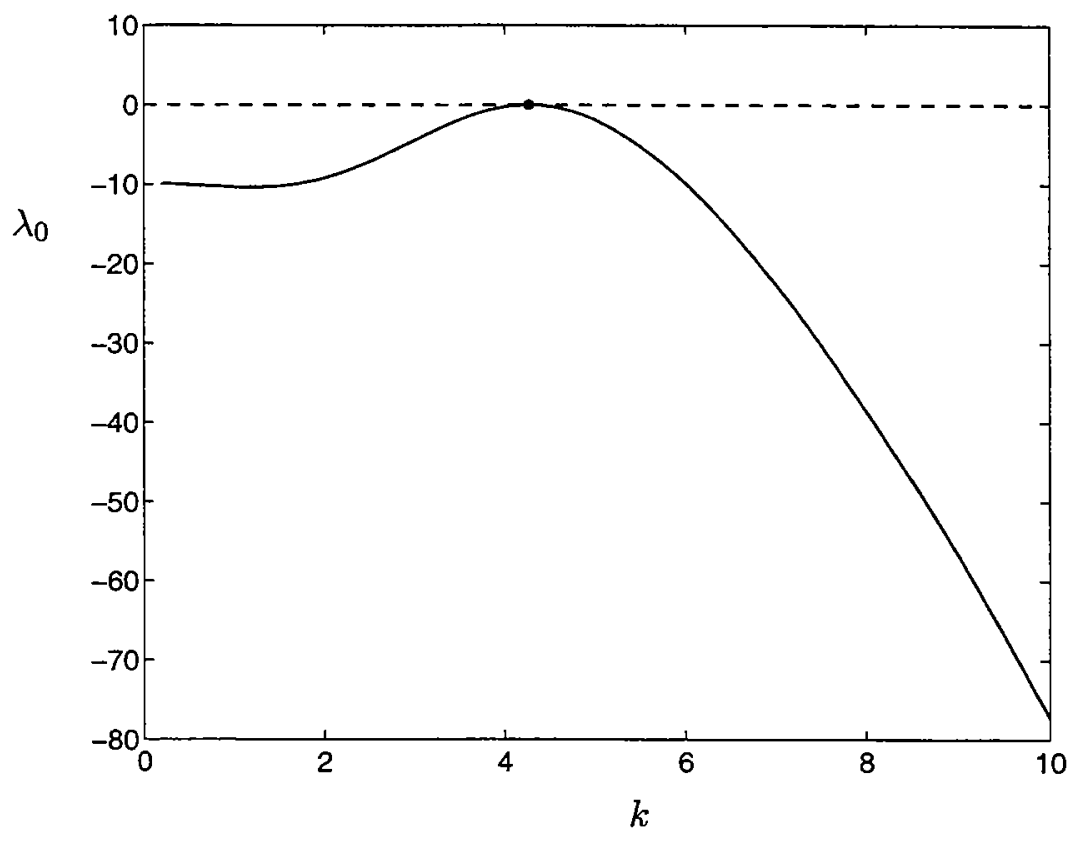

Figure 3: The spectrum of Equations (33-35) at $R a=10^{9}$ and optimal boundary thickness $\delta_{c}=1.3457 \times 10^{-3}$.

- Fix $R a$.

- For fixed $\delta$ calculate $\lambda_{0}(k)$ and find its maximum $\lambda_{0}^{*}$

- Vary $\delta$ until $\lambda_{0}^{*}=0$

The envelope of the eigenvalue spectrum $\lambda_{0}(k)$ is found to have a unique maximum. We denote the wavenumber at which the maximum occurs by $k_{c}$. This procedure leads us to the critical $\delta$ for which condition (36) is marginally satisfied. It is the same $\delta$ as calculated by the numerical scheme used by Otero. Figure 3 shows an example of the spectrum at $R a=10^{9}$.

\subsection{Comparison of Numerics}

For comparison of our numerical technique with that of Otero we calculate $\delta_{c}$ at six points in $\log (R a)$-space between 4 and 9 . The results are shown in Figure 5. The solid upper line is plotted using data supplied by Otero. Our calculation is shown as circles which fall reassuringly well on top of Otero's data. Also shown is Chan's optimal upper bound with a $1 / 3$ scaling taken directly from his 1971 publication. Also of interest here are the crosses which are associated to a lower bound on the optimal upper bound calculated here to explore the nature of the duality which exists between the Howard-Chan method and the Doering-Otero method. We took the eigenfunctions for $w$ and $\theta$ associated with the critical 


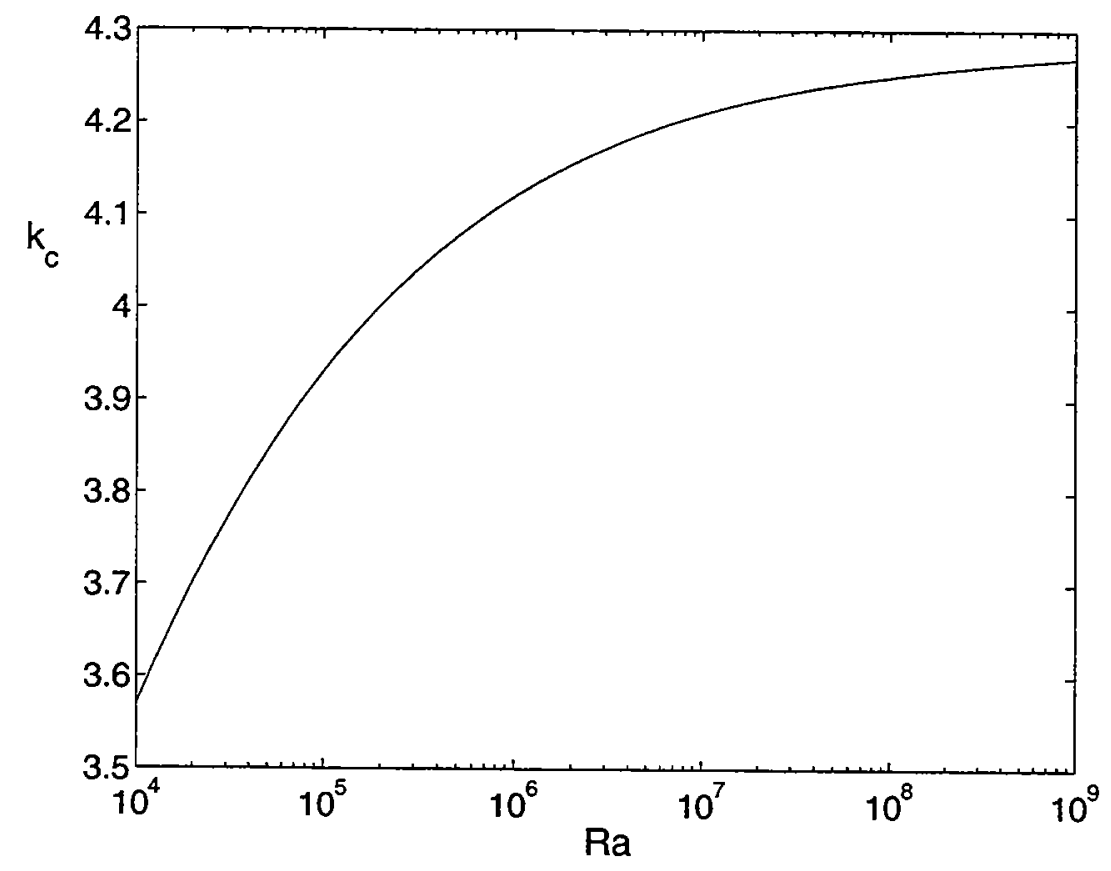

Figure 4: The critical wavenumber for the optimising piecewise linear profiles. This shows that $k_{c} \sim O(1)$.

wavenumber $k_{c}$ when $\lambda_{0}^{*}=0$ and evaluated Chan's homogeneous functional (19) at these points. This procedure guarantees a lower bound on Chan's optimal upper bound. However, this lower bound is very poor and this naive experiment reveals that the eigenfunctions (see Figure 7) do not well approximate the structure of Chan's optimal solutions (see Figure 6) and are thus poor test functions for the Howard-Chan variational problem. One notices, for example, that there is no boundary layer in the product $w \theta$ for our eigenfunctions.

Interestingly the critical wavenumber of the optimal piecewise linear profile remains order one for all $R a$ studied here (see Figure 4).

\section{Discussion}

To summarise we have confirmed Otero's numerical calculation and we have shown that the duality shared between the two variational problems discussed here is worth further investigation.

In all other cases, for example plane Couette flow, pipe flow and arbitrary Prandtl number convection, where piecewise linear test profiles have been applied within the Background method the correct optimal scaling of the upper bound was achieved. In this case Chan's result presents $1 / 3$ as the optimal scaling of $N u$ with $R a$ however the piecewise linear profiles are capturing $2 / 5$ instead. We note briefly that we have solved this problem without setting $b=2$ and have found that optimising the upper bound over the balance parameter $b$ does not alter the $2 / 5$ scaling of this upper bound result.

To conclude we would like to propose that future work on this problem should be 


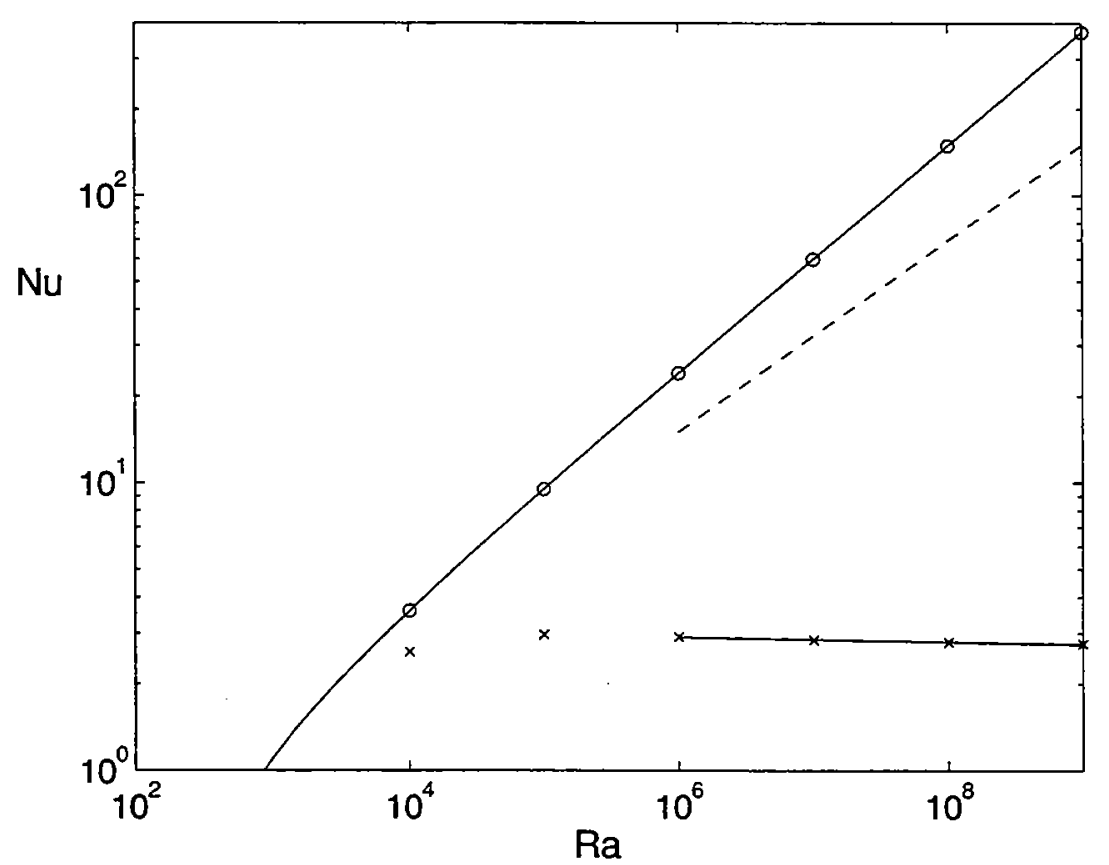

Figure 5: The numerical upper bound for the Background method with piecewise linear profile. The upper solid line is the upper bound calculated by Otero. Circles are the points calculated during this study. The dashed line is Chan's multi- $\alpha$ upper bound. The crosses are a lower bound on the optimal bound which was calculated by evaluating Chan's functional with the eigenfunctions associated to the critical wavenumbers. 


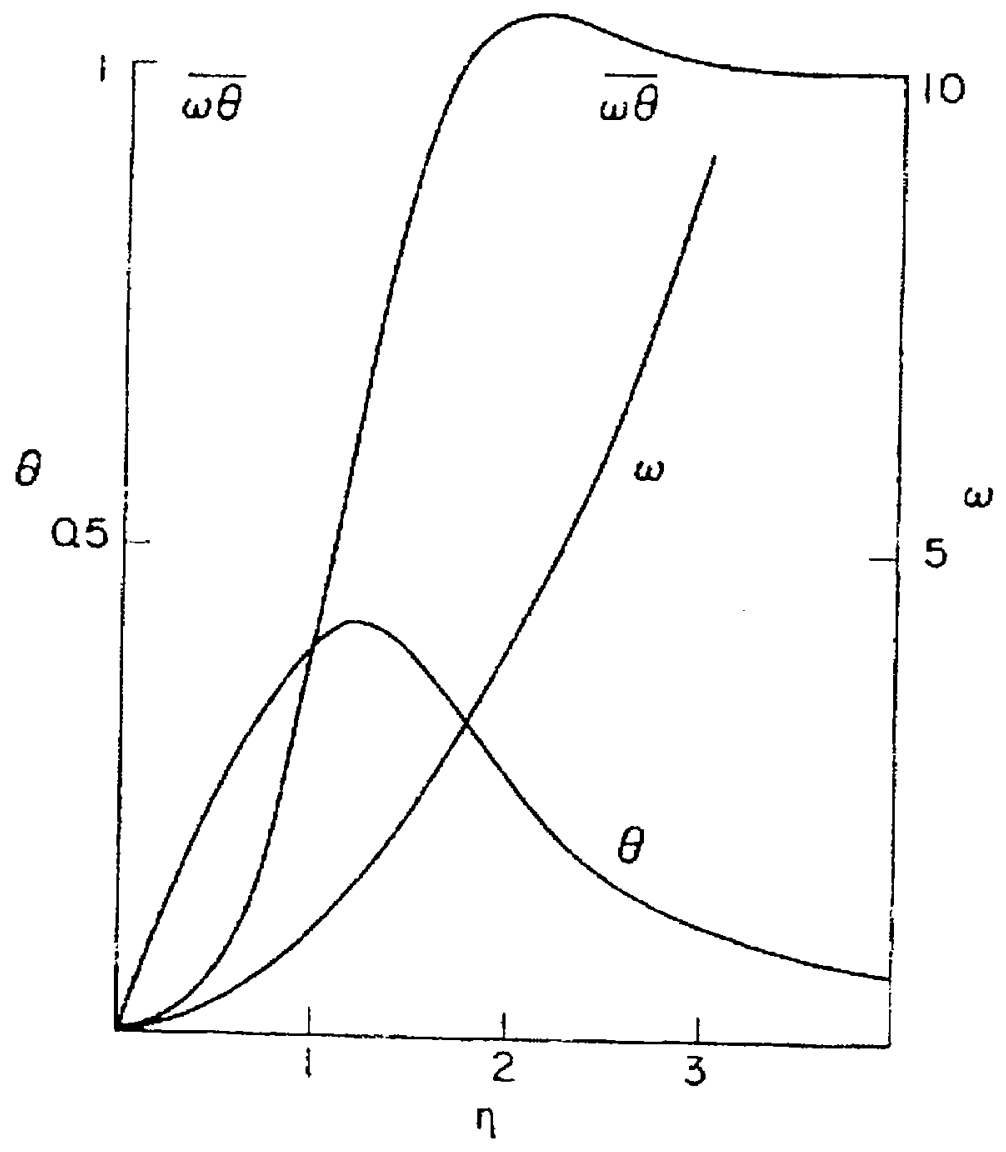

Figure 6: The $z$-structure of Chan's optimal solutions.

concerned with solving the full optimal Background problem, as done recently in [10] for the problem of plane Couette flow, or as an intermediate step, to study how more sophisticated test profiles, such as profiles with non-zero internal gradients, can improve the upper bound.

This work has been completed with the help of C. Doering, L. Howard, G. Ierley, R. Kerswell and J. Otero. I thank all of them for their insightful advice and their enthusiasm for this problem. I would also like to thank all of the students and staff of the Woods Hole program who together made the Summer an unforgettable experience. Finally I hope that the legendary GFD Dynamos softball team have many more triumphant years to come under the auspices of George Veronis.

\section{References}

[1] L. N. Howard, "Heat transport by turbulent convection," J. Fluid Mech. 17, 405 (1963).

[2] L. N. Howard, "Bounds on flow quantities," Annu. Rev. Fluid Mech. 4, 473 (1972). 


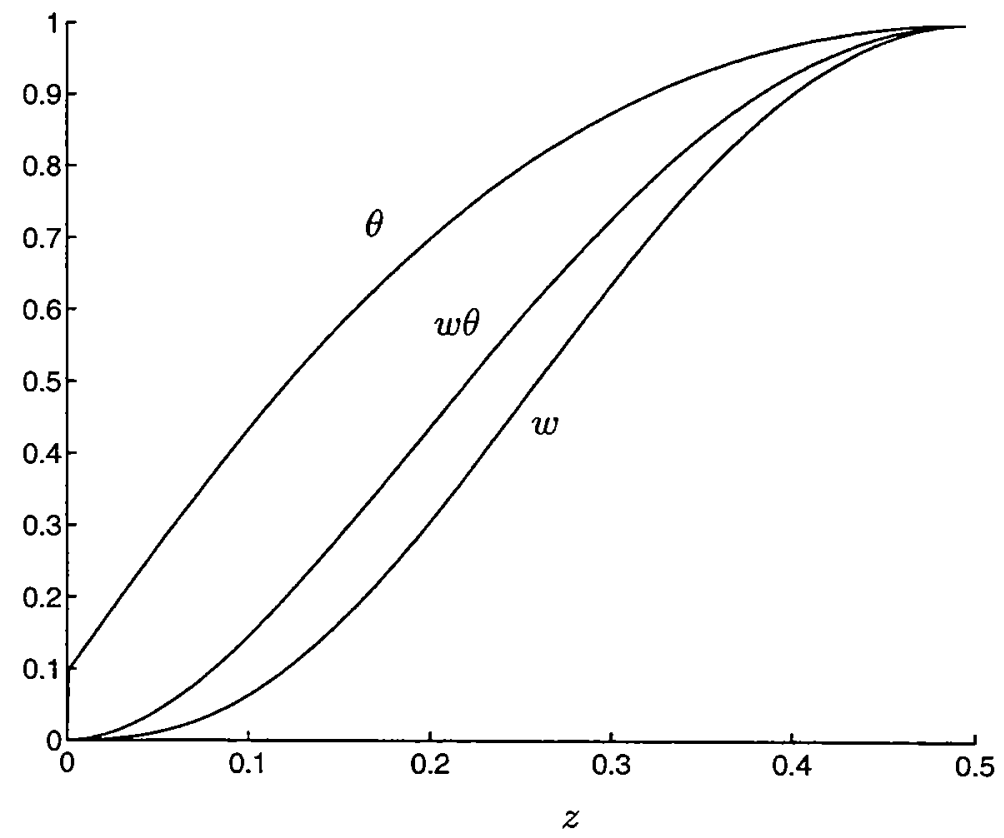

Figure 7: The normalised $z$-structure of the eigenfunctions associated with the critical wavenumber in the piecewise linear optimal upper bound. 
[3] F. H. Busse, "On Howard's upper bound for heat transport by turbulent convection," J. Fluid Mech. 37, 457 (1969).

[4] S.-K. Chan, "Infinite Prandtl Number Turbulent Convection," Stud. Appl. Math. 50, 13 (1971).

[5] C. R. Doering and P. Constantin, "Variational bounds on energy dissipation in incompressible flows. III. Convection," Phys: Rev. E 53, 5957 (1996).

[6] R. R. Kerswell, "New results in the variational approach to turbulent Boussinesq convection," Phys. Fluids 13, 192 (2001).

[7] C. R.Doering and P. Constantin, "On upper bounds for infinite Prandtl number convection with or without rotation," J. Math. Phys. 42, 784 (2001).

[8] P. Constantin and C. R. Doering, "Infinite Prandtl number convection," J. Statist. Phys. 94, 159 (1999).

[9] J. Otero, Ph.D. thesis, University of Michigan, 2002.

[10] S. C. Plasting and R. R. Kerswell, in press (unpublished). 
University of California, San Diego

SIO Library 0175C

9500 Gilman Drive

La Jolla, CA 92093-0175

Hancock Library of Biology \& Oceanography

Alan Hancock Laboratory

University of Southern California

University Park

Los Angeles, CA 90089-0371

Gifts \& Exchanges

Library

Bedford Institute of Oceanography

P.O. Box 1006

Dartmouth, NS, B2Y 4A2, CANADA

NOAA/EDIS Miami Library Center

4301 Rickenbacker Causeway

Miami, FL 33149

Research Library

U.S. Army Corps of Engineers

Waterways Experiment Station

3909 Halls Ferry Road

Vicksburg; MS 39180-6199

Marine Resources Information Center

Building E38-320

MIT

Cambridge, MA 02139

Library

Lamont-Doherty Geological Observatory

Columbia University

Palisades, NY 10964

Library

Serials Department

Oregon State University

Corvallis, OR 97331

Pell Marine Science Library

University of Rhode Island

Narragansett Bay Campus

Narragansett, RI 02882

Working Collection

Texas A\&M University

Dept. of Oceanography

College Station, TX 77843
Fisheries-Oceanography Library

151 Oceanography Teaching Bldg.

University of Washington

Seattle, WA 98195

Library

R.S.M.A.S.

University of Miami

4600 Rickenbacker Causeway

Miami, FL 33149

Maury Oceanographic Library

Naval Oceanographic Office

Building 1003 South

1002 Balch Blvd.

Stennis Space Center, MS, 39522-5001

Library

Institute of Ocean Sciences

P.O. Box 6000

Sidney, B.C. V8L 4B2

CANADA

National Oceanographic Library

Southampton Oceanography Centre

European Way

Southampton SO14 3ZH

UK

The Librarian

CSIRO Marine Laboratories

G.P.O. Box 1538

Hobart, Tasmania

AUSTRALIA 7001

Library

Proudman Oceanographic Laboratory

Bidston Observatory

Birkenhead

Merseyside L43 7 RA

UNITED KINGDOM

IFREMER

Centre de Brest

Service Documentation - Publications

BP 7029280 PLOUZANE

FRANCE 


\begin{tabular}{|c|c|c|}
\hline $\begin{array}{l}\text { REPORT DOCUMENTATION } \\
\text { PAGE }\end{array}$ & $\begin{array}{l}\text { 1. REPORT NO. } \\
\text { WHUI-2003-06 }\end{array}$ & 3. Recipient's Accession No. \\
\hline \multirow{2}{*}{\multicolumn{2}{|c|}{$\begin{array}{l}\text { 4. Title and Subtitle } \\
\text { Bounds on Turbulent Transport }\end{array}$}} & $\begin{array}{l}\text { 5. Report Date } \\
\text { July } 2003\end{array}$ \\
\hline & & 6. \\
\hline \multicolumn{2}{|c|}{$\begin{array}{l}\text { 7. Author(s) John A. Whitehead, Director; Friedrich Busse, Louis Howard, Charles Doering, } \\
\text { Peter Constantin, Colm-cille Caulfield, Richard Kerswell, Principal Lecturers }\end{array}$} & $\begin{array}{l}\text { 8. Pertorming Organization Rept. No. } \\
\text { WHOI-2003-06 }\end{array}$ \\
\hline \multirow{2}{*}{\multicolumn{2}{|c|}{$\begin{array}{l}\text { 9. Pertorming Organization Name and Address } \\
\text { Woods Hole Oceanographic Institution } \\
\text { Woods Hole, Massachusetts } 02543\end{array}$}} & 10. Project/Task/Work Unit No. \\
\hline & & $\begin{array}{l}\text { 11. Contract(C) or Grant(G) No. } \\
\text { (C) NOOO14-97-1-0934 } \\
\text { (G) OCE 98-10647 }\end{array}$ \\
\hline \multirow{2}{*}{\multicolumn{2}{|c|}{$\begin{array}{l}\text { 12. Sponsoring Organization Name and Address } \\
\text { Office of Naval Research }\end{array}$}} & $\begin{array}{l}\text { 13. Type of Report \& Period Covered } \\
\text { Technical Report }\end{array}$ \\
\hline & & 14. \\
\hline
\end{tabular}

This report should be cited as: Woods Hole Oceanog. Inst. Tech. Rept., WHOI-2003-06.

16. Abstract (Limit: 200 words)

The subject for the 2002 GFD programs was "Bounds on Turbulent Transport". This topic was introduced and reviewed in a series of ten lectures by six lecturers who constitute almost every contributor to this subject. The subject was introduced and foundations were laid by five lectures by F. H. Busse. In the middle of the first week, L. Howard reviewed his historical first approach to this subject and described more recent advances. Additional lectures by P. Constantine, R. Kerswell, C. Caulfield and C. Doering provided modern advances. We trust that the lecture notes will constitute a timely review of this promising subject. The following weeks had many highlights with approximately 40 additional lectures. The mini symposium on rotating convection in early July included presentations of experimental, ocean, atmospheric, and planetary observations. During the rest of the program, participants and visitors who have studied turbulence, convection, and instability in numerous geophysical situations with application to the ocean, the earth's atmosphere and planetary circulation made numerous contributions. The ten fellows reports were delivered during the last week of the program. Their projects involved original work that was both initiated and brought to some focus during the program. The lecture notes and fellows reports are listed at http://www.whoi.edu/education/gfd/proceedings.html.

17. Document Analysis a. Descriptors turbulent transport convection planetary observations

b. Identifiers/Open-Ended Terms

c. COSATI Field/Group

18. Availability Statement

Approved for public release; distribution unlimited.

\begin{tabular}{|l|l|}
\hline $\begin{array}{c}\text { 19. Security Class (This Report) } \\
\text { UNCLASSIFIED }\end{array}$ & $\begin{array}{l}\text { 21. No. of Pages } \\
317\end{array}$ \\
\hline 20. Security Class (This Page) & 22. Price \\
\hline & $\begin{array}{l}\text { OPTIONAL FORM 272 (4-77) } \\
\text { (Formerly NTIS-35) } \\
\text { Department of Commerce }\end{array}$ \\
\hline
\end{tabular}

